\title{
IntechOpen
}

\section{Energy Technology and Management}

\author{
Edited by Tauseef Aized
}

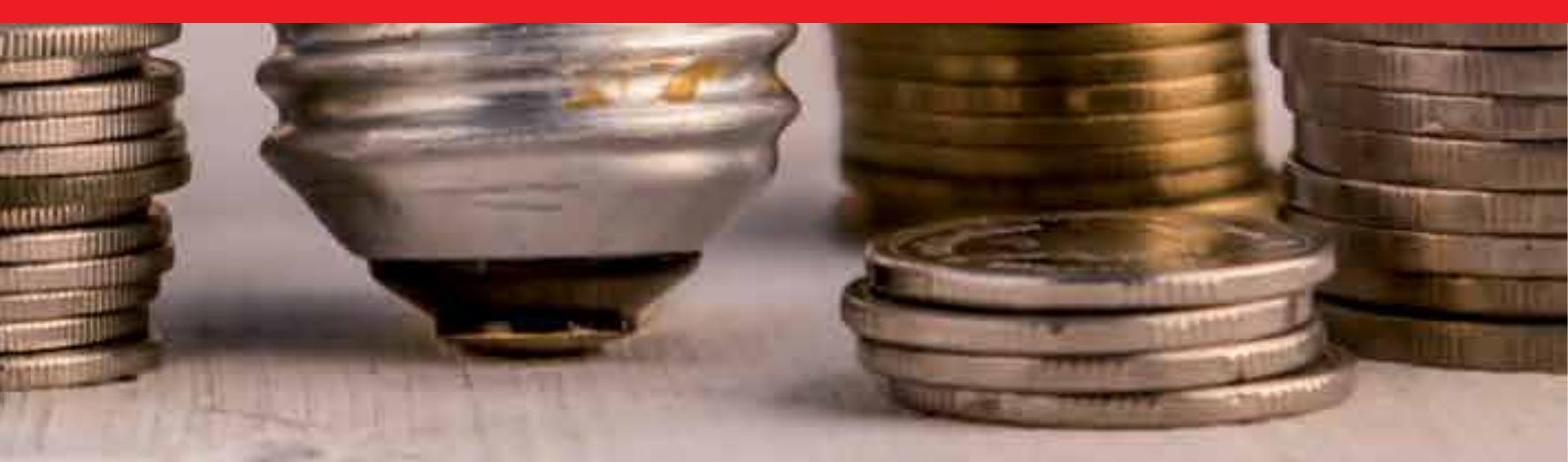





\section{ENERGY TECHNOLOGY AND MANAGEMENT}

Edited by Tauseef Aized 


\section{Energy Technology and Management}

http://dx.doi.org/10.5772/1819

Edited by Tauseef Aized

\section{Contributors}

Nattachote Rugthaicharoencheep, Somporn Sirisumrannukul, Behdad Kiani, Xiaodan Yu, Chen Huanfei, Liu Zhao, Jia Hongjie, Joseph Yakubu Oricha, Bei Gou, Weibiao Wu, Yi Xie, Rocky Kow-Chuen Chang, Xiapu Luo, Yuqiu Yang, Hiroyuki Hamada, Zoltán Mucsi, Péter Ábrányi-Balogh, Imre Csizmadia, Vasily Moshnyaga, Luca Andreassi, YoungMyoung Kim, Young-Woo Lee

\section{(c) The Editor(s) and the Author(s) 2011}

The moral rights of the and the author(s) have been asserted.

All rights to the book as a whole are reserved by INTECH. The book as a whole (compilation) cannot be reproduced, distributed or used for commercial or non-commercial purposes without INTECH's written permission.

Enquiries concerning the use of the book should be directed to INTECH rights and permissions department (permissions@intechopen.com).

Violations are liable to prosecution under the governing Copyright Law.

\section{(cc) BY}

Individual chapters of this publication are distributed under the terms of the Creative Commons Attribution 3.0 Unported License which permits commercial use, distribution and reproduction of the individual chapters, provided the original author(s) and source publication are appropriately acknowledged. If so indicated, certain images may not be included under the Creative Commons license. In such cases users will need to obtain permission from the license holder to reproduce the material. More details and guidelines concerning content reuse and adaptation can be foundat http://www.intechopen.com/copyright-policy.html.

\section{Notice}

Statements and opinions expressed in the chapters are these of the individual contributors and not necessarily those of the editors or publisher. No responsibility is accepted for the accuracy of information contained in the published chapters. The publisher assumes no responsibility for any damage or injury to persons or property arising out of the use of any materials, instructions, methods or ideas contained in the book.

First published in Croatia, 2011 by INTECH d.o.o.

eBook (PDF) Published by IN TECH d.o.o.

Place and year of publication of eBook (PDF): Rijeka, 2019.

IntechOpen is the global imprint of IN TECH d.o.o.

Printed in Croatia

Legal deposit, Croatia: National and University Library in Zagreb

Additional hard and PDF copies can be obtained from orders@intechopen.com

Energy Technology and Management

Edited by Tauseef Aized

p. cm.

ISBN 978-953-307-742-0

eBook (PDF) ISBN 978-953-51-6064-9 


\section{We are IntechOpen, \\ the world's leading publisher of Open Access books}

Built by scientists, for scientists

\section{$4,100+$}

Open access books available

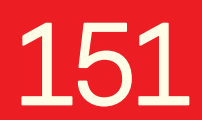

Countries delivered to
$116,000+$

International authors and editors
$120 \mathrm{M}+$

Downloads

Our authors are among the

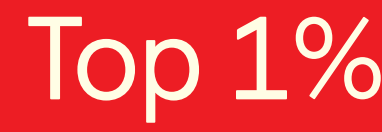

most cited scientists

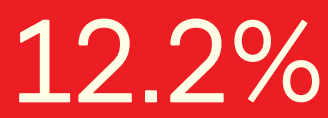

Contributors from top 500 universities

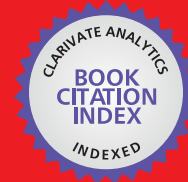

WEB OF SCIENCE ${ }^{\mathrm{TM}}$

Selection of our books indexed in the Book Citation Index in Web of Science ${ }^{\mathrm{TM}}$ Core Collection (BKCI)

Interested in publishing with us?

Contact book.department@intechopen.com

Numbers displayed above are based on latest data collected.

For more information visit www.intechopen.com

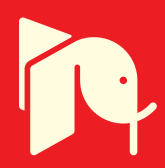





\section{Meet the editor}

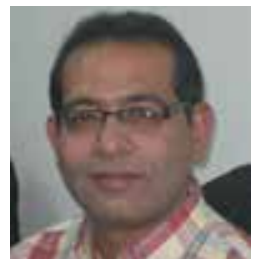

Dr. Tauseef Aized received his Ph.D. from Tokyo Institute of Technology. He held two research fellowships; first Endeavour Research Fellowship at Monash University and the second Commonwealth Fellowship at the University of Cambridge. He has a number of papers in reputed journals and conferences. His teaching and research interest include energy technology and policy, computer integrated manufacturing and operations management. Currently, he is a professor and chair of department of mechanical engineering, UET-KSK campus, Lahore-Pakistan. 



\section{Contents}

Preface XI

Part 1 Energy Technology 1

Chapter 1 Centralizing the Power Saving Mode for $\mathbf{8 0 2 . 1 1}$ Infrastructure Networks 3

Yi Xie, Xiapu Luo and Rocky K. C. Chang

Chapter 2 A Study on Design of Fiber-Reinforced Plastic (FRP) Tubes as Energy Absorption Element in Vehicles 25

Yuqiu Yang and Hiroyuki Hamada

Chapter 3 Optimal Feeder Reconfiguration with Distributed

Generation in Three-Phase Distribution System by

Fuzzy Multiobjective and Tabu Search $\mathbf{5 9}$

Nattachote Rugthaicharoencheep and Somporn Sirisumranukul

Chapter 4 Energy Managements in the Chemical and Biochemical World, as It may be Understood from the Systems Chemistry Point of View 79

Zoltán Mucsi, Péter Ábrányi Balogh, Béla Viskolcz

and Imre G. Csizmadia

Chapter 5 Energy Planning for Distributed Generation Energy System:

The Optimization Work 111

Behdad Kiani

Chapter 6 Network Reconfiguration for Distribution System with Micro-Grid 125

Yu Xiaodan, Chen Huanfei, Liu Zhao and Jia Hongjie

Chapter 7 A Camera-Based Energy Management

of Computer Displays and TV Sets 137

Vasily G. Moshnyaga 
Chapter 8 Enhancement of Power System State Estimation 157

Bei Gou and Weibiao Wu

Chapter 9 Smart Home Services for a Smart Grid 171

Young-Myoung Kim and Young-Woo Lee

Part 2 Energy Management 185

Chapter 10 Management Crisis in Partial Deregulation of Energy Sector and Modeling the Technical and Economic Results of Organizational Management Structure 187

Joseph Yakubu Oricha

Chapter 11 Methodology Development for a Comprehensive and Cost-Effective Energy Management in Public Administrations 201

Capobianchi Simona, Andreassi Luca, Introna Vito, Martini Fabrizio and Ubertini Stefano 


\section{Preface}

Energy is one of the most important issue of modern civilization. All material developments are strongly linked with energy availability and efficient utilization. Unfortunately, energy resources are not unlimited, especially conventional energy resources are depleting at an enormous pace. Hence, efficient utilization of available resources and development of new energy resources are extremely important in order to maintain material development of human civilization. Energy management, saving and efficient utilization are important in the backdrop of current energy shortfalls. Additionally, energy studies have a wider scope than merely concentrating on technological issues of energy resource development and also include energy policy and planning issues.

This book is compiled to address both technology and policy issues and presents a collection of articles from experts belonging to different parts of the world. The articles range from policy to technological issues of energy development and efficient utilization. In order to comprehend this book, some background of energy related issues is required. Students, researchers, academics, policy makers and practitioners may get benefit from this book.

Prof. Tauseef Aized

University of Engineering and Technology (UET)

Lahore

Pakistan 



\section{Part 1}

Energy Technology 



\title{
Centralizing the Power Saving Mode for 802.11 Infrastructure Networks
}

\author{
Yi Xie ${ }^{1}$, Xiapu Luo ${ }^{2}$ and Rocky K. C. Chang ${ }^{2}$ \\ ${ }^{1}$ Department of Computer Science, Xiamen University \\ ${ }^{2}$ Department of Computing, The Hong Kong Polytechnic University \\ ${ }^{1}$ China \\ ${ }^{2}$ Hong Kong, SAR China
}

\section{Introduction}

With the rapid development of wireless networks, efficient energy management for wireless LAN (WLAN) has become an important problem, because mobile devices' availability is determined by their stringent batteries power. Quite a few sources of energy consumption have been identified (Narseo et al., 2010), among which the wireless communication component uses up a significant amount of energy. For instance, the Motorola Droid phone consumes around $200 \mathrm{~mW}$ with the backlight off, close to $400 \mathrm{~mW}$ with the backlight on, and over $800 \mathrm{~mW}$ when the Wi-Fi radio is active (Zeng et al., 2011). This chapter focuses on improving the energy efficiency of wireless communication component, because they may consume up to $50 \%$ of the total energy.

Various mechanisms have been proposed to balance between communication quality and energy consumption for wireless devices, for example, power saving mode (PSM) that puts an idle client into a low-power mode (Gast, 2005), transmission power control (Nuggehalli et al., 2002), packet transmission scheduling (Qiao et al., 2003; Tarello et al., 2005), and some cross-layer methods (Anastasi et al., 2007). They investigate the trade-off between energy consumption and throughput (Gao et al., 2010; Zhang \& Chanson, 2003), delay (Guha et al., 2010; Nuggehalli et al., 2002; 2006), or network utility (Chiang \& Bell, 2004). In this chapter, we propose a centralized PSM (C-PSM), an AP-centric deployment of the IEEE 802.11 PSM, to optimize power saving and multiple performance metrics for infrastructure networks which are widely deployed in enterprise, campus, and metropolitan networks. In these networks, wireless clients (e.g., laptops, PDAs and mobile phones) using the IEEE 802.11 infrastructure mode connect to the Internet through an access point (AP). The experiment results show that significant improvements can be obtained from the new deployment of C-PSM.

The IEEE 802.11 PSM, widely used in WLAN, allows an idle client to go into a sleep mode. Hereafter, we use PSM to refer to the IEEE 802.11 PSM. The clients save energy by sleeping while wakes up periodically to receive beacon frames from AP. The beacon frame, sent by an access point (AP) every beacon interval (BI), indicates whether clients have frames buffered at the AP. Each client's wake-up frequency is determined by a PSM parameter listen interval (LI). Both BI and LI are configurable, and their settings directly influence the PSM's performance shown by the analysis of section 4 . Unfortunately, the protocol does not prescribe how the BI 
and LI should be configured in PSM; therefore, default values are often used. Obviously, the PSM using default settings cannot adapt to the traffic and configuration dynamics inherent in typical wireless networks. Worse yet, the PSM was reported to have adverse impact on application performance, such as short TCP connections (Krashinsky \& Balakrishnan, 2005). To address these shortcomings, a number of new power-saving schemes that put idle clients into sleep have been proposed. A class of them (e.g., (Nath et al., 2004); (Qiao \& Shin, 2005); (Krashinsky \& Balakrishnan, 2005)) enables each client to save energy by reducing the number of unnecessary wake-ups ( i.e., design an optimal wake-up schedule). These user-centric schemes, however, do not address energy consumption due to channel contention which, as we will show in section 3 , is another major source of energy wastage. Another class adopts an AP-centric approach which exploits AP to improve the energy efficiency of all clients in the network. Within this class, some schemes design a packet transmission schedule to minimize channel contention (e.g., (Lin et al., 2006); (He et al., 2007); (Zeng et al., 2011)). Others redesign beacon frame and poll clients one by one, which totally avoids channel contention (Lee et al., 2006). However, most AP-centric schemes are not compatible with the standard PSM scheme or difficult to implement, because they employ precise transmission schedule.

Unlike the previous works on the power saving mode (PSM), our C-PSM optimizes the beacon interval, listen interval, minimal congestion window, and sequence of first wake-up time for each device according to the traffic characteristics. Firstly, the AP chooses the optimal BI and LIs for clients based on the pattern of arriving packets to reduce energy consumption due to both unnecessary wake-ups and channel contentions. Especially, the energy wasted in channel contentions could be very significant, because all clients involved cannot go to sleep throughout the contention period which could be very long. Secondly, the AP assigns congestion windows to the clients which are involved in collisions, such that a client that wakes up less frequently will be able to retransmit earlier. Finally, C-PSM provides an additional wakeup schedule to further reduce simultaneous wakeups of clients.

Having the AP control the PSM parameters, C-PSM is therefore able to maximize the total energy efficiency for all clients and facilitates various aspects of network management and operations. Our extensive simulation results show that the C-PSM is very promising under four traditional distributions of inter-frame arrival times: deterministic, uniform, exponential, and Pareto. For example, under exponential traffic, the C-PSM can reduce power consumption by at least $50 \%$ compared with the standard PSM (S-PSM). At the same time, C-PSM also decreases the frame buffering delay at the AP by 30\%. The wake-up schedule can further save the energy consumption by another $22 \%$. Moreover, the C-PSM's advantage of energy saving is robust in a wide range of operational scenarios. For example, the C-PSM's energy saving remains effective for a large number of clients, heavier network workloads, and other configuration settings. In contrast, a client randomly selecting PSM parameters cannot obtain any long-term benefit in terms of performance or energy consumption.

Our C-PSM is different from other AP-centric schemes in three important aspects. First, C-PSM conforms to PSM, whereas other AP-centric schemes, such as (Belghith et al., 2007), do not. The only additional mechanism required for C-PSM is to notify the clients of their optimal LIs which could be accomplished through the beacon transmission channel. Second, C-PSM does not rely on computational-expensive packet scheduling which is employed in (He et al., 2007); (Lin et al., 2006); (Lee et al., 2006). Instead, the AP in C-PSM simply observes the statistics of the packet arrival patterns. Third, C-PSM is designed independent of the upper-layer protocols. Therefore, it could be used for any mix of network protocols. However, some AP-centric schemes, such as (Anastasi et al., 2004), are designed only for TCP traffic. 
The rest of this chapter is organized as following. In section 2, we summarize previous energy-saving schemes for IEEE802.11 infrastructure networks. The system models and a PSM simulator are described in section 3. We motivate C-PSM by discussing the impacts of $\mathrm{BI}$ and LIs on energy efficiency and other performance metrics in section 4 . Next, section 5 presents the design of C-PSM, and section 6 evaluates the performance of C-PSM based on extensive simulation experiments. The results lend a strong support to the efficiency of C-PSM. For example, compared with PSM, C-PSM reduces significantly more energy (up to $76 \%$ ), achieves higher energy efficiency (up to $320 \%$ ), and reducing AP buffering delay (up to $88 \%$ ). The results also show that the improvements of C-PSM over S-PSM mainly depend on the wake-up energy consumption and the ratio of idle power to sleep power. Finally, section 7 concludes this chapter with future work.

\section{Related work}

Several enhancements adopt a user-centric approach to let each client determine when it will sleep and wake up. For example, Nath et al. (Nath et al., 2004) proposed a dynamic wake-up period in which each client chooses its LI according to the round-trip time of its current TCP connection. The Bounded Slowdown Protocol (Krashinsky \& Balakrishnan, 2005), another user-centric method, allows a client to increase its LI when the period of idleness increases. In Smart PSM (Qiao \& Shin, 2005), each client determines whether it will enter into the PSM depending on the traffic condition. After the client enters into the PSM, the LI can be dynamically adjusted. Although the user-centric methods are quite effective in reducing a client's energy, they do not address the power consumption due to channel contention. Moreover, it is not clear whether these schemes remain effective when some other clients do not employ them.

An AP-centric approach, on the other hand, lets the AP deploy the PSM operations. The power-saving management proposed in (Anastasi et al., 2004) saves a client's energy by extending its sleep period and reducing unnecessary wakeups when the AP and the single client communicate using Indirect-TCP. Most AP-centric schemes support multiple clients, and try to totally eliminate channel contention. For example, the wake-up schedule proposed in (Lin et al., 2006) redesigns the TIM to let only one client to retrieve its buffered frame. The AP in the scheduled PSM (He et al., 2007) assigns slices of a BI for the clients' buffered frames. The scheme proposed in (Lee et al., 2006) computes an optimal BI and design an energy-efficient scheduler for frame transmissions within one BI. Network-Assisted Power Management (NAPman) (Rozner \& Navda, 2010) for WiFi devices leverages AP virtualization and uses a new energy-aware fair scheduling algorithm to minimize client energy consumption and unnecessary retransmissions. It is also helpful of ensuring fairness among competing traffic. Although these schemes generally perform well, their computation-intensive scheduling algorithms introduce high cost. In contrast, our C-PSM computes optimal BI and LIs jointly to reduce unnecessary wake-ups and channel contention, and optionally uses a wake-up schedule to further decrease the energy consumption.

Other powers-saving schemes based on sleeping even abandon the frame retrieving process of PSM. In the Once Poll PSM (Belghith et al., 2007), the frames buffered at the AP are forwarded upon the reception of a single PS-Poll. In the PSM-throttling (Tan et al., 2007), which does not use beacon frames, a wireless client wakes up at the beginning of each traffic burst, because it can identify bandwidth throttling connections and reshape the TCP traffic into periodic bursts. A power-saving multi-channel MAC protocol (PSM-MMAC) (Wang et al., 2006) was 
designed to reduce the collision probability and the waiting time in the 'awake' state of a node. The medium access probability is optimized by estimating the number of active links, queue lengths and channel conditions, such that PSM-MMAC results in improved throughput, delay performance, and energy efficiency.

\section{Models and notations}

\subsection{The IEEE802.11 PSM}

The PSM allows a wireless client to sleep instead of staying in active state all the time and asks the AP to buffer the frames for them. Let the AP's BI be $\beta$ millisecond (ms). The AP broadcasts a beacon frame every $\beta$ ms to announce the buffer status of all PSM-enabled clients in the Traffic Indication Map (TIM) that uses one bit to indicate empty or nonempty buffer for each client. On the other hand, each PSM-enabled client's LI is a multiple of BI; therefore, the BI actually determines the LI's granularity. Let the value of LI be $\gamma \times \beta \mathrm{ms}$, where $\gamma \geq 1$. For the PSM, the default settings are $\beta=100 \mathrm{~ms}$ and $\gamma=1$.

Figure 1 (Gast, 2005) illustrates the PSM operation for two wireless clients $s_{1}$ and $s_{2} . s_{1}$ has a LI of 2 while $s_{2}$ has a LI of 3 . The first (second) bit in the TIM indicates the buffer status for client $s_{1}\left(s_{2}\right)$. The shaded region shows that the client is in the active state. After waking up for the first time, $s_{1}$ is notified of its frames being buffered at the AP through the TIM. The client then sends a PS-Poll frame to retrieve the first frame. If the More Data bit in the received frame is not set, it will return to sleep; otherwise, it will send another PS-Poll frame. The same data exchange repeats until all buffered frames are sent and then the client goes to sleep. However, if a client wants to send data, it may wake up any time to transmit them. We will come back to this figure in section 4 to analyze the energy consumption sources.

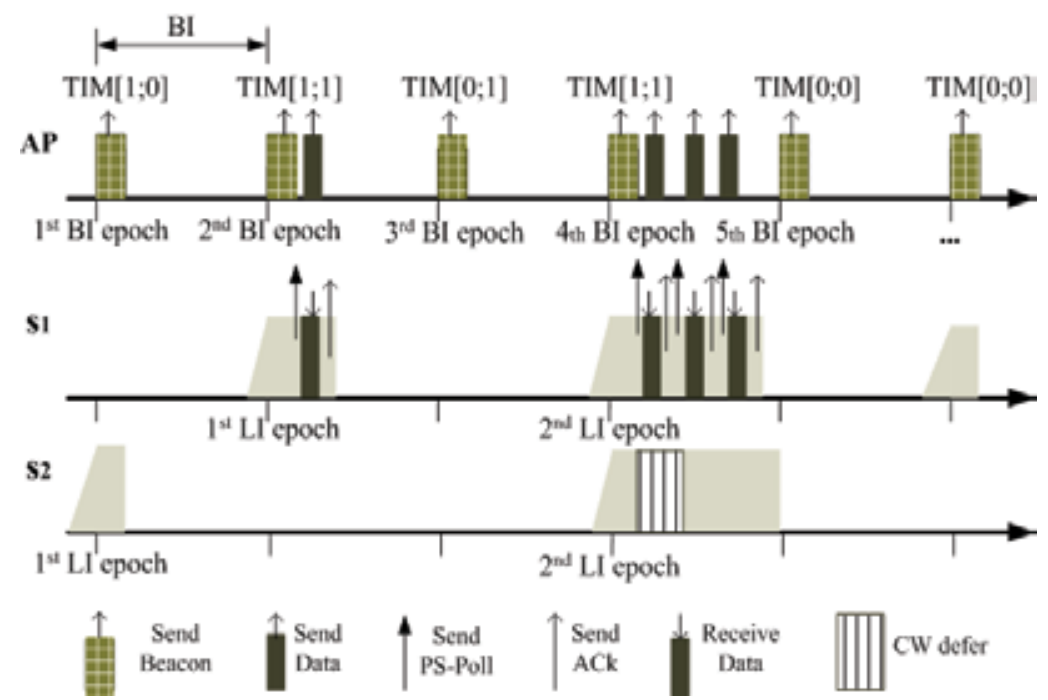

Fig. 1. An example of the PSM operation for two wireless clients.

\subsection{System model}

Our model consists of an AP and $c$ PSM-enabled wireless clients $s_{j}, j=1, \ldots, c$, which run on IEEE $802.11 \mathrm{~b}$ with a transmission rate of $11 \mathrm{Mps}$. We have chosen IEEE $802.11 \mathrm{~b}$ over 
IEEE $802.11 \mathrm{a} / \mathrm{g}$ for its less complex protocols. Moreover, IEEE $802.11 \mathrm{a} / \mathrm{g}$ 's rate adaptation algorithms will make the analysis much more difficult. The model considers only downlink traffic (i.e., those from the AP to clients). We do not consider the uplink traffic, because the PSM is effective only for downlink-dominant communication patterns. Furthermore, we model the traffic arriving at the AP from different sources, which are affected by applications, upper-layer protocols, and network path properties.

There are two random variables in our models: inter-frame arrival time and frame size. Let $T_{j}$ be the inter-frame arrival time for $s_{j}$ 's traffic source and the mean of $T_{j}$ be $\delta_{j}$. Let the vector of the client's mean inter-frame arrival times be $\Delta=\left[\delta_{1}, \ldots, \delta_{c}\right]$. Our current study allows $T_{j}$ to take on four types of distributions: deterministic (DET), uniform (UNI), exponential (EXP), and Pareto (PAR). On the other hand, the frame size distribution is either deterministic and uniform. Besides $\beta$, the AP in C-PSM can also configure the LIs for all clients $\left(\Gamma=\left[\gamma_{1}, \ldots, \gamma_{c}\right]\right)$ and the minimal congestion windows for all clients $\left(\Theta=\left[\theta_{1}, \ldots, \theta_{c}\right]\right)$.

\subsection{A simulator}

We have adopted simulation as the major tool to study the problem, because simulation can capture many fine details than analytical models. Unfortunately, many publicly available simulators, such as J-SIM (Tyan, 2002), have not implemented most operations for infrastructure networks and the PSM, such as the beacon frames and PS-Poll frames. Even for the de facto simulator ns-2 (Berkeley et al., 1996), it is surprising that its PSM module (Krashinsky \& Balakrishnan, 2005) supports only a single client. This prompted us to write our own simulator using MATLAB which provides an easy-to-use language and other supporting facilities to model the MAC sublayer accurately and effectively. There are also other MATLAB-based IEEE802.11 simulators, such as for IEEE802.11a (MATLAB Central, 2003) and the PHY layer of IEEE802.11b (MATLAB Central, 2009).

Our simulator implements the details of the IEEE 802.11b DCF with PSM, including the PS-Poll, beacon frames (with TIM), backoff algorithm, and congestion window. But we have excluded other nonessential elements (e.g., authentication and (de)association) and the RTS/CTS mechanism which is often turned off to increase the performance. We have used the simulation parameters in Table 1 for the experiments conducted in this chapter ${ }^{1}$. The values of power and energy consumption, which belong to the power consumption model $\mathcal{A}$ (shown in Table 10), have been widely used (Feeney \& Nilsson, 2001; Margi, 2006) ${ }^{2}$. We will explain why the simulator selects these values in subsection 6.4.

The simulator produces detailed trace files which record frame exchanges, channel collisions, and clients' mode transitions. By carefully analyzing the trace files, we have validated the correctness of the PSM simulation. Moreover, we have obtained the following performance metrics from the trace files:

1. $P$ : the total power consumed by the clients by watt (W).

2. $T$ : the total client throughput by bits per second (bps).

3. $R_{T / P}: \frac{T}{P}$, the total energy efficiency metric by bits per joule (bpJ).

\footnotetext{
${ }^{1}$ The simulator uses only the long preamble, $192 \mu$ s. The transmission time of each frame is therefore equal to $192+\frac{\text { size } * 8}{\text { transmission rate }} \mu s$.

2 The data for LUCENT IEEE 802.11 WAVELAN PC card were provided by the manufacturer and evaluated in (Feeney \& Nilsson, 2001).
} 


\begin{tabular}{|l|l|}
\hline Simulation parameters & Values \\
\hline Number of clients & 1 to 20 \\
Data transmission rate (DTR) & $11 \mathrm{Mbps}$ \\
Basic transmission rate (BTR) & $2 \mathrm{Mbps}$ \\
\hline Data frame size (DFS) & 512 bytes \\
Beacon frame size (BFS) & 28 bytes \\
PS-Poll frame size (PFS) & 14 bytes \\
ACK frame size (AFS) & 14 bytes \\
\hline Transmission power & $1.4 \mathrm{~W}$ \\
Reception power & $0.9 \mathrm{~W}$ \\
Idle power & $0.7 \mathrm{~W}$ \\
Sleeping power & $0.060 \mathrm{~W}$ \\
Wake-up energy & $0.003 \mathrm{~J}$ \\
\hline slotTime & $20 \mu \mathrm{s}$ \\
SIFS & $10 \mu \mathrm{s}$ \\
DIFS & $50 \mu \mathrm{s}$ \\
\hline
\end{tabular}

Table 1. Simulation parameters used in this chapter.

4. $R_{c / t}: \frac{N_{c}}{N_{t}}$, where $N_{t}$ is the total number of transmission attempts by AP and all clients, and $N_{c}$ is the total number of collided frames by AP and all clients.

5. $R_{u / w}: \frac{N_{u}}{N_{w}}$, where $N_{w}$ is the total number of wake-ups by all clients, and $N_{u}$ is the total number of unnecessary wake-ups by all clients.

6. $R_{b B / B, k}: \frac{N_{b B, k}}{N_{B}}$, where $N_{B}$ is the total number of BIs, and $N_{b B, k}$ is the total number of BIs in which $k$ clients are involved in channel contention, $k \geq 2$.

7. $d_{j}$ : the frame buffering delay of $s_{j}$ 's frames at the AP by ms.

We have simulated for $c=2, \ldots, 20$ in an increment of two. Each experiment was run for at least 20 seconds in simulation time after observing the time of convergence from several preliminary experiments. we have repeated for each simulation setting for 20 times and report their average values. All the results reported in the paper fall within a $95 \%$ confidence level.

\section{A preliminary analysis}

To motivate the design of C-PSM, we first analyze the impact of the BI and that of the LIs on the energy consumption of two wireless clients. There are two main sources of energy wastage: unnecessary wake-ups and channel contention. Clearly, the individual LI has a direct impact on the number of unnecessary wake-ups; an overly-frequent wake-ups will consume a significant amount of energy. For example, as shown in Figure 1, s2 wakes up at the first epoch but finds no frames buffered for it.

Energy wastage due to channel contention, on the other hand, is more complicated. Back to Figure 1 again, $s_{2}$ wakes up the second time to find the frames buffered at the AP. However, it loses to $s_{1}$ after contending for the channel during the PS-Poll transmissions. Client $s_{2}$ then stays in the active mode during the backoff process which could take a long time. Therefore, rescheduling the active clients' wake-ups to nonoverlapping epoches will reduce such energy wastage. Note that reducing the BI value will also help, because, as mentioned before, the BI value determines the LI's granularity. Besides, the clients' congestion windows will also contribute to this energy consumption source. 


\subsection{Evaluating the impact of beacon and listen intervals}

Since Nedevschi et al. (2008) has shown that the sleeping mechanism is valuable when the network utilization $(\rho)$ is less than $30 \%$, we select $\Delta$ to let the network be lightly loaded. More precisely, we consider only the $\Delta$ s that satisfy

$$
\begin{aligned}
\rho & =b_{\min } \sum_{j=1}^{c} 1 / \delta_{j}<30 \%, \text { where } \\
b_{\min } & =\frac{D F S}{D T R}+\frac{P F S+A F S}{B T R}+D I F S+2 \text { SIFS. }
\end{aligned}
$$

where $b_{\min }$ is the minimum transmission time for one data frame (i.e., without using PSM or suffering from channel contention). According to the DCF and Table $1, b_{\min }$ is around $1.13 \mathrm{~ms}$ when $c=2$ and $\Delta=[15 ; 25] \mathrm{ms}$ (i.e., $\rho \approx 12 \%<30 \%$ ).

Impact of BI We investigate the impact of $\beta$ when it changes from $10 \mathrm{~ms}$ to $200 \mathrm{~ms}$ with the default PSM settings: $\Gamma=[1 ; 1]$ and $\Theta=[31 ; 31]$. As shown in Figure 2(a), $P$ is high when $\beta$ is too small, because much energy is wasted on clients' frequent wake-ups. When $\beta$ is too large, many frames are accumulated at the AP. Consequently, energy is wasted on channel contention. The optimal $\beta$ in this example is $50 \mathrm{~ms}$, instead of the default value of $100 \mathrm{~ms}$. Moreover, since $R_{T / P}$ is inversely proportional to $P$, Figure 2(b) shows a similar trend as Figure 2(a).

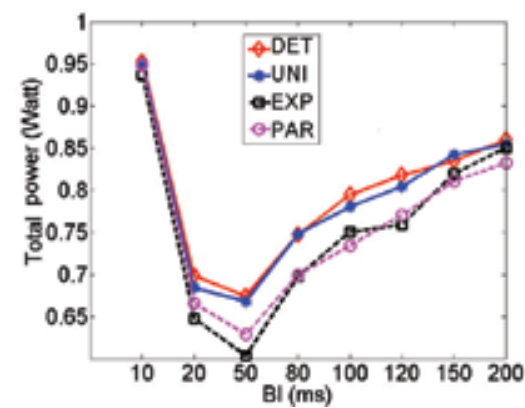

(a) $P$ verses $\beta, \gamma_{1}=\gamma_{2}=1$.

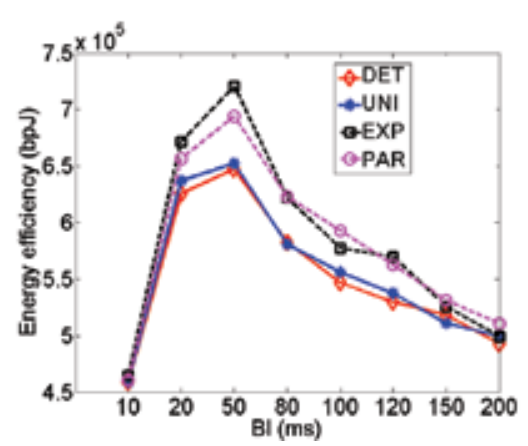

(b) $R_{T / P}$ verses $\beta, \gamma_{1}=\gamma_{2}=1$.

Fig. 2. Impact of BI for $c=2$ and $\Delta=[15 ; 25] \mathrm{ms}$.

Impact of LIs $\Gamma$ can also influence the clients' energy consumption and communication performance. Lei \& Nilsson (2007) obtained optimal LIs to maximize energy efficiency based on $\mathrm{M} / \mathrm{G} / 1$ and $\mathrm{D} / \mathrm{G} / 1$ queuing models. However, the assumptions used in these models generally do not hold for the PSM. In our simulation study, we fix $\beta$ to 50ms and consider different $\Gamma$ s for two clients. Table 2 for the EXP distribution clearly shows that the default case of $[1 ; 1]$ is not optimal for the six performance metrics. The best value for each metric is underlined. Overall, the case of $[1 ; 2]$ achieves the best performance. It is worth noting that $\frac{\gamma_{1}}{\gamma_{2}}$ is closest to $\frac{\delta_{1}}{\delta_{2}}$ for $\Gamma=[1 ; 2]$. We have observed similar results for the other three inter-frame arrival time distributions. 


\begin{tabular}{|l|l|l|l|l|l|l|l|}
\hline$\Gamma$ & $R_{c / t}$ & $R_{u / w}$ & $R_{b B / B, 2}$ & $P(\mathrm{~W})$ & $R_{T / P}\left(10^{5} \mathrm{bpJ}\right)$ & $d_{1}(\mathrm{~ms})$ & $d_{2}(\mathrm{~ms})$ \\
\hline$[1 ; 1]$ & $1.54 \%$ & $11.51 \%$ & $81.37 \%$ & 0.6109 & 7.1578 & 37.4 & 32.3 \\
{$[1 ; 2]$} & $1.04 \%$ & $4.97 \%$ & $\frac{42.32 \%}{0.5487}$ & $\frac{0.9674}{25}$ & $\underline{29.8}$ & 60.0 \\
{$[2 ; 1]$} & $1.07 \%$ & $12.24 \%$ & $46.79 \%$ & 0.6032 & 7.2316 & 81.0 & 28.0 \\
{$[2 ; 2]$} & $1.25 \%$ & $\underline{1.67 \%}$ & $49.16 \%$ & 0.7470 & 5.8260 & 125.4 & 61.3 \\
\hline
\end{tabular}

Table 2. Simulation results for different $\Gamma$ s under EXP inter-frame arrival distribution for $c=2, \beta=50 \mathrm{~ms}$, and $\Delta=[15 ; 25] \mathrm{ms}$.

\section{Centralized PSM}

\subsection{The main algorithm}

This section presents the centralized PSM (C-PSM) scheme that allows the AP to determine and deploy optimal PSM settings for itself and all clients. The AP first decides optimal $\beta$ (denoted by $\beta^{*}$ ) and optimal $\Gamma$ (denoted by $\Gamma^{*}$ ) based on the client's frame arrival patterns. These optimal settings are expected to bring significant improvement to the energy efficiency, because the intervals are selected to reduce the number of unnecessary wake-ups and channel contention. The AP also obtains a $\Theta$ (denoted by $\Theta^{*}$ ) to ensure that any client will not be denied channel access for too long.

The inputs to the main algorithm include $\Delta, \beta_{\min }, \epsilon_{\beta}$, and $\epsilon_{\Theta} . \beta_{\min }$ is a lower bound of $\beta^{*}$, and $\epsilon_{\beta}$ and $\epsilon_{\Theta}$ are the step sizes for searching $\beta^{*}$ and $\Theta^{*}$, respectively. The algorithm executes the following steps to yield $\beta^{*}, \Gamma^{*}$, and $\Theta^{*}$.

Step 1 (Determining the candidates of $\beta^{*}$ and $\Gamma^{*}$ ) The purpose of this step is to obtain a number of $\beta^{*}$ and $\Gamma^{*}$ candidates for the second step.

We first consider a $\Gamma^{*}$ candidate: $\left[L_{1} ; \ldots ; L_{c}\right]$. Let $L_{j}=\alpha_{j} \times \delta_{j}$, where $\alpha_{j} \geq 1$, is an integer scaling factor. To reduce unnecessary wake-up, the probability that an awaken client finds an empty buffer at the AP (denoted by $P r_{0}$ ) should be less than a given threshold $0<\xi \leq 1$. The choice of this threshold reflects the tradeoff between the number of unnecessary wake-ups and the period of channel contention. If $\xi$ is too large, the LI may be short and a lot of unnecessary wake-ups will occur. If $\xi$ is too small, the frames buffered during the long LI may cause channel contention. After running a number of empirical simulations, we let $\xi=0.05$. Then, $\alpha_{j}$ is the smallest integer which satisfies $P r_{0} \leq 0.05$. Table 3 shows examples of determining $\alpha_{j}$ under the four inter-frame arrival time distributions. For example, for the EXP distribution, $L_{j}$ is three times of $\delta_{j}$.

To determine the $\beta^{*}$ candidates, following the guideline in (Nath et al., 2004), we set $\beta_{\text {min }}$ to $10 \mathrm{~ms}$. To determine the upper bound of $\beta$, we note that BI should not be larger than any client's LI. Therefore, the upper bound of $\beta$ is given by $\min _{\forall j} L_{j}$. We then select $n+1$ BI candidates uniformly within the range of $\left[\beta_{\min }, \min _{\forall j} L_{j}\right]$, where $n=\left\lfloor\left(\min _{\forall j} L_{j}-\beta_{\min }\right) / \epsilon_{\beta}\right\rfloor$. Moreover, for each $\beta^{*}$ candidate $\beta_{i}$, we consider three $\Gamma^{*}$ candidates:

1. $\Gamma_{i, 1}=\left[\left\lceil L_{1} / \beta_{i}\right\rceil ; \cdots ;\left\lceil L_{c} / \beta_{i}\right\rceil\right]$,

2. $\Gamma_{i, 2}=\left[\left\langle L_{1} / \beta_{i}\right\rangle ; \cdots ;\left\langle L_{c} / \beta_{i}\right\rangle\right]$, where $\langle x\rangle$ gives the round-off value of a real number $x$, and

3. $\Gamma_{i, 3}=\left[\left\lfloor L_{1} / \beta_{i}\right\rfloor ; \cdots ;\left\lfloor L_{c} / \beta_{i}\right\rfloor\right]$.

Step 2 (Determining $\beta^{*}$ and $\Gamma^{*}$ ) The purpose of this step is to obtain the best $\beta^{*}$ and $\Gamma^{*}$ from a set of candidates identified in Step 1. The criterion is based on minimizing the number of simultaneous wake-ups. There are two sub-steps to achieving the goal.

In the first sub-step, we search for the best $\Gamma$ for each $\beta^{*}$ candidate obtained in Step 1 . That is, for a given $\beta_{i}$ obtained in Step 1, we select the best $\Gamma$ from $\Gamma_{i, 1}, \Gamma_{i, 2}$, and $\Gamma_{i, 3}$ that minimizes the 


\begin{tabular}{|l|l|l|l|l|l|}
\hline Distribution & \multicolumn{5}{|c|}{$\operatorname{Pr}_{0}$} \\
& $\alpha=1$ & $\alpha=2$ & $\alpha=3$ & $\alpha=4$ & $\alpha=5$ \\
\hline DET & $\underline{0}$ & 0 & 0 & 0 & 0 \\
UNI & 0.5 & $\underline{0}$ & 0 & 0 & 0 \\
EXP & 0.3679 & 0.1353 & $\underline{\mathbf{0 . 0 4 9 8}}$ & 0.0183 & 0.0067 \\
PAR $(\mathrm{k}=1 / 3)$ & 0.2963 & 0.0787 & $\underline{0.0315}$ & 0.0156 & 0.0089 \\
\hline
\end{tabular}

Table 3. Empty probability vs. scaling factor under different traffic distributions.

number of simultaneous wake-ups. Since $\gamma_{j}$ s are integers, we can compute the least common multiple (LCM) for all the elements of each $\Gamma^{*}$ candidate. Note that the LCM gives the minimal number of BIs for which two or more clients wake up simultaneously. Therefore, a larger LCM means a smaller number of simultaneous wake-ups. We therefore choose the best $\Gamma$ based on the largest LCM and denote it as $\Gamma_{i}^{*}$.

In the second sub-step, given $\left(\beta_{i}, \Gamma_{i}^{*}\right), i=1, \ldots, n+1$, we select the best $\Gamma$ from the $\Gamma_{i}^{*} \mathrm{~s}$ that minimizes simultaneous wake-up. The criterion is based on the largest spread of their elements from one another which is measured by the ratio of the standard deviation and the mean of the elements in $\Gamma_{i}^{*}$. Therefore, $\Gamma^{*}$ is given by the $\Gamma_{i}^{*}$ that gives the highest ratio, and $\beta^{*}$ is the corresponding $\beta_{i}$.

Step 3 (Determining $\Theta^{*}$ ) The final step determines $\Theta^{*}$ based on the $\Gamma^{*}$ obtained in the last step. The motivation is to mitigate the possible unfairness in the frame buffering delay experienced by the clients. We assign a smaller $\theta_{j}^{*}$ to the client with a larger $\gamma_{j}^{*}$. In this way, the client that wakes up less frequently will have a higher priority to retrieve its frames during channel contention. To do so, we assign the default value to $s_{j}$ if its $\gamma_{j}^{*}$ is the largest (i.e. $\theta_{j}^{*}=31$ ). We then increase other clients' $\theta_{j}$ by $\epsilon_{\Theta}$ when their $\gamma_{j}^{*}$ decrease. They are given by $\theta_{j}^{*}=$ $31+\epsilon_{\Theta}\left(\max _{\forall j}\left(\gamma_{j}^{*}\right)-\gamma_{j}^{*}\right)$.

\subsection{The optimal wake-up schedule}

Besides the main algorithm, the AP in C-PSM may also obtain the optimal wake-up schedule (WS). This optional step is to schedule the first wake-up times of the clients, so that the maximal number of waking clients at one BI epoch is minimized. The optimization problem is given by

$$
\min _{\mathbf{r}}: \max _{v=0,1,2, \ldots} N\left(v, \mathbf{r}, \Gamma^{*}\right)
$$

The vector $\mathbf{r}$ presents the sequence of the first wake-up times where the client $s_{j}$ first wakes up at the $r_{j}$ th BI epoch and $r_{j} \in\left[0, \operatorname{LCM}\left(\Gamma^{*}\right)-1\right]$ is an integer. The function $N\left(v, \mathbf{r}, \Gamma^{*}\right)$ is the number of waking clients at the $v$ th BI epoch when the clients wake up according to $r$ and $\mathbf{L I}^{*}$. WS consists of the optimal solution denoted as $\mathbf{r}^{*}$ which will further decrease the simultaneous wake-ups if two or more elements of $\Gamma^{*}$ are the same or have the same common factor.

Since the optimization problem (3) can be decomposed into a series of wake-up scheduling problems (WSPs) (Lin et al., 2006), we solve it by developing an algorithm based on the stepwise solving method for WSP.

When a new PSM-enabled client $s_{j}$ joins an infrastructure network (including a set of $m$ clients, $\mathcal{S}$ ) in the $v$ th BI epoch, WSP is formulated to minimize the maximal number of wake-up clients 
in the following BI epoches. The optimization problem of WSP is given by

$$
\min _{k_{j}(v)}: \max _{u=1,2, \ldots}\{N(v+u)\}
$$

where $N(v+u)$ is the number of waking clients at the $(v+u)$ th BI epoch and the wake-up counter $k_{j}(v)$ records the remaining BIs that the client $s_{j}$ will wake up. Moreover, $N(v+u)$ equals to $\sum_{i \in \mathcal{S} \cup j} w_{i}(v+u)$ where the wake-up indicator $w_{i}(v+u)$ is 1 if $s_{i}$ wakes up at the $(v+u)$ th BI epoch; otherwise, it is 0 . Given the LI parameter of each client $\gamma_{i}, 0 \leq k_{i}(v) \leq$ $\gamma_{i}-1, i \in \mathcal{S} \cup j$, the stepwise solving method (Lin et al., 2006) can calculate $k_{j}^{*}(v)$, the optimal wake-up counter of $s_{j}$. And $k_{j}^{*}(v)$ is a function $f\left(j, \gamma_{j}, m, w_{i}(v), k_{i}(v), \gamma_{i}\right), i=1, \ldots, m$. It is easy to see that $k_{j}^{*}(v)$ is the optimal first wake-up time of $s_{j}$ when $v=0$, i.e. $r_{j}^{*}=k_{j}^{*}(0)$.

According to $\Gamma^{*}$, our algorithm obtains $k_{j}^{*}(0)$ for each client at $v=0$. Therefore, we determines WS as $\mathbf{r}^{*}=\left[k_{1}^{*}(0), \ldots, k_{c}^{*}(0)\right]$. The client $s_{j}$ first wakes up optimally at the $r_{j}^{*}$ th BI epoch, $\forall j=1, \ldots, c$. For example, if $r_{j}^{*}=1, s_{j}$ will miss the first beacon frame at the beginning of simulation but wake up for the first time after $\beta^{*}$. The detail steps of our algorithm for obtaining $\mathbf{r}^{*}$ is given below:

1. Initialize the following variables: $v=0, \mathcal{S}=\left\{s_{1}\right\}, m=1, w_{1}(v)=1, k_{1}(v)=0, r_{1}^{*}=0$ and $j=2$.

2. If $j>c$, return $\mathbf{r}^{*}$ and exit; else, go to step 3 .

3. Find the optimal wake up time of $s_{j}$, where

$$
k_{j}^{*}(v)=f\left(j, \gamma_{j}^{*}, m, w_{i}(v), k_{i}(v), \gamma_{i}^{*}\right), i=1, \ldots, m .
$$

4. Update variables: $r_{j}^{*}=k_{j}^{*}(v), m=m+1$ and $\mathcal{S}=\mathcal{S} \cup s_{j}$.

5. If $r_{j}^{*}=0$, then $w_{j}(v)=1$; else, $w_{j}(v)=0$.

6. Increase $j$ by 1 and go back to step 2 .

\section{Performance evaluation}

We evaluate the performance of C-PSM and compare it with the PSM with default parameters (which is referred to as standard PSM or S-PSM). We do not compare C-PSM with other user-centric/AP-centric PSM schemes, because the design objectives and the study scopes are different. For example, C-PSM improves energy efficiency for all clients, whereas the user-centric PSM schemes consider only a single client. On the other hand, C-PSM is standard-compliant, but most AP-centric schemes are not compatible with the PSM scheme.

\subsection{Evaluation methodology}

In order to evaluate the effectiveness of different components of C-PSM, we examine three different versions. The first one is a "full version" which includes the optional optimal wake-up sequence discussed in the last section. The other two, Scheme- 1 and Scheme-2, on the other hand, exclude this option and adopt the default congestion window size. Moreover, Scheme-2 adopts the default $\Gamma$ value. To sum up, we compare the following schemes in reference to S-PSM.

1. C-PSM: $\beta^{*}, \Gamma^{*}, \Theta^{*}, \mathbf{r}^{*}$; 
2. Scheme-1: $\beta^{*}, \Gamma^{*}, \theta_{j}=31, r_{j}=0$;

3. Scheme-2: $\beta^{*}, \gamma_{j}=1, \theta_{j}=31, r_{j}=0$;

4. S-PSM: $\beta=100 \mathrm{~ms}, \gamma_{j}=1, \theta_{j}=31, r_{j}=0$.

We use the following four performance indices for comparing C-PSM, Scheme- 1 , and Scheme-2 against S-PSM: power saving (index $\eta_{P}$ ), throughput (index $\eta_{T}$ ), energy efficiency (index $\eta_{T / P}$ ), and frame buffering delay (index $\eta_{D}$ ). The notations with a superscript $S-P S M$ refers to S-PSM, whereas that without refer to C-PSM, Scheme-1, or Scheme-2. For easy comparison, a positive value indicates improvement over S-PSM.

$$
\begin{aligned}
\eta_{P} & =\left(P^{S-P S M}-P\right) / P^{S-P S M} \times 100 \%, \\
\eta_{T} & =\left(T-T^{S-P S M}\right) / T^{S-P S M} \times 100 \%, \\
\eta_{T / P} & =\left(R_{T / P}-R_{T / P}^{S-P S M}\right) / R_{T / P}^{S-P S M} \times 100 \%, \\
\eta_{D} & =\frac{1}{c} \times \sum_{j=1}^{c}\left(d_{j}^{S-P S M}-d_{j}\right) / d_{j}^{S-P S M} \times 100 \% .
\end{aligned}
$$

\subsection{Two clients}

We first evaluate C-PSM in the two-client system. Given $\Delta=[15 ; 25] m s$, the AP obtains the optimal parameters of C-PSM under different traffic distributions, as shown in Table 4.

\begin{tabular}{|l|l|l|l|l|l|}
\hline$\Delta(\mathrm{ms})$ & distribution & $\beta^{*}(\mathrm{~ms})$ & $\Gamma^{*}$ & $\Theta^{*}$ & $\mathbf{r}^{*}$ \\
\hline$[15 ; 25]$ & DET & 10 & {$[2 ; 3]$} & {$[39 ; 31]$} & {$[0 ; 0]$} \\
& UNI & 26 & {$[1 ; 2]$} & {$[39 ; 31]$} & {$[0 ; 0]$} \\
& EXP,PAR & 38 & {$[1 ; 2]$} & {$[39 ; 31]$} & {$[0 ; 0]$} \\
\hline$[20 ; 30 ; 30]$ & DET & 16 & {$[1 ; 2 ; 2]$} & {$[39 ; 31 ; 31]$} & {$[0 ; 0 ; 1]$} \\
& UNI & 30 & {$[1 ; 2 ; 2]$} & {$[39 ; 31 ; 31]$} & {$[0 ; 0 ; 1]$} \\
& EXP,PAR & 46 & {$[1 ; 2 ; 2]$} & {$[39 ; 31 ; 31]$} & {$[0 ; 0 ; 1]$} \\
\hline
\end{tabular}

Table 4. Optimal parameters of C-PSM $\left(\epsilon_{\beta}=2 \mathrm{~ms}\right.$ and $\left.\epsilon_{\Theta}=8\right)$.

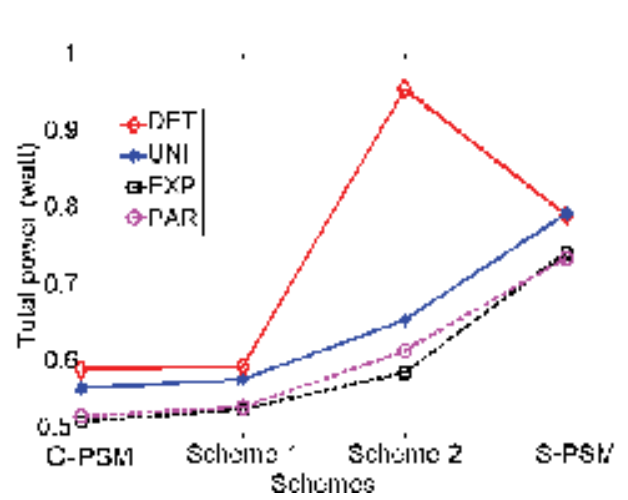

(a) Total power, $P$.

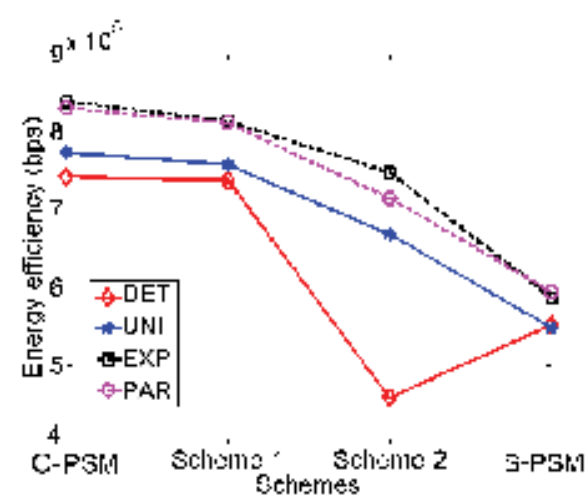

(b) Total energy efficiency, $R_{T / P}$.

Fig. 3. A comparison of the four PSM schemes with $\Delta=[15 ; 25] m s$. 
Figure 3 depicts that C-PSM outperforms S-PSM on saving energy and improving energy efficiency under different distributions. C-PSM achieves lowest $P$ and highest $R_{T / P}$ among the four schemes. Scheme-1 performs a little worse than C-PSM, since it does not adopt $\Theta^{*}$. Comparing with scheme-1, scheme- 2 increases power and decreases energy efficiency, since it does not use $\Gamma^{*}$. Adopting $\beta^{*}$, Scheme-2 still outperforms S-PSM under all traffic distributions except the DET distribution. Scheme-2 is the worst under deterministic traffic, because two clients wake up every $\beta^{*}=10 \mathrm{~ms}$ and they both waste energy on the frequent unnecessary wake-ups. Note that, the WS is not adopted, since $\mathbf{r}^{*}$ is a zero vector when $\gamma_{j}(\forall j)$ are relative prime. In this case, all clients wake up at the beginning of simulation.

\begin{tabular}{|l|l|l|l|l|l|}
\hline index, \% & scheme & DET & UNI & EXP & PAR \\
\hline$\eta_{P}$ & C-PSM & 25.41 & 28.75 & 29.73 & 28.13 \\
& Scheme-1 & 24.91 & 27.28 & 27.53 & 26.47 \\
& Scheme-2 & -20.82 & 17.52 & 21.10 & 16.48 \\
\hline$\eta_{T} / P$ & C-PSM & 34.63 & 41.18 & 43.01 & 39.76 \\
& Scheme-1 & 33.71 & 38.33 & 38.65 & 36.57 \\
& Scheme-2 & -16.88 & 21.95 & 27.38 & 20.30 \\
\hline$\eta_{D}$ & C-PSM & 82.33 & 68.79 & 54.80 & 54.18 \\
& Scheme-1 & 82.08 & 68.15 & 53.07 & 53.56 \\
& Scheme-2 & 94.54 & 79.79 & 69.88 & 68.75 \\
\hline$\eta_{T}$ & C-PSM & 0.41 & 0.59 & 0.50 & 0.45 \\
& Scheme-1 & 0.40 & 0.60 & 0.48 & 0.42 \\
& Scheme-2 & 0.43 & 0.58 & 0.50 & 0.47 \\
\hline
\end{tabular}

Table 5. Indices of C-PSM, Scheme-1 and Scheme-2, $\Delta=[15 ; 25] m s$.

As shown in Table 5, all indices of C-PSM are positive and the improvements of C-PSM over S-PSM are significant under different distributions. For example, compared with S-PSM, C-PSM reduces power consumption by $29.37 \%$, improves energy efficiency by $43.01 \%$ and reduces average buffering delay by $54.8 \%$ under the EXP distribution of traffic. We also find that C-PSM has largest $\eta_{P}$ and $\eta_{T / P}$. That is, C-PSM which employs $\beta^{*}, \Gamma^{*}$ and $\Theta^{*}$ together, performs the best in saving power and increasing energy efficiency.

In C-PSM, the benefit of adopting $\beta^{*}$ and $\Gamma^{*}$ is significant whereas the improvement due to $\Theta^{*}$ is minor. Scheme-1 using $\beta^{*}$ and $\Gamma^{*}$ has obtained large positive indices. Its indices are slightly less than C-PSM's indices. For example, the energy efficiency is improved by $38 \%$ while the $\eta_{T / P}$ of C-PSM is $43.01 \%$. Therefore, the usage of $\Theta^{*}$ is helpful to save energy but not much. Moreover, $\beta^{*}$ and $\Gamma^{*}$ jointly play the major roles in improving PSM performance. In contrast, the PSM performance greatly degrades without using $\Gamma^{*}$. The $\eta_{P}$ and $\eta_{T / P}$ of Scheme-2 are much smaller than the ones of C-PSM and Scheme-1. Scheme-2 therefore is worse than these two schemes. It is even worse than S-PSM, because of its negative indices under the DET distribution of traffic.

Next, we compare C-PSM and S-PSM under the EXP distribution of traffic. As shown in Figure 4(a), the AP buffering delay is shortest in C-PSM, whereas it is longest in S-PSM. Consequently, the clients using C-PSM can return to sleep mode earlier, because the frames with shorter buffering delay are retrieved faster than those in S-PSM. Moreover, C-PSM improves the fairness of clients, since it greatly decreases the delay difference of the two clients. C-PSM speeds up the retrieval of frames in the fast client, because the delay of $s_{1}$ is one sixth of that in S-PSM. At the same time, it does not degrade the slow client, since the 


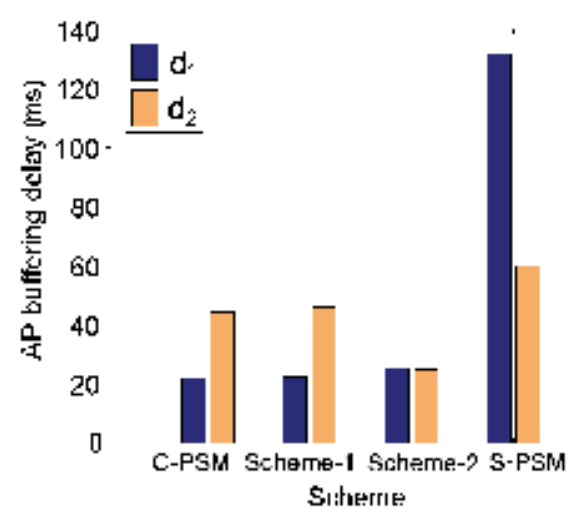

(a) The AP buffering delay.

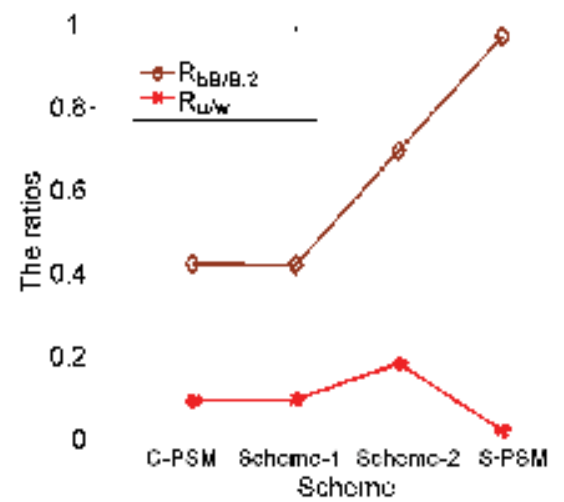

(b) The ratios of unnecessary wake-ups and simultaneous wake-ups.

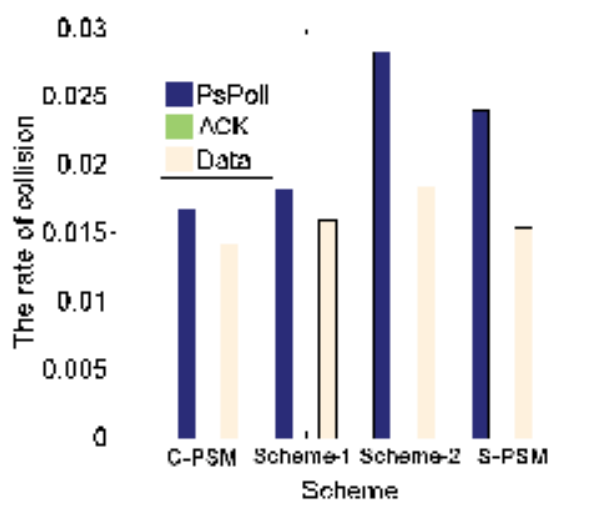

(c) The collision ratios.

Fig. 4. A comparison of the four schemes under the EXP distribution with $\Delta=[15 ; 25] m s$.

delay of $s_{2}$ reduces a little. Figure 4(b) shows that C-PSM greatly reduces the chances that two clients simultaneously wake up to compete for channel, since its $R_{b B / B, 2}$ is lowest. In contrast, S-PSM lets two clients wake up simultaneously at a large proportion of BI epochs, since its $R_{b B / B, 2}$ is near 1 . The clients using C-PSM spend less time on channel contention, consume less energy on idle mode and then achieve higher energy efficiency.

Figure 4 illustrates that C-PSM and Scheme- 1 have similar performance metrics. The collision ratios of PS-Poll and data frames in C-PSM are less than those in Scheme-1. It means that $\Theta^{*}$ is useful for reducing channel collisions. That is why C-PSM performs better than Scheme-1 with slightly higher indices.

Scheme-2 outperforms S-PSM under the EXP distribution of traffic, since it achieves shorter AP buffering delay and less simultaneous wake-up ratio than S-PSM. Scheme-2 is less energy-efficient than C-PSM and Scheme-1, because it spends more energy on unnecessary wake-ups and channel contention. The clients in Scheme- 1 frequently wake up every $\beta^{*}$ but nearly $20 \%$ of wake-ups are unnecessary while the unnecessary wake-ups ratio is less than $10 \%$ in C-PSM. Without using $\Gamma^{*}$, the clients in Scheme-2 spend more energy on idle mode 
when they simultaneously wake up to compete channel with a higher probability. Shown in Figure $4(\mathrm{~b})$, they are involved in channel contention at $68 \%$ of BIs, i.e. $R_{b B / B, 2}=68 \%$ while the $R_{b B / B, 2}$ in C-PSM is only $40 \%$. Furthermore, the collision ratios in Scheme-2 are higher than those in C-PSM and Scheme-1, shown in Figure 4(c). For example, the PS-Poll collision ratio in Scheme-2 is the highest, nearly 1.5 times of that in C-PSM. Therefore, the clients in Scheme-2 have to spend more energy to handle the collisions. On the other hand, Scheme-2 has shorter delay for the slow client $s_{2}$ than C-PSM and Scheme-2. However, its benefit is too small to affect the performance.

Additionally, the collision ratios of ACK are almost zero in Figure 4(c). The reason is that an awaken client always returns ACK after it has finished receiving a data frame and a SIFS has elapsed. The channel is rarely occupied by the other client or the AP within such a short SIFS. Therefore, ACKs rarely suffer from collisions especially when $c=2$. If the number of clients increases, the probability of ACK collisions will increase as the channel contention intensifies.

\subsection{More than two clients}

We have applied C-PSM to a network with more than two clients. Firstly, we evaluate C-PSM when the number of clients is 3 and two clients have the same mean of inter-frame arrival times. According to $\Delta=[20 ; 30 ; 30]$ where $\rho=13.18 \%<30 \%$, the main algorithm obtains the optimal parameters of C-PSM, also shown in Table 4. Note that when the elements of $\Gamma^{*}$ are not relative prime numbers or even the same, C-PSM must adopt WS. In this case, $\gamma_{2}^{*}$ and $\gamma_{3}^{*}$ have a greatest common divisor 2 , and then C-PSM uses WS, i.e., $\mathbf{r}^{*}=[0 ; 0 ; 1] . s_{1}$ and $s_{2}$ wake up at the beginning of simulation while $s_{3}$ defers the first wake-up time for one BI.

\begin{tabular}{|l|l|l|l|l|l|}
\hline index, $\%$ & scheme & DET & UNI & EXP & PAR \\
\hline$\eta_{P}$ & C-PSM & 36.38 & 39.08 & 36.78 & 36.31 \\
& C-PSM not WS & 29.00 & 30.08 & 26.43 & 27.33 \\
\hline$\eta_{T / P}$ & C-PSM & 59.86 & 65.92 & 59.11 & 58.00 \\
& C-PSM not WS & 43.22 & 44.56 & 36.73 & 38.41 \\
\hline$\eta_{D}$ & C-PSM & 84.00 & 68.69 & 52.16 & 51.98 \\
& C-PSM not WS & 81.80 & 64.62 & 45.23 & 46.37 \\
\hline$\eta_{T}$ & C-PSM & 1.71 & 1.08 & 0.60 & 0.63 \\
& C-PSM not WS & 1.69 & 1.07 & 0.59 & 0.58 \\
\hline
\end{tabular}

Table 6. Indices of C-PSM with/without WS, $\Delta=[20 ; 30 ; 30] \mathrm{ms}$.

The positive indices in Table 6 show that C-PSM outperforms S-PSM in terms of power saving, energy efficiency and AP buffering delay while keeping or slightly increasing throughput in the three-client network. For example, C-PSM reduces power consumption by $36.78 \%$, improves the energy efficiency by $59.11 \%$ and shortens the average buffering delay by $52.16 \%$ under the EXP distribution of traffic while total throughput remains almost the same. On the other hand, the indices of C-PSM without WS are less than the ones of C-PSM under all traffic distributions. For example, without using WS, the $\eta_{T / P}$ Of C-PSM is decreased at most by $22 \%$ under the EXP distribution of traffic. Therefore, WS is much helpful to improve energy efficiency when the symmetric clients exist.

Next, we compare the simulation results of C-PSM, C-PSM without WS and S-PSM to explain the above findings. As an example, we study these three schemes under the EXP distribution of traffic. 


\begin{tabular}{|l|ccc|}
\hline metrics & \multicolumn{3}{|c|}{ C-PSM C-PSM not WS S-PSM } \\
\hline$P$ (Watt) & 0.8242 & 0.9591 & 1.3037 \\
$T\left(10^{5} \mathrm{bps}\right)$ & 4.7538 & 4.7536 & 4.7257 \\
$R_{T / P}\left(10^{5} \mathrm{bpJ}\right)$ & 5.7675 & 4.9563 & 3.6248 \\
$d_{1}(\mathrm{~ms})$ & 34.4 & 36.3 & 234.2 \\
$d_{2}(\mathrm{~ms})$ & 55.8 & 63.2 & 84.5 \\
$d_{3}(\mathrm{~ms})$ & 54.9 & 64.7 & 87.4 \\
\hline$R_{c / t}$ & $1.44 \%$ & $1.78 \%$ & $2.14 \%$ \\
$R_{u / w}$ & $10.06 \%$ & $10.66 \%$ & $4.86 \%$ \\
$R_{b B / B, 2}$ & $83.83 \%$ & $10.47 \%$ & $7.64 \%$ \\
$R_{b B / B, 3}$ & $\mathbf{0}$ & $39.05 \%$ & $92.29 \%$ \\
\hline
\end{tabular}

Table 7. A comparison of three schemes under the EXP distribution with $\Delta=[20 ; 30 ; 30] \mathrm{ms}$.

C-PSM saves energy by shortening the period of channel contention, shown in Table 7. All the clients' frame buffering delays of C-PSM are smaller than those of other two schemes. That is, each client can receive its buffered frames most quickly and then enter to sleep instead of spending much energy and time on idle mode during channel contention. C-PSM also saves energy by reducing channel contentions. It totally avoids all-client simultaneous wake-ups and $R_{b B / B, 3}$ is zero. On the other hand, in S-PSM, three clients wake up together to receive data in almost all BIs and $R_{b B / B, 3}$ is as high as $92.29 \%$. At the same time, C-PSM consumes a small amount of energy on unnecessary wake-ups, since the total ratio of unnecessary wake-ups $R_{u / w}$ is near $10 \%$. It also decreases the channel collisions where the total ratio of collisions $R_{c / t}$ is reduced by about one-third. From what has been discussed above, C-PSM outperforms S-PSM.

C-PSM without WS obviously outperforms S-PSM but is worse than C-PSM. Without using WS, the total power increases, the total energy efficiency decreases and three awaken clients compete for receiving data in $39.05 \%$ of BIs. However, C-PSM can totally avoid the situation of all-client simultaneous wake-ups. On the other hand, the $R_{b B / B, 2}$ in the C-PSM without WS is smaller than the one in C-PSM. It is helpful to save energy but does not determine the energy consumption of all clients. The reason is that more energy is consumed on channel contention when all of three clients wake up simultaneously. In a global view, C-PSM saves more energy and achieves higher energy efficiency after using $\mathbf{r}^{*}$. Therefore, WS is helpful to improve energy efficiency, because the number of clients which wake up at the same beacon epoch has been minimized.

Furthermore, we find that C-PSM is applicable for a large scale network and saves more energy when the number of clients increases. These two sets of simulations evaluate the performance of our scheme when the number of PSM-enabled clients increases up to 20.

In the first set of simulations, we let $\delta_{j}=10 c(\mathrm{~ms}), j=1, \ldots, c$. The total amount of traffic of all symmetric clients will not change with $c$, and the total arrival rate of frames $\lambda$ always equals 100 frames per second. The traffic workload is light, when $\rho=11.3 \%<30 \%$. Under light traffic, S-PSM can save energy, since the average power of each client is always lower than its idle power. For example in Figure 5, $P^{S-P S M}$ is much less than the $c$ times of client's idle power under the EXP distribution of traffic. C-PSM scheme can further reduce energy consumption, since the power difference between $P^{S-P S M}$ and $P^{C-P S M}$ is always larger than zero. Moreover, this power difference increases with $c$. Therefore, C-PSM saves more energy when the number of clients increases. 


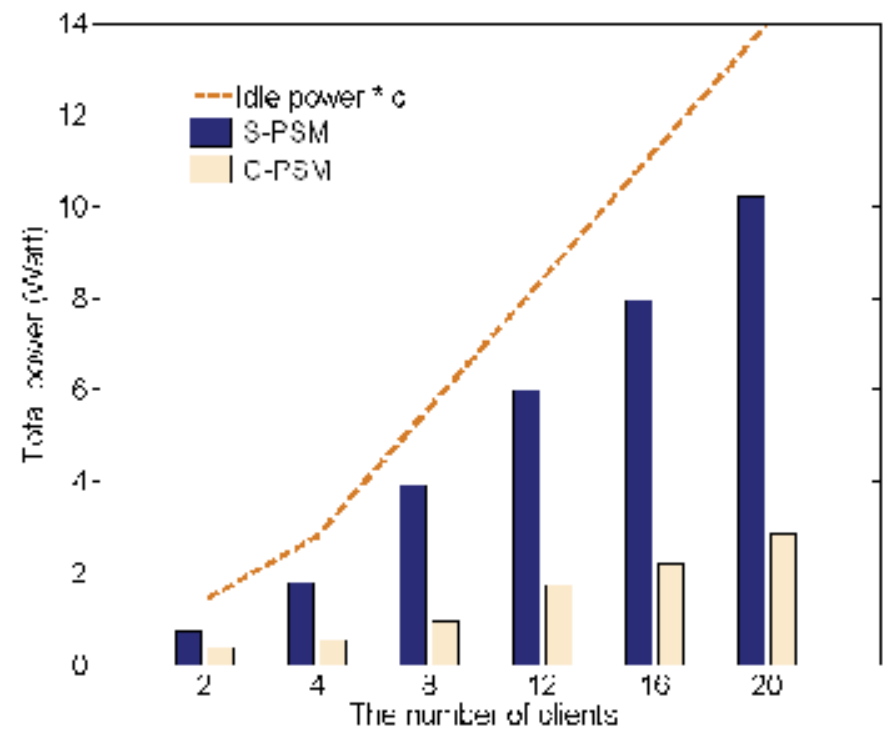

Fig. 5. Total power verses $c$ under the EXP distribution with $\delta_{j}=10 c(\mathrm{~ms})$.

\begin{tabular}{|l|l|l|l|l|l|l|l|}
\hline Index,$\%$ & $\mathrm{~T}_{j}$ & $\mathrm{c}=2$ & $\mathrm{c}=4$ & $\mathrm{c}=8$ & $\mathrm{c}=12$ & $\mathrm{c}=16$ & $\mathrm{c}=20$ \\
\hline$\eta_{P}$ & DET & 22.30 & 63.75 & 79.20 & 71.54 & 75.52 & 72.98 \\
& UNI & 45.37 & 72.52 & 78.82 & 70.58 & 71.74 & 72.22 \\
& EXP & 51.09 & 70.33 & 76.07 & 70.98 & 72.27 & 72.14 \\
& PAR & 50.76 & 70.43 & 76.68 & 69.77 & 70.91 & 70.33 \\
\hline$\eta_{R_{T / P}}$ & DET & 29.07 & 177.76 & 396.05 & 263.65 & 325.31 & 286.14 \\
& UNI & 83.50 & 265.28 & 384.96 & 251.95 & 270.96 & 281.65 \\
& EXP & 105.04 & 238.69 & 327.07 & 257.23 & 277.64 & 281.02 \\
& PAR & 103.89 & 239.39 & 338.50 & 241.89 & 260.80 & 255.76 \\
\hline$\eta_{D}$ & DET & 92.35 & 87.63 & 85.78 & 87.47 & 90.18 & 89.21 \\
& UNI & 73.94 & 70.37 & 84.07 & 85.57 & 87.28 & 88.11 \\
& EXP & 64.09 & 70.89 & 82.68 & 85.02 & 87.04 & 88.43 \\
& PAR & 62.93 & 69.48 & 82.25 & 85.78 & 87.10 & 88.19 \\
\hline$\eta_{T}$ & DET & 0.29 & 0.70 & 3.17 & 3.51 & 4.10 & 4.32 \\
& UNI & 0.24 & 0.40 & 2.72 & 3.55 & 4.83 & 6.02 \\
& EXP & 0.29 & 0.48 & 2.19 & 3.68 & 4.73 & 6.14 \\
& PAR & 0.40 & 0.37 & 2.27 & 3.36 & 4.94 & 5.55 \\
\hline
\end{tabular}

Table 8. The indices of C-PSM verses the number of clients when $\delta_{j}=10 c(\mathrm{~ms}), j=1, \ldots, c$.

Table 8 has shown that C-PSM achieves significant improvements of four main metrics in a large network. For example, the indices of $\eta_{P}, \eta_{T / P}$ and $\eta_{D}$ in are as high as $76.07 \%, 327.07 \%$ and $82.68 \%$ individually when $c=8$ under the EXP distribution of traffic. C-PSM scheme also improves clients' throughput in a large network, since $\eta_{T}$ increases slightly with the number of clients.

In the second set of simulations, we let $\delta_{j}=10+5 j(\mathrm{~ms}), j=1, \ldots, c$. The total packet arrival rate $\lambda$ increases with the number of clients where $\rho$ increases from $13.18 \%$ to $49.51 \%$. When $c \geq 8$, the traffic is not light any more, since $\rho \geq 32 \%$.

We firstly find that C-PSM has a wider applicability than the standard PSM. It is effective to save energy when the network supports many clients whose workload is not light. For 


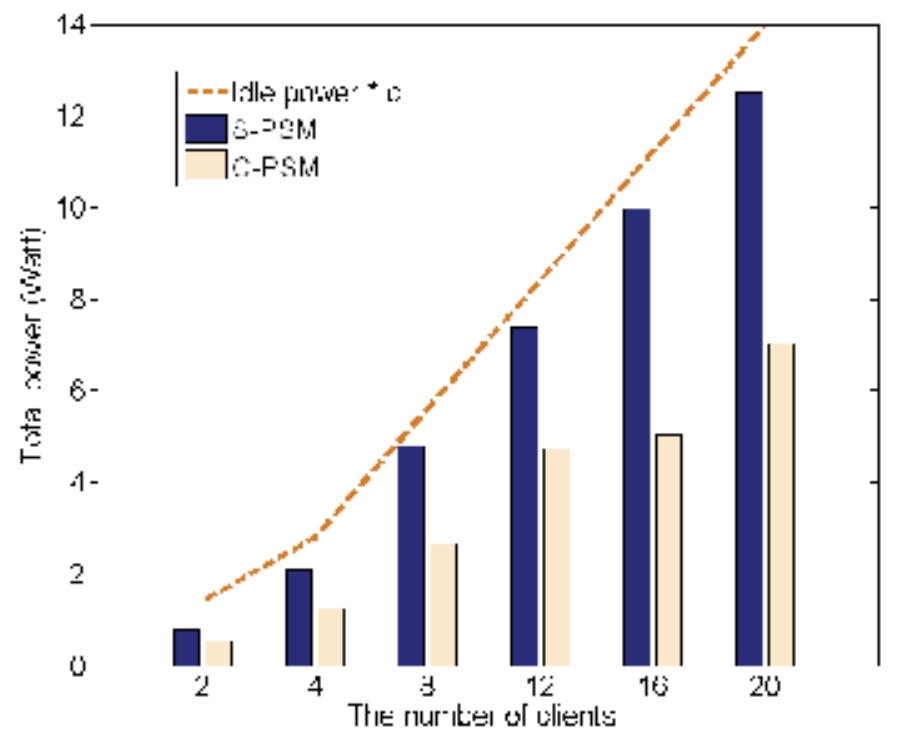

Fig. 6. Total power verses $c$ under the EXP distribution with $\delta_{j}=10+5 j(\mathrm{~ms}), j=1, \ldots, c$.

example in Figure 6 under the EXP distribution of traffic, $P^{S-P S M}$ is much close to the total power of $c$ idle clients, when $c \geq 8$. It is obvious that these clients are too busy to sleep and S-PSM cannot save much energy. After using C-PSM, $P^{C-P S M}$ is much less than the total power of $c$ idle clients even when $c$ increases to 20. That is, C-PSM is still effective to save energy even when the network workload is as high as $\rho \approx 50 \%$. Moreover, C-PSM saves more energy when the number of clients increases, since that the power difference $P^{S-P S M}-P^{C-P S M}$ increases with $c$. Although not shown here, the similar simulation results are obtained under other traffic distributions.

\begin{tabular}{|l|l|l|l|l|l|l|l|}
\hline Index, $\%$ & $T_{j}$ & $\mathrm{c}=2$ & $\mathrm{c}=4$ & $\mathrm{c}=8$ & $\mathrm{c}=12$ & $\mathrm{c}=16$ & $\mathrm{c}=20$ \\
\hline$\eta_{P}$ & DET & 1.01 & 29.04 & 42.17 & 44.31 & 51.49 & 52.71 \\
& UNI & 21.93 & 37.81 & 44.29 & 37.34 & 51.98 & 50.97 \\
& EXP & 28.98 & 39.73 & 44.20 & 36.01 & 49.44 & 43.89 \\
& PAR & 25.64 & 37.59 & 44.19 & 35.65 & 48.85 & 43.49 \\
\hline$\eta_{R_{T / P}}$ & DET & 0.55 & 55.54 & 132.72 & 174.52 & 248.45 & 286.48 \\
& UNI & 28.79 & 76.14 & 138.29 & 137.83 & 245.38 & 255.72 \\
& EXP & 41.51 & 75.73 & 124.41 & 115.23 & 201.97 & 172.88 \\
& PAR & 35.07 & 71.90 & 126.80 & 114.65 & 203.60 & 173.49 \\
\hline$\eta_{D}$ & DET & 96.60 & 94.98 & 95.59 & 93.39 & 94.00 & 91.36 \\
& UNI & 76.05 & 79.90 & 85.27 & 85.56 & 86.88 & 85.72 \\
& EXP & 66.12 & 69.74 & 77.41 & 77.21 & 79.52 & 76.21 \\
& PAR & 65.05 & 69.82 & 77.69 & 77.10 & 79.66 & 76.30 \\
\hline$\eta_{T}$ & DET & 0.45 & 10.38 & 34.58 & 52.88 & 69.04 & 82.76 \\
& UNI & 0.55 & 9.54 & 32.75 & 49.03 & 65.84 & 74.41 \\
& EXP & 0.49 & 5.91 & 25.23 & 37.74 & 52.68 & 53.12 \\
& PAR & 0.43 & 7.29 & 26.58 & 38.13 & 55.28 & 54.56 \\
\hline
\end{tabular}

Table 9. The indices of C-PSM verses the number of clients when $\delta_{j}=10+5 j(\mathrm{~ms})$, $j=1, \ldots, c$. 
Table 9 also shows that C-PSM scheme outperforms S-PSM on saving power, improving energy efficiency, shortening delay and increasing throughput. When the traffic is not light (i.e. $c \geq 8$ ), C-PSM improves energy efficiency a lot, since it not only reduces power consumption but also increases throughput greatly. For example, compared with S-PSM, C-PSM saves $49.44 \%$ of power, increases $52.68 \%$ of throughput and then finally achieves $201.97 \%$ higher energy efficiency in the sixteen-client system under the EXP distribution of traffic.

\subsection{Effects of power consumption model on C-PSM}

The power profile of wireless device has a great impact on the performance of energy-saving scheme using sleeping (Nedevschi et al., 2008). This profile includes the power consumption of client in transmission, reception, idle mode, sleeping mode and mode transition (when the client wakes up from sleeping mode to active mode), as well as the wake-up time. Additionally, the energy consumed on client's wake-up is the product of wake-up power ${ }^{3}$ and wake-up time. The set of these above parameters are defined as a power consumption model in this chapter.

We adopt model $\mathcal{A}$ (Feeney \& Nilsson, 2001; Margi, 2006) in our simulator, which is widely used. Model $\mathcal{A}$ is comparable to the hardware characteristics of many popular wireless interface cards. The ratio of transmission power to reception power in model $\mathcal{A}$ is approximately $160 \%$ which is similar to the ratioes of ORiNOCO 11a/b/g ComboCard (Proxim Wireless Corporation, 2006a), ORiNOCO 11a/b/g PCI card (Proxim Wireless Corporation, 2006b), CISCO AIRONET 802.11A/B/G Wireless Cardbus adapter (Cisco Systems, Inc., 2004), CISCO AIRONET 350 Series Wireless LAN Client Adapters (Cisco Systems, Inc., 2005) and Aironet's PC4800 PCMCIA NIC (Ebert et al., 2002). The reception power is near to the idle power in model $\mathcal{A}$ which is the same in CISCO AIRONET 802.11A/B/G Wireless Cardbus adapter (Cisco Systems, Inc., 2004) and Aironet's PC4800 PCMCIA NIC (Ebert et al., 2002). Moreover, the sleep power is about an order of magnitude lower than the idle power in model $\mathcal{A}$. The ratio of idle power to sleep power denoted as $R_{I / S}$ equals to $1167 \%$ which is common in many popular wireless network interface cards.

\begin{tabular}{|l|l|l|l|l|l|}
\hline State & model $\mathcal{A}$ & model $\mathcal{B}$ & model $\mathcal{C}$ & model $\mathcal{D}$ & model $\mathcal{E}$ \\
\hline Transmission power & $1.4 \mathrm{~W}$ & $1.65 \mathrm{~W}$ & $0.75 \mathrm{~W}$ & $1.3 \mathrm{~W}$ & $0.85 \mathrm{~W}$ \\
Reception power & $0.9 \mathrm{~W}$ & $1.4 \mathrm{~W}$ & $0.75 \mathrm{~W}$ & $0.95 \mathrm{~W}$ & $0.85 \mathrm{~W}$ \\
Idle power & $0.7 \mathrm{~W}$ & $1.15 \mathrm{~W}$ & $0.75 \mathrm{~W}$ & $0.79 \mathrm{~W}$ & $0.85 \mathrm{~W}$ \\
Sleeping power & $0.06 \mathrm{~W}$ & $0.045 \mathrm{~W}$ & $0.05 \mathrm{~W}$ & $0.17 \mathrm{~W}$ & $0.005 \mathrm{~W}$ \\
$R_{I / S}$ & $1167 \%$ & $2556 \%$ & $1500 \%$ & $468 \%$ & $17000 \%$ \\
\hline Wake-up power & $0.7^{*} 2 \mathrm{~W}$ & $1.15^{*} 2 \mathrm{~W}$ & $0.75 \mathrm{~W}$ & $0.51 \mathrm{~W}$ & $0.85^{*} 2 \mathrm{~W}$ \\
Wake-up Time & $2 \mathrm{~ms}$ & $2 \mathrm{~ms}$ & $2 \mathrm{~ms}$ & $13 \mathrm{~ms}$ & $2 \mathrm{~ms}$ \\
Wake-up Energy & $0.003 \mathrm{~J}$ & $0.005 \mathrm{~J}$ & $0.0015 \mathrm{~J}$ & $0.0066 \mathrm{~J}$ & $0.0034 \mathrm{~J}$ \\
\hline
\end{tabular}

Table 10. Five power consumption models.

Next, we compare model $\mathcal{A}$ with other power consumption models model $\mathcal{B}$ (Jung \& Vaidya, 2002; Simunic et al., 2000), model $\mathcal{C}$ (Anastasi et al., 2007; Krashinsky \& Balakrishnan, 2005), model D (Jeong et al., 2004) and model $\mathcal{E}$ (C-Guys, Inc., 2004) listed in Table 10. In order to study

\footnotetext{
${ }^{3}$ During the mode transition, the client's power consumption is near or higher than transmission power (Stemm \& Katz, 1997). It could be estimated as two times of idle power (Jung \& Vaidya, 2002), for example the models $\mathcal{A}, \mathcal{B}$ and $\mathcal{E}$.
} 
the effects of power consumption model on the performance of C-PSM, we deploy S-PSM and C-PSM in the two-client network $(\Delta=[15 ; 25] \mathrm{ms})$ (shown in section 4$)$ under the five different power consumption models.

The improvements of C-PSM over S-PSM mainly depend on the wake-up energy consumption and $R_{I / S}$. Comparing the power saving index $\eta_{P}$ and the energy efficiency index $\eta_{R_{T / P}}$ in Table 11, C-PSM outperforms S-PSM greatly with the highest indices in model $\mathcal{C}$, since the wake-up energy consumption is minimal and $R_{I / S}$ is as high as $1500 \%$. Model $\mathcal{C}$ and model $\mathcal{A}$ has the similar $R_{I / S}$ over $1000 \%$, the indices of $\eta_{P}$ and $\eta_{R_{T / P}}$ decrease with the wake-up energy consumption. When the energy consumption is the same like model $\mathcal{A}$ and model $\mathcal{E}$, the indices increase with $R_{I / S}$. That is, the advantages of C-PSM over S-PSM is more outstanding when the wake-up energy consumption decreases and $R_{I / S}$ increases. Model $\mathcal{D}$ achieves the worst performance, since it has the maximal wake-up energy consumption and the lowest $R_{I / S}$. For example, C-PSM is worse than S-PSM with negative indices under the DET distribution. Therefore, C-PSM is not efficient when clients consume much energy on waking up or the sleeping power is close to the idle power.

\begin{tabular}{|l|l|l|l|l|l|l|}
\hline Index & $T_{j}$ & $\mathcal{A}$ & $\mathcal{B}$ & $\mathcal{C}$ & $\mathcal{D}$ & $\mathcal{E}$ \\
\hline$\eta_{P}$ & DET & 26.16 & 30.86 & 46.46 & -4.86 & 38.44 \\
& UNI & 27.72 & 32.32 & 42.12 & 5.65 & 38.73 \\
& EXP & 28.99 & 33.72 & 39.63 & 12.69 & 39.58 \\
& PAR & 29.56 & 34.37 & 40.31 & 13.02 & 40.30 \\
\hline$\eta_{R_{T / P}}$ & DET & 36.25 & 45.52 & 87.91 & -4.05 & 63.44 \\
& UNI & 39.01 & 48.46 & 73.58 & 6.48 & 63.98 \\
& EXP & 41.29 & 51.37 & 66.17 & 14.91 & 66.04 \\
& PAR & 42.30 & 52.71 & 67.93 & 15.24 & 67.91 \\
\hline
\end{tabular}

Table 11. Indices of C-PSM in different power consumption models when $c=2, \Delta=[15 ; 25]$.

\section{Conclusions and future works}

We propose the centralized PSM (C-PSM) to increase the energy efficiency of all wireless clients in an infrastructure wireless network. C-PSM is traffic-aware and inherits the operations of standard PSM except for using optimal parameters. According to the traffic characteristics, C-PSM instructs the AP to compute the optimal beacon interval, optimal listen intervals, optimal minimal congestion windows and optimal sequence of first wake-up times. C-PSM achieves the significant improvements over standard PSM because (1) the jointly optimized intervals can reduce unnecessary wake-ups and channel contentions which collectively translate into the energy saving and reduction in the buffering delay; (2) the optimal minimal congestion windows are effective to balance the delay among clients; and (3) the first wake-up times reduce the simultaneous wake-ups to alleviate channel contention. Moreover, C-PSM has a wider applicability than S-PSM. It is effective even when the workload of the network is not light. The improvements of C-PSM over S-PSM increases when the number of clients increases, the wake-up energy consumption decreases or the ratio of idle power to sleep power increases. In future works, we will further improve optimization algorithms and extend C-PSM to support more traffic models. 


\section{Acknowledgement}

We acknowledge the financial support from the Fundamental Research Funds for the Central Universities of the Republic of China (No.2010121066) and the National Defense Basic Scientific Research Program of China under Grant (B1420110155).

\section{References}

Anastasi, G., Conti, M., Gregori, E. \& Passarella, A. (2004). A performance study of power-saving polices for Wi-Fi hotspots, The International Journal of Computer and Telecommunications Networking 45(3): 295-318.

Anastasi, G., Conti, M., Gregori, E. \& Passarella, A. (2007). 802.11 power-saving mode for mobile computing in Wi-Fi hotspots: Limitations, enhancements and open issues, ACM/Springer Wireless Networks .

Belghith, A., Belghith, A. \& Molnar, M. (2007). Enhancing psm efficiencies in infrastructure 802.11 networks, International Journal of Computing and Information Sciences (IJCIS) 5(1): 13-23.

Berkeley, U., LBL, USC/ISI \& PARC, X. (1996). The network simulator - ns-2, http: / / www . isi.edu/nsnam/ns/.

C-Guys, Inc. (2004). SD-Link 11b specifications, http://www.embeddedworks.net/ c-guysusa/wwwroot/SD-Link11b.html.

Chiang, M. \& Bell, J. (2004). Balancing supply and demand of bandwidth in wireless cellular networks: Utility maximization over powers and rates, Proc. IEEE INFOCOM.

Cisco Systems, Inc. (2004). Data sheet of cisco aironet $802.11 \mathrm{a} / \mathrm{b} / \mathrm{g}$ wireless cardbus adapter, http: //www. winncom.com/pdf/Ciscoclient.pdf.

Cisco Systems, Inc. (2005). Cisco Aironet Wireless LAN Client Adapters Installation and Configuration Guide (Technical Specifications).

Ebert, J., Aier, S., Kofahl, G., Becker, A., Burns, B. \& Wolisz, A. (2002). Measurement and simulation of the energy consumption of an WLAN interface, Technical report, Technical University Berlin, Telecommunication Networks Group.

Feeney, L. \& Nilsson, M. (2001). Investigating the energy consumption of a wireless network interface in an ad hoc networking environment, Proc. IEEE INFOCOM.

Gao, Y., Zeng, Z. \& Kumar, P. R. (2010). Joint random access and power selection for maximal throughput in wireless networks, Proc. IEEE INFOCOM.

Gast, M. (2005). 802.11 Wireless Networks: The Definitive Guide, 2 edn, O’Reilly Media, Inc.

Guha, S., Chau, C. K. \& Basu, P. (2010). Green wave: Latency and capacity-efficient sleep scheduling for wireless networks, Proc. IEEE INFOCOM.

He, Y., Yuan, R., Ma, X., Li, J. \& Wang, C. (2007). Scheduled PSM for minimizing energy in wireless lans, Proc. IEEE ICNP.

Jeong, Y., Park, J., Ma, J. \& Kim, D. (2004). An enhanced power save mode for ieee 802.11 station in ad hoc networks, Proc. IFIP International Federation for Information, pp. 414-420.

Jung, E. \& Vaidya, N. H. (2002). An energy efficient MAC protocol for wireless LANs, Proc. IEEE INFOCOM.

Krashinsky, R. \& Balakrishnan, H. (2005). Minimizing energy for wireless web access with bounded slowdown, Wireless Networks 11: 135-148. 
Lee, J., Rosenberg, C. \& Chong, E. (2006). Energy efficient schedulers in wireless networks: design and optimization, Mobile Networks and Applications 11(3).

Lei, H. \& Nilsson, A. (2007). Queuing analysis of power management in the IEEE 802.11 based wireless lans, IEEE Transactions on Wireless Communications 6(4).

Lin, H., Huang, S. \& Jan, R. (2006). A power-saving scheduling for infrastructure-mode 802.11 wireless LANs, Computer Communications 29: 3483-3492.

Margi, C. (2006). Energy Consumption Trade-offs in Power Constrained Networks, PhD thesis, University of California Santa Cruz.

MATLAB Central (2003). IEEE 802.11a WLAN model, http://www.mathworks.com/ matlabcentral/fileexchange/3540.

MATLAB Central (2009). 802.11b PHY matlab code, http://www. mathworks.com/ matlabcentral/fileexchange/3213/.

Narseo, V. R., Pan, H., Jon, C. \& Andrew, R. (2010). Exhausting battery statisticsunderstanding the energy demands on mobile handsets, Proc. MobiHeld.

Nath, S., Anderson, Z. \& Seshan, S. (2004). Choosing beacon periods to improve response times for wireless HTTP clients, Proc. the ACM International Workshop on Mobility Management and Wireless Access (MobiWac).

Nedevschi, S., Popa, L. \& Iannaccone, G. (2008). Reducing networking energy consumption via sleeping and rate-adaptation, Proc. the 5th USENIX Symposium on Networked Systems Design and Implementation.

Nuggehalli, P., Srinivasan, V. \& Rao, R. (2002). Delay constrained energy efficient transmission strategies for wireless devices, Proc. IEEE INFOCOM.

Nuggehalli, P., Srinivasan, V. \& Rao, R. (2006). Energy efficient transmission scheduling for delay constrained wireless networks, IEEE Transactions on Wireless Communications $5(3)$.

Proxim Wireless Corporation (2006a). Data sheet of orinoco 11a/b/g combocard, http://www.proxim.com/downloads/products/cp/DS_0806_11a_b_ gComboCard_USHR.pdf.

Proxim Wireless Corporation (2006b). Data sheet of orinoco pci card, http: / / www . proxim . com/downloads/products/cp/DS_0806_11a_b_gPCI_USHR.pdf.

Qiao, D., Choi, S., Jain, A. \& Shin, K. (2003). MiSer: An optimal low-energy transmission strategy for IEEE $802.11 \mathrm{a} / \mathrm{h}$, Proc. ACM MOBICOM.

Qiao, D. \& Shin, K. (2005). Smart power-saving mode for IEEE 802.11 wireless lans, Proc. IEEE INFOCOM.

Rozner, E. \& Navda, V. (2010). Napman: Network-assisted power management for wifi devices, Proc. ACM MobiSys.

Simunic, T., Benini, L., Glynn, P. \& Micheli, G. (2000). Dynamic power management for portable systems, Proc. ACM MOBICOM.

Stemm, M. \& Katz, R. (1997). Measuring and reducing energy consumption of network interfaces in hand-held devices, IEICE Transactions on Communications E80-B(8): 1125-31.

Tan, E., Guo, L., Chen, S. \& Zhang, X. (2007). PSM-throttling: Minimizing energy consumption for bulk data communications in WLANs, Proc. IEEE ICNP.

Tarello, A., Sun, J., Zafer, M. \& Modiano, E. (2005). Minimum energy transmission scheduling subject to deadline constraints, Proc. IEEE WIOPT.

Tyan, H. (2002). Design, realization and evaluation of a component-based compositional software architecture for network simulation, http://www. j-sim.org/. 
Wang, J. F., Fang, Y. G. \& Wu, D. P. (2006). A power-saving multi-radio multi-channel mac protocol for wireless local area networks, Proc. IEEE INFOCOM.

Zeng, Z., Gao, Y. \& Kumar, P. R. (2011). Sofa: A sleep-optimal fair-attention scheduler for the power-saving mode of wlans, Proc. IEEE ICDCS.

Zhang, F. \& Chanson, S. (2003). Throughput and value maximization in wireless packet scheduling under energy and time constraints, Proc. IEEE RTSS. 


\title{
A Study on Design of Fiber-Reinforced Plastic (FRP) Tubes as Energy Absorption Element in Vehicles
}

\author{
Yuqiu Yang and Hiroyuki Hamada \\ Kyoto Institute of Technology \\ Matsugasaki, Sakyo-ku, Kyoto \\ Japan
}

\section{Introduction}

To date, the global automotive industry is arguably the largest and most complex undertaking in industrial history. However, where cars multiply twice as fast as people, the automobile accidents old as automobiles themselves increased correspondingly. A car accident is a road traffic incident which usually involves at least one road vehicle being in collision with which may result in injury, property damage, sometimes even death at serious situations. Up to now, road traffic injuries represent about $25 \%$ of worldwide injuryrelated deaths as the leading cause. Facing the transport safety problem, policymakers in government of all over the world are doing their efforts e.g. NHTSA of USA. On the other hand, the automakers are also putting their emphasis in the increasing of the production quality particularly their crashworthiness and crash compatibility. Till now, many products including bumper, seat belt, airbag, anti-lock braking system (ABS) are proved useful to secure the occupant from a collision or a sudden stop and therefore already required as the mandatory equipments. Additionally, for scientists and engineers, in the late of 1990's, a particular international conference and a journal publication on crashworthiness well known as ICRASH and IJCRASH were formed and provide them a platform to discuss and present their works in the field of structural crashworthiness and impact biomechanics. Besides safety, the automobile have another serious problem i.e. pollution. Most automobiles in use today are propelled by gasoline or diesel. When it runs on the road, it creates a lot of exhaust gas such as $\mathrm{NO}_{\mathrm{x}}$ that pollute the air and $\mathrm{CO}_{2}$ which is one major causes of global warming. With the increasing demands both for energy and environment protection, economic cooperation (ECO) cars which have high fuel efficiency therefore are desirable. It is considered that the improvement of the fuel efficiency can be realized by reducing the vehicle's weight. For a traditional metallic vehicle, however, it is difficult to realize both light weight and high crashworthiness. Therefore, people are considered use new materials system to instead of metal to manufacture next generation automobile. In the area, fiber reinforced composites (FRPs) are found to be attractive [1-28].

Research into the use of structural components such as bumper for energy management is not new. As a result, the energy absorbing characteristics of metals are fairly well understood. However, until the late 1970 's, attempts at understanding the energy absorbing capabilities 
and crushing mechanisms of FRPs have been made [1-4]. Most of the existing work on the energy absorption capability of FRPs has concentrated on the behaviors of the tubular specimens. In general, FRP tubes do not exhibit the ductile failure mechanism associated with metals. Instead, the brittle nature of most fibers tends to generate a brittle mode of failure. That is to say unlike metals, which absorb energy through plastic deformation, most FRP tubes are found to absorb energy through progressive crushing mode by a combination of multi microfracture and friction [5-10]. It is this fundamental difference which gives rise to the very distinct energy absorption characteristics of FRPs. As the above researched showing, the energy absorption capacity of FRP tubes is more attractive than metals. However, the adoption of composite as energy absorbing structural elements is limited at present. There are many main reasons. One of them is that the crushing performances and the energy absorption mechanisms of FRP tubes are complicated as compared to metal. Referring to the energy absorption capability, there are many important variables. Although a lot of researches have been done on the inhere materials (reinforced fiber, matrix and the bonding between them) [5, 10, 11-15] and the reinforcement form (roving, cloth, braiding, knitting, chopped, and so on) [16-24], there is still an urgent need of database from various composite tubes and a complete understanding of the energy management from the multi micro fractures. Secondly, the manufacturing cost of a FRP tube is rather higher than that of metal. Cost is considered to be the key to apply the FRP into practice. Therefore the effects on the cost down are always warm welcome. Additionally, research on actual application of composites is absent. Apart from the understanding of the energy absorption capacity of the FRP tubes, a reasonable design such as the geometry, length, located position and collapse trigger is required to transcend the experimental stage and cross over to true application studies. Therefore, current studies elaborate some experiments on this aspect. Three improvement methods including the design of the bending energy of the tube fronds and the design of the fiber fractures energy were proposed. Firstly, in this paper, the crushing behavior of FRP tube was linked with the appearance of the bending behavior of beam. Then mechanism model of a bending beam was used to simulate the bending fronds of FRP tube. Based on the founding that the bending energy is related directly to the geometry of transversal cross section, design of the bending energy though the design of the geometry of the FRP tubes was carried out. In details, a FRP tube with general square transversal cross section was designed to mimic the circular one through applying a big radius on the corners. Additionally, a special shape which utilizing both circular and square geometry in transversal cross section was designed.

Up to now, much of the preceding discussion on the energy absorption capability of FRP tubes had been carried out to investigate the effects of the internal components and the structures. At the same time, researchers have found that the energy management has been shown to be dependent on a number of external factors, for example, strain rate i.e. crushing speed and collapse trigger mechanism. The influence of strain rate would appear to be one of the most contentious issues relating to the energy absorption of FRPs [6,9,13,28,30-35]. As an energy absorption element in automobiles, the crushing performance and the energy management in dynamic condition is eagerly to be clarified. However, until now there is not a clear understanding about the relationship between the energy management and crushing speed. Some authors have reported increases in specific energy absorption (Es, i.e. the absorbed energy per unit mass of the material) with loading rate, others decreases. Farley [30] observed a rise in specific energy absorption of up to $35 \%$ for carbon-epoxy tubes over a range of crushing speeds between $0.01 \mathrm{~m} / \mathrm{s}$ and $12 \mathrm{~m} / \mathrm{s}$. However, the energy absorption of carbonepoxy tubes with a structure of $[0 / \pm \theta]^{2}$ was not found to be sensitive to crushing speed and 
showed no increase. He suggests that energy absorption dependence on crushing speed will be related to the energy absorbing mechanism which controls the crushing process. If the mechanism is a function of strain rate then energy absorption will be a function of crushing speed. Hull [6] supported results of the increased energy management under impact test and suggested that friction in the crush zone may be another reason why energy absorption varies with crushing speed. It has been found that frictional effects generate a considerable amount of heat and it is likely that the magnitude of energy absorbed by this mechanism will vary with loading rate. Conversely, Mamalis et al. $[28,31]$ report that the energy management of thick walled random chopped glass mat / polyester tubes exhibited a clear decrease under high speed crushing (up to $24 \mathrm{~m} / \mathrm{s}$ ). However, there was no clear evidence of such a decrease for thin-walled (<4 mm tubes). Similarly to Farley [1], Mamalis et al [28] attribute the differences in energy absorption under static and dynamic conditions to the changes in the crushing mechanisms. In particular, the resin was found to become increasingly brittle under elevated strain rates. Schmueser \& Wickcliffe [13] also report that energy management decreased in dynamic $(5-6 \mathrm{~m} / \mathrm{s})$ tests than in comparable static ones for carbon-epoxy, glass-epoxy and aramid-epoxy tubes. In some cases, dynamic energy absorption was only approximately $75 \%$ of equivalent static values. Although the above observations may at first sight appear contradictory, it should be recognized that they are all based on isolated examples of specific material and geometric configurations. As such, it is difficult to draw any overall conclusions. In general, the effect of strain rate on the energy absorption capability of a given composite structure is likely to be a complex function of the particular material system, fiber orientation, geometry employed and other external factors.

On the other hand, FRP tubes, no matter which components are contained, what configuration of the fibers is, or under which crushing speed, generally need a collapse trigger to trigger progressive crushing rather than a sudden catastrophic type of failure. Progressive crushing is a typical crushing performance of FRP materials in which the crushing load keeps almost constant during the whole compression process with a relative high value. However, before progressive crushing forms, there is another important stage, i.e. the initial crushing period, although the energy absorption during the initial period some times are ignored in rough calculation the $E s$ values. In the case of without any trigger, the crushing load of FRP tube increases linearly in initial compression stage. When the crushing load exceeds the critical strength of the whole tube, catastrophic failures occurs. Several previous studies have investigated such collapse trigger with some modification at one end of the tube $[3,5,36-39]$ and found that they performed well in initiating progressive crushing. The collapse trigger mechanism is to generated high stress concentration at the modified part from where the fracture is initiated or triggering before the load increase to the critical strength of the whole tube. Defects of the above triggers are involved extra cost and difficulty during the assembling procedure because of the angular edge.

\section{Foundation thinking for optimization design}

\subsection{Bending energy}

Based on the accident data analysis, it is known that about $70 \%$ accidents are from the full lap and offset impacts. Therefore, such a design of energy absorption structures is carried on in order to realize the minimum deformation of the cabin for saving the occupant's space and a small accelerate when vehicles are involved in crashes. In particular, a tubular component, termed "Front Side Member"(Fig. 1), is designed to be installed behind the 
bumper as a special kind of energy absorption element which can absorb most of the impact energy through the fracture of itself in the chapter in particularly.

For a FRP tube which was fractured through progressive crushing mode, the energy absorbing mechanism can be clarified from the observation on the axial cross sections through the crush zone. Based on the summarized the observation results from the above reference papers, it is found that FRP absorb energy by multi micro-fractures. A sample cited from [12, 25] is given in Fig.2. The tube wall was split into pieces of fronds and bend to both sides of the tube. A wedge of debris was formed by the fractured fiber and resin. Under the wedge of debris, a central crack propagated. In the bending fronds, the delaminations and the fiber fractures can be observed. Based on the observations, the total absorbed energy $\left(U_{T}\right)$ is considered using formulation (1).

$$
U_{T}=U_{\text {split }}+U_{c c}+U_{d e}+U_{b e n d}+U_{f f}+U_{f r}
$$

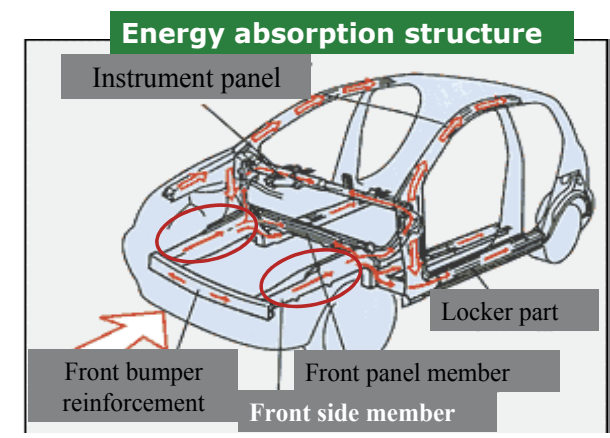

the deformation for saving the occupant's space $\longleftarrow$ absorb a lot of energy
the acceleration experienced by the vehicle during an impact $\longleftarrow$ progressively

Fig. 1. Schematic illustration of energy absorption structure in a vehicle illustrating the component of front side member

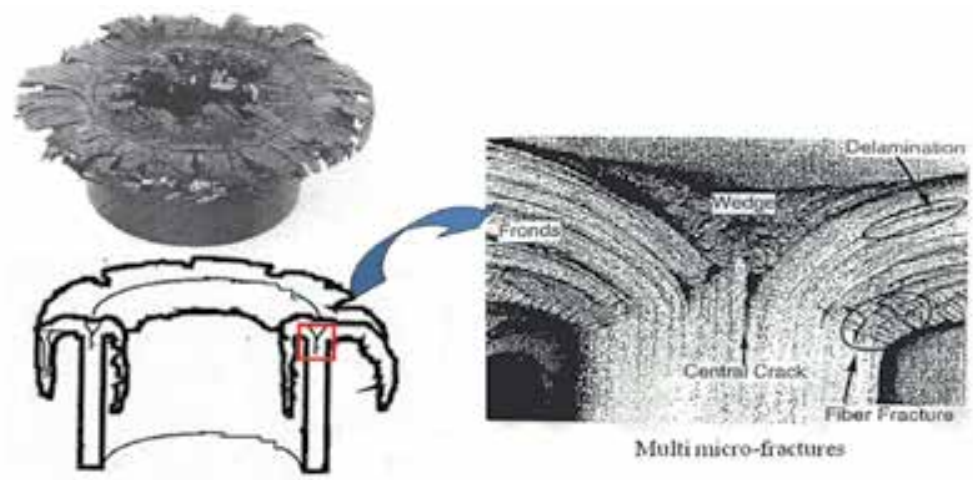

Fig. 2. Observation results on a carbon/PEEK circular tube illustrating multi micro fractures (cite from reference list $[12,25]$ )

Here, $U_{\text {split }}$ is the energy absorbed by splitting the integrated tube wall into pieces of fronds. $U_{c c}$ is the energy for initiation and propagation of the central crack; $U_{d e}$ is the energy for 
delaminations; $U_{b e n d}$ is the energy of the bending of fronds; $U_{f f}$ is the energy required for fiber fracture and $U_{f r}$ is the energy associated with friction. Because these fractures occur simultaneously and they correlate and affect each other, it is difficult to evaluate individual energy absorption contribution and construct a design criterion.

In these multi-micro fractures, it is noticed that the FRP tubes show the bending behavior as the essential performance. The propagation of the central crack or delaminations and the fracture of the fibers or resin are considered to be generated during the bending process. Therefore, a solution for the design of FRP tube's energy management was proposed though design $U_{\text {bend }}$. Here the bending frond is considered as a bending beam by an external force. The mechanism model for such a beam with a height of $l$ which is pulled by an external force $F$ is illustrated in Fig.3. The displacement along longitudinal direction is the $s$ and the $y_{\max }$ is the maximum distance in $y$ direction. The deformation energy i.e. the bending energy $U_{\text {bend }}$ can be express by formula 2 .

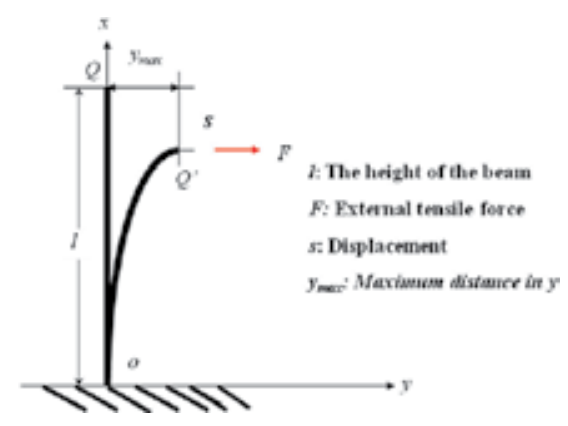

Fig. 3. Mechanism model for a beam which is pulled by an external force.

$$
U_{b e n d}=\int_{0}^{l} \frac{M^{2}(x)}{2 E I} d x
$$

Here $M$ is the bending moment of the beam ( $y$ trial) and $x$ is any point in $x$ direction from 0 to $s$ displacement. According to the relationship between external force $F$ and $y_{\max }$ and the relationship between displacement $s$ and maximum distance $y_{\max }$, the bending energy can be express by formula 3. For the given material (with a modulus $E$ and a height $l$ ) bent to a displacement compression (s), the bending energy $U_{b e n d}$ is affected directly by the inertia moment $(I)$. In a word, $U_{\text {bend }}$ is in a function of $I$.

$$
\begin{aligned}
\because U_{\text {bend }} & =\frac{F^{2}}{2 E I} \int_{0}^{l}(l-x)^{2} d x \\
& =\frac{F^{2}}{2 E I} \int_{0}^{l}(x-l)^{2} d x \\
& =\left.\frac{F^{2}}{2 E I} \cdot \frac{1}{3}(x-l)^{3}\right|_{l} ^{0} \\
& =\frac{F^{2} l^{3}}{6 E I}
\end{aligned}
$$

and

$$
F=-\frac{3 E I y_{\max }}{l^{3}}
$$




$$
\therefore \quad U_{\text {bend }}=\frac{3 E I\left(y_{\max }\right)^{2}}{2 l^{3}}=\frac{5 E \cdot I \cdot s}{2 l^{2}}
$$

An example about the effect of cross section geometry on $I$ is given in Fig.4. For a given material with the same section area $\left(A: 50 \mathrm{~mm}^{2}\right)$ and thickness $(t: 4 \mathrm{~mm})$, making the structure to have cross section geometry in corner or flat wall shape, different $I$ would be obtained. Here the calculation method of $I$ should be noted. In Fig.4(a), corner geometry is illustrated $\left(A\right.$ : cross section area of $50 \mathrm{~mm}^{2} ; R$ : radius of outside of corner $10 \mathrm{~mm} ; r$ : radius of inside of corner $6 \mathrm{~mm}$; $t$ : thickness $4 \mathrm{~mm}$ ). It is assumed to be split to two parts as outer and inner fronds during compression process along the broken line located in the middle of the thickness. The $I$ of the corner region, i.e. $I_{\text {corner }}$ can be calculated by formulae (4) and (5)

$$
\begin{gathered}
I_{\text {corner }}=I_{z c \text { (outer frond in corner geometry) }}+I_{z c \text { (inner fornd in corner geometry) }} \\
I_{z c \text { (outer/inner frond in corner geometry) }}=\frac{\pi+2}{16}\left(R^{4}-r^{4}\right)-\frac{8\left(R^{3}-r^{3}\right)^{2}}{9 \pi\left(R^{2}-r^{2}\right)}
\end{gathered}
$$

Here, $R$ and $r$ are the radii of the curvatures of the corner. Therefore, $r$ plus thickness $(t)$ amounts $R$. Additionally, for flat wall geometry (Fig.3b, A: cross section area of $50 \mathrm{~mm}^{2} ; t$ : thickness $4 \mathrm{~mm}$; $w$ : length $12.5 \mathrm{~mm})$, the I of flat wall, i.e. $I_{\text {flat wall }}$ also can be calculated by (6) and (7)

$$
\begin{gathered}
I_{\text {flat wall }}=2\left(I_{z c(\text { outer frond in flat wall geometry })}\right)=2\left(I_{z c \text { (inner fornd in flat wall geometry })}\right) \\
I_{z c \text { (outer } / \text { inner frond in flat wall geometry })}=\frac{1}{12} w t^{3}
\end{gathered}
$$

From the calculation results listed in the table in Fig.4(c), it is understood that $I_{\text {corner }}\left(40 \mathrm{~mm}^{4}\right)$ is bigger than $I_{\text {flat wall }}\left(16.8 \mathrm{~mm}^{4}\right)$, although they have same cross section area and thickness. According to formula (3), under the same displacement (s), big bending energy would be generated in the structure which has the transverse cross section in corner geometry.

From previous experiences [7, 26-29], it is reported that square or rectangular tubes are generally less effective at absorbing energy than a comparable circular tube. However, square or rectangular tubes have the geometrical advantage because their flat wall can assembled with other component easily. A pure circular tube can be considered as a combination of four pieces of corner geometry while a pure square or rectangular tube consists of four pieces of flat wall regions in cross section. $I$ of pure circular tube is quite different with that of a pure square tube even they have same cross section area and height. It is considered that much big $I$ in circular geometry is one of the reasons why circular tube have higher energy absorption capability as compared to square tube. Based on the above thinking, therefore, the following methods were proposed aiming improved the energy absorption capacity of square and rectangular tubes, which would be designed to have reasonable consisting of 4 pieces of flat wall and 4 corners in the transversal cross section. 


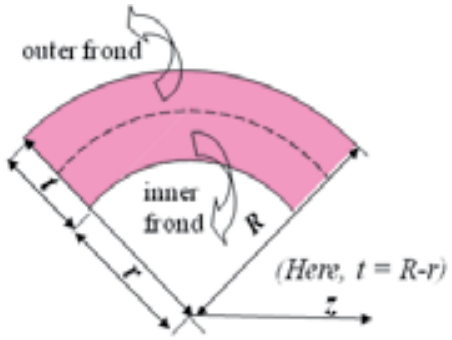

(a) Comer

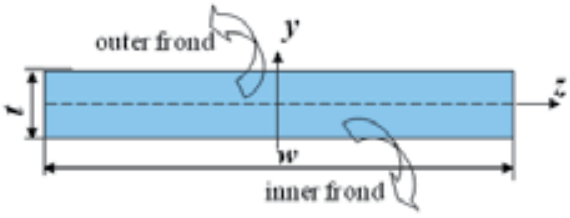

(b) Flat wall

(c) Calculation results

$$
\begin{aligned}
& \text { Example: } \\
& R=10 \mathrm{~mm} \\
& r=6 \mathrm{~mm} \\
& t=4 \mathrm{~mm} \\
& A_{\text {comet }}=50 \mathrm{~mm}^{2} \\
& A_{\text {Aut }}=50 \mathrm{~mm}^{2}
\end{aligned}
$$

\begin{tabular}{|c|c|c|c|c|}
\hline & parameters & outer frond & inner frond & Total \\
\hline \multirow{3}{*}{ corner } & inertial moment $\left(\mathrm{mm}^{4}\right)$ & 25.6 & 14.4 & 40 \\
\cline { 2 - 5 } & area $\left(\mathrm{mm}^{2}\right)$ & 28.3 & 22.0 & 50 \\
\hline \multirow{2}{*}{ flat wall } & inertial moment $\left(\mathrm{mm}^{4}\right)$ & 8.4 & 8.4 & 16.8 \\
\cline { 2 - 5 } & area $\left(\mathrm{mm}^{2}\right)$ & 25.1 & 25.1 & 50 \\
\hline
\end{tabular}

Fig. 4. Calculation of Inertial moments $(I)$ of different geometries (A: cross section area; R: radius of outside of corner; $r$ : radius of inside of corner; $t$ : thickness)

\subsection{Fiber fracture energy}

Many researches indicated that FRP tubes absorb energy by multi micro-fractures. During the initial compression stage, a wedge of debris was formed by the fractured fiber and resin. Under the wedge of debris, a central crack propagated. Then the tube wall was split into pieces of fronds and bend to both sides of the tube (two-side-bending) along the central crack. During the bending process, delaminations and the fractures of both fiber and resin generate simultaneously. These fractures occur at the same time and they correlate and affect each other, which lead to the complication in designing the energy management. In this study, based on the consideration that the energy absorbed by fiber fractures $\left(U_{f f}\right)$ can contributed to the total absorbed energy $\left(U_{T}\right)$ significantly, an attempt to design of $U_{f f}$ is carried out. From mechanism fracture theory, it is known that fiber fracture is affected by stresses $(\sigma)$ directly. Therefore, a method to increase $\sigma$ of the fronds during bending process was proposed as illustrated in Fig.5. According to equation 8, an increased thickness and a small bending curvature are helpful to obtain a high $\sigma$.

$$
\sigma=\frac{E t}{2 r^{\prime}}
$$

Here, $E$ is the modulus of the composite. $t$ and $r^{\prime}$ are the thickness and bending curvature of the bending wall, respectively. The max magnitude of these stresses $(\sigma)$ can be obtained on the surfaces layers in particular bottom layers of the bending tube wall, where the radius of curvature might be smallest and the thickness largest.

Usually, the FRP tube wall, with the above mentioned collapse triggers, is split to two parts and through two-side-bending mode under the flat compression plate of testing machine to 
absorb energy. At that case, before the delamination occurs, $t$ is half of the tube wall. If some device can force the tube wall to be bent towards only one way (one-side-bending mode), a double thicker thickness of the frond is possible to be achieved as compared to that in twoside-bending mode. At the same time, small bending curvature could be realized through design the device. Therefore, in this study, connected with the aim of design of bending stress, device, as a new collapse trigger mechanism, is proposed. Here in this antecedent foundation investigation, the FRP tube with the transversal cross section geometry in circular and square are focused.

For the circular FRP tubes, the devices shown in Fig.6(a) are of four kinds (C-Inner 3, C-Inner 5, C-Outer 3, and C-Outer 5). The concave part of Inner type is in circular geometry with a diameter of $55 \mathrm{~mm}$ while that of the convex part in Outer type device is $50 \mathrm{~mm}$. Specially, a radius $\left(R^{\prime}\right)$ of 3 or $5 \mathrm{~mm}$ was modified around the circular shoulder of concave or convex part in each kind of device where the top end of the FRP tube touches the device. That is to say, the collapse of FRP tube is expected to be triggered from the $R^{\prime}$ region. On the other hand, S-Inner 2 (Fig.6b) was made for square FRP tube. The concave part is in a square transversal cross section with a size of $50 X 50 \mathrm{~mm}^{2}$. Similarly, a radius of $2 \mathrm{~mm}\left(R^{\prime} 2\right)$ was chamfered on the concave part where the square end of the FRP tube contacts with it.

$$
\begin{array}{r}
\mathbf{U}_{\mathbf{T}}=\mathbf{U}_{\text {split }}+\mathbf{U}_{\mathbf{c c}}+\mathbf{U}_{\mathbf{d e}}+\mathbf{U}_{\text {bend }}+{\widehat{\mathbf{U}_{\text {ff }}}}_{U}+\mathbf{U}_{\text {fr }} \\
U_{f f}=f(\sigma) \\
\text { Design of } \sigma \\
\sigma=\frac{E t}{2 r}
\end{array}
$$

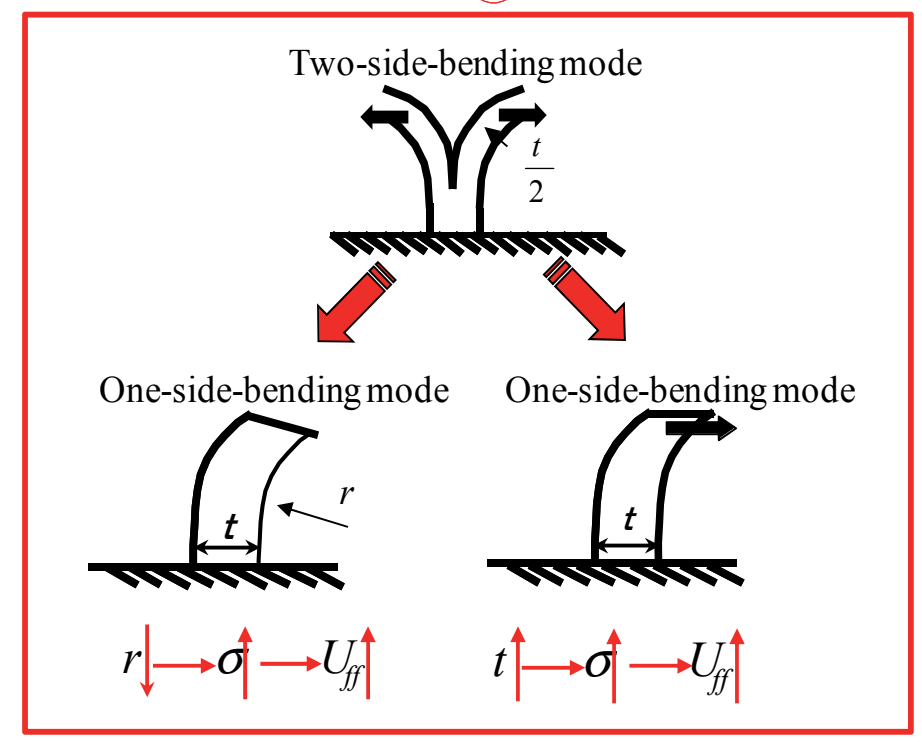

Fig. 5. Design method of fiber fracture energy 


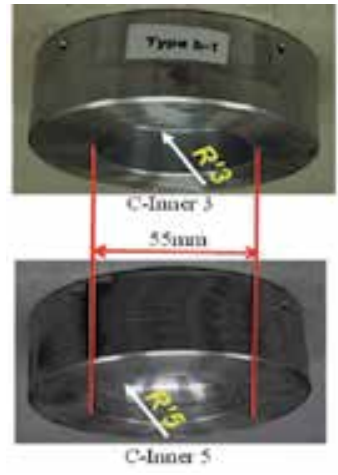

(a) four kinds of devices for circular FRP tubes

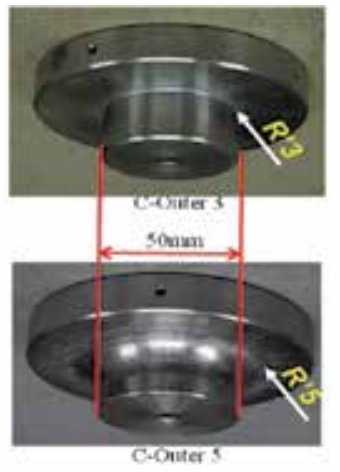

(b) S-Inner 2 devices for square FRP tubes

Fig. 6. Photographs of devices (a):four kinds of devices for circular FRP tubes illustrating the diameters of concave or convex part and the radius on the shoulder; (b) S-Inner 2 devices for square FRP tubes illustrating the size of the concave part $\left(50 \times 50 \mathrm{~mm}^{2}\right)$ and the radius on the shoulder.

\section{Mimic of square to circular}

\subsection{Material and experiment}

Metal mandrels with a rectangular transversal cross section of $36 \mathrm{mmX} 24 \mathrm{~mm}$ were used to fabricate the FRP tubes with a shape of rectangular in the transversal cross section. In order to investigate the effect of design of $I$ of FRP tubes, two kinds of mandrels ( $\mathrm{r} 3$ and $\mathrm{r} 9$ mandrels) are employed, where a radius of $3 \mathrm{~mm}(\mathrm{r} 3)$ or $9 \mathrm{~mm}(\mathrm{r} 9)$ was modified on the corners of the mandrel respectively.

Referring to the reinforcement form of FRP specimens in the mimic square to circular method experiments, 2.5D braids fabricated by an experimental braiding machine (Murata machinery, Ltd) were adopted. Carbon fiber (T-300 for braiding yarns and T-1000 for middle-end-fiber from Toray industries, INC.) and Epoxy (XNR 6805 from Nagase ChemteX Corporation) were used as reinforcement and matrix. 96 of the braiding yarns and 40 of middle-end-fibers were fabricated to form each layer of 2.5D braided structure given in Table 1.

The fabrication process of the braided performs includes:

1. Fabricate the preforms on the above metal mandrels according to the fiber architecture listed in Table 1.

2. Secondly, an additional braided layer was fabricated on the outside of the above preforms in order to retain the shape during subsequent impregnation process. (The additional braided layer was fabricated by traditional braiding machine with 48 bundles of braiding yarn without middle-end-yarns in a braiding angle of 60.)

3. Then these braids were impregnated with Epoxy resin by Vacuum Assisted Resin Transfer Molding process (VARTM). Finally, they were cured in an oven at $80^{\circ} \mathrm{C}$ for 8 hours. After cool naturally, the FRP composite tubes are drawn from the mandrels.

Depending on the mandrel shape, the carbon/epoxy braided composite tubes were divided into two groups. $\mathrm{r} 3$ group tubes, braided on the $\mathrm{r} 3$ mandrel, comprised of two different braid architectures named as r3-45 and r3-18 (45 and 18 are the value of the braiding angle). Here the braiding angle is the angle between the longitudinal axes and the braiding yarn. The $\mathrm{r} 9$ group tubes consisting of $\mathrm{r} 9-45$ and $\mathrm{r} 9-18$ were fabricated on R9 mandrel with the similar fiber braided architectures as r3-45 and r3-18. 
The specifications of the fabricated composite tubes are summarized in Table 1.These CFRP tubes with a fiber volume fraction of about $50 \%$ were segmented into individual specimens with a length of $50 \mathrm{~mm}$. In order to initiate progressive crushing, one end of each specimen was chamfered to a taper with a 45 degree angle shown in Fig.7. Specially, the transversal cross section of $\mathrm{r} 3$ and $\mathrm{r} 9$ specimens are compared together to illustrate the difference in the geometry on the corners.

Quasi-static tests were performed on an INSTRON (4206) universal testing machine at a constant crosshead speed of $5.0 \mathrm{~mm} / \mathrm{min}$. The composite tubes were axially crushed between parallel steel flat platens. Three replicate tests were conducted for each kind of braided composite tubes to verify the stability of the energy absorption capability. The experiment commenced when the compression platen touched smoothly the chamfered taper. Every second, five data points were recorded to follow the track of the load during the compression procedure.

\begin{tabular}{|c|c|c|c|c|c|c|c|}
\hline & & \multicolumn{2}{|c|}{ Preform } & \multicolumn{4}{|c|}{ FRP tubes (2.5D braided Carbon/Epoxy) } \\
\hline & specimen & $\begin{array}{l}\text { Braiding } \\
\text { angle }^{\circ} *\end{array}$ & $\begin{array}{l}\text { Number } \\
\text { of layers }\end{array}$ & $\begin{array}{c}\text { Thickness of long } \\
\text { flat wall }(\mathrm{mm})\end{array}$ & $\begin{array}{c}\text { Thickness of short } \\
\text { flat wall }(\mathrm{mm})\end{array}$ & $\begin{array}{l}\text { Thickness of } \\
\text { corner (mm) }\end{array}$ & $\begin{array}{r}\text { Density } \\
\left(\mathrm{g} / \mathrm{cm}^{3}\right) \\
\end{array}$ \\
\hline \multirow{2}{*}{$\begin{array}{l}\text { r3 } \\
\text { group }\end{array}$} & r3-45 & 45 & 2 & 2.2 & 2.7 & 2.4 & 1.33 \\
\hline & r3-18 & 18 & 3 & 2.8 & 2.6 & 2.3 & 1.28 \\
\hline \multirow{2}{*}{$\begin{array}{l}\mathrm{r} 9 \\
\text { group }\end{array}$} & r9-45 & 45 & 2 & 2.3 & 2.9 & 2.7 & 1.20 \\
\hline & r9-18 & 18 & 3 & 2.7 & 2.3 & 2.5 & 1.29 \\
\hline
\end{tabular}

(Braiding angle*: the angle between the longitudinal axes and the braiding yarn)

Table 1. Specifications of carbon 2.5D braided preforms and FRP tubes which are with a rectangular transversal cross section

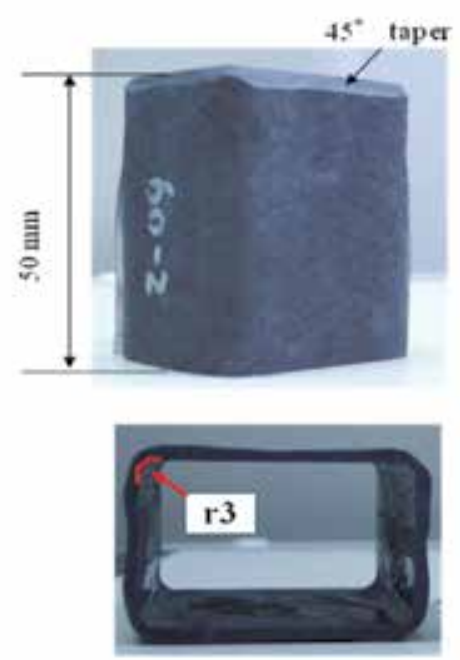

(a) Fabricated on $\mathrm{r}$ mandrel
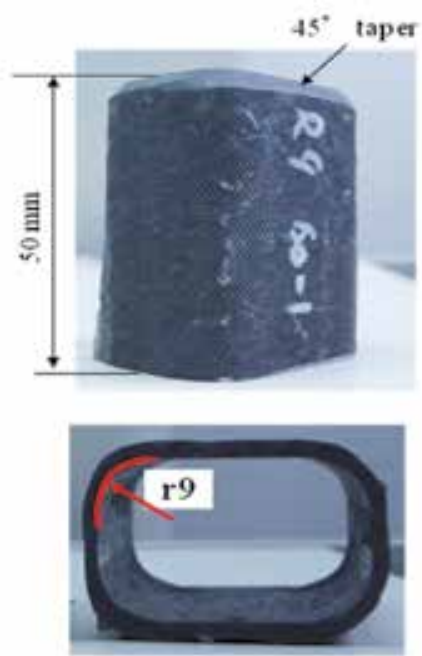

(b) Fabricated on 19 mandrel

Fig. 7. Carbon fiber/Epoxy 3D braided composite tubes, illustrated the length, taper with a 45 degree angle, and corner geometry. 


\subsection{Results and discussion}

At the initial compression stage, the taper was compressed and crushed to the inner side of the tube. With the advancement of the compression platen, the tube wall was mainly split into 4 parts along the flat wall. And each split part was bent towards both sides of the tube known as external and internal fronds. Under the compression, the split tube wall was delaminated to pieces furthermore. During the crushing procedure, a noise, that seemed to emanate from crack propagation and fractures, was heard. After approximately $30 \mathrm{~mm}$ crushing, compression was stopped and the compression platen was returned back. The fronds sprang back with the relaxation of the compression in a way. It can be seen from Fig.8 that a wedge of debris consisting of the crushed resin and fibers formed on the top and between the external and internal fronds.

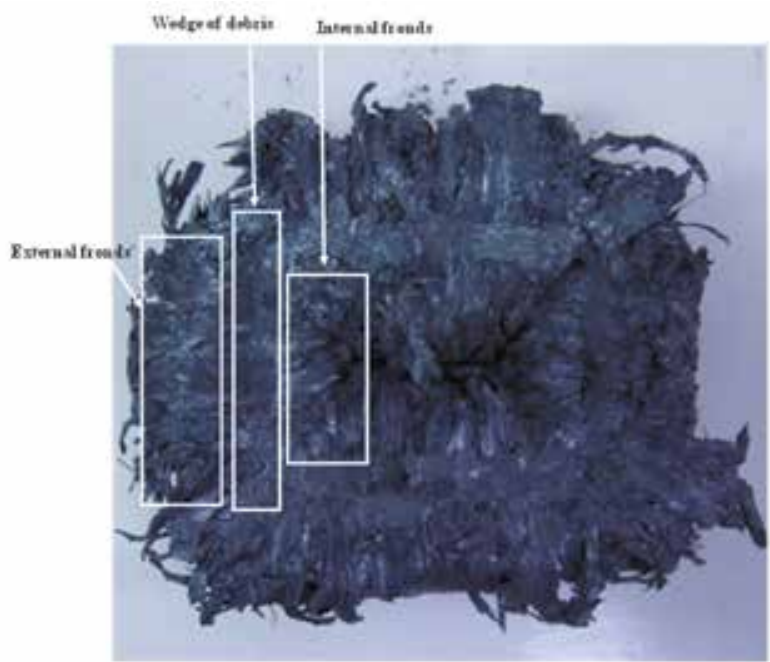

Fig. 8. Top view of the specimen after axial quasi-static compression test at a constant speed of $5 \mathrm{~mm} / \mathrm{min}$. The tube was split into many parts along the flat wall and each split part includes internal and external fronds.

In order to find the difference in the crushing behavior between the $\mathrm{r} 3$ and $\mathrm{r} 9$ group tubes, the load-displacement curves of both groups are compared in Fig. 9 under the same braiding angle. Here, the load per unit cross section (crushing stress) is used as the longitudinal axes to reduce the influence of thickness. For all of the specimens, a common feature of load during compression process is that the loads rapidly increased to peak at the initial stage and then show the characteristics of the progressive crushing mode. From these figures, it could be seen that the specimens fabricated on $\mathrm{R} 9$ mandrel achieved a relatively more stable crushing performance with a higher average stress in both of the braided texture structures. The parameters of energy absorption capability, which were calculated from the above loaddisplacement curves, are summarized in Fig.10. Specific energy absorption $(E s)$, defined as the absorbed energy per unit mass of the crushed material, is employed to evaluate the energy absorption capacity for both groups of tubes, which is often used in the automotive industry when studying the energy absorption. Among both $\mathrm{r} 3$ and $\mathrm{r} 9$ group tubes, the Es values were increased with the decrease in braiding angle from $45^{\circ}$ to $18^{\circ}$. With the braiding angle decreasing from $45^{\circ}$ to $18^{\circ}$, the main fiber orientation is being more and more close to the axial. Longitudinal fibers sustain the axial compressive load effectively. Therefore, the 
mechanical property in axial was enhanced in the tubes with a small braiding angle. Additionally, the values of $E s$ of the $\mathrm{r} 9$ group tubes were higher as compared to that of the r3 group tubes under the same fiber architecture i.e. R9-45 and r9-18 attained about $18 \%$ and $10 \%$ higher Es than that of r3-45 and r3-18, respectively.
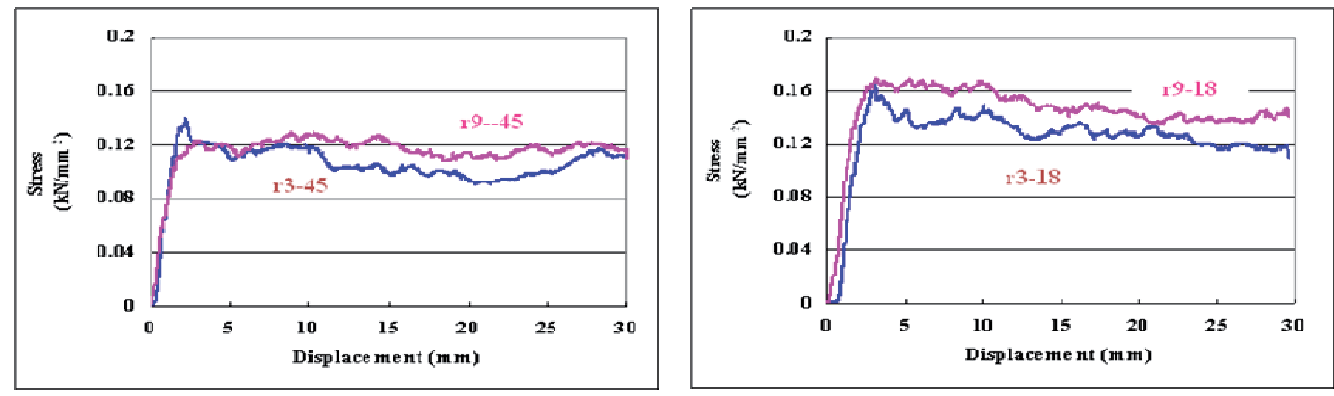

Fig. 9. Typical load-displacement curves of $\mathrm{r} 3$ and $\mathrm{r} 9$ specimens (Here, the stress is used as the longitudinal axes to reduce the influence of thickness) Obviously, r9 specimens obtained higher crushing stress during the crushing process as compared with r3 specimens under the same braiding angle i.e. same braiding structure.

\begin{tabular}{|c|c|c|}
\hline specimen & $\begin{array}{c}\text { Cross section } \\
\left(\mathrm{mm}^{2}\right)\end{array}$ & $\begin{array}{c}\text { Es } \\
(\mathrm{kJ} / \mathrm{kg})\end{array}$ \\
\hline r3-45 & 300 & 78.4 \\
\hline r9-45 & 301 & 92.6 \\
\hline r3-18 & 325 & 96.6 \\
\hline r9-18 & 304 & 106.3 \\
\hline
\end{tabular}

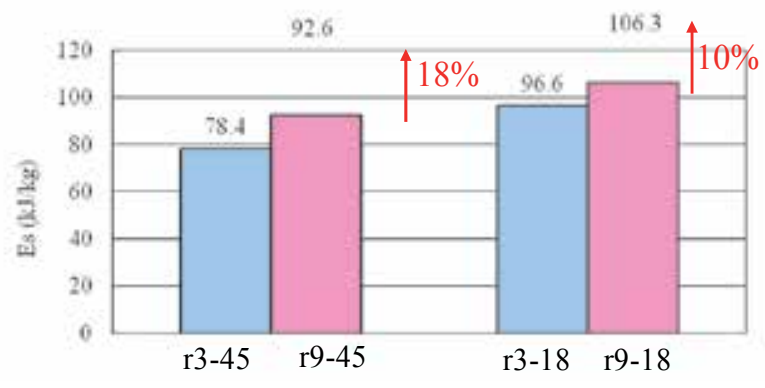

Fig. 10. Comparison of specific energy absorption between $\mathrm{r} 3$ and $\mathrm{r} 9$ specimens

As illustrated in Fig. 11, with the design of the corner, the geometry of the FRP tubes changed. Here, according to the fracture fashion the FRP tubes, the tube was separated into 8 parts consisting of 2 pieces of long flat walls, 2 pieces of short flat walls and 4 pieces of corners. The $I$ of each part ( $I_{\text {long flat wall }}, I_{\text {short flat wall }}$ and $I_{\text {corner }}$ ) was calculated separately to get a total $I$ of the FRP tube i.e. $I_{\text {total }}$.

$$
I_{\text {total }}=4\left(I_{\text {flat wall }}+I_{\text {corner }}\right)=2\left(I_{\text {long flat wall }}+I_{\text {short flat wall }}\right)+4\left(I_{\text {corner }}\right)
$$

Here, $I_{\text {long flat wall }}, I_{\text {short flat wall }}$ and $I_{\text {corner }}$ are $I_{z c}$ of the long flat wall, short flat wall and corner respectively calculated based on formulas $(4 \sim 7)$. For corner part, $r$ (inner radius of corner) is considered as $3 \mathrm{~mm}$ or $9 \mathrm{~mm}$ according to the used metal mandrel's shape. In this case, while $R$ (outside radius of corner) is measured from each specimen, because it is found that the thickness of long flat wall is different with that of short flat wall as shown in Table 
1. The length $(w)$ of long flat wall or short flat wall in $r 3$ group tubes is $36-(2 \times 3)$ i.e. 30 or $24-$ $2 \times 3$ i.e. 18. Similarly, $w$ of long flat wall or short flat wall in $\mathrm{r} 9$ group specimens is 18 or 6 accordingly.

The detailed calculation results are summarized in the table in Fig.11. For $\mathrm{r} 9$ specimens, all of $I_{\text {flat wall }}$ were decreased a little with an amount of $5.32 \mathrm{~mm}^{4}$ overall as compare to the $\mathrm{r} 3$ specimens. However, the increase of $I_{\text {corner }}\left(144.12 \mathrm{~mm}^{4}\right)$ was too much than the decease part of flat wall region in $\mathrm{r} 9$ specimen. Therefore, $I_{\text {total }}$ of $\mathrm{r} 9$ specimen is much higher than that of $\mathrm{r} 3$ specimen. According to formula (3), high $I$ means high $U_{\text {bend }}$. It is considered that the increased $U_{\text {bend }}$ contributed to the total absorbed energy directly or indirectly which led to a higher Es in $\mathrm{r} 9$ speicmens.
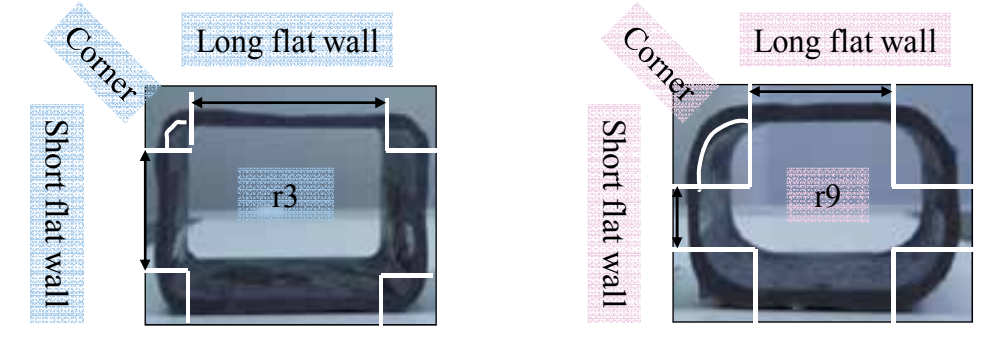

\begin{tabular}{|c|c|c|c|c|c|}
\hline & & $\boldsymbol{I}_{\text {Long flat wall }}$ & $\boldsymbol{I}_{\text {Short flat wall }}$ & $\boldsymbol{I}_{\text {Corner }}$ & \multirow{2}{*}{$\boldsymbol{I}_{\text {total }}$} \\
\hline \multirow{2}{*}{$\mathrm{r} 3$} & Outer & 4.32 & 2.59 & 2.51 & \multirow{2}{*}{43.08} \\
\cline { 2 - 6 } & Inner & 4.32 & 2.59 & 1.35 & \\
\hline \multirow{2}{*}{$\mathrm{r} 9$} & Outer & 3.22 & 1.03 & 23.38 & \multirow{2}{*}{176.56} \\
\cline { 2 - 6 } & Inner & 3.22 & 1.03 & 16.51 & \\
\hline
\end{tabular}

Fig. 11. Parameter about the geometry of each part of R3 and R9 specimens.

\section{The combining of both circular and square}

\subsection{Materials and experiments}

The mandrel was designed into three parts (Fig. 12a) i.e. pure circular tube part, cone part and general square tube parts. The beginning circular tube part is for high-efficient energy absorption capability. The end square tube part is to conveniently assemble with other components in assembling process in the automobile manufacture. And a gradual cone part as a joint part between circular and square parts. The mandrel is approximate $400 \mathrm{~mm}$ long, in which the circular tube part is about $250 \mathrm{~mm}$ long, the cone part is about $25 \mathrm{~mm}$ and the square tube is about $125 \mathrm{~mm}$. The diameter of circular tube part and the side length of the square tube part are $50 \mathrm{~mm}$. On the corners of the square tube part, there is a radius of $9 \mathrm{~mm}$. In addition, in order to combine the circular and square parts smoothly, there are some modifications on the cone part. That is to say the cone part is not with a cure cone shape. Here, the study at present is concentrated in both circular and square tube parts only.

Concerning about the fabrication process of preforms, firstly, 48 braiding yarn and 24 middle-end-fibers of Carbon fibers as reinforcement material were used to fabricate braided preforms on the above new designed mandrel by a braiding machine (Murata machinery, 
Ltd). With different braiding architecture, three kinds of prefoms (Type 15-15; Type 15-60 and Type 60-60) are made. The former and later numbers represent the braiding angles in the circular part and square part respectively. In details, in Type 15-15 and Type 60-60, the braiding angles are the same in each parts of the tube, i.e. 15 degree or 60, was applied in the whole tube. However, In Type15-60, 15 degree of braiding angle was applied in the circular tube part firstly. When going to the cone area, decrease the moving speed of both bundle and pultrusion of fabricated braids to fabricate the braids with a 60 degree braiding angle in square tube parts. Those fabricated carbon fiber braided preforms i.e. Type 15-15; Type 1560 and Type 60-60 are compared in Fig. 12b to show the difference of braided structure in circular and square parts. Such braiding process was repeated 4 times to accumulate 4 layers in order to get a suitable thickness of braids. Then, a skin braided layer mentioned in the above sections was fabricated on the most-outside of all of preforms in order to retain the shape during the impregnation process. Finally, the preforms were impregnated with Epoxy resin (XNR 6805 from Nagase ChemteX Corporation) by VARTM and were cured in an oven at $80^{\circ} \mathrm{C}$ for 10 hours.

The braided composites were drawn out from the mandrel and cut into approximate $300 \mathrm{~mm}$ long specimens as shown in Fig.13. Similar to the mandrel shape, the specimens have $200 \mathrm{~mm}$ in circular tube part; $25 \mathrm{~mm}$ in cone part, and $75 \mathrm{~mm}$ in square tube part. The specifications of the specimens are given in Table 2. Because the change of the shape, the geometry and area of cross section is changed from the circular tube part, cone tube part to square tube part. Apart from the shape's change, the density also changed because of the change of braiding architecture. Therefore, one piece of the circular tube part and square tube part were segmented to measure the weight and thickness in order to get the density of both parts in each specimen. The fiber volume fraction of all specimens was about $50 \%$. Additionally, a $45^{\circ}$ taper was chamfered at top end of the circular tube part of each specimen before compression axially in order to initiate progressive crushing.

An INSTRON 4206 universal testing machine with the maximum load cell of $100 \mathrm{kN}$ was employed in quasi-static compressions. The composite tubes were crushed between parallel steel flat platens from the circular tube part at a constant speed of $50 \mathrm{~mm} / \mathrm{min}$. A 50points/second data sampling rate was chosen to record the track of the load during the whole crushing process.

\subsection{Results and discussion}

For Type 15-15, the braided FRP specimen was crushed in a splaying mode as an example shown in Fig. 14. The crushed tube wall was split into pieces and bent towards both inside and outside of the tube like a splaying flower during the whole crushing process. From the load-displacement curve of Type 15-15 shown in Fig.14, it could be said that the braided composite tube was crushed in progressive crushing because their crushing load fluctuated with a small oscillation particularly in circular tube part. However during the crushing process through the cone and square tube parts, the load drops twice from $85 \mathrm{kN}$ to $50 \mathrm{kN}$. On the other hand, for Type 15-60 (Fig. 15), crushing fashion is similar to the former one, i.e. many splitting are formed and bend to both sides of the tube wall. The different crushing performance between Type 15-15 and Type 15-60 is concentrated in the period during crushing of the cone part and square tube part. It is obvious that the load curve of Type 15 has dropped twice (from $85 \mathrm{kN}$ to $50 \mathrm{kN}$ ) while it did not occur in Type 15-60. On the contrary, the load of Type 15-60 show an increase trend during the crushing period from cone tube part to square tube part. For Type 60-60, there is quite different crushing 


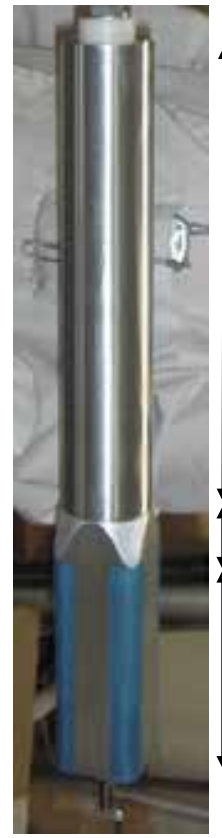

Circular Part

Diameter:50mm

Height: over $250 \mathrm{~mm}$

\section{Conepart}

About $25 \mathrm{~mm}$

Square Part

Side length: $50 \mathrm{~mm}$

Height: About $125 \mathrm{~mm}$

Radius on corner:9mm

(a)mandrel

Type 60-60

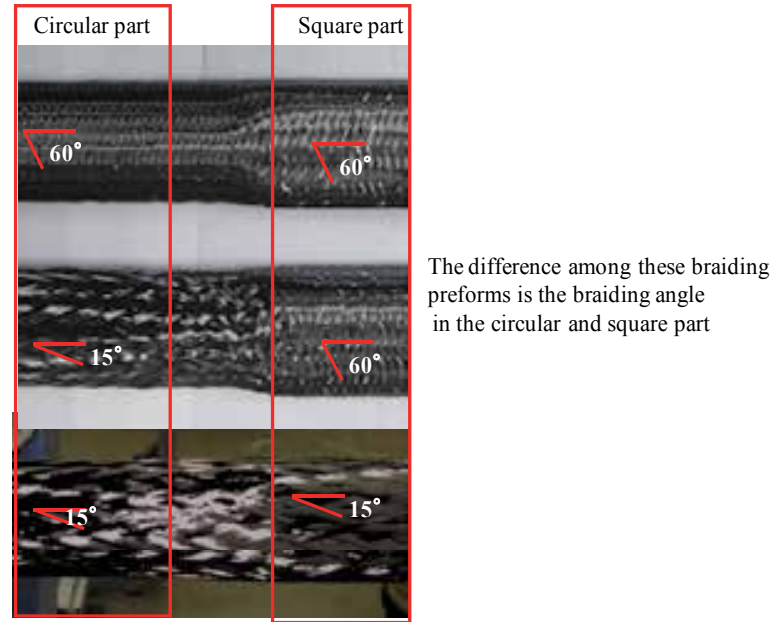

(b) Carbon fiber braided performs

Fig. 12. (a) a mental mandrel with a novel three-phases geometry: circular part of $250 \mathrm{~mm}$, cone part of $25 \mathrm{~mm}$ and square part of $125 \mathrm{~mm}$; (b) Carbon fiber braided performs of Type $15-$ 15; Type 15-60 and Type 60-60 showing the difference in braided angle between circular part and square part.

performance. When the compression commences, fracture initiates from the taper region. Then the tube wall was crushed into many fragments which is different with the splitting fronds in the above two specimens. When the tube was compressed to the displacement of 
$50 \mathrm{~mm}$, it can be clearly observed that buckling fracture generated under these fragments (Fig. 16 (a)). When the tube was compressed to the placement of $100 \mathrm{~mm}$, serious buckling fractures occurred in the cone tube part (Fig.16(b)) and the load decreased rapidly. It is considered that the specimen of 60-60 did not fracture in a stable progressive crushing mode.

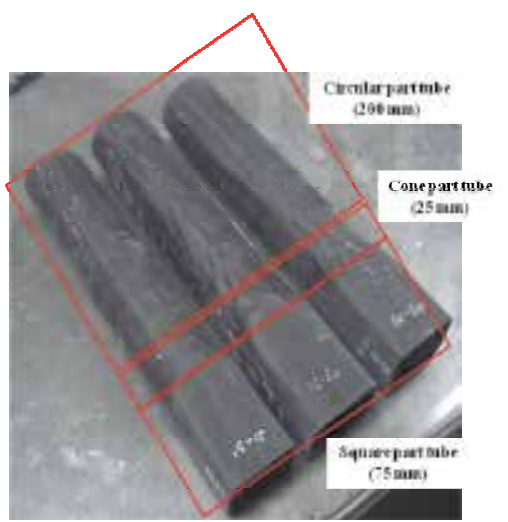

Fig. 13. Fabricated carbon braided FRP tubes with a novel three-phases geometry: circular part of $200 \mathrm{~mm}$, cone part of $25 \mathrm{~mm}$ and square part of $75 \mathrm{~mm}$

\begin{tabular}{|c|c|c|c|c|c|c|c|c|}
\hline & \multicolumn{3}{|c|}{ Circular tube part } & \multicolumn{3}{|c|}{ Square tube part } & \multirow{2}{*}{\begin{tabular}{|c|} 
Weight of the \\
whole \\
specimen $(\mathrm{g})$
\end{tabular}} & \multirow{2}{*}{$\begin{array}{c}\text { Height of the } \\
\text { whole specimen } \\
(\mathrm{mm})\end{array}$} \\
\hline & $\begin{array}{c}\text { Thickness } \\
(\mathrm{mm})\end{array}$ & \begin{tabular}{|c|}
$\begin{array}{c}\text { Cross section } \\
\left(\mathrm{mm}^{2}\right)\end{array}$ \\
\end{tabular} & $\begin{array}{l}\text { Density } \\
\left(\mathrm{g} / \mathrm{cm}^{3}\right)\end{array}$ & \begin{tabular}{|c} 
Thickness \\
$(\mathrm{mm})$
\end{tabular} & \begin{tabular}{|c|} 
Cross section \\
$\left(\mathrm{mm}^{2}\right)$
\end{tabular} & $\begin{array}{l}\text { Density } \\
\left(\mathrm{g} / \mathrm{cm}^{3}\right)\end{array}$ & & \\
\hline Type 15-15 & 2.55 & 420.8 & 1.51 & 2.24 & 429.2 & 1.51 & 179.6 & 300.5 \\
\hline Type 15-60 & 2.56 & 421.7 & 1.50 & 2.87 & 555.5 & 1.57 & 204.6 & 300 \\
\hline Type 60-60 & 3.52 & 587.6 & 1.57 & 2.92 & 570.5 & 1.57 & 259.4 & 299 \\
\hline
\end{tabular}

Table 2. Specification of specimens (carbon braided FRP tubes with novel three-phases geometry)

In this case, specific energy absorption i.e. Es was calculated from the mean crushing load according to the below simplified calculation formula (10)

$$
\text { specific energy absorption }(E s)=\frac{W}{A \cdot s \cdot \rho} \approx \frac{\bar{P} \cdot s^{\prime}}{A \cdot s \cdot \rho}=\frac{\bar{P}}{A \cdot \rho}
$$

Where, $W$ is the work done i.e. the total absorbed energy, $A$ is the transverse cross sectional area of the tube, $s$ is the crush displacement, $\rho$ is the density of the material, and $\bar{P}$ is the average load during progressive crushing, $s^{\prime}$ is the approximate crushing displacement $s$ which ignore the displacement during the initial crushing period.

For Type 15-15 and Type 15-60 which had progressive crushing performance, their Es values of both circular and square tube parts based on formula (10) were calculated and list in Table 3. (As mentioned before, the study at present is concentrated on both circular and square parts. Additionally in this new designed geometry, the cone part is not a strict cone in mathematics. In order to simplify discussion, the discussion on cone region is omitted.) Compared to Type15-15, the mean crushing load and Es of Type 15-60 in the circular tube 
part did not show difference, i.e. the energy absorption capability of Type 15-15 and Type 15-60 are similar in the circular tube part. However, referring to the square tube part, in Type 15-60, the load kept almost stable during the whole crushing process from circular tube part to cone and square tube part in Type 15-60. While there were two load-downs from $85 \mathrm{kN}$ to $50 \mathrm{kN}$ in Type $15-15$. Additionally, the decrease of square tube part compared to the circular tube part in Type $15-15$ is $18 \%$ while that is $13 \%$ in Type $15-60$. Therefore, braided FRP with the specific transversal geometry can be enhanced with appropriate braiding texture design. It is considered that with a big braiding angle of 60 degree in the cone part, the main fiber orientation is close to circumference. Axial fibers can sustain the axial compressive load effectively but hard to prevent propagation of the longitudinal central crack. On the contrary, circumferential fiber can prevent the spreading out of the axial fibers until shearing fractures occur. Thus, when crushing the cone part and square tube part, cracks seemed controlled in a way in Type 15-60 which resulted in no apparent drop of the load and higher energy management consequently.

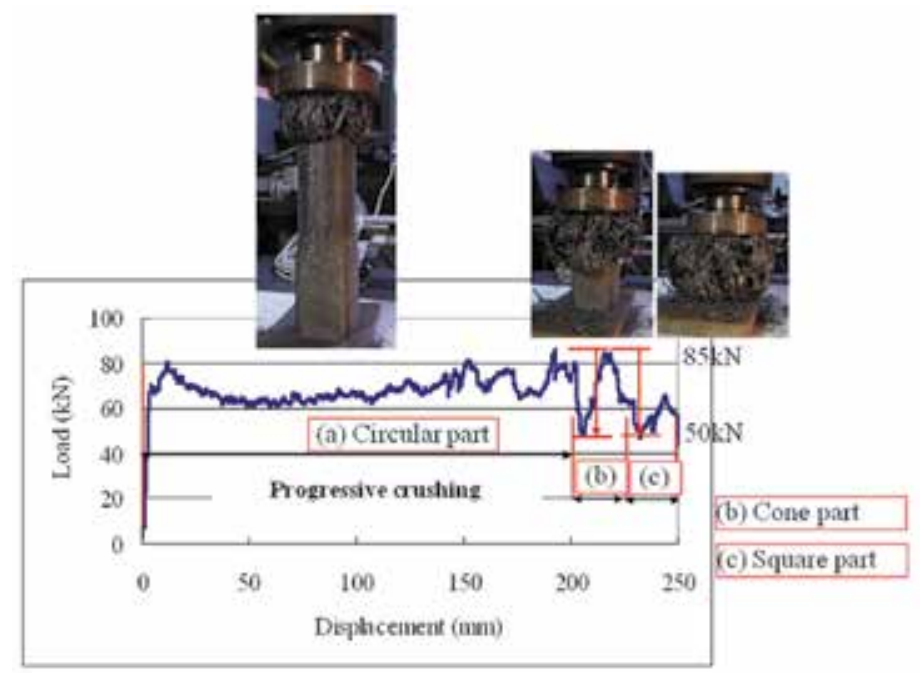

Fig. 14. The load-displacement curve and crushing fashion of Type15-15 during the crushing process (a) circular tube part; (b) cone tube part; (c) square tube part.

\begin{tabular}{|c|c|c|c|c|}
\hline & \multicolumn{2}{|c|}{ Type 15-15 } & \multicolumn{2}{c|}{ Type 15-60 } \\
\hline & Circular & Square & Circular & Square \\
\hline$A\left(\mathrm{~mm}^{2}\right)$ & 420.8 & 429.2 & 421.7 & 555.5 \\
\hline$\rho\left(\mathrm{g} / \mathrm{cm}^{3}\right)$ & 1.51 & 1.51 & 1.50 & 1.56 \\
\hline $\bar{P}_{(\mathrm{kN})}$ & 68.59 & 57.42 & 67.22 & 79.69 \\
\hline $\boldsymbol{E s}(\mathbf{k J} / \mathbf{k g})$ & $\mathbf{1 0 7 . 9}$ & $\mathbf{8 8 . 6}$ & $\mathbf{1 0 6 . 3}$ & $\mathbf{9 2 . 0}$ \\
\hline
\end{tabular}

Table 3. Parameters and energy absorption capability of the specimens carbon braided FRP tubes with novel three-phases geometry 


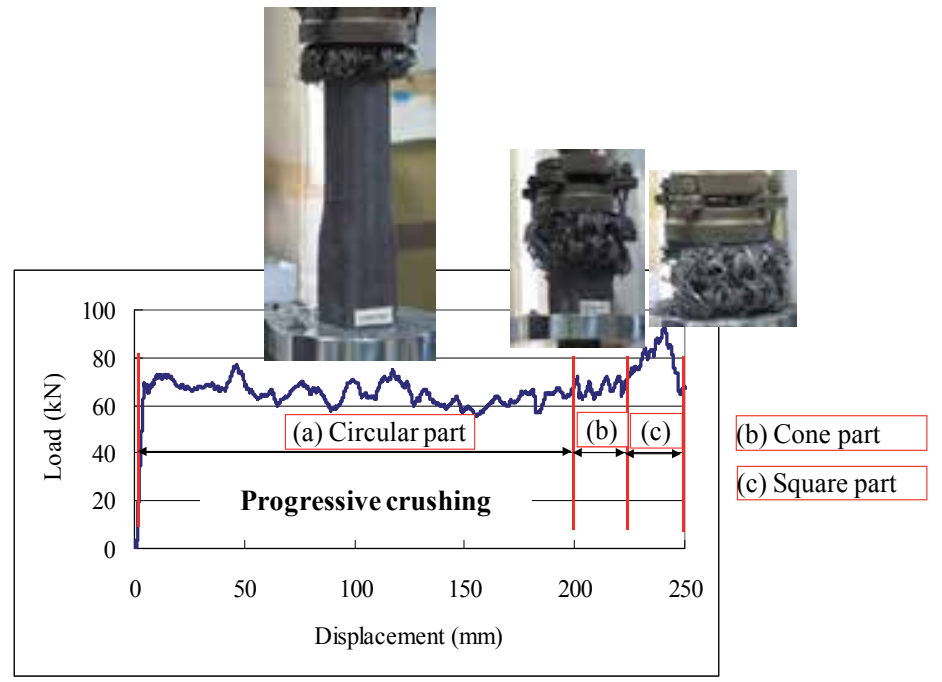

Fig. 15. The load-displacement curve and crushing fashion of Type15-60 during the crushing process (a) circular tube part; (b) cone tube part; (c) square tube part.

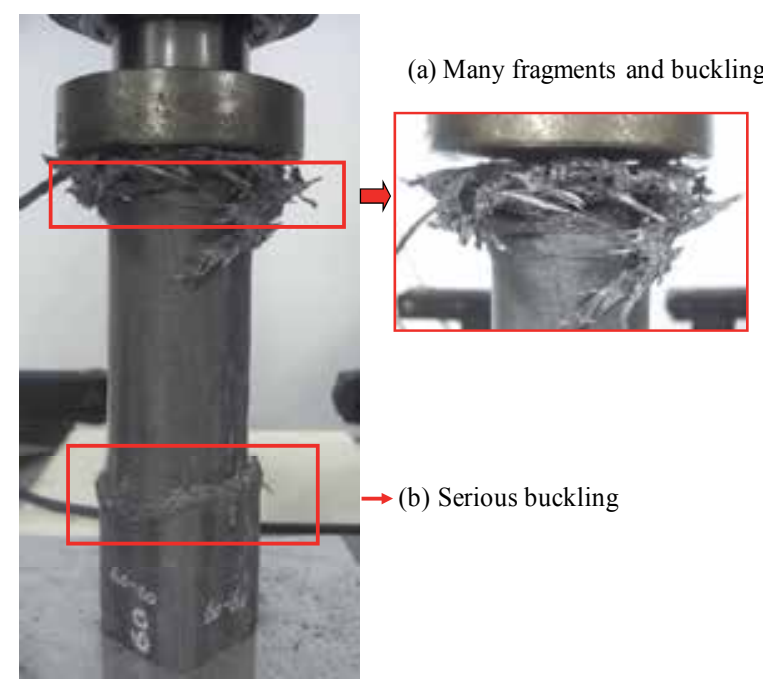

Fig. 16. The crushing fashion of Type 60-60 during the crushing process (a) buckling on the top of the circular tube part; (b) serious buckling in cone tube part.

\section{FRP tubes compressed under designed devices}

\subsection{Materials and experiments}

\subsubsection{FRP tube with circular transversal cross section}

Prepreg yarn consisting of epoxy resin and carbon fiber bundles made of 12,000 filaments with a diameter of $6.8 \mu \mathrm{m}$ was used to fabricate FRP tube with circular transversal cross section by braiding technology. The fabrication process of braiding structure is listed as followings: 
1. Initially, 48 bundles of prepreg yarn as braiding yarns were fabricated on a circular metallic mandrel with a braiding angle of $60^{\circ}$. (As illustrated in Fig.17 (a), braiding angle is the angle between the braiding yarn and axial direction.)

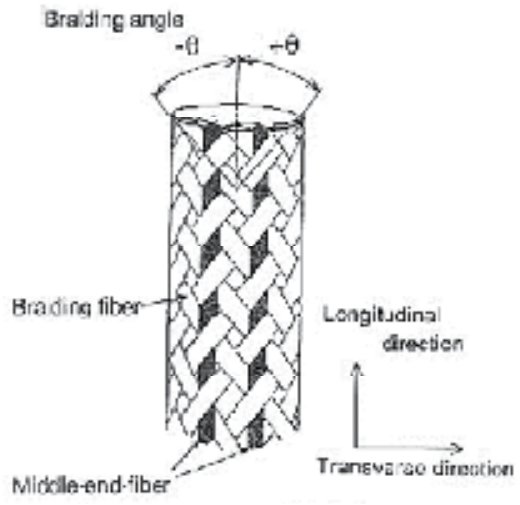

(a) Schematic illustration of a circular braided structure

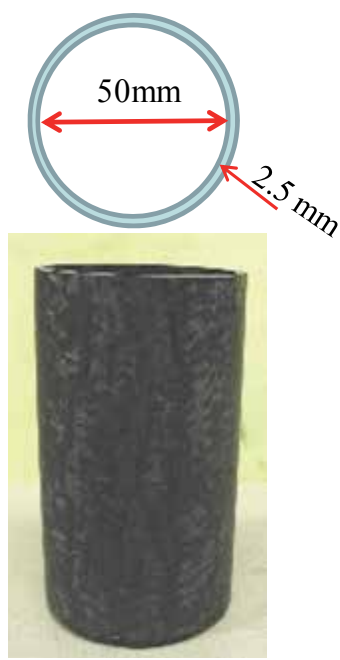

(b) Photograph of a circular braided carbon/Epoxy tube

Fig. 17. Braided carbon/Epoxy circular composite tube with an inner diameter of $50 \mathrm{~mm}$ and a thickness of $2.5 \mathrm{~mm}$.

2. Secondly, 48 braiding yarns and 24 middle-end-fibers were used to fabricate the $2^{\text {nd }}$ to $5^{\text {th }}$ layers with a braiding angle of $15^{\circ}$. (Here, middle-end-fiber is the yarn located paralleled to the axial direction while braiding yarn are alternately cross over middleend-fibers with an angle of 15.)

3. Then the $6^{\text {th }}$ layer with a same fiber architecture as the $1^{\text {st }}$ one was fabricated in the outermost layer. The $1^{\text {st }}$ and $6^{\text {th }}$ layers are the skin layers of braiding preform in order to get a smooth surface during curing process.

4. After braiding fabrication, shrink tape was wrapped on the surface of the braided preforms under an appropriate pressure to get a high fiber volume fraction and low void fraction. After that, it was cured in an oven at a constant temperature of $130^{\circ} \mathrm{C}$ for 4 hours. (The temperature was increased from room temperature to $130^{\circ} \mathrm{C}$ with a rate of $\left.5^{\circ} \mathrm{C} / \mathrm{min}\right)$

5. Finally, after the shrink tape was removed, the composite pipes (Fig.17(b)) were drawn out from the mandrels. The fabricated braided carbon/epoxy composite pipes were with a fiber volume fraction of about $52 \%$ and a thickness of $2.5 \mathrm{~mm}$, an inner diameter of $50 \mathrm{~mm}$.

In order to assess the viability of these new kinds of collapse triggers, the Fig. 6 devices were compared against the taper trigger which is the most common trigger involved FRP tubes. According to the collapse trigger, the segmented circular tubes with a height of $50 \mathrm{~mm}$ are divided into two groups. Taper group, was composed of the braided carbon/Epoxy circular tubes of Taper-15, Taper-45 and Taper-75. The flat end of one side of those specimens were chamfered to sharp edge in $15^{\circ}, 45^{\circ}$ or $75^{\circ}$. For the Device group tubes, there was not any 
modification on their ends. However, the afore explained devices are capped onto the FRP tubes before compression test. Depending on the device type, the specimens were named. For example, the braided carbon/Epoxy circular tube, which was capped C-Inner 3 type device, was called Inner-3. In the same way, Inner-5, Outer-3 and Outer-5 were named correspondingly.

\subsubsection{FRP tube with square transversal cross section}

Two kind of FRP tubes with a similar square transversal cross section were employed to investigated the energy absorbing mechanism under the effect of S-Inner 2 device in both quasi-static and dynamic compression condition. One is Carbon UD tube which has unidirectional carbon fiber as reinforcement and polyester as matrix. The other is Carbon MWK tube which is involved carbon multi-axial warp knitted (MWK) fabric. The laminate lay-ups of Carbon MWK tube consists of glass sward mat (Glass SM, 360g/ $\mathrm{m}^{2}$ ), carbon MWK fabric $\left(600 \mathrm{~g} / \mathrm{m}^{2}\right)$, glass roving (Glass UD, $4450 \mathrm{~g} / \mathrm{Km}$ ), and another carbon MWK layer $\left(600 \mathrm{~g} / \mathrm{m}^{2}\right)$ from outside to inside in the thickness. Here, in the MWK carbon fabric in both FRP tubes, two unidirectional carbon fiber layers i.e. $90^{\circ} / 0^{\circ}$ were combined by knitted yarn. Both of the square FRP tubes are fabricated by pultrusion process. Unidirectional fiber bundles or MWK fabrics are drawn together through the resin rain. Then the resin coated fibers are drawn through specific heated die where the fibers are impregnated with resin and cured to have a desirable geometry similar to the die. After cooled, the FRP tubes are cut into pieces in any ordered length.

The introduction about square FRP specimens is given in Table 4 as well as their photographs. Referring to the square geometry in transversal cross section, the FRP tubes have an outside length of $50 \mathrm{~mm}$ and a thickness of $4.2 \mathrm{~mm}$ in the flat wall. In particular, there are the internal corners with a radius of $6 \mathrm{~mm}$ and external radius of $2 \mathrm{~mm}$ respectively. Specimens were prepared in $100 \mathrm{~mm}$ length for quasi-static and $300 \mathrm{~mm}$ for impact tests. Similarly to the circular FRP tubes, the energy absorption capabilities of square FRP tubes are compared under both S-Inner 2 device and taper triggers with an angle of $45^{\circ}$.

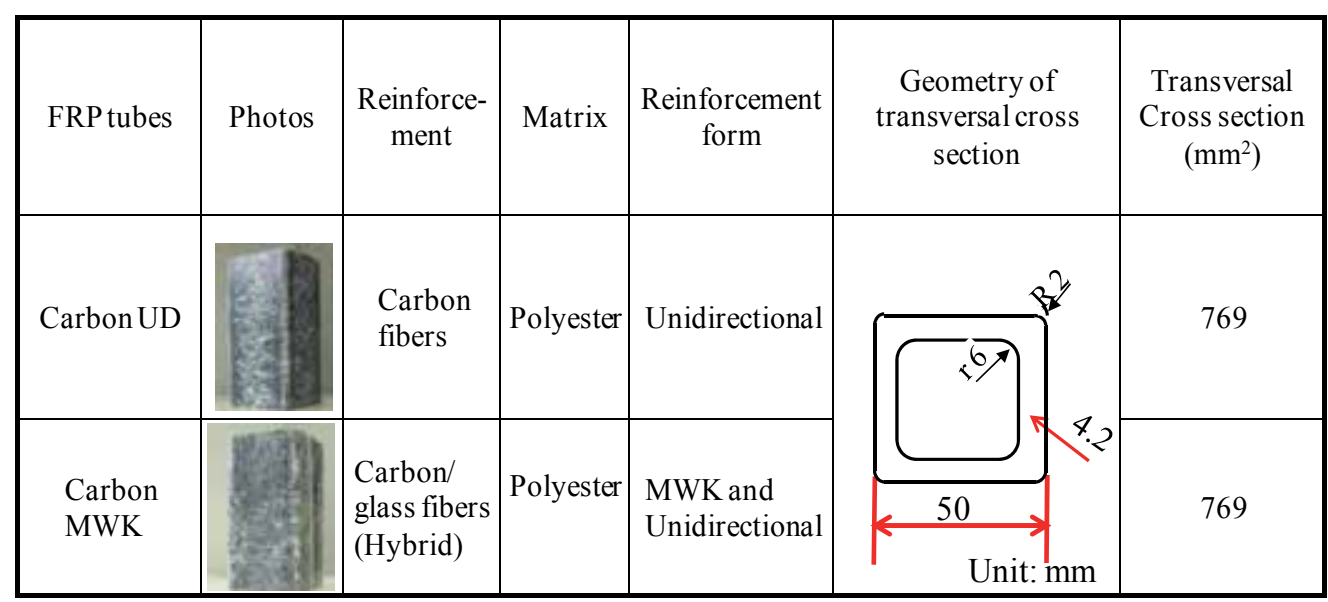

Table 4. Specifications and photographs of the FRP specimens with square transversal cross section 


\subsubsection{Experiments}

Quasi-static compression tests were performed on an INSTRON (4206) universal testing machine at a constant crosshead speed of $5.0 \mathrm{~mm} / \mathrm{min}$ for both circular and square FRP tubes. The test started when the flat compression plate of the machine slightly touched the taper of FRP tube or the device which was capped on one end of the tube. During the compression procedure, five data points every second were recorded to follow the track of the load. Later, three replicate tests were conducted to verify the stability of the energy absorption capability.

On the other hand, impact tests were carried out commercially (in Tokai Techno-research Company) for square FRP tubes additionally. An example of Carbon UD specimen with the usage of S-Inner 2 device as collapse trigger is given in Fig.18 to illustrate the preparation of impact test. After the specimen is fixed on the test machine, the impactor of $120 \mathrm{~kg}$ which located from $12 \mathrm{~m}$ height place fall freely to create the impact velocity of $55 \mathrm{~km} / \mathrm{h}(15.3 \mathrm{~m} / \mathrm{s})$ when it crash the FRP tube. During the impact process, the sampling rate of load is selected $50 \mu \mathrm{s}(20 \mathrm{kHz})$ to obtain a series data of loads. Here something regrettable should be claimed. In case of S-Inner 2 device usage, several duplicate specimens were not done in dynamic compression. The reason will be explain in the following Discussions Section.

\subsection{Results}

\subsubsection{Circular braided carbon/Epoxy FRP tubes}

For Taper group tubes i.e. in the cases of taper triggers, the representative loaddisplacement curves obtained from the axial quasi-static compression tests are shown in Fig.19 (a). The common feature of all of the curves is that the loads increased rapidly in the initial stage, reach a peak value and then dropped slightly. After that, the loads increased again, and finally showed the characteristics of progressive crushing. Although, Taper-15 had the highest peak value at the initial crushing stage and a little different inclination compared Taper-45 and Taper-75, the figures clearly indicate that the Taper group tubes exhibit similar crushing load, in particularly, during the progressive crushing period. However, as shown in Fig.19 (b), the situation is complicated for the Device group tubes. That is, these tubes with different device type displayed distinct mean crush loads. In detailed, the mean crush loads are $82.7 \mathrm{kN}$ for Inner-3; $35.8 \mathrm{kN}$ for Inner-5; $44.3 \mathrm{kN}$ for Outer-3 and $19.2 \mathrm{kN}$ for Outer-5. In addition, the mean loads of the tubes capped outer type device were lower than their initial peak values. On the other hand, for the tubes with the inner type trigger, the mean loads were retained at a higher level than the initial peak.

The usage situation of different triggers on the circular braided carbon/Epoxy FRP tubes in quasi-static compression tests are summarized in Table 5. It is found that in the cases of taper trigger, the crushing fashions of the circular FRP tubes are almost the same i.e. the crushed walls were spread out towards both sides of the tube in two-side-bending mode like a spreading flower. However, in the cases of device trigger usage, the fronds show oneside-bending mode. The crush wall was split into pieces and bent inwards by Inner type device or spread outwards by the Outer type device. Those internal fronds seem superposed together tightly whereas, the external fronds are found to be separated. In addition, the Es values, calculated from the above Load-displacement curves, is also given in Table 5. Apart from the crushing performance, such closed Es values from 86.8 to 94.3 illustrate that the Taper group tubes have the similar energy management. However, quite different $E s$ values were obtained with different devices usage. It seems that Inner type device or a small radius associates to higher energy absorption than Outer type device or a big radius. In the usage 
of inner type device with a radius of $3 \mathrm{~mm}$, the highest value of 110.8 is achieved in C-Inner 3 specimen even compared to that of Taper group tubes.

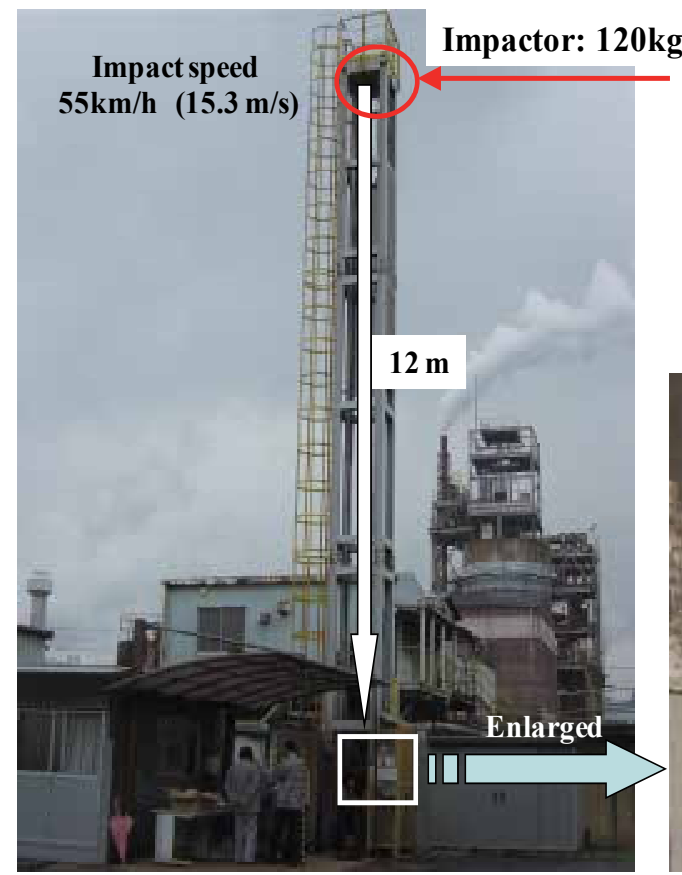

(a) Impact test machine

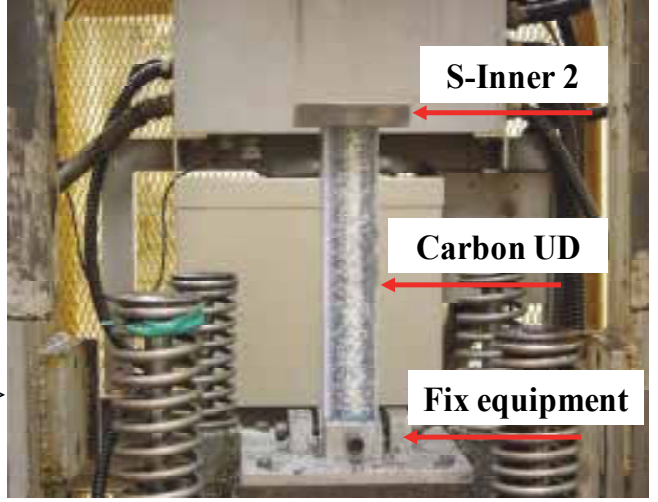

(b) Fixed Carbon UD specimen capped S-Inner 2 device

Fig. 18. Impact test machine and an example of Carbon UD specimen with the usage of SInner 2 device as collapse trigger.

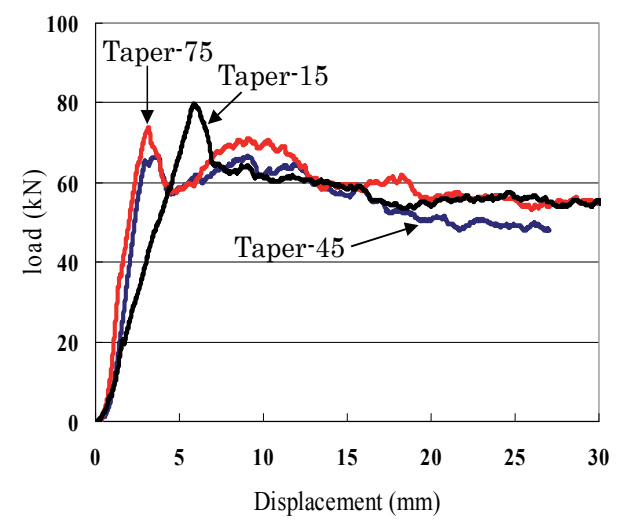

(a) Taper trigger

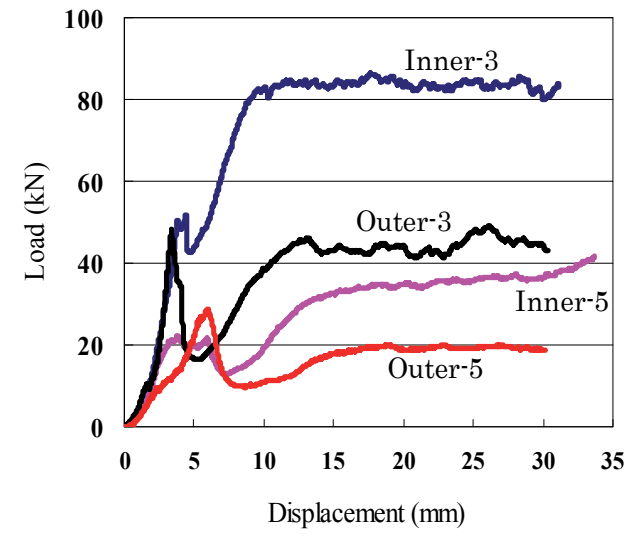

(b) device trigger

Fig. 19. Typical load-displacement curves of circular braided carbon/Epoxy tubes with different collapse triggers in quasi-static compression tests. 


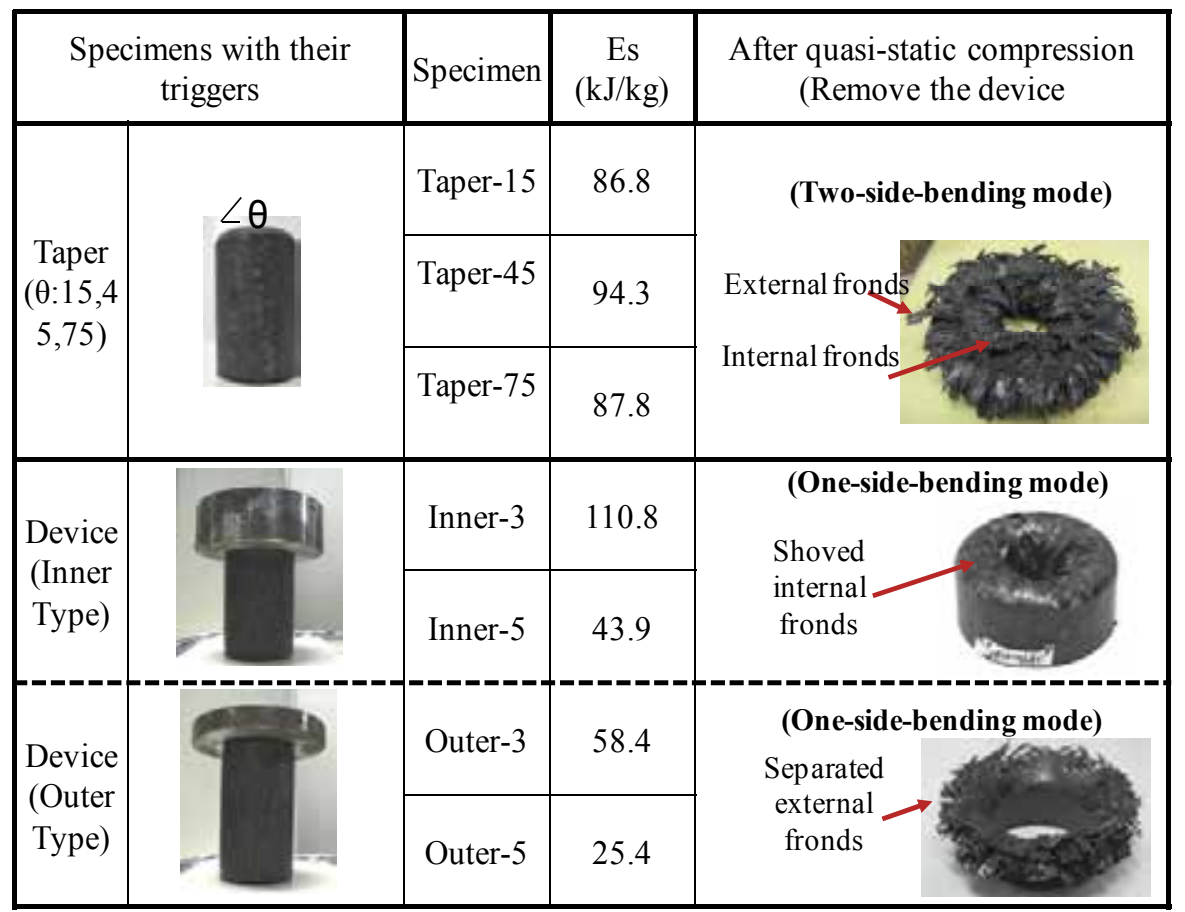

Table 5. Fracture performance of circular braided carbon/Epoxy tube with different collapse trigger in quasi-static compression test

\subsubsection{Square carbon UD and carbon MWK tubes}

The typical Load-displacement curves of Carbon UD and Carbon MWK tubes from both quasi-static and impact tests are shown in Fig.20. The tubes were almost compressed until $50 \mathrm{~mm}$ in quasi-static compression and more than $150 \mathrm{~mm}$ in impact test. It is found that no matter which triggers usage, almost the FRP tubes show the progressive crushing performance in both quasi-static and dynamic compression conditions. In particularly, in both quasi-static and impact test, the FRP tubes show the trend that relative higher crushing load can be obtained with the usage of device trigger than that of taper trigger except Carbon UD tube. Carbon UD tube had a relative higher peak load which is over $120 \mathrm{kN}$. After peak value, the load of Carbon UD tube decreased without stopping in dynamic test with the usage of S-inner 2 device and even lower than that of taper trigger after $100 \mathrm{~mm}$ displacement. That is to say, there is not a satisfied progressive crushing occurred in Carbon UD tube in the condition associated with device and impact. In Fig.21, the photographs of Carbon UD and Carbon MWK tubes with device as collapse trigger in impact tests were compared. As shown in Fig.21(a), the tube wall of Carbon UD was split into many pieces but part of them couldn't be embedded into the inside of the device. That is considered as the main reason why the load got down continuously. On the other hand, as illustrated in Fig.21(b), unlike the former one, the Carbon MWK tube showed a relatively successful crushing performance i.e. almost of the split tube wall were bent into the inside of the device. On the other hand, in the case of the most common collapse trigger i.e. taper, all of the specimens are crushed in two-side-bending mode, i.e. the tube wall was split to many 
pieces and bent towards both sides of the tubes. Compared the fronds bending with a small bending curvature in quasi-static test in Fig.22(a/b), the split tube wall sprang back greatly and bending with a relative big bending curvature (Fig.22 (c/d)) in impact test. It is thought that parts of the bending walls did not fracture but still under flexural deformation in impact test.

(a) Carbon UD
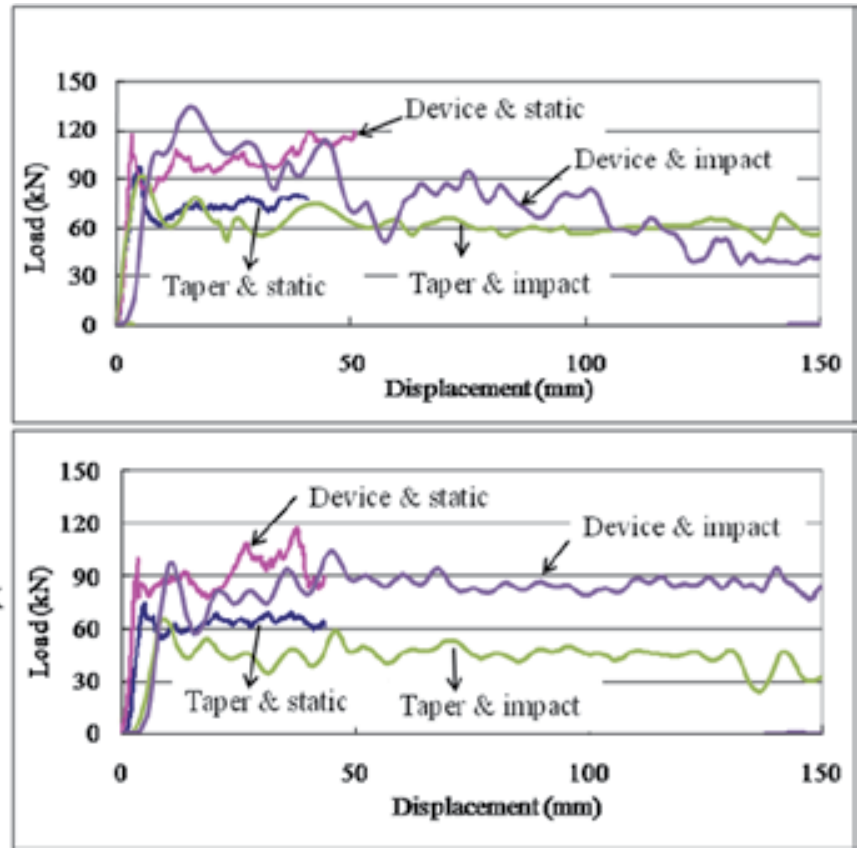

Fig. 20. Typical load-displacement curves of square Carbon UD and Carbon MWK tubes with different collapse triggers in both quasi-static and impact tests.

Their energy managements were discussed based on the Es value listed in Table 6. Because that the Carbon UD tube which with the device trigger could not generate progressive

\begin{tabular}{|c|c|c|c|c|c|}
\hline FRP tube & Trigger & Quasi-static & Impact & $\begin{array}{l}\text { Impact vs. } \\
\text { Static }\end{array}$ & $\begin{array}{c}\text { Inner Device } \\
\text { vs. Taper }\end{array}$ \\
\hline \multirow{2}{*}{ Carbon UD } & $45^{\circ}$ Taper & 55.0 & 52.1 & $94.7 \%$ (taper) & $145.5 \%$ (static) \\
\hline & $\begin{array}{c}\text { S-Inner } 2 \\
\text { device }\end{array}$ & 80.0 & 59.6 & $74.5 \%$ (device) & $114.4 \%$ (impact) \\
\hline \multirow{2}{*}{$\begin{array}{l}\text { Carbon } \\
\text { MWK }\end{array}$} & $45^{\circ}$ Taper & 47.7 & 32.2 & $67.5 \%$ (taper) & $142.6 \%$ (static) \\
\hline & $\begin{array}{c}\text { S-Inner } 2 \\
\text { device }\end{array}$ & 68.0 & 71.3 & $104.9 \%$ (device) & $221.4 \%$ (impact) \\
\hline
\end{tabular}

Table 6. Energy management of square FRP tube with different collapse trigger in both quasi-static and impact test. 
crushing in impact compression test owing to the decreasing load, Es of all the specimens are calculated based on the data from 0 displacement to $150 \mathrm{~mm}$ in dynamic and 0 to $50 \mathrm{~mm}$ in quasi-static compression. Additionally, ratios of impact vs. quasi-static test and the one of device vs. taper are calculated to find the lost or increase under the different compression conditions. It is known that Es values in dynamic compression test are lower than that in quasi-static compression tests for almost the specimens except Carbon MWK tube with usage of device trigger. For Carbon MWK tubes, there is distinct trend between device and taper trigger. In details, in taper trigger, the Es value in dynamic is only $67 \%$ of that in quasistatic condition. That is to say, more that $30 \%$ is lost in impact test compared the equivalent static values. However, with the usage of device trigger, on the contrary, a similar or a relatively higher Es was obtained. From the ratios of Device vs. Taper, it can be concluded that no matter which reinforcement form and compression speed, higher Es values can be achieved with the Inner type device trigger usage than that of the taper trigger.

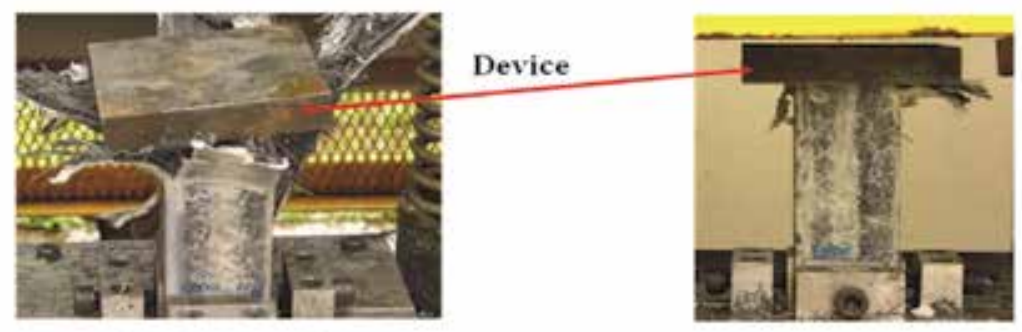

(a) Carbon UD tube

(b) Carbon MWK tube

Fig. 21. Photograph of square FRP tubes in impact test with the device as collapse trigger.

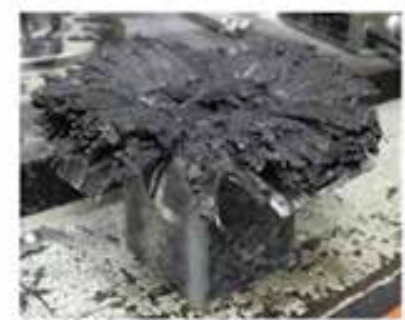

(a) Carbon UD after static test

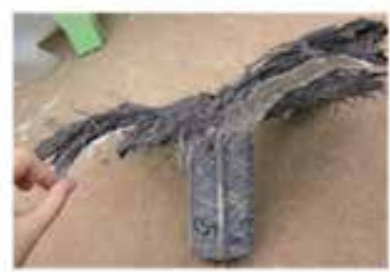

(c) Carbon UD after impact test

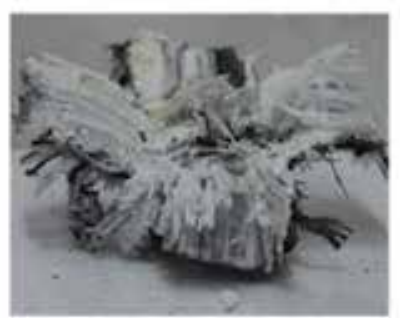

(b) Carbon MTVK after static test

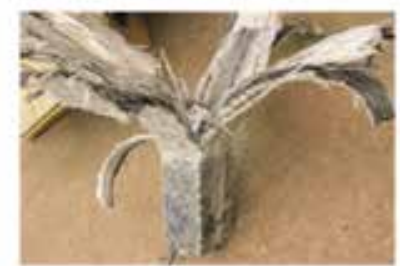

(d) Carbon MWK after impact test

Fig. 22. Photograph of square FRP tubes with taper as trigger after compression test (quasistatic and dynamic) 


\subsection{Discussions}

\subsubsection{One-side-bending vs. two-side-bending}

From their crushing performances, it is well understood that with the usage of device, the tube wall of FRP is possible to be in One-side-bending mode rather than Two-side-bending in the case of taper trigger. As illustrated in Fig. 5, the tube fronds in One-side-bending mode have a double thick thickness of that in Two-side-bending mode. If the thickness of the tube wall is given, forcing it to be in one-side bending mode would be more effectively in bending behavior. According to the formula (3), the bending energy of fronds is directly affected by $I$. Owing to the double size thickness in one-side-bending mode, section modulus will increase greatly in any kind of shape. For an example, for a piece of flat wall of FRP tube assumed as a beam with a rectangular transversal cross section of (length $w \mathrm{X}$ thickness $t$ ), the bending energy can be expressed used formula 11.

$$
U_{\text {bend }}=\frac{5 E \cdot I_{\text {flatwall }} \cdot s}{2 l^{3}}=\frac{5 E \cdot s \cdot \frac{1}{12} w t^{3}}{2 l^{3}}
$$

Here, $E, I, s$ and $l$ are the modulus, inertia moment, compression displacement and the height of the beam. In one-side-bending mode, the section modulus will increase 8 times because of double $t$. Therefore the total $U_{\text {bend }}$ i.e. the whole bending energy of all of the fronds would increases 4 times. It is considered that the increase $U_{\text {bend }}$ contributes to the enhanced energy absorption capability indirectly. On the other hand, the most important effect is that with the increased thickness, the bending stresses are increased correspondingly according to the formula (8) and affect the fractures of fiber as well as resin directly. In details, during the bending process, the fronds experience high tensile stresses on the up layers and compressive stresses on the bottom layers. When the stresses exceed the strength of fiber, many fibers fractured.

To clarify the energy absorbing mechanisms, micro-observations were carried out furthermore. The specimens after compression tests were immerged into the polyester resin and then cured in order to maintain the fracture morphology. After that, cured specimens were cut along the axial direction by the diamond cutter and some appropriated axial cross sections through the crush zone were selected and polished for observation on a metallic microscope (reflection type). Here the difficulty of digging the tested specimens from the S-Inner 2 in the case of Inner type device usage in particular after impact test should be mentioned. During the compression process, the FRP tube had expanded and clung to the device. Additionally, the bending fronds compacted tightly in the concave part of Inner type device. As a result, the specimens after compression were very difficult to be taken out from the Inner type device even with a hammer. To hit the FRP tube with a big strength had been avoided, because the morphology of fractures might be damaged. Appropriated strength was applied to the hammer to hit the FRP tube walls carefully and alternately. In some cases, however, such specimens which did not be controlled successfully by the inner type device after impact test had to be destroyed. Those fragments which are filled in the concave part of the Inner type device in an extreme compacted situation were solved by acetone so that the Inner type device could be released for the next experiments. That is the reason why several repeat impact tests couldn't be implemented within the limited stipulated time. 
Here the observation results on the flat wall of Carbon MWK composite specimens after impact test were illustrated in Fig. 23 (taper trigger) \&24 (device trigger) as examples to compare the effect of triggers on the energy absorbing mechanisms of FRP tubes. In the case of taper trigger, as shown in Fig.23, two-side-bending behaviors could be seen. Although some fiber fractures could be found in the inside bending fronds, many intro/inter delaminations generated in the middle and outside fronds instead. Additionally, a big distance could be found between middle and outside layers after the compression was released. It is considered that the middle fronds sprung back because of the lack of fiber fractures. On the other hand, as illustrated in Fig.24, the flat wall of carbon MWK tube shows these features: bending of tube walls towards inside only; many inter and introdelaminations in the middle layers; many transversal cracks and fiber fractures in both surfaces layers. Here, an attention should be paid that although many inter or intro delaminations occurred, those independent fronds did not separated each other even after it was released from the control of device. They touch and bent in a similar bending curvature towards inside.

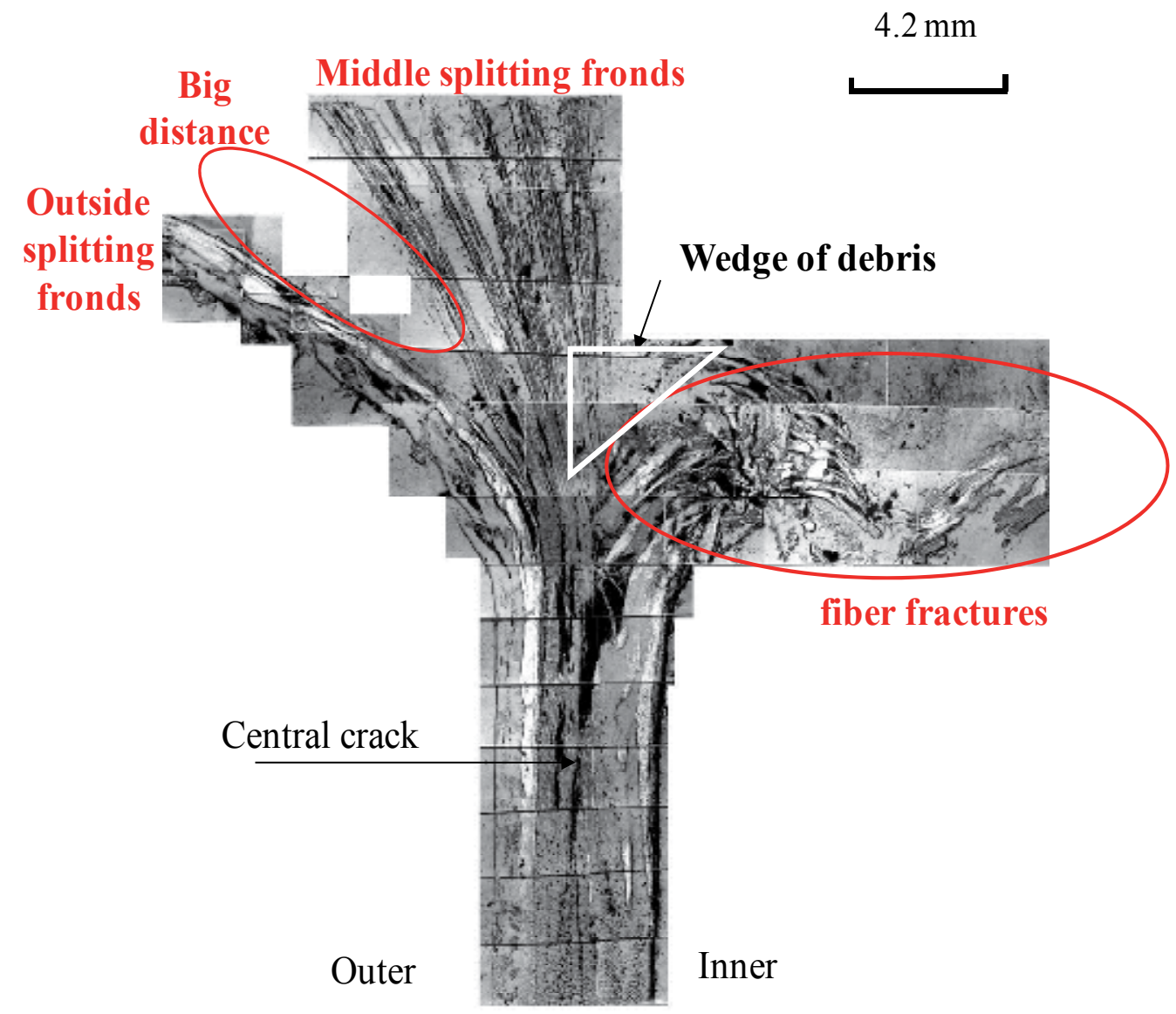

Fig. 23. Observation of flat wall of Carbon MWK tube after impact test illustrating two-sidebending behavior, many splitting frond and some fiber fractures (taper trigger) 


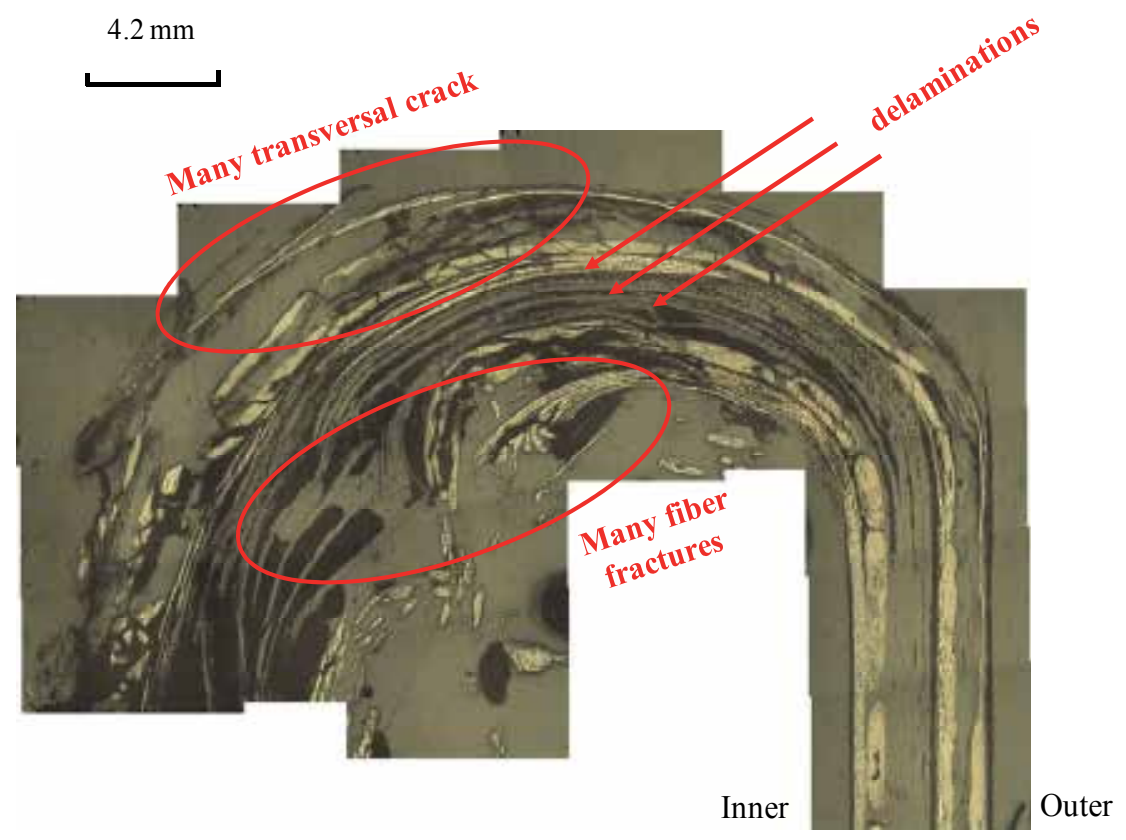

Fig. 24. Observation of flat wall of Carbon MWK tube after impact test illustrating that the fronds were being bent towards inside only and many fracture generated (S-Inner 2 device trigger).

\subsubsection{Effects of the radius $\left(R^{\prime}\right)$ and the type of device}

From the quasi-static compression experimental results of the braided carbon circular tubes, it can be said that devices can trigger progressive crushing similar to taper. However, unlike taper, device can also change the energy absorption capacity of the FRP tubes significantly. Inner type could be said to be better than outer type and a smaller $R^{\prime}$ confers better energy absorption capability.

Here Inner-3 and Inner-5 specimens were compared their energy absorbing mechanisms in Fig.25. The observation focused on the axial cross section through the crush zone where had been under the $R^{\prime}$ region of the devices. The common characteristic of both specimens is that the tube walls were delaminated into several layers and bends were towards inside. It is considered that at the beginning of the crushing process, with the limitation imposed by device, the tube wall was forced to bend inwards only. Shearing stresses are induced to split the integrated tube wall into pieces. Braiding confers the characteristic that fibers are oriented continuously. Intra-laminar cracks are therefore difficult to grow. On the other hand, the bonding of each braided fabric layer is relatively weaker, so with the advancement of the crushing platen, inter-laminar cracks (delaminations) occurred and were propagated. However, the propagation of delaminations are different, which is deep in Inner- 5 while seemed restrained in Inner-3. Additionally, compared Inner-5, Inner-3 had a relatively smaller radius of bending curvature. During the process of bending the pieces of tube wall over a sharp radius of curvature, it is considered that the radius of curvature $\left(r^{\prime}\right)$ is controlled by the radius $\left(R^{\prime}\right)$ of the device. Therefore, fronds of Inner-3 experienced relative higher tensile stresses on the convex side and compressive stresses on the concave side. 
Subsequently, fibers together with resin would be broken and contributed to higher energy absorption capability.

For a given tube compressed under Inner or Outer types devices with same $R^{\prime}$, quite different energy managements were found. According to the formulae of (3) and (8), they should have similar bending energy and bending stresses. However, obviously Inner type device show greater contribution to energy absorption of FRP tubes. Here the fracture space is considered as the major factor. When the tube wall has to be bent inwards or outwards, the fracture space will be shortened in the case of Inner type or expanded in that case of Outer type device. After the tube wall shears or splits into pieces of "independent" fronds, in the case of inner type device, these "independent" fronds have to be superposed each other owing to the shortened fracture space and shoved the adjacent ones below it with the advancement of the compression process, whereas that will not occur in the case of outer type device because those fronds are separated in an extended fracture space. When the adjacent fronds shove each other, they touch tightly and slide in order to get a space and bend through almost the same bending curvature during the whole compression process, which will cause frictional stresses on the touched surfaces between any two adjacent fronds. Frictional stresses action on the surface of fronds, break fiber as well as resin or generate heat energy at some degree. Apparently, this kind of friction generated by inner type device contributes to the total absorbed energy greatly. Fairfull and Hull [39] had investigated the energy absorbed by the frictional processes in the axially crushing of glass cloth/epoxy tubes. They claimed that frictional energy could account for more than $50 \%$ of the total energy absorbed. If the influences of bending curvatures of fronds are ignored temporarily, it could be inferred that the inner type device is better than taper trigger, and taper trigger is better than outer type device, because the friction effect from taper trigger is considered a blend of half from the inner type device and half from the outer type device.

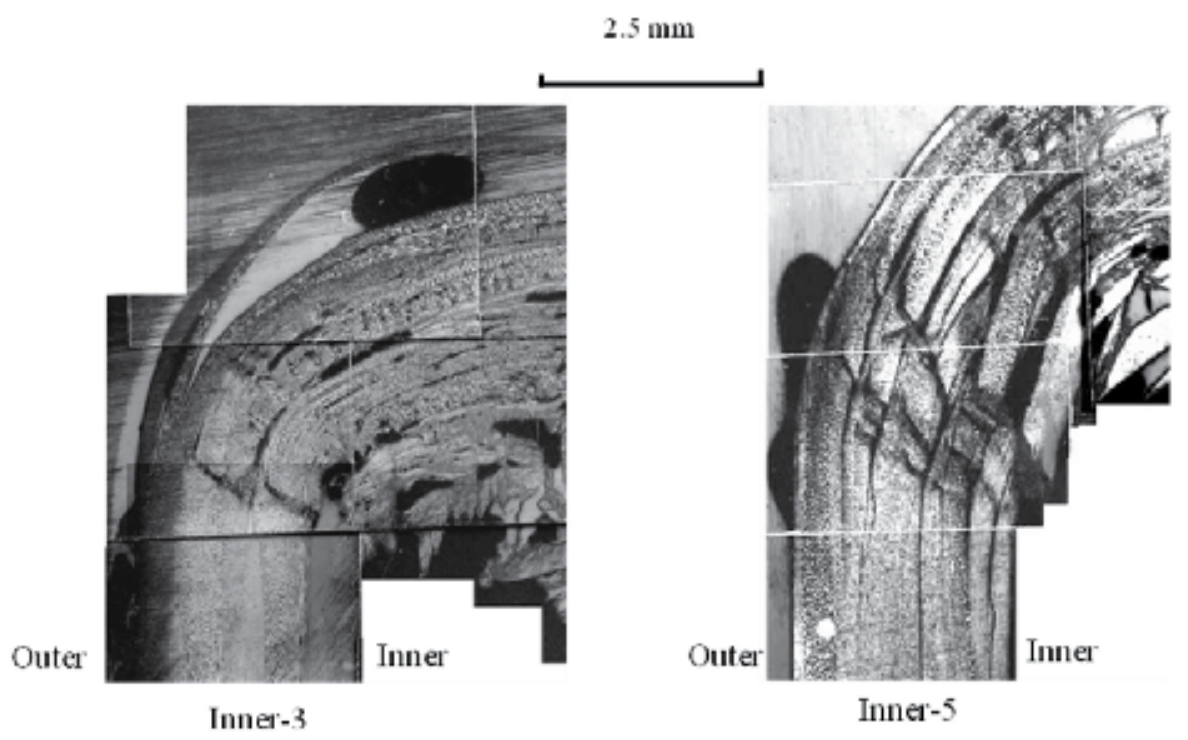

Fig. 25. Observation on the cross sections of Inner-3 \& Inner-5 specimens (the crush zone which had been under the $R$ region of device illustrates the different radii of bending curvatures and the propagation of delaminations.) 


\section{Conclusions}

FRP tubes have a very complicated energy absorption mechanism during progressive crushing process. It is considered that the energy absorbed by fiber fracture can contribute to the total absorbed energy significantly. Therefore, an attempt of design of fiber fractures was carried out in current study with an attempt to apply the FRP tubes as energy absorption component in a vehicle.

Crushing behavior of FRP tube was considered related in appearance of the bending behavior of beam. Here mechanism model of a bending beam was used to simulate the bending fronds of FRP tube. It is found that the bending energy is in direct proportion of $I$, i.e. the moments of inertia of the section area which is determined by the geometry. Therefore two improvement methods based on design of the bending energy through the geometry design were proposed. In method of mimic square to circular, two types of mandrels (r3 and $\mathrm{r} 9$ mandrels) with $3 \mathrm{~mm}$ or $9 \mathrm{~mm}$ radius modification on the corners were employed to determine the effect of design of the geometry. It is found that Es of the CFRP tubes fabricated on the $\mathrm{r} 9$ mandrel were improved significantly as compared to that fabricated on the $\mathrm{r} 3$ mandrel even with the same fiber architecture and similar size of cross section area. The influence of the geometry is discussed in terms of $I$. It is found that trough a reasonable geometry design, the energy absorption management of FRP tube with a bigger I can be improved.

In the method of the combining both circular and square, the CFRP tube with a new geometry, which utilizing both circular cross-section for its higher energy absorption capability and square cross-section for its assembling convenience with other components, was designed. Additionally, braiding texture is used as fiber reinforcement form based on the considerations of cost and preforms fabrication. It was also shown that the braiding texture is helpful to improve the crushing performance of FRP tube with this kind of geometry, particularly during the crushing different cross section process such as from circular tube part to cone part or from cone part to square tube part.

Additionally, a method aims a thicker thickness and a very small bending curvature of bending fronds according to the formula of bending stress. On the other hand, FRP tubes generally need a collapse trigger mechanism to generate stable, progressive, high energy crushing. Therefore, in the study, bending stress was under consideration with collapse trigger mechanism. The target of present experiments is to design a new collapse trigger for the practical application of FRP tubes which is possible to enhance their energy absorption capability.

Four types of devices are designed and used in the axial quasi-static compression tests of braided carbon/epoxy tube with a circular transversal cross section. They include inner and outer types with a radius of $3 \mathrm{~mm}$ or $5 \mathrm{~mm}$. It was found that the devices could trigger progressive crushing similar to taper. However, unlike taper, devices can change the energy absorption capacity of the crushed materials significantly. Inner type device could be said to be better than outer type device because of high friction and a smaller radius confers better energy absorption capability because of high bending stresses.

Additionally an inner type device with a square transverse cross section and $R^{\prime} 2$ was designed for the FRP tube which has a square transversal cross section. The square FRP tubes were compressed in impact test with the usage of device in order to investigate the effect of device under dynamic condition. The results are evaluated in comparison with both taper and equivalent quasi-static values in order to find the effect from the reinforcement 
form in the impact test with device. It is found that texture structure (such as multi-axial warp knitted fabric) is better than unidirection in the case of usage of device, because the texture structure can control fiber layers well in bending behavior. Because of the double size thickness in one-side bending, apart from the increased the bending energy, bending stresses are also increased significantly. Many fibers were broken consequently and the fiber fracture energy i.e. $U_{f f}$ increased greatly. As result, the higher energy absorption capability could be obtained.

\section{Acknowledgments}

This study was conducted as part of the Japanese National Project "R\&D of Carbon FiberReinforced Composite Materials to Reduce Automobile Weight" supported by NEDO (New Energy and Industrial Technology Development Organization). The authors would like to thank Dr. T. Uozumi @ Murata machinery Ltd and Dr. K. Yamaguchi @ Toray Industries, for their supplied materials and cooperation of fabrication of the specimens.

\section{Appendix}

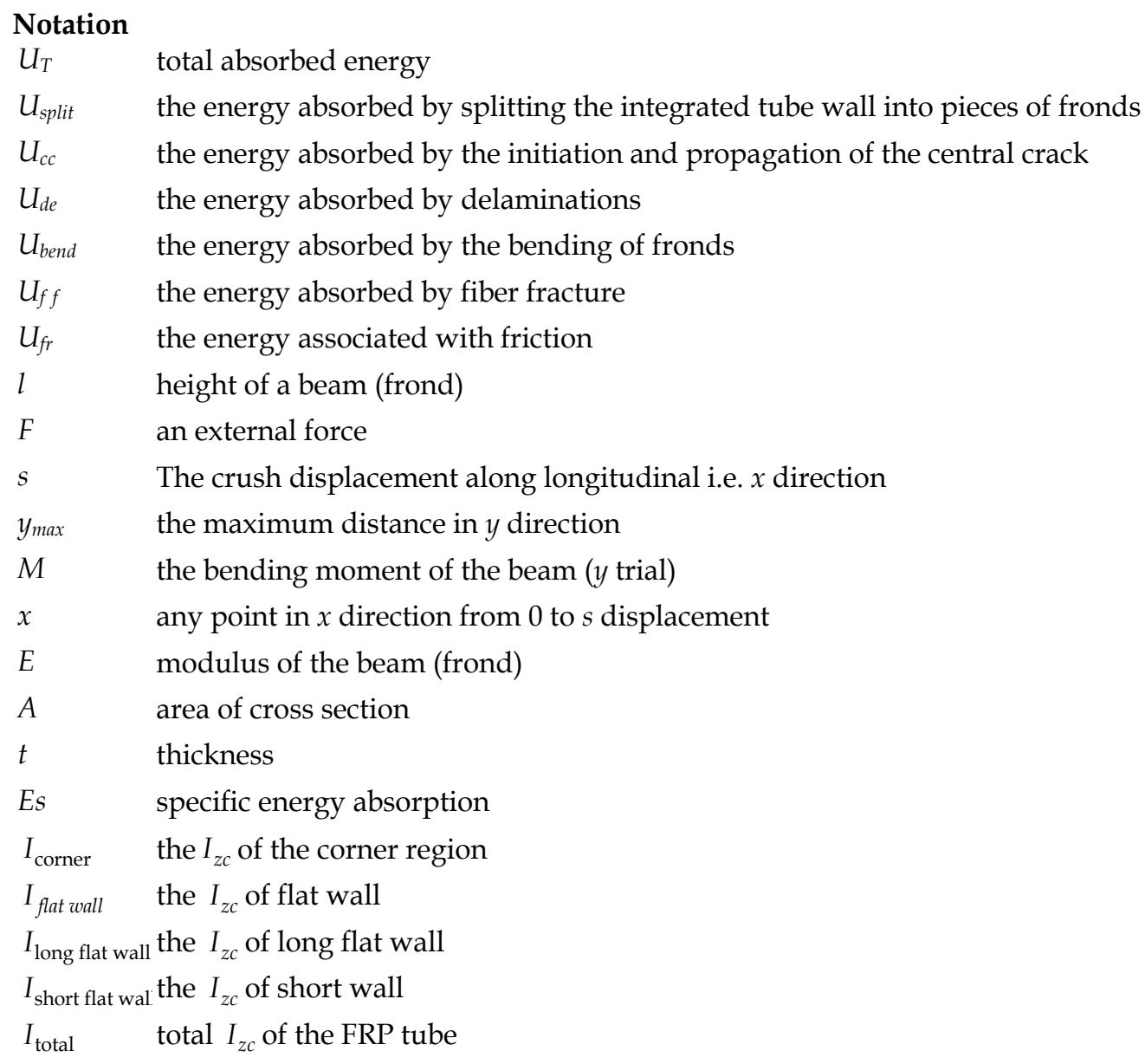


$w \quad$ length of flat wall

$R \quad$ outside radius of the curvatures of the corner

$r \quad$ inside radius of the curvatures of the corner

$W \quad$ the work done i.e. the total absorbed energy

$\rho \quad$ the density of the material

$\bar{P} \quad$ the average load during progressive crushing

$s^{\prime} \quad$ the approximate crushing displacement $s$ which ignore the initial displacement

$\sigma \quad$ bending stresses

$r^{\prime} \quad$ radius of the bending curvature of the frond (beam)

$R^{\prime} \quad$ radius of the curvature of the concave or convex part on the device

$w$ length of flat wall

\section{References}

[1] Rosen, R.W., Mechanics of composite strengthening. In Fiber Composite Materials, American Society for Metals, Metals Park, OH, pp.37-76, 1965.

[2] Thornton, P.H. \& Magee, C.L., "The Interplay of Geometric and Materials Variables in Energy Absorption", Journal of Engineering Materials and Technology, Vol. 99, pp. 114-120, (1977).

[3] Thornton, P. H. "Energy Absorption in Composite Structures," Journal of Composite Materials, Vol. 13, pp. 247-263 (July 1979)

[4] Cronkhite, J.D., Hass, T.J., Berry, V.L. \& Winter, R., Investigation of the crash impact characteristics of advance airframe structure. USARLT-TR-79-11, 1979.

[5] Farley, G.L., "Energy Absorption of Composite Materials", Journal of Composite Materials, Vol. 17, pp. 267-279, (1983).

[6] Hull, D., "A Unified Approach to Progressive Crushing of Fibre-Reinforced Composite Tubes", Composites Science \& Technology, Vol. 40, pp. 377-421, (1991).

[7] Thornton, P.H. \& Edwards, P.J., "Energy Absorption in Composite Tubes", Journal of Composite Materials, Vol. 16, pp. 521-545, (1982).

[8] Farley, G.L. \& Jones, R.M., "Crushing Characteristics of Continuous FiberReinforcedComposite Tubes", Journal of Composite Materials, Vol. 26, pp. 37-50, (1992).

[9] Farley, G.L., "The Effect of Fiber and Matrix Maximum Strain on the Energy Absorption Capability of Composite Materials", Journal of Composite Materials, Vol. 20, pp. 322334, (1986).

[10] Hamada, H., Coppola, J.C. \& Hull, D., "Effect of Surface Treatment on Crushing Behaviour of Glass Cloth / Epoxy Composite Tubes", Composites, Vol. 23, No. 2, pp. 93-99, (1992).

[11] Hull, D. \& Clyne, T.W., An Introduction to Composite Materials, Second Edition, Cambridge University Press, Cambridge, (1996).

[12] Hamada, H., Ramakrishna, S. \& Satoh, H., "Crushing Mechanism of Carbon Fibre /PEEK Composite Tubes", Composites, Vol. 26, No. 11, pp. 749-755, (1995). 
[13] Schmueser, D. \& Wickliffe, L.E., "Impact Energy Absorption of Continuous Fibre Composite Tubes", Journal of Engineering Materials and Technology, Vol. 109, pp. 7277, (1987).

[14] Dorey, G., "Impact and Crashworthiness of Composite Structures", Structural Impact and Crashworthiness, Ed. Davies, G.A.O., Vol. 1, pp. 155-192, Elsevier Applied Science Publishers, London, (1984).

[15] Tao, W.H., Robertson, R.E. \& Thornton, P.H., "Effects of Material Properties and Crush Conditions on the Crush Energy Absorption of Fiber Composite Rods", Composites Science and Technology, Vol. 47, pp. 405-418, (1993).

[16] Karbhari, Vistasp M., Falzon, Paul J., and Herzberg, Israel "Energy Absorption Characteristics of Hybrid Braided Composite Tubes," Journal of Composite Materials, Vol. 31, No. 12. pp.1165-1186, (1997).

[17] Farley, Gary L and Jones, Robert M. "Crushing Characteristics of Continuous FiberReinforced Composite Tubes," Journal of Composite Materials, Vol. 26, No. 1, pp. 3750 (1992).

[18] Hull, D. "Axial crushing of fiber reinforced composite tubes", In structural crashworthiness, ed. Jones, N. \& Wierzibichi, T., Butterworths, London, pp. 118135, (1983).

[19] Hamada, H., Coppola, J.C., Hull, D., Maekawa, Z, \& Sato, H., “Comparison of Energy Absorption of Carbon / Epoxy and Carbon / PEEK Composite Tubes", Composites, Vol. 23, No. 4, pp. 245-252, (1992).

[20]. Hamada, H., Ramakrishna, S. \& Sato, H., "Effect of Fiber Orientation on the Energy Absorption Capability of Carbon Fiber / PEEK Composite Tubes", Journal of Composite Materials, Vol. 30, No. 8, pp. 947-963, (1996).

[21] Schmueser, D. W. and Wickliffe, L. E. "Impact Energy Absorption of the Continuous Fiber Composite Tubes," Journal of Engineering Materials and Technology, Vol. 109, pp. $72-77$ (January 1987).

[22] Karbhari, Vistasp M. "Progressive Crush of Resin Transfer Molded Square Tube Stiffened Beam Elements," Journal of Composite Materials, Vol. 31, No. 10. pp.9811001 (1997).

[23] Hamada, H., Nakai, A., Kameo, K., and Takeda, N. “Crushing Performance of Braided Composites," Proceedings of the Eighth Japan-U. S. Conference on Composite Materials, September 24-25, pp. 467-475 (1998)

[24] Mamalis, A.G., Manolakos, D.E., Viegelahn, G.L., Yap, S.M. \& Demosthenous, G.A., "On the Axial Crumpling of Fibre-Reinforced Composite Thin-Walled Conical Shells", International Journal of Vehicle Design, Vol. 12, No. 4, pp. 450-467, (1991).

[25] Hamada, H., Ramakrishna, S. \& Sato, H., "Effect of Fiber Orientation on the Energy Absorption Capability of Carbon Fiber / PEEK Composite Tubes", Journal of Composite Materials, Vol. 30, No. 8, pp. 947-963, (1996).

[26] Farley, G.L. \& Jones, R.M., "Crushing Characteristics of Composite Tubes with "NearElliptical" Cross Sections", Journal of Composite Materials, Vol. 26, No. 12, pp. 17411751, (1992).

[27] Kindervater, C.M., "Energy Absorption of Composites as an Aspect of Aircraft Structural Crash-Resistance", Developments in the Science and Technology of Composite Materials, Ed.s Fuller, J. et al., pp. 643-651, Elsevier Applied Science Publishers, London, (1990). 
[28] Mamalis, A.G., Yuan, Y.B. \& Viegelahn, G.L., “Collapse of Thin-Wall Composite Sections Subject to High Speed Axial Loading", International Journal of Vehicle Design, Vol. 13, No. 5-6, pp. 564-579, (1992).

[29] Y. Yang, Y. Nishikawa, A. Nakai, U. S. Ishiaku, H. Hamada, “Effect of cross-sectional geometry on the energy absorption capability of unidirectional carbon fiber reinforced composite tubes", Science and Engineering of Composite Materials. Vol.15, pp. 249-263, 2008.

[30] FARLEY, G.L., "The Effects of Crushing Speed on the Energy-Absorption Capability of Composite Tubes", Journal of Composite Materials, Vol. 25, pp. 1314-1329, (1991).

[31] MAMALIS, A.G., MANOLAKOS, D.E., DEMOSTHENOUS, G.A. \& IOANNIDIS, M.B., "Axial Collapse of Thin-Walled Fibreglass Composite Tubular Components at Elevated Strain Rates", Composites Engineering, Vol. 4, No. 6, pp. 653-677, (1994).

[32] A.G. Mamalis, D.E. Manolakos, M.B. Ioannidis and D.P. Papapostolou, 'On the response of thin-walled CFRP composite tubular components subjected to static and dynamic axial compressive loading: experimental', Composite Structures, Vol.69 pp.407-420, (2005).

[33] D. Hull, 'Energy Absorption of Composite Materials under Crash Conditions', Proceedings of the Fourth International Conference on Composite Materials (ICCM IV), 1982, 1, 861-870.

[34] J. Berry and D. Hull, 'Effect of speed on progressive crushing of epoxy-glass cloth tubes', Conf. Ser. Inst. Phys, Vol.70, pp.463-470, (1984).

[35] MAMALIS, A.G., MANOLAKOS, D.E., DEMOSTHENOUS, G.A., IOANNIDIS, M.B., The static and dynamic axial collapse of fibreglass composite automotive frame rails. Composite Structure, Vol.34, pp.77-90, (1996).

[36] Sigalas, I., Kumosa, M., and Hull, D., Trigger mechanisms in energy-absorbing glass cloth/epoxy tubes, Composite science and technology, Vol.40, pp.265-287,(1991).

[37] Czaplicki, M.J. et al, Comparison of Bevel and Tulip Triggered Pultruded Tubes for Energy Absorption, Composite Science and Technology, Vol.40, pp.31-46,(1991).

[38] Saito, H. et al, Crushing properties of pultruded glass reinforced square tubes, International Journal of Crashworthiness, Vol. 7, No.1, pp.21-33 (2002).

[39] Fairfull, A.H. and Hull, D., Energy Absorption of Polymer Matrix Composite Structures: Frictional Effects, Structural Failure, Ed.s WIERZBICKI,T. and JONES,N., pp.255279,(1988). 


\title{
Optimal Feeder Reconfiguration with Distributed Generation in Three-Phase Distribution System by Fuzzy Multiobjective and Tabu Search
}

\author{
Nattachote Rugthaicharoencheep and Somporn Sirisumranukul \\ King Mongkut's University of Technology North Bangkok \\ Thailand
}

\section{Introduction}

Distribution systems are normally configured radially for effective coordination of their protective devices (Kashem et al., 2006). Two types of switches are generally found in the system for both protection and configuration management. These are sectionalizing switches (normally closed switches) and tie switches (normally opened switches) (Su \& Lee, 2003). By changing the status of the sectionalizing and tie switches, the configuration of distribution system is varied and loads are transferred among the feeders while the radial configuration format of electrical supply is still maintained and all load points are not interrupted. This implementation is defined as feeder reconfiguration. The advantages obtained from feeder reconfiguration are, for example, real power loss reduction, balancing system load, bus voltage profile improvement,(Baran \& $\mathrm{Wu}, 1989)$ increasing system security and reliability, and power quality improvement (Kashem, et al., 2000).

Over the last decade, distribution systems have seen a significant increase in small-scaled generators, which is known as distributed generation (DG). Distributed generators are gridconnected or stand-alone electric generation units located within the distribution system at or near the end user. Recent development in DG technologies such as wind, solar, fuel cells, hydrogen, and biomass has drawn an attention for utilities to accommodate DG units in their systems (Gil \& Joos, 2008, Jones \& Chowdhury, 2008, Quezada, et al., 2006, Carpaneto, et al., 2006). The introduction of DG units brings a number of technical issues to the system since the distribution network with DG units is no longer passive.

The practical aspects of distribution system should also be considered for the implementation of feeder reconfiguration. The actual distribution feeders are primarily unbalanced in nature due to various reasons, for example, unbalanced consumer loads, presence of single, double, and three-phase line sections, and existence of asymmetrical line sections. The inclusion of system unbalances increases the dimension of the feeder configuration problem because all three phases have to be considered instead of a single phase balanced representation. Consequently, the analysis of distribution systems necessarily required a power flow algorithm with complete three-phase model.

This paper emphasizes on the implementation of feeder reconfiguration to the distribution system with distributed generators. Three objectives to be minimized are real 
power loss, feeder load balancing, and number of switching operations of tie and sectionalizing switches. Each objective is modeled by fuzzy set to specify its membership value which represents the satisfaction of the objective. The optimal on/off patterns of the switches that compromise the three objectives while satisfying specified constraints is determined using fuzzy multiobjective and Tabu search algorithm. The effectiveness of the methodology is demonstrated by a practical sized distribution system consisting of 69 bus and 48 load points.

\section{Feeder reconfiguration}

Feeder Reconfiguration is a very important and usable operation to reduce distribution feeder losses and improve system security. The configuration may be varied via switching operations to transfer loads among the feeders. Two types of switches are used: normally closed switches (sectionalizing switches) and normally open switches (tie switches) (Baran $\& \mathrm{Wu}, 1989)$. By changing the open/close status of the feeder switches load currents can be transferred from feeder to feeder. During a fault, switches are used to fault isolation and service restoration. There are numerous numbers of switches in the distribution system, and the number of possible switching operations is tremendous. Feeder reconfiguration thus becomes a complex decision-making process for dispatchers to follow. There are a number of closed and normally opened switches in a distribution system. The number of possible switching actions makes feeder reconfiguration become a complex decision-making for system operators. Figure 1 shows a schematic diagram of a simplified primary circuit of a distribution system (Baran \& Wu, 1989). In the figure, CB1CB6 are normally closed switches that connect the line sections, and CB7 is a normally open switch that connects two primary feeders. The two substations can be linked by CB8, while CB9, when closed, will create a loop.

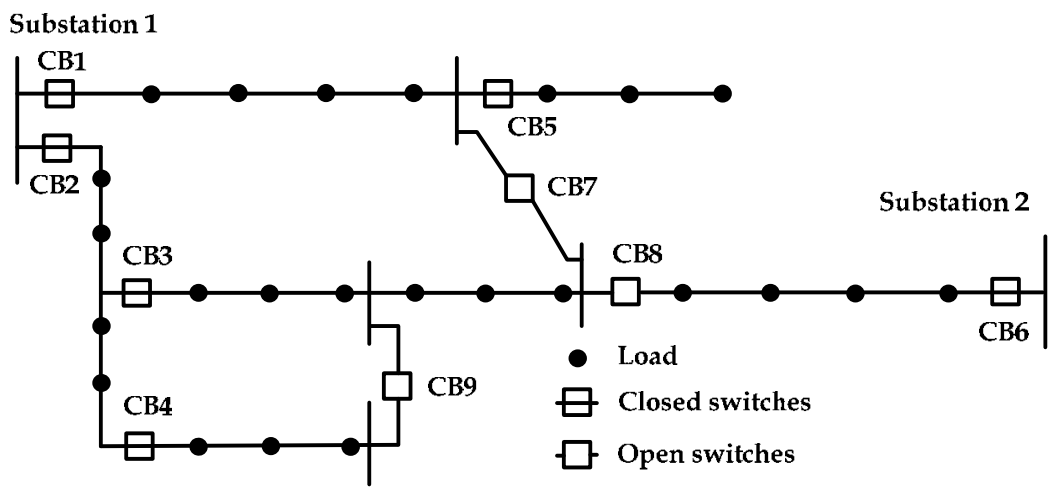

Fig. 1. Schematic diagram of a distribution system

Optimum operation of distribution systems can be achieved by reconfiguring the system to minimize the losses as the operating conditions change. Reconfiguration problem essentially belongs to combinatorial optimization problem because this problem is carried out by taking into account various operational constraints in large scale distribution systems. It is, therefore, difficult to rapidly obtain an exact optimal solution on real system (Chung-Fu, 2008). A flowchart for feeder reconfiguration algorithm is shown in Fig 2. 


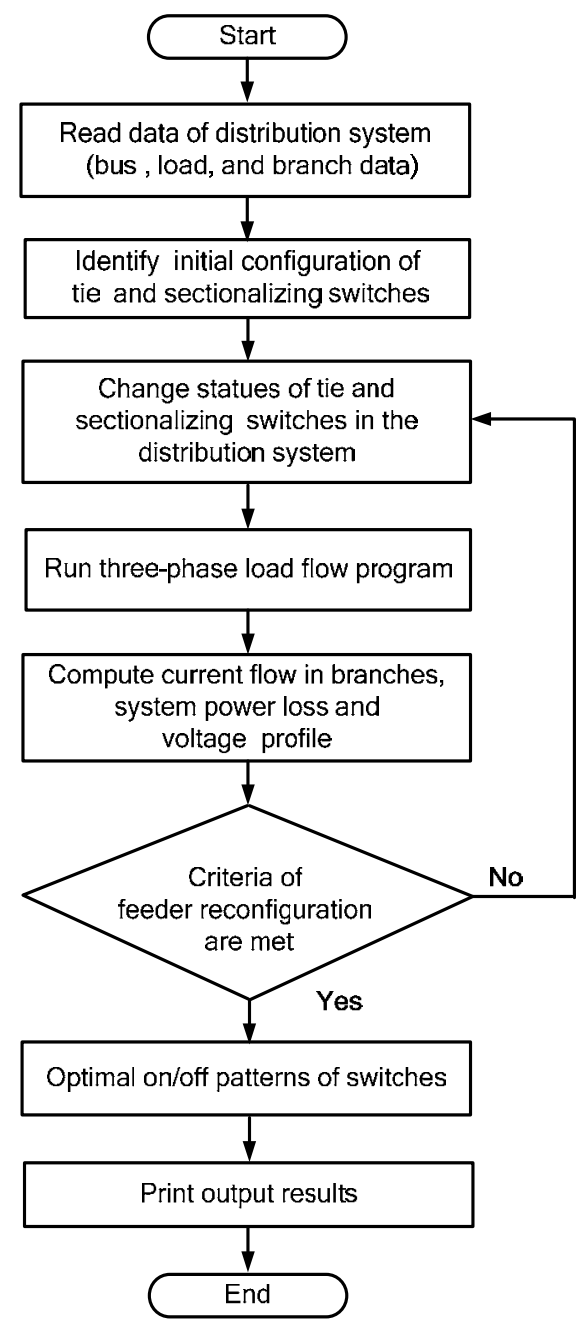

Fig. 2. Flowchart of feeder reconfiguration for loss reduction

\section{Tabu search}

\subsection{Background}

Tabu search is a meta-heuristic that guides a local heuristic search strategy to explore the solution space beyond local optimality. Tabu search was developed by Glover and has been used to solve a wide range of hard optimization problems, such as resource planning, telecommunications, financial analysis, scheduling, space planning, and energy distribution (Dengiz \& Alabas, 2000). The basic idea behind the search is a move from a current solution to its neighborhood by effectively utilizing a memory to provide an efficient search for optimality. The memory is called "Tabu list", which stores attributes of solutions. In the search process, the solutions are in the Tabu list cannot be a candidate of the next iteration. As a result, it helps inhibit choosing the same solution many times and avoid being trapped into cycling of the solutions (Glover, 1989). The quality of a move in solution space is 
assessed by aspiration criteria that provide a mechanism (see Fig. 3) for overriding the Tabu list. Aspiration criteria are analogous to a fitness function of the genetic algorithm and the Bolzman function in the simulated annealing.

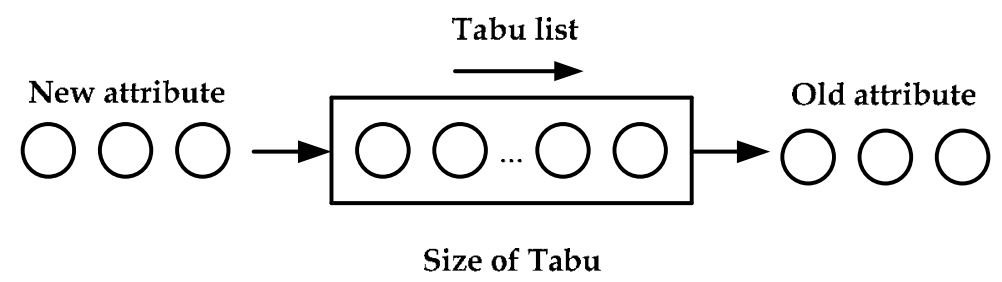

Fig. 3. Mechanism of Tabu list

\subsection{Neighborhood}

In the search process, a move to the best solution in the neighborhood, although its quality is worse than the current solution, is allowed. This strategy helps escape from local optimal and explore wider in the search space. A Tabu list includes recently selected solutions that are forbidden to prevent cycling. If the move is present in the Tabu list, it is accepted only if it has a better aspiration level than the minimal level so far. Fig. 4 shows the main concept of a search direction in Tabu search (Mori \& Ogita, 2002).

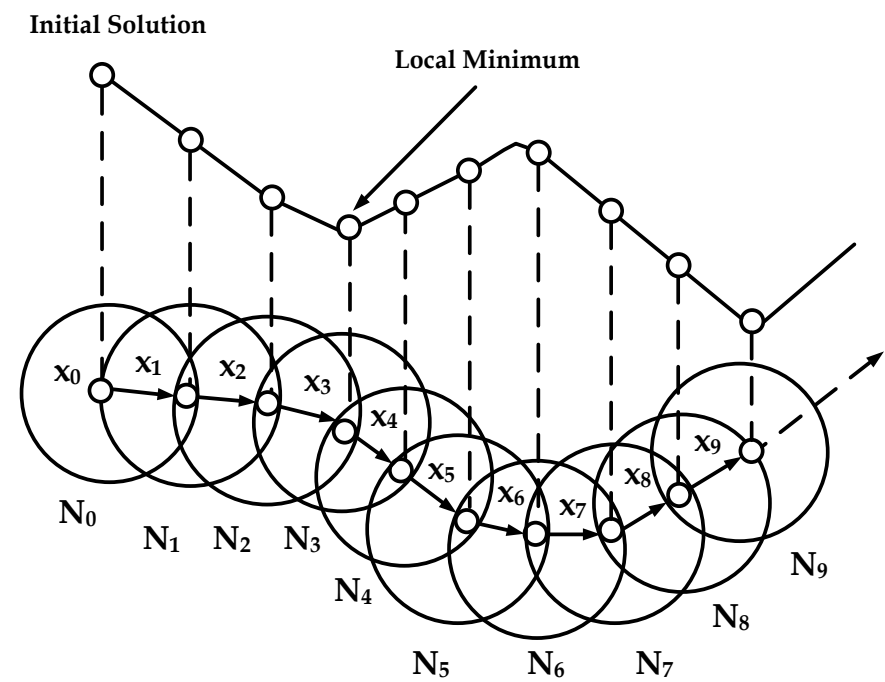

Fig. 4. Search direction of Tabu search

An application of the Tabu search algorithm is shown by a three-feeder distribution system in Fig. 5 (Su, C. T. \& Lee, C. S. 2003). The system consists of 16 buses, 13 load points, 13 normally closed switches, and 3 normally open switches. The initial configuration states that switches located on branch No. 14, No. 15 and No. 16 are open. With this configuration, the initial power loss is $511.44 \mathrm{~kW}$. Fig. 6 shows moves from the current solution to two feasible solutions generated by the Tabu search: neighborhood solutions 1 and 2 . The moves to solutions 1 and 2 give a power loss of $676.63 \mathrm{~kW}$ and $483.87 \mathrm{~kW}$, respectively. The same process 
continues until 100 iterations. The optimal solution indicates that switch No. 16 remains open and the statuses of switches No. 7 and 8 are changed from 'closed' to 'open', giving a real power loss of $466.12 \mathrm{~kW}$.

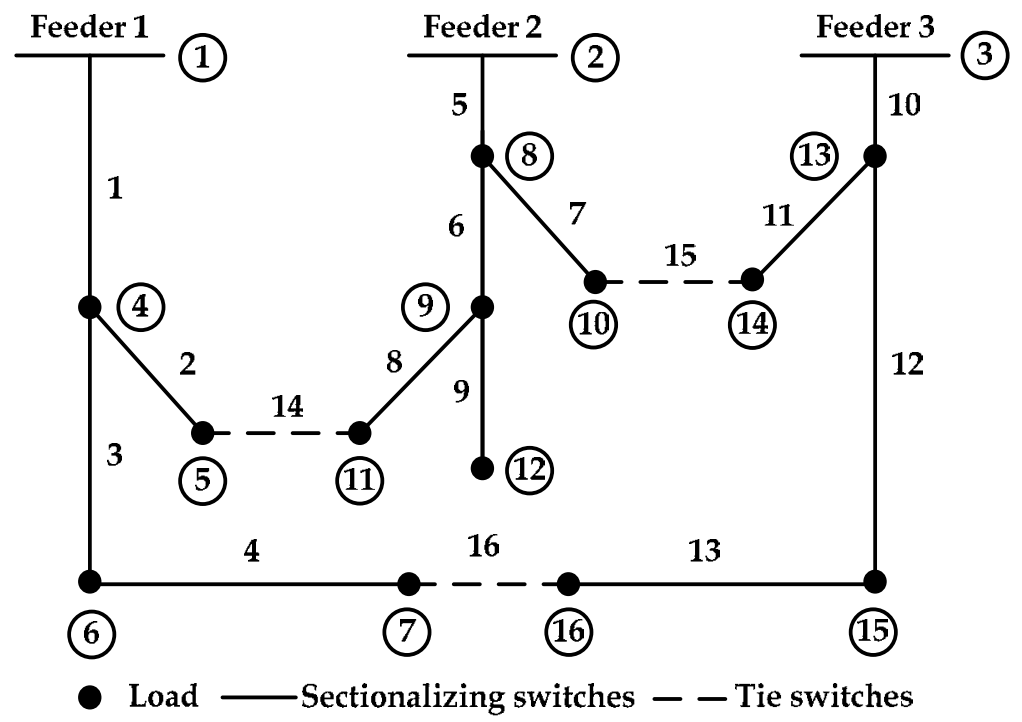

Fig. 5. Single-line diagram of 16-bus distribution system

$$
\begin{aligned}
& S_{k}=\text { Switch to be opened during reconfiguration } \\
& (k=1,2, \ldots, 16)
\end{aligned}
$$

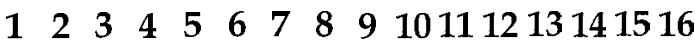

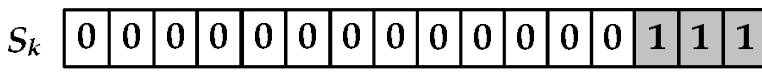

$$
\begin{aligned}
& \text { Current solution }
\end{aligned}
$$

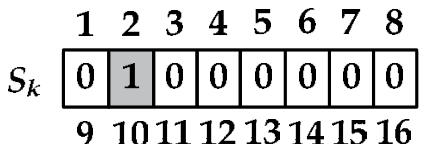

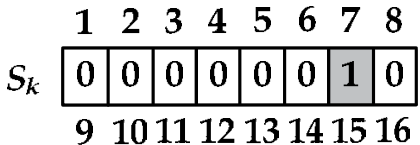

$$
\begin{aligned}
& \begin{array}{l|l|l|l|l|l|l|l|l|}
S_{k} & \mathbf{0} & \mathbf{0} & \mathbf{0} & \mathbf{0} & \mathbf{0} & \mathbf{0} & \mathbf{1} & \mathbf{1} \\
\hline
\end{array} \\
& \begin{array}{ll|l|l|l|l|l|l|l|}
S_{k} & 0 & 0 & 0 & 0 & 0 & 1 & 0 & 1 \\
\hline
\end{array} \\
& \text { Neighborhood solution } 2
\end{aligned}
$$

\section{0 : switch closed, 1 : switch open}

Fig. 6. Neighborhood search for tie and sectionalizing switches

\section{Membership function of objective}

\section{A. Membership function for power loss}

The power loss is calculated by 


$$
\mathrm{P}_{\text {LOSS }}=\sum_{\mathrm{k}=1}^{1}\left|\mathrm{I}_{\mathrm{k}}\right|^{2} \mathrm{R}_{\mathrm{k}}
$$

Where

$\mathrm{P}_{\text {LOSS }}=$ total power loss

$\mathrm{I}_{\mathrm{k}}=$ current flow in branch $\mathrm{k}$

$\mathrm{R}_{\mathrm{k}}=$ resistance of branch $\mathrm{k}$

$1=$ number of feeders

Let us define the ratio of power loss as (Das, 2006).

$$
\mathrm{PL}_{\mathrm{t}}=\frac{\mathrm{P}_{\mathrm{LOSS}, \mathrm{t}}}{\mathrm{P}_{\mathrm{LOSS}, 0}}
$$

The membership function of power loss is assigned to be trapezoidal fuzzy number demonstrated in Fig. 7. It is fully satisfied if the system loss is smaller than $P L_{\min }$. Between $\mathrm{PL}_{\min }$ and $\mathrm{PL}_{\max }$, the satisfaction level declines as the system loss becomes wider and unacceptable if exceeding $\mathrm{PL}_{\max }$, thus the zero membership value given for this point.

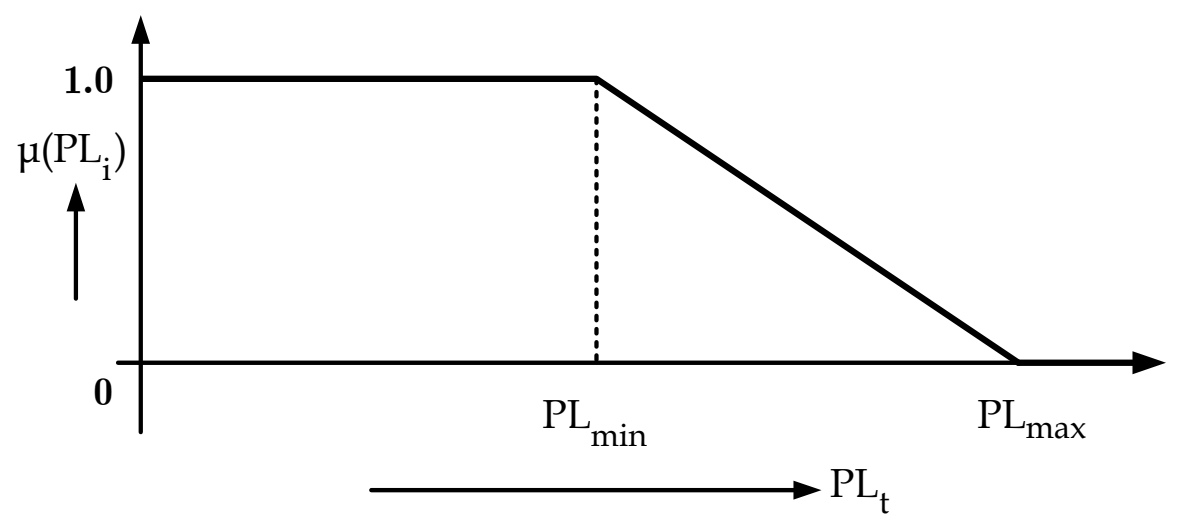

Fig. 7. Membership function for power loss reduction

The membership value $\mu\left(\mathrm{PL}_{\mathrm{t}}\right)$ derived by this membership function can be written as

$$
\mu\left(P L_{t}\right)= \begin{cases}\frac{\left(P L_{\max }-P L_{t}\right)}{\left(P L_{\max }-P L_{\min }\right)} & \text { for } P L_{\min }<P L_{t}<P L_{\max } \\ 1 & \text { for } P L_{t} \leq P L_{\min } \\ 0 & \text { for } P L_{t} \leq P L_{\max }\end{cases}
$$

\section{B. Membership function for load balancing}

Loading balance index (LBI) represents the degree of loading among feeders. This index measures how much a branch can be loaded without exceeding the rated capacity of the branch indicates (Kashem et al., 2006). LBI may be defined as (Peponis \& Papadopoulos, 1995). 


$$
\mathrm{B}_{\mathrm{t}}=\sum_{\mathrm{k} \in \mathrm{B}}\left(\frac{\left|\mathrm{I}_{\mathrm{k}, \mathrm{t}}\right|}{\mathrm{I}_{\mathrm{k}}^{\mathrm{max}}}\right)^{2}
$$

Where

$B_{t}=$ load balancing index for feeder reconfiguration pattern $t$

$\mathrm{B}=$ set of net work branches forming loops

$\mathrm{I}_{\mathrm{k}, \mathrm{t}}=$ current capability of branch $\mathrm{k}$ for feeder reconfiguration pattern $\mathrm{t}$

$\mathrm{I}_{\mathrm{k}}^{\mathrm{max}}=$ maximum current capability of branch $\mathrm{k}$

The load balancing index (LBI) in (4) is normalized by

$$
\mathrm{LB}_{\mathrm{t}}=\frac{\mathrm{B}_{\mathrm{t}}}{\mathrm{B}_{0}}
$$

Where

$\mathrm{LB}_{\mathrm{t}}=$ normalized LBI for feeder reconfiguration pattern $\mathrm{t}$

$\mathrm{B}_{0}=\mathrm{LBI}$ before feeder reconfiguration

The membership function presented in Fig. 8 is used for load balancing objective. As can be seen, the load balancing index is expected to be less than $\mathrm{LB}_{\mathrm{min}}$ and not greater than $\mathrm{LB}_{\max }$. Therefore, the allowable range for $\mathrm{LB}_{\mathrm{t}}$ varies from 0 to $\mathrm{LB}_{\max }$.

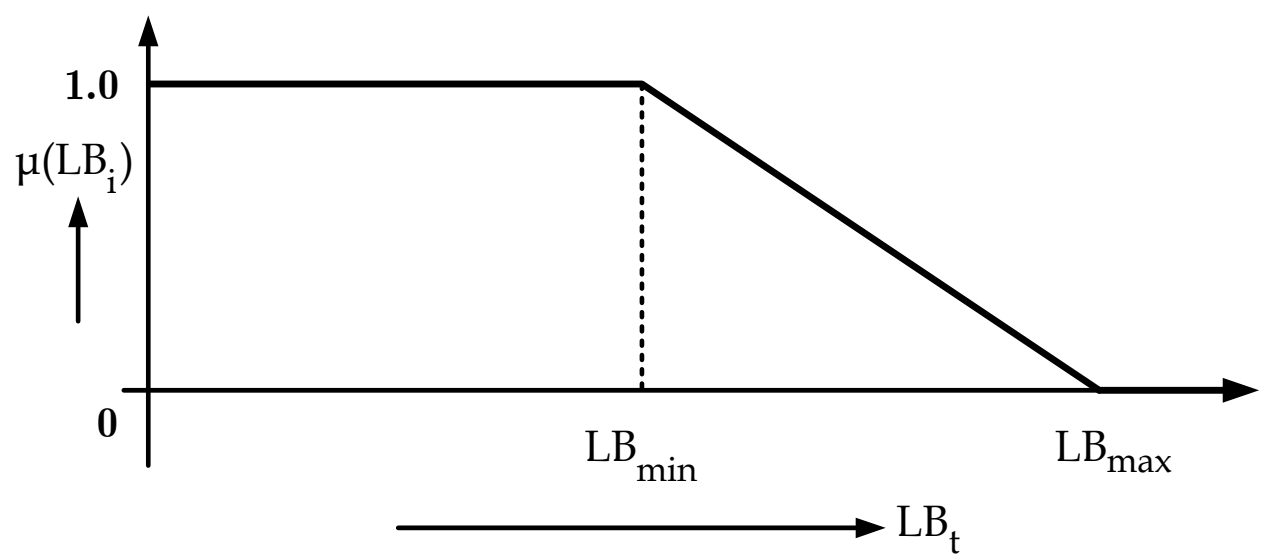

Fig. 8. Membership function for load balancing

$\mathrm{LB}_{\mathrm{t}}$ is employed to compute $\mu\left(\mathrm{LB}_{\mathrm{t}}\right)$ using the membership function given below.

$$
\mu\left(\mathrm{LB}_{\mathrm{t}}\right)= \begin{cases}\frac{\left(\mathrm{LB}_{\text {max }}-\mathrm{LB}_{\mathrm{t}}\right)}{\left(\mathrm{LB}_{\max }-\mathrm{LB}_{\min }\right)} & \text { for } \mathrm{LB}_{\min }<\mathrm{LB}_{\mathrm{t}}<\mathrm{LB}_{\max } \\ 1 & \text { for } \mathrm{LB}_{\mathrm{t}} \leq \mathrm{LB}_{\min } \\ 0 & \text { for } \mathrm{LB}_{\mathrm{t}} \geq \mathrm{LB}_{\max }\end{cases}
$$




\section{Membership function for number of switching operations}

The membership value for the number of switching operations of sectionalizing and tie switches is represented by Fig. 9. The figure states that as long as the number of switching operations is less than $\mathrm{SW}_{\min }$, unity membership value is assigned. The membership function linearly deceases if $\mathrm{SW}_{\mathrm{t}}$ lies between $\mathrm{SW}_{\min }$ and $\mathrm{SW}_{\max }$. A zero membership value is assigned to $S W_{t}$ if it is greater than $S W_{\max }$.

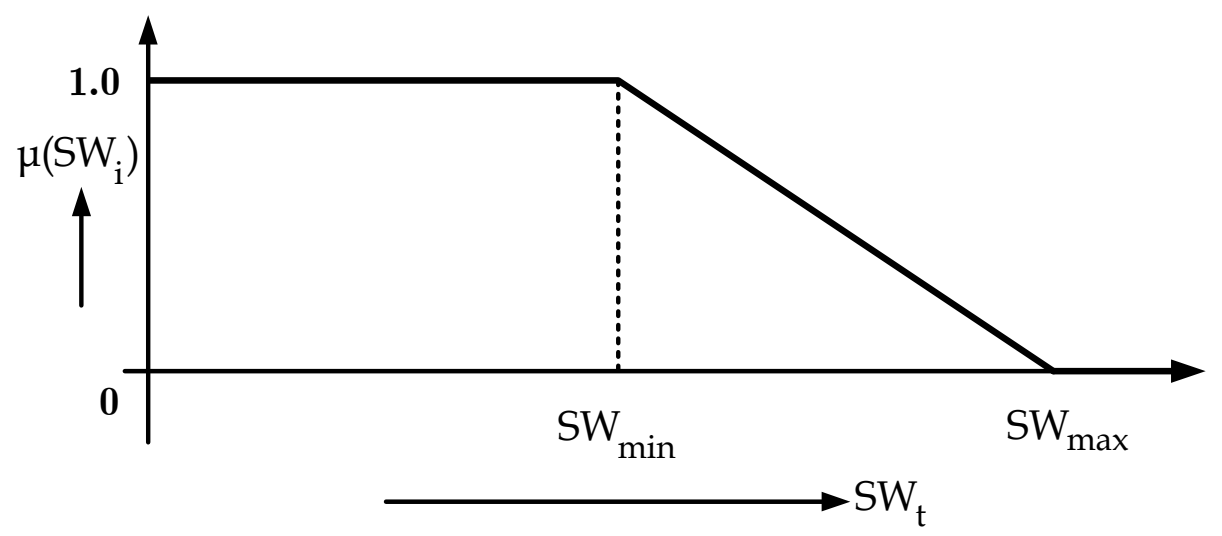

Fig. 9. Membership function for number of switching operation index

The membership function for the number of switching operations is expressed as

$$
\mu\left(S W_{t}\right)= \begin{cases}\frac{\left(S W_{\text {max }}-S W_{t}\right)}{\left(S W_{\text {max }}-S W_{\text {min }}\right)} & \text { for } S W_{\text {min }}<S W_{t}<S W_{\text {max }} \\ 1 & \text { for } S W_{t} \leq S W_{\min } \\ 0 & \text { for } S W_{t} \geq S W_{\max }\end{cases}
$$

Where

$\mathrm{SW}_{\mathrm{t}}=$ switching operation for feeder reconfiguration pattern $\mathrm{t}$

\section{Three-phase power flow}

Power flow is an essential tool for the steady state operational analysis of power systems. The main objective of power flow analysis is to calculate the real and reactive powers flow in each line as well as the magnitude and phase angle of the voltage at each bus of the system for the specific loading conditions (Subrahmanyam, 2009). Certain applications, particularly in distribution automation and optimization, require repeated power flow solutions. As the power distribution networks become more and more complex, there is a higher demand for efficient and reliable system operation. Consequently, power flow studies must have the capability to handle various system configurations with adequate accuracy and speed.

In general, power systems in steady state analysis are operated with balanced three-phase generation and loads by the transposition of transmission lines. However, it is not always 
the case, particularly for radial distribution systems, because of single-phase, two-phase and three-phase loads. As a result, the models based on single phase analysis are not adequate to represent unbalanced three phase networks. The method employed as a major tool to solve the unbalanced power flow problem is based on actual phase quantities with all the relevant equipment modelled in phase coordinates. Thus, power flow solution for unbalanced case and, hence special treatment is required for solving such networks (Ranjan, et. Al., 2004).

The equivalent circuit for each line section is represented by the nominal $\pi$-equivalent model as shown in Fig. 10, which shows a schematic representation of a line connected between bus $\mathrm{i}$ and bus $\mathrm{j}$. This model has one series and two parallel components. The series component stands for the total line impedance consisting of the line resistance and reactance. The parallel components represent the total line capacitance, which is distributed along the line. In the pi-equivalent line representation, the total line capacitance is equally divided into two parts: one lumped at the receiving end bus and the other at sending end bus while the series line impedance is lumped in between. The series impedance and the shunt capacitance for a three-phase line are $3 \times 3$ complex matrices which take into account the mutual inductive coupling between the phases (Zimmerman, 1992).

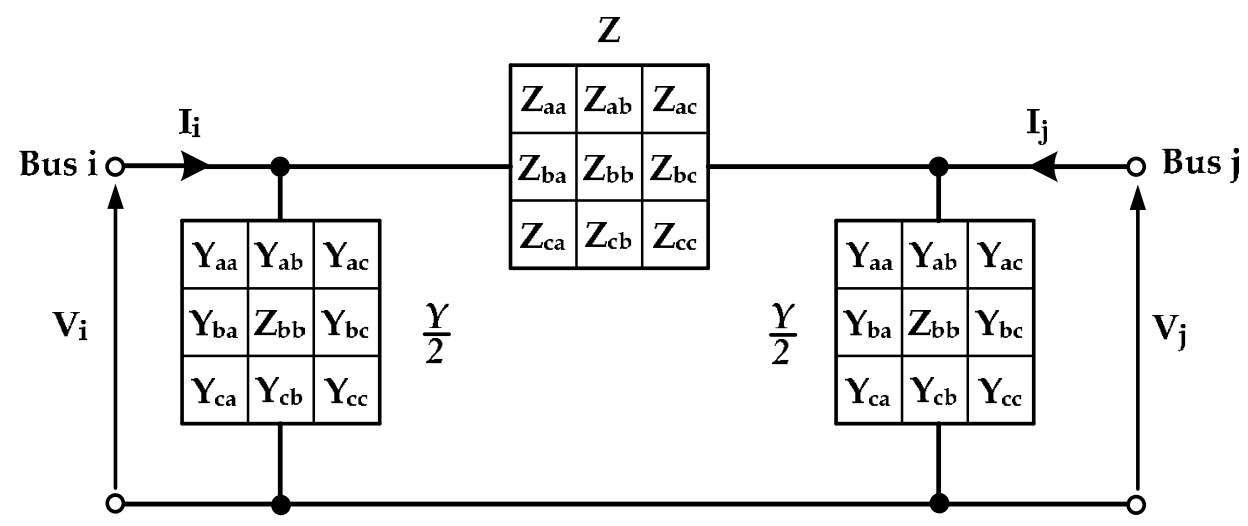

Fig. 10. Compound $\pi$-equivalent model for three-phase

If $Z$ and $Y$ are the $3 \times 3$ matrices representing the series impedance and shunt admittance, respectively, then the admittance matrix for a three-phase conductor between buses $i$ and $j$ is the $6 \times 6$ matrix in equation (8).

$$
Y_{i j}=\left[\begin{array}{cc}
Z^{-1}+\frac{1}{2} Y & -Z^{-1} \\
-Z^{-1} & Z^{-1}+\frac{1}{2} Y
\end{array}\right]
$$

The voltages and currents labeled by the $3 \times 1$ vectors $V_{i}, V_{j}, I_{i}$ and $I_{j}$ in Fig 10. are related by

$$
\left[\frac{I_{i}}{I_{j}}\right]=Y_{i j}\left[\frac{V_{i}}{V_{j}}\right]
$$


Given a system with a total of $\mathrm{n}$ buses, a bus voltage vector $\left(\mathrm{V}_{\mathrm{bus}}\right)$ and a bus injection current vector ( $\left.\mathrm{I}_{\text {bus }}\right)$, are defined as

$$
\begin{gathered}
\mathrm{V}_{\text {bus }}=\left[\mathrm{V}_{1}^{\mathrm{a}}, \mathrm{V}_{1}^{\mathrm{b}}, \mathrm{V}_{1}^{\mathrm{c}}, \mathrm{V}_{2}^{\mathrm{a}}, \mathrm{V}_{2}^{\mathrm{b}}, \mathrm{V}_{2}^{\mathrm{c}}, \ldots, \mathrm{V}_{\mathrm{n}}^{\mathrm{a}}, \mathrm{V}_{\mathrm{n}}^{\mathrm{b}}, \mathrm{V}_{\mathrm{n}}^{\mathrm{c}}\right]^{\mathrm{T}} \\
\mathrm{I}_{\text {bus }}=\left[\mathrm{I}_{1}^{\mathrm{a}}, \mathrm{I}_{1}^{\mathrm{b}}, \mathrm{I}_{1}^{\mathrm{c}}, \mathrm{I}_{2}^{\mathrm{a}}, \mathrm{I}_{2}^{\mathrm{b}}, \mathrm{I}_{2}^{\mathrm{c}}, \ldots, \mathrm{I}_{\mathrm{n}}^{\mathrm{a}}, \mathrm{I}_{\mathrm{n}}^{\mathrm{b}}, \mathrm{I}_{\mathrm{n}}^{\mathrm{c}}\right]^{\mathrm{T}}
\end{gathered}
$$

where $V_{i}^{p}$ and $I_{i}^{p}$ are complex values representing the voltage and injected current, respectively, of phase $\mathrm{p}$ at bus $\mathrm{i}$.

$$
\mathrm{I}_{\text {bus }}=\mathrm{Y}_{\text {bus }} \mathrm{V}_{\text {bus }}
$$

where $Y_{\text {bus }}=\left[Y_{i j}^{p m}\right]$ is a $3 n \times 3 n$ complex matrix whose element relates the voltage $V_{j}^{m}$ to the current $\mathrm{I}_{\mathrm{i}}^{\mathrm{p}}$.

Rewriting (12) as a summation of the individual matrix and vector components gives the injected current of phase $\mathrm{p}$ at bus i. Equation (12) thus becomes (13)

$$
I_{i}^{p}=\sum_{j=1}^{n} \sum_{m=a}^{c} Y_{i j}^{p m} V_{j}^{m}
$$

Active and reactive powers for phase $\mathrm{p}$ at bus i presented in terms of the phase voltage magnitudes and angle are described in (14)

$$
\begin{gathered}
I_{i}^{p}=\sum_{j=1}^{n} \sum_{m=a}^{c} Y_{i j}^{p m} V_{j}^{m} \\
S_{i}^{p}=P_{i}^{p}+Q_{i}^{p} \\
P_{i}^{p}=\left|V_{i}^{p}\right| \sum_{j=1}^{n} \sum_{m=a}^{c}\left|V_{j}^{m}\right|\left[G_{i j}^{p m} \cos \theta_{i j}^{p m}+B_{i j}^{p m} \sin \theta_{i j}^{p m}\right] \\
Q_{i}^{p}=\left|V_{i}^{p}\right| \sum_{j=1}^{n} \sum_{m=a}^{c}\left|V_{j}^{m}\right|\left[G_{i j}^{p m} \sin \theta_{i j}^{p m}-B_{i j}^{p m} \cos \theta_{i j}^{p m}\right]
\end{gathered}
$$

Where

$\mathrm{p}=$ phases $\mathrm{a}, \mathrm{b}$, and $\mathrm{c}$

$P_{i}^{p}, Q_{i}^{p}$ active and reactive power for phase $a, b$, and $c$ at bus $i=1,2,3, \ldots, n$

$Y_{i j}^{p m}=G_{i j}^{p m}+j_{i j}^{p m}$ 
$\mathrm{V}_{\mathrm{i}}^{\mathrm{p}}=$ voltage for phase $\mathrm{a}, \mathrm{b}$, and $\mathrm{c}$ of bus $\mathrm{i}$

$\theta_{\mathrm{ij}}^{\mathrm{pm}}=\theta_{\mathrm{i}}^{\mathrm{p}}-\theta_{\mathrm{j}}^{\mathrm{m}}$

\section{Problem formulation}

The objective functions to be minimized are the system power loss, the load balancing index, and the number of switching operations of sectionalizing and tie switches. Tabu search are employed to generate on/off patterns of the switches. The three objectives of each pattern can then be computed. Each objective is fuzzified using the membership function presented in Section 4 The max-min principle is applied to determine the optimal solution. The objective function can be written as

$$
\begin{gathered}
\mathrm{Z}=\max \left\{\mathrm{T}_{\mathrm{h}}\right\} \mathrm{h}=1,2,3, \ldots, \mathrm{NT} \\
\mathrm{T}_{\mathrm{h}}=\min \left\{\mu\left(\mathrm{PL}_{\mathrm{t}}\right), \mu\left(\mathrm{LB}_{\mathrm{t}}\right), \mu\left(\mathrm{SW}_{\mathrm{t}}\right)\right\} \\
\text { for } \mathrm{t}=1,2,3, \ldots, \mathrm{N}_{\text {neighbor }}
\end{gathered}
$$

Where

$\mathrm{Z}=$ fuzzy decision for an optimal solution

$\mathrm{T}_{\mathrm{h}}=$ fuzzy decision for the objectives being considered

NT =number of solutions in Tabu list

$\mu\left(\mathrm{PL}_{\mathrm{t}}\right)=$ membership value for power loss of feeder reconfiguration pattern $\mathrm{t}$

$\mu\left(\mathrm{LB}_{\mathrm{t}}\right)=$ membership value for load balancing of feeder reconfiguration pattern $t$

$\mu\left(S W_{t}\right)=$ membership value for the number of switching operations of feeder reconfiguration pattern $\mathrm{t}$

$\mathrm{N}_{\text {neighbor }}=$ number of solutions neighborhood

The objective function is subjected to the following constraints.

1) Power flow equations

2) Bus voltage limits:

$$
\mathrm{V}^{\mathrm{p}, \mathrm{min}} \leq \mathrm{V}_{\mathrm{i}}^{\mathrm{p}} \leq \mathrm{V}^{\mathrm{p}, \mathrm{max}}
$$

3) Feeder capability limits:

$$
\left|\mathrm{I}_{\mathrm{k}}^{\mathrm{p}}\right| \leq \mathrm{I}_{\mathrm{k}}^{\mathrm{p}, \max } \mathrm{k} \in\{1,2,3, \ldots 1\}
$$

4) Radial configuration format

5) No load-point interruption

Where

${ }_{\mathrm{V}}^{\mathrm{p} \text {, min }} \mathrm{V}^{\mathrm{p} \text {, max }}=$ minimum and maximum voltage for phase $\mathrm{a}, \mathrm{b}$, and $\mathrm{c}$

$\mathrm{I}_{\mathrm{k}}^{\mathrm{p}, \max }=$ maximum current capability for phase $\mathrm{a}, \mathrm{b}$, and $\mathrm{c}$ of branch $\mathrm{k}$ 


\section{Algorithm by Tabu search}

The Tabu search algorithm is applied to solve the optimal or near optimal solution of the feeder configuration problem by taking the following steps:

Step 1: Read the bus, load and branch data of a distribution system including all the operational constraints.

Step 2: Randomly select a feasible solution from the search space: $S_{0} \in \Omega$, where $S_{0}$ is an initial solution and $\Omega$ is the search space.

Step 3: Set the size of a Tabu list, maximum iteration and iteration index $\mathrm{m}=1$.

Step 4: Let the initial solution obtained in step 2 be the current solution and the best solution: $S_{\text {best }}=S_{0}$, and $S_{\text {current }}=S_{0}$, where $S_{\text {best }}$ is the best solution in the search space and $S_{\text {current }}$ is the current solution in search space.

Step 5: Perform a power flow analysis to determine power loss, bus voltages, and branch currents.

Step 6: Determine the membership values of all the objectives.

Step 7: Calculate $T_{h}=\min \left\{\mu\left(\mathrm{PL}_{\mathrm{t}}\right), \mu\left(\mathrm{LB}_{\mathrm{t}}\right), \mu\left(\mathrm{SW}_{\mathrm{t}}\right)\right\}$. The value of $\mathrm{T}_{\mathrm{h}}$ represents the decision in a fuzzy environment that can be viewed as the intersection of the membership functions of objectives (Zimmermann, 1987). The intersection of membership functions is defined by the minimum operation.

Step 8: Calculate the objective function of $S_{\text {best }}$ by $f\left(S_{\text {best }}\right)=Z=\max \left\{T_{h}\right\}$. The value of $\mathrm{Z}$ indicates the highest degree of membership in the fuzzy decision (Zimmermann, 1987) and is assigned to be the aspiration level.

Step 9: Generate a set of solutions in the neighborhood of $S_{\text {current }}$ by changing the switch numbers that should be opened. This set of solutions is designated as $S_{\text {neighbor }}$.

Step 10: Calculate the aspiration level for each member of $S_{\text {neighbor }}$ and choose the one that has the highest aspiration level, $S_{\text {neighbor_best }}$.

Step 11: Check whether the attribute of the solution obtained in step 10 is in the Tabu list. If yes, go to step 12 , or else $S_{\text {current }}=S_{\text {neighbor_best }}$ and go to step 13 .

Step 12: Accept $S_{\text {neighbor_best }}$ if it has a better aspiration level than $\mathrm{f}_{\text {best }}$ and set $S_{\text {current }}=S_{\text {neighbor_best }}$, or else select a next-best solution that is not in the Tabu list to become the current solution.

Step 13: Update the Tabu list and set $\mathrm{m}=\mathrm{m}+1$.

Step 14: Repeat steps 7 to 13 until a specified maximum iteration has been reached.

Step15: Report the optimal solution.

\section{Case study}

The test system for the case study is a radial distribution system with 69 buses, 7 laterals and 5 tie-lines (looping branches), as shown in Fig. 11. The current carrying capacity of branch No.1-9 is 400 A, No. 46-49 and No. 52-64 are 300 A and the other remaining branches including the tie lines are 200 A. Four DG units are located at buses 14, 35, 46, 
and 53 with capacities of 300, 200, 100, and $400 \mathrm{~kW}$, respectively. The base values for voltage and power are $12.66 \mathrm{kV}$ and $100 \mathrm{MVA}$. Each branch in the system has a sectionalizing switch for reconfiguration purpose. The load data are given in Table 1 and Table 2 provides branch data (Savier \& Das, 2007). The initial statuses of all the sectionalizing switches (switches No. 1-68) are closed while all the tie-switches (switch No. 69-73) open. The total loads for this test system are 3,801.89 kW and 2,694.10 kVAr. The minimum and maximum voltages are set at 0.95 and 1.05 p.u. The maximum iteration for the Tabu search algorithm is 100 . The fuzzy parameters associated with the three objectives are given in Table 3.

\begin{tabular}{|c|c|c|c|c|c|}
\hline $\begin{array}{c}\text { Bus } \\
\text { Number }\end{array}$ & $\begin{array}{c}\mathrm{P}_{\mathrm{L}} \\
(\mathrm{kW})\end{array}$ & $\begin{array}{c}\mathrm{QL}_{\mathrm{L}} \\
(\mathrm{kVAr})\end{array}$ & $\begin{array}{c}\text { Bus } \\
\text { Number }\end{array}$ & $\begin{array}{c}P_{\mathrm{L}} \\
(\mathrm{kW})\end{array}$ & $\begin{array}{c}\mathrm{QL}_{\mathrm{L}} \\
\text { (kVAr) }\end{array}$ \\
\hline 6 & 2.60 & 2.20 & 37 & 26.00 & 18.55 \\
\hline 7 & 40.40 & 30.00 & 39 & 24.00 & 17.00 \\
\hline 8 & 75.00 & 54.00 & 40 & 24.00 & 17.00 \\
\hline 9 & 30.00 & 22.00 & 41 & 1.20 & 1.00 \\
\hline 10 & 28.00 & 19.00 & 43 & 6.00 & 4.30 \\
\hline 11 & 145.00 & 104.00 & 45 & 39.22 & 26.30 \\
\hline 12 & 145.00 & 104.00 & 46 & 39.22 & 26.30 \\
\hline 13 & 8.00 & 5.00 & 48 & 79.00 & 56.40 \\
\hline 14 & 8.00 & 5.50 & 49 & 384.70 & 274.50 \\
\hline 16 & 45.50 & 30.00 & 50 & 384.70 & 274.50 \\
\hline 17 & 60.00 & 35.00 & 51 & 40.50 & 28.30 \\
\hline 18 & 60.00 & 35.00 & 52 & 3.60 & 2.70 \\
\hline 20 & 1.00 & 0.60 & 53 & 4.35 & 3.50 \\
\hline 21 & 114.00 & 81.00 & 54 & 26.40 & 19.00 \\
\hline 22 & 5.00 & 3.50 & 55 & 24.00 & 17.20 \\
\hline 24 & 28.00 & 20.00 & 59 & 100.00 & 72.00 \\
\hline 26 & 14.00 & 10.00 & 61 & $1,244.00$ & 888.00 \\
\hline 27 & 14.00 & 10.00 & 62 & 32.00 & 23.00 \\
\hline 28 & 26.00 & 18.60 & 64 & 227.00 & 162.00 \\
\hline 29 & 26.00 & 18.60 & 65 & 59.00 & 42.00 \\
\hline 33 & 14.00 & 10.00 & 66 & 18.00 & 13.00 \\
\hline 34 & 19.50 & 14.00 & 67 & 18.00 & 13.00 \\
\hline 35 & 6.00 & 4.00 & 68 & 28.00 & 20.00 \\
\hline 36 & 26.00 & 18.55 & 69 & 28.00 & 20.00 \\
\hline
\end{tabular}

Table 1. Load data of 69-bus distribution system 


\begin{tabular}{|c|c|c|c|c|}
\hline $\begin{array}{l}\text { Branch } \\
\text { Number }\end{array}$ & $\begin{array}{l}\text { Sending } \\
\text { end bus }\end{array}$ & $\begin{array}{l}\text { Receiving } \\
\text { end bus }\end{array}$ & $\begin{array}{c}\mathrm{R} \\
(\Omega)\end{array}$ & $\begin{array}{c}X \\
(\Omega)\end{array}$ \\
\hline 1 & 1 & 2 & 0.0005 & 0.0012 \\
\hline 2 & 2 & 3 & 0.0005 & 0.0012 \\
\hline 3 & 3 & 4 & 0.0015 & 0.0036 \\
\hline 4 & 4 & 5 & 0.0251 & 0.0294 \\
\hline 5 & 5 & 6 & 0.3660 & 0.1864 \\
\hline 6 & 6 & 7 & 0.3811 & 0.1941 \\
\hline 7 & 7 & 8 & 0.0922 & 0.0470 \\
\hline 8 & 8 & 9 & 0.0493 & 0.0251 \\
\hline 9 & 9 & 10 & 0.8190 & 0.2707 \\
\hline 10 & 10 & 11 & 0.1872 & 0.0619 \\
\hline 11 & 11 & 12 & 0.7114 & 0.2351 \\
\hline 12 & 12 & 13 & 1.0300 & 0.3400 \\
\hline 13 & 13 & 14 & 1.0440 & 0.3450 \\
\hline 14 & 14 & 15 & 1.0580 & 0.3496 \\
\hline 15 & 15 & 16 & 0.1966 & 0.0650 \\
\hline 16 & 16 & 17 & 0.3744 & 0.1238 \\
\hline 17 & 17 & 18 & 0.0047 & 0.0016 \\
\hline 18 & 18 & 19 & 0.3276 & 0.1083 \\
\hline 19 & 19 & 20 & 0.2106 & 0.0690 \\
\hline 20 & 20 & 21 & 0.3416 & 0.1129 \\
\hline 21 & 21 & 22 & 0.0140 & 0.0046 \\
\hline 22 & 22 & 23 & 0.1591 & 0.0526 \\
\hline 23 & 23 & 24 & 0.3463 & 0.1145 \\
\hline 24 & 24 & 25 & 0.7488 & 0.2475 \\
\hline 25 & 25 & 26 & 0.3089 & 0.1021 \\
\hline 26 & 26 & 27 & 0.1732 & 0.0572 \\
\hline 27 & 3 & 28 & 0.0044 & 0.0108 \\
\hline 28 & 28 & 29 & 0.0640 & 0.1565 \\
\hline 29 & 29 & 30 & 0.3978 & 0.1315 \\
\hline 30 & 30 & 31 & 0.0702 & 0.0232 \\
\hline 31 & 31 & 32 & 0.3510 & 0.1160 \\
\hline 32 & 32 & 33 & 0.8390 & 0.2816 \\
\hline 33 & 33 & 34 & 1.7080 & 0.5646 \\
\hline 34 & 34 & 35 & 1.4740 & 0.4873 \\
\hline 35 & 3 & 36 & 0.0044 & 0.0108 \\
\hline 36 & 36 & 37 & 0.0640 & 0.1565 \\
\hline 37 & 37 & 38 & 0.1053 & 0.1230 \\
\hline
\end{tabular}




\begin{tabular}{|c|c|c|c|c|}
\hline 38 & 38 & 39 & 0.0304 & 0.0355 \\
\hline 39 & 39 & 40 & 0.0018 & 0.0021 \\
\hline 40 & 40 & 41 & 0.7283 & 0.8509 \\
\hline 41 & 41 & 42 & 0.3100 & 0.3623 \\
\hline 42 & 42 & 43 & 0.0410 & 0.0478 \\
\hline 43 & 43 & 44 & 0.0092 & 0.0116 \\
\hline 44 & 44 & 45 & 0.1089 & 0.1373 \\
\hline 45 & 45 & 46 & 0.0009 & 0.0012 \\
\hline 46 & 4 & 47 & 0.0034 & 0.0084 \\
\hline 47 & 47 & 48 & 0.0851 & 0.2083 \\
\hline 48 & 48 & 49 & 0.2898 & 0.7091 \\
\hline 49 & 49 & 50 & 0.0822 & 0.2011 \\
\hline 50 & 8 & 51 & 0.0928 & 0.0473 \\
\hline 51 & 51 & 52 & 0.3319 & 0.1114 \\
\hline 52 & 9 & 53 & 0.1740 & 0.0886 \\
\hline 53 & 53 & 54 & 0.2030 & 0.1034 \\
\hline 54 & 54 & 55 & 0.2842 & 0.1447 \\
\hline 55 & 55 & 56 & 0.2813 & 0.1433 \\
\hline 56 & 56 & 57 & 1.5900 & 0.5337 \\
\hline 57 & 57 & 58 & 0.7837 & 0.2630 \\
\hline 58 & 58 & 59 & 0.3042 & 0.1006 \\
\hline 59 & 59 & 60 & 0.3861 & 0.1172 \\
\hline 60 & 60 & 61 & 0.5075 & 0.2585 \\
\hline 61 & 61 & 62 & 0.0974 & 0.0496 \\
\hline 62 & 62 & 63 & 0.1450 & 0.0738 \\
\hline 63 & 63 & 64 & 0.7105 & 0.3619 \\
\hline 64 & 64 & 65 & 1.0410 & 0.5302 \\
\hline 65 & 11 & 66 & 0.2012 & 0.0611 \\
\hline 66 & 66 & 67 & 0.0047 & 0.0014 \\
\hline 67 & 12 & 68 & 0.7394 & 0.2444 \\
\hline 68 & 68 & 69 & 0.0047 & 0.0016 \\
\hline \multicolumn{5}{|c|}{ Tie line } \\
\hline 69 & 11 & 43 & 0.5000 & 0.5000 \\
\hline 70 & 13 & 21 & 0.5000 & 0.5000 \\
\hline 71 & 15 & 46 & 1.0000 & 0.5000 \\
\hline 72 & 50 & 59 & 2.0000 & 1.0000 \\
\hline 73 & 27 & 65 & 1.0000 & 0.5000 \\
\hline
\end{tabular}

Table 2. Branch data of 69-bus distribution system 


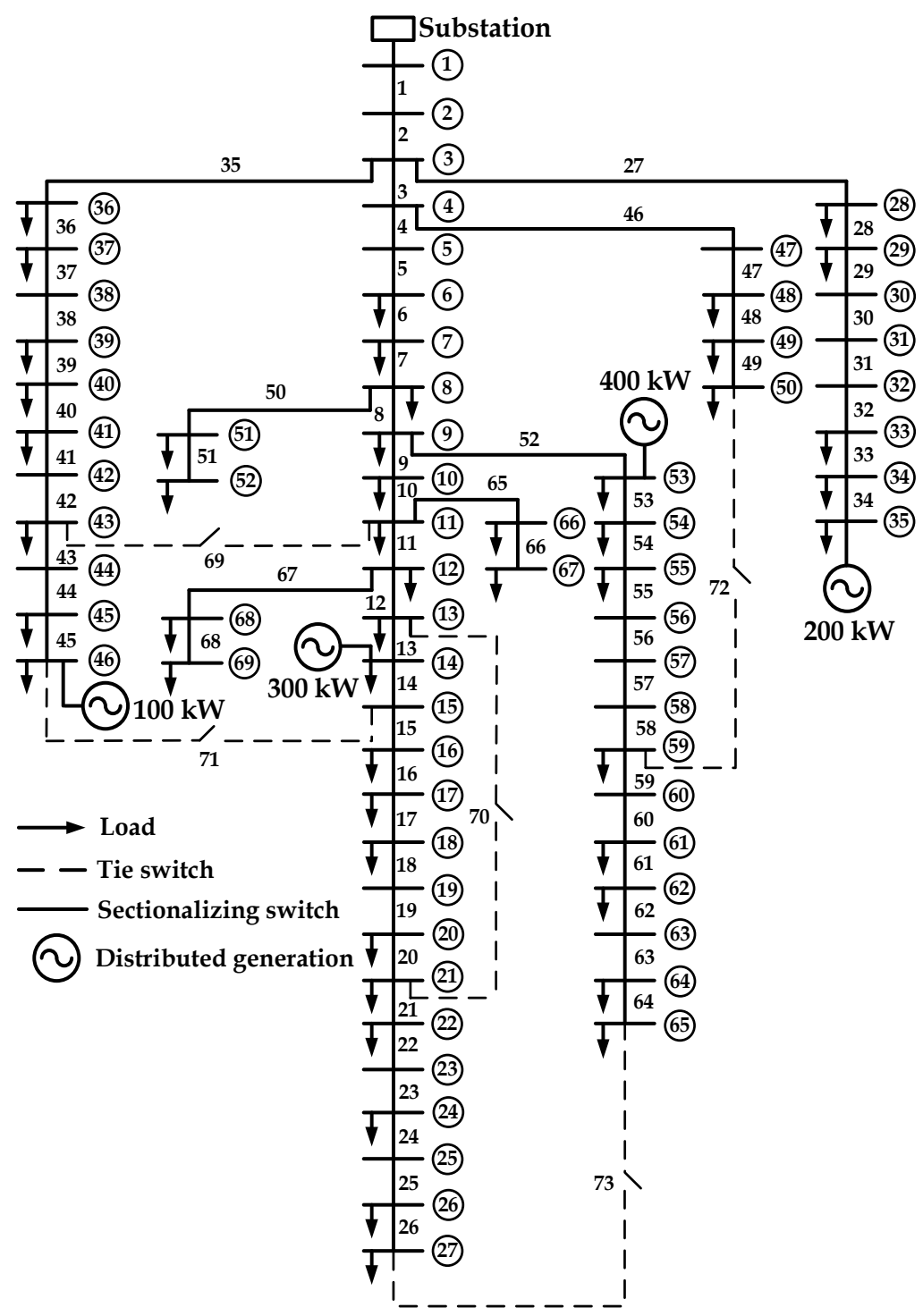

Fig. 11. Single-line diagram of 69-bus distribution system with distributed generation

Six cases are examined as follows:

Case 1: The system is without feeder reconfiguration

Case 2: The system is reconfigured so that the system power loss is minimized.

Case 3: The system is reconfigured so that the load balancing index is minimized.

Case 4: The same as case 2 with a constraint that the number of switching operations of sectionalizing and ties switches must not exceed 4.

Case 5: The system is reconfigured using the solution algorithm described in Section 4.

Case 6: The same as case 5 with system $20 \%$ unbalanced loading, indicating that the load of phase $b$ is $20 \%$ higher than that of phase but lower than that in phase $c$ by the same amount. 


\begin{tabular}{cc}
\hline Fuzzy parameters & Value \\
\hline $\mathrm{PL}_{\text {min }}$ & 0.5 \\
$\mathrm{PL}_{\text {max }}$ & 1.0 \\
$\mathrm{LB}_{\min }$ & 0.7 \\
$\mathrm{LB}_{\max }$ & 1.0 \\
$\mathrm{SW}_{\min }$ & 4.0 \\
$\mathrm{SW}_{\max }$ & 10 \\
\hline
\end{tabular}

Table 3. Fuzzy parameters for each objective

The numerical results for the six cases are summarized in Table 4. In cases 1-5 (balanced systems), the system power loss and the LBI are highest, and the minimum bus voltage in the system violates the lower limit of 0.95 per unit. The voltage profile of case 1 is shown in Fig. 12. It is observed that the voltages at buses 57-65 are below 0.95 p.u. because a large load of 1,244 kW are drawn at bus 61 . Without the four DG units, the system loss would be $673.89 \mathrm{~kW}$. This confirms that DG units can normally, although not necessarily, help reduce current flow in the feeders and hence contributes to power loss reduction, mainly because they are usually placed near the load being supplied. In cases 2 to 5 , where the feeders are reconfigured and the voltage constraint is imposed in the optimization process, no bus voltage is found violated (see Figs.12 and 13).

\begin{tabular}{|c|c|c|c|c|c|c|}
\hline & Case 1 & Case 2 & Case 3 & Case 4 & Case 5 & Case 6 \\
\hline $\begin{array}{c}\text { Sectionalizing switches to be } \\
\text { open }\end{array}$ & - & $\begin{array}{c}12,20, \\
52,61\end{array}$ & $\begin{array}{c}42,14, \\
20,52,61\end{array}$ & 52,62 & $13,52,63$ & 12,5261 \\
\hline Tie switches to be closed & - & $\begin{array}{c}70,71, \\
72,73\end{array}$ & $\begin{array}{c}69,70, \\
71,72,73\end{array}$ & 72,73 & $71,72,73$ & $71,72,73$ \\
\hline Power loss (kW) & 586.83 & 246.33 & 270.81 & 302.37 & 248.40 & 290.98 \\
\hline Minimum voltage (p.u.) & 0.914 & 0.954 & 0.954 & 0.953 & 0.953 & 0.965 \\
\hline Load balancing index (LBI) & 2.365 & 1.801 & 1.748 & 1.921 & 1.870 & 2.273 \\
\hline $\begin{array}{c}\text { Number of switching } \\
\text { operations }\end{array}$ & - & 8 & 10 & 4 & 6 & 6 \\
\hline
\end{tabular}

Table 4. Results of case study

As expected, the system power loss is at minimum in case 2, the LBI index is at minimum in case 3 , and the number of switching operations of switches is at minimum in case 4 . It is obviously seen from case 5 that a fuzzy multiobjective optimization offers some flexibility that could be exploited for additional trade-off between improving one objective function and degrading the others. For example, the power loss in case 5 is slightly higher than in case 2 but case 5 needs only 6, instead of 8 , switching operations. Although the LBI of case 3 is better than that of case 5, the power loss and number of switching operations of case 3 are greater. Comparing case 4 with case 5 , a power loss of about $18 \mathrm{~kW}$ can be saved from two more switching operations. It can be concluded that the fuzzy model has a potential for solving the decision making problem in feeder reconfiguration and offers decision makers some flexibility to incorporate their own judgment and priority in the optimization model. 
The membership value of case 5 for power loss is 0.961 , for load balancing index is 0.697 and for number of switching operations is 0.666 .

When the system unbalanced loading is $20 \%$ in case 6 , the power loss before feeder reconfiguration is about $624.962 \mathrm{~kW}$. The membership value of case 6 for power loss is 0.840 , for load balancing index is 0.129 and for the number of switching operations is 0.666 . The voltage profile of case 6 is shown in Fig. 14 .

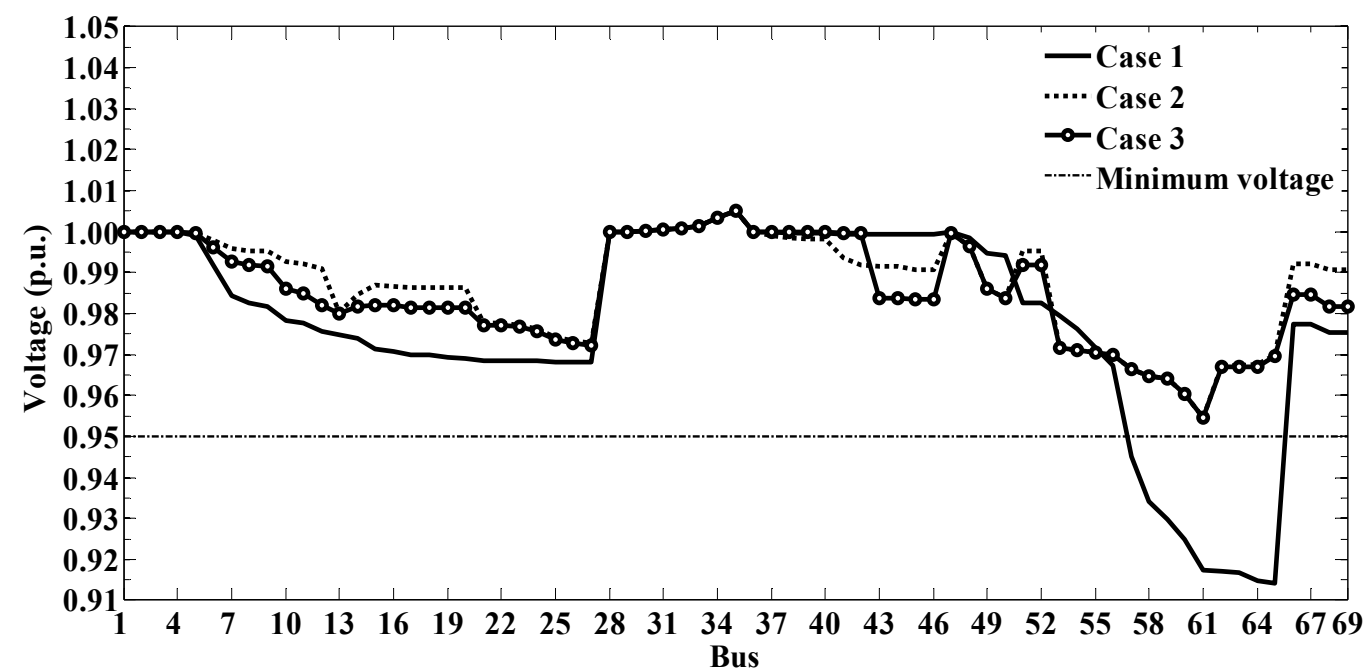

Fig. 12. Bus voltage profile in cases 1, 2 and 3

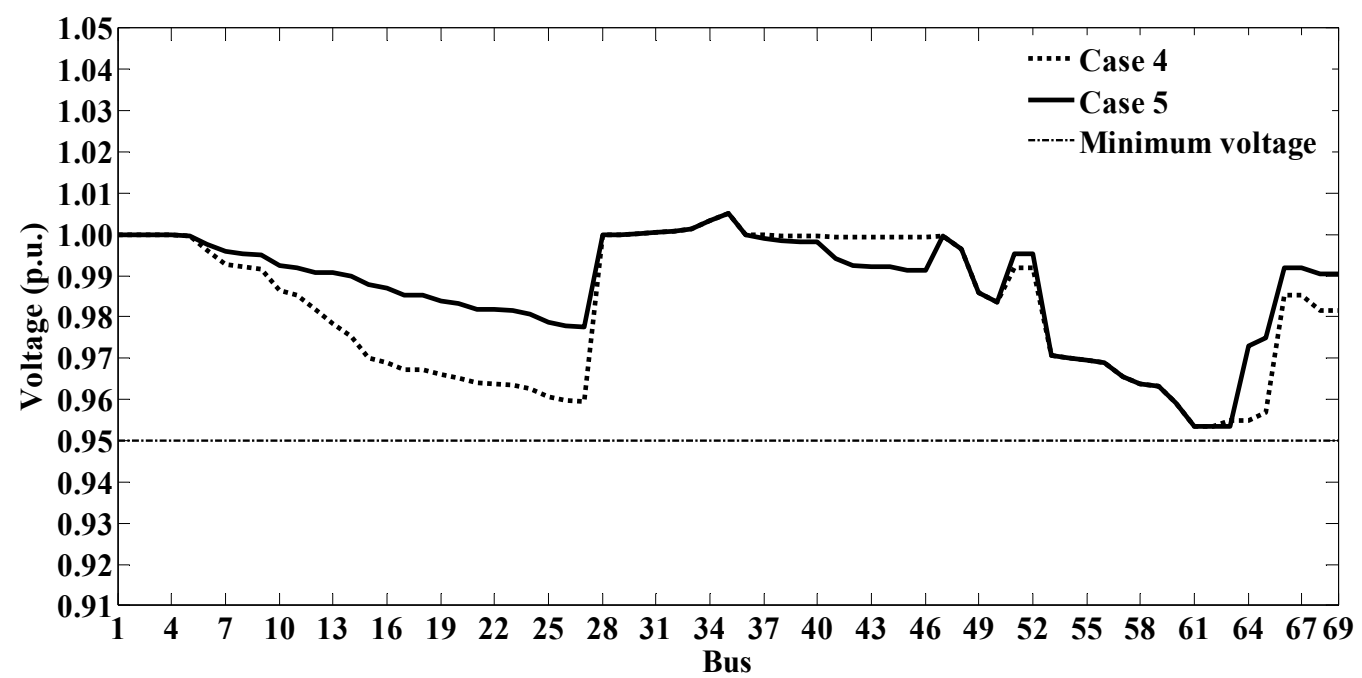

Fig. 13. Bus voltage profile in cases 4 and 5 


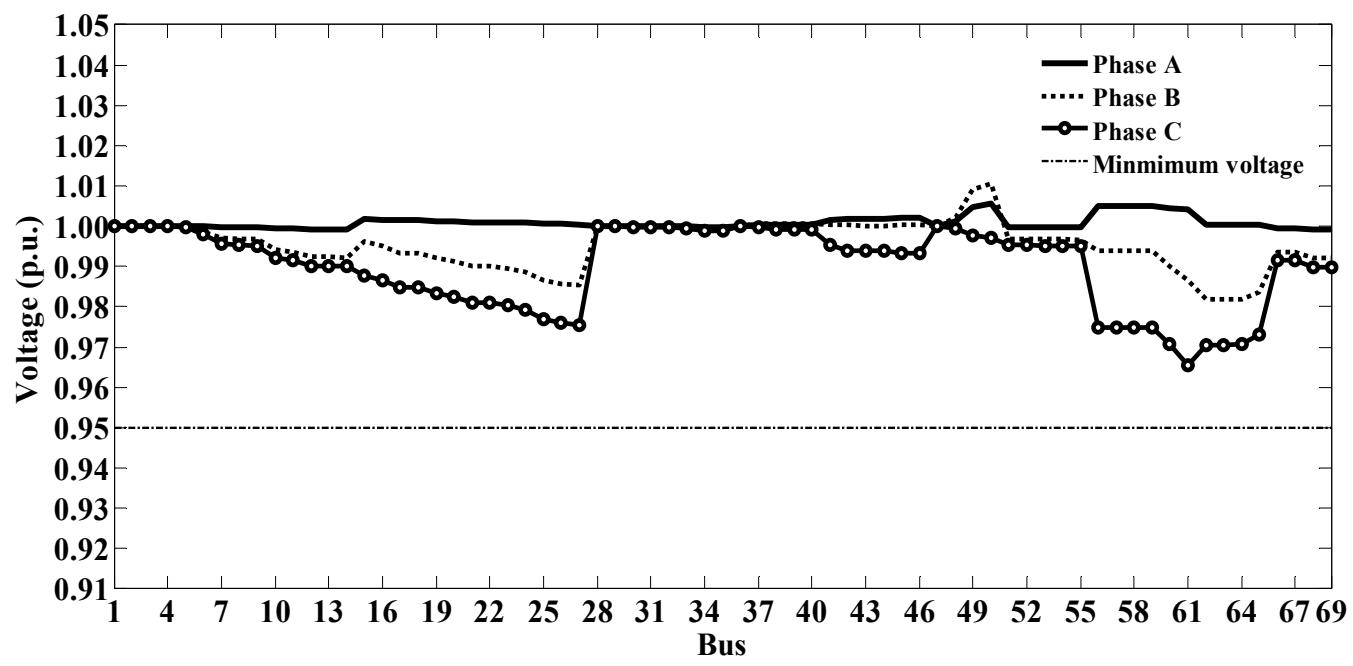

Fig. 14. Bus voltage profile in cases 6

\section{Conclusion}

A fuzzy multiobjective algorithm has been presented to solve the feeder reconfiguration problem in a distribution system with distributed generators. The algorithm attempts to maximize the satisfaction level of the minimization of membership values of three objectives: system power loss, load balancing index, and number of switching operations for tie and sectionalizing switches. These three objectives are modeled by a trapezoidal membership function. The search for the best compromise among the objectives is achieved by Tabu search. On the basis of the simulation results obtained, the satisfaction level of one objective can be improved at the expense of that of the others. The decision maker can prioritize his or her own objective by adjusting some of the fuzzy parameters in the feeder reconfiguration problem.

\section{References}

Kashem, M. A.; Ganapathy V. \& Jasmon, G. B. (1999). Network reconfiguration for load balancing in distribution networks. IEE Proc.-Gener. Transm. Distrib., Vol. 146, No. 6, (November) pp. 563-567.

$\mathrm{Su}$, C. T. \& Lee, C. S. (2003). Network reconfiguration of distribution systems using improved mixed-integer hybrid differential evolution. IEEE Trans. Power Delivery, Vol. 18, No. 3, (July) pp. 1022-1027.

Baran, M. E. \& Wu, F. F. (1989). Network reconfiguration in distribution systems for loss reduction and load balancing. IEEE Trans. on Power Delivery, Vol. 4, No. 2, (April) pp. 1401-1407.

Kashem, M.A.; Ganapathy V. \& Jasmon, G.B. (2000). Network reconfiguration for enhancement of voltage stability in distribution networks. IEE Proc.-Gener. Transm. Distrib., Vol. 147, No. 3, (May) pp. 171-175. 
Gil, H. A. \& Joos, G. (2008). Models for quantifying the economic benefits of distributed generation, IEEE Trans. on Power Systems, Vol. 23, No. 2, (May) pp. 327-335.

Jones, G. W. \& Chowdhury, B. H. (2008). Distribution system operation and planning in the presence of distributed generation technology. Proceedings of Transmission and Distribution Conf. and Exposition, (April) pp. 1-8.

Quezada, V. H. M.; Abbad, J. R. \& Roman, T. G. S. (2006). Assessment of energy distribution losses for increasing penetration of distributed generation. IEEE Trans. on Power Systems, Vol. 21, No. 2, (May) pp. 533-540.

Carpaneto, E. G.; Chicco, \& Akilimali, J. S. (2006). Branch current decomposition method for loss allocation in radial distribution systems with distributed generation. IEEE Trans. on Power Systems, Vol. 21, No. 3, (August) pp. 1170-1179.

Chung-Fu Chang. (2008). Reconfiguration and capacitor placement for loss reduction of distribution systems by ant colony search algorithm. IEEE Trans. on Power Systems, Vol. 23, No. 4, (November) pp. 1747-1755.

Dengiz, B. \& Alabas, C. (2000). Simulation optimization using tabu search. Proceedings of Winter Simulation Conf., pp. 805-810.

Glover, F. (1989). Tabu search-part I. ORSA J. Computing, Vol. 1, No.3,

Mori, H. \& Ogita, Y. (2002). Parallel tabu search for capacitor placement in radial distribution system. Proceedings of Power Engineering Society Winter Meeting Conf., Vol. 4, pp 2334-2339.

Das, D. (2006). A fuzzy multiobjective approach for network reconfiguration of distribution systems. IEEE Trans. on Power Delivery, Vol. 21, No. 1, (January) pp. 1401-1407

Peponis, G. \& Papadopoulos M. (1995). Reconfiguration of radial distribution networks: application of heuristic methods on large-scale networks. IEE Proc.-Trans. Distrib., Vol. 142, No. 6. (November) pp. 631-638.

Subrahmanyam, J. B. V. (2009). Load flow solution of unbalanced radial distribution systems. J. Theoretical and Applied Information Technology, Vol. 6, No. 1, (August) pp. 40-51

Ranjan, R.; Venkatesh, B.; Chaturvedi , A. \& Das, D. (2004). Power flow solution of threephase unbalanced radial distribution network. Electric Power Components and Systems, Vol. 32, No.4, pp.421-433.

Zimmerman, R. D. (1992). Network reconfiguration for loss reduction in three-phase power distribution system. Thesis of the Graduate School of Cornell University, May

Zimmermann, H. J. (1987). Fuzzy set decision making, and expert systems. Kluwer Academic Publishers

Savier, J. S. \& Das, D. (2007). Impact of network reconfiguration on loss allocation of radial distribution systems. IEEE Trans. on Power Delivery, Vol. 22, No.4, (October) pp. 2473-2480. 


\title{
Energy Managements in the Chemical and Biochemical World, as It may be Understood from the Systems Chemistry Point of View
}

\author{
Zoltán Mucsi, Péter Ábrányi Balogh, Béla Viskolcz and Imre G. Csizmadia \\ University of Szeged \\ Hungary
}

\section{Introduction}

If anyone compares biochemical and industrial processes from energetic point of view, it may well be concluded that the bio-production of any living entity exhibits far greater energy efficiency than any human controlled industrial production. Most of the bioreactions take place at the same cell at the same temperature, within a narrow range, without external heating or cooling system. In contrast to that, industrial chemical processes usually proceed separately at various reaction temperatures from $-80{ }^{\circ} \mathrm{C}$ to $+200{ }^{\circ} \mathrm{C}$. Furthermore, these reactions require significantly larger energy input, which is taken in either as external heating or internal molecular energy of active reagents (high energy reagents, like acylhalogenides and $\mathrm{LiBH}_{4}$ ), meanwhile the large excess of energy waste, released during the reaction, must be led away.

Behind the high efficacy of biological processes compared to man-made processes there are two energetic reasons. At first, biological reactions used to start from low energy intermediates and proceed by means of very well designed catalysts, such as enzymes, therefore activation energy gaps are low (Figure 1, green line), consequently reaction can be carried out at ambient temperature. Secondly, reagents used by living organism, like NAD ${ }^{+}$, FAD, ATP and other bio-reagents are so effectives under enzymatic conditions, that they need to store only slightly more than the necessary energy within their structures to carry out the reaction, resulting low energy waste, or in other word, reagents balance the reaction energy by their internal molecular energy. Two non-catalyzed laboratory processes (black dashed and red lines) are compared with a enzyme catalyzed biological process (green line) schematically in Figure 1 and Table 1. For any reaction to proceed, sufficient reagent has to be chosen, which at Gibbs free energy level is higher than the Gibbs free energy level of the product. The Gibbs free energy difference between the row material and product $\left(G_{I} \rightarrow G_{F}\right)$ is called built-in energy. To prepare active reagent from row material, some energy needs to be invested $\left(\mathrm{G}_{\mathrm{I}} \rightarrow \mathrm{G}_{1}\right.$ and $\left.\mathrm{G}_{\mathrm{I}} \rightarrow \mathrm{G}_{3}\right)$. Under laboratory conditions I (black, dashed line), instead of the addition of high energy and very active reagents, we react only low energy reagent $\left(\right.$ at $\left.\mathrm{G}_{1}\right)$, therefore thermal energy via increased reaction temperature need to be input $\left(G_{1} \rightarrow G_{5}\right)$, consequently the waste energy is high. In laboratory condition II (red line), normally high energy and active reagent is reacted via low transition state $\left(G_{3} \rightarrow G_{4}\right)$, it does not require high reaction temperature. However, the overall waste energy remained 
significant, due to the large investment energy to prepare active reagents from row materials. In contrast with the previous processes, biological system (green line) uses low energy reagents (at $\left.G_{1}\right)$ joint with effective enzyme catalyst $\left(G_{I} \rightarrow G_{2}\right)$, therefore the resultant waste energy is minimal.

\begin{tabular}{ccccccc}
\hline Processes & $\begin{array}{c}\text { Type of the } \\
\text { process }\end{array}$ & $\begin{array}{c}\text { Invested } \\
\text { energy }\end{array}$ & $\begin{array}{c}\text { Transition } \\
\text { state energy }\end{array}$ & $\begin{array}{c}\text { Waste } \\
\text { energy }\end{array}$ & $\begin{array}{c}\text { Reaction } \\
\text { rate }\end{array}$ & $\begin{array}{c}\text { Product } \\
\text { efficacy }\end{array}$ \\
\hline $\begin{array}{c}\text { Laboratory I } \\
\text { (black dashed) }\end{array}$ & non-catalysed & $\begin{array}{c}\text { low } \\
\mathrm{G}_{1}-\mathrm{G}_{\mathrm{I}}\end{array}$ & $\begin{array}{c}\text { high } \\
\mathrm{G}_{5}-\mathrm{G}_{1}\end{array}$ & $\begin{array}{c}\text { high } \\
\mathrm{G}_{\mathrm{F}}-\mathrm{G}_{5}\end{array}$ & low & low \\
\hline $\begin{array}{c}\text { Laboratory II } \\
\text { (red line) }\end{array}$ & non-catalysed & $\begin{array}{c}\text { high } \\
\mathrm{G}_{3}-\mathrm{G}_{\mathrm{I}}\end{array}$ & $\begin{array}{c}\text { low } \\
\mathrm{G}_{4}-\mathrm{G}_{3}\end{array}$ & $\begin{array}{c}\text { high } \\
\mathrm{G}_{\mathrm{F}}-\mathrm{G}_{4}\end{array}$ & high & high \\
\hline $\begin{array}{c}\text { Biological } \\
\text { (green) }\end{array}$ & catalysed & $\begin{array}{c}\text { Low } \\
\mathrm{G}_{1}-\mathrm{G}_{\mathrm{I}}\end{array}$ & $\begin{array}{c}\text { low } \\
\mathrm{G}_{2}-\mathrm{G}_{1}\end{array}$ & $\begin{array}{c}\text { low } \\
\mathrm{G}_{\mathrm{F}}-\mathrm{G}_{2}\end{array}$ & high & high \\
\hline
\end{tabular}

Table 1. Summary of the comparison of two laboratory and a biological processes from energy management point of view, joining to Figure 1.
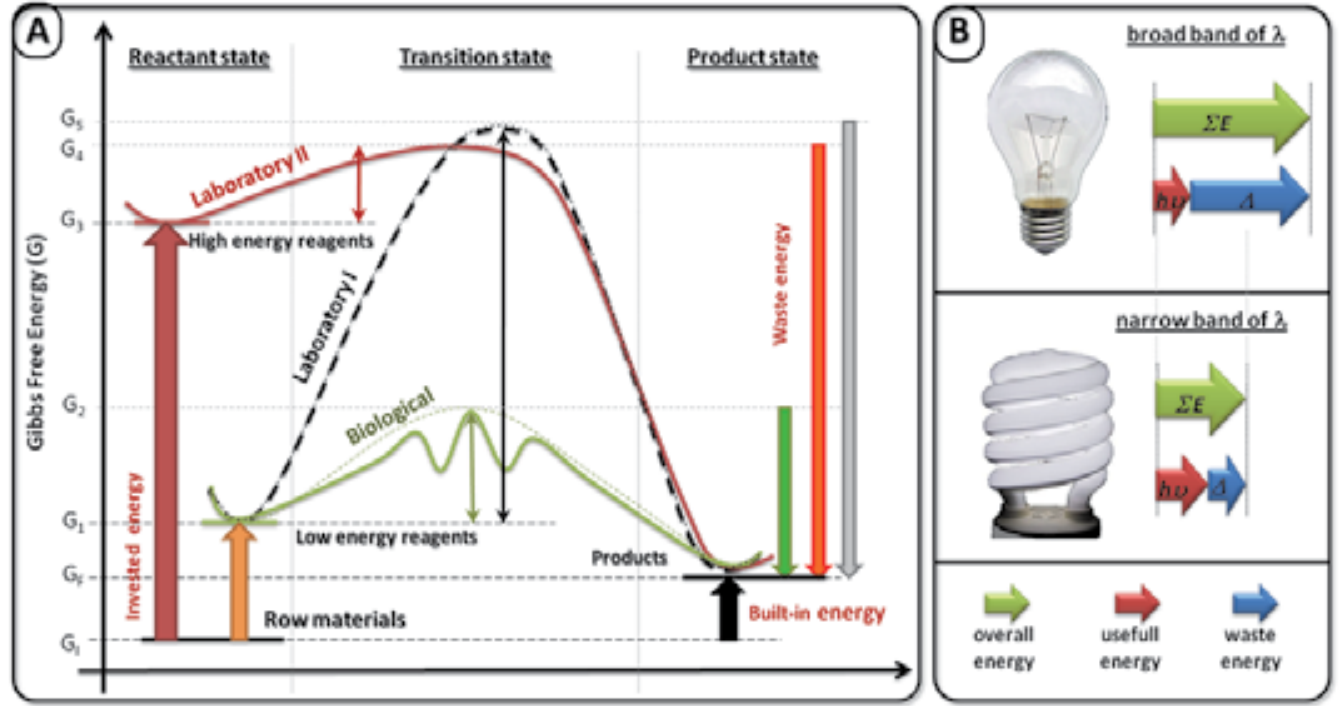

Fig. 1. (A) Relative Gibbs free energy profiles for a reaction carried out at laboratory I. (black dashed line, low energy reagent, non-catalyzed process, therefore high energy transition state and large energy waste), biological (green line, low energy reagent, enzymatic catalysis, therefore low energy transition state and low energy waste) and laboratory II. conditions (red line, high energy reagent, non-catalyzed process, but low energy transition state and high energy waste). The biological reaction is the most energy efficient due to the smallest invested and waste-energies. $\mathrm{G}_{\mathrm{I}}=$ initial Gibbs free energy; $\mathrm{G}_{\mathrm{F}}=$ final Gibbs free energy; from $G_{1}$ to $G_{5}=$ different Gibbs free energy levels. (B) A schematic comparison of an incandescent light bulb with a modern 'energy-saving bulbs' being in analogy with the manmade reaction and natural processes. 
By symbolic analogy, one may compare the influence of structure on energy loss in many synthetic reactions to that of an incandescent light bulb; the latter losing (as 'side product' wavelengths and heat) $\sim 70 \%$ of energy input to produce the desired product 'white light' (Figure 1B). Yield of white light may be improved by optimizing each of the systemic components, where even shape contributes to efficient excitation of filament-gas to populate a narrow band of desired energy levels; as in modern 'energy-saving bulbs'.

Nowadays, in modern organic and medicinal chemistry a typical molecule may involve several analogous functional groups, which are able to react with a reagent dissimilarly, resulting in different products, therefore the fast determination or at least estimation of the reactivity of these functional groups is essential for planning synthetic routes. Nevertheless, in the case of theoretical methods, which can predict reactivities by modeling the reaction mechanism, it is typical that behind a seemingly simple chemical reaction, the real mechanism is quite complex, involving many species in each individual elementary step, like reactants, reagents, solvent molecules, catalysts, and acid or base as co-reagents [1-5]. All these species should be involved in the calculation to investigate the real and detailed mechanism, in order to obtain a correct and accurate view of the reaction taking place in a real media. In fact, determination of the minimal size of the appropriate chemical model (e.g. number of explicit solvent molecules necessary) is very difficult, time and resource consuming [1]. Moreover, an incorrect chemical model provides not only inaccurate energy values, but frequently absolutely wrong or opposite results, questioning the competence of theoretical methods in the applied science [1]. Reactions taking place in media usually require the consideration of a base or an acid as catalyst together with many solvent molecules in an appropriate 3D arrangement $[1,2,5]$. Taking into consideration of all of these criteria, it seems nearly impossible to model even a simple acylation reaction. It was demonstrated earlier that the computation of one or a few, easily and quickly computable quantum mechanical (QM) descriptors, such as aromaticity [6-9], amidicity [10-12], carbonylicity,[13,14] olefinicity,[15-17] and others can predict properly and somewhat quantitatively certain reactivity and selectivity issues. The global and complex view of these descriptors was defined as the concept of systems chemistry [18], wherein molecules are described as strategically located functional components within molecular frameworks, 'valued' at more than their components' sum, acting in unison to effect efficient energy management.

\section{The concept and methodology of systems chemistry}

\subsection{General remarks}

Every organic structure and their energy content can be modeled at three levels of organization. This deconvolution of the total energy into three components is illustrated by

Figure 2. The first level takes into consideration only the $\sigma$ skeleton of a molecule, the energy content of this level can be calculated by the sum of the average sigma bond type. The second level summarizes the $\pi$ scaffold, summing up the $\pi$ energy content of the double bonds (i.e. double bond energy - single bond energ). It is known that adjacent double bonds get into interactions by overlapping between their atomic orbitals. However, the estimation of the energy content of the resonance level is not trivial. In simpler cases, where the number and the types of the $\sigma$ and the $\pi$-bonds do not change the resonance energy is turned out to be a reliable measure of the overall relative energetic of the process. In order to measure it, a novel concept and therefore a novel discipline was defined, wherein molecules are 
<smiles>O=c1ccnc2ccccn12</smiles>
a complex molecule

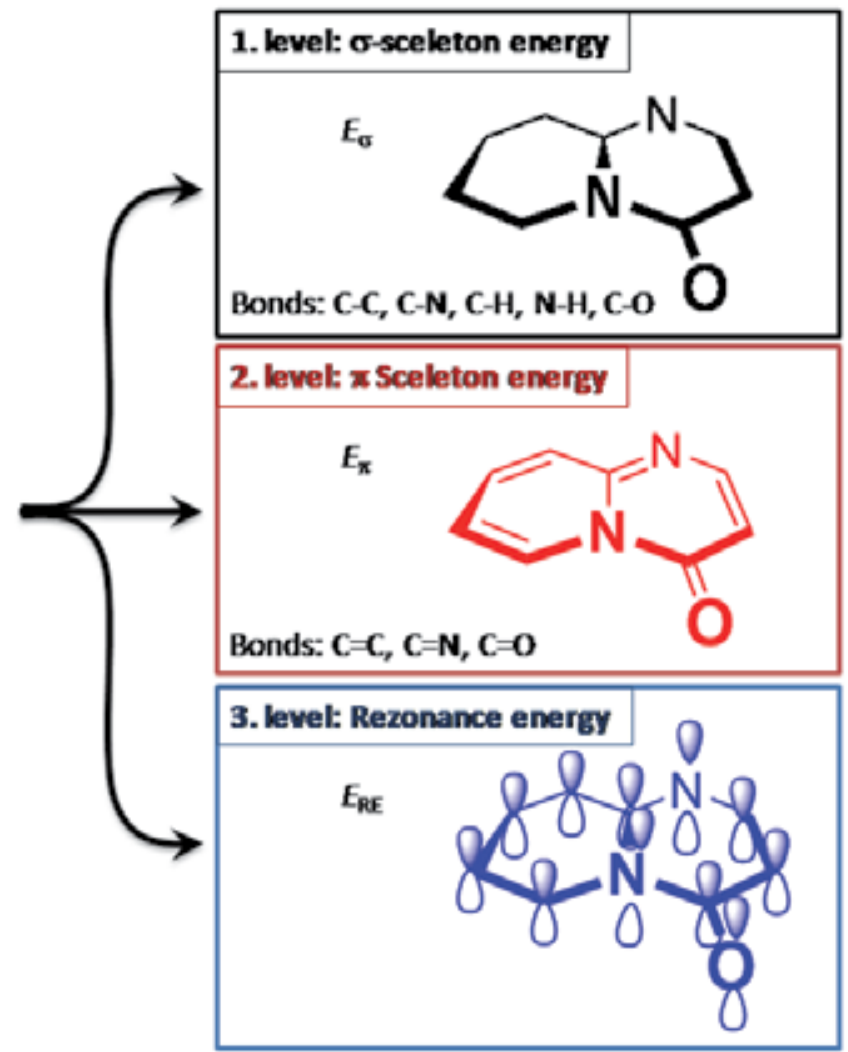

Fig. 2. A schematic illustration of how the internal molecular energy may be deconvoluted to $\sigma, \pi$ and resonance energy.

described as frameworks of strategically located functional components within molecular frameworks, acting in unison to effect efficient energy management. The term 'Systems Chemistry' effectively serves to define the phenomena of an assembly of atoms and functional groups (a molecule) having systemic properties 'valued' at more than their component sum. Systems Chemistry focuses on the framework of component functional groups and atoms within a given molecule acting in unison to orchestrate a variety of chemical phenomena. Molecular properties, such as reactivity and stability, are a result of the relative spatial orientation(s) of constituent atoms, mediated by environmental and statistical factors (e.g. solvent and concentration/bulk, respectively). Systems Chemistry is a discipline wherein component functionalities are not segregated, in a reductionist fashion, but rather where they are considered as integrated parts of a whole system of interacting functional groups; yet, reductionist component resolution is retained.

This implies that functional molecular systems are more than just assemblies of atomic and functional components. To attain Nature's efficiency, one must approach chemical phenomena as systems rather than as single entities. Systems Chemistry has in-hand the types and locations of organic functional groups (e.g. ortho, meta, para substitutions, catalystligand identities) and aims to quantifying their relationships and influence on one another. Coupling between components of a chemically or biologically important molecule, such as aromatic rings, amide groups, olefins, carbonyls and metal-ligands, are central to the 
molecules' chemical efficiency. With the quantification of these couplings in mind, we recently introduced the molecular descriptors: aromaticity,[6] amidicity,[10] carbonylicity,[13] olefinicity,[15] each of which in a surrogate thermodynamic function, contributing to the characterization of the mechanisms by which Nature fine-tunes and stores reaction energies to attain hyper-efficiency.

\subsection{Aromaticity}

Chemical structures and transition states are often influenced by aromatic stabilizing or antiaromatic destabilizing effects, which are not easy to characterize either experimentally or theoretically. The exact description and precise quantification of the aromatic characteristics of ring structures is difficult and requires special theoretical investigation. A novel, yet simple method to quantify both aromatic and antiaromatic qualities on the same linear scale, by using the enthalpy of hydrogenation reaction of the compound has been examined. A reference hydrogenation reaction is also considered on a corresponding nonaromatic reference compound in order to cancel all secondary structure destabilization factors, such as ring strain or double bond strain. From these data the relative enthalpy of hydrogenation may easily be calculated [6]:

$$
\Delta \Delta H_{\mathrm{H} 2}=\Delta H_{\mathrm{H} 2} \text { (examined) }-\Delta H_{\mathrm{H} 2} \text { (reference) } .
$$

In the present work concept, the $\Delta \Delta H_{\mathrm{H} 2}$ value of benzene defines the perfect or completely aromatic character $(+100 \%)$, while the closed shell of the singlet cyclobutadiene represents maximum antiaromaticity $(-100 \%)$.

Aromaticity and antiaromaticity are characterised by a common and universal linear scale based on the heat of hydrogenation $\left(\Delta \mathrm{H}_{\mathrm{H} 2}(\mathrm{I})\right.$, Eq. 2; Figure 3) when cyclobutadiene (1) and benzene (2) are considered as $-100 \%$ and $+100 \%$, respectively. This methodology compares the hydrogenation reaction of the examined compound [3 $\rightarrow 6, \Delta H_{\mathrm{H} 2}(\mathrm{I})$, Eq. 2] with that of a properly chosen reference reaction $\left[\mathbf{9} \rightarrow \mathbf{1 2}, \Delta H_{\mathrm{H} 2}\right.$ (II), Eq. 3]. The difference between the two enthalpy values $\left[\Delta \Delta H_{\mathrm{H} 2}(\mathrm{AR})\right.$, Eq. 4$]$ is transformed to aromaticity percentage (AR \%; Eq. 5), which is the basis of the calculation of the resonance enthalpy $\left[H_{\mathrm{RE}}(\mathrm{AR})\right.$; Eq. 6]. Some aromatic compounds may exhibit larger aromaticity values, than $100 \%$, meaning to a larger resonance enthalpy (RE) inside the system. Typical case is the double ring naphthalene and its analogues, where this larger value is the sum of the resonance enthalpies of the two rings.

$$
\begin{gathered}
\Delta H_{\mathrm{H} 2}(\mathrm{I})=H[\mathbf{6}]-\left\{H[3]+H\left(\mathrm{H}_{2}\right)\right\} \\
\Delta H_{\mathrm{H} 2}(\mathrm{II})=H[\mathbf{1 2}]-\left\{H[\mathbf{9}]+H\left(\mathrm{H}_{2}\right)\right\} \\
\Delta \Delta H_{\mathrm{H} 2}(\mathrm{AR})=\Delta H_{\mathrm{H} 2}(\mathrm{I})-\Delta H_{\mathrm{H} 2}(\mathrm{II}) \\
\mathrm{AR} \%=\mathrm{m}_{\mathrm{AR}} \Delta \Delta H_{\mathrm{H} 2}(\mathrm{AR})+\mathrm{b}_{\mathrm{AR}} \\
H_{\mathrm{RE}}(\mathrm{AR})=\mathrm{AR} \% / \mathrm{m}_{\mathrm{AR}}
\end{gathered}
$$

The various compounds for which aromaticity and antiaromaticity values were determine form a "spectra" of such aromatic/antiaromatic characters are illustrated by Figure 4.

Interesting examples can be found in phosphorous organic compounds [7-9,19], one of them is exemplified in Figure 5. The aromaticity of phospholes was questionable for a long time and the commonly accepted view was that they have a very weak aromatic character. The 
Examined reaction

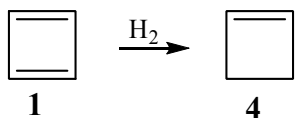<smiles>c1ccccc1</smiles>

2<smiles>CC1C=CCCC=C1</smiles>

5<smiles>C1=CC=CC=CC=C1</smiles>

3

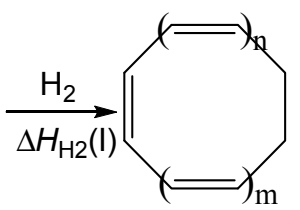

6
Ref erence reaction
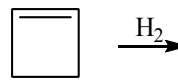<smiles>C1CCC1</smiles>

10<smiles>C1=CCCCC1</smiles>

8

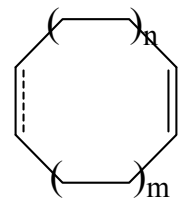

9
Degree of aromaticity

$-100 \%$

$100 \%$

Fig. 3. $\Delta H_{\mathrm{H} 2}$ vales calculated for an antiaromatic and aromatic species.

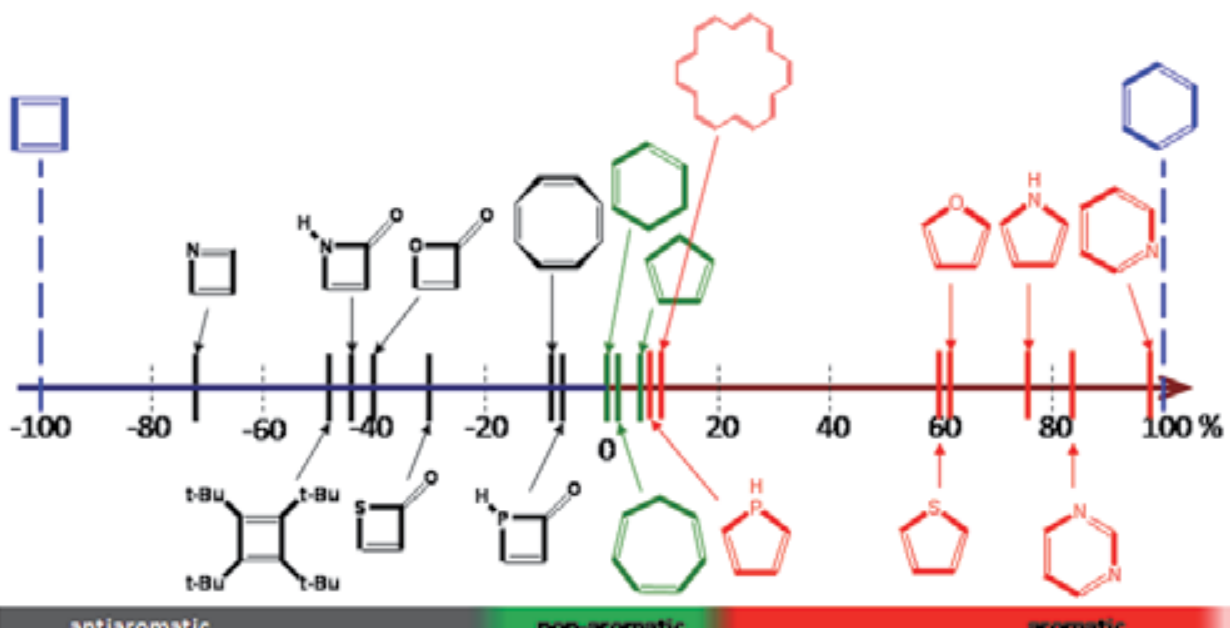

Fig. 4. Combined aromaticity and antiaromaticity spectrum with some representative compounds.

weak aromaticity may be due to the pyramidal geometry around the $\mathrm{P}$ atom since the lone electron pair cannot effectively participate in the delocalization. Several studies revealed that contrary to the stability of phosphole (13), phosphole oxide derivatives (14) exhibit an unusual instability. The phosphole oxides (14) obtained on oxidation of the phospholes (13), undergo a Diels-Alders type [4+2] dimerization reaction to afford 15 (upper line of Figure 5). Other experimental findings revealed that an other phosphole derivative $\mathbf{1 6}$ is stable for days, but their oxidized derivative $\mathbf{1 7}$ is unstable under the same conditions and is 
rearranged to $\mathbf{1 8}$ (lower line of Figure 5). The instability of $\mathbf{1 7}$ was explained by the existing weak antiaromaticity $[9,19]$.

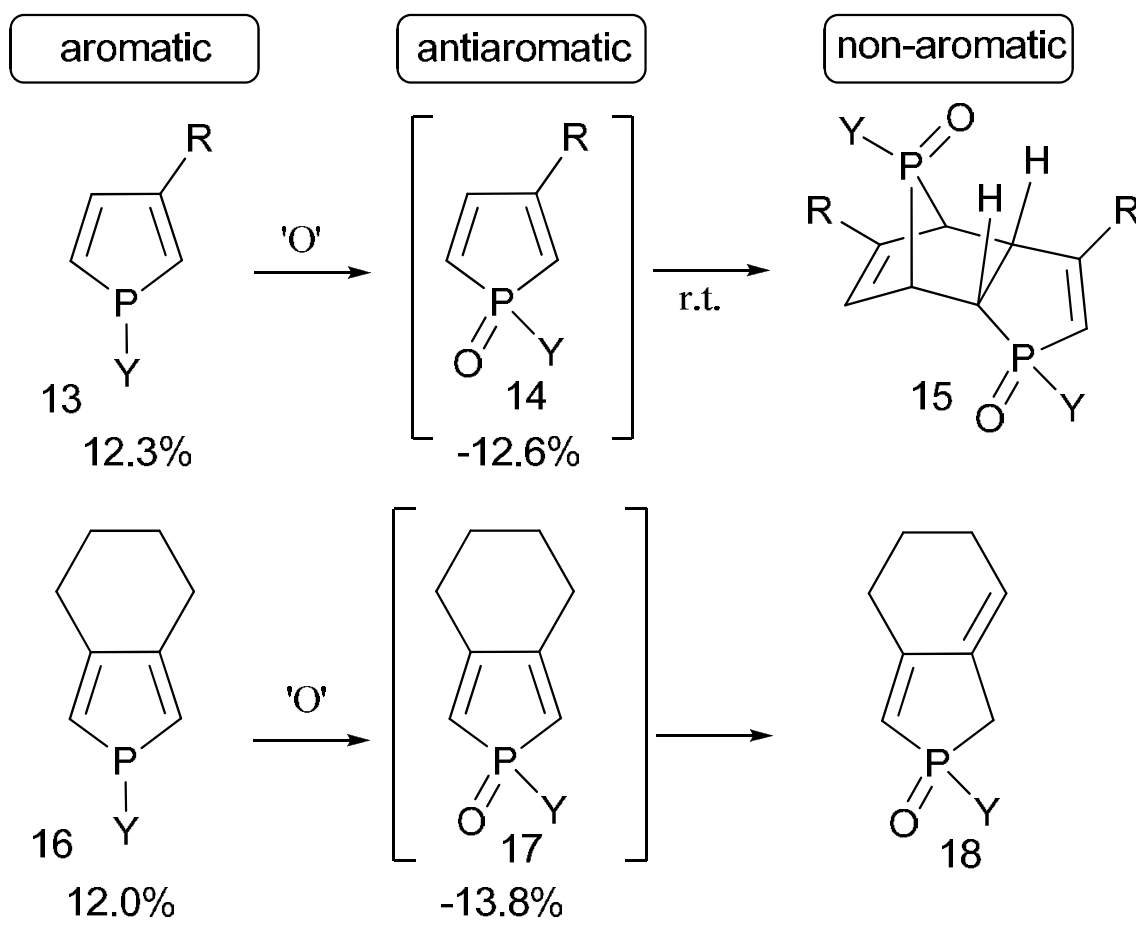

Fig. 5. $\Delta H_{\mathrm{H} 2}$ vales calculated for selected antiaromatic and aromatic species containing phosphorous.

\subsection{Carbonylicity and amidicity}

The carbonyl group is one of the most pervasive moieties in organic, bioorganic and industrial chemistry. Ketons, aldehydes as well as carboxylic acids, their halogenides, amides, esters, acyl anhydrides and other derivatives are also so-classified, commonly found in peptides/proteins, lipids/membranes and other biologically active compounds, such as Penicillin, drugs and toxins. They may be characterized as being very stable and resilient (amides, esters, acids), as well as very reactive systems (carboxyl acid halogenids, and thiol derivatives). There are numerous examples in the field of organic and biochemistry, where the carbonyl derivatives undergoes nucleophilic addition reaction, such as esterification, transesterification, amidation, transamidation, anhydride formation, aldol addition, among others. Examples also include the near-spontaneous or enzymatic hydrolysis of ester and amide bonds. Reduction of the carbonyl group by complex metal hydrides has significant synthetic importance in obtaining various alcohols, amines and other compounds (Figure 6). The large variability in the chemical reactivity of the carbonyl group may be attributed to the potential for fine-tuning of the bond strength, facilitated by attached substituent groups. Stronger conjugation, implies a larger contribution of resonance stabilization (lowering overall energy), with an associated increase in system stability. The extent of conjugation, predetermines its specific chemical reactivity; analogous to the situation in amide systems [13]. 


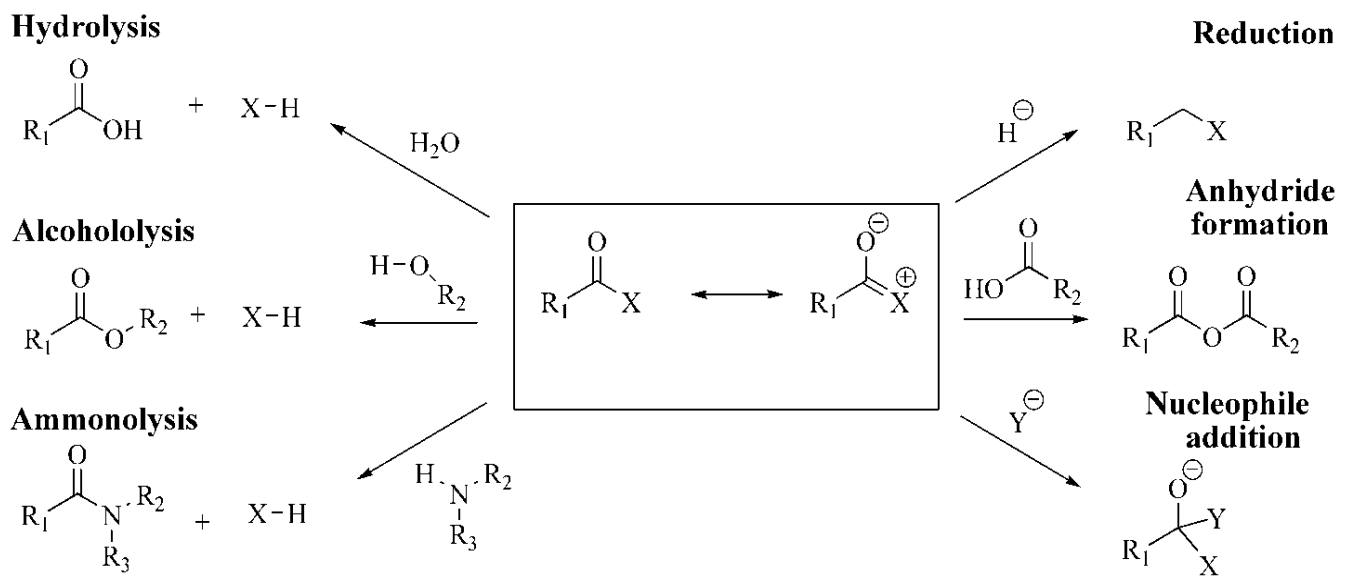

Fig. 6. A schematic illustration of the variety of reactions of carbonyl derivatives.

The large variability in the chemical reactivity of the amide bond may be attributed to the potential for fine-tuning of the bond strength, facilitated by the attached substituent groups. The amide bond strength of a general amide compound, as illustrated by its associated resonance structures, determines its specific chemical reactivity; essential to the biological activity of biochemical compounds. A stronger amide bond is more resistant to attack by nucleophilic agents (e.g. $\mathrm{HO}^{-}, \mathrm{H}_{2} \mathrm{O}$, amines, metal hydrides or the hydroxyl groups of serine-proteases), whereas a weaker amide bond is correspondingly more reactive. For a stronger amide bond, the conjugation between $\mathrm{N}$ and the $\mathrm{C}$ of the carbonyl group is more extensive, meaning that the contribution of the two most significant resonance structures are more closely balanced between the two structures, than in a weaker amide bond. In the case where there is no significant conjugation, the preferred resonance structure is represented by the left structure in the box in Fig. 6. [10].

\subsubsection{Amidicity and carbonylicity percentages and its resonance enthalpies (AM\% and $\mathrm{CA} \%$ :}

The "amidicity scale", quantifying amide bond (Figure 7) strength on a linear scale, based on the computed enthalpy of hydrogenation $\left[\Delta H_{\mathrm{H} 2}(\mathrm{AM})\right.$; Eq. 7; Figure 8-TOP] of the compound examined, comparing to reference compounds 19 and 20. The $\Delta H_{\mathrm{H} 2}(\mathrm{AM})$ value for dimethylacetamide (19) is used to define perfect amidic character (Eq. 8.; AM \% = $+100 \%$ ), while azaadamantane-2-on (20) represents complete absence of amidic character $(\mathrm{AM} \%=0 \%)$ [10]. The amidicity value is transformed to the resonance enthalpy $\left[H_{\mathrm{RE}}(\mathrm{AM})\right.$; Eq. 9]. However, amidicity is not limited to the values between $0 \%$ and $100 \%$. Some amide compounds exhibit extreme amidicity values, either below $0 \%$ or above $100 \%$, and referring to the cases when the amide bond may be weaker than that in $\mathbf{2 0}$ or stronger than that in 19, respectively.

$$
\begin{gathered}
\Delta H_{\mathrm{H} 2}(\mathrm{AM})=H[22]-\left\{H[21]+H\left(\mathrm{H}_{2}\right)\right\} \\
\mathrm{AM} \%=\mathrm{m}_{\mathrm{AM}} \Delta H_{\mathrm{H} 2}(\mathrm{AM})+\mathrm{b}_{\mathrm{AM}} \\
H_{\mathrm{RE}}(\mathrm{AM})=\mathrm{AM} \% / \mathrm{m}_{\mathrm{AM}}
\end{gathered}
$$




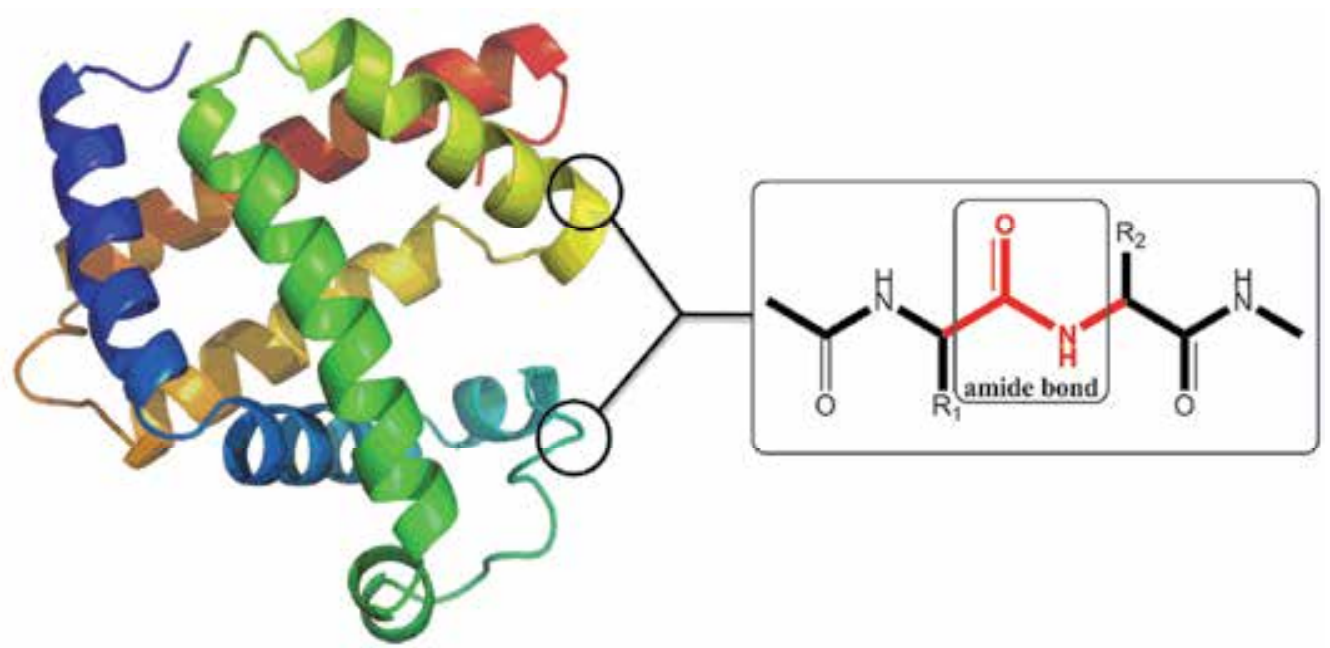

Fig. 7. A schematic illustration of the occurance of an amide bond within protein secondary structures.
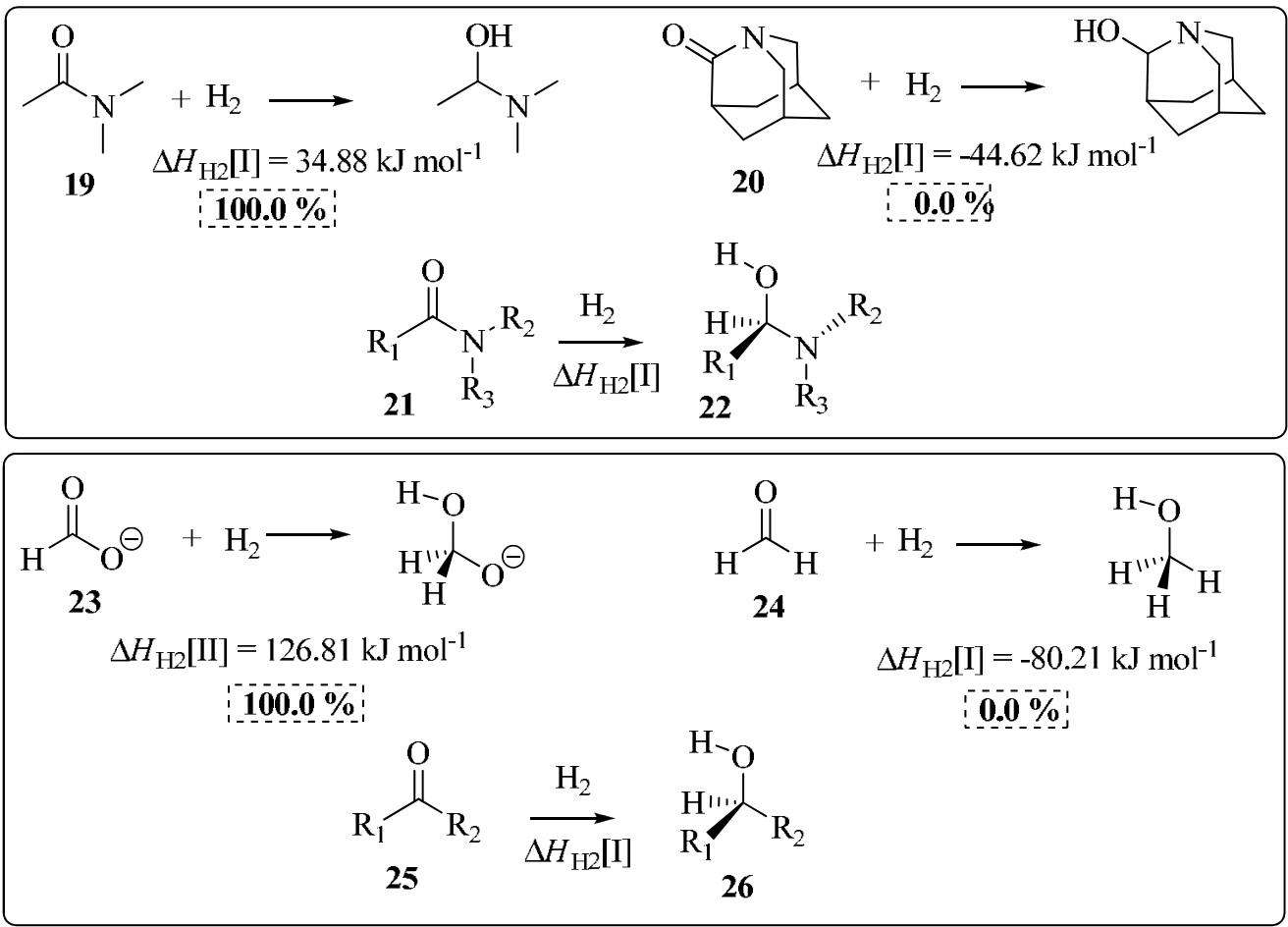

Fig. 8. The definition of the amidicity (TOP) and carbonylicity percentages (BOTTOM) based on the enthalpy of hydrogenation $\left(\Delta H_{\mathrm{H} 2}\right)$ of the carbonyl group. Values were obtained from the B3LYP/6-31G $(\mathrm{d}, \mathrm{p})$ geometry-optimized structures. In structure $\mathbf{2 2}$ and 26, the O$\mathrm{C}-\mathrm{X}-\mathrm{R}^{3}$ and the $\mathrm{H}-\mathrm{O}-\mathrm{C}-\mathrm{X}$ dihedral angles are chosen to be in the anti orientation. 
Analogously, the "carbonylicity scale", quantifying carbonyl bond strength on a linear scale, based on the computed enthalpy of hydrogenation $\left[\Delta H_{\mathrm{H} 2}(\mathrm{CA})\right.$; Eq. 10; Figure 8-BOTTOM] of the compound examined, comparing to reference compounds 23 and 24 . The $\Delta H_{\mathrm{H} 2}(\mathrm{CA})$ value for formiate anion (23) is used to define perfect conjugation (Eq. 11.; CA \% $=+100 \%$ ), while formaldehyde (24) represents complete absence of a conjugation (CA \% $=0 \%$ ) [13]. To calculate the carbonylicity value of compound 25 can be calculated by the hydrogenation reaction to 26, using Eq. 10-12. The carbonylicity value is transformed to the resonance enthalpy $\left[H_{\mathrm{RE}}(\mathrm{CA}) ; \mathrm{Eq}\right.$. 12]. Here the carbonylicity value is also not limited to the values between $0 \%$ and $100 \%$.

$$
\begin{gathered}
\Delta H_{\mathrm{H} 2}(\mathrm{CA})=H[25]-\left\{H[26]+H\left(\mathrm{H}_{2}\right)\right\} \\
\mathrm{CA} \%=\mathrm{m}_{\mathrm{CA}} \Delta H_{\mathrm{H} 2}(\mathrm{CA})+\mathrm{b}_{\mathrm{CA}} \\
H_{\mathrm{RE}}(\mathrm{CA})=\mathrm{CA} \% / \mathrm{m}_{\mathrm{CA}}
\end{gathered}
$$

Figure 9 shows, in a combined fashion the amidicity (TOP) and carbonylicity (BOTTOM) scale. Note that the two set of values represent different scales, than the amidicity is a special section of the carbonylicity scale.

Amidicity percentage for example is able to predict whether a transamidation reaction is taking place under the given conditions or not [10-12] and it can also point out the most reactive amide bond of a molecule. It was shown that carbonyl groups exhibiting a lower amidicity value are more reactive toward nucleophilic reagents (like amines) than carbonyl groups having a larger value. Moreover, when more products can be deduced it was demonstrated that the difference between the sum of amidicity percentages of products and the sum of those values in the reactants indicates the direction of a transamidation reaction. If this difference is positive, the reaction is energetically favored, while in the case of a negative value the reaction is disadvantageous from the driving force point of view. The reaction route, where the sum of amidicity percentages for products is larger than that for other possible reaction routes, is predicted to be the favorable one.

A very similar conclusion was drawn for acyl transfer reactions using carbonylicity as the descriptor [13]. It should be noted, however, that these simple views of the reaction do not consider the kinetic consequences, which sometimes perturb the simplest and quickest conclusion. For example, as presented in an earlier work [13], in acyl transfer reactions it is not enough to find the lowest carbonylicity value, but one of the carbonyl groups should also be a good leaving group.

\subsection{Olefinicity}

The olefinic group, illustrated in Figure 10, may be considered as one of the most important moieties in the organic and bioorganic chemistry. Substituted olefines, such as enamines, vinyl eters and other derivatives can be ranked among this category. Most of them are common in the field of the biochemistry such as proteins, lipids, nucleinic acids and other biologically active compounds like drugs and toxins. Their chemical reactivity may be characterised as very stable and resistant chemical systems, (simple olefines), as well as very active and reactive compounds (enamines, vinyl esters, etc.). There are numerous examples in the field of organic and biochemistry, where the olefinic derivatives undergo electrophilic or nucleophilic reactions [15]. 

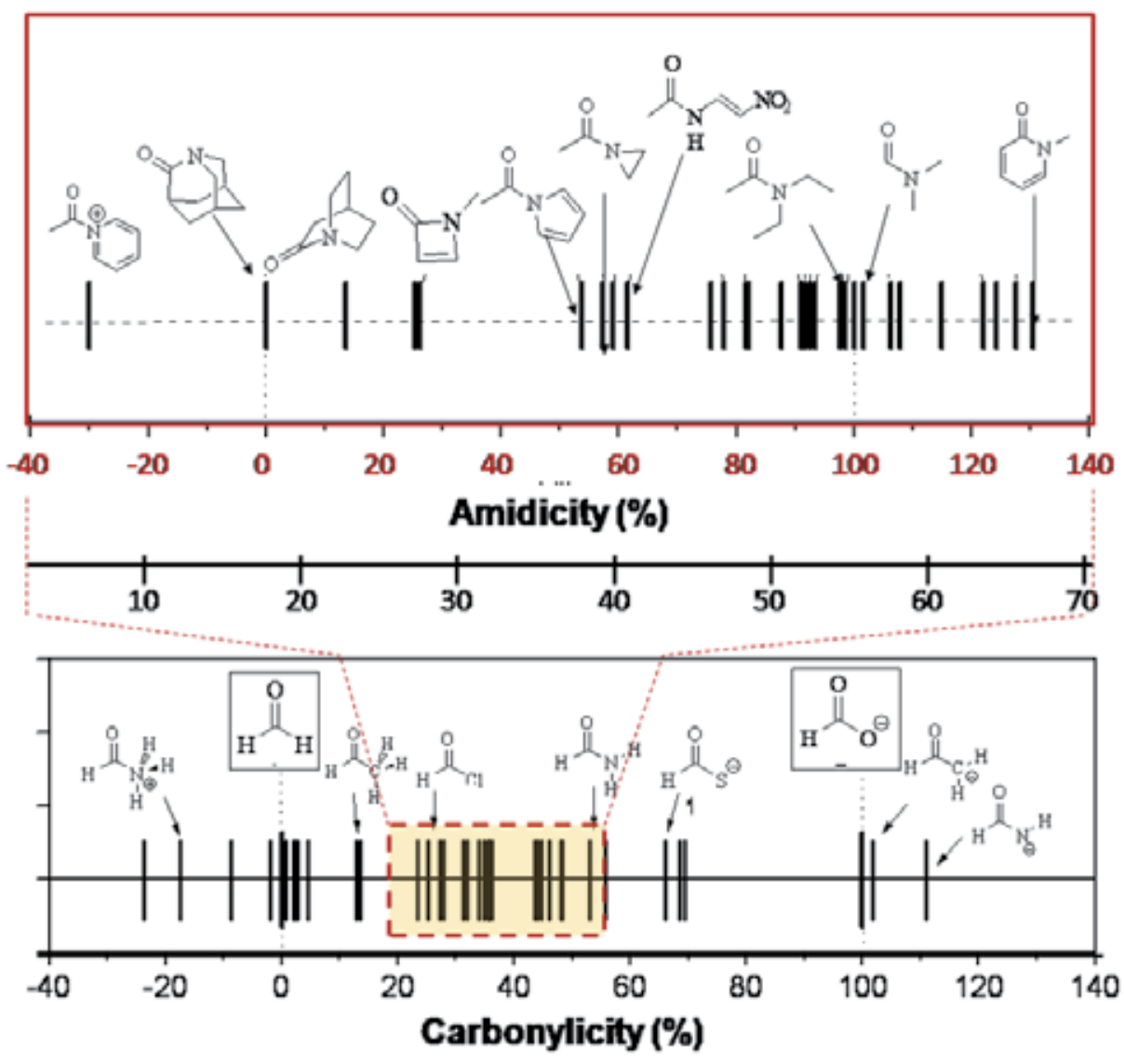

Fig. 9. A schematic representation of the theoretical amidicity and carbonylicity values of given compounds on the carbonylicity and amidicity spectrum, illustrating, that the amidicity spectrum is a small section of the carbonylicity spectrum. 
The large variability in the chemical reactivity of the olefin group may be attributed to the potential fine-tuning ability of the bond conjugation, facilitated by the attached substituent groups. The extent of conjugation of a general olefin compound, as illustrated by its associated resonance structures (Figure 10), predetermines its specific chemical reactivity [15].

\section{Hydration}

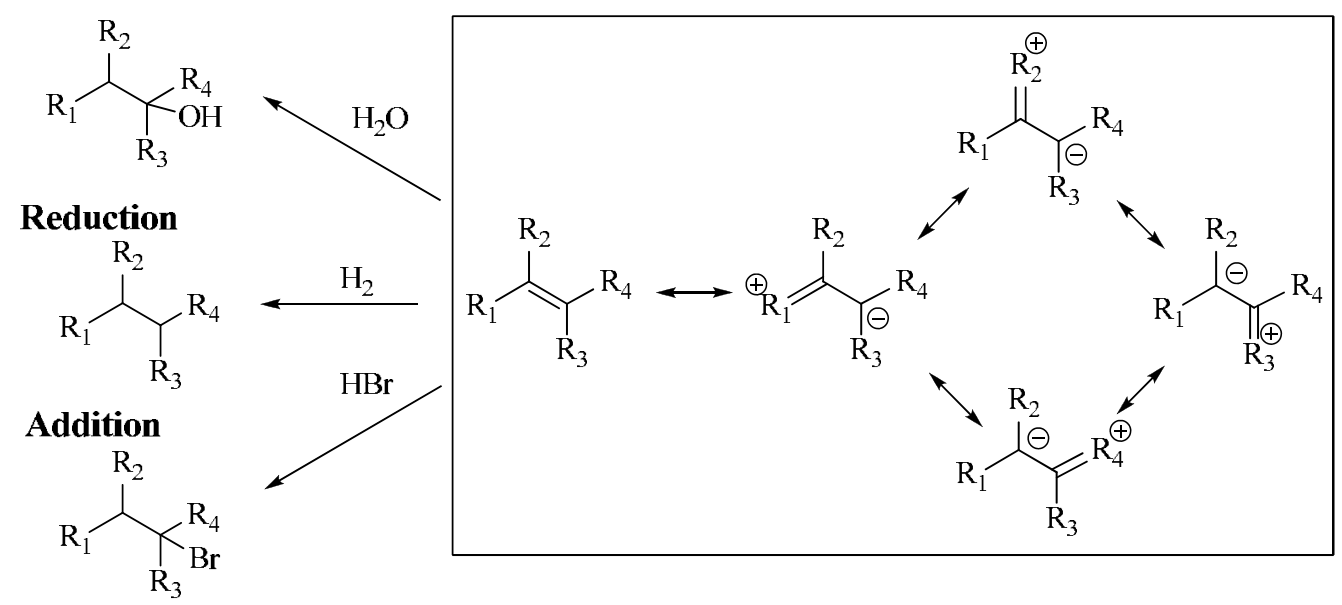

Fig. 10. Some selected typical reactions of the olefinic moiety.

\subsubsection{Olefinicity percentage and its resonance enthalpy (OL \%):}

The "olefinicity scale", quantifying alkene bond strength (Figure 11) on a linear scale, based on the computed enthalpy of hydrogenation $\left[\Delta \mathrm{H}_{\mathrm{H} 2}(\mathrm{OL})\right.$; Eq. 13] of the compound examined (29), comparing to reference compounds 27 and 28 (Eq. 14) [15]. The $\Delta H_{\mathrm{H} 2}(\mathrm{OL})$ value for allyl anion (27) is used to define equivalent conjugation (OL \% $=+100 \%$ ), while ethylene (28) represents complete absence of conjugation (OL \% $=0 \%$ ), by Eq. 15. This olefinicity value is transformed to resonance enthalpy $\left[H_{\mathrm{RE}}(\mathrm{OL}) ; \mathrm{Eq} .15\right]$.

$$
\begin{gathered}
\Delta H_{\mathrm{H} 2}(\mathrm{OL})=H[\mathbf{T}]-\left\{H[\mathbf{S}]+H\left(\mathrm{H}_{2}\right)\right\} \\
\mathrm{OL} \%=\mathrm{m}_{\mathrm{OL}} \Delta H_{\mathrm{H} 2}(\mathrm{OL})+\mathrm{b}_{\mathrm{OL}} \\
H_{\mathrm{RE}}(\mathrm{OL})=\mathrm{OL} \% / \mathrm{m}_{\mathrm{OL}}
\end{gathered}
$$

\section{Energetic study of industrial and biochemical reactions}

Due to the enormously large variety of chemical reactions, in this chapter only acyl transfer reactions, including transamidation and reduction-oxidation reactions are exemplified, which are also essential both in industrial chemistry and biochemistry. In order to understand the energy flow and determine the direction of such reactions, thermodynamic selection rule and driving force should be clarified. Based on Systems Chemistry approach, measuring numerically the resonance energy of functional groups inside the molecule, a relatively simple protocol is provided for practicing organic chemists to predict the outcome of an experiment. The change of specific values over the course of a reaction made it 
possible to see that the process is favorable or unfavorable. A series of examples are analyzed in the order of the complexity, from simple single value change to multi value changes.<smiles>CCCC(C)C(C=O)C=CC=O</smiles>

27<smiles>C=C</smiles><smiles>CC</smiles>

28

$$
\begin{gathered}
\Delta H_{\mathrm{H} 2}[\mathbf{I}]=-133.08 \mathrm{~kJ} \mathrm{~mol}^{-1} \\
\mathbf{0} . \overline{\mathbf{0}} \% \\
\hdashline \%
\end{gathered}
$$<smiles>[R4]C([R1])=C([R4])[R]</smiles>

29

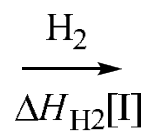

$\Delta H_{\mathrm{H} 2}[\mathbf{I}]$

\section{$100.0 \%$}

Fig. 11. The definition of the olefinicity percentage based on the enthalpy of hydrogenation $\left(\Delta H_{\mathrm{H} 2}\right)$ of the double bond. Values were obtained from the B3LYP/6-31G $(\mathrm{d}, \mathrm{p})$ geometryoptimized structures.

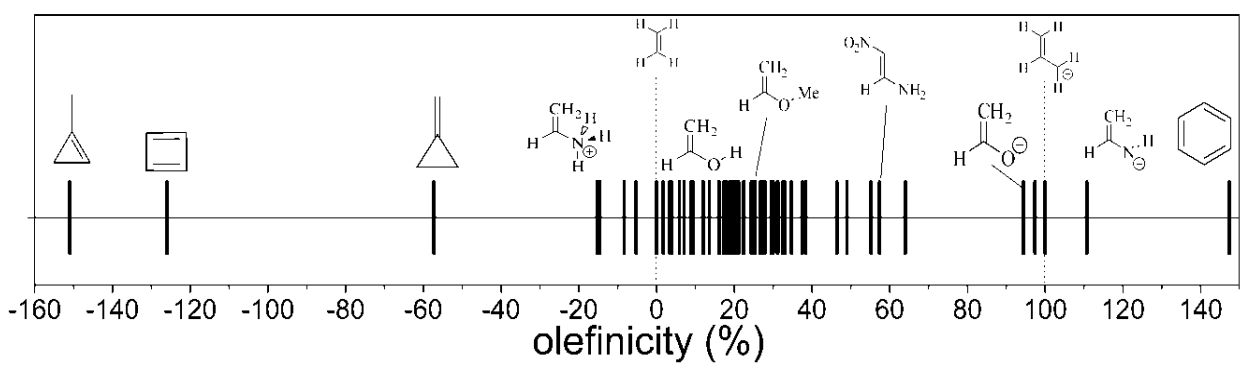

Fig. 12. A schematic representation of the theoretical olefinicity values of given compounds on the olefinicity spectrum.

\subsection{General remarks for acyl transfer reactions}

In the following paragraph, some very important acyl transfer processes are studied from energy management point of view comparing the human and biochemical solutions. The first studied reaction is a simple amide and ester formation from simple amine or alcohol as reactants via different ways. Acyl transfer reactions have a significant interest from preparative and biological points of view. For simple acyl halogenides and acyl anhydrides are widely used in common synthesis. Here we introduce $\Delta$ carbonylicity or $\Delta C A(\%)$ value, which represent the difference between the carbonylicity values of the starting molecules and the products (Eq. 16), illustrated by Figure 13 [13]. 
Reagent<smiles>[X]C(C)=O</smiles>

$\mathrm{CA} \%($ reagent)
Product

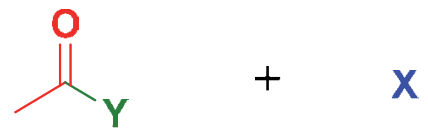

Fig. 13. A general acyl transfer reaction, where the active acyl reagent reacts with reactant $Y$, producing acyl product and X.

$$
\Delta \mathrm{CA}(\%)=\mathrm{CA} \%(\text { product })-\mathrm{CA} \%(\text { starting material })
$$

If the resultant $\triangle \mathrm{CA}$ value is positive, then the reaction is favored from the 'carbonylicity point of view'. Of course, a reaction may have several other parameters, which determine if a reaction is favored or not, such as steric hindrance, kinetic consequences, side-reaction; therefore a positive carbonylicity value does not mean automatically the occurance of a reaction. Nevertheless, the $\Delta$ carbonylicity represents a thermodynamic driving force of an acyl transfer reaction, analogously to the role of amidicity (AM\%) in the case of the transamidation reactions (Figure 14). The change in the amidicity value gives information about the direction of a transamidation reaction, described by Eq. 17. In the following part, this new methodology is applied on the field of peptide chemistry, especially for the peptide bond formation [10].

\section{Reagent}<smiles>[Y10]C(C)=O</smiles>

$\mathrm{AM} \%($ reagent)
Product

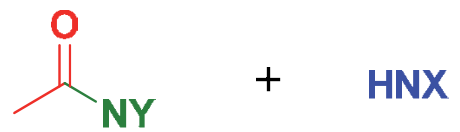

AM\%(product)

Fig. 14. A general transamidation reaction, where the active amide reagent reacts with amine reactant $Y$, producing amide product and $X$.

$$
\Delta \mathrm{AM}(\%)=\mathrm{AM} \%(\text { product })-\mathrm{AM} \%(\text { starting material })
$$

As was mentioned, amide and ester functionalities play crucial role in chemistry constructing proteins, nucleic acids, polyhydrocarbons, vitamins, lipids, drugs, plastics and many other important materials. The simplest chemical reagent to form amide or ester bonds is carboxylic acides. However, carboxylic acids are typically not able to effectively form the desired amide product and the ester formation is also very slow under normal conditions. In this case the slow ester formation reaction can be explained by the low carbonylicity change.

The unproductive amide formation in the case of carboxylic acids is due to the deprotonation of the acid reagents to an unreactive reagent by the amine, being in an acidbase equilibrium. Carboxylate anion exhibits very large carbonylicity value (106\%), which makes this reaction to very endothermic, consequently unsuccessful. From Figure $\mathbf{1 5}$ it is 
clear that in order to produce an ester or an amide the acid has to be activated or in other word has to prepare a high energy reagent. One of the simplest protocols for activation is the chlorine exchange of the hydroxyl group, but it can be done via different methods. The first method for reagent formation of Figure 16 clearly indicates that the $\mathrm{HCl}$ molecule is not energetic enough to carry out the necessary activation. In the second method of Figure 16, where the high energy content phosphoryl chloride $\left(\mathrm{POCl}_{3}\right)$ is already sufficiently strong for activate the carboxylic acid. Finally, high energy active reagent, acid chloride can readily react with ammonia in an exothermic reaction.
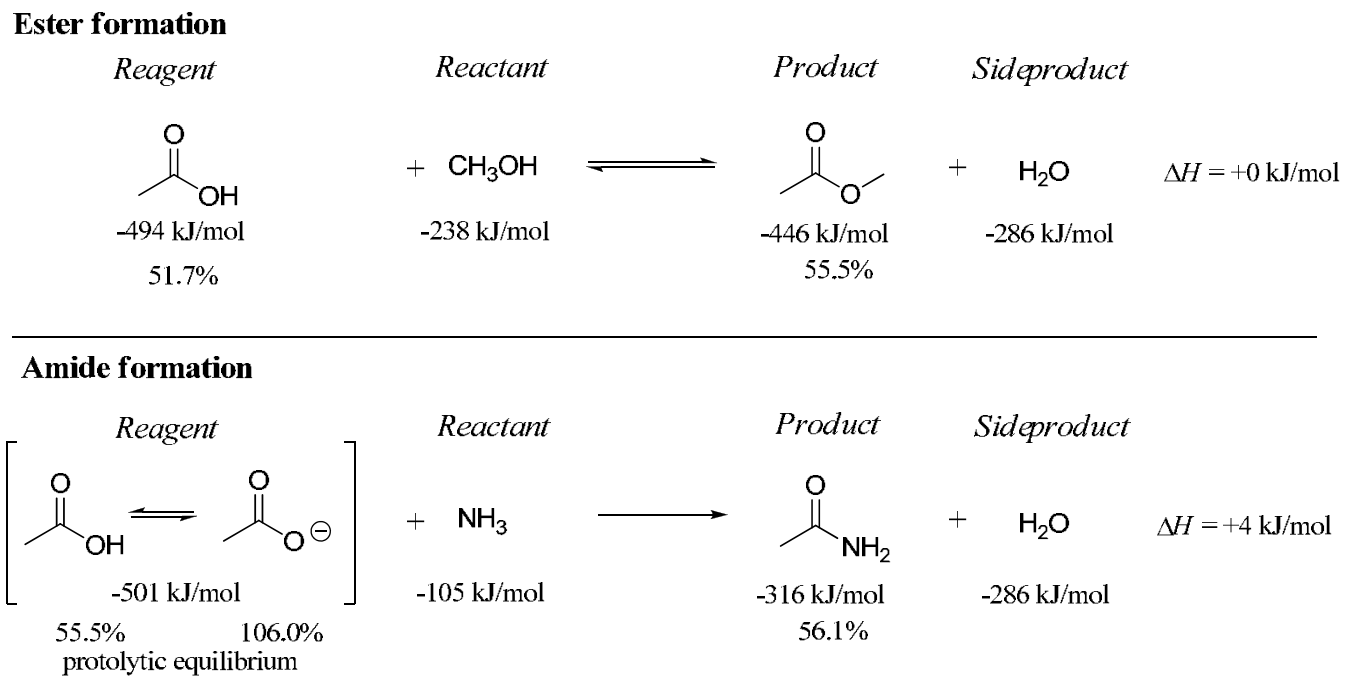

Fig. 15. Thermodynamics of simple ester and amide formation. Data were taken from the National Institute of Standards and Technology (NIST).

\subsubsection{Acyltransfer reactions making amide bonds}

An amide or peptide bond can be formed by different ways and each method starts with the activation of the acid reactant, followed by the nucleofil attack of the amine reactant. From the carbonylicity point of view, the reaction between an acid (e.g. 31) and an amine (e.g. 34) is thermodynamically advantageous, in the present example the reaction exhibit $+3.9 \%$ of $\Delta$ carbonylicity, which means $\Delta$ carbonylicity $/ \mathrm{m}=3.9 / 0.4830=8.1 \mathrm{~kJ} / \mathrm{mol}$ increase in resonance energy. However, as was discussed before, an acid is not able to react with an amine due to the high carbonylicity value of the forming inactive carboxylate anion in the protonation-deprotonation equilibrium. To form amide 35, the acid reagent need to be activated somewhat, that is to be transformed to a more active carbonyl reagent (36) having lower carbonylicity value. In all of the activation methods, this high carbonylicity value of 31 are lowered significantly, consequently the reactivity of the acid is enhanced [10-12]. 


\section{Reagent formation, method A (not feasible)}

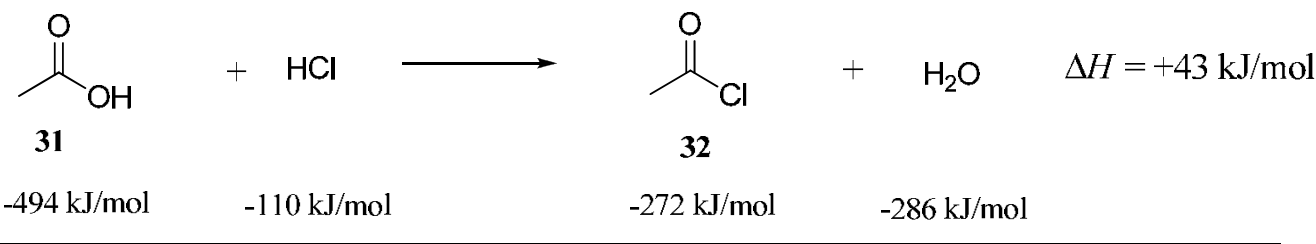

\section{Reagent formation, method B (feasible)}

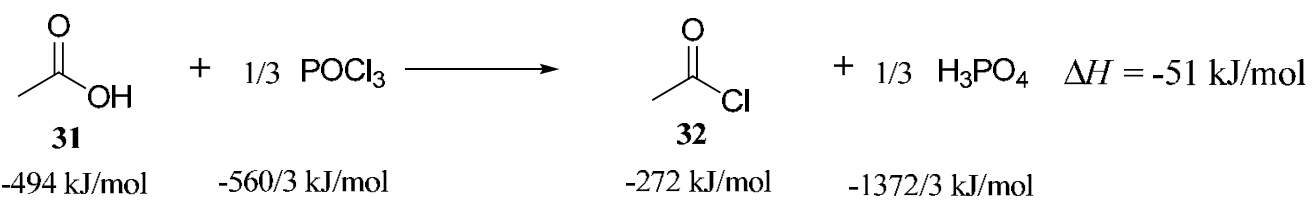

\section{Amide formation}

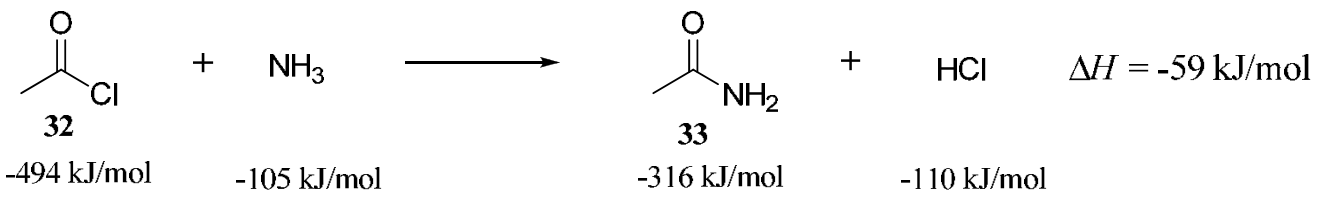

Fig. 16. Formation and utilization of an active (i.e. high energy) reagent. Data were taken from the National Institute of Standards and Technology (NIST).

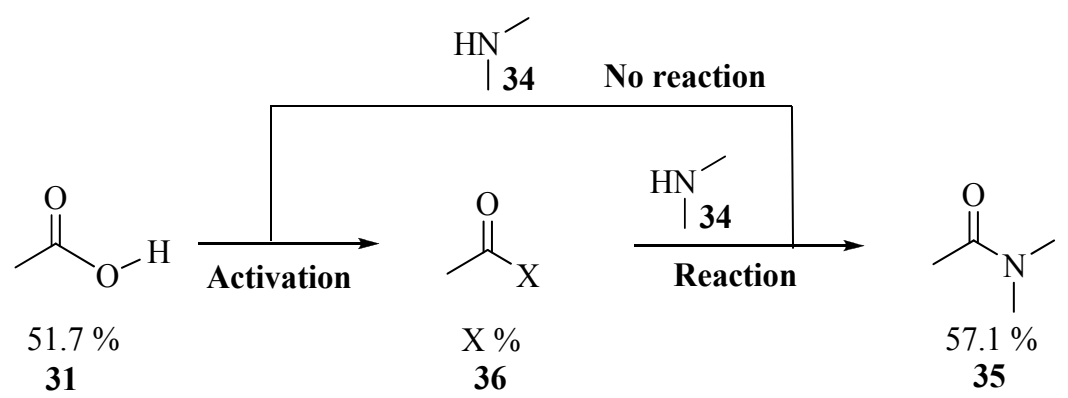

Fig. 17. Amide formation through reactant activation

Five different activation methods are considered and studied here; involving acylchloride (R-I), anhydride (R-II), active ester (R-III, R-IV, R-V). Also, activation by 1-hydroxy benztriazole derivatives (BOP and HBTU, R-VI) and by dicyclohexyl carbodiimide (DCC, $\mathbf{R}-$ V). The most widely known amide forming reagent is the acyl chloride (R-I; 37 in Figure 18) exhibiting as low carbonylicity value as $23.7 \%$. In the course of reaction with an amine (34), the change in carbonylicity is very significant $(\Delta \mathrm{CA}=+33.4 \%)$, yielding 35 [10].

In the case of the peptide bond formation via mixed anhydrides (R-II), the acid (31) is reacted by isobutyl-chlorophormate (38, in Figure 19), resulting a mixed anhydride (39) with low carbonylicity value on the original carbonyl functionality $(29.8 \%)$. This active species may easily react with an amine (34), leading to the desired product $35(57.1 \%)$ and side- 
product $40(55.6 \%)$, which decomposes to isobutylene, $\mathrm{CO}_{2}$ and $\mathrm{H}_{2} \mathrm{O}$. Although, in the activation step $(31+38 \rightarrow 39)$ the change in the carbonylicity value is small, but negative but small $(-4.4 \%)$, the $\mathrm{HCl}$ elimination and the salt formation with the applied base provide a strong driving force. The active mixed anhydride reagent (39) exhibits low carbonylicity at $\mathrm{C} 2$, indicating a significant reactivity toward 34 , however $\mathrm{C} 4$ atom possesses a larger carbonylicity, which is not so reactive, therefore only products $\mathbf{3 5}$ and $\mathbf{4 0}$ form exclusively and not 41 and $\mathbf{4 2}$, which route is not preferred from either thermodynamic and kinetic point of view [10].

\section{R-I}

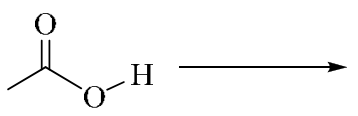

$51.7 \%$

$31 \Delta \mathrm{CA}=-28.0 \%$<smiles>CC(=O)Cl</smiles>

$23.7 \%$

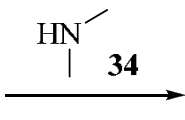

$\Delta \mathrm{CA}=+33.4 \%$<smiles>CC(=O)N(C)C</smiles>

$57.1 \%$

35

Fig. 18. Amide formation through activated acid chloride

\section{R-II}<smiles>[13CH3]OC(=O)Cl</smiles><smiles>CC(=O)O[Hg]Cl</smiles><smiles>CC(=O)OC(=O)O[13CH3]</smiles>

$51.7 \%$ 31 $\Delta \mathrm{CA}=-9.8 \%$
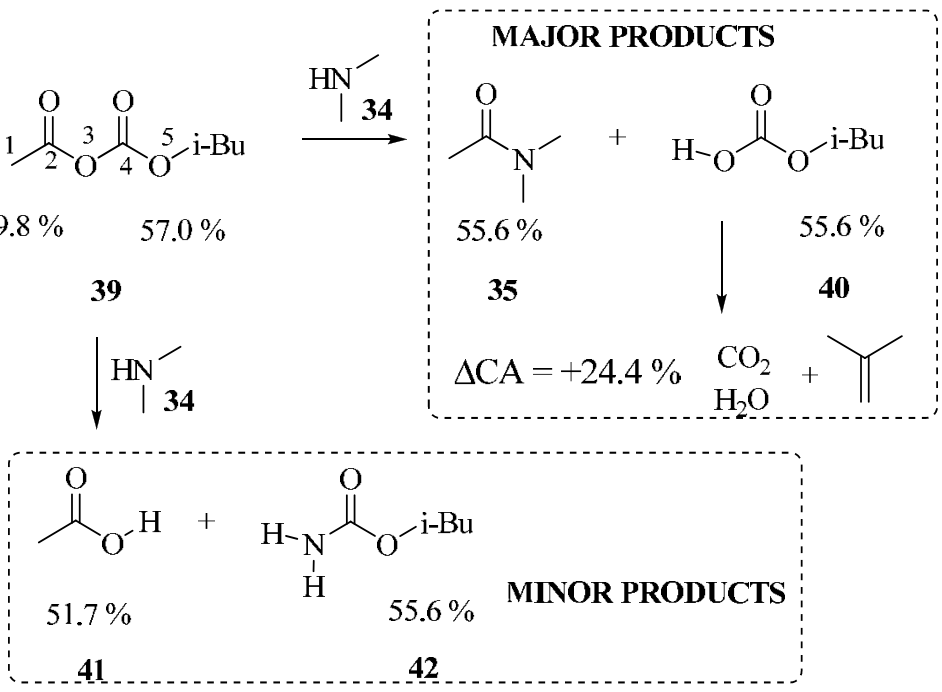

Fig. 19. Amide formation through activated mixed anhydride

Originally, an alkyl ester (43) is able to transform to the corresponding amide 35 and 44, but due to the high carbonylicity value of the ester $\mathbf{4 3}$ and the small change in $\Delta$ carbonylicity in R-III (Figure 20), the reaction requires usually high temperature or Lewis acid catalyst (e.g. $\left.\mathrm{AlMe}_{3}\right)$ to proceed. Active esters, which are usually aryl esters, however exhibit lower carbonylicity values, which allow a smooth reaction under convenient circumstances. 


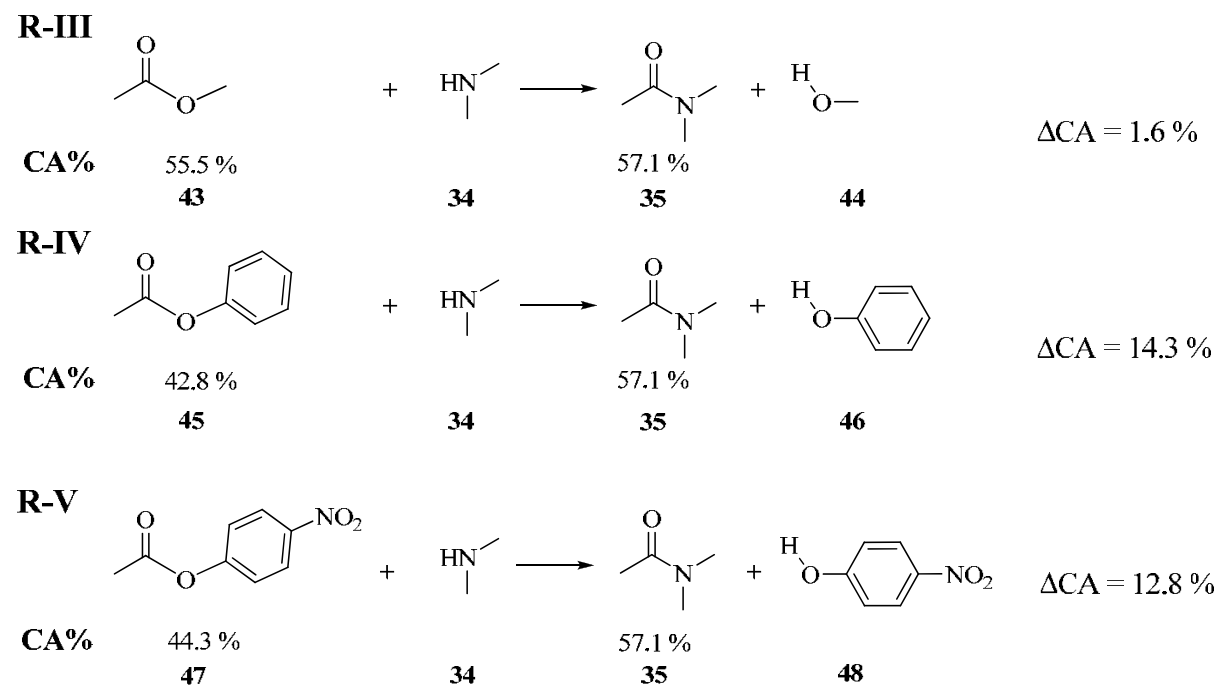

Fig. 20. Amide formation from various esters

In R-IV and R-III (Figure 20), two known coupling procedures are presented, which were used earlier to prepare peptide bond. In both cases, the significant increase in the carbonylicity values predicts a smooth reaction of the aryl ester $(45,47)$ with 34 , resulting amide 35 , beside 46 and 48 as by-products [10].

However, these active esters proved to be not so efficient due to the relatively high reaction temperature and long reaction time, which may be attributed to the not too significant carbonylicity changes. More modern coupling reagents in the peptide chemistry, such as benzotriazole-1-yl-oxy-tris-(dimethylamino)-phosphonium hexafluorophosphate (BOP, 49a, R-VIa in Figure 21) and $O$-benzotriazole- $N, N, N^{\prime}, N^{\prime}$-tetramethyl-uronium-hexafluorophosphate (HBTU, 49b, R-VIb) provide more rapid peptide bond formations in smooth conditions. In both cases, in the first step, is the elimination of the 1-hydroxy-benztriazole moiety (50) of the reagent, leading to a very active acylating agents $51 \mathbf{a}(25.5 \%), \mathbf{5 1 b}(28.3$ $\%$ ), which reacts with $\mathbf{5 0}$, forming a common, less active, but active enough intermediate 52 (36.4 \%). Finally, this intermediate 52 takes part in an acyl-exchange reaction with 34, furnishing the formulation of a new peptide bond in 35. Due to the higher carbonylicity change during the reaction, the reaction rate is faster even at room temperature. Moreover, the corresponding carbonylicity values for 51a, 51b during the reaction sequences may explain the experimental observation that the BOP reagent (49a) is usually provide faster reaction than HBTU (49b) [10].

The one of the most efficient peptide bond forming reagents is the $N, N^{\prime}$ dicylclohexylcarbodiimide (DCC, 53), which readily reacts with the carboxylic acid (e.g. 31), forming a very active species $\mathbf{5 4}$ (38.7 \%), as shown by R-VII in Figure 22. Subsequently, this intermediate furnishes a reaction with amines (34), meanwhile $N, N^{\prime}$-dicyclohexylurea (DCU, 55) leaves the molecule, yielding the amide 35.

The most impressive usage of DCC may well be the synthesis of penicillin (Figure 22, RVIII/a), where the last step of cyclization was carried out using this reagent. According to literature data, this cyclization of the open chain mono-deprotonated penicillin derivative (56) was successful only in basic condition (aqueous $\mathrm{KOH}$ ). After the reaction between 56 
and DCC (53), the carbonylicity value $51.7 \%$ decreases dramatically to $36.0 \%$, in the resulting intermediate 57 . Due to the slightly higher carbonylicity value of the penicillin product $58(37.1 \%)$, is the reason that intermediate 57 can in fact cyclize to form penicillin 58. However, this small, but positive difference in the carbonylicity $(37.1 \%-36.0 \%=+1.1$ $\%)$ is not sufficient to provide enough driving force to complete the reaction, therefore the experimental yield is rather low (10-12\%) [10].

Many unsuccessful experiments were carried out in order to cyclize penicillin in neutral or slightly more acidic conditions in the hope to improve the yield (Figure 22, R-VIII/b). In this case, the starting compound is in neutral form (59), which reacts with DCC, furnishing intermediate 60 (carbonyilicity value $=36.0 \%$ ), having the same value, than it was obtained for 57. However, here the penicillin product is neutral (61), which exhibits much lower carbonylicity value $(22.6 \%)$, therefore the reaction is unable to proceed, due to the negative $\Delta$ carbonylicity value $(22.6 \%-36.0 \%=-13.4 \%)$ [10].

In the triglyceride synthesis (R-IX in Figure 23) the starting fatty or oleic acid forms (62) an ester bond with a glycerin or its derivative (67). Living organism follow an analogue strategy as the human synthesis, namely acid (62) is activated by ATP (63) in the form of phosphorous anhydride (64), when the carbonylicity value of the carbonyl group is decrease to as low as $37.1 \%$. This already active species presumably is in a too active form, it can hydrolyze in the aqueous media rapidly, therefore it is transformed to a somewhat stabilized reagent by means of CoA (65), yielding a little bit more stable a tioester derivative of fatty acid (66). This fatty acid derivative, finally can enter in an acyl transfer reaction by glycerine, providing the final product as glycerine ester 68 [10].

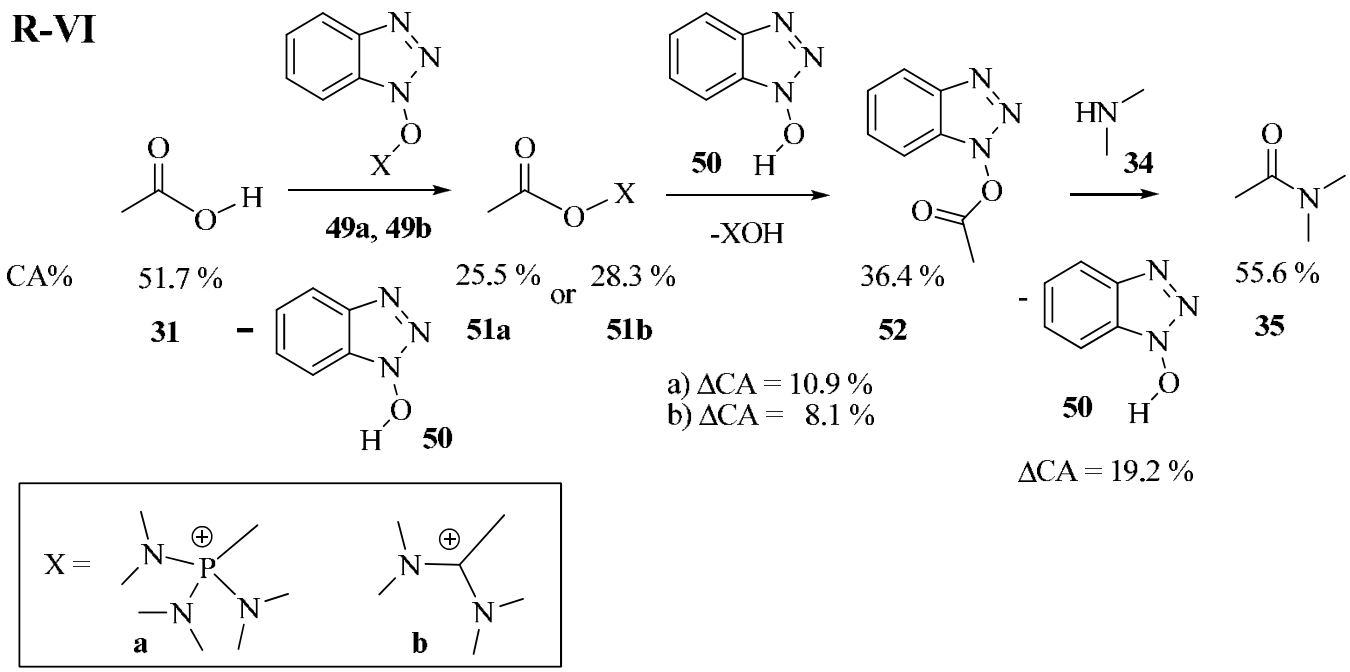

Fig. 21. Amide formation through carboxylic acid activation using 1-hydroxy-benztriazole derivative 
R-VII

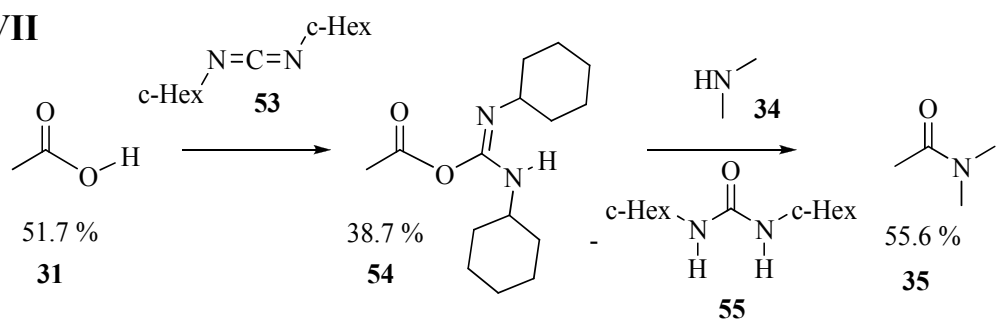

\section{R-VIII/a}

$$
\Delta \mathrm{CA}=16.9 \%
$$

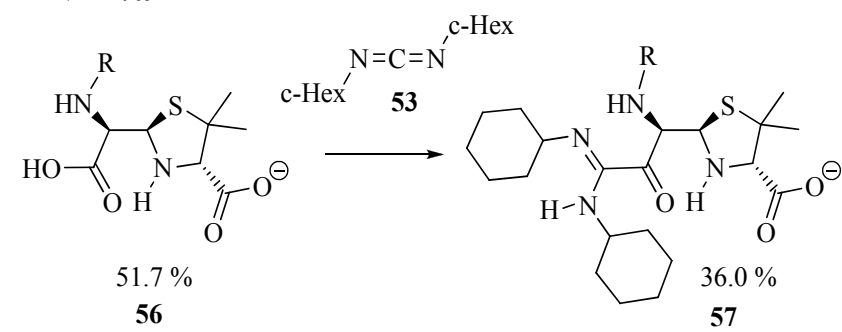

R-VIII/b

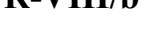

59<smiles>[R]N[C@H](C(=O)/C(=N/C1CCCCC1)NC1CCCCC1)[C@@H]1SC(C)(C)[C@@H](C(=O)O)N1[R]</smiles>

60

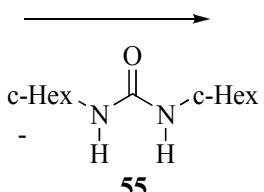

$\triangle \mathrm{CA}=1.1 \%$<smiles>[R]N[C@H]1C(=O)N2[C@@H](C(=O)[O-])C(C)(C)S[C@H]12</smiles>

$37.1 \%$

Fig. 22. Amide formation from carboxylic acid through activation by DCC

\section{R-IX}

Inactive reagent

Activated reagent

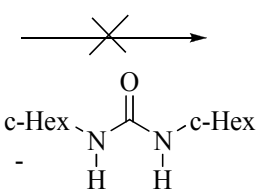

55

$\Delta \mathrm{CA}=-13.4 \%$<smiles>[R]N[C@H]1C(=O)N2[C@@H]1C(C)(C)S[C@@H]2C(=O)O</smiles>

$22.6 \%$

61<smiles>[R]C(=O)O[Al]</smiles>

62

\section{Stabilized active reagent}

Product

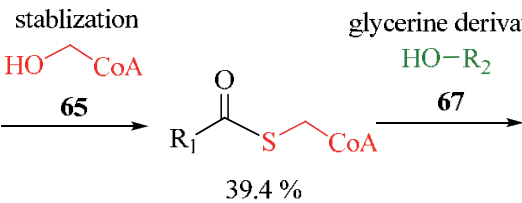

66<smiles>[R]OC([R])=O</smiles>

$55.5 \%$

68

Fig. 23. Tri-gliceride formation from fatty acides via thioester activation.

From chemical point of view, the in vivo peptide or protein synthesis is based on similar strategy (R-X in Figure 24), where the free amino acid (69) is activated via analogous phosphorylation process $(69 \rightarrow 70)$ by means of ATP $(63)$, resulting primary active reagent 70, which reacts with a hydroxyl group on a well-defined site of tRNS (71), stabilizing the 
active species in a less, but still active ester from (72). This AA-tRNS is the main active intermediate in this process, resulting finally the polypeptide chain (74) [10].

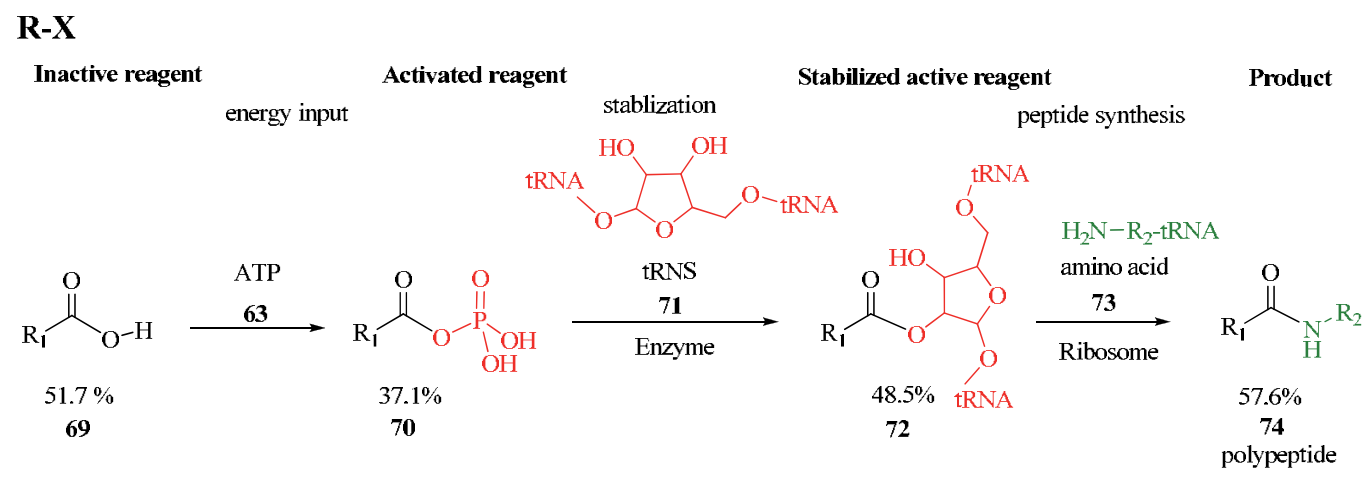

Fig. 24.

\subsubsection{Transamidation reactions}

The amide bond may be considered as one of the most important chemical building blocks, playing an important role not only in living organisms, but in organic chemistry as well. Amide bonds may be considered as a one of the most important chemical moieties in biological organisms, common in peptides/proteins and lipids/membranes and other biochemical systems. Amides also play an important role in selected biologically active compounds, such as Penicillin-like antibiotics, drugs and toxins. They are characterised as being very stable chemical bonds, with half-lives in neutral aqueous solution exceeding hundreds of years.

In contrast to their general resistant to reactivity, there are numerous examples in the field of organic and biochemistry, where the amide bond undergoes nucleophilic reaction. Examples include the spontaneous or enzymatic hydrolysis of amide bond in peptides, proteins. Perhaps the most famous small biogen amides are the Penicillin-like antibiotics, which inhibit penicillin binding proteins such as transpeptidase and carboxylpeptidase through an acylation of a serine residue. In this way, the bacterial cell wall synthesis stops, leading to higher susceptibility to osmotic effect and cell burst.

The reduction of the amide bond by complex metal hydrides has significant synthetic importance to obtain various amines. Some amide compounds are able to react with amines, called as an acyl transfer or transamidation reaction. These processes represent very useful transformations in synthetic organic chemistry to obtain various amide structures from amino compounds, selectively. The most notable application is the Traube synthesis of heterocycles. In many biological or pharmaceutical cases, Mother Nature or the practicing chemist must find the appropriate balance between the reactivity and stability of the amide bond. If the amide bond is too reactive, it may have an increased activity, but may also be metabolised prior to reaching its intended target (the enzyme). If however, the amide bond is less reactive, with an increased stability in aqueous solutions and bodily fluids, it will be difficult for such a compound to react with efficacy when it encounters the target (the enzyme). The Penicilin-like antibiotics ${ }^{5}$ presents a good example for the above mentioned natural design; the $\beta$-lactam ring is highly reactive due to its strained four-membered ring, which may open easily in the presence of nucleophilic reagents, such as the hydroxyl group 
of an enzyme side-chain. The reactivity of the amide bond can be fine-tuned by using different substituents, obtaining an appropriate molecule, which survives the aqueous body fluid and finds the targeted enzyme.

Unsubstituted amides such as 75 and 33 exhibit a reduced value of amidicity (Figure 21) relative to mono-substituted or di-substituted ones, such as 77 and 35 (97-103\%); one may therefore predict a transamidation proceeding between them. Mono- and di-substituted amines (e.g. 34) are shown to react readily with formamide (75, R-XI) and acetamide (35, RXII) at RT or above, as used in the Traube synthesis. The formylation of benzylamine and $\mathrm{N}$ methylbenzylamine furnished by $\mathbf{7 5}$ proceeded very smoothly, however, in the case of $\mathbf{3 3}$, $\mathrm{AlCl}_{3}$ was required in order to attain an acceptable rate, which is due to the high activation energy of the sterically hindered reaction center [10-12].

\section{R-XI}
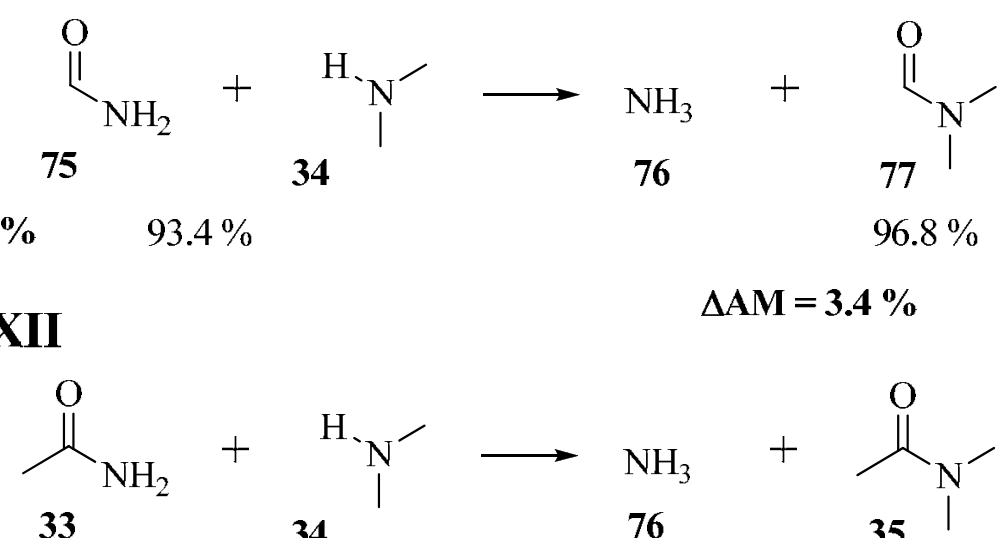

AM\% $\quad 96.1 \%$

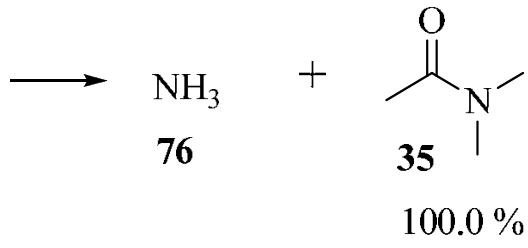

$\Delta \mathrm{AM}=\mathbf{3 . 9} \%$

Fig. 21. Examples for transamidation involving secondary amine.

Compounds 78 and 82 represent mild acylating agents (Figure 22) taking part in transamidation reactions with amines (e.g. 79 for R-XIII and R-XIV), forming amide 81 and 80 and 83 as side-products. The acylating properties of these compounds can be attributed to the competition between the aromatic ring and the amide group of the $\mathrm{N}$ atom lone pair, which decreases both the amidicity and aromaticity percentages of the $\mathbf{7 8}$ and $\mathbf{8 2}$. The main driving force of these reactions is the significant increase of the amidicity value during the acylation reaction. Compound $\mathbf{8 4}$ in R-XV (Figure 22) exhibits an extremely low amidicity percentage $(-30.2 \%)$, making this molecule an excellent acylating agent, prepared in situ from $\mathrm{AcCl}$ and pyridine. Thus, 84 readily reacts with amines (e.g. 85 for $\mathbf{R}-\mathbf{X V}$ ), with an extremely large $\Delta \mathrm{AM}$ value (Figure 22) even at low temperature. In R-XVI, the acetanilide derivatives (e.g. 88) with lowered amidicity values are also shown to be acylating compounds, transferring their acyl group to alkyl amines (e.g. 34 in Figure 22). The not too high $\triangle \mathrm{AM}$ value may be one of the underlying reasons that these types of reactions are not often referred to in the literature. The reaction between $\mathbf{8 8}$ and $\mathbf{3 4}$ is very slow, even in the presence of $\mathrm{AlCl}_{3}$ at high temperature, but it may be due to the larger steric hindrance of the carbonyl group in 82 [10-12]. 

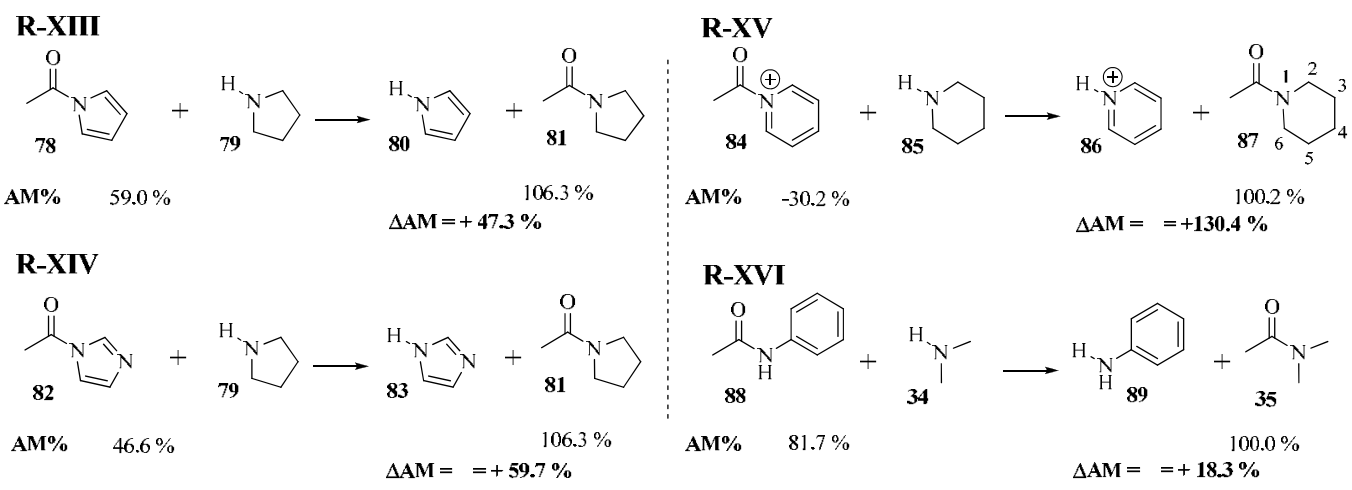

Fig. 22. Example for transamidation involving cyclic secondary amines

Using amidicity values, one may explain the inactivity of some commonly used organic amide-type solvents, such as $N, N$-dimethylformamide (77, DMF, R-XVII in Figure 23) and $\mathrm{N}$-methylpirrolydinone (91, NMP, R-XVII, Figure 23). In both cases the $\Delta \mathrm{AM}$ values are negative, making the reaction 'amidically unfavorable', therefore no reaction can be observed between 77 or 91 with 85 or 34 , even at $180^{\circ}$ and in the presence of $\mathrm{AlCl}_{3}$ as catalyst [10-12].

\section{R-XVII}<smiles>CN(C=O)C=O</smiles><smiles>CNC1CCCCC1</smiles>

$97.0 \%$ $95.6 \%$

R-XVIII

$$
\Delta \text { Amidicity }=-\mathbf{1 . 4} \%
$$<smiles>CN1CCCC1=O</smiles>

91

$120.3 \%$<smiles>CNC</smiles>

34<smiles>CC(C)(C)C(C)(C)C</smiles><smiles>CNCCCC(=O)N(C)C</smiles>

$104.0 \%$

\section{$\Delta$ Amidicity $=-16.3 \%$}

Fig. 23. Attempted transamidation reactions involving fully substituted amides and secondary amines

The use of the amidicity change or the change in stabilization enthalpy leads to a thermodynamic selection rule, allowing for the reactions to be categorized as being either thermodynamically favorable or unfavorable. This principle was illustrated as being operative in cases of differing reactions. Such a thermodynamic selection rule may be used to predict the selectivity of reactions in the presence of competing functional groups. The selectivity of the transamidation reactions was applied to molecular systems having an 
additional, but another type of amino group (93). Molecule 93 has two opportunities for acylation, where in principle it could have yielded two types of mono-amide compounds (94-95 and 96-97) or a single diamide compound (98 or 99) as represented in Figure 24. As the amidicity change $(\triangle \mathrm{AM})$ indicated, only the alkyl amine group could react with 75 and 33, therefore only compounds 94 and 95 were formed, whereas 96 and 97 as well as 98 and 99 were not produced, even in traces amounts [10-12].

\section{R-XIX}<smiles>[R]C(N)=O</smiles>

$$
\begin{aligned}
& \mathrm{R}=\mathrm{H}, \mathbf{7 5}(93.4 \%) \\
& \mathrm{R}=\mathrm{Me}, \mathbf{3 3}(96.1 \%)
\end{aligned}
$$<smiles>[R]C(=O)NCc1ccc(N)cc1</smiles><smiles>Cc1ccc(C)c(NC(=O)CC2CC3CCC32)c1CN</smiles><smiles>[R]C(=O)NCc1c(NC([R])=O)ccc(C)c1OC([R])C</smiles>

Fig. 24. Selectivity of transamidation reactions

From the numerous biochemical processes, involving transamidation reaction, only few, but very representative examples are presented here. The first example is taken from the multistep process of the blood clotting. In the last thirteenth step ( $\mathbf{R}-\mathbf{X X}$ in Figure 25) of the process, the two final protein intermediates 100 and 101 are jointed to each other through forming a side-chain amide bond. This process is spontaneous, therefore does not require external energy input. From system chemistry point of view, it is due to the positive $\Delta \mathrm{AM}$ value of the process, where the initial $96.0 \%$ is increased to $101 \%$. This small change provides a driving force for this reaction, but it is not enough to exhibit high reaction rate, therefore it is catalyzed by an enzyme transamidinaze [21].

\section{R-XX Precursores}

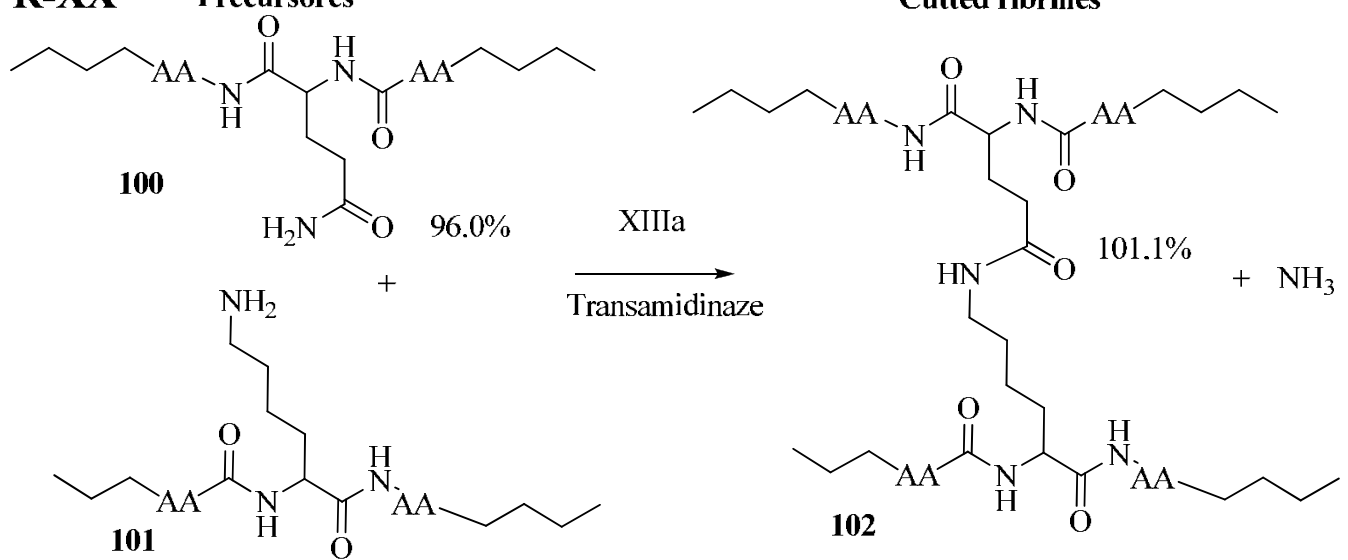

Fig. 25. 
The intein-mediated protein splicing (R-XXI) is, relatively speaking, a newly discovered biological process (Figure 26) [21]. In this case however two amides are involved in transamidation process rather than me amide and one amine as before. Protein splicing is so rapid that the precursor protein is rarely observed in native systems. The intein peptide sequence usually contains no sufficient information and it is supposed to be originated from a virus, which inserted into the original DNA sequence producing the protein. The original broken protein sequence is named as extein. The intein plus the first C-extein residue contain sufficient information for splicing in foreign proteins, which involves four basic chemical steps. For the sake of simplicity, here we have presented only the starting and the ending states of the reaction and the amidicity values of the amide bonds were calculated for the functional groups in question.

\section{R-XXI}

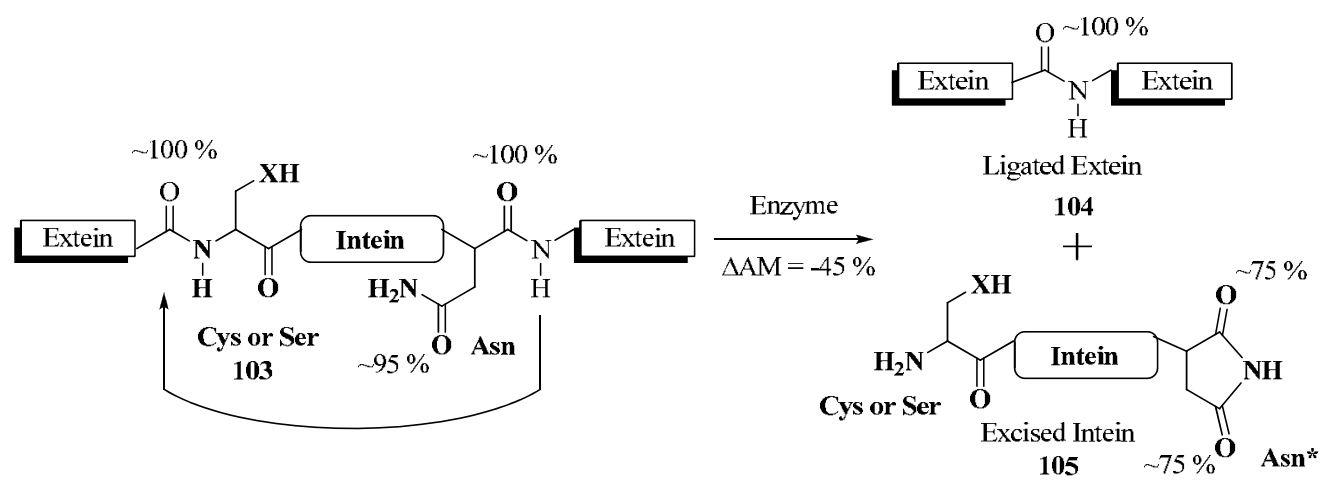

Fig. 26. Protein splicing as a special type of transamidation reaction.

The overall process exhibits large negative $\Delta \mathrm{AM}$ value $(-45 \%)$, which may result an endothermic reaction. However, the folding of the spliced protein supposed to be more favorable, than that of the original, intein containing protein, which may provide an other driving force covering the energy demand of the overall process.

\subsection{Complex analysis: Comparison of redox reactions of NAD and FAD with human made redox reaction [18]}

Biochemical reactions are exceptionally energy-efficient relative to laboratory synthetic processes. Liberation and subsequent loss of heat in exothermic reactions (e.g. redox processes) is a mismanagement of energy and would be biologically detrimental as are highly endothermic ones, thus are avoided in nature. To support such a hypothesis, laboratory redox reactions were compared to those occurring in biological organisms. Evidently, Nature is able to store reactive potential within the molecular system, in carefully designed chemical structures which act as reversible energy carriers; effectively molecular free-energy capacitors. This modus operandi of Nature implies that during the evolutionary process, only those molecular that fulfilled near thermo-neutral requirements were retained. In an oxygen-containing atmosphere the development of aerobic life required appropriate reducing/oxidising agents. Thus, long ago Nature implemented the coenzymes dinucleotides Nicotinamide Adenine Dinucleotide $\left(\mathrm{NAD}^{+}, 106\right)$ and Flavin Adenine Dinucleotide (FAD, 107; for full structures see Figure 27, where the $\mathrm{R}$ groups later are 
simplified to Me) together with their respective redox pairs $\mathrm{NADH}$ and $\mathrm{FADH}_{2}$ to mediate the redox processes in all known living cells. These bioreagents play crucial energy storage roles, which act as 'energy catalysts', storing reductive potential until required.
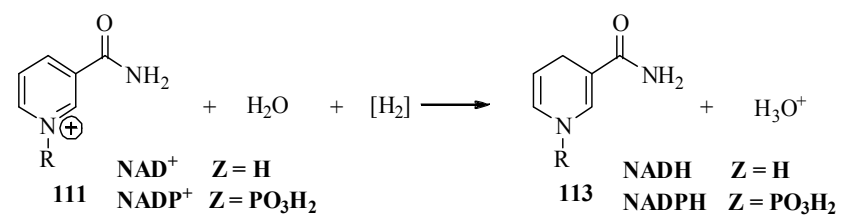<smiles>Cc1cc2nc3c(=O)[nH]c(=O)nc-3n(CC(O)C(O)C(O)[In])c2cc1C</smiles><smiles>[AlH2][AlH2]</smiles>

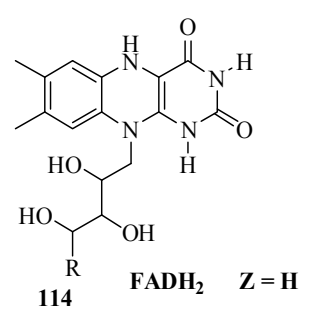

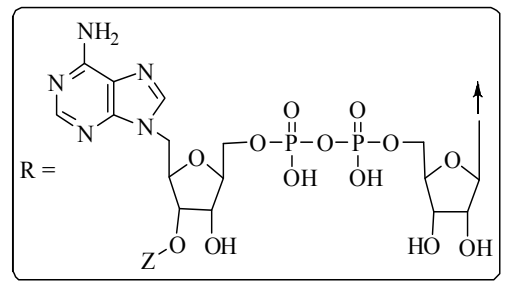

Fig. 27. The complete structures and reactions of $\mathrm{NAD}^{+}(\mathbf{1 1 1})$ and FAD (112). The apostrophises at the compound numbers are emphasizing that these are the full structures rather than the model compounds on which the computations were performed.

These typical examples were selected, due to their published enthalpy of formation data. The preparation of reagent $\mathrm{H}_{2}$ requires high amount of external energy (enthalpy), usually via electrolysis (R-XXII/a in Figure 28), while the reduction of pyruvate (108) to lactate (109) releases or waste also the same order of magnitude enthalpy (c.a. $-200 \mathrm{~kJ} / \mathrm{mol} ; \mathbf{R}-\mathbf{X X I I} / \mathbf{b}$ in Figure 28). Other human applied reductive reagents, like $\mathrm{NaBH}_{4}$ also serves analogues examples, where both the preparation and the usage of these reagents are not very energy efficient.

In the Nature side, the same reduction of pyruvate (108) to lactate (109) by using NADH (108) as bioreagent wastes only c.a. $30 \mathrm{~kJ} / \mathrm{mol}$ enthalpy (R-XXIII/b in Figure 28). Moreover, the preparation of this active reducing reagent NADH (108) from $\mathrm{NAD}^{+}(\mathbf{1 1 1})$ by means of maleate (110) and oxalacetate (111) redox equilibrium (R-XXIII/a in Figure 28).

Comparing these human- (R-XXII) and bioprocesses (R-XXIII), both the preparation (a) and reaction $(\mathbf{b})$ of the NADH (bio)reagent proceed with $1 / 10^{\text {th }}$ the enthalpy change observed in the laboratory exercise, consequently this bioprocess can avoid the large endo- and exothermic changes in the course of the reaction, does not requiring intense external heating or cooling of the living organism. Such systemic chemical principles may be universally applied in all life-related processes. Coupling between components of a chemically or biologically important molecule, such as aromatic rings, amide groups, olefins, carbonyls and metal-ligands, are central to the molecules' chemical efficiency.

The reduction of $\mathrm{NAD}^{+}$and $\mathrm{FAD}$ is complimented by an enthalpy transfer between organic functional components (aromatic ring, amide and olefinic functionalities), yet, the sum of the overall energy values (the total system) remains nearly constant irrespective of what direction the redox reaction proceeds. From this aspect, both $\mathrm{NAD}^{+}$and FAD operate as real chemical systems of atoms and functional groups, working together within the individual molecules to store the reaction enthalpy as resonance enthalpy, rather than manifesting it as emitted or absorbed heat. In this way, the thermo-neutral reaction of the wet combustion occurring in all living cells is made possible by an internal "cooling process". 
Concerning $\mathrm{NAD}^{+}(\mathbf{1 0 6})$ and FAD (107), two questions may be phrased: (Q1) What is the role of the amide functionality and why the meta-substituted $\mathrm{NAD}^{+}$structure was selected during molecular evolution? (Q2) Why a complex, three-ring structure is necessary for proper function and catalytic efficacy of the FAD molecular system? A systemic approach is required to answer these. Making use of the recently established methodology ${ }^{15-19}$, hydrogenation reactions were used to determine aromaticity, amidicity and olefinicity values.

\section{R-XXII}

Reducing agent preparation [a]:

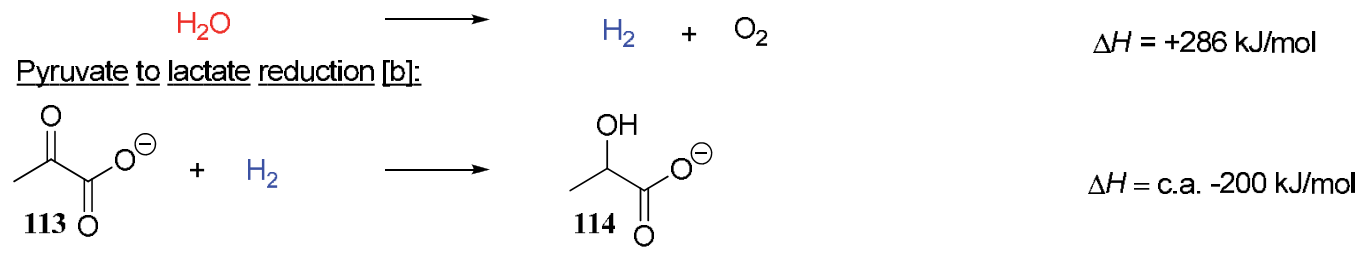

R-XXIII

Reducing agent preparation [a]:
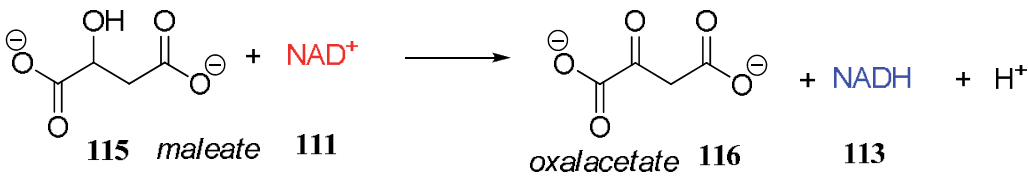

$\Delta H=$ c.a. $+26 \mathrm{~kJ} / \mathrm{mol}$

Pyruvate to lactate reduction [b]:

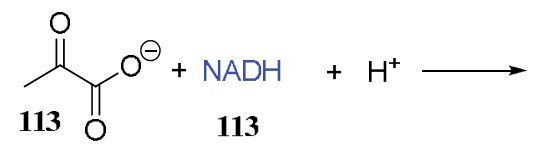<smiles>[13CH3]C(=O)C(O)=[18O]</smiles>

Fig. 28. A schematic depiction of the order of magnitude difference in energetic efficiency between synthetic and natural processes. The preparation of the reducing agents $[\mathrm{I} / \mathrm{a}$ and II/a] and redox processes [I/b and II/b] in the case of a certain, biologically significant reaction are used as examples.

\subsubsection{NAD Coenzyme and its models}

From the Systems Chemistry point of view, many organic molecules are comprised of aromatic, amidic and olefinic portions, linked together by the global electronic structure of the system. These organic functional groups may easily be described by the concept of 'conjugativicity'; a term analogous to aromaticity, ${ }^{15}$ amidicity, ${ }^{16,17}$ carbonylicity ${ }^{18}$ and olefinicity. ${ }^{19}$ Accordingly, the structure of $\mathrm{NAD}^{+}$and related models I-III $(\mathbf{1 0 6}, \mathbf{1 1 2}, \mathbf{1 1 4}, \mathbf{1 1 6})$ are composed of an aromatic (pyridine) and most of them an amidic part; each described using aromaticity and amidicity parameters, respectively. The structure of NADH and its related models I-III $(\mathbf{1 0 7}, \mathbf{1 1 3}, \mathbf{1 1 5}, \mathbf{1 1 7})$ are composed of one amidic and two olefinic parts, described by one amidicity and two olefinicity parameters, respectively.

Figure 29 provides a comparison of resonance enthalpy (RH) change in the naturally occurring nicotinic amide $\left(\mathrm{NAD}^{+}, \mathbf{1 0 6}\right)$ as well as its model congeners [models I (112), II (114 
and III (116)]. Table 2 shows the naturally occurring meta isomer having the greatest RH 'benefit' (+42.5 kJ mol-1), manifested as an exothermic $-42.5 \mathrm{~kJ} \mathrm{~mol}^{-1}$ reaction enthalpy. Our novel Systems Chemistry analysis shows that the principle RH component, originally stored as aromaticity $\left(145.8 \mathrm{~kJ} \mathrm{~mol}^{-1}\right)$ in the pyridine ring of $\mathrm{NAD}^{+}(\mathbf{1 0 6})$, is partly transferred postreduction $(\mathbf{1 0 6} \rightarrow \mathbf{1 0 8})$, to the two olefinicities $a$ and $b$.

\begin{tabular}{|c|c|c|c|c|c|c|c|c|}
\hline & & \multicolumn{6}{|c|}{ Systems Chemistry } & \multirow[b]{3}{*}{$\mathrm{kJ} / \mathrm{mol}$} \\
\hline & & \multicolumn{2}{|c|}{ aromaticity } & \multicolumn{2}{|c|}{ amidicity } & \multicolumn{2}{|c|}{ olefinicity } & \\
\hline & & $\%$ & $\mathrm{~kJ} / \mathrm{mol}$ & $\%$ & $\mathrm{~kJ} / \mathrm{mol}$ & $\%$ & $\mathrm{~kJ} / \mathrm{mol}$ & \\
\hline Natural & 106 & 95.1 & 145.8 & 35.2 & 28.1 & 0.0 & 0.0 & \\
\hline \multirow[t]{3}{*}{ form } & 108 & 0.0 & 0.0 & 109.0 & 86.7 & $34.6+54.4$ & 129.5 & \\
\hline & & & +145.8 & & -58.7 & & -129.5 & -42.5 \\
\hline & 112 & 91.6 & 140.8 & 36.4 & 29.0 & 0.0 & 0.0 & \\
\hline \multirow[t]{3}{*}{ Model I } & 113 & 0.0 & 0.0 & 103.7 & 86.7 & $36.1+32.1$ & 97.6 .5 & \\
\hline & & & +140.8 & & -53.5 & & -97.6 & -10.6 \\
\hline & 114 & 101.0 & 154.8 & 66.1 & 52.6 & 0.0 & 0.0 & \\
\hline \multirow[t]{3}{*}{ Model II } & 115 & 0.0 & 0.0 & 98.2 & 78.1 & $38.1+38.1$ & 109.0 & \\
\hline & & & +154.8 & & -25.5 & & 109.0 & +20.2 \\
\hline & 116 & 94.9 & 145.5 & 0.0 & 0.0 & 0.0 & 0.0 & \\
\hline \multirow[t]{2}{*}{ Model III } & 117 & 0.0 & 0.0 & 0.0 & 0.0 & $32.6+32.6$ & 93.4 & \\
\hline & & & +145.5 & & 0.0 & & -93.4 & +52.1 \\
\hline
\end{tabular}

Table 2. Summary of different "icity" values (aromaticity, amidicity and olefinicity) and related resonance energies in $\mathrm{kJ} / \mathrm{mol}$ calculated for 106,108, 112-117. For details see Figure 29.

This resulted in $16.8 \mathrm{~kJ} \mathrm{~mol}^{-1}$ less than complete energy recovery. However, the change in amidicity of the amide functionality provides $58.7 \mathrm{~kJ} \mathrm{~mol}^{-1}$ to $\mathrm{RH}$, covering not only the 'missing' $16.8 \mathrm{~kJ} \mathrm{~mol}^{-1}$, but also makes the overall process $42.5 \mathrm{~kJ} \mathrm{~mol}^{-1}$ advantageous from an RH point of view. In comparison, both the ortho (model I, 112 $\rightarrow$ 113) and para (model II, $114 \rightarrow \mathbf{1 1 5}$ ) isomers of $\mathrm{NAD}^{+}$are not able to provide overall $\mathrm{RH}$ 'recovery' (Table 2, Figure 29) during the reduction; the principle reason that the natural form 106 uses the meta isomer of $\mathrm{NAD}^{+}$as a part of this redox system. Not only is the meta position the most suitable, it holds the most biologically available functional group, supporting the notion of 'molecular selection' being operative during the evolution of redox biochemistry of all life.

Our study also indicated that the entire amide functionality is crucial from the Systems Chemistry point of view, as the structurally simpler model III (117, Table 2, Figure 29) cannot recover the loss of RH from the loss of aromaticity during the reduction $(\mathbf{1 1 6} \rightarrow \mathbf{1 1 7})$, due to the lack of an electron withdrawing group (EWG) in the meta position. The biologically more prevalent $\mathrm{COOH}$ functionality, mostly existing as $\mathrm{COO}^{-}$at biological $\mathrm{pH}$ 6-8 (COO- is an electron donating group, or EDG), would be far less effective than the $\mathrm{CONH}_{2}$ EWG. Due to the highly effective energy recovery in both the forward and reverse reactions, is the principle reason that the $\mathrm{NAD}^{+} / \mathrm{NADH}$ redox pair $(\mathbf{1 0 6 / 1 0 8})$ works as a near-thermoneutral bio-reagent in biochemeical reactions. 


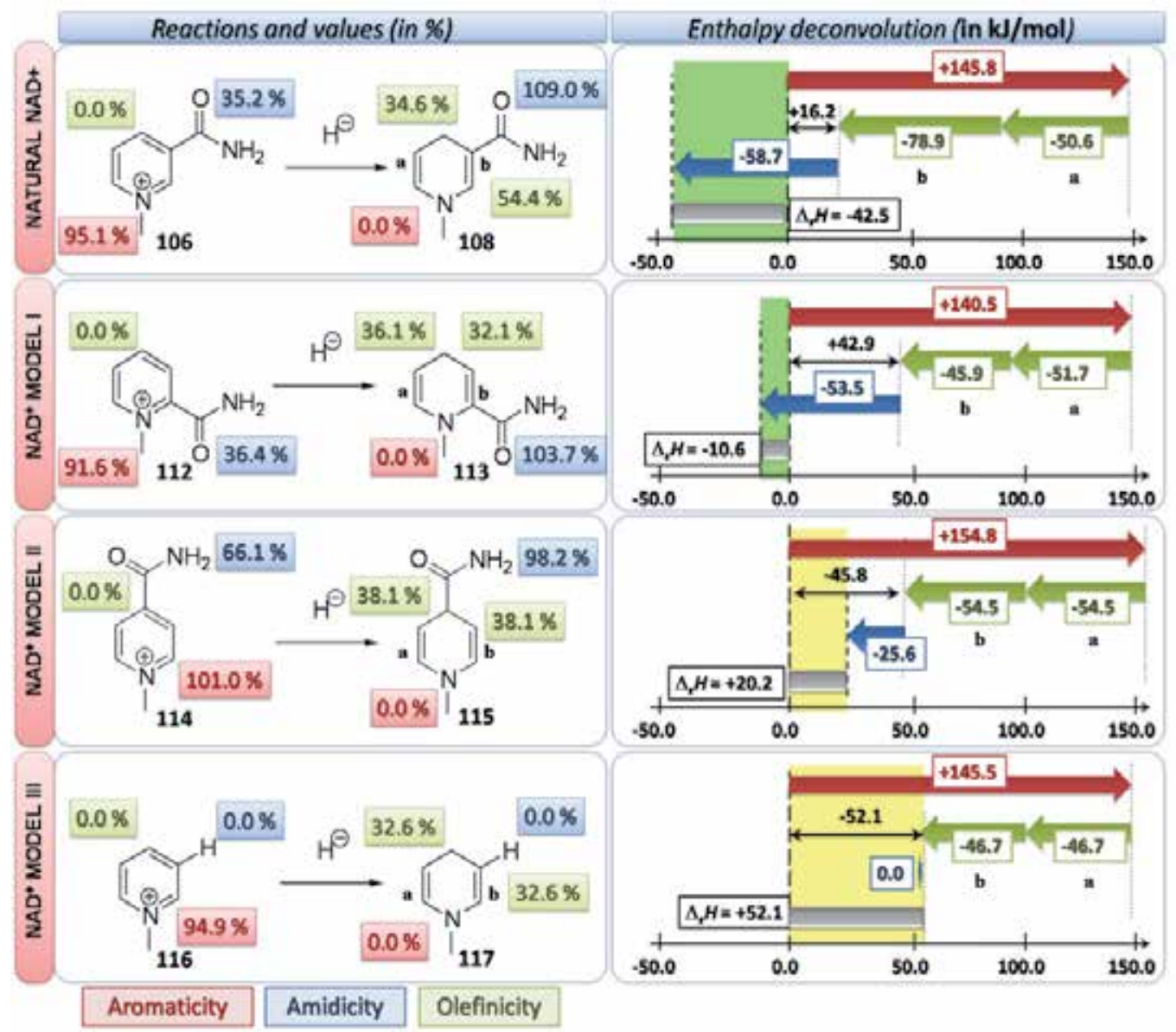

Fig. 29. LEFT: Redox reactions of the natural form of $\mathrm{NAD}^{+}$and related pyrimidium ion congener model systems $(\mathbf{1 0 6}, \mathbf{1 1 2}, \mathbf{1 1 4}, \mathbf{1 1 6})$ and their respective reduced products $(\mathbf{1 0 8}, \mathbf{1 1 3}$, $115,117)$, including percentages of conjugative aromaticity (red), amidicity (blue) and olefinicity (green) values. RIGHT: Changes in resonance enthalpy $(\mathrm{RH})$ values related to conjugativity, indicated by arrows using the same colour scheme as used at left, including both the sign and magnitude of change.

\subsubsection{Systems chemistry analysis of FAD}

Analogously, the three ring FAD (107) and $\mathrm{FADH}_{2}(109)$ can be dissected to an aromatic portion composed of two rings ( $\mathrm{A}$ and $\mathrm{B}$ ) in addition to two-amide bond forming ring $\mathrm{C}$, accounted for by a combined aromaticity value and two amidicity parameters, respectively. In this study some modified models of $\mathrm{FAD}-\mathrm{FADH}_{2}$ system are also considered as 118-119; 120-121 and 122-123. It may be demonstrated that all components of the FAD system (112) (tricyclic form with ring A, B and C, involving two amide bonds; $a$ and $b$; see Figure 30 and Table 3) are essential. Thus such natural arrangement of functional groups is requisite for proper function. From the Systems Chemistry point of view, FAD (107) may be considered as being similar to $\mathrm{NAD}^{+}(\mathbf{1 0 6})$. The oxidized form (108) is comprised of two fused-rings in 
the aromatic portion (ring A and B), characterized by a single $131.2 \%$ aromaticity value $\left(\mathrm{RH}=201.2 \mathrm{~kJ} \mathrm{~mol}^{-1}\right)$. The aromatic component is connected to a $3^{\text {rd }}$ ring, itself substituted by two amide bonds having $111.6 \%$ and $71.0 \%$ amidicity values $\left(\mathrm{RH}=88.7 \mathrm{~kJ} \mathrm{~mol}^{-1}\right.$ and $56.4 \mathrm{~kJ} \mathrm{~mol}^{-1}$ ), respectively (first line of Figure 30 and Table 3). The aromaticity in the double-ring of the reduced form (109) is lowered to $100.9 \%\left(\mathrm{RH}=154.7 \mathrm{~kJ} \mathrm{~mol}^{-1}\right)$, while amidicity rises in both amide bonds to $140.1 \%$ and $125.4 \%$, respectively $\left(\mathrm{RH}=111.9 \mathrm{~kJ} \mathrm{~mol}^{-}\right.$ 1 and $\left.99.7 \mathrm{~kJ} \mathrm{~mol}^{-1}\right)$. Loss of aromaticity upon reduction $\left(\mathbf{1 0 7}+\mathrm{H}_{2} \rightarrow \mathbf{1 0 9}\right)$, is rationalized by an observed antiaromatisation of ring $B$ (6 electron pairs in rings $A$ and $B$ ), manifested as a bent, non-planar, 3D fused-ring structure (109). However, amidicity values increase significantly, resulting in an overall $\mathrm{RH}$ change of $+20.1 \mathrm{~kJ} \mathrm{~mol}^{-1}$, representing a relatively large exothermic change $\left(-20.1 \mathrm{~kJ} \mathrm{~mol}^{-1}\right)$ in the systemic $\mathrm{RH}$.

The aromatic ring $\mathrm{A}$ of the FAD system serves to buffer the antiaromatisation of ring $\mathrm{B}$, appearing as a non-planar 3D structure of 107 and 118. This is exemplified on reduction of FAD model I (118 $\rightarrow$ 119; second line Figure 30 and Table 3), wherein ring A is removed, resulting in a corresponding $\Delta$ aromaticity $=-72.9 \%$ in contrast to the $-30.3 \%$ change in the natural form.

\begin{tabular}{|c|c|c|c|c|c|c|c|c|}
\hline & & \multicolumn{6}{|c|}{ Systems Chemistry } & \multirow[b]{3}{*}{$\mathrm{kJ} / \mathrm{mol}$} \\
\hline & & \multicolumn{2}{|c|}{ aromaticity } & \multicolumn{2}{|c|}{ amidicity (a) } & \multicolumn{2}{|c|}{ amidicty (b) } & \\
\hline & & $\%$ & $\mathrm{~kJ} / \mathrm{mol}$ & $\%$ & $\mathrm{~kJ} / \mathrm{mol}$ & $\%$ & $\mathrm{~kJ} / \mathrm{mol}$ & \\
\hline \multirow{3}{*}{$\begin{array}{l}\text { Natural } \\
\text { form }\end{array}$} & 107 & 131.2 & 201.1 & 111.6 & 89.1 & 71.0 & 56.7 & \\
\hline & 109 & 100.9 & 154.7 & 140.1 & 111.8 & 125.4 & 100.1 & \\
\hline & & & +46.5 & & -23.3 & & -43.3 & -20.1 \\
\hline \multirow{3}{*}{ Model I } & 118 & 41.2 & 63.2 & 111.4 & 88.9 & 72.4 & 57.8 & \\
\hline & 119 & -31.7 & -48.6 & 144.8 & 115.6 & 126.4 & 100.9 & \\
\hline & & & +111.9 & & -26.6 & & -42.9 & +42.4 \\
\hline \multirow{3}{*}{ Model II } & 120 & 126.2 & 193.5 & 122.2 & 97.6 & 59.2 & 47.3 & \\
\hline & 121 & 111.5 & 170.9 & 111.2 & 88.8 & 49.7 & 39.7 & \\
\hline & & & +22.5 & & +8.8 & & +7.6 & +38.8 \\
\hline \multirow{3}{*}{$\begin{array}{l}\text { Model III } \\
\text { Natural }\end{array}$} & 122 & 124.7 & 191.2 & 51.5 & 41.2 & 0.0 & 0.0 & \\
\hline & 123 & 103.2 & 158.2 & 80.0 & 63.9 & 0.0 & 0.0 & \\
\hline & & & +32.9 & & -22.7 & & 0.0 & +10.3 \\
\hline
\end{tabular}

Table 3. Summary of different "icity" values (aromaticity, amidicity and olefinicity) and related resonance energies in kJ/mol calculated for 107,109, 118-123. For details see Figure 30.

This is due to the large degree of antiaromaticity in the heterocycle component of $\mathbf{1 1 9}$ (aromaticity $=-31.7 \%$ ), which is not offset by a corresponding, stabilizing increase in amidicity; $\Delta$ amidicity for $\mathbf{1 1 8} \rightarrow \mathbf{1 1 9}$ is similar to that of the $\mathbf{1 0 7} \rightarrow \mathbf{1 0 9}$ and the overall $\mathrm{RH}$ for the reduction of model I is no longer favorable $\left(+42.4 \mathrm{~kJ} \mathrm{~mol}^{-1}\right)$.

The $b$ amide component also serves a crucial systemic role, helping stabilize the system upon reduction through a $54.4 \%$ amidicity increase in the natural form $(\mathbf{1 0 7} \rightarrow \mathbf{1 0 9} ; 71.0 \% \rightarrow$ $125.4 \%$ ). Its removal (FAD model II (120); third line in Figure 30 and Table 3) 'softens' the destabilizing reduction of aromaticity $(\mathbf{1 2 0} \rightarrow \mathbf{1 2 1} ; 124.7 \% \rightarrow 103.2 \%=-21.5 \%)$ relative to the natural process $(\mathbf{1 0 7} \rightarrow \mathbf{1 0 9}=-30.3 \%)$. However, the absence of this secondary -CONHfunctionality excludes its thermodynamically advantageous amidicity increase, resulting in an overall unfavorable process $\left(\mathbf{1 2 0} \rightarrow \mathbf{1 2 1}=+10.3 \mathrm{~kJ} \mathrm{~mol}^{-1}\right)$. 
The role of the chemical linkage between ring II and amide B was also examined using the truncated FAD model III $(\mathbf{1 2 2} \rightarrow \mathbf{1 2 3}$; fourth row in Figure $\mathbf{3 0})$. Removal of the bond destroys ring $\mathrm{C}$ and raises the overall $\mathrm{RH}$, making reduction $+58.9 \mathrm{~kJ} \mathrm{~mol}^{-1}$ less favorable relative to the natural system, due to the absence of amidicity increase in amide II. In the natural compound (107), ring $C$ buffers the appearing antiaromaticity, manifested in its own increased aromaticity and amidicity values (109). Truncation of this very important bond eliminates the systemic linkage between ring $\mathrm{B}$ and amide $b$. In the reduction of FAD, only the ring $\mathrm{B}$ and amide $a$ components take chemical part in the reaction, together receiving two electrons and two protons. One may therefore conclude that the role of all other

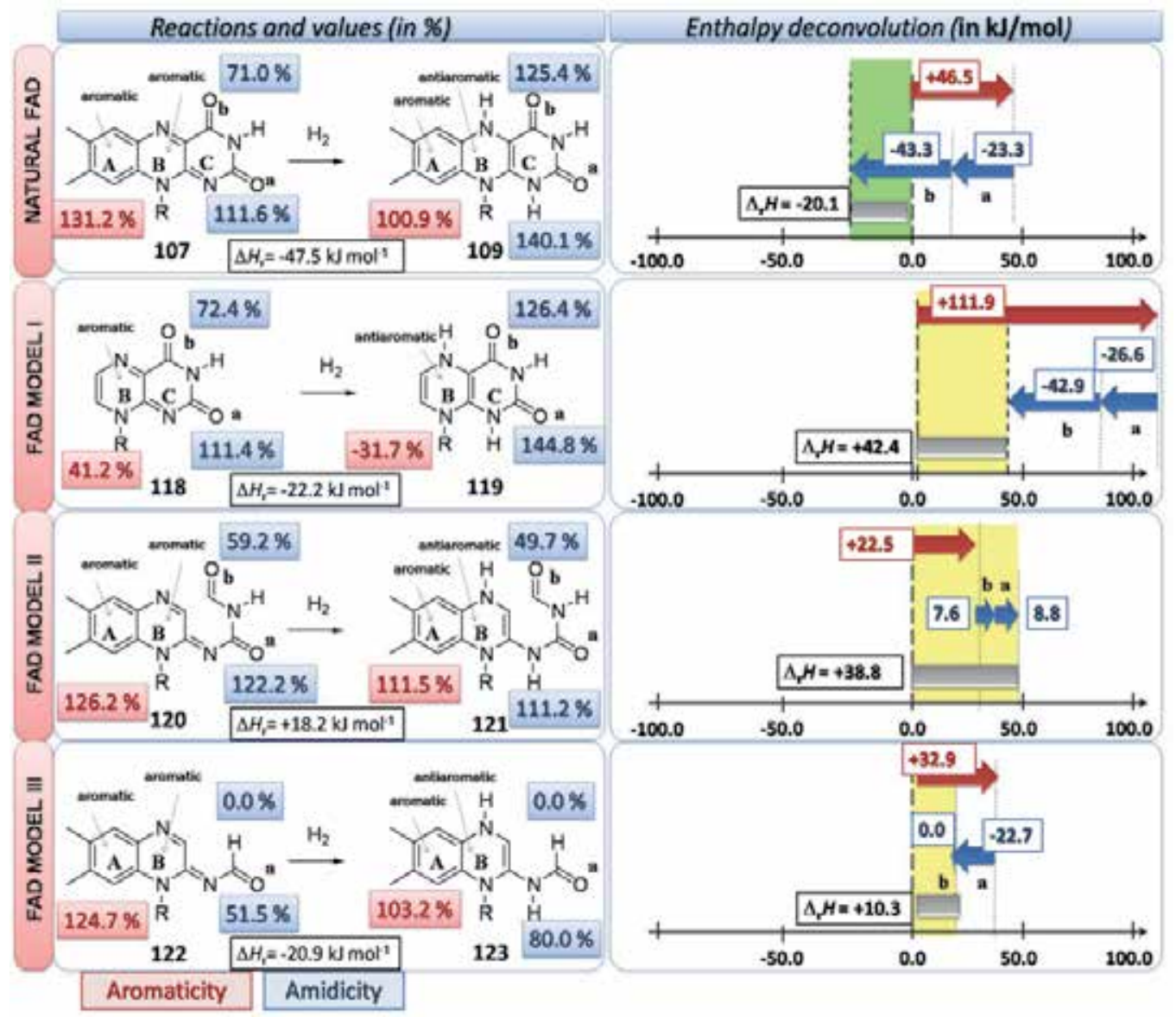

Fig. 30. LEFT: Redox reactions of the natural form of FAD $(\mathbf{1 0 7})$ and their different models (I, 118; II, 120 and III, 122) leading to reduced products $(109,119,121,123)$ and their percentages of their respective conjugative [aromaticity (red), amidicity (blue)] values.

RIGTH: Changes of resonance enthalpy $(\mathrm{RH})$ values related to conjugativity [indicated by arrows with the same color as was used left, referring to both the sign and the amount of these]. FAD (107) and its models 118, 120, 122 as well as $\mathrm{FADH}_{2}(\mathbf{1 0 9})$ together with its congeners $(\mathbf{1 1 9}, \mathbf{1 2 1}, \mathbf{1 2 3})$ is composed of an aromatic ring (aromaticity, ring A and B) and two amide functionalities (amidicity, amide $a$ and $b$ ). 
functional components is systemic, compensating for (reducing) the antiaromatization of ring $B$. This leads to the thought that, were Nature truly so efficient, it would remove the antiaromatization and the need for other components; unless antiaromatization itself were essential, playing a secondary role in the process. Considering only the reductive part of FAD (model III, 122, last line in Figure 30 and Table 3), removes the need to compensate the disadvantageous antiaromaticity. The reductive power of the resultant small molecule $(\mathbf{1 2 2} \rightarrow \mathbf{1 2 3})$ is extremely large, meaning that by means of specialized enzymes, it has the ability to reduce each reducable compound in the cell. However the associated large exothermic reaction enthalpy would call for an intensive cooling process to bring the temperature under control; antiaromaticity providing the 'counterbalance' to the process and a means by which energy may be stored.

\section{References}

[1] Z. Mucsi, A. Szabo, I. Hermecz, Á. Kucsman, I.G. Csizmadia, J.Am. Chem Soc. 2005, 127, 7615-7631.

[2] E. Buncel, R.A.Stairs, H. Wilson, In The Role of the Solvent in Chemical Reactions; Oxford University Press: Oxford, 2003.

[2] M. Nonn, L. Kiss, E. Forró, Z. Mucsi, F. Fülöp Tetrahedron, 2011, 67. 4079-4085.

[3] F. Ruff, I.G. Csizmadia, In Organic Reactions: Equilibria, Kinetics and Mechanism; Elsevier: Amsterdam, 1994; Chapter 8. pp 232-239.

[4] J.C. Cramer, In Essentials of Computational Chemistry; John Wiley Sons Ltd.: West Sussex, 2001; Chapter 7. p 433.

[5] E. Frank, Z. Mucsi, I. Zupkó, B. Réthy, G. Falkay, Gy. Schneider, J. Wölfling, J. Am. Chem. Soc. 2009, 131, 3894-3904.

[6] Z. Mucsi, B. Viskolcz, I. G. Csizmadia, J. Phys. Chem. A. 2007, 111, 1123-1132.

[7] Z. Mucsi, T. Körtvélyesi, B. Viskolcz, I. G. Csizmadia, T. Novák, G. Keglevich, Eur. J. Org. Chem. 2007, 1759-1767.

[8] Z. Mucsi, I. Hermecz, B. Viskolcz, I. G. Csizmadia, G. Keglevich Tetrahedron, 2008, 64, 1868-1878.

[9] Z. Mucsi, G. Keglevich Eur. J. Org. Chem. 2007, 1759-1767.

[10] Z. Mucsi, A. Tsai, M. Szori, G. A. Chass, B. Viskolcz, I. G. Csizmadia, J. Phys. Chem. A. 2007, 111, 13245-13254.

[11] T. R. Varga, P. Nemes, Z. Mucsi, P. Scheiber Tetrahedron Letters, 2007, 48, 1159-1161.

[12] Z. Mucsi, G. A. Chass, I. G. Csizmadia, J. Phys. Chem. B. 2008, 112, 7885-7893.

[13] Z. Mucsi, G. A. Chass, B. Viskolcz, I. G. Csizmadia, J. Phys. Chem. A. 2008, 112, $9153-$ 9165.

[14] M. Porcs-Makkay, B. Volk, Z. Mucsi, Gy. Simig Tetrahedron, 2010, 66. 7017-7027.

[15] Z. Mucsi, G. A. Chass, B. Viskolcz, I. G. Csizmadia, J. Phys. Chem. A. 2009, 113, 7953-7962.

[16] M. Pilipecz, Z. Mucsi, T. Varga, P. Scheiber, P. Nemes, Tetrahedron 2008, 64, 5545-5550.

[17] T. Novák, Z. Mucsi, B. Balázs, L. Keresztély, G. Blaskó, M. Nyerges, Synlett, 2010, 16, 2411-2414.

[18] Z. Mucsi, G. A. Chass, I. G. Csizmadia, J. Phys. Chem. B. 2009, 113, 10308-10314.

[19] Z. Mucsi, I.G. Csizmadia, Current Organic Chemistry 2008, 12, 83-96.

[20] J. M. Berg, J. L. Tymoczko, L. Stryer, In Biochemistry, 5th edition; Freeman and Company, New York, 2002.

[21] Y. Anraku, R. Mizutani, Y. Satow, IUBMB Life 2005, 57, 563-574. 


\title{
Energy Planning for Distributed Generation Energy System: The Optimization Work
}

\author{
Behdad Kiani \\ Institute for Integrated Energy Systems, University of Victoria \\ Canada
}

\section{Introduction}

Behind the public eye a quiet revolution is taking place, one that will permanently alter our relationship with energy. Most people today have heard about deregulation of the electric utility industry. Recently, privatization of most important energy sectors (electricity) in Iran has turned former monopolies into free market competitors. This has been specially the case with the unbundling of vertically integrated energy companies in the electricity sector where generation, transmission, and distribution activities have been split. Community consciousness of fossil fuel resource depletion and environmental impact caused by large scale power plants is growing. Because of large land area, losses in Iran power transmission network are significant. These reasons caused greater interest in distributed generation (DG) - small scale, demand site - technologies based on renewable energy sources.

Energy planning has to be carried out by modeling all sectors of energy system from primary energy sources (fossil fuels, renewable) to end use technologies for determination of optimal configuration of energy systems. Energy planning is a powerful tool for showing the effects of certain energy policies, which helps decision makers choose the most appropriate strategies in order to expand DG technologies and taking into account environmental impacts and costs to the community. Energy planning is carried out in Iran's energy system. Therefore, we have defined a reference energy system for Iran.

The aim of this paper is to evaluate the contribution of DG technologies when energy planning is carried out. For this purpose, the energy system optimization model MESSAGE has been utilized to take into account the presence of DG technologies. To provide a detailed description of DG production, a power grid scheme is considered. Planning procedure follows an optimization process based on the cost function minimization in the presence of technical and energy-policy and environmental constraints.

In Section 2, a brief explanation of model MEESAGE is given. In this section you will know main parts and aim of the model. In section 3, a brief review of the spread of DG technologies is reported. In Section 4, the reference energy system of Iran relating to the proposed optimization procedure and structure of model MESSAGE is illustrated. In section 5 , Model validation is studied. The test results of several scenarios applied to Iran's energy system are reported in Section 6. 


\section{Overview of model MESSAGE}

MESSAGE (Model for Energy Supply Strategy Alternatives and their General Environmental Impacts) is a system engineering optimization model used for medium-term to long-term energy system planning (i.e. energy supplies and utilization), energy policy analysis, and scenario development. The model was originally developed at International Institute for Applied Systems Analysis (IIASA). The underlying principle of MESSAGE model is optimization of an objective function under a set of constraints that define the feasible region containing all possible solutions of the problem. In general categorization, MESSAGE belongs to the class of mixed integer programming models as it has the option to define some variables as integer. The model provides a framework for representing an energy system with the most important interdependencies from resource extraction, imports and exports, conversion, transport, and distribution, to the provision of energy end-use services such as agriculture sector, residential and commercial space conditioning, industrial production processes, and transportation. A set of standard solvers (e.g., GLPK, OSLV2, OSLV3, CPLEX, and MOSEK) can be used to solve the MESSAGE model. The degree of technological detail in the representation of an energy system is flexible and depends on the geographical and temporal scope of the problem being analyzed. A typical model application is constructed by specifying performance characteristics of a set of technologies and defining a reference energy system (RES) that includes all the possible energy chains that the model can make use of. In the course of a model run MESSAGE will then determine how much of the available technologies and resources are actually used to satisfy a particular end-use demand, subject to various constraints, while minimizing total discounted energy system costs which include investment costs, operation cost and any additional penalty costs defined for the limits, bounds and constraints on relations. For all costs occurring at later points in time, the present value is calculated by discounting them to the base year of the case study. MESSAGE is designed to formulate and evaluate alternative energy supply strategies consonant with the user-defined constraints such as limits on new investment, fuel availability and trade, environmental regulations and market penetration rates for new technologies. Environmental aspects can be analyzed by accounting, and if necessary limiting, the amounts of pollutants emitted by various technologies at various steps in energy supplies. This helps to evaluate the impact of environmental regulations on energy system development. For more details on the model and the mathematical representation of the reference energy system see [4],[5].

\section{Overview of distributed generation technologies}

The term distributed generation is defined in this paper as power generation technologies below $10 \mathrm{MW}$ electrical outputs that can be sited at or near the load they serve or designed to deliver production to low voltage or medium voltage electricity networks. So, small hydro power plant, wind-powered generator, photovoltaic cells (PV), geothermal and solar-thermal power plants have been considered as DG technologies. In recent years, there has been a considerable expansion of DG technologies in Iran, thanks to progress in reliability and government policies. Despite the remarkable progress attained over the past decades, nowadays there are a few DG facilities in Iran (less than $0.5 \%$ of all electricity generation is supplied by DG facilities [1]). But DG facilities are expanding at high rate. It's predicted that $20 \%$ of demand for electricity will be supplied by DG 
facilities at 2030. The presence of DG facilities brings benefits both to the electric power system and the total energy system. With DGs energy can be generated directly where it is consumed. As a result, transmission and distribution networks are less charged; safety operation margins increase, and transmission costs and power losses are reduced [6], [7]. Since with most DG options renewable based technologies are used, there is a lower environmental impact. At the very least, the spread of DG technologies enhances supply safety in the energy field by reducing dependence on fossil fuels. Therefore, Renewable energy technologies are emerging as potentially strong rivals for more widespread use. Some DG technologies have already achieved a significant market share in comparison with other DGs in Iran. For example, Small hydropower systems are well established. Wind generators, which have been going through intense technology and market development, have achieved considerable market share, even though further technological improvements need to be made. Solar thermal power plants are also developed. But the solar photovoltaic and geothermal market is comparatively small. DG technologies are commonly connected to power distribution network.

\section{The reference energy system}

Fig. 1 illustrates the MESSAGE RES of Iran. As you can see, large conventional power plants production and DGs are assumed to be at the secondary and final level respectively. The ability of technology substitution is maximized by considering many end-use technologies. A few technologies have not been shown in fig. 1 because lack of space. The balance of primary energy sources is reported in table 1 [1].

\begin{tabular}{|c|c|c|c|c|c|c|c|}
\hline & $\begin{array}{l}\text { Electric } \\
\text { energy } \\
\text { (mboe) }\end{array}$ & $\begin{array}{c}\text { Crude oil } \\
\text { and Oil } \\
\text { products } \\
\text { (mboe) }\end{array}$ & $\begin{array}{l}\text { Natural } \\
\text { Gas } \\
\text { (mboe) }\end{array}$ & $\begin{array}{c}\text { Coal } \\
\text { (mboe) }\end{array}$ & $\begin{array}{l}\text { Biomass } \\
\text { (mboe) }\end{array}$ & $\begin{array}{l}\text { Hydro } \\
\text { (mboe) }\end{array}$ & $\begin{array}{c}\text { Renewables } \\
\text { (mboe) }\end{array}$ \\
\hline Production & - & 1595.4 & 688.7 & 7.5 & 25.4 & 10.7 & 0.07 \\
\hline Imports & 1.5 & 121.9 & 39.5 & 2.3 & - & - & - \\
\hline Exports & -1.6 & -1115.7 & -36.1 & -0.3 & - & - & - \\
\hline $\begin{array}{c}\text { International } \\
\text { Marine Bunkers }\end{array}$ & - & -0.2 & - & - & - & - & - \\
\hline TPES & -0.1 & 619.4 & 692 & 8.5 & 25.4 & 10.7 & 0.07 \\
\hline TFC & 86.4 & 485.1 & 401.9 & 3.2 & 25.4 & - & - \\
\hline $\begin{array}{c}\text { Residential and } \\
\text { commercial }\end{array}$ & 44.5 & 90.5 & 263.6 & 0.07 & 25.4 & - & - \\
\hline Industry & 28.7 & 60.7 & 107.1 & 1 & - & - & - \\
\hline Transport & 0.08 & 267 & 3.3 & - & - & - & - \\
\hline Agriculture & 10.4 & 26.1 & 0.3 & - & - & - & - \\
\hline Non-specified & 2.7 & - & - & - & - & - & - \\
\hline Non-energy use & - & 40.8 & 37.6 & 2.1 & - & - & - \\
\hline
\end{tabular}

Table 1. Primary and End-use consumption energy source balance at the reference year in Iran 


\subsection{General information}

We assumed that base year to be 2006 and time horizon to be 20 years. Model years were assumed to be 2010, 2014, 2018, 2022 and 2026. So, we have 4 periods for optimization. Discount rate is assumed to be $11 \%$ in Iran. The units for energy and power are MWyr and MW. All monetary values are given in dollars of 2006. (1\$=8200 IRR - Iranian Rail -)

\subsection{Load region}

For those energy forms that cannot be stored such as electricity and heat, it is vital to model variation in demand within a year rather than considering only annual demand. The MESSAGE model allows modeling of variations in energy demand within a year with seasons, types of days or time of a day. This requires additional parameters to form the pattern of the energy demand. Parts of a year are referred to as load regions while energy demand pattern as per time-division, is termed as load curve. We assumed 4 seasons in this model, which every season contains 2 types of the day: holiday and workday. Load curves for some demands like space heat or space chill that their values depend on season are considered. For example it is assumed that demand of energy for space heating at winter is $50 \%$ of total annual demand of energy for space heating.

\subsection{Energy forms and levels}

We assumed 6 levels in this model. Each level contains some energy forms which are shown in fig. 1.

Effect of $\mathrm{CO}_{2}, \mathrm{SO}_{2}$ and $\mathrm{NO}_{\mathrm{x}}$ emissions from large conventional power plants has been considered by adding a dummy energy form at the final level which is named environmental impacts. First the monetary damage costs for $\mathrm{SO}_{2}, \mathrm{NO}_{\mathrm{x}}$ and $\mathrm{CO}_{2}$ per $\mathrm{kWh}$ electricity generated are derived. Emissions of $\mathrm{CO}_{2}, \mathrm{SO}_{2}$ and $\mathrm{NO}_{\mathrm{x}}$ due to electricity production and Social costs of $\mathrm{CO}_{2}, \mathrm{SO}_{2}$ and $\mathrm{NO}_{\mathrm{x}}$ emissions to air are reported in tables 2-3 [1]. We have defined some relations for electric output of power plants and emissions to the air according to the values in table 2. Costs of emissions are added to objective function. Therefore, minimization of objective function means to minimize emissions.

We have defined a dummy demand at the useful level to consider the exports in model According to table 1 . We derived share of export of each energy carrier in total primary energy supply. For example, about $60 \%$ of oil production has been exported at the reference year. So we assumed that $60 \%$ of oil production can be exported in model years. The monetary values for export have been entered with negative sign.

\begin{tabular}{ccccccc}
\hline & \multicolumn{2}{c}{$\mathrm{CO}_{2}$} & \multicolumn{2}{c}{$\mathrm{NO}_{x}$} & \multicolumn{2}{c}{$\mathrm{SO}_{2}$} \\
\cline { 2 - 7 } & Ton & $\frac{g r}{k W h}$ & Ton & $\frac{g r}{k W h}$ & Ton & $\frac{g r}{k W h}$ \\
\hline Steam power plant & 58110093 & 628.346 & 90005 & 0.973 & 120211 & 1.300 \\
\hline Gas power plant & 32249656 & 782.089 & 51609 & 1.252 & 52567 & 1.275 \\
\hline $\begin{array}{c}\text { Combined-cycle } \\
\text { power plant }\end{array}$ & 19677900 & 487.766 & 30379 & 0.753 & 18934 & 0.469 \\
\hline Diesel & 172120 & 743.178 & 338 & 1.459 & 1021 & 4.408 \\
\hline Hydro power plant & 120464 & 6.595 & 0 & 0 & 0 & 0 \\
\hline Renewable & 0 & 0 & 0 & 0 & 0 & 0 \\
\hline Total & 110330233 & - & 172332 & - & 192733 & - \\
\hline Average & - & 572.603 & - & 0.894 & - & 1.000 \\
\hline
\end{tabular}

Table 2. Emissions to air at the reference year due to electricity production in Iran 

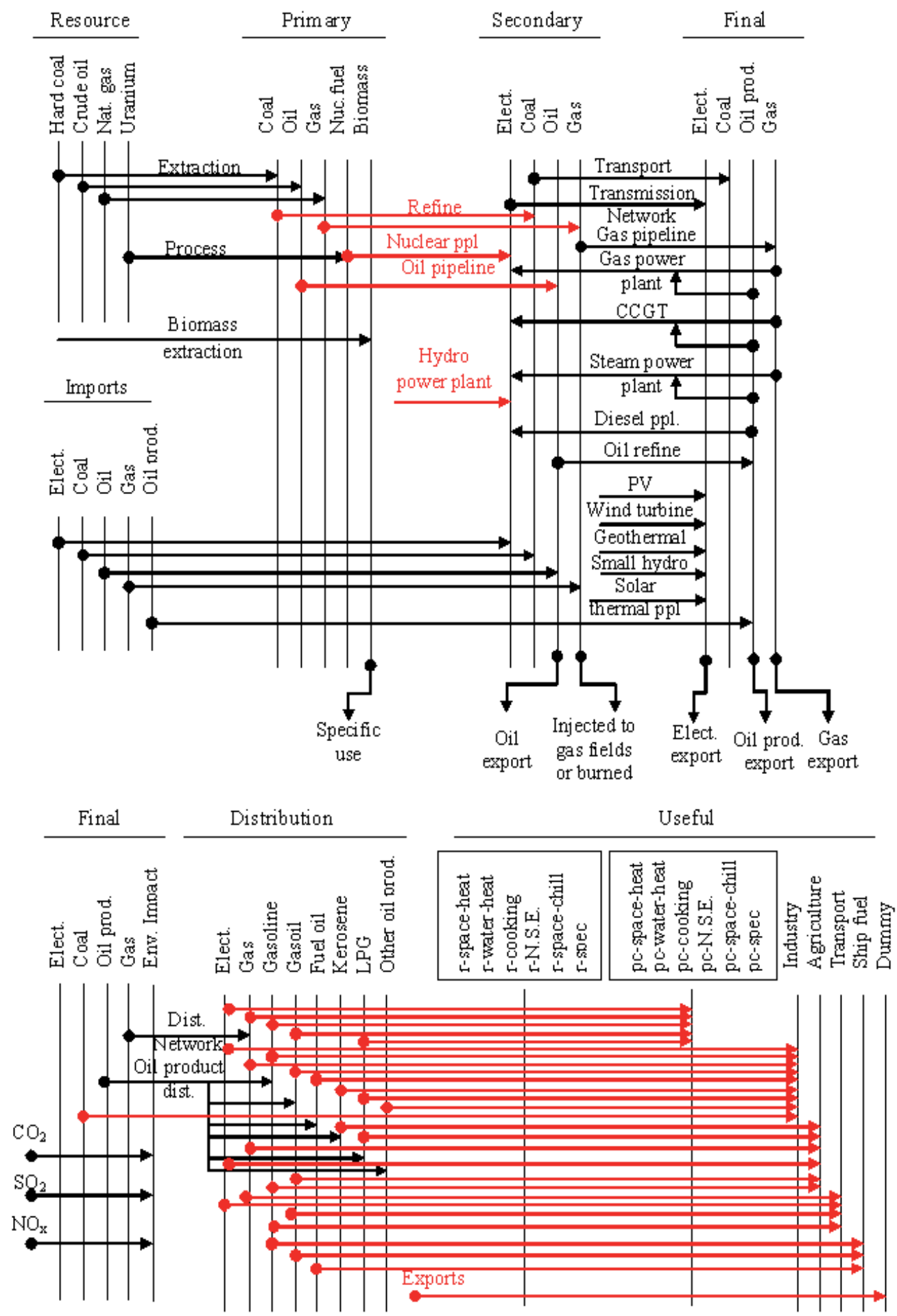

Fig. 1. Reference energy system of Iran 


\begin{tabular}{llll}
\hline & $\mathrm{CO}_{2}$ & $\mathrm{NO}_{\mathrm{x}}$ & $\mathrm{SO}_{2}$ \\
\hline$\frac{\text { Cent }}{k W h}$ & 1.297 & 0.65 & 0.1 \\
\hline
\end{tabular}

Table 3. Social costs of $\mathrm{CO}_{2}, \mathrm{SO}_{2}$ and $\mathrm{NO}_{\mathrm{x}}$ emissions to air at the reference year (Cent per $\mathrm{kWh}$ electricity generated)

\subsection{Demands}

We assumed three types of demand: energy demands, non-energy demands and energy sector demands. Direct energy demands contains residential and commercial, industry, agriculture, transport sectors demands. In each sector share of different oil products is denoted and reported in table 4. End-use consumption at the reference year is reported in table 1. Energy carrier prices for end use technologies are reported in table 5. Annual growth rates of electricity demand and industry sector demand and other sectors demand are set at $8 \%, 10 \%$ and $2.6 \%$ respectively.

\begin{tabular}{cccccc}
\hline & gasoline & kerosene & gasoil & Fuel oil & LPG \\
\hline Residential & 0 & 6705494 & 848894 & 0 & 4456489 \\
\hline $\begin{array}{c}\text { Public and } \\
\text { commercial }\end{array}$ & 107698 & 389908 & 1859630 & 1723850 & 26789 \\
\hline Agriculture & 12572 & 38804 & 4150757 & 0 & 0 \\
\hline Transport & 26669302 & 0 & 16407472 & 0 & 193085 \\
\hline Ship fuel & 39477 & 0 & 475239 & 490687 & 0 \\
\hline Industry & 37922 & 60546 & 2979076 & 5853445 & 0 \\
\hline
\end{tabular}

Table 4.Oil products demand at the reference year in $\operatorname{Iran}\left(\mathrm{m}^{3}\right)$

\begin{tabular}{|c|c|c|c|}
\hline Energy Carrier & Sector & Unit & Price \\
\hline \multirow{6}{*}{ Natural Gas } & residential & \multirow{6}{*}{$\frac{\text { Cent }}{m^{3}}$} & 0.976 \\
\hline & Commercial & & 2.439 \\
\hline & Public & & 2.439 \\
\hline & Industry & & 1.689 \\
\hline & Power plants & & 0.357 \\
\hline & Transport & & 0.732 \\
\hline \multirow{5}{*}{ electricity } & residential & \multirow{5}{*}{$\frac{\text { Cent }}{k W h}$} & 1.255 \\
\hline & Public & & 2.216 \\
\hline & Industry & & 2.444 \\
\hline & Agriculture & & 0.259 \\
\hline & Other sectors & & 6.599 \\
\hline \multirow{5}{*}{ Oil products } & Gasoline & \multirow{5}{*}{$\frac{\text { Cent }}{\text { Lit }}$} & 9.756 \\
\hline & Kerosene & & 2.012 \\
\hline & Fuel oil & & 1.152 \\
\hline & Gasoil & & 2.012 \\
\hline & LPG & & 0.386 \\
\hline Crude oil & - & $\frac{\$}{L i t}$ & 60 \\
\hline
\end{tabular}

Table 5. Energy carrier prices at the reference year in Iran 


\subsection{Resources}

Hard coal, natural gas and crude oil resources as reported in [1] are $1.2 \times 10^{9}$ tons, 28.13 trillions $\mathrm{m}^{3}$ and $138.2 \times 10^{9}$ barrels respectively.

\subsection{Technologies}

We have defined more than 110 technologies in our model. These technologies cover all part of Iran's energy system from extraction to end use. We can divide all technologies into 9 parts: extraction, refinery, transport, distribution, export, import, power grid, power plants and end use technologies. Most important technologies are shown in fig. 1. Most of technical and monetary information for technologies belong to Iran. Most of information in this subsection is extracted from [1]. For those that we don't have enough information, MENA or world data are used. Technical and monetary information about electric energy sector which contains power plants, transmission and distribution network and etc. are reported in tables 6-8. Data are extracted from [1], [2], [3], [8].

\begin{tabular}{ccc}
\hline & Installed capacity (MW) & Activity (GWh) \\
\hline Steam power plant & 15553.4 & 92481 \\
\hline Gas power plant & 14860.9 & 41235.3 \\
\hline $\begin{array}{c}\text { Combined-cycle power } \\
\text { plant }\end{array}$ & 7675.5 & 40342.9 \\
\hline Diesel & 417.9 & 231.6 \\
\hline Hydro power plant & 6572.2 & 18265.6 \\
\hline Renewable ( wind and solar) & 58.9 & 125.4 \\
\hline Total generation capacity & 45138.8 & - \\
\hline
\end{tabular}

Table 6.Installed electric generation capacities and activity at the reference year in Iran

\begin{tabular}{ccc}
\hline & unit & value \\
\hline $\begin{array}{c}\text { Gross production } \\
\begin{array}{c}\text { Transmission and subtransmission } \\
\text { network losses }\end{array}\end{array}$ & GWh & 192681.8 \\
\hline Distribution network losses & $\%$ & 4.9 \\
\hline Own use (power plants) & $\%$ & 17.5 \\
\hline Net electric energy import & GWh & 4.2 \\
\hline Net electric energy export & GWh & 2540 \\
\hline End-use Consumption & GWh & 2775 \\
\hline
\end{tabular}

Table 7. Electric energy grid balance at the reference year in Iran 


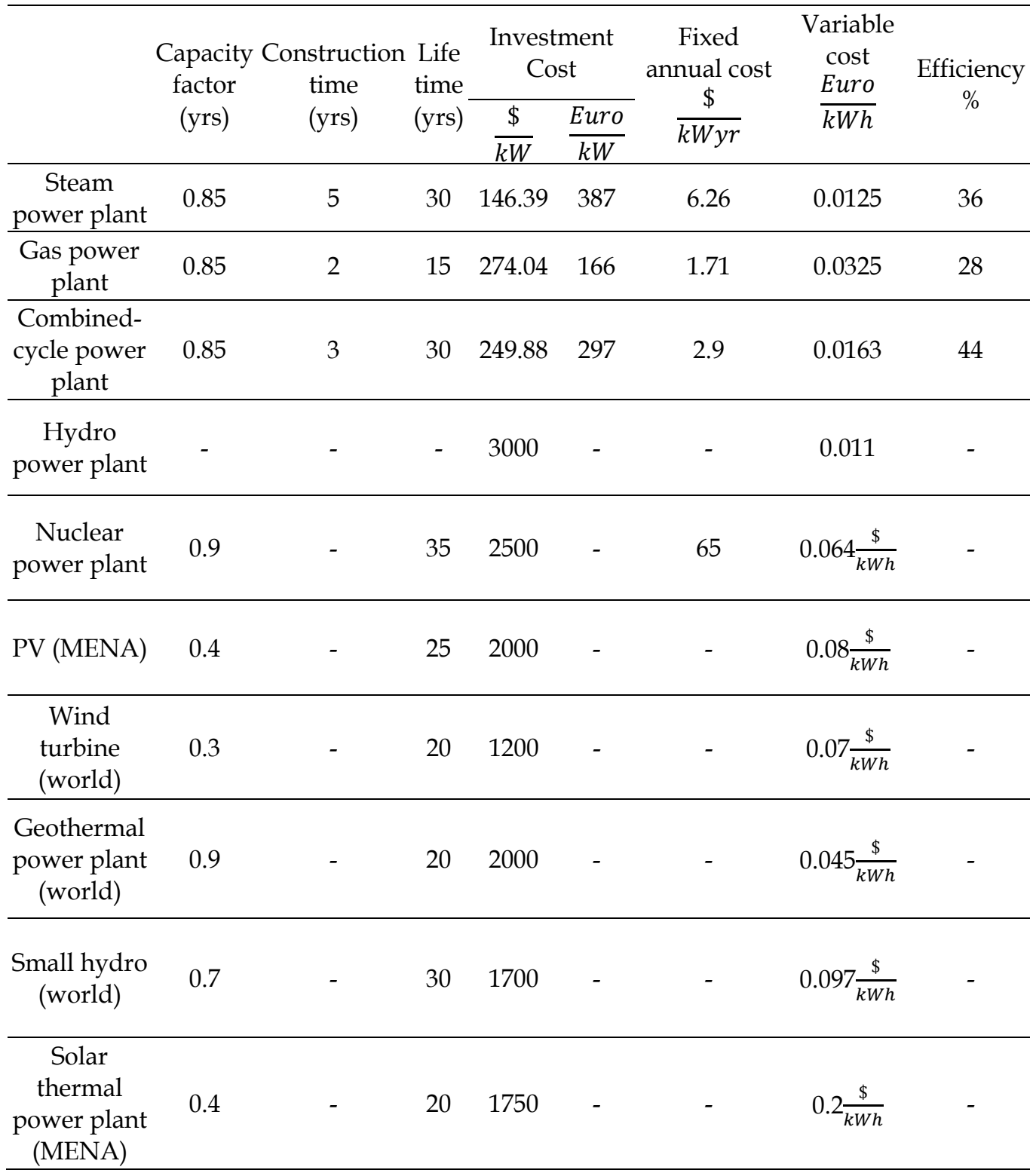

Table 8. Main Cost and technology parameters of power plants in Iran (base year values)

\begin{tabular}{cccc}
\hline & $\mathrm{CO}_{2}$ & $\mathrm{NO}_{x}$ & $\mathrm{SO}_{2}$ \\
\cline { 2 - 4 } & kton & kton & kton \\
\hline Total & 110800 & 170.3 & 187.6 \\
\hline
\end{tabular}

Table 9. Emissions to air due to electricity production (Model Validation case study) 


\section{Model validation}

In order to examine model validation, we assumed that all demands to be constant in all years. We have defined fixed bounds on activities of technologies. Demands and activities at all years are equal to base year. So, no optimization is done. In this case, Results of model should be same as real energy system. Emissions to air, in this case, are reported in table 9. If we compare results in table 9 (Model results) and data in table 2 (real data), we will see that they are very close together and it's what we expected. Maximum relative error is less than $3 \%$.

In other case we have eliminated all constraints. It's obvious that in this case cost function should be decreased. The results show that cost function reduces about $67 \%$. When no constraint is considered, with the aim of minimizing the cost function, model uses specific technologies and many technologies remain unused.

\section{Results and discussion}

In order to show the effectiveness of proposed reference energy system and procedure several scenarios have been analyzed for a time horizon of 20 years. Electric energy is estimated at $2427.1 \frac{k c a l}{k W h}$ for primary uses [1].

In DG-low scenario, DG technologies are not taken into account. No minimum level of expansion is imposed on DG technologies and share of DGs in total electricity production is assumed to be $0.5 \%$ and constant.

In DG-med scenario, the percentage of electricity production relating to DG technologies must reach $10 \%$ of total production by end of planning horizon.

In DG-max scenario, the percentage of electricity production relating to DG technologies must reach $20 \%$ of total production by end of planning horizon.

In all scenarios we assumed that DG technologies market penetrations on activities to be $100 \%$ which mean a growth rate of 2 .

Results for each scenario are reported in tables 10-16. We see that in DG-max scenario transmission losses decrease 15\% in comparison with DG-min scenario (from $4641 \mathrm{MWyr}$ to 3930 MWyr). Also emissions to air decrease about 19.7\% (from 305900 kton to 245600 kton). Emissions to air and transmission network losses are shown in fig. 2 and fig. 3 for different scenarios. In fig. 4 total installed capacity of DG technologies in different scenarios is reported. In DG-min scenario total installed capacity of DG technologies with a growth equal to $164 \%$ reaches $500 \mathrm{MW}$ at the end of time horizon. In DG-max scenario total installed capacity of DG technologies reaches $27.1 \mathrm{GW}$ at the end of time horizon. In DGmed scenario we see a constant growth rate in capacities in opposition to DG-max scenario. In fig. 5 total installed capacity of conventional power plants in different scenarios is reported. We can see that total installed capacity of conventional power plants growth equally in all scenarios until 2018. It means that in current situation which less than $0.5 \%$ of total electricity production belong to DG facilities, it lasts 8 years to DG technologies affect growth rate of conventional power plants and coordinate with consumption growth. In DGmin scenario total installed capacity of conventional power plant reaches $97.7 \mathrm{GW}$ at the end of time horizon. In DG-min and DG-med scenarios total installed capacity of conventional power plant increase in all year, but in DG-max scenario a reduction in capacities occur from 2024 to 2026 which means that we don't need new capacities to be installed and we can discard old power plants which their life is finished. 


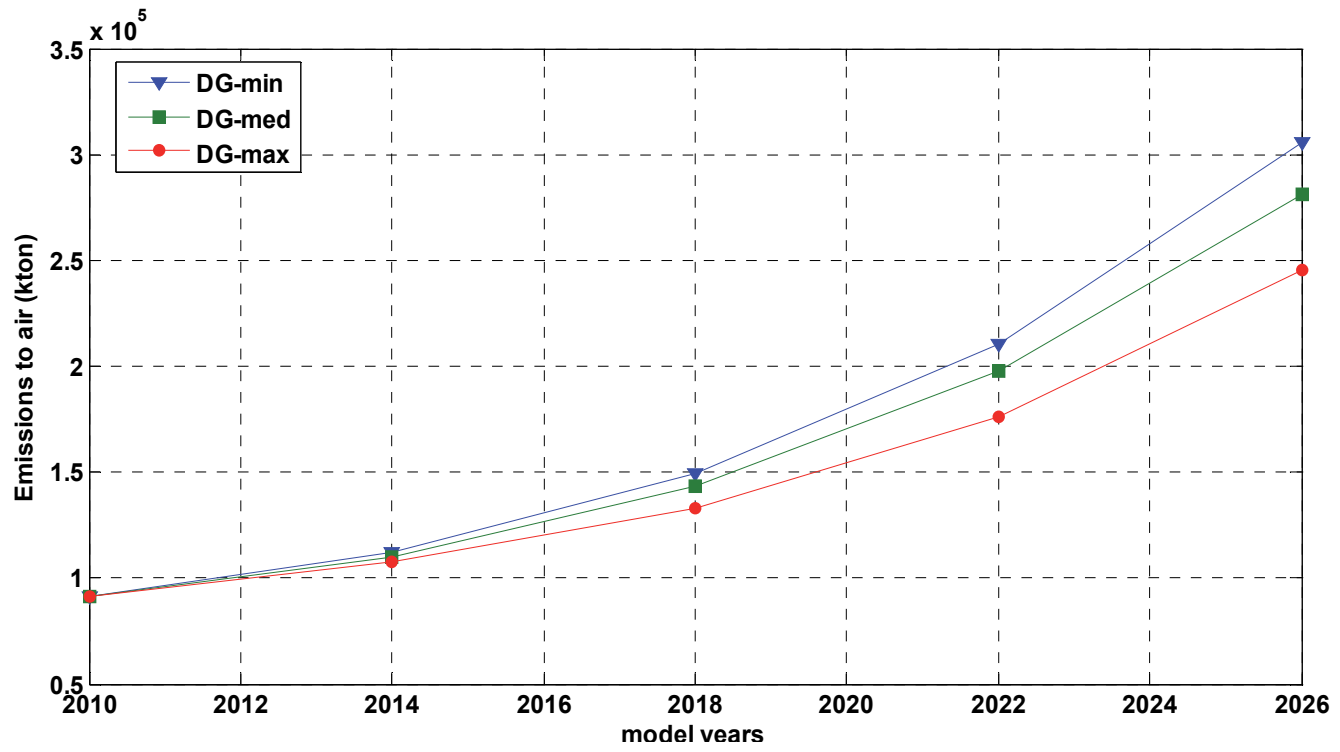

Fig. 2. Greenhouse gas Emissions

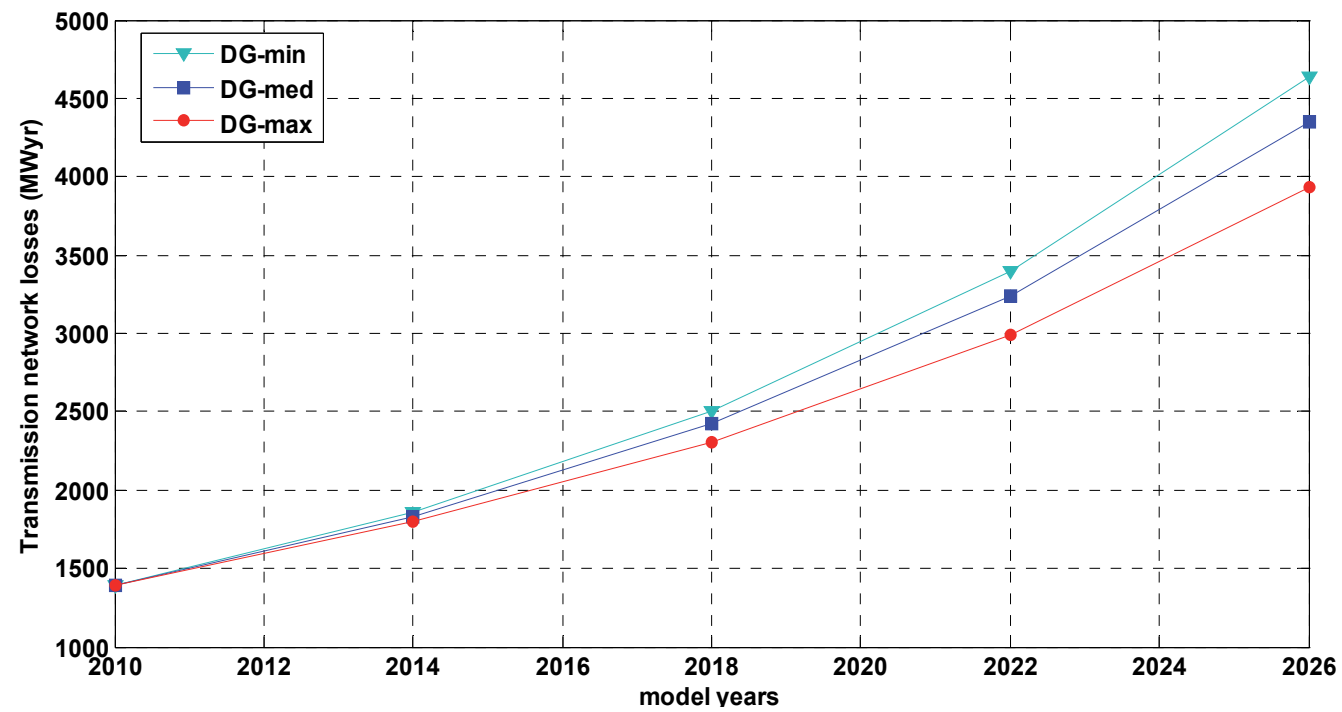

Fig. 3. Transmission network losses 


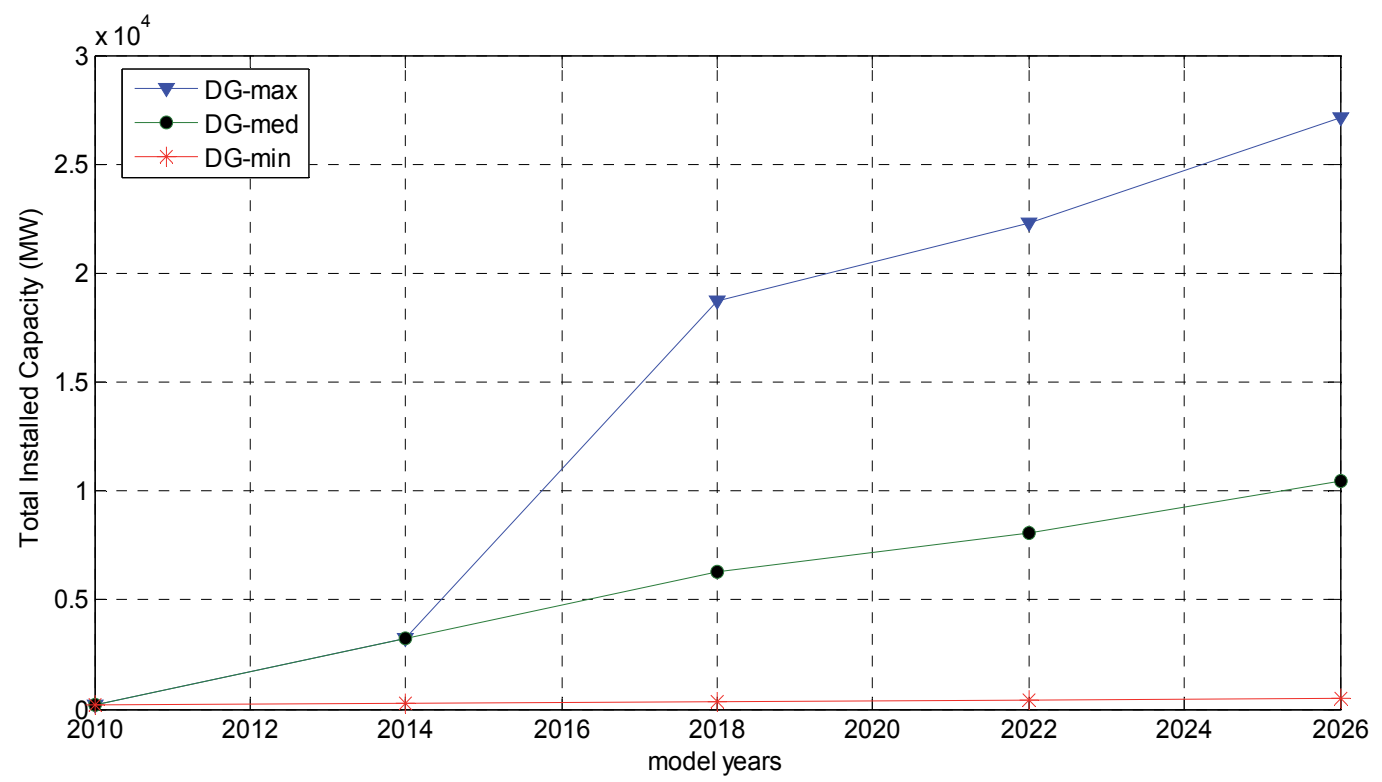

Fig. 4. Total installed capacity of DGs

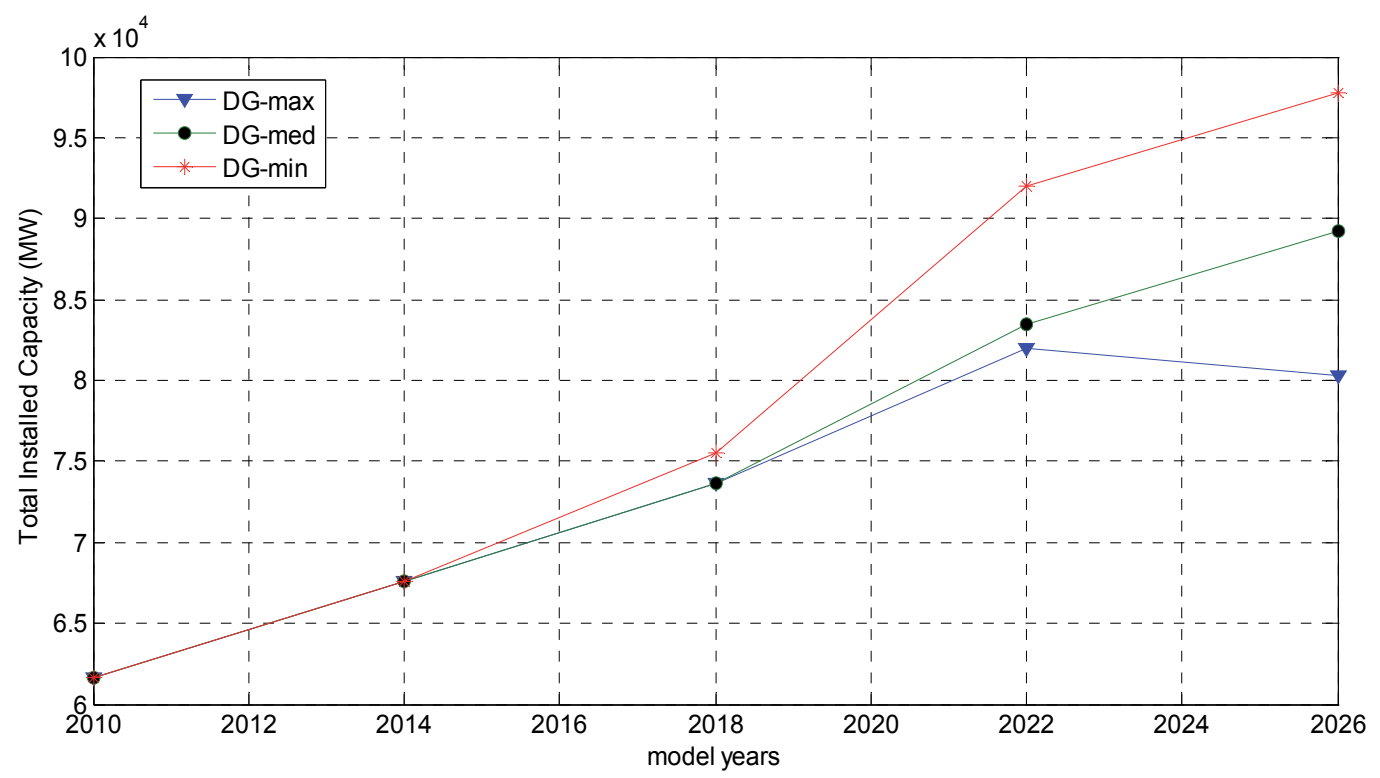

Fig. 5. Total installed capacity of conventional power plants 


\begin{tabular}{cccc}
\hline & DG-min & DG-med & DG-max \\
\hline 2010 & 91303.1 & 91303.1 & 91303.1 \\
\hline 2014 & 112248 & 110294.8 & 107644.6 \\
\hline 2018 & 149450.8 & 143303.4 & 132765.8 \\
\hline 2022 & 210770.8 & 197652.1 & 176185 \\
\hline 2026 & 305892.6 & 281445.5 & 245590.7 \\
\hline
\end{tabular}

Table 10. Emissions to air (kton)

\begin{tabular}{ccccccccc}
\hline & $\begin{array}{c}\text { Gas } \\
\text { power } \\
\text { plant }\end{array}$ & $\begin{array}{c}\text { Nuclear } \\
\text { power } \\
\text { plant }\end{array}$ & $\begin{array}{c}\text { electricity } \\
\text { imports }\end{array}$ & $\begin{array}{c}\text { Combined } \\
\text {-cycle } \\
\text { power } \\
\text { plant }\end{array}$ & $\begin{array}{c}\text { Steam } \\
\text { power } \\
\text { plant }\end{array}$ & $\begin{array}{c}\text { Hydro } \\
\text { power } \\
\text { plant }\end{array}$ & Diesel & Total \\
\hline 2010 & 3274.8 & 250 & 263.9 & 7455.6 & 6549.5 & 9925.2 & 26.4 & 27745.4 \\
\hline 2014 & 4382.5 & 0 & 352.7 & 7632.2 & 8765 & 15925.2 & 27.5 & 37085.1 \\
\hline 2018 & 5909.9 & 0 & 475.6 & 9850.9 & 11819.7 & 21925.2 & 28.6 & 50009.9 \\
\hline 2022 & 8026.3 & 0 & 645.9 & 15239.6 & 16052.6 & 27925.2 & 29.8 & 67919.4 \\
\hline 2026 & 10968.2 & 0 & 882.7 & 25070.9 & 21936.5 & 33925.2 & 31 & 92814.5 \\
\hline
\end{tabular}

Table 11. Activity of large conventional power plants and electricity imports (MWyr) - DG$\min$

\begin{tabular}{ccccccc}
\hline & PV & $\begin{array}{c}\text { Wind } \\
\text { turbine }\end{array}$ & $\begin{array}{c}\text { Geotherma } \\
1\end{array}$ & $\begin{array}{c}\text { Small } \\
\text { hydro }\end{array}$ & $\begin{array}{c}\text { Solar thermal } \\
\text { power plant }\end{array}$ & Total \\
\hline 2010 & 0.2 & 44.6 & 0 & 32 & 0 & 76 \\
\hline 2014 & 0 & 44.6 & 0 & 101.4 & 0 & 146.08 \\
\hline 2018 & 0 & 44.6 & 0 & 152.4 & 0 & 197 \\
\hline 2022 & 0 & 44.6 & 0 & 222.9 & 0 & 267.54 \\
\hline 2026 & 0 & 20.3 & 0 & 345.4 & 0 & 365.61 \\
\hline
\end{tabular}

Table 12. Activity of DG technologies (MWyr) - DG-min

\begin{tabular}{ccccccccc}
\hline & $\begin{array}{c}\text { Gas } \\
\text { power } \\
\text { plant }\end{array}$ & $\begin{array}{c}\text { Nuclear } \\
\text { power } \\
\text { plant }\end{array}$ & $\begin{array}{c}\text { electricity } \\
\text { imports }\end{array}$ & $\begin{array}{c}\text { Combined- } \\
\text { cycle power } \\
\text { plant }\end{array}$ & $\begin{array}{c}\text { Steam } \\
\text { power } \\
\text { plant }\end{array}$ & $\begin{array}{c}\text { Hydro } \\
\text { power } \\
\text { plant }\end{array}$ & Diesel & Total \\
\hline 2010 & 3274.8 & 250.00 & 263.9 & 7455.6 & 6549.5 & 9925.2 & 26.44 & 27745.4 \\
\hline 2014 & 4382.5 & 0.00 & 348.3 & 7175.8 & 8765 & 15925.2 & 27.51 & 36624.2 \\
\hline 2018 & 5905.9 & 0.00 & 461.8 & 8414.7 & 11819.7 & 21925.2 & 28.63 & 48559.9 \\
\hline 2022 & 8026.3 & 0.00 & 616.5 & 12174.4 & 16052.6 & 27925.2 & 29.79 & 64824.8 \\
\hline 2026 & 10968.2 & 0.00 & 827.8 & 19359.2 & 21936.5 & 33925.2 & 31.00 & 87047.9 \\
\hline
\end{tabular}

Table 13. Activity of large conventional power plants and electricity imports (MWyr) - DGmed 


\begin{tabular}{ccccccc}
\hline & PV & $\begin{array}{c}\text { Wind } \\
\text { turbine }\end{array}$ & Geothermal & $\begin{array}{c}\text { Small } \\
\text { hydro }\end{array}$ & $\begin{array}{c}\text { Solar thermal } \\
\text { power plant }\end{array}$ & Total \\
\hline 2010 & 0.2 & 44.64 & 0 & 32 & 0 & 76.8 \\
\hline 2014 & 0.4 & 271.9 & 150 & 162 & 0 & 584.33 \\
\hline 2018 & 0 & 271.9 & 483.9 & 820 & 0 & 1575.96 \\
\hline 2022 & 0 & 271.9 & 483.9 & 2454.7 & 0 & 3210.5 \\
\hline 2026 & 0 & 247.6 & 483.9 & 5118.3 & 0 & 5849.7 \\
\hline
\end{tabular}

Table 14. Activity of DG technologies (MWyr) - DG-med

\begin{tabular}{ccccccccc}
\hline & $\begin{array}{c}\text { Gas } \\
\text { power } \\
\text { plant }\end{array}$ & $\begin{array}{c}\text { Nuclear } \\
\text { power } \\
\text { plant }\end{array}$ & $\begin{array}{c}\text { electricity } \\
\text { imports }\end{array}$ & $\begin{array}{c}\text { Combined- } \\
\text { cycle power } \\
\text { plant }\end{array}$ & $\begin{array}{c}\text { Steam } \\
\text { power } \\
\text { plant }\end{array}$ & $\begin{array}{c}\text { Hydro } \\
\text { power } \\
\text { plant }\end{array}$ & Diesel & Total \\
\hline 2010 & 3274.8 & 250.00 & 263.9 & 7455.6 & 6549.5 & 9925.2 & 26.44 & 27745.4 \\
\hline 2014 & 4382.5 & 0.00 & 342.4 & 6556.6 & 8765 & 15925.2 & 27.51 & 35999.1 \\
\hline 2018 & 5909.9 & 0.00 & 438.2 & 5952.6 & 11819.7 & 21925.2 & 28.63 & 46074.2 \\
\hline 2022 & 8026.3 & 0.00 & 568.3 & 7158.7 & 16052.6 & 27925.2 & 29.79 & 59761 \\
\hline 2026 & 10968.2 & 0.00 & 747.4 & 10981.8 & 21936.5 & 33925.2 & 31.00 & 78590.1 \\
\hline
\end{tabular}

Table 15. Activity of large conventional power plants and electricity imports (MWyr) - DG$\max$

\begin{tabular}{ccccccc}
\hline & PV & $\begin{array}{c}\text { Wind } \\
\text { turbine }\end{array}$ & $\begin{array}{c}\text { Geotherma } \\
1\end{array}$ & $\begin{array}{c}\text { Small } \\
\text { hydro }\end{array}$ & $\begin{array}{c}\text { Solar thermal } \\
\text { power plant }\end{array}$ & Total \\
\hline 2010 & 0.2 & 44.64 & 0 & 32 & 0 & 76.8 \\
\hline 2014 & 2.6 & 714.2 & 150 & 162 & 150 & 1178.8 \\
\hline 2018 & 0 & 714.2 & 2255.5 & 820.1 & 150 & 3939.9 \\
\hline 2022 & 0 & 714.2 & 3010.1 & 4151.9 & 150 & 8026.3 \\
\hline 2026 & 0 & 689.9 & 3010.1 & 10043.1 & 150 & 13893.1 \\
\hline
\end{tabular}

Table 16. Activity of DG technologies (MWyr) - DG-max

\section{Conclusion}

A reference energy system for Iran has been adopted to investigate DG diffusion in energy planning studies. The proposed approach is based on model MESSAGE that details the exploitation of primary energy sources, defined technologies, end-use sectors and emissions. Particular care has been given to the description of DG technologies and their energy injections in the electric grid. To this purpose, a representation of the electric grid with transmission and distribution network has been considered. The contribution of DG facilities in electricity generation under different policies has been shown by carrying out simulations on a realistic energy system of Iran. Test results have proved that energy policies aimed at reducing environmental impact of electricity production can be supported 
by DG technologies (mainly small-hydro and wind turbine). By promoting exploitation of DG technologies, reduction in conventional power plants production has occurred with a decrease in transmission losses and emissions.

\section{References}

[1] Iran Ministry of Energy, Deputy of Electricity and Energy Affairs. Energy Balance at 2006 (ترازنامه انرزي سال 1385), ISBN 978-964-91272-4-8 (http://pep.moe.org.ir/), 2008 (in Persian)

[2] World Energy Outlook 2005, Middle East and North Africa in sight. International Energy Agency. (www.iea.org)

[3] World Energy Outlook 2006. International Energy Agency. (www.iea.org)

[4] Messner S, Strubegger M. User's Guide for MESSAGE III. WP-95-69, International Institute for Applied Systems Analysis (IIASA), Laxenburg, 1995.

[5] MESSAGE V User manual. International Atomic Energy Agency. October 2003.

[6] El-Khattam W, Salama M. M. A. Distributed generation technologies, definitions and benefits. Electric Power Systems Research 71 (2004) 119-128

[7] Pepermans G, Driesen J, Haeseldonckx D, Belmans R, D'haeseleer W. Distributed generation : definitions, benefits and issues. Energy Policy 33 (2005) 787-798

[8] 2007 Survey of Energy Resources. World Energy Council 2007. ISBN: 0946121265. (www.worldenergy.org) 


\title{
Network Reconfiguration for Distribution System with Micro-Grid
}

\author{
Yu Xiaodan, Chen Huanfei, Liu Zhao and Jia Hongjie \\ School of Electrical Engineering and Automation, Tianjin University
}

China

\section{Introduction}

Nowadays, technologies of distributed generation (DG) and distributed energy resource (DER) are developing rapidly. More and more DG devices, such as photovoltaic(PV), micro-turbine, wind generator, $\mathrm{CCHP}$, energy storage, have been installed to the traditional power system (especially to the distribution system). How to draw more benefits from such DG devices has been paid even more attention than before (EPRI, 2007; IEEE, 2003; EPRI, 2001). A possible solution vision is micro-grid (Barnes et al, 2007; Khan \& Iravani, 2007; Dimeas \& Nikos, 2005). A micro-grid is a portion of power system that includes one or more DG units capable of operating either parallel with or independent from a distribution system. It is demonstrated to be more reliable and economical that DGs are integrated into a distribution system through micro-grid. So, more and more micro-grids will occur in the distribution system in the future.

Targets of the network reconfiguration in traditional distribution system are to reduce power loss (Civanlar et al, 1988; Baran \& Wu, 1989; Song et al, 1997; Kashem et al, 2001; Carpaneto \& Chicco, 2004; Sua et al, 2005), balance power supplying and consuming, improve power quality, isolate fault components and restore system quickly under some emergencies (Tu \& Guo, 2006; Bhattacharya \& Goswami, 2008; Carreno et al, 2008), et al through optimizing the sectionalizing and tie switchers on the feeders. Just as we know, traditional distribution system was constructed and operated radially. In such network, any load only had a single supplying source and power flow on any feeder was in one-way. However, things will be changed once some micro-grids exist in the distribution system. Since a micro-grid may contain various DGs, such as PV, CCHP, wind generator, it can be considered as a power source or a consuming load at different time so that power flow on some feeders will be bidirectional under some conditions (Chen et al, 2008; Yu et al, 2009). It is obvious that reconfiguration for the traditional distribution system and reconfiguration for the distribution system with micro-grids are very different.

In this chapter, we mainly concern the impact of micro-grids on the distribution system reconfiguration. A reconfiguration model suitable for the distribution system with microgrids is presented. Once a fault occurs, it can be applied to construct some islands. Any island contains one or more micro-grids so as to guarantee power supplying for some important customers and to reduce the power loss at the same time. The problem is then decomposed into a capacity sub-problem and a reconfiguration sub-problem. The former is used to determine the optimal capacity of each island, while the latter is used to find the optimal reconfiguration with less power loss. Finally, some typical distribution systems are employed to validate the effectiveness of the presented method. 
Rest of this chapter is organized as following: Section 2 gives the model of the distribution system with micro-grids used in this chapter. Section 3 provides a suitable reconfiguration model and discusses its solving method. Numerical studies and conclusions are given by Section 4 and Section 5.

\section{Distribution system model}

In this chapter, we will consider the distribution system with parallel operating micro-grids as shown in Fig.1. In the figure, two micro-grids are connected to system at node $N_{i}$ and $N_{j}$. Just as we know, if DG devices are directly installed into the distribution system, they will be tripped quickly once a fault occurs in the system according to the standard of IEEE-1547 (IEEE, 2003) in order to keep the equipments and persons safe. However, if various DGs are first integrated into a micro-grid, and then the micro-grid is connected to the distribution system as a whole, more benefits will be drawn. e.g. if a fault causes some feeder outage, a micro-grid can operate as an isolated island so that it can supply power to some important customers nearby (Barnes et al, 2007; Khan \& Iravani, 2007; Dimeas \& Nikos, 2005). In this chapter, our aim is to find the optimal islanding scheme so as to guarantee power supplying for more customers with less power loss at the same time.

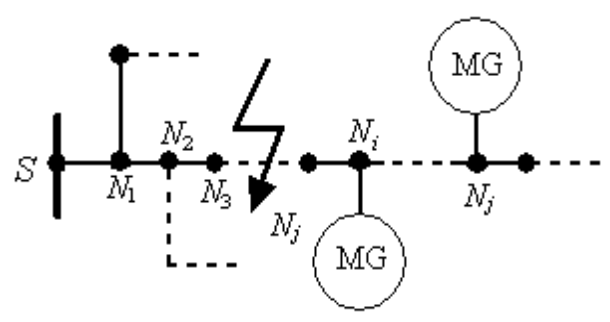

Fig. 1. Distribution system with micro-grids

For the system as shown in Fig.1, we use $S$ to denote the source node and use $\boldsymbol{N}, \boldsymbol{B R}, \boldsymbol{M G}$ for the set of nodes, branches and micro-grids in the system.

$$
\begin{gathered}
\boldsymbol{N}=\left\{N_{1}, N_{2}, N_{3}, \cdots, N_{n}\right\} \\
\boldsymbol{B} \boldsymbol{R}=\left\{B R_{1}, B R_{2}, B R_{3}, \cdots, B R_{m}\right\} \\
\boldsymbol{M G}=\left\{M G_{i}\left(N_{j}\right)\right\}, i=1,2, \ldots, k ; N_{j} \in \boldsymbol{N}
\end{gathered}
$$

Where, $n, m, k$ are numbers of the system nodes, branches and micro-grids. In Eq.(3) $M G_{i}\left(N_{j}\right)$ means that the $i$-th micro-grid is connected to node $N_{j}$. Normally, distribution system is operated radially, so the following equation holds $n=m+1$. Further $\overline{\boldsymbol{U}}, \underline{\boldsymbol{U}}$ are used for the upper and lower voltage limits of $N$, and $\overline{\boldsymbol{S B}}$ for the upper power limit of $\boldsymbol{B R}$.

$$
\begin{aligned}
& \overline{\boldsymbol{U}}=\left\{\bar{U}_{1}, \bar{U}_{2}, \bar{U}_{3}, \cdots, \bar{U}_{n}\right\} \\
& \underline{\boldsymbol{U}}=\left\{\underline{U}_{1}, \underline{U}_{2}, \underline{U}_{3}, \cdots, \underline{U}_{n}\right\} \\
& \overline{\boldsymbol{S B}}=\left\{\bar{S}_{1}, \bar{S}_{2}, \bar{S}_{3}, \cdots, \bar{S}_{m}\right\}
\end{aligned}
$$


A micro-grid can be treated as a load or a generator under different operating conditions. When it is operated as a load, it only draws power from distribution system just like a normal load. While, if it is operated as a generator, it can send power into the distribution system. Once a fault occurs in the distribution system, some loads may be interrupted without micro-grid. However, if there are some micro-grids connecting to the system, things may be changed. A micro-grid with "extra power" can form an island and send its extra power to some nearby loads temporarily just like a local generator. And, loads interruption may be avoided. In this chapter, we use $\boldsymbol{S M G}$ to denote the maximum extra power (maximum capacity) of the micro-grids that can be used under a fault condition.

$$
\boldsymbol{S M G}=\left\{S M G_{1}, S M G_{2}, S M G_{3}, \cdots, S M G_{k}\right\}
$$

Further, $\boldsymbol{S S}, \boldsymbol{T S}$ is used to denote sets of the sectionalizing switchers and tie switchers as following:

$$
\begin{gathered}
\boldsymbol{S S}=\left\{S S_{i}\left(B R_{j}\right)\right\}, i=1,2, \ldots, K_{s}, B R_{j} \in \boldsymbol{B} \boldsymbol{R} \\
\boldsymbol{T S}=\left\{T S_{i}\left(N_{j}, N_{k}\right)\right\}, i=1,2, \ldots, K_{t}, N_{j}, N_{k} \in \boldsymbol{N}
\end{gathered}
$$

where $K_{s}, K_{t}$ are numbers of the sectionalizing switchers and tie switchers. $S S_{i}\left(B R_{j}\right)$ means the $i$-th sectionalizing switcher is located on branch $B R_{j}$, and $T S_{i}\left(N_{j}, N_{k}\right)$ means the $i$-th tie switcher is located between node $N_{j}$ and $N_{k}$.

\section{Network reconfiguration}

\subsection{Reconfiguration model}

Switchers of $\boldsymbol{S S}, \boldsymbol{T S}$ can be optimized so as to reduce the power loss and the customer interruption at the same time in an emergency condition. The reconfiguration model used in this chapter is given as following:

$$
\begin{gathered}
\min \left\{W_{1}\left[\sum_{i=1}^{I S}\left(S I S_{i}-L D I S_{i}\right)\right]+W_{2}\left(P_{\text {loss }}^{s y s}+\sum_{i=1}^{I S} P_{\text {loss }, i}^{I S \operatorname{land}}\right)\right\} \\
\text { s.t. } n_{0}=m_{0}+1 \\
n_{i}=m_{i}+1, \quad i=1,2,3, \ldots, I S \\
I S \leq k \\
S_{i} \leq \bar{S}_{i}, B R_{i} \in \boldsymbol{B R} \\
\underline{V}_{i} \leq V_{i} \leq \bar{V}_{i}, N_{i} \in \boldsymbol{N} \\
\operatorname{SIS}_{i}-\mathrm{LDIS}_{i} \geq 0, \quad i=1,2,3 \ldots, I S
\end{gathered}
$$

where, IS is number of the islands formed by the micro-grids. An island can consist of more than one micro-grid, so $I S \leq k, k$ is number of the micro-grids. $S I S_{i}$ is the total extra power of 
the $i$-th island. When there is a single micro-grid in the island, $S I S_{i}$ equals to its $S M G$. While, if there are more than one micro-grid, $S I S_{i}$ equals to the $S M G$ sum of all micro-grids in the island. ${ }^{2} D I S_{i}$ is the total loads in the $i$-th island. $P_{\text {loss }}^{s y s}$ is the power loss of the distribution system exclusive of all islands, and $P_{\text {loss }, i}^{\text {Isand }}$ is the power loss of the $i$-th island.

It can be found that, in the above model, there are two optimal objects: one is to maximize the uninterrupted loads and the other is to minimize the power loss of the whole system, including distribution system exclusive of micro-grids and all islands. In the model, Eq.(11) and Eq.(12) guarantee that the distribution system exclusive of micro-grids and all islands are operated radially. Eq.(14) and Eq.(15) guarantee all system limits not to be violated. Eq. (16) guarantees that there is no load interrupted in any island, i.e. power supply is larger than the power demand in any island.

\subsection{Solving of the reconfiguration model}

Since the reconfiguration model used in this chapter is a multi-objective optimization model, it can be decomposed into two sub-problems: capacity sub-problem and reconfiguration sub-problem.

Capacity sub-problem is a typical combinatorial optimization model. It is used to determine the optimal capacity of each island, i.e. optimal values of $\operatorname{LDIS}_{i}$ and SIS $_{i}$ for each island. The model is given as below:

$$
\begin{gathered}
\min \sum_{i=1}^{I S}\left(S I S_{i}-L D I S_{i}\right) \\
\text { s.t. } n_{0}=m_{0}+1 \\
n_{i}=m_{i}+1, \quad i=1,2,3, \ldots, I S \\
S I S_{i}-\text { LDIS }_{i} \geq 0, \quad i=1,2,3 \ldots, I S
\end{gathered}
$$

After optimization, the capacity sub-problem will yield the islanding scheme $I S L D_{i}^{o}, i=1,2,3, \ldots, I S$. It tells us which micro-grid and which node are included in an island. Reconfiguration sub-problem is used to minimize the power loss of whole system including the rest distribution system exclusive of micro-grids and all islands. The model is given as following:

$$
\begin{gathered}
\min \left(P_{\text {loss }}^{s y s}+\sum_{i=1}^{I S} P_{\text {loss }, i}^{\text {Illand }}\right) \\
\text { s.t. } I S L D_{i}=I S L D_{i}^{o}, i=1,2,3, \ldots, I S \\
n_{0}=m_{0}+1 \\
n_{i}=m_{i}+1, \quad i=1,2,3, \ldots, I S \\
S_{i} \leq \bar{S}_{i}, B R_{i} \in \boldsymbol{B R}
\end{gathered}
$$




$$
\underline{V}_{i} \leq V_{i} \leq \bar{V}_{i}, N_{i} \in N
$$

Since the rest distribution system exclusive of all micro-grids and all islands in the above model are all operated radially, Eq.(21)-Eq.(26) just form a typical distribution network reconfiguration model. Its objective is to minimize the power loss of the whole system. It can be solved effectively by some existed methods (Civanlar et al, 1988; Baran \& Wu, 1989; Song et al, 1997; Kashem et al, 2001; Carpaneto \& Chicco, 2004; Sua et al, 2005; Tu \& Guo, 2006; Bhattacharya \& Goswami, 2008; Carreno et al, 2008). In this chapter, we just use an improved branch exchange method given by (Kashem et al, 2001) to solve this problem. Details of the method can be referred to (Kashem et al, 2001; Baran \& Wu, 1989).

The above two sub-problems are called iteratively, the whole reconfiguration problem given by Eq.(10)-Eq.(16) can be solved finally (Chen et al, 2008; Yu et al, 2009).

\section{Case studies}

In this chapter, IEEE 33-node system and PG\&E 69-node system(Baran \& Wu, 1989, Chen et al, 2008; Yu et al, 2009) are employed to validate the presented method.

\subsection{IEEE 33-node system}

IEEE 33-node system is shown in Fig.2. It consists of 33 nodes and 5 tie lines all with switchers. The first node is treated as the source node. And, it is assumed that all branches have sectionalizing switchers. In this chapter, a fault occurring on branch 11-12 is considered. It will cause this branch out of service after fault.

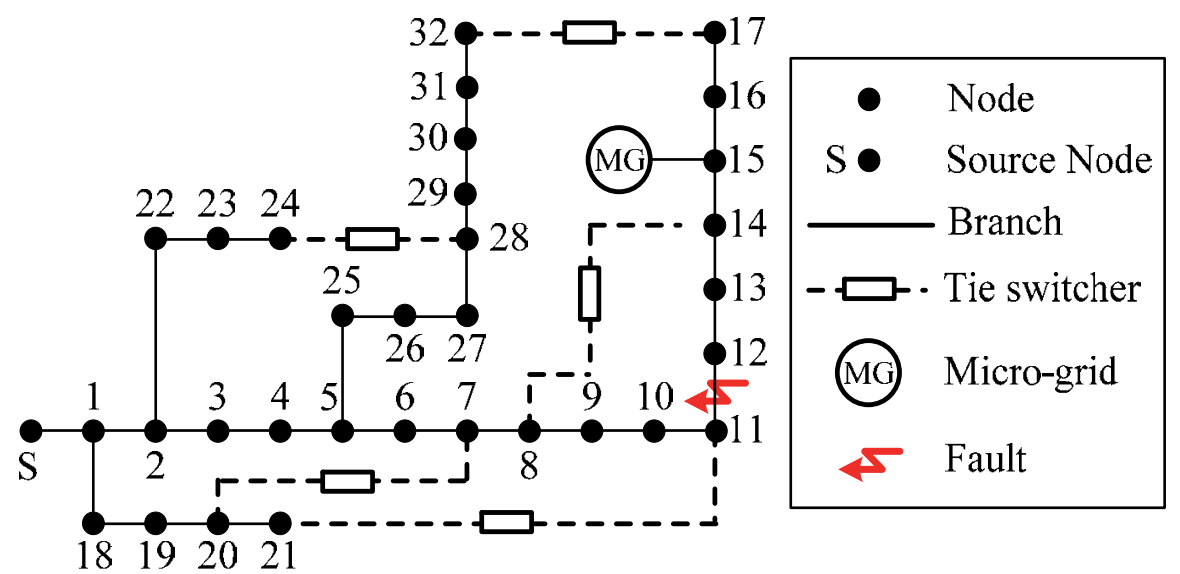

Fig. 2. IEEE 33-node system

\section{Reconfiguration without micro-grid}

When there is no micro-grid in the system, we can get the reconfiguration result as shown in Fig.3. Five sectionalizing switchers are opened after optimization. They are switchers of 6-7, 8-9, 11-12, 14-15, 27-28, and all tie switchers are closed at the same time. Power loss changes from $134.98 \mathrm{~kW}$ to $153.14 \mathrm{~kW}$ after reconfiguration. The power loss increasing is caused by the fault. 


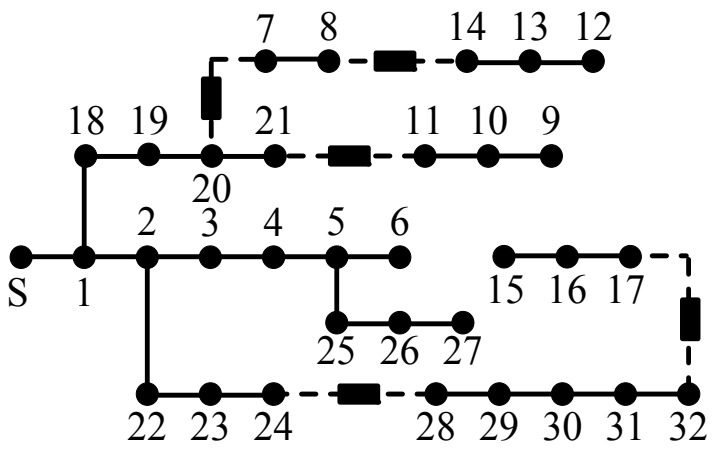

Fig. 3. Reconfiguration result of IEEE 33-node system without micro-grid

\section{Reconfiguration with a micro-grid and $S M G=900 \mathrm{~kW}$}

When a micro-grid with $S M G=900 \mathrm{~kW}$ is installed to node 15 just as shown in Fig.2. After reconfiguration, we can get the optimization result shown in Fig.4. It can be found that an island is formed. It consists of the micro-grid and 9 nodes: 8, 12, 13, 14, 15, 16, 17, 31 and 32 . The rest part consists of all the other nodes and is supplied by the original source. Power loss after reconfiguration turns to $80.03 \mathrm{~kW}$, which is less than the one without micro-grid. And, the lowest voltage is also changed from 0.9143 p.u.(without micro-grid) to $0.9545 \mathrm{p} . \mathrm{u}$ (with a micro-grid).

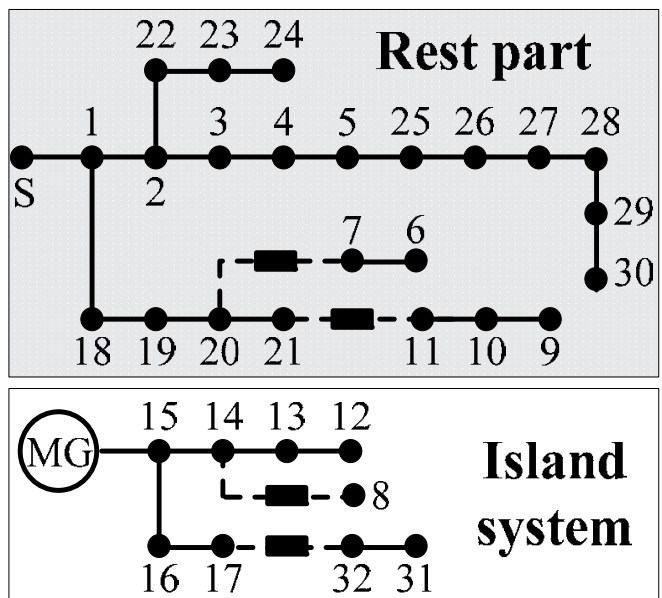

Fig. 4. Reconfiguration result of IEEE 33-node system with a micro-grid and $S M G=900 \mathrm{~kW}$

\section{Reconfiguration results with a micro-grid and various $S M G$ values}

When there is a single micro-grid in the system and its SMG changes in the range $0 \sim 1700 \mathrm{~kW}$, reconfiguration results are shown in Tab.1, Fig.5 and Fig.6. Following conclusions can be drawn from the calculation results:

1. When there is a micro-grid in the distribution system, it can form an island so as to supply power to the nearby loads under the emergency condition. Comparing with the result without micro-grid, we can find that the power loss is reduced and lowest voltage is improved at the same time. 


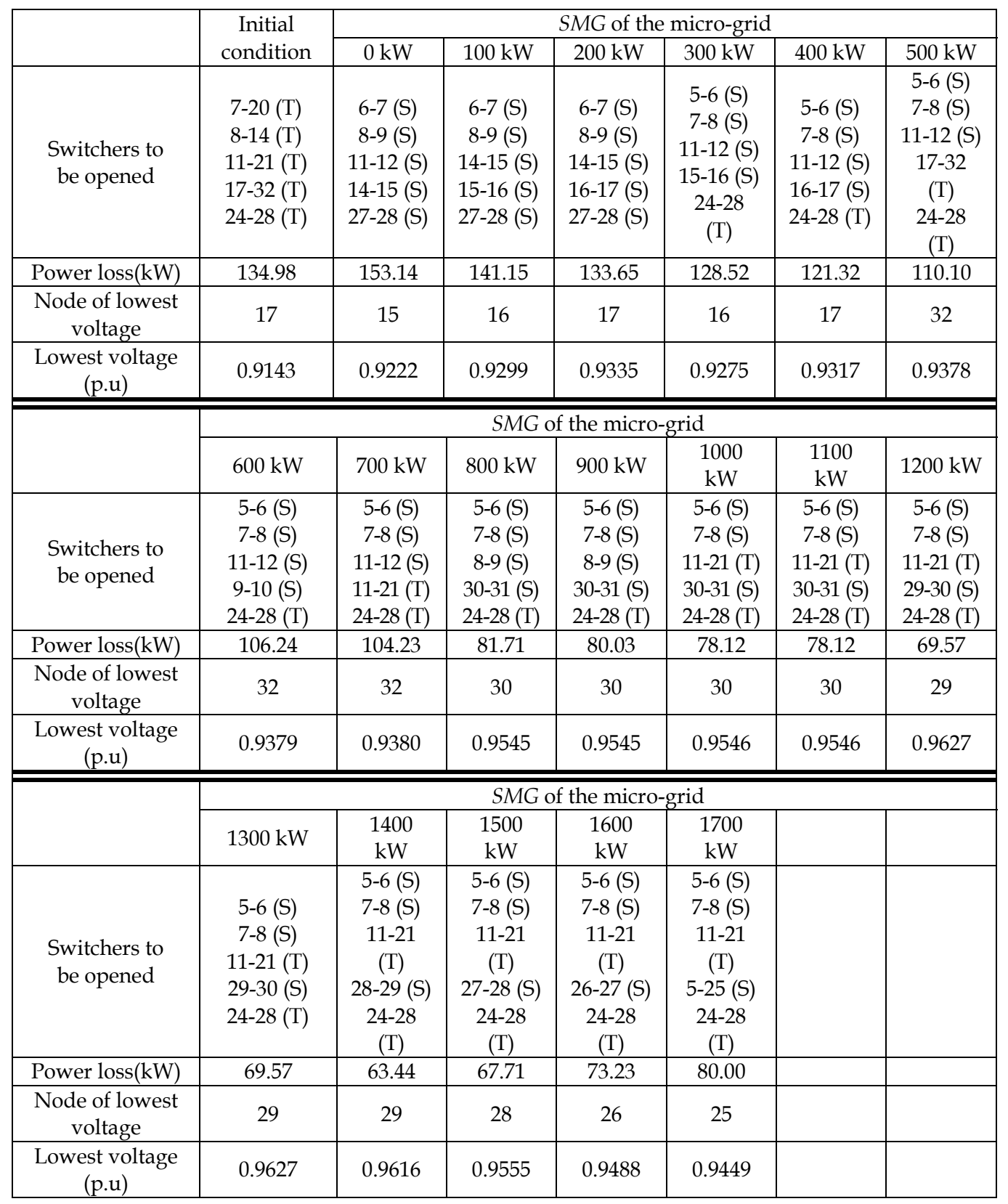

* In Tab.1, if a switcher is marked by (T), it is a tie switcher, e.g. 11-21(T) means a tie switcher between node 11 and 21. If a switcher is marked by (S), it is a sectionalizing switcher, e.g. 5-6 (S) means a sectionalizing switcher between node 5 and 6 .

Table 1. Reconfiguration results for IEEE 33-node system with a single micro-grid and different SMG values. 


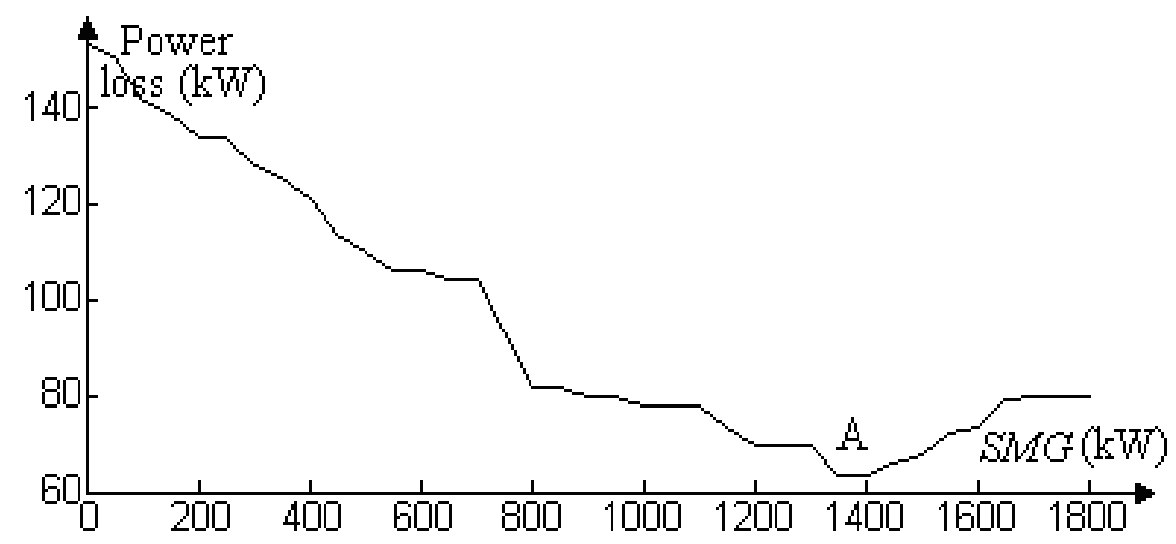

Fig. 5. Power loss changes with SMG (IEEE 33-node system)

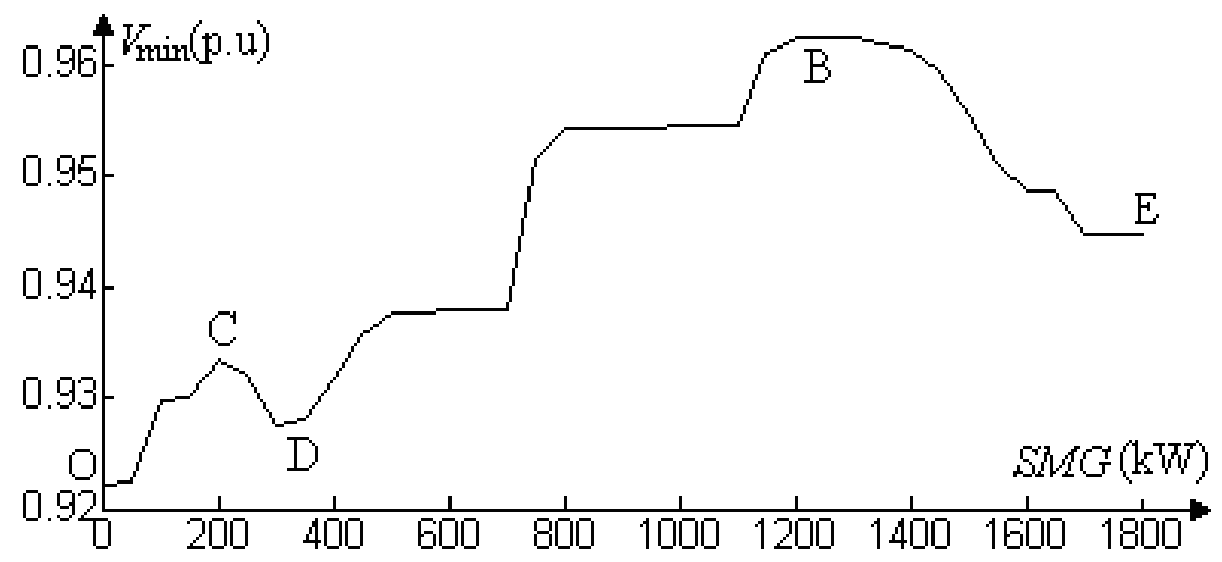

Fig. 6. Lowest voltage changes with SMG (IEEE 33-node system)

2. Power loss and lowest voltage $\left(V_{\min }\right.$ in Fig.6) both change with the SMG value of microgrid. For power loss, the minimum value occurs at point A in Fig. 5 with $(1400 \mathrm{~kW}$, $63.44 \mathrm{~kW})$, while the maximum value of $V_{\min }$ occurs at point B with $(1200 \mathrm{~kW}$, 0.9627 p.u.). It is very interesting that the $S M G$ values of point $\mathrm{A}$ and point $\mathrm{B}$ are not equal.

3. Power loss and $V_{\min }$ both change with $S M G$ nonlinearly. e.g. In Fig.6, when SMG increases in the interval of O-C and D-B, $V_{\min }$ also increases. While $S M G$ increases in the interval of C-D and B-E, $V_{\min }$ decreases. For power loss, it is interesting that there is a minimum point at $S M G=1400 \mathrm{~kW}$. When the $S M G$ is less than $1400 \mathrm{~kW}$, the power loss decreases with $S M G$ increasing. When $S M G$ is larger than $1400 \mathrm{~kW}$, the power loss increases with $S M G$ increasing.

\subsection{PG\&E 69-node system}

PG\&E 69-node system consists of 69 nodes, 5 tie lines all with tie switchers. All branches have sectionalizing switchers. Details of the system can be referred to (Baran \& Wu, 1989, 
Chen et al, 2008; Yu et al, 2009). It is assumed that there is a micro-grid connecting to node 25 . When a fault occurs, it causes branch 14-15 out of service. Using the presented method in section 3, we calculate the power loss and lowest voltage when SMG of the micro-grid changes. The result is shown in Tab.2, Fig.7 and Fig.8. Discussion to the result is similar to that of IEEE 33-node system, which is omitted here for simplification.

\begin{tabular}{|c|c|c|c|c|c|c|c|}
\hline & \multirow{2}{*}{$\begin{array}{c}\text { Initial } \\
\text { Condition }\end{array}$} & \multicolumn{6}{|c|}{$S M G$ of the micro-grid } \\
\hline & & $0 \mathrm{~kW}$ & $100 \mathrm{~kW}$ & $200 \mathrm{~kW}$ & $300 \mathrm{~kW}$ & $400 \mathrm{~kW}$ & $500 \mathrm{~kW}$ \\
\hline \multirow{5}{*}{$\begin{array}{l}\text { Switchers to } \\
\text { be opened }\end{array}$} & $11-66(\mathrm{~T})$ & $11-66(\mathrm{~T})$ & $11-66(\mathrm{~T})$ & $11-66(\mathrm{~T})$ & $11-66(\mathrm{~T})$ & $11-66(\mathrm{~T})$ & $11-66(\mathrm{~T})$ \\
\hline & $13-21(\mathrm{~T})$ & $13-21(\mathrm{~T})$ & $21-22(S)$ & $14-15(S)$ & 13-21(T) & 13-21(T) & 13-21(T) \\
\hline & 15-69(T) & 14-15(S) & 15-69(T) & 18-19(S) & 15-69(T) & 15-69(T) & 63-64(S) \\
\hline & 27-54(T) & 50-51(S) & 27-54(T) & 27-54(T) & 27-54(T) & 53-54(S) & 53-54(S) \\
\hline & 39-48(T) & $47-48(S)$ & 47-48(S) & $47-48(S)$ & $47-48(S)$ & $47-48(S)$ & 47-48(S) \\
\hline $\begin{array}{l}\text { Power } \\
\text { loss(kW) }\end{array}$ & 188.53 & 129.08 & 121.96 & 118.15 & 117.32 & 110.32 & 110.01 \\
\hline $\begin{array}{l}\text { Node of lowest } \\
\text { voltage }\end{array}$ & 54 & 50 & 54 & 54 & 54 & 53 & 53 \\
\hline \multirow[t]{3}{*}{$\begin{array}{l}\text { Lowest voltage } \\
\text { (p.u) }\end{array}$} & 0.9140 & 0.9236 & 0.9254 & 0.9261 & 0.9261 & 0.9297 & 0.9297 \\
\hline & \multicolumn{7}{|c|}{ SMG of the micro-grid } \\
\hline & $600 \mathrm{~kW}$ & $700 \mathrm{~kW}$ & $800 \mathrm{~kW}$ & $900 \mathrm{~kW}$ & $\begin{array}{l}1000 \\
\mathrm{~kW}\end{array}$ & $\begin{array}{l}1100 \\
\mathrm{~kW}\end{array}$ & $\begin{array}{l}1200 \\
k W\end{array}$ \\
\hline \multirow{5}{*}{$\begin{array}{l}\text { Switchers to } \\
\text { be opened }\end{array}$} & $11-66(\mathrm{~T})$ & $11-66(\mathrm{~T})$ & $11-66(\mathrm{~T})$ & $11-66(\mathrm{~T})$ & $11-66(\mathrm{~T})$ & $11-66(\mathrm{~T})$ & $11-66(\mathrm{~T})$ \\
\hline & $13-21(\mathrm{~T})$ & $13-21(\mathrm{~T})$ & $21-22(S)$ & 14-15(S) & $21-22(S)$ & 14-15(S) & $10-11(S)$ \\
\hline & $3-59(S)$ & 15-69(T) & $15-69(\mathrm{~T})$ & 3-59(S) & 3-59(S) & $3-59(\mathrm{~S})$ & 67-68(S) \\
\hline & $53-54(S)$ & $52-53(S)$ & $51-52(S)$ & $50-51(S)$ & $50-51(S)$ & $50-51(S)$ & $50-51(\mathrm{~S})$ \\
\hline & $47-48(S)$ & $47-48(S)$ & $47-48(S)$ & $47-48(S)$ & $47-48(S)$ & $47-48(S)$ & $47-48(S)$ \\
\hline $\begin{array}{c}\text { Power } \\
\operatorname{loss}(k W)\end{array}$ & 109.60 & 83.77 & 83.78 & 83.80 & 83.80 & 83.81 & 84.81 \\
\hline $\begin{array}{c}\text { Node of lowest } \\
\text { voltage }\end{array}$ & 53 & 52 & 51 & 50 & 50 & 50 & 50 \\
\hline $\begin{array}{l}\text { Lowest voltage } \\
\text { (p.u) }\end{array}$ & 0.9297 & 0.9412 & 0.9416 & 0.9425 & 0.9425 & 0.9425 & 0.9426 \\
\hline
\end{tabular}

Table 2. Reconfiguration results for PG\&E 69-node system with a single micro-grid and different SMG values. 


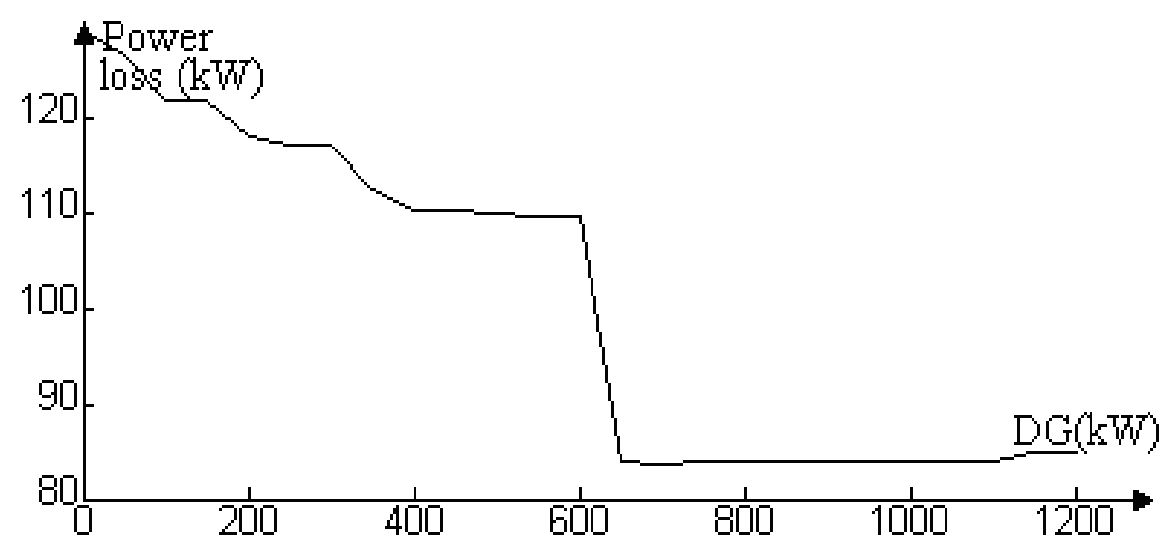

Fig. 7. Power loss changes with SMG (PG\&E 69-node system)

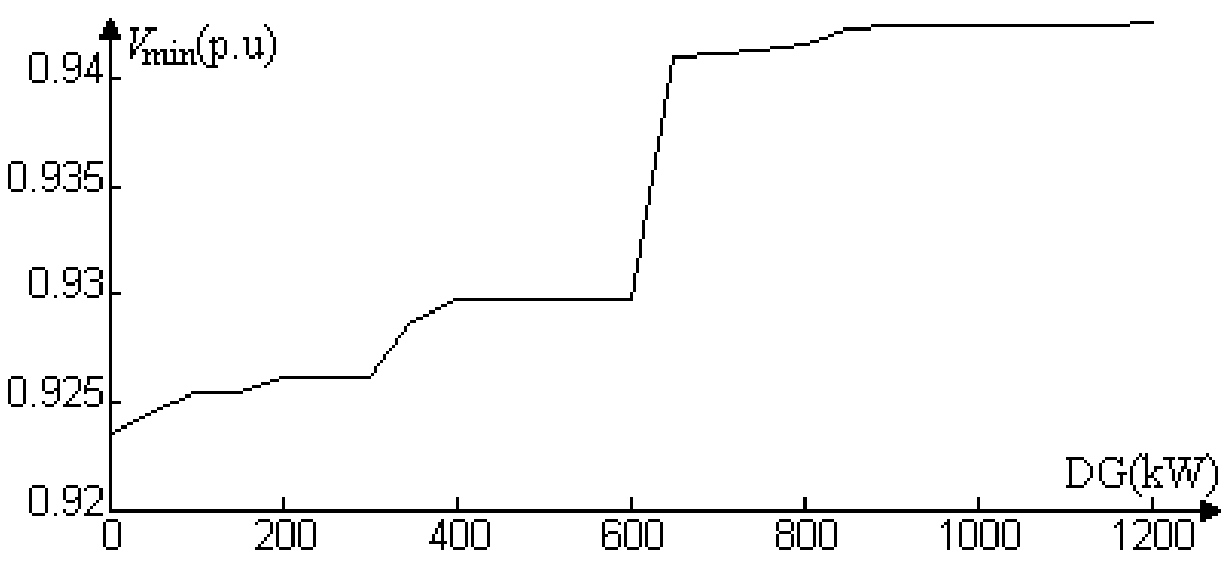

Fig. 8. Lowest voltage changes with SMG (PG\&E 69-node system)

\section{Conclusion}

This chapter presents an optimal model for reconfiguration analysis of distribution system with micro-grids. Once a fault occurs in a distribution system, it can be applied to construct some optimal islands so as to guarantee power supplying to some important loads and to reduce power loss at the same time. The model is then decomposed into a capacity sub-problem and a reconfiguration sub-problem. The former is used to determine the optimal capacity for each island, and the latter is used to find the optimal reconfiguration with less power loss. Two sub-problems are called iteratively to get the optimization solution. Finally, IEEE 33-node system and PG\&E 69-node system are employed to validate the effectiveness of the presented method. Studies of this chapter are helpful to find the optimal integration scheme for various DG devices connecting to distribution system in the future. 


\section{References}

EPRI(2007). Renewable energy technical assessment guide-TAG-RE: 2006. EPRI Final Report, Palo Alto, CA USA: 2007. 1012722

EPRI(2001). Integrating distributed resources into electric utility distribution systems, EPRI Final Report, Palo Alto, CA USA: 2001. 1004061

IEEE(2003). IEEE standard for interconnecting distributed resources with electric power systems, IEEE Std 1547-2003.

Chen, H. F.; Liu, Z.; Jia, H. J.; Yu, X. D. Bender's decomposition based reconfiguration for distribution network with distributed generation. Automation of Electric Power Systems, 2008, 32(1): 6-10.

Yu, X. D.; Jia, H. J.; Wang, C.S.; et al. Network reconfiguration for distribution system with micro-grids, Proc. of 1st SUPERGEN, 2009, April 6-7, Nanjing, China. Article No: 5348219.

Barnes M; Kondoh, J; Asano, H; et al. (2007). Real-world microgrids: an overview, Proc of IEEE International Conference on System of Systems Engineering, San Antonio, TX USA, 2007.4. 16-4.18, pp.1-8.

Khan M. S.; Iravani, M. R. (2007). Supervisory hybrid control of a micro grid system, Proc. of IEEE 2007 Electrical Power Conference, 2007, Oct.25-26, Montréal, Québec, Canada, pp.20-24.

Dimeas, A. L.; Nikos, D. H. (2005). Operation of a multiagent system for micro-grid control. IEEE Trans. on Power Systems, 2005, 20(3): 1447-1455.

Civanlar, S.; Grainger, J. J.; Yin H.; et al. (1988). Distribution feeder reconfiguration for loss reduction. IEEE Trans. on Power Delivery, 1988, 3(3): 1217-1223.

Baran, M. E.; Wu, F. F. (1989). Network reconfiguration in distribution systems for loss reduction and load balancing, IEEE Trans. on Power Delivery, 1989, 4(2): 1401 1407.

Song, Y. H.; Wang, G. S.; Johns, A. T.; Wang, P. Y. (1997). Distribution network reconfiguration for loss reduction using fuzzy controlled evolutionary programming, IEE Proc.-Generation, Transmission and Distribution, 1997, 144 (4): 345-350.

Kashem, M. A.; Ganapathy, V; Jasmon, G. B. (2001). A geometrical approach for network reconfiguration based loss minimization in distribution systems, Int. J. of Electrical Power and Energy Systems, 2001, 23(4):295-304.

Carpaneto, E.; Chicco, G. (2004). Ant-colony search-based minimum losses reconfiguration of distribution systems, Proc. of the 12th IEEE Mediterranean, MELECON, 2004, May 9-12, Dubrovnik, Croatia, pp. 971-974.

Sua, C.T.; Changb, C. F.; Chiou, J. P. (2005). Distribution network reconfiguration for loss reduction by ant colony search algorithm, Electric Power Systems Research, 2005, 75(2-3):190-199.

Tu, Q.; Guo, Z. Z. (2006). Median current moment method for dynamic reconfiguration in distribution network, Proc. of Int. Conference on Power System Technology, 2006, 22-26 Oct. Chongqing, China, pp.1-4.

Bhattacharya, S. K.; Goswami, S. K. (2008). Distribution network reconfiguration considering protection coordination constraints, Electric Power Components and Systems, 2008, 36(11):1150-1165. 
Carreno, E. M.; Romero, R.; Padilha F. A. (2008). An efficient codification to solve distribution network reconfiguration for loss reduction problem, IEEE Trans. on Power Systems, 2008, 23(4): 1542-1551. 


\title{
A Camera-Based Energy Management of Computer Displays and TV Sets
}

\author{
Vasily G. Moshnyaga \\ Fukuoka University \\ Japan
}

\section{Introduction}

With increase in image quality and screen sizes, the energy consumption of computer displays and television sets has significantly grown. In a typical personal computer or PC, display accounts for 30\% 50\% of the total PC energy consumption [Mahesri 2005, Robertson 2002]. For instance, typical 19 inch LCD monitor, such as Sony SDM-S93 (1280x1024 pixels), burns in active mode $50 \mathrm{~W}$ or almost $38 \%$ of the total desktop system power $(130 \mathrm{~W})$. With large popularity of video and gaming applications, LCD makers are being called on to cut power consumption while providing better images. Rapid utilization of multiple displays -each consuming tens of watts -- throughout homes, offices and buildings increases cost and environmental impact of energy consumption significantly. Although most PC displays support power management, new robust methods are needed for evolving display usage scenarios.

For TV sets, the quest for efficient display energy management is more severe, because modern TVs have much bigger screens than computer displays and therefore consume more power. Although the LCD TVs are more efficient than their cathode-ray tubes (or CRT) TVs, recently emerged plasma television sets are twice as bigger and about four times more energy than their cathode-ray tube equivalents [Coughlan, 2006]. A 50-inch flat-screen plasma HDTV now burns over 500Watts of power [Plasma TV, 2006]; consuming almost the same amount of energy as dishwasher or in-room air-conditioner. The problem however does not relate only to plasma television sets. The LCD TV sets also consume a lot. A typical 42" LCD TV takes 169-250Watts of power per each hour [TV Power Consumption, 2008]. According to Nielsen Media Research Inc. [Nielsen 2009] over 99\% of all households in US have TV sets, with 2.24 TVs per household in average. Since TV is ON for almost 5 hours in an average US home a day [Television \& Health], it has become one of the largest energy consumers. Due to emerging problems of global warming and fossil fuel shortage, reducing TV energy is very important.

Up to the date, reducing energy consumption of LCD displays has been tackled mainly through system and circuit optimizations, which either ignore the user, assuming fixed and stable demands on system operation or rely on very simplified policies, which eventually lead to large energy losses. Generally, there are two sources of energy losses in a device: intrinsic losses and the user-related losses. The intrinsic energy losses are caused by the engineering design, technology and materials used in construction of the device. For example, a plasma TV intrinsically dissipates more energy than a LCD TV, etc. The user- 
related losses are associated with varying and inefficient device usage. Keeping a TV ON when nobody watches it, for example, causes energy loss associated with bad device usage. Existing energy management policies are device centric; that is they either ignore the user, assuming unchangeable operational environment for the device or rely on very simplified polices. Take a TV for example. Take a TV for example. A variety of methods has been proposed to reduce energy consumption of TVs. Majority of them, however, target the intrinsic energy losses, without considering the viewer. As a result, the television sets produce bright and high quality pictures independently whether there is any viewer or not. According to [Gram-Hansen, 2003], the energy consumption of consumer electronic devices can differ by a factor of two due to usage. Some experts estimate that $26 \%-36 \%$ of the total domestic energy consumption are losses related to unreasonable usage of appliances [Elias 2007]. Clearly, in order to reduce the losses, we must make the device energy management user-centric, i.e. adaptable to the varying user behavior. No energy should ever be spent uselessly.

In this chapter we present a new approach to LCD display and TV set energy management, which unlike existing methods employs a video camera to bind the display power state to the actual user's attention. We discuss implementation of this novel approach and show the results of its experimental evaluation.

The chapter is organized as follows. In the next section we survey related research. Section 3 describes the proposed camera-based display energy management approach. Section 4 presents implementation features for PC display and TV set. Section 5 summarizes our findings and outlines work for the future.

\section{Related research}

The core technology to manage power consumption of display in modern personal computers is Advanced Configuration and Power Interface (or ACPI in short) developed by HP, Intel, Microsoft, Phoenix, and Toshiba. The OS-based ACPI specifies one or more power states (e.g. standby, sustain, etc.) that are intermediate between on and off turning the display to low power state after a specified period of inactivity on mouse and/or keyboard. Each power state corresponds to proper level of display brightness and power consumption. The main problem with ACPI is that it strongly depends on inactivity intervals, either set as default or by the user. From one hand, if the inactivity intervals are improperly short, e.g. 1 or 2 minutes, the ACPI can be quite troublesome by shutting the display off when it must be on. From another hand, if the inactivity intervals are set to be long, the ACPI efficiency decreases. Because modifying the intervals requires system setting, most users however never adjust the power management of their PCs for fear that it will impede performance [Fujitsu-Siemens]. Those who do the adjustment, usually assign long intervals. HP inspected 183,000 monitors worldwide and found that almost a third was not set to take advantage of the energy saving features. Just enabling these features after 20 minutes of inactivity can save up to $381 \mathrm{kWh}$ for a monitor per year [Hewlett-Packard 2006]. Evidently, to prevent such a problem the PC energy management must employ more efficient user monitoring. Several techniques have been proposed to improve presence detection of computer users. Extending touch-pad function beyond pointer movement to provide user-presence

\footnotetext{
${ }^{1}$ Apple PCs employ power management technology that is distinct from but similar to ACPI (see [Nordman 1996], [Nordman 1997] for more details
} 
identification is proposed in [Park, 1999]. [Dai 2003] suggests using thermal sensors placed around display screen to detect user's presence by comparing temperature fluctuation the sensors during a sample interval. When user is present, the temperature fluctuation is consistent with a normal fluctuation pattern of human breathing.

TV sets also employ screen brightness dimming technologies for energy saving. Nowadays TV viewers can modify the screen brightness level by selecting one of three operation modes: the "standard mode" delivers the highest level of brightness; the "saving mode" refers to the dimmed screen and "no brightness mode" reflects the dark screen. The brightness level in the saving mode can also be changed. Sensing light is already a feature of many TVs to enable dimming based on ambient light level. However, unless the light is changed or the viewer changes the mode, the TV maintains same brightness.

Many efforts have been put recently on brightness/contrast adjustment techniques to lower display energy consumption in active mode. The reason is that transmissive and transflective color TFT LCD panels [Sharp 2002] do not illuminate itself but filter a backlight, the primary source of display energy dissipation. Because simply dimming the backlight degrades the display visibility, Choi, et al [Choi 2002] proposed to maintain brightness or contrast of the LCD panel when the backlight is dimmed down. To reduce the average energy demands of the backlight, Gatti, et al [Gatti 2002] suggested the backlight auto regulation scheme. Cheng and Pedram [Cheng 2004] showed that a concurrent brightness and contrast scaling (CBCS) technique further enhances image fidelity with a dim backlight, and thus saves an extra power. Chang, et al [Chang 2005] introduced a dynamic luminance scaling or DLS technique that dimmed the backlight while allowing more light to pass through the screen panel to compensate for the loss of brightness in the original image. Shim, et al [Shim 2004] combined the DLS technique with dynamic contrast enhancement and applied it for transflective TFT LCD panels. Pasricha, et al [Pasricha 2004] presented an adaptive middleware-based technique to optimize backlight power when playing streaming video. Iranli, et al [Iranli 2006] presented HVS-aware dynamic backlight scaling in TFT LCD. A modification of the LCD panel to permit zoned backlighting has been discussed in [Flin 1999]. There is also a variety of techniques for automated adjustment of brightness in high dynamic range panoramic images, e.g. [Pattanai 2000], [Tumblin 1999]. These techniques dynamically brighten or darken image regions depending on the scene content and average local luminance.

Despite differences, the proposed brightness and/or contrast adjustment techniques have one feature in common. Namely, they work independently on the viewer attention. While the techniques are able to decrease the TV energy consumption in active mode, they can not change the modes. So TV screen remains active even if nobody watches it. Similarly to computer users, majority of TV viewers do not change brightness or power mode for energy savings, fearing that it affects picture quality. Besides, viewers usually watch TV while doing other activities: reading books, working on PC, preparing food, chatting with friends, etc. According to statistics, a TV is ON for almost 5 hours in an average US home a day [3]. As Kaiser Family Foundation [Generation M2, 2010] reports, 39\% of 8-18 year olds in US say they keep TV on while doing other things "most of the time"; $29 \%$ say they do so "some of the time." In other words, a TV frequently wastes energy for producing high quality pictures when nobody watching them.

Leading TV produces have recently started to embed "user sensors" into TV sets in order adjust power consumption to the user behavior. For example, the VIERA ${ }^{\circledR}$ plasma TV from Panasonic senses the user through the remote controller. If the time from the last use of 
remote controller exceeds a pre-defined time interval (e.g. 1, 2 or 3 hours), the TV automatically powers off. The latest Bravia ${ }^{\circledR}$ HDTV from Sony incorporates an infra-red motion sensor, which switches the TV off when no motion has been detected in front of it over a period of time (e.g. $5 \mathrm{~min}, 30 \mathrm{~min}$ or 1 hour) pre-set by the user. Also Hitachi and Toshiba use hand gesture sensors to control TV.

Sensing the viewer explicitly (through fingers, hands or body motion) has several problems: it is either incorrect (a moving dog or a tree in the window can keep the TV on) or troublesome, i.e. requires the user either to push the remote control or move in front of the screen frequently or to enlarge the allowed duration of inactivity interval to prevent shutting the TV down. Also, because modern PCs monitor the user' fingers not eyes, they can not distinguish whether the user looks at the screen or not. Therefore, they may either switch the screen off inappropriately, i.e. when someone looks at the screen without pressing the keys, or lose energy by staying in active mode while idling. We claim that ignorance of the viewer's attention is the main cause of the user-related energy losses of existing displays in PCs and TVs. While the display operation mode must depend on the viewer, existing displays, up to our knowledge, do not take the viewer's focus into account. We propose a method which can solve this problem.

\section{The proposed display energy management technology}

The main goal of our approach is to increase the energy efficiency of display by enabling it to "watch" its viewer and lower down the display power whenever the viewer is detracted from the screen. We assume that the screen of computer display or TV is enabled with a video camera. This can be a special camera embedded in display for viewer monitoring, an infra-red camera or a general purpose video camera (e.g. SONY VAIO visual communication camera) connected via USB port for video capture, conferencing, etc. The camera is located at the top of display or TV. When a viewer looks at the screen, it faces the camera frontally. Also we assume that computer display and TV have a number of backlight intensity levels with the highest level corresponding to the largest power consumption and the lowest level to the smallest power, respectively. The highest level of backlight intensity is enabled either initially or whenever the user looks at the screen. In all cases we assume that the viewer monitoring mode is optional to the user.

The idea behind our display energy management is simple. When the user of PC or TV looks at screen, the screen is kept active in "power-up" mode to provide the best visibility. If the user detracts his/her attention from the screen, the screen is dimed off to decrease energy consumption. Finally, if nobody looks at the screen for long time or disappears from the camera's range, the screen enters the "sleep" mode or is turned off to save energy.

In order to make the technology effective, the camera based viewer tracking system must satisfy a number of requirements, such as initialization simplicity, be non-intrusive, support unrestricted head movements, real time operation and low energy consumption. The latter requirement is especially important for battery operated personal computers because it keeps the PC battery lifetime and weight reasonable. Majority of currently available viewer tracking systems, unfortunately, do not satisfy these requirements making energy management a real challenge. For instance, some systems need calibration procedures; the others require users to wear head-mounted gears (a hat or helmet or glasses on which camera is mounted) or restrict head positions within a quite narrow area. Those systems which meet the requirements are unfortunately very energy consuming [Ji 2002], [Baluja 1994], [Ohno 2003], [Theocharides 2004], [Park 2005], [Kawato 2005]. 


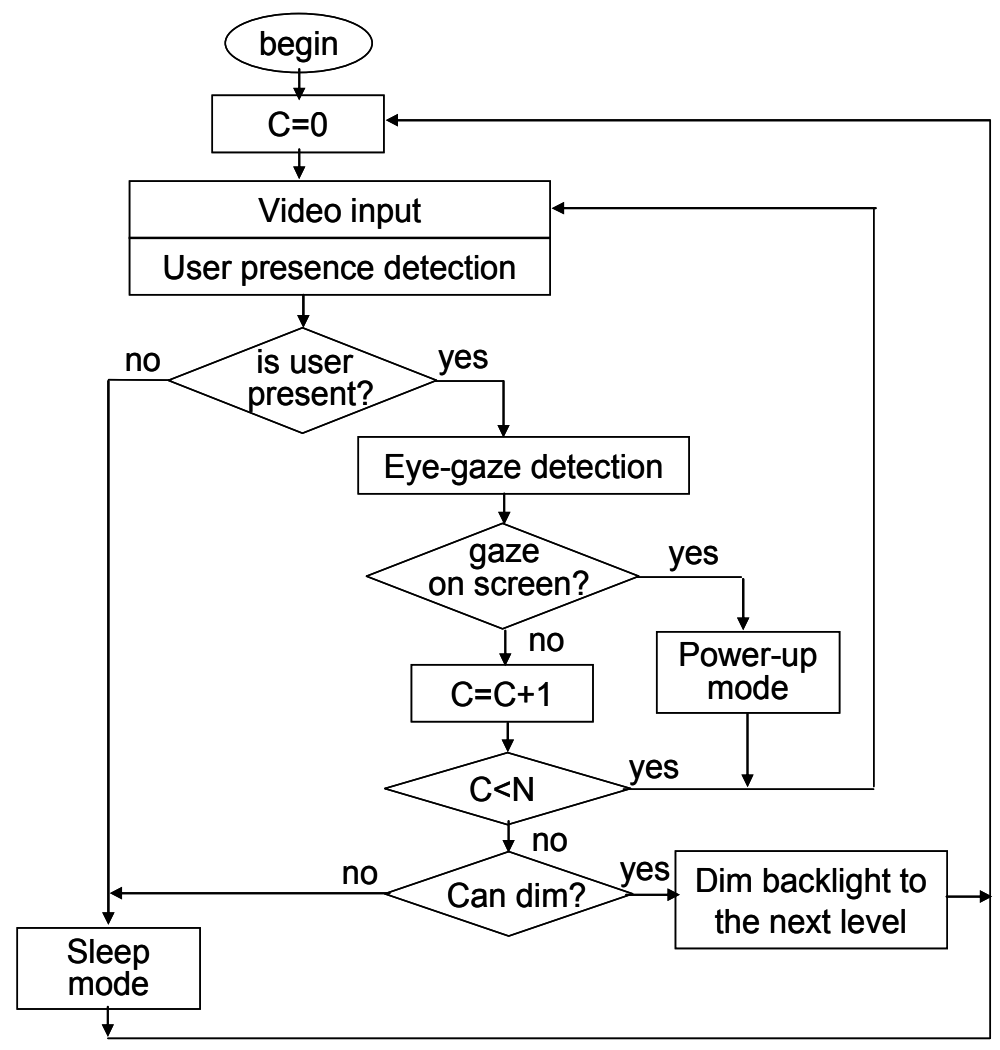

Fig. 1. The flowchart of the proposed camera-based computer display energy management

Below we present techniques which ensure real-time camera-based energy management with low-energy overhead. Although the same methodology can be used for tracking viewers of computer display and TV set, we optimize the techniques by the application to reduce the energy overhead.

\subsection{The camera based computer PC display energy management}

Figure 1 shows the flowchart of our camera-based computer display energy management. Here, $\mathrm{C}$ counts the number of consecutive image frames in which the user's gaze is off the screen. We assume that the camera is located at the top of display. If no user is detected in the $\mathrm{N}$ consecutive video frames, the display is turned to the sleep mode. Otherwise, the technology tracks the user's eye-gaze. If the gaze has been off the screen for more than $\mathrm{N}$ consecutive frames, the backlight luminance is dimmed down to the next level. Any onscreen gaze reactivates the initial backlight luminance by moving the display onto power up mode. However, if no on-screen gaze has been detected for more than $\mathrm{N}$ frames and the backlight luminance has already been lowest, the display enters the sleep mode. Returning back from sleep mode is done automatically whenever the user gaze is back on screen. The user may also switch the display OFF whenever he or she wishes. Re-activating the screen from the OFF state requires pushing the ON button. 

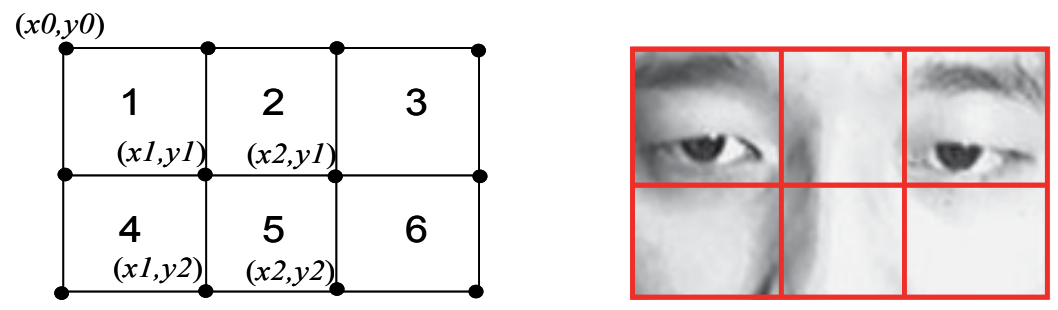

Fig. 2. An illustration of the SSR filter

\subsubsection{User presence detection}

The goal of this task is to determine from the camera (or CMOS sensor) readings whether or not the user is currently present in front of display. To detect the user's presence in front of the display, we first localize the face search by applying background subtraction and skincolor segmentation to the RGB representation of input image. The skin is defined by the following criteria [Douchamps 2002]: $0.55<\mathrm{R}<0.85,1.15<\mathrm{R} / \mathrm{G}<1.19,1.15<\mathrm{R} / \mathrm{B}<1.5$ and $0.6<(\mathrm{R}+\mathrm{G}+\mathrm{B})<1.8$.

To accelerate the face-area extraction, we use two filters. The first one limits the size of the head in reasonable range. The second one verifies that the face contains a minimum of $25 \%$ of skin colored pixels. Thus, if the total number of pixels in the derived face area exceeds the threshold (W), the user is assumed present.

\subsubsection{Eye-gaze detection}

The eye-gaze detector implements the algorithm proposed by [Kawato 2005], which scans the Six Segment Rectangular filter (SSR) over the integral representation [Viola 2001] input image. to define the Between The Eyes (BTE) point of human face (see Fig.2) and then searches the regions 1 and 3 from the left and right side of the BTE point to locate the eyes. The algorithm does not depend on illumination, face occlusion, eye closure. It is more stable, robust and less complex than the other eye-tracking formulations. However, it is still very computationally demanding. In a quest to locate all faces (without restriction on face size, motion and rotation) the algorithm repeats scan six times over the whole image frame performing over 28 Million operations, such as addition or subtraction per frame $(640 \times 480$ pixels frame size). Though such a full search might be necessary in some applications, it seems redundant when tracking eyes of the PC user.

In our eye-tracking application we can assume that:

1. The target object is a single PC user. The user sits in front of PC at a relatively close distance $(50-70 \mathrm{~cm})$.

2. The user's motion is slow relatively to the frame rate.

3. The background is stable and constant.

Based on these assumptions, we apply the following optimizations to reduce eye-tracking complexity:

a. single SSR filter scan;

b. fixed SSR filter size;

c. three pixel displacement of the SSR filter during the scan;

d. low frame processing rate (5-10 fps); 


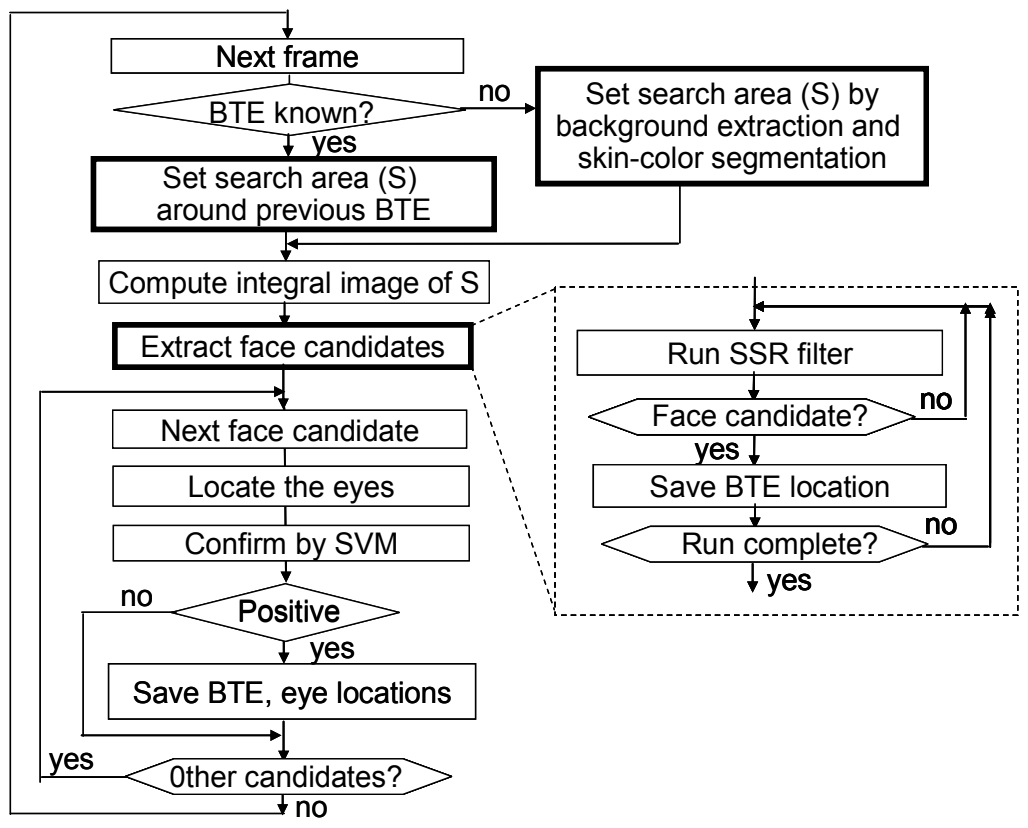

Fig. 3. The modified eye-tracking algorithm

As our study shows [Yamamoto 2009], such enhancements reduce computational complexity considerably without affecting the quality of the results.

Fig.3 shows the modified algorithm. For the first frame or any frame in which the search for BTE candidate was unsuccessful, we search the image area reduced by background and skincolor extraction; otherwise the search is restricted to a small area (S) of \pm 8 pixels around the BTE point. For the chosen area, the algorithm first computes the integral image and then scans it by the Six Segment Region filter (SSR) to select the BTE candidate. If the BTE candidate is found, the system uses the BTE as a starting point to locate eyes. If eyes have been detected, the user is assumed to be looking at screen; else it is not. If no face candidate has been found for $\mathrm{N}$ consecutive frames, the user is considered not present in front of the display.

The BTE search is organized as follows. The green component of the search area is transformed to integral image representation [Viola 2001] and scanned by the six-segment rectangular (SSR) filter (Fig.2). At each location, the SSR filter compares the integral sums of the segments as follows: At each location, the SSR filter compares the integral sums of the segments as follows:

$$
\begin{aligned}
& \operatorname{Sum}(1)<\operatorname{Sum}(2) \& \operatorname{Sum}(1)<\operatorname{Sum}(4) \\
& \operatorname{Sum}(3)<\operatorname{Sum}(2) \& \operatorname{Sum}(3)<\operatorname{Sum}(6)
\end{aligned}
$$

If the above criteria (1) are satisfied, the SSR is considered to be a candidate for the BTE pattern (i.e. face candidate) and two local minimum (i.e. dark) points each are extracted from the regions 1 and 3 of the SSR for left and right eyes, respectively.

In the search for eyes, we ignore 2 pixels at the boarder of the regions to avoid effects of eyebrows, hair and beard. Also, because the eyebrows have almost the same grey level as the eyes, the search starts from the lowest positions of regions 1 and 3. 

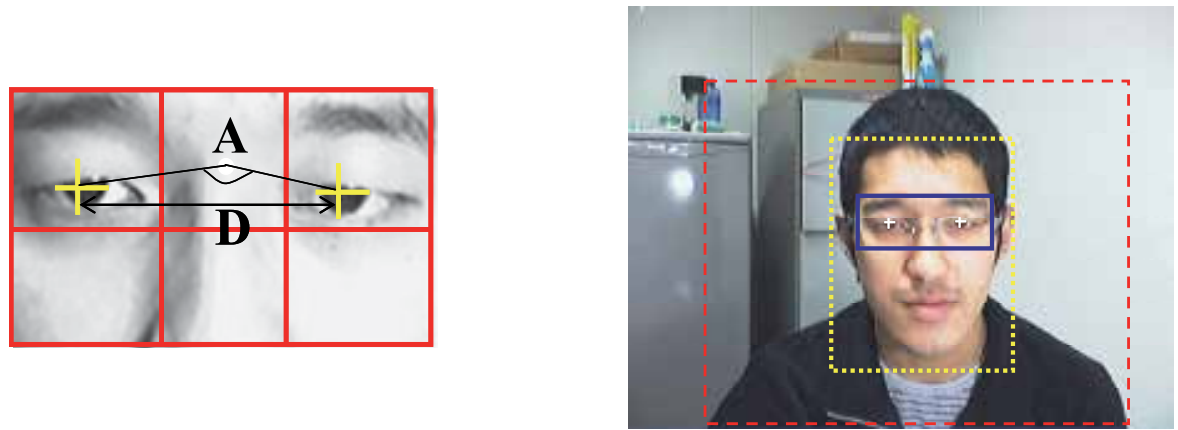

Fig. 4. An illustration of the eye detection heuristics (left) and the search area reduction

The eye localization procedure is organized as a scan over the green plane representation of regions 1 and 3 for a continuous segment of dark pixels (i.e. whose value is lower than the threshold k). As in [Kawato 2000], we assume that eyes are located if the distance between the located eyes $(D)$ and the angle $(A)$ at the center point of the BTE area 2 (see Fig.4, left) satisfy the following:

$$
30<\mathrm{D}<42 \& 115^{\circ}<\mathrm{A}<180^{\circ}
$$

If both eyes are detected, the user's gaze is considered to be on screen. Otherwise, it is assumed to be off the screen. The eye positions found in the current frame are then used to reduce complexity of processing the successive frames. Since the face/eye motion of computer user is slow in practical circumstances, the search in the next frame is limited to a small region, which spans by 8 pixels in vertical and horizontal direction around the eye points of the current frame.

Fig. 4(right) demonstrates the search area reduction by our algorithm: dashed line shows the area defined by background extraction; dotted line depicts the area obtained by skin-color segmentation; the plain (dark line) shows the area around the previous BTE point; white crosses show the computed locations of eyes.

If for any frame, the user gaze is detected to be on screen, the backlight luminance is set-up to the highest level by resetting the backlight voltage to the initial value. If the gaze has been detected off the screen for $\mathrm{N}$ consecutive frames, the backlight voltage is reduced to the next level to dim the backlight luminance down. If the lowest level of the backlight voltage supply has been reached and the user still is not found in the camera readings, the system switches the display off to save energy.

\subsubsection{Implementation}

Fig. 5 outlines the block-diagram of the proposed computer display power management system. The user tracking unit receives an RGB color image and outputs two logic signals, $\mathrm{u}_{1}, \mathrm{u}_{0}$. If the user is detected in the image, the signal $\mathrm{u}_{0}$ is set to 1 ; otherwise it is 0 . The zero value of $\mathrm{u}_{0}$ enforces the voltage converter to shrink the backlight supply voltage to 0 Volts, dimming the display off. If the eye-gaze detector determines that the user looks at screen, it sets $\mathrm{u}_{1}=1$. When both $\mathrm{u}_{0}$ and $\mathrm{u}_{1}$ are 1 , the display operates as usual. If the user's gaze has been off the screen for more than $N$ consecutive frames, $u_{1}$ becomes 0 . If $u_{0}=1$ and $u_{1}=0$, the input voltage $\left(\mathrm{V}_{\mathrm{b}}\right)$ of the high-voltage inverter is decreased by $\Delta \mathrm{V}$. This voltage drop lowers backlight luminance and so shrinks the power consumption of the display. Any on-screen 


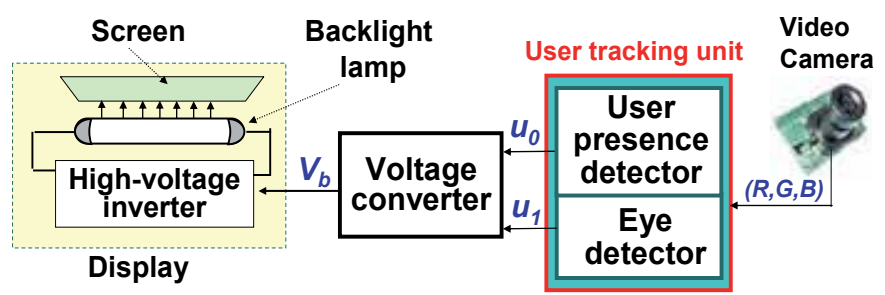

Fig. 5. Block diagram of the camera-based display energy management system

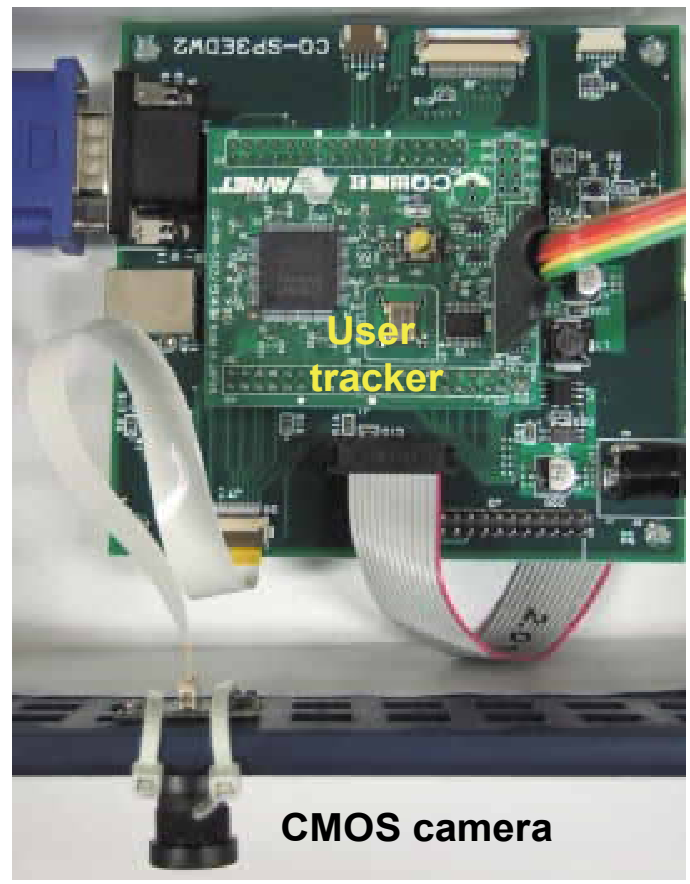

Fig. 6. The user-tracking hardware

gaze in this low power mode reactivates the initial backlight luminance and moves the display onto normal mode. However, if no on-screen gaze has been detected for $N$ consecutive frames and the backlight luminance has already reached the lowest level, the display is turned onto sleep mode.

We implemented the system in hardware (see [Moshnyaga 2009] for details). Fig.6 illustrates the hardware design of the user tracking unit. The design was synthesized in Verilog HDL using Synopsis Design Compiler and realized on a single FPGA (Xilinx XC3S250E) board. The board is connected to VGA CMOS camera, through parallel I/O interface. Table 1 summarizes parameters of the FPGA design. The user tracking unit operates at $48 \mathrm{MHz}$ frequency using $3.3 \mathrm{~V}$ external voltage and provides user presence detection at $20 \mathrm{fps}$ rate. Due to capacity limitations of the on-chip SRAM memory, input images were 160x120 pixels in size. The SSR filter was 30x20 pixels in size. The total power consumption of the gaze detector design was $150 \mathrm{~mW}$, which is 35 times less than software implementation of the user presence detector on desktop PC (Pentium4@2.53GHz) [Moshnyaga 2005]. 


\begin{tabular}{|l|c|}
\hline \multicolumn{1}{|c|}{ Parameter } & Value \\
\hline System clock frequency & $48 \mathrm{MHz}$ \\
\hline External voltage & $3.0 \mathrm{~V}$ \\
\hline Internal voltage & $1.2 \mathrm{~V}$ \\
\hline System gate count & 250000 \\
\hline Logic cell count & 18508 \\
\hline Memory size & $216 \mathrm{~Kb}$ \\
\hline Frame size (pixels) & $160 \times 120$ \\
\hline Detection rate & $20 \mathrm{fps}$ \\
\hline Power & $150 \mathrm{~mW}$ \\
\hline
\end{tabular}

Table 1. FPGA design parameters

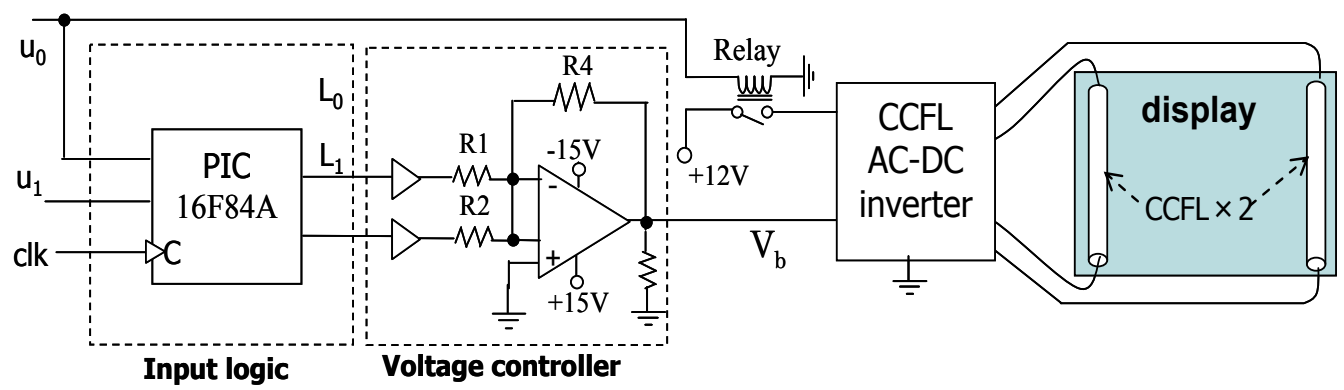

Fig. 7. The modified voltage converter

Next, to change the display brightness dynamically, we modified the voltage converter by adding the input logic, the voltage controller, and a relay, as shown in Fig.7. In TFT LCD displays, the Pulse Width Modulation (PWM) scheme links brightness of the cold cathode fluorescent lamps (CCFL) to the input voltage, $\mathrm{Vb}$, of the CCFL AC-DC inverter. Usually, the display brightness is the highest when $\mathrm{Vb}=0$ and the lowest when $\mathrm{V}_{\mathrm{b}}=5 \mathrm{~V}$. In our design, the voltage $V_{b}$ is controlled by two inputs $\left(u_{0}, u_{1}\right)$ whose levels are defined by the eye-gaze detector (see fig.5). When $\mathrm{u}_{0}=0$, the relay disconnects the $12 \mathrm{~V}$ power supply to the display switching it OFF. Turning the display ON automatically sets $\mathrm{u}_{0}$ to 1 , which enforces $+12 \mathrm{~V}$ voltage supply to display. When $\mathrm{u} 0=1$, the display power consumption is controlled by the output $\left(\mathrm{V}_{\mathrm{b}}\right)$ of the voltage controller. Namely, when $\mathrm{u}_{1}=1$, the counter (cnt) nulls outputs $\mathrm{L}_{1}, \mathrm{~L}_{2}$ setting $\mathrm{V}_{\mathrm{b}}$ to $0 \mathrm{~V}$. When $\mathrm{u}_{1}=0$, the counter increments its state with each new rise of signal $c l k$, thus setting the voltage converter to increment its output $\mathrm{V}_{\mathrm{b}}$ by approximately $0.7 \mathrm{~V}$. Table 2 outlines the display voltage control and the power consumption at the corresponding four voltage levels. As it clearly shows, changing the brightness leads to large savings of display power. 


\begin{tabular}{|cl|c|c|c|}
\hline $\mathrm{u} 1$ & $\mathrm{u} 0$ & $\mathrm{Vb}(\mathrm{V})$ & Brightness & Power $(\mathrm{W})$ \\
\hline 1 & 1 & 0 & bright & 34.03 \\
\hline 1 & 0 & 1.2 & Level 1 & 27.43 \\
\hline 1 & 0 & 2.4 & Level 2 & 22.21 \\
\hline 1 & 0 & 3.6 & Level 3 & 17.54 \\
\hline 1 & 0 & 4.8 & Level 4 & 14.62 \\
\hline 0 & - & - & OFF & 0.01 \\
\hline
\end{tabular}

Table 2. Display voltage control parameters

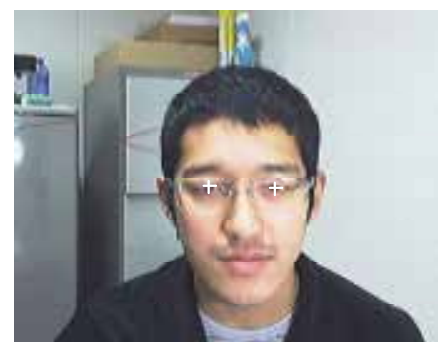

a)

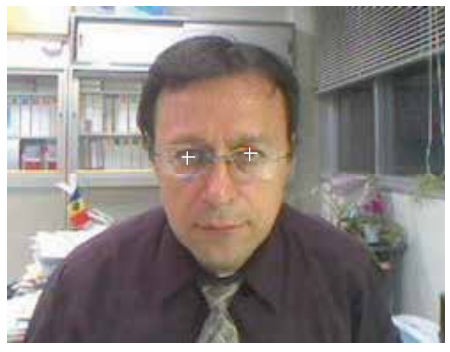

c)

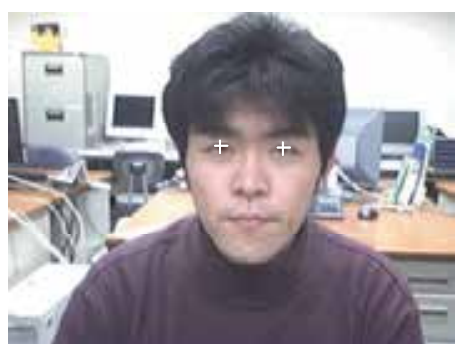

b)

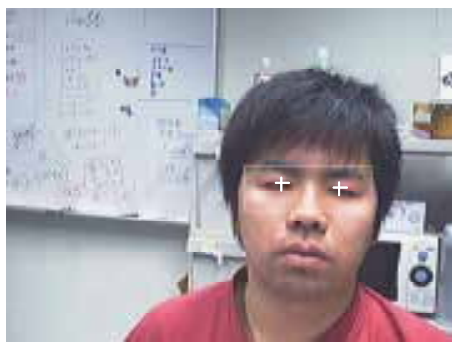

d)

Fig. 8. Examples of true detection

\subsubsection{Evaluation}

To evaluate accuracy of the gaze detector, we ran four different tests each of each conducted by different users. The users were free to look at the camera/display, read from the materials on the table, type text, wear eyeglasses, move gesticulate or even leave the PC whenever wanted. Fig. 8 illustrates the detection results on 4 images. The + marks depict positions where the system assumes the eyes to be. As we see, even though the lighting conditions of faces vary, the results are correct. Ordinary pairs of glasses (see Fig.8) have no bad effect on the performance for frontal faces. In some face orientations, however, the frame of pair of glasses can hide a part of eye ball, causing the system to loose the eye. Or sometimes it takes eyebrow or hair as an eye and tracks it in the following frames.

Table 3 summarizes the results. Here, the second column depicts the total number of frames considered in the test; columns marked by 'True' and 'False' reflect the number of true and false detections, respectively; the Accuracy column shows the ratio of true decisions to the 


\begin{tabular}{|c|c|c|c|c|}
\hline Test & Frames & True & False & Accuracy (\%) \\
\hline 1 & 151 & 133 & 18 & 88 \\
\hline 2 & 240 & 214 & 26 & 89 \\
\hline 3 & 100 & 90 & 10 & 90 \\
\hline 4 & 180 & 152 & 28 & 84 \\
\hline Average & 167 & 147 & 20 & 88 \\
\hline
\end{tabular}

Table 3. Results of evaluation on test sequences

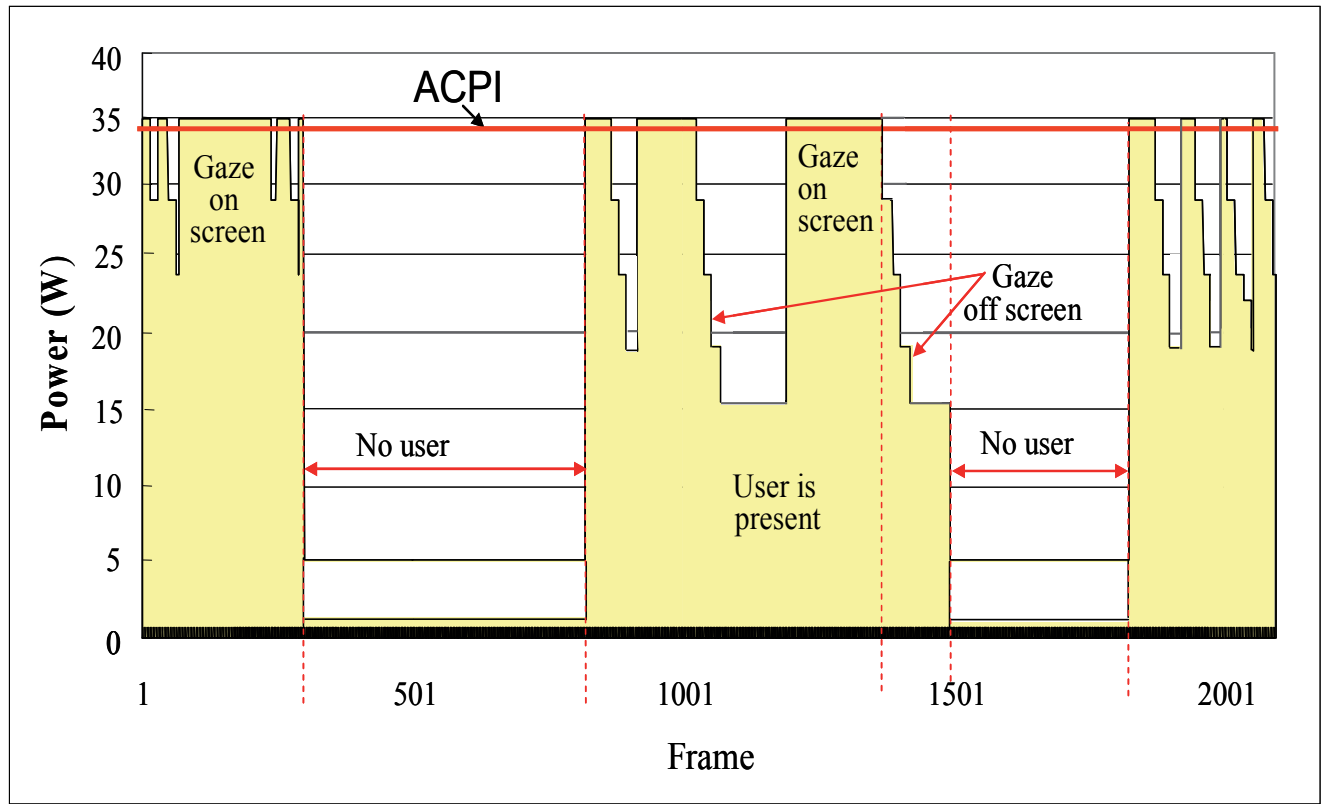

Fig. 9. Power consumption vs. image frame for the tested display

total number of all decisions made. As the tests showed, the eye detection and tracking accuracy of proposed hardware is quite high ( $88 \%$ on average).

Next, we estimated the energy efficiency of the proposed system by measuring the total power consumption taken from the wall by the system itself and the 17" IO-DATA TFT LCD display (35W on peak, eight different levels of screen brightness) controlled by the system. Fig. 9 profiles the results measured per frame on a 100sec (2000frames) long test. In the test, the user was present in front of the display (frames 1-299, 819-1491, 1823-2001); moved a little from the display but still present in the camera view (frames 1300 to 1491); and stepped away from the PC disappearing from the camera (frames 300-818, 1492-1822). The system was set to step down from the current power level if the eye-gaze off the screen was continuously detected for more than 15 frames (i.e. almost $1 \mathrm{sec}$ ). The ACPI line shows the power consumption level ensured by the conventional ACPI power management.

We observe that our technology is very effective. It changes the display power accordingly to the user behavior; dimming the display when the user gaze is off the screen and illuminating the screen (by elevated power) when the user looks on it. Changing the 

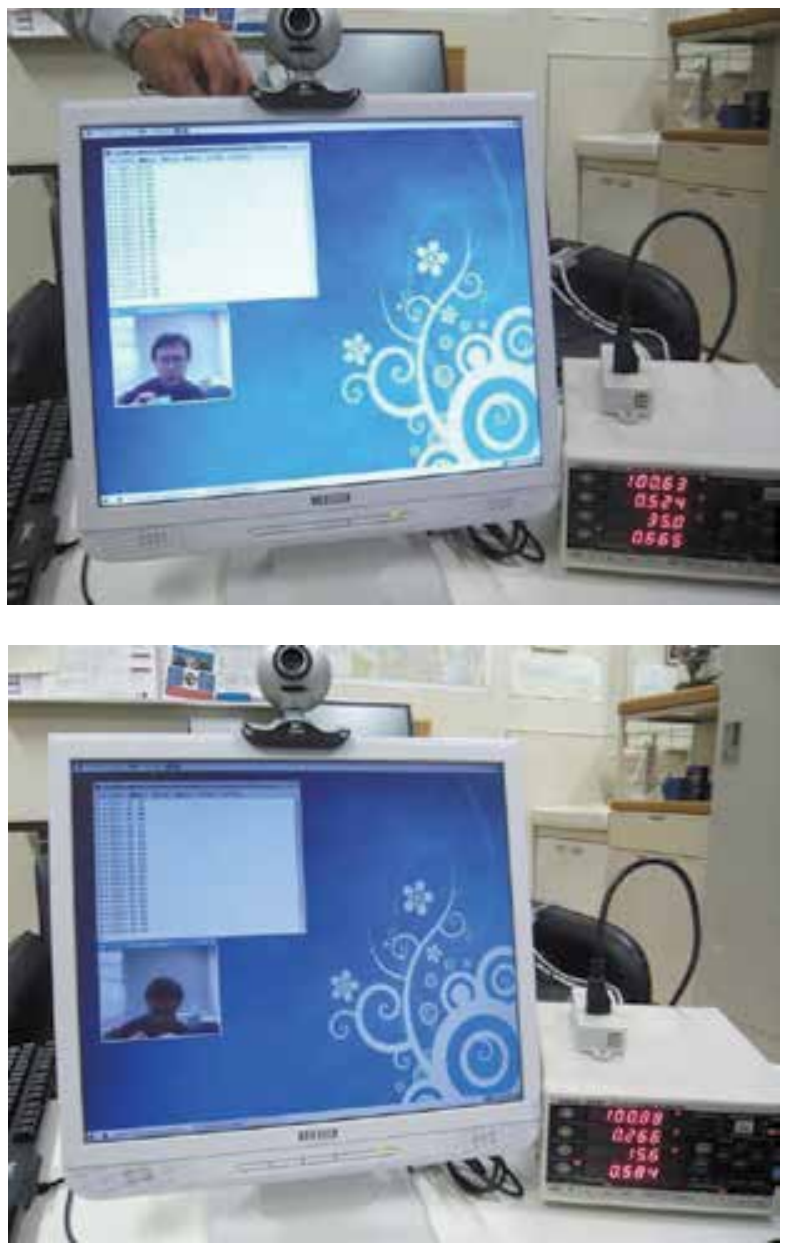

Fig. 10. Screenshots of display and corresponding power consumption: when the user looks at screen, the screen is bright (power: $35 \mathrm{~W}$ ); else the screen is dimmed (power: $15.6 \mathrm{~W}$ )

brightness from one power level to another in our system takes only $20 \mathrm{~ms}$, which is unobservable for the user. Fig. 10 shows the brightness of the screenshots and the corresponding power consumption level (see the figures which are displayed on the downright corner of the pictures; the second row from the bottom shows the power).

The total power overhead of the system is $960 \mathrm{~mW}$. Even though the system takes a little more power than ACPI (see horizontal line in Fig.9) in active mode on this short test, it saves $36 \%$ of the total energy consumed by the display. In environments when users frequently detract their attention from the screen or leave computers unattended (e.g. school, university, office) the energy savings could be significant.

\subsection{The camera based TV energy management}

\subsubsection{An overview}

The methodology used for camera-based TV energy management is similar to that discussed in Section 3.1 with the difference that for TV it monitors multiple viewers simultaneously. 


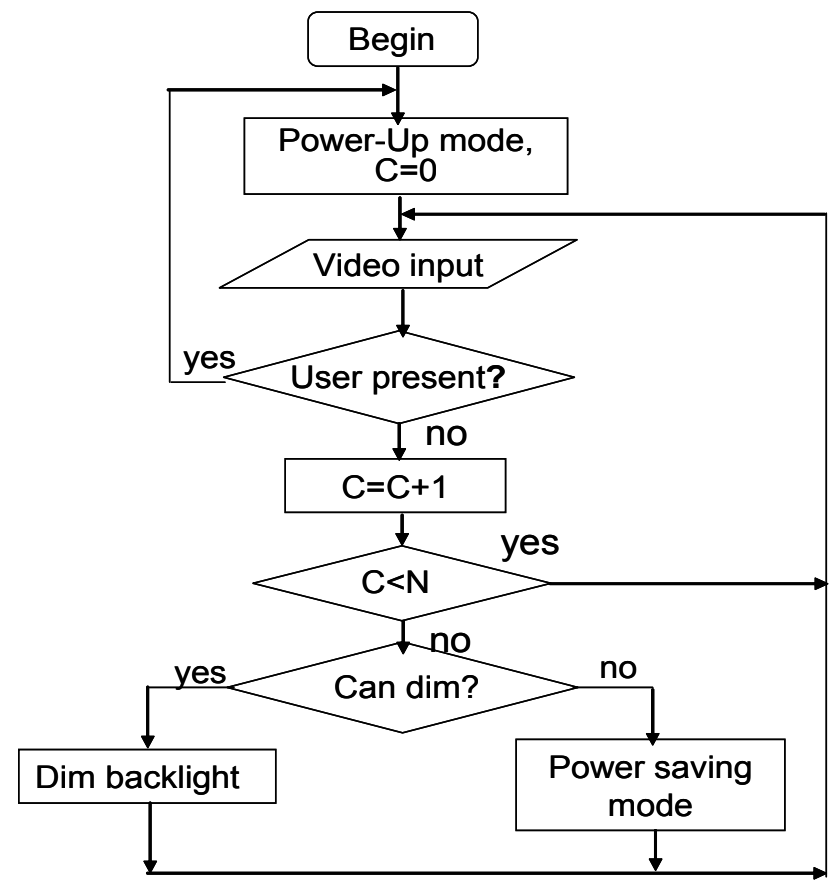

Fig. 11. Flowchart of the camera-based TV energy management

Also, because TV screens are much larger than PC displays, the TV viewers can be on a far distance from camera than the PC users. As result, detecting gaze of TV viewers correctly based on a typical CMOS sensor or web camera actually becomes impossible. Therefore for $\mathrm{TV}$, we track faces (no eyes), assuming that viewers, who watch TV, face the screen (and camera) frontally. Besides, people sometime watch TV in darkness when ordinary CMOS image sensors are almost useless. In such conditions, the only device which can be used is an infra-red camera or IR-vision sensor.

Fig. 11 shows the flowchart of the proposed TV energy management. Here C denotes a counter and $\mathrm{N}$ is a given threshold. By default, the TV screen is bright providing the best picture quality. Every image frame captured by the camera is scanned to detect a human face. If neither motion nor human face is detected (i.e. no viewer is present or present but not looking at the TV screen) in $\mathrm{N}$ consecutive frames, the screen is dimmed to a lower level of screen brightness. If the screen brightness has already reached the lowest level and can not be dimmed anymore while no human face has been detected for $\mathrm{N}$ consecutive frames, the screen is turned to the sleep mode (i.e. black screen) while the audio system (i.e. sound) is kept ON. Any on-screen face reactivates the initial backlight luminance by moving the screen onto the normal mode.

\subsubsection{Implementation and evaluation}

To detect a human face we developed in Linux OS a face-detection software by using opensource C-language image processing library "Open-CV" [OpenCV] from Intel. The program detects faces based on a boosted cascade of simple Haar-like features, proposed by Viola and Jones [Viola, 2001]. It provides correct detection of human faces located up to 4 meters away from the camera and \pm 40 degrees in horizontal and vertical directions. 


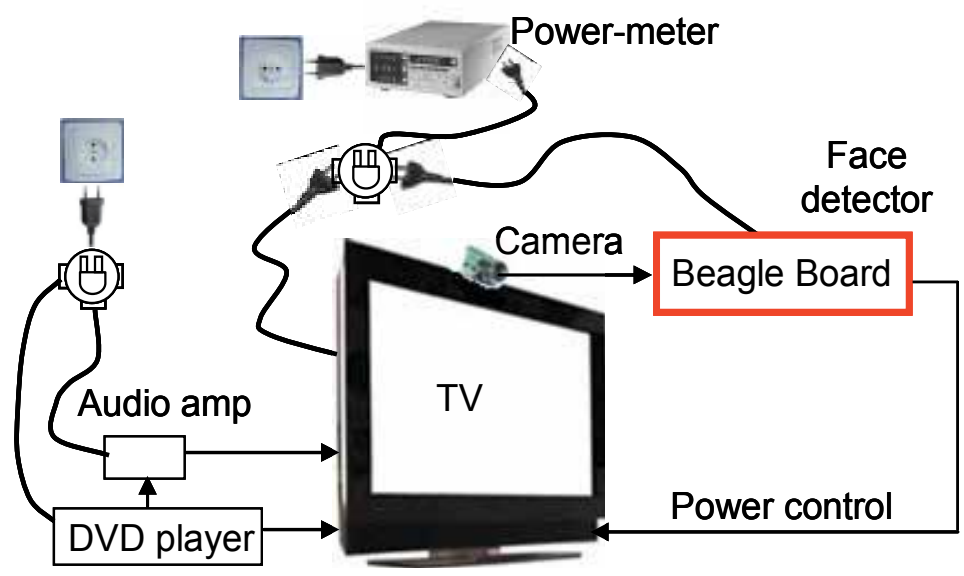

Fig. 12. Experimental system of camera-based TV management

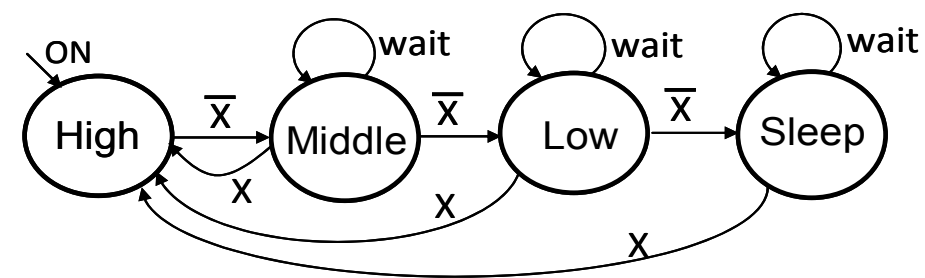

Fig. 13. TV State Transition Diagram

To evaluate the efficiency of the proposed approach, we developed prototype camera-based TV management system illustrated in Fig.12. The core of the system is ARM-based BEAGLE-Board, which runs face-detection and TV power control in Ubuntu OS. The board is connected through RS-232C serial port to 42in NEC LCD V421 TV and through parallel port to video camera $(640 \times 480$ pixel resolution, 30fps) placed at the top of the TV. Images captured by the camera are processed in real time to detect whether there is at least one viewer of the TV screen or not. Based on the detection results, the board generates commands that change the TV brightness and power or even set the TV off. To facilitate experimental measurement, we connect the TV to a DVD player which runs a tested video film. Additionally, to keep the TV's audio system ON while screen is OFF (such mode unfortunately is not supported by the TV), we use a separate audio amplifier connected to the TV.

Fig.13 shows the state transition diagram of the TV control implemented by the board. Here, $\mathrm{X}$ corresponds to a positive result of face detection; 'High', 'Middle' and 'Low' denote states corresponding to the brightness levels 100, 50 and 0, respectively (see Fig.14); 'Sleep' represents the state with dark screen (backlight off) and audio ON. The wait time in each state was set to $5 \mathrm{sec}$ in our system. The transition time from a higher brightness state to a lower brightness state was a few milliseconds; the time of High-brightness state reactivation from the Sleep state was also $5 \mathrm{sec}$. According to our measurement, the Beagle-Board consumed $4 \mathrm{~W}$ of power when running the face detection. The camera consumed $0.5 \mathrm{~W}$. Therefore the overhead of our software based implementation of face detection was less than 5 Watt. 


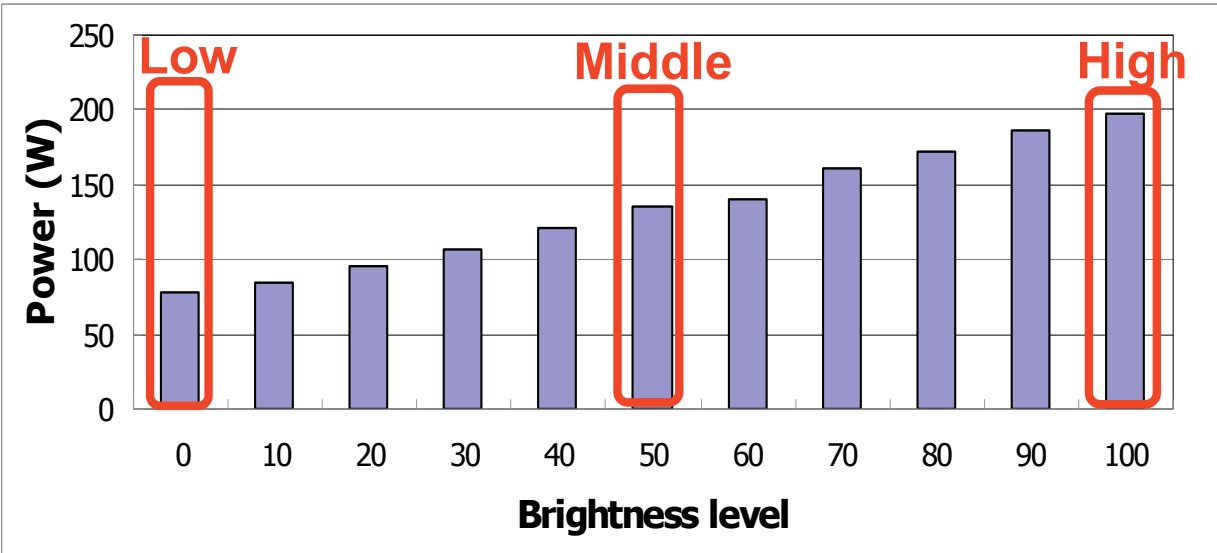

Fig. 14. The dependency of TV power consumption on brightness. The brightness levels corresponding to selected power states are shown in red.

To evaluate energy efficiency of the proposed approach, we performed a number of tests, each of each differed by the number of viewers, viewer behavior, the duration of time the TV was viewed, the activities simultaneously done while watching TV, etc. (More details about the tests can be found in [Moshnyaga 2011]). In all these tests, we measured the total energy taken from the wall by all components of our system (TV, Beagle-board and camera) and compared it to the energy consumed by TV in the motion-based screen-off mode, which was set to the shortest ( $5 \mathrm{~min}$ ) period of inactivity.

The results reveal that the proposed energy management technology performs better then Motion-Based Power Management (MBPM) when the TV users are either frequently detracted from the screen by other activities or use it mainly for listening (as radio), not watching. Even with the shortest time setting, MBPM technique was unable to save energy most of the time because of the viewer's motion. In contrast, the energy saving achieved by our method are high (up to 50-90\%). Obviously, the savings depend on the user behavior.

If the viewer is not disrupted from TV by other activities, the proposed method adds 5 Watt per hour overhead to the TV energy consumption. However, in comparison to TV power of $200 \mathrm{~W}$ it is quite small. Moreover, whenever a $200 \mathrm{~W}$ TV is left unwatched for longer than 1.2 min per hour, the proposed camera-based energy management works better than existing motion-based user sensing. Fig.15 shows the screenshots of TV screen, camera readings on PC display and the power meter: when there is a TV viewer, the screen is in High Brightness mode (power: 206.4W); else the screen is dimmed and eventually enters sleep mode- bottom picture (power: $5.2 \mathrm{~W}$ ).

Fig.16 exemplifies the TV power consumption during typical 2 hours long TV watching by two users. The power bursts in the figure correspond to the screen activation when the viewer returns his gaze to the screen. Notice, the MBPM takes around 200W all the time independently of the viewer behavior. Even though the power savings achieved by our CBPM system in comparison to MBPM on this test were not as impressive as on the other tests there was quite large: $29 \%$. 

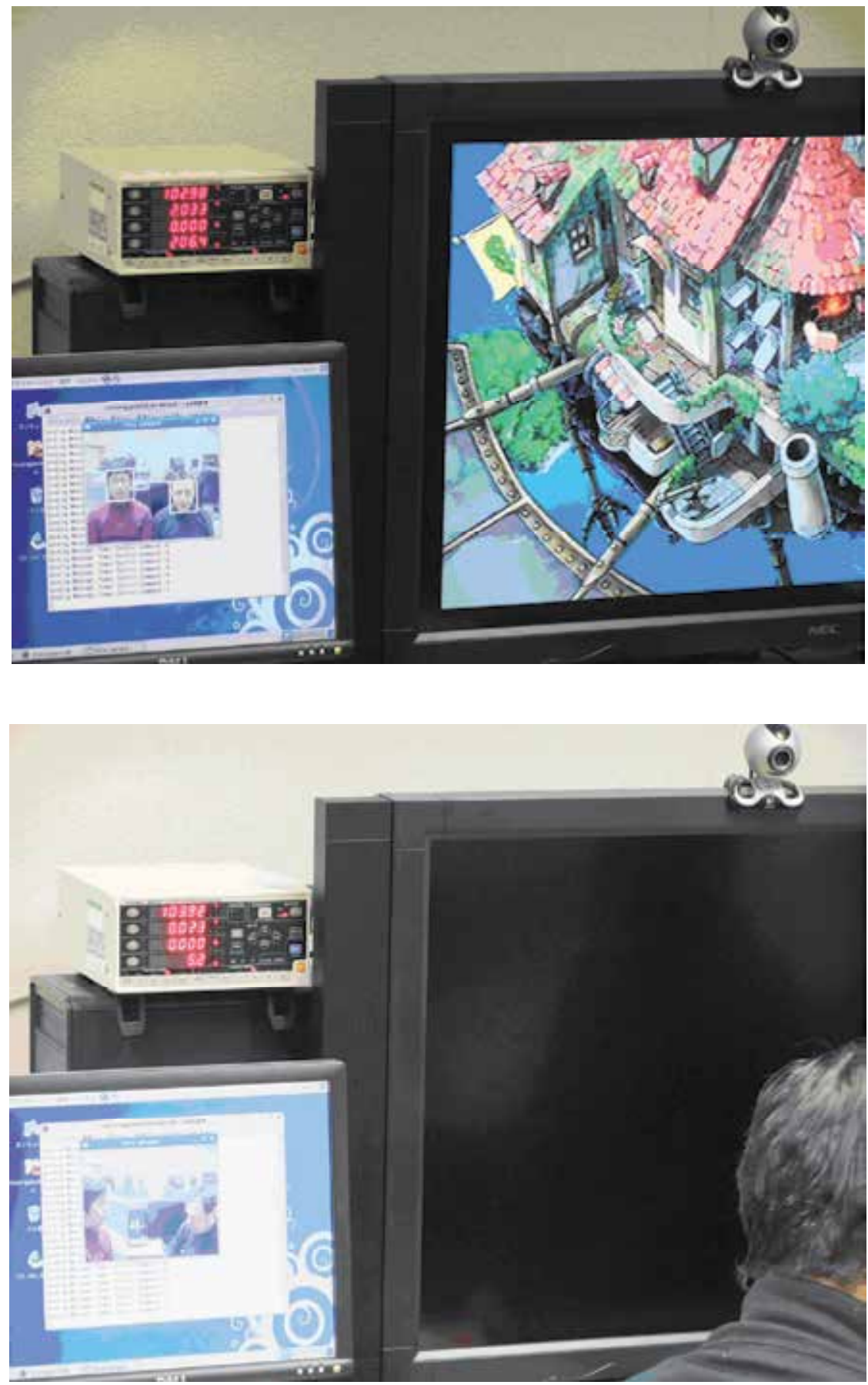

Fig. 15. Screenshots of TV and corresponding power consumption: when viewers looks at screen, the screen is bright (power: $206.4 \mathrm{~W}$ ); else the screen is dimmed (power: $5.2 \mathrm{~W}$ ) 


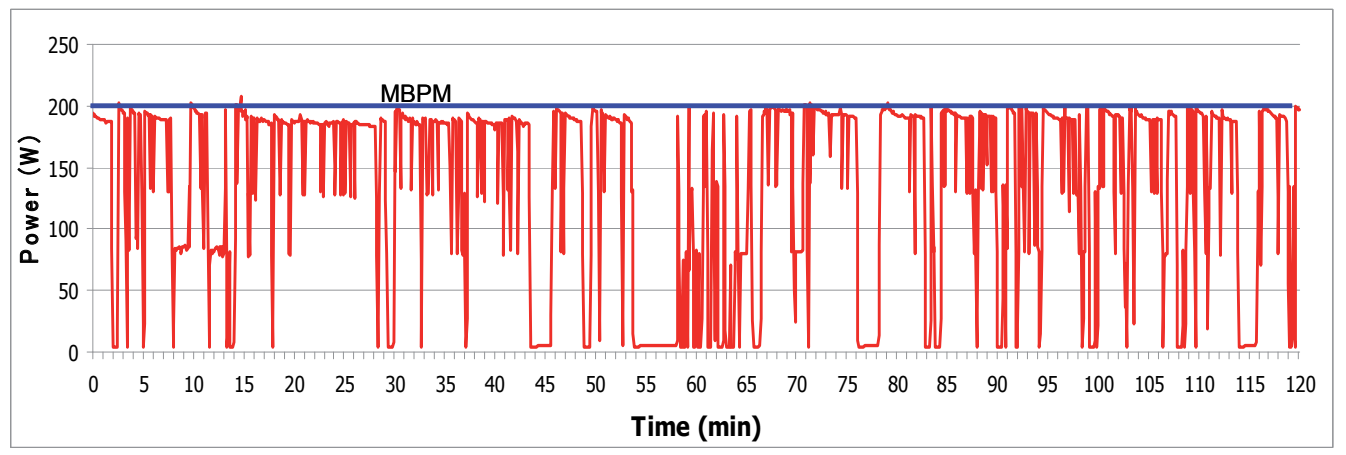

Fig. 16. A profile of power consumed by the proposed camera based power management (CPBM) system in comparison to motion based power management (MBPM) during 2 hours long typical TV watching.

\section{Conclusion}

In this paper we presented a new technology for energy management in computer display and TV set based on camera-based viewer monitoring. For the PC display, we track eyes of the user, while for the TV set -- faces of its viewers, keeping the screen active only when someone looks at it. Experiments showed that the technology saves more energy than existing schemes monitoring viewers behavior in real-time with high accuracy. The current implementation of PC display energy management in FPGA consumes only $1 \mathrm{~W}$ of power while implementation of camera-based TV energy management in low-power embedded system (Beagle-Board) takes only $5 \mathrm{~W}$.

A possible solution to reduce power overhead could be in designing a custom LSI chip for viewer detection, similarly to those implemented in photo camera. This will push the energy overhead to the mW level.

The research presented here is a work in progress and the list of things to improve it is long. In the current work on PC energy management, we restricted ourselves to a simple case of a singular user. However, when talking about the user-gaze monitoring in general, some critical issues arise. For instance, how to handle more than PC user? The main PC user might not look at screen while the others do. Concerning this point, we believe that a feasible solution is to keep the display active while there is someone looking at the screen.

The TV viewer monitoring also has several challenging issues. First, the viewers can be positioned quite far from the TV set. Second, the viewers can watch TV when laying on a bed or a sofa, so the viewer's face can rotate on a large angle. Third, the face illumination condition may change from a very bright to a complete darkness. In these conditions, the correct real-time face monitoring with low-energy overhead becomes really difficult. Our future study will cover the use of IR-camera, impact of face orientation, face color and other issues.

\section{Acknowledgment}

The work was sponsored by The Ministry of Education, Culture, Sports, Science and Technology of Japan under Regional Innovation Cluster Program (Global Type, 2nd Stage) and Grant-in-Aid for Scientific Research (C) No.21500063. 


\section{References}

ACPI: Advanced Configuration and Power Interface Specification, Rev.3.0, Sept.2004, http://www.acpi.info/spec.htm

BeagleBoard: System Reference Manual, Rev.4, available from http:/ / beagleboard.org/

Baluja, S., Pomerlau, D. (1994) Non intrusivegaze tracking using artificial neural networks, Technical report CMU-CS-94-102.

Chang N., Choi I., and Shim H. (2004) DLS: dynamic backlight luminance scaling of liquid crystal display, IEEE Trans. VLSI Systems, vol.12, no.8, pp.837-846.

Cheng W.-C. , Pedram M. (2004) Power minimization in a Backlight TFT-LCD display by concurrent brightness and contrast scaling, Proceedings of the Design Automation and Test in Europe, Vol. 1, pp. 16-20.

Choi L., Shim H., Chang N. (2002) Low power color TFT LCD display for hand-held embedded systems, Proceedings of International Symposium on Low-Power Electronics and Design, pp.112-117.

Coughlan S., (2006) Do flat-screen TVs eat more energy? BBC News, 7 Dec.2006 http://news.bbc.co.uk/2/hi/uk_news/magazine/6188940.stm

Dai X. and Raychandran K., (2003) Computer screen power management through detection of user presence, US Patent 6650322, Nov.18.

Douxchamps D., Campbell N., Robust real time face tracking for the analysis of human behavior, in Machine Learning for multimodal Interaction, LNCS 4892, 1-10, 2002.

Elias E. W. A., Dekoninck E. A., Culley S. J., (2007) The Potential for Domestic Energy Savings through Assessing User Behaviour and Changes in Design, $5^{\text {th }}$ International Symposium on Environmentally Conscious Design and Inverse Manufacturing, Tokyo, Japan, 2007.

Fujitsu-Siemens (2006) Energy savings with personal computers, from http://www.fujitsusiemens.nl/aboutus/sor/energy_saving/prof_desk_prod.html

Flinn J., and Satyanarayanan S. (1999) Energy-aware adaptation for mobile applications, Proceedings of the Symposium on Operating Systems Principles, pp.48-63..

Gatti F., Acquaviva A., Benini L., Ricco B. (2002) Low-power control techniques for TFT LCD displays. Proceedings of the International Conference on Compilers, Architecture and Synthesis for Embedded Systems, pp.218-224

Hewlett-Packard Co. (2006), Global Citizenship Report", available from www.hp.com/ hpinfo/globalcitizenship/gcreport/pdf/hp2006gcreport_lowres.pdf

Generation M2: Media in the lives of 8-18 years old. A Kaiser Family Foundation Study, (2010, June). Henry J. Kaiser Family Foundation, Menlo Park, California, (79 pages)

Gram-Hansen K. (2003), Domestic electricity consumption - consumers and appliances, Nordic Conference on Environmental Social Sciences.

Iranli A., Lee W., Pedram M., (2006)“HVS-Aware Dynamic Backlight Scaling in TFT-LCDs,” IEEE Trans. on Very Large-Scale Integration Systems, Vol. 14, No. 10, pp. 1103-1110.

Ji Q., Zhu Z. (2002) Eye and gaze tracking for interactive graphic display. Proceedings of the ACM Int. Symposium on Smart Graphics.

Kawato S., and Ohya J. (2000) Two-step approach for real-time eye-tracking with a new filtering technique. IEEE Int. Conf. of Systems, Man \& Cybernetics, 1366-1371.

Kawato S., Tetsutani N., Osaka K. (2005) Scale-adaptive face detection and tracking in real time with SSR filters and support vector machine, IEICE Trans. Information $\mathcal{E}$ Systems, E88-D, (12) 2857-2863.

Lee C.G. and Moshnyaga V.G. (2011) TV Energy management by Camera-Based User Monitoring, Proceedings of the IEEE International Symposium on Circuits and Systems. 
Mahesri A., Vardhan V. , (2005) Power Consumption Breakdown on a Modern Laptop, Proceedings of the Power Aware Computer Systems, LNCS, vol.3471,pp.165-180,2005.

Moshnyaga V.G., Morikawa E. (2005) LCD Display Energy Reduction by User Monitoring, Proc. Int. Conf. on Computer Design, pp.94-97.

Moshnyaga V.G., Hashimoto K., Suetsugu T., Higashi S. (2009) A hardware implementation of the user-centric display energy management, LNCS 5953, Springer, 56-65.

Nielsen Media Research Inc., (2009) Television Audience 2009. The Nielsen Company

Nordman, Bruce, Mary Ann Piette, and Kris Kinney. 1996. Measured Energy Savings and Performance ofPower-Managed Personal Computers and Monitors. LBL-38057. Lawrence Berkeley National Lab., available at http://eetd.lbl.gov/EA/Reports/38057/

Nordman, Bruce, Mary Ann Piette, Kris Kinney, and Carrie Webber. 1997. User Guide to Power Management for PCs and Monitors. LBNL-39466. Lawrence Berkeley National Lab., available at: http:/ / eetd.lbl.gov/EA/Reports/39466/

Ohno T., Mukawa N., Kawato S. (2003) Just blink your eyes: a head-free gaze tracking system. Proceedings of the CHI 2003, 950-951.

Open CV: Open Computer Vision Library, available at http://ubaa.net/shared/processing/opencv/

Park, W.I., (1999) Power saving in a portable computer, EU Patent, EP0949557, 1999

Park, R, Kim, J. (2005) Real-time facial and eye gaze tracking system, IEICE Transaction on Information E Systems., E88-D (6), 1231-1238.

Pasricha S. Luthra M., Mohapatra S., Dutt N., and Venkatasubramanian N. Dynamic backlight adaptation for low-power handheld devices, IEEE Design and Test Magazine, Sept/Oct. 2004, pp. 398-405.

Pattanai K.S.N., Tumblin J.E., Yee H., and Greenberg D.P. (2000) Time dependent visual adaptation for realistic image display", Proceedings of the SIGGRAPH, pp.47-54.

Robertson J. , Homan G.K., Mahajan A., et al, (2002) Energy use and power levels in new monitors and personal computers", LBNL-48581, UC Berkeley, July 2002

Plasma TV: Performance Test Results - Power consumption Tests (2006), http://www.plasmadisplaycoalition.org/results/power.php

Sharp Microelectronics of the Americas, (2002), Display Modes :Transmissive/Reflective/ Transflective, available from: http://www.sharpsma.com/sma/Products/ displays/AppRefGuide/DisplayModes.htm

Shim H., Chang N., and Pedram M. (2004 Sept/Oct) A backlight power management framework for the battery-operated multi-media systems. IEEE Design and Test Magazine, pp. 388-396.

Theocharides T., Link G., Vijakrishnan N., Irwin M.J., Wolf W. (2004) Embedded Hardware Face Detection, 17th IEEE Int. Conf. VLSI Design, pp.133-138.

Tumblin J.E. , Hodgins J.K. , and Guenter B.K. (1999) Two methods for display of high contrast images. ACM Transactions on Graphics, Vol.18, no.1, pp. 56-94, Jan.1999.

TV Power Consumption: Is There a Problem? (and Can LCD TVs Help?) LCD TV Association, 2008, available from www.LCDTVAssociation.ORG

Television \& Health, http:/ / www.csun.edu/science/health/docs/tv\&health.html

Viola P. and Jones M. (2001) Rapid object detection using a boosted cascade of simple features. Proceedings of the IEEE International Conference on Computer Vision and Pattern Recognition.

Yamamoto S. and Moshnyaga V.G. (2009) Algorithm optimizations for low-complexity eye tracking. Proceedings of the IEEE International Conference on Systems, Man and Cybernetics, 18-22. 


\title{
Enhancement of Power System State Estimation
}

\author{
Bei Gou ${ }^{1}$ and Weibiao $\mathrm{Wu}^{2}$ \\ ${ }^{1}$ Department of Electrical and Computer Engineering, North Dakota State University \\ ${ }^{2}$ Department of Statistics, University of Chicago \\ USA
}

\section{Introduction}

Power Utility companies use the state estimator to provide system operating status to the operators of their control center to allow them to manage and to take appropriate measures to prevent the loss of electricity. The unavailability of state estimation solution may cause the occurrence of cascading failures or blackouts in local and/or regional areas for considerable time periods, if disturbance occurs during the period of unavailability and thus can not be closely monitored. The robustness and reliability of state estimation is a critical issue and concern of power utilities.

The Weighted Least Square (WLS) method is the commonly used state estimation methodological approach in power industry. If one or more gross errors are contained in the measurements the WLS state estimator may not reach a solution and diverge. A well-known example when the WLS did not converge due to the existence of a topology error was a indirect contributing factor to the August Blackout in Northeastern U.S. in 2003. According to the President's Task Force the operator could not determine the status of the system because of a computer program 'glitch'. This 'glitch' was a failure of the WLS method to converge and give a solution to the State Estimation. Task Force comments noted the 'unacceptability' of such computer program errors when the economic impact of the consequential blackout was so dramatic. The economic damage of the 2003 blackout was reported to be in excess of $\$ 10$ Billion dollars.

The following figure shows the convergence property of WLS state estimation. This figure was obtained on IEEE-118 bus system. WLS state estimation has been simulated on 5000 different patterns of load levels for IEEE 118-bus system. It is clear to see that WLS state estimation will be completely unfunctional after the load level reaches a specific amount. Details of this simulation will be explained later in the chapter.

The need to detect the gross errors is a critical and challenging issue for WLS state estimation. Many researchers have tried to develop algorithms to detect gross errors for WSL state estimation without dramatic success. Most of the detection techniques proposed so far are based on a solution of WLS state estimation. The dilemma is that detecting gross errors requires a solution of state estimation under the presence of gross errors that solution may not occur.

Topology errors are classified in two categories: branch status errors and substation configuration errors (Abur and A.G. Exposito, 2004). The analysis of conditions upon which topology errors can be detected was presented in (K. A. Clements and A. Simoes-Costa, 1988 
and F. F. Wu and E. H. E. Liu, 1989). A geometric interpretation of the measurement residuals for topology errors identification was provided in (K. A. Clements and A. SimoesCosta, 1988) which also proposed a systematic analysis of the normalized residuals to detect the bus configuration errors. Ref. (F. F. Wu and E. H. E. Liu, 1989) presented the effect of measurement equations when including topology errors and proposed a method to detect the topology errors by residual analysis. A method based on the number of measurements labeled as bad data was proposed in (H. J. Koglin et al 1986, H. H. J. Koglin and H. T. Neisius, 1990, and H. J. Koglin and H. T. Neisius, 1993). A robust Huber estimator based on an approximate decoupled model was proposed in (L. Mili et al, 1999) as a means of prechecking the assumed system topology. Effects of topology errors can be considered explicitly by representing the circuit breakers in terms of the real and reactive power flows (Monticelli and A. Garcia, 1991, Monticelli, 1993, and Monticelli, 1993). Observability of breaker flows and cases of undetectable breaker status errors are identifies by the WLAV estimator (Abur et al, 1995). LAV was also used to detect the topology errors in (H. Singh and F. L. Alvarado, 1995). A generalized state estimation was proposed to identify topology errors in (E. M. Lourenco, et al, 2004, and O. Alsac, et al, 1998).

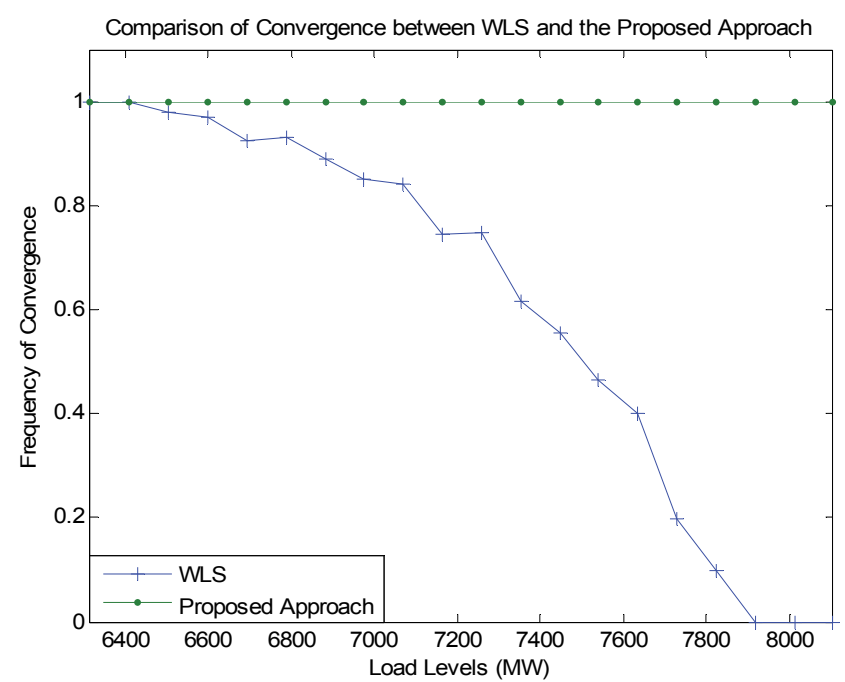

Fig. 1. Divergence rate of WLS state estimator for different load levels in IEEE 118 bus test system.

The newly developed disruptive state estimator is based on a totally different philosophy that does not require a solution of state estimation. As the divergence of the WLS state estimation occurs far too frequently it is to the new approach's merit that a solution of the system is not needed. This new innovative approach also is able to provide a reasonable state estimation solution under any circumstance.

\section{Proposed bad data processing algorithm}

For a transmission line, if the voltage at one end and parameters of the line are known, then the voltage of the other end can be uniquely calculated from the power flow on this line. The 
idea can be applied to the entire system: if a tree formed by branch flow measurements and the root voltage is known, then the voltages of the whole system can be uniquely calculated (P. Bonanomi and G. Gramberg, 1983). The idea is re-studied in this paper.

The proposed algorithms in this paper are totally different from the one in (P. Bonanomi and G. Gramberg, 1983):

1. The tree defined above in (P. Bonanomi and G. Gramberg, 1983) does not always exist and the authors of (P. Bonanomi and G. Gramberg, 1983) did not solve this problem (see discussion in (P. Bonanomi and G. Gramberg, 1983)). This paper solves this problem by introducing an Extended Solving Tree. With suitable adjustment, the PI's proposed algorithms of observability analysis (Bei Gou, 2007, Bei Gou and Ali Abur, 2000, Bei Gou and Ali Abur, 2001, Bei Gou, 2006) can be used to find an extended solving tree and the redundant measurements for all the measurements in the extended solving tree;

2. The bad data detection method is totally different: (P. Bonanomi and G. Gramberg, 1983) made use of KCL and KVL laws and this paper uses the residuals of redundant measurements which is clearer and more efficient in bad data detection;

3. This paper proposes an non-iterative robust state estimation which is equivalent to the weighted least square, and therefore the best estimates of the states can always be obtained under any circumstances.

\subsection{Extended solving tree}

If there does not exist a tree of measurements to connect all the buses in an island (subnetwork), then this island can be processed individually and solved by using WLS. Then the extended solving tree is defined to be a tree that contains not only transmission lines assigned by measurements but also islands whose sizes are minimized.

In the following context, we will still use solving tree for the description, but it should be note that the description is also true for the extended solving tree.

\section{Definitions}

Before the description, we give the following definitions:

- $\quad$ Bus Distance: the Bus Distance between buses $i$ and $j$ is defined as

$$
d_{i j}=\left|V_{i}-V_{j}\right|=\sqrt{\left(\left|V_{i}\right|-\left|V_{j}\right|\right)^{2}+\left(\theta_{i}-\theta_{j}\right)^{2}} .
$$

- $\quad$ Parent Bus: bus $A$ is called a parent bus of bus $B$ when bus $B$ can be directly solved from bus $A$. A bus can only have one parent bus in a solving tree.

- Children Buses: Bus $A$ is called a children bus of bus $B$ when bus $A$ can be directly solved from bus $B$. A bus can have multiple children buses in a solving tree.

- Ancestor Buses: ancestor buses of bus $A$ are defined to be all the buses solved before bus $A$. Ancestor buses also forms an island.

- Descendent Buses: descendent buses of bus $A$ are defined to be all the buses that can be solved only after bus $A$ is solved. Descendent buses also forms an island.

- Recovered Power Flows of a solving tree: are defined to be the power flows and power injections that are calculated from the solution of the solving tree.

\subsection{Error propagation}

For a solving tree, it is obvious to see that an error present in any of the measurement in the solving tree will be propagated to its descendent buses. We will show that the following Theorem is true. 
Lemma 1: For a given set of redundant measurements, if this set of measurements is perfect, then the solutions of any possible solving trees are identical, and equal to the one when all the measurements are used.

Theorem 2: If a bad data appears in a measurement of a solving tree, then all the recovered power flows corresponding to the redundant measurements of this measurement contain a gross error.

\section{Proof:}

Let us assume all the measurements are perfect except a gross error in a flow measurement $S_{k m}$ (see Fig. 1 for the explanation) that is included in a solving tree $l . S_{k m}$ is a measurement connecting two islands: one is formed by the ancestor buses of $S_{k m}$ and the other is formed by the descendent buses of $S_{k m}$. Suppose a gross error appear in $S_{k m}$. So the voltage $V_{m}$ contains an error. Assume one of the redundant measurements of $S_{r}$ is recovered and equal to $\tilde{S}_{r}$. Now we need to prove that $\tilde{S}_{r}$ is different from $S_{r}$ which is perfect.

We assume that $\tilde{S}_{r}$ equals $S_{r}$

Now if we form a new solving tree $l_{1}$ by including $S_{r}$ in $l$ and discarding $S_{k m}$. The new solving tree forms a tree and can still solve the whole system. Since $\tilde{S}_{r}=S_{r}$, so the solving tree $l_{1}$ obtains the same solution as that of $l$. That means that voltage $V_{m}$ at bus $m$ solved from $l_{1}$ is the same as the voltage solved from the solving tree $l$. And $V_{m}$ contains an error due to the error appearing in $S_{k m}$ in $l$.

However, since all the measurements in the solving tree $l_{1}$ are perfect, Lemma 1 shows that we should obtain an exact solution. That means that the voltage at bus $m$ should be accurate. We reach a contradiction! Therefore, our assumption is wrong. $\tilde{S}_{r}$ does not equal to $S_{r}$. We conclude the proof.

\section{Remarks:}

1. Theorem 2 implies that all the voltages at the descendent buses of a measurement $S_{k m}$ are pushed in-group to a wrong place by the error in $S_{k m}$;

2. Theorem 2 implies that any error including bad data in a measurement of the solving tree, topology error or parameter error in a line of the solving tree, will cause obvious errors in the residuals of the redundant measurements of that measurement.

\section{Examples for theorem 2}

\section{A) Gross error in measurement}

Let us look at an example. In this example, we introduced a gross error (change the sign) to the real power measurement on branch 4-7. In Fig. 2, we can see that some of the voltages showed by ' + ' and ' $O$ ' are overlapped, while other voltages showed by ' + ' are moved down, which indicates the approximately same error is attached to all the descendent buses of bus 4 .

Detection: The recovered power flows, which correspond to the redundant measurements of this measurement, should have big deviations from the redundant measurements. This feature can be used to detect errors in the measurements.

\section{B) Error in branch parameter}

In the same system and measurement configuration, we added an error in the parameter of branch 7-9. The comparison of voltages with and without parameter error is shown in Fig. 3. Detection: Assume the measurement be perfect on the branch 7-9 that has a parameter error. If the measurement on branch 7-9 is replaced by one of its redundant measurements to form 


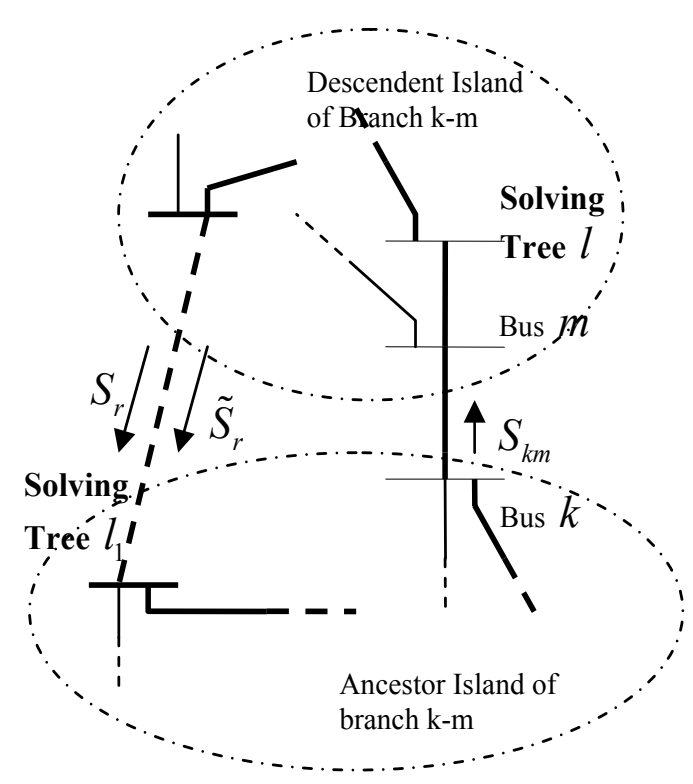

Fig. 2. Explanation of the proof

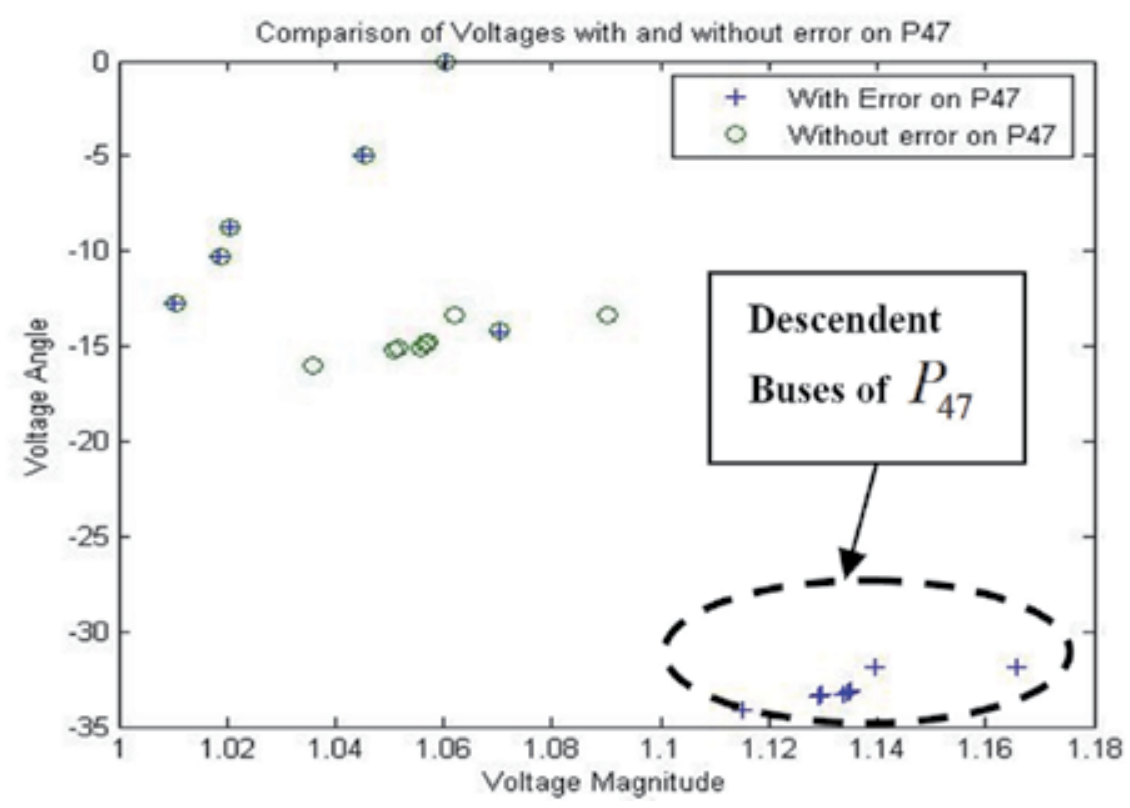

Fig. 3. Comparison of Voltages with and without Errors 
a new solving tree, then the recovered power flow of branch 7-9, calculated from the solution of the new solving tree, should be equal to the measurement on branch 7-9. This feature can be used to detect the branch parameter errors.

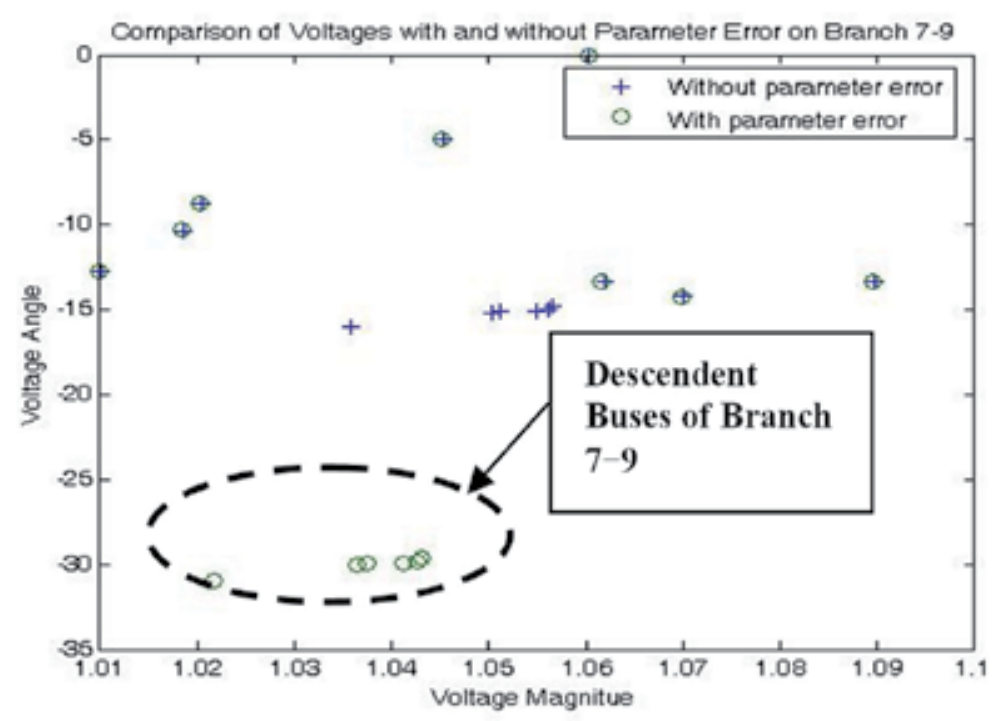

Fig. 4. Comparison of Voltages with and without Parameter Error

C) Topology error

Furthermore, we added a topology error on branch 13-14 that is wrongly considered to be closed while it is actually open. The comparison of voltage with and without topology error shows that only the voltages at the descendent buses of branch 13-14 have errors, which is shown in Fig. 4.

Detection: Assume the injection measurement at bus 14 is error-free. Then the assigned flow measurement on branch 12-13. The voltages between these two buses have very close voltages showing in Fig. 4. This feature can be used to detect the topology error.

\section{Bad data detection}

Once the recovered measurements are obtained from the solution of a solving tree, we are able to detect the bad data. The main idea to detect the bad data is to use the redundant measurements. Here we assume that there are no critical measurements and critical pairs. For a solving tree, every measurement (real flow or reactive flow) has at least two redundant measurements that connect the ancestor island and descendent island of this measurement. The residuals of these redundant measurements (the difference between the redundant measurements and their recovered power flows) can be directly used to detect the bad data. Hypothesis Testing Technique (L. Mili, et al 1984) is used to detect the bad data.

The multiple interacting and confirming bad data can be detected by the proposed bad data detection algorithm. We do not need to study it separately.

\section{Critical measurement and critical pairs}

Comparing the maximum historical bus distance with the bus distance calculated from the solving tree is possibly able to detect the bad data in the measurements. 


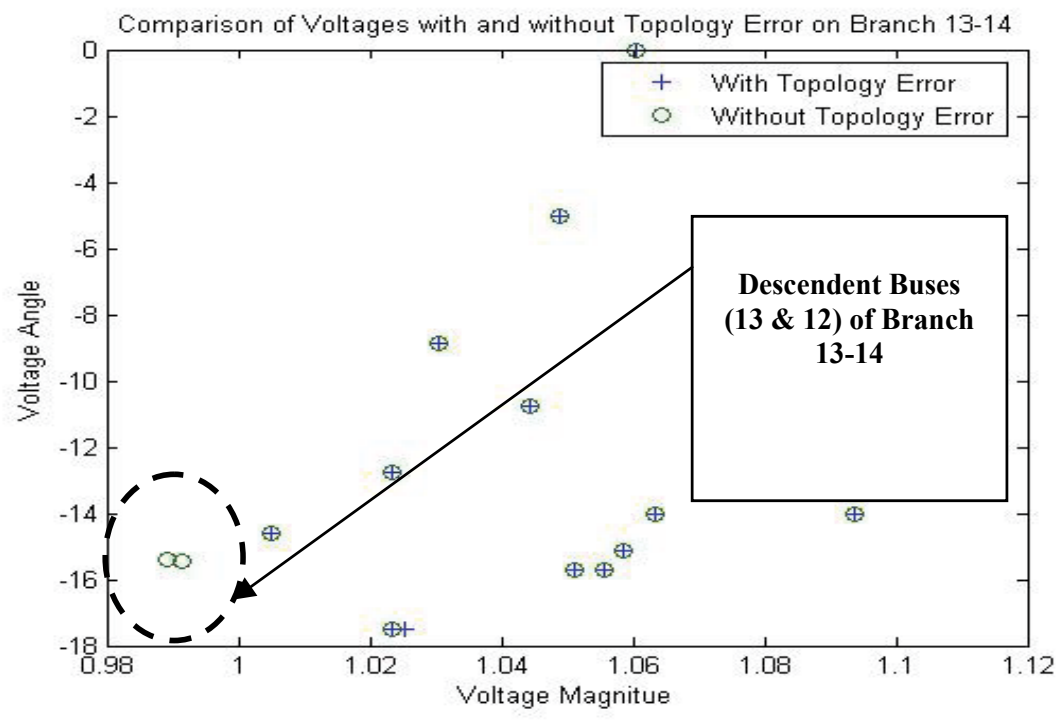

Fig. 5. Comparison of Voltage with and without Topology Error

\section{A numerical test example}

IEEE 14 bus system is used to test the proposed robust state estimator. A solving tree $l$ is found which is given in Figures 1 and 2.

Three gross errors are added: 1 ) a topology error on branch 4-7: an open breaker on branch 4-7 is wrongly considered to be closed; 2 ) a sign change on real power measurement $P_{79}$; and 3) a sign change on reactive power measurement $Q_{9,10}$. The redundant measurements are flows on branches 2-4, 4-2, 4-5, 5-4, 4-9, 9-4, 6-11, 11-6, 6-12, 12-6, 6-13, 13-6, 9-7; injections at buses

A solution is obtained from the solving tree and power flows are recovered. Comparing the residuals of original measurements and the recovered power flows, we found the following measurements having biggest residuals: real and reactive power flow measurements on branches: 2-4, 4-2, 4-5, 5-4, 4-9, 9-4, 6-11, 11-6, 6-12, 12-6, 6-13, 13-6; and real power flow measurement $P_{97}\left(P_{79}\right.$ is a measurement in the solving tree).

Big residuals on branches 2-4, 5-4, 6-11, 6-12, 6-13 show that there must be a gross error in the measurement (injection at bus 3 ) that is assigned to branch 3-4. We removed the injection measurement at bus 3 , and add the measurement on branch 2-4 $\left(P_{24}\right.$ and $\left.Q_{24}\right)$ to form a new solving tree $l_{1}$.

Solve the system by using $l_{1}$ and calculate the residuals of original redundant measurements and their recovered power flows. We found the following branches having big residuals: real and reactive power flow measurements on branches 4-9, 6-11, 6-12, 6-13, and the real power flow $P_{97}$ on branch 9-7. It is obvious that those branches indicate a gross error in the measurement on branch 7-9.

We removed $P_{79}, Q_{79}$ and add $P_{97}, Q_{97}$ on the branch 7-9 to form a new solving tree $l_{2}$.

Solve the system and calculate the residuals, we found $Q_{11,6}$ and $Q_{10,9}$ have big residuals. Their corresponding measurement in $l_{2}$ is $P_{9,10}$ and $Q_{9,10}$. We replaced them with their 
redundant measurements $P_{6,11}$ and $Q_{6,11}$ to form a new solving tree $l_{3}$. The residuals from the solution of solving tree $l_{3}$ are all very small.

Then we concert all the redundant measurements to the solving tree, for example, branch flows on 6-12 and 6-13 were converted to measurements on 1-2, injection at bus 10 was converted to flows on 9-10, voltage magnitude measurement at bus 14 was converted to bus 1 , etc. After all the redundant measurements were converted, we calculated the voltages at all the buses by using the updated solving tree $l_{3}$ and reached the best estimates of IEEE 14bus system (see Table I). White noises having zero mean and 0.001 standard deviation were added to all the measurements.

\begin{tabular}{|c|c|c|}
\hline \multirow{2}{*}{ Bus } & \multicolumn{2}{|c|}{ Tree } \\
\cline { 2 - 3 } & $|V|$ & $\theta$ \\
\hline $\mathbf{1}$ & 1.0610 & 0.0 \\
\hline $\mathbf{2}$ & 1.0459 & -4.980 \\
\hline $\mathbf{3}$ & 1.0109 & -12.710 \\
\hline $\mathbf{4}$ & 1.0193 & -10.320 \\
\hline $\mathbf{5}$ & 1.0211 & -8.770 \\
\hline $\mathbf{6}$ & 1.0708 & -14.210 \\
\hline $\mathbf{7}$ & 1.0624 & -13.370 \\
\hline $\mathbf{8}$ & 1.0903 & -13.370 \\
\hline $\mathbf{9}$ & 1.0568 & -14.950 \\
\hline $\mathbf{1 0}$ & 1.0514 & -15.110 \\
\hline $\mathbf{1 1}$ & 1.0570 & -14.800 \\
\hline $\mathbf{1 2}$ & 1.0549 & -15.080 \\
\hline $\mathbf{1 3}$ & 1.0502 & -15.160 \\
\hline $\mathbf{1 4}$ & 1.0361 & -16.030 \\
\hline
\end{tabular}

Table 1. Estimates of system states from the solving tree

\section{Simulation results}

The comparison between WLS and our new approach has been performed on IEEE 118 bus system. Three test scenarios that include two random bad data, two random interacting and conforming bad data, and two random topology errors, have been examined. Identical sets of measurements were tested for both approaches. Under light load levels, the new approach is several percent better [99.7\%] than the WLS method [97\%] in detecting topology errors. Under heavy loads where the WLS method frequently fails to reach a solution and the new approach is very superior. 
To validate the new approach we also examined the new approach on a real power system with 5145 buses. A clear delineation between the two methods at a certain load level is apparent, which shows the similar characteristics as IEEE-118 bus system.

A) Comparison-local redundancy method vs. WLS

In this comparison random two bad data were simulated on IEEE-118 bus system with 5000 different load levels.

Random bad data

\begin{tabular}{|c|c|c|}
\hline Method & \# of Divergence & \# of Bad Data Detected \\
\hline $\begin{array}{c}\text { New } \\
\text { Approach }\end{array}$ & $0 \%$ & $91.2 \%$ \\
\hline WLS & $61.5 \%$ & $36.2 \%$ \\
\hline
\end{tabular}

Table 2. Comparison of convergence of the proposed algorithm with the WLS state estimation

The simulation results are displayed in above table, which shows that the new approach always converges and is able to detect the bad data for most of the cases. Fig. 5 shows the comparison of bad data detection between WLS and the new approach. It is obvious to see that the new approach is much more robust than WLS for all different load levels.

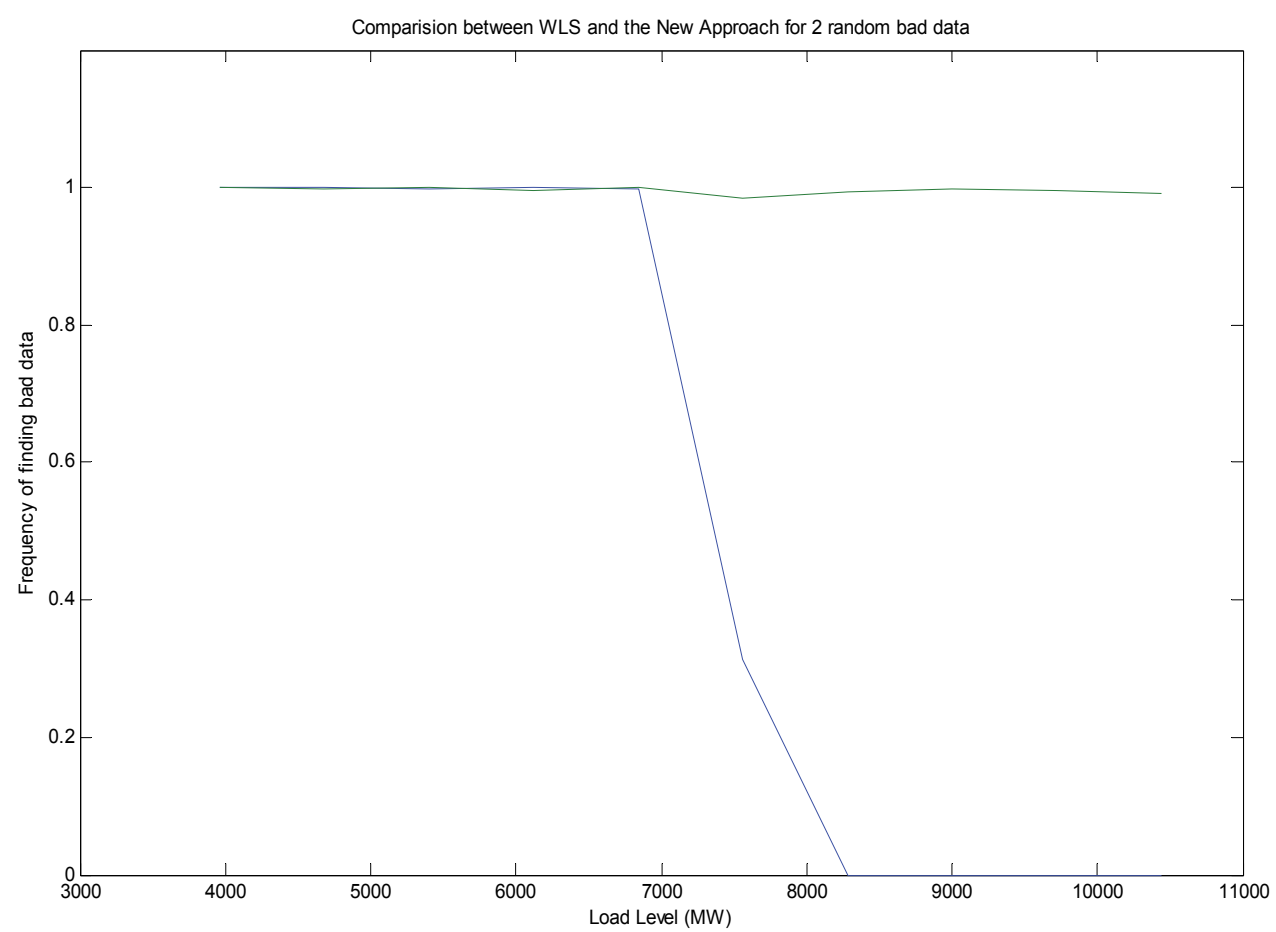

Fig. 6. Comparison of bad data detection between the new approach and WLS 
Interacting and conforming bad data

It is known that WLS state estimation has difficulty in detecting the interacting and conforming bad data. This simulation is aimed to test the detection capability of the new approach and its comparison to WLS state estimation. The following table shows the results of comparison. The comparison indicates that the new approach is much more robust than the traditional WLS stat estimation.

\begin{tabular}{|c|c|c|}
\hline Method & \# of Divergence & \# of Bad Data Detected \\
\hline $\begin{array}{c}\text { New } \\
\text { Approach }\end{array}$ & $0 \%$ & $89.8 \%$ \\
\hline WLS & $50.2 \%$ & $38.1 \%$ \\
\hline
\end{tabular}

Table 3. Comparison of convergence of the proposed algorithm with the WLS state estimation

Fig. 6 shows the comparison of multiple interacting and conforming bad data detection between the new approach and WLS state estimation. The comparison implies that the new approach is much more robust than traditional WLS state estimation and has higher percentage of error detection in light load levels.

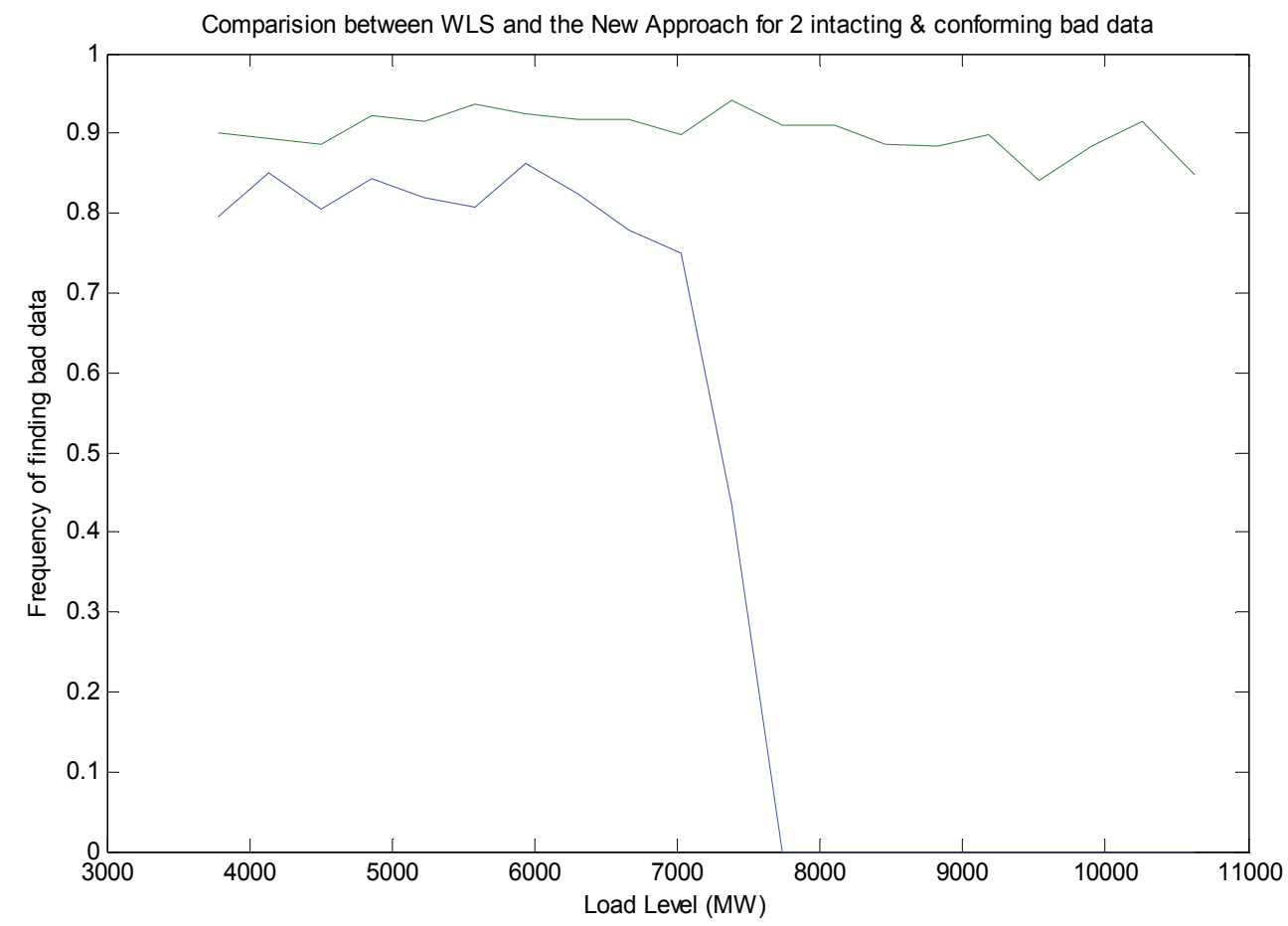

Fig. 7. Comparison of interacting and conforming bad data detection between the new approach and WLS 
Topology errors

Topology error is currently the biggest concern of power system operators, especially after the occurrence of North American Blackout in August 2003. Topology errors are severe gross errors, which can make the state estimation fail to converge. This simulation is to test the capability of the new approach in detecting topology errors. The following table shows the comparison of topology error detection between traditional WLS state estimation and the new approach. It is obvious to see that the new approach is much more robust.

Fig. 7 shows the comparison of topology error detection between traditional WLS state estimation and the new approach, under different load levels. The results indicates that WLS state estimation has good detection capability only when the system load is light; the topology error detection capability of WLS drops very fast if the load level reaches a certain amount. On the other hand, the new approach has a very stable capability of topology error detection. It is obvious that the new approach is much more robust than traditional WLS state estimation.

\begin{tabular}{|c|c|c|}
\hline Method & \# of Divergence & \# of Bad Data Detected \\
\hline $\begin{array}{c}\text { New } \\
\text { Approach }\end{array}$ & $0 \%$ & $99.5 \%$ \\
\hline WLS & $53 \%$ & $46.7 \%$ \\
\hline
\end{tabular}

Table 4. Comparison of convergence of the proposed algorithm with the WLS state estimation

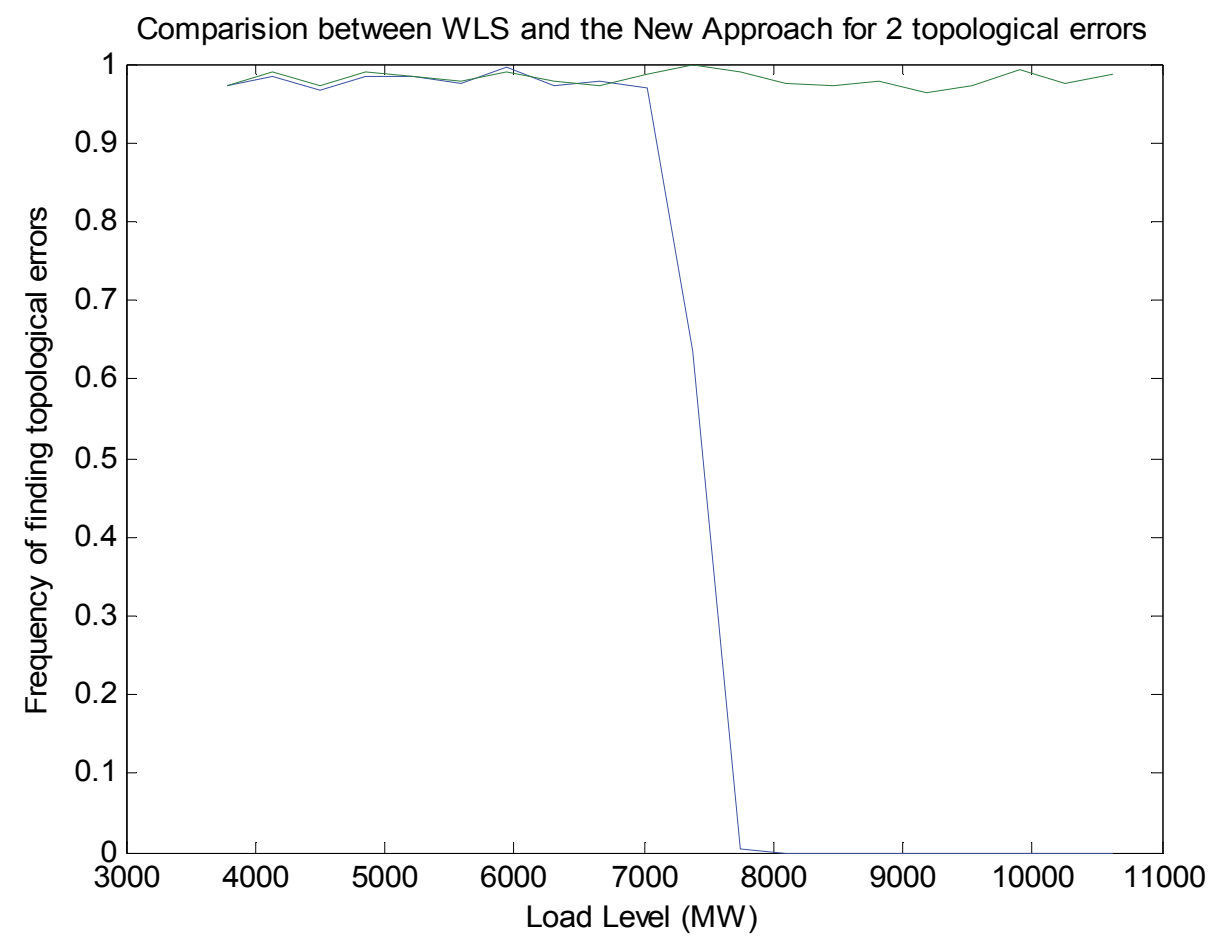

Fig. 8. Comparison of topology error detection between the new approach and WLS 
Comparison on a real system with 5145 buses

A real power system with 5145 buses was used to test the new approach. Because from the above tests we found that the divergence of WLS state estimation is the main reason of nonrobustness of WLS state estimation, we only compare the convergence ability of the new approach and WLS state estimation on this real system. In this test 6658 branch flow measurements, 1000 injection measurements and 500 voltage measurements are used. 500 sets of measurements were generated by randomly selecting the locations of all the measurements. Here are the results of this test.

The new approach

The new approach was able to get convergent and accurate solutions for these 500 sets of measurements.

\section{WLS state estimation}

WLS state estimation failed to get solutions for 169 sets of measurements, and converged to unacceptable solution for 165 sets of measurements, and only converged to accurate solutions for 165 sets of measurements.

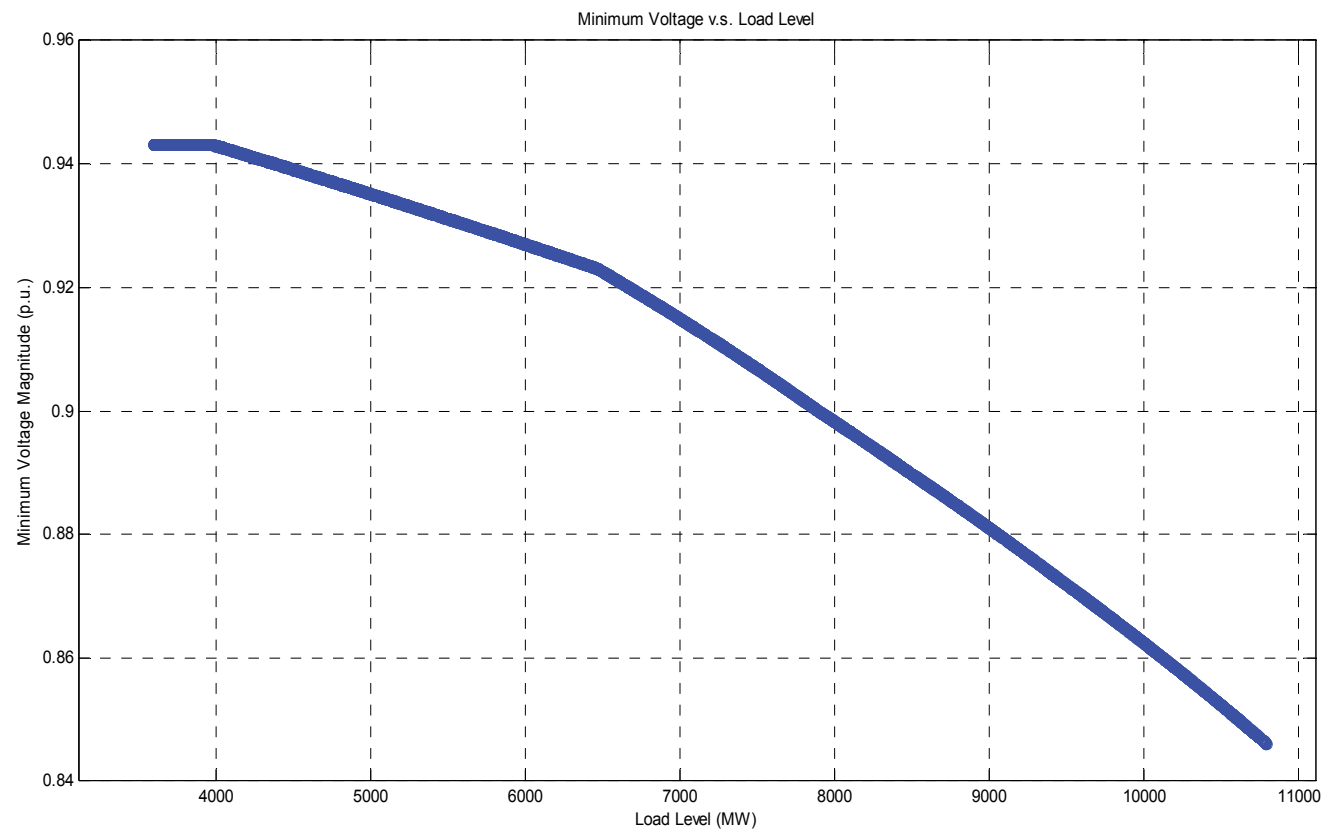

Fig. 9. Minimum voltage magnitudes for 500 sets of measurements.

\section{Conclusion}

Since topology error is currently the main concern of power system operators, it is very important to develop a robust state estimation that is robust and not dependent on a solution of state estimation. A new approach of topology error detection has been proposed in this paper. The newly developed disruptive state estimator is based on a totally different philosophy that does not require a solution of state estimation. As the divergence of the WLS 
state estimation occurs far too frequently it is to the new approach's merit that a solution of the system is not needed. Various tests have been conducted on IEEE 118-bus system and a real system with 5145 buses. The results show that the new approach is much more robust than traditional WLS state estimation, and it is ready to be applied in power industry.

\section{References}

Abur and A.G. Exposito, (2004). Power System State Estimation: Theory and Implementation, Marcel Dekker, ISBN 0824755707

K. A. Clements and A. Simoes-Costa, (1988). Detection and Identification of Topology Errors in Electric Power Systems, IEEE Trans. on Power Systems, Vol. 3, No. 4, pp. 17481753

F. F. Wu and E. H. E. Liu, (1989). Detection of Topology Errors by State Estimation, IEEE Trans. on Power Systems, Vol. 4, pp. 176-183

H. J. Koglin, D. Oeding and K. D. Schmitt, (1986). Identification of Topology Errors in State Estimation, IEE International Conference on Power System Monitoring and Control,

Durham, pp. 140-144 H. H. J. Koglin and H. T. Neisius, (1990). Treatment of Topology Errors in Substations, Proc. 10 th Power Systems Computation Conference, Butterworth, Graz, pp. 1045-1053

H. J. Koglin and H. T. Neisius, (1993). A Topology Processor Based on State Estimation, Proceedings of the 11th Power Systems Computation Conference, pp. 633-638, Avignon

L. Mili, G. Steeno, F. Dobraca, and D. French, (1999). A Robust Estimation Method for Topology Error Identification, IEEE Transactions on Power Systems, Vol. 14, No. 4, pp. $1469-1476$

Monticelli and A. Garcia, (1991). Modeling Zero Impedance Branches in Power System State Estimation, IEEE Trans. on Power Systems, Vol. 6, No. 4, pp. 1561-1570

Monticelli, (1993). Modeling Circuit Breakers in Weighted Least Square State Estimation, IEEE Trans. on Power Systems, Vol. 8, No. 3, pp. 1143-1149

Monticelli, (1993). The Impact of Modeling Short Circuit Branches in State Estimation, IEEE Trans. on Power Systems, Vol. 8, No. 1, pp. 364-370

Abur, H. Kim and M. Celik, (1995). Identifying the Unknown Circuit Breaker Status in Power Networks, IEEE Trans. on Power Systems, Vol. 10, No. 4, pp. 2029-2037

H. Singh and F. L. Alvarado, (1995). Network Topology Determination Using Least Absolute Value State Estimation, IEEE Trans. on Power Systems, Vol. 10, No. 3, pp. 1159-1165

E. M. Lourenco, A. S. Costa and K. S. Clements, (2004). Bayesian-based hypothesis testing for topology error identification in generalized state estimation, IEEE Trans. on Power Systems, Vol. 9, No. 2, pp. 1206-1215

O. Alsac, N. Vempati, B. Stott and A. Monticelli, (1998). Generalized state estimation, IEEE Trans. on Power Systems, Vol. 13, No. 3, pp. 1069-1075

P. Bonanomi and G. Gramberg, (1983). Power System Data Validation and State Calculation by Network Search Techniques, IEEE Trans. on Power Apparatus and Systems, Vol. PAS-102m No. 1, pp. 238-249

Bei Gou, (2007). Observability Analysis for State Estimation Using Hactel's Augmented Matrix Method, Electric Power Systems Research, Vol. 77, No. 7, pp: 865-875 
Bei Gou and Ali Abur, (2000). A direct numerical method for observability analysis, IEEE Trans. on Power Systems, Vol. 15, No. 2, pp. 625-630

Bei Gou and Ali Abur, (2001). An improved measurement placement algorithm for network observability, IEEE Trans. on Power Systems, Vol. 16, No. 4, pp. 819-824

Bei Gou, (2006). Jacobian matrix-based observability analysis for state estimation, IEEE Trans. on Power Systems, Vol. 21, No. 1, pp. 348-356

L. Mili, Th. van Cutsem, and M. Ribbens-Pavella, (1984). Hypothesis Testing Identification: a New Method for Bad Data Analysis in Power System State Estimation, IEEE Transactions on Power Apparatus and Systems, Vol. PAS-104, No. 11, pp. 32393252 


\title{
Smart Home Services for a Smart Grid
}

\author{
Young-Myoung Kim and Young-Woo Lee \\ KT (Korea Telecom) \\ Korea
}

\section{Introduction}

A smart grid is a next-generation intelligent power grid, created by grafting ICT (Information Communication Technology) onto the current grid, which allows power suppliers and consumers to communicate on a two-way basis in real-time and to improve energy efficiency. Current grids are based on a one-way communication structure where power is generated in plants (utilities) and consumed in homes or buildings. However, in a smart grid, energy delivery and consumption is optimized using a real-time power supply and consumption data based on a two-way interactive communication structure. Therefore, as illustrated in Figure 1, significant improvements are expected throughout the value chain from generation to consumption in a smart grid.

For example, at the generation stage, even unstable intermittent renewable energy sources like solar energy and wind energy can be connected to the grid and large-scale renewable plants can be established. In addition, consumers would be able to generate electricity by building solar or wind generation systems in their own home or building. At the transmission/substation/distribution stages, information on devices and electricity in the grid would be delivered in real time and reliable fault prediction, efficient outage management and automatic self-repair (self healing) would become possible, ensuring a supply of stable electricity.

The most significant change will take place in the consumers' (home/building) stage, where consumers will now be able to use or sell the electricity generated by distributed generation using an energy storage device. Consumers will also be able to buy electricity when the price $^{1}$ is low and sell when the price is high. Consumers will then be able to participate in not only consumption, but also in generation and sales; they will be able to monitor the price of electricity and the information on their energy consumption in real time and change their consumption behavior accordingly.

However, in order to induce real participation from the consumer's side, HAN (Home Area Network) and home energy management systems have to be established first, and the service has to be designed to be user-friendly and based on consumers' values and needs.

In this study, we explored the perception of consumers and the value they experienced when using electricity at home, to identify their unmet needs and suggest a few new services.

\footnotetext{
${ }^{1}$ Utilities are expected to turn to new pricing schemes that adjust the price of electricity based on consumption patterns. Utilities will curb consumption during peak periods when electricity is most expensive, and shift consumption to those times when it is less expensive.
} 
Afterwards, we carried out a survey to evaluate consumers' willingness to use our suggested services and lastly we made suggestions for a successful home service on a smart grid.

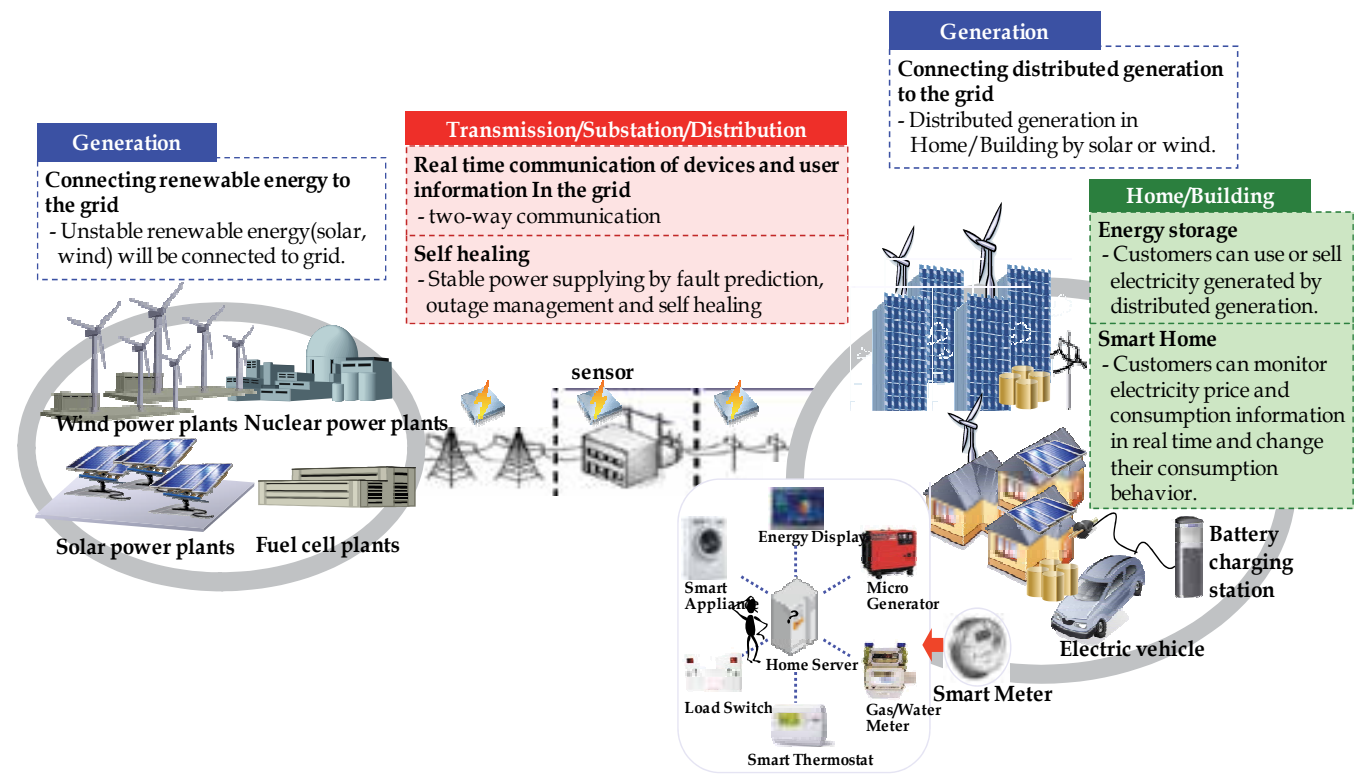

Fig. 1. The characteristic of a smart grid

\section{Outline of the survey}

There were two parts to our study. First, we explored the perception of consumers when using electricity at home and the values they would like to experience qualitatively using a HV (Home Visit) and a FGD (Focus Group Discussion). Afterwards, we carried out a quantitative survey to evaluate our consumers' willingness to use the suggested services. Both studies were carried out in Seoul, Korea.

The HV was performed according to a FLC (Family Life Cycle)-young couples with no children, families with children in K-5th grade, families with children in 6th-12th grade, families with children in college and old couples in an empty nest. The HV was performed in two stages; in the first stage, participants' electricity usage behavior was examined through observational studies and interviews and, in the second stage, changes in perception and attitude toward electricity usage were explored after the participants used electricity-saving products (an electricity inspector and an outlet timer) for some time.

The FGD was also carried out and the group was divided according to FLC in the same way as the above. However, since the participants in these groups were all housewives, we added one more group solely consisting of professional males. In the FGD, we completed a cognitive map to show the value of using electricity.

Lastly, a quantitative survey was carried out with 300 participants who reside in Seoul, Korea and use the electricity service. The sample was selected based on FLC and the survey was completed by interviewing the participants face-to-face. The main purpose of the survey was to identify the key values of using smart grid services and to test people's willingness to use our suggested services. 


\section{Customer perception and their values when using electricty}

Three main perceptions and values when using electricity were derived by using a cognitive map method. First, consumers perceived electricity as an 'indispensable necessity' that makes the use of home appliances possible. Second, consumers perceived electricity as a 'potential danger' that emits harmful electromagnetic waves, causes short circuits and electric shocks and suggested that they need to be safely protected from them. Lastly, consumers perceived electricity as a 'costly energy' that needs to be saved on the user's side and they want the use of electricity to be stress-free.

Figure 2 shows a cognitive map of how consumers perceive electricity as an 'indispensable necessity'. Consumers regarded electricity itself as an 'indispensable necessity', but that indispensability was often conceived as being connected to other home necessities such as a refrigerator, PC, air-conditioner or TV. Consumers displayed positive perceptions using words like warm, cool, bright and convenient toward electricity as it was deemed to be an indispensable necessity in their lives.

Customers also exhibited some negative perception towards electricity; they regarded electricity as a 'potential danger' that emits harmful electromagnetic waves and causes short circuits and electric shocks. As a result of the above, consumers expressed the need for safety during electricity usage. Figure 3 shows a cognitive map of how consumers perceive electricity as a 'potential danger'. In response to the various dangers that electricity usage poses, consumers constantly try to mitigate the threat by placing charcoal or plants known to absorb harmful electromagnetic waves in the house, dusting off outlets and unplugging unused appliances.

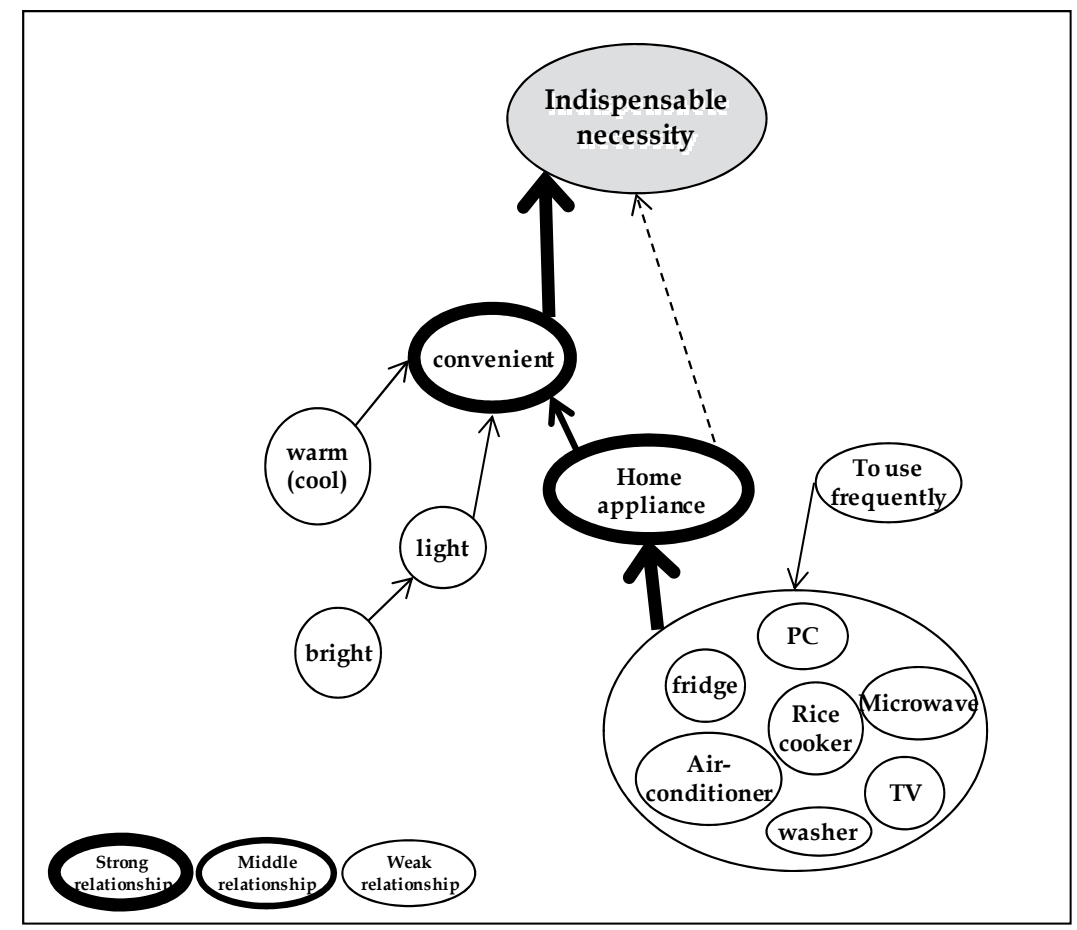

Fig. 2. Cognitive map (1) : Indispensable necessity 


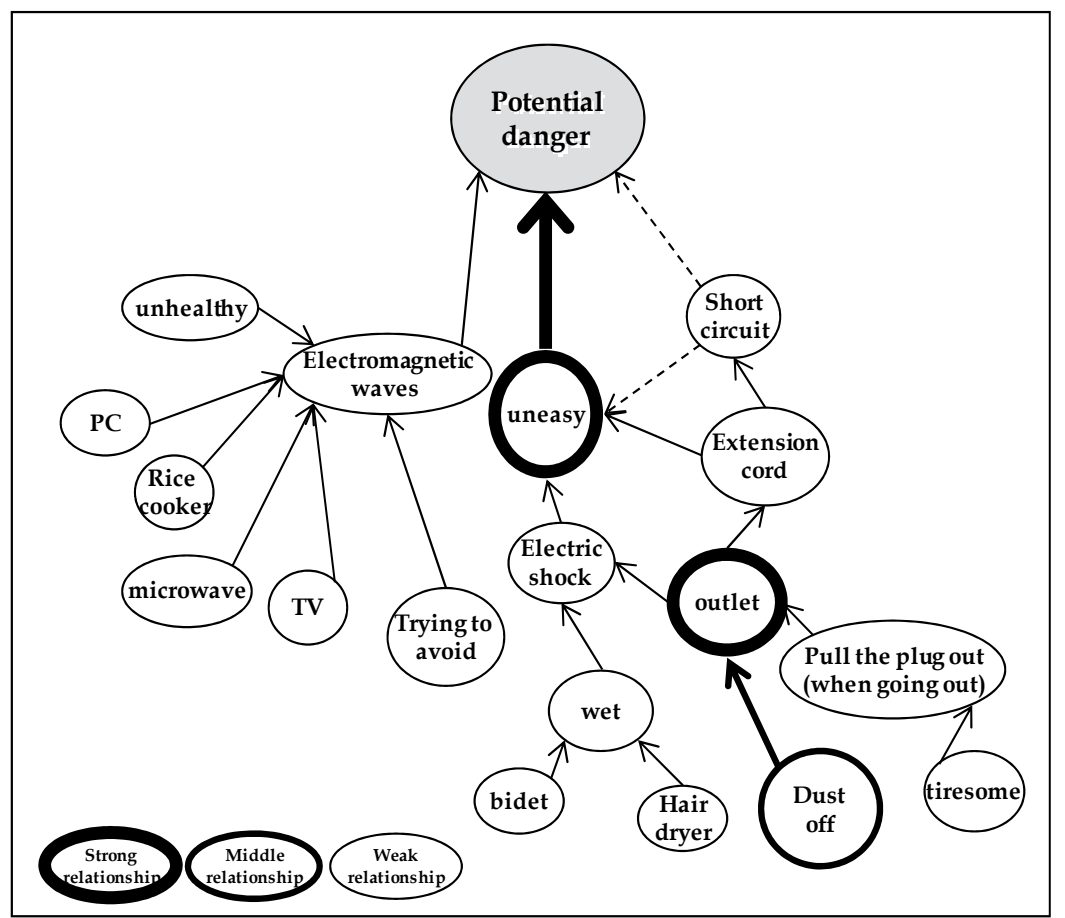

Fig. 3. Cognitive map (2) : Potential danger

Lastly, Figure 4 shows how consumers perceive electricity as a 'costly energy' that needs to be saved on the consumer side. In Korea, electricity rates are cumulative, which means consumers with a higher electricity consumption are charged at a higher rate. For example, the electricity price per kwh when consumers use over $500 \mathrm{kWh}$ is 11 times greater than the price per $\mathrm{kWh}$ when consumers use under $100 \mathrm{kWh}$. In this system, those who use more electricity are charged at a more expensive rate. Because of this system, most housewives in Korea are well aware of the cost of using electricity and feel the need to save money by wisely consuming electricity. Each housewife has their own way to save electricity usage but they are also feel tired of the pressure of being wise electricity consumer and are irked by the wasteful usage of other family members.

\section{Electricity usage behavior and unmet needs}

\subsection{Reducing electricity usage}

Home appliances can be grouped in three categories based on how frequently they are used; constant use, frequent use and occasional use. The first group is constantly used all throughout the day, thereby needing the power to be on all the time, like a refrigerator. The second group is used frequently throughout the day like a TV and PC and the last group is used occasionally like a hair dryer. Consumers show concerns about the power usage of appliances that are frequently used and try to turn off the power or unplug the cord in an attempt to save electricity usage. Their persistence in their attempts to reduce electricity usage depends on the nature of the appliance and its energy efficiency. For example, if an appliance consumes a lot of electricity (e.g., an air conditioner) or it is simple to unplug, they 


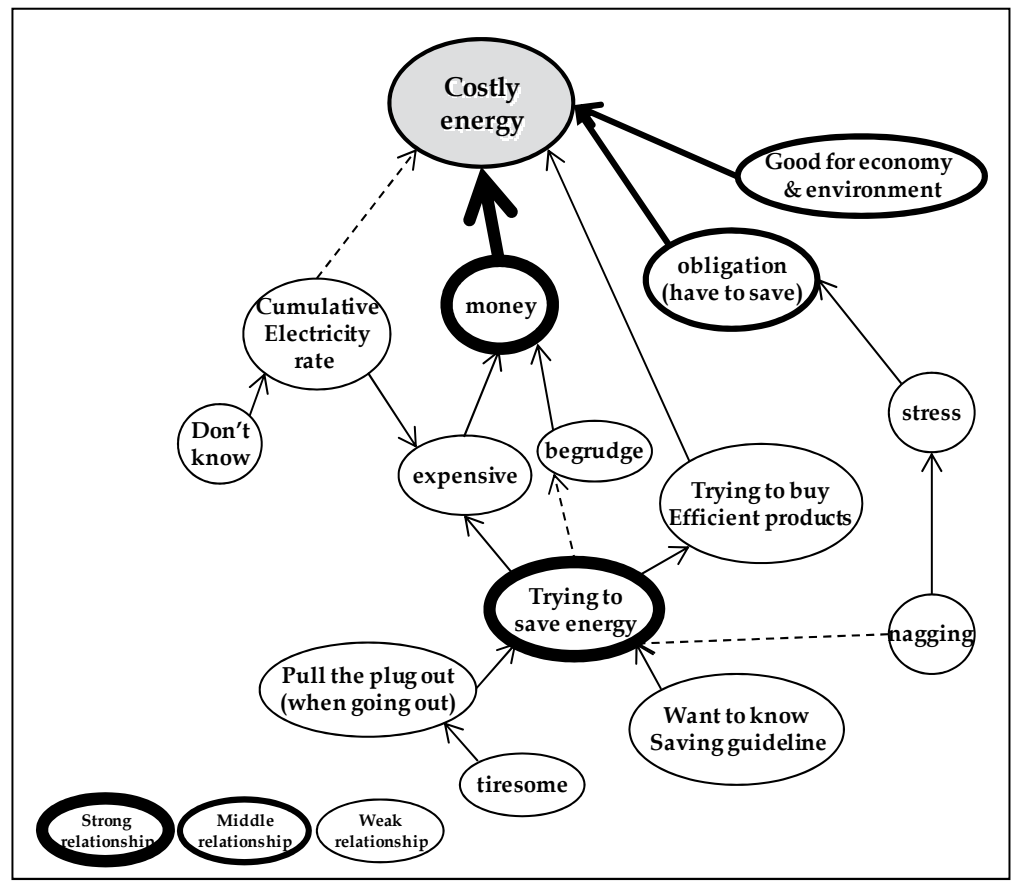

Fig. 4. Cognitive map (3) : Costly energy

just pull the plug out. However, sometimes laziness or fear of electric shocks, particularly from appliances like TVs or PCs that have many cables and complicated power arrangements, discourages consumers from unplugging them. Consumers often read their electronic bills thoroughly when the billed amount is higher than usual and compare their bill with that of their neighbors in order to find what caused a rise in the bill. Consumers try to reduce the use of appliances that use up much electricity and react sensitively toward reducing electricity usage. However, they confess to feeling tired from nagging other family members who react indifferently toward reducing electricity usage.

With regards to saving energy (reducing the use of electricity), most participants agreed with its importance but evaluated themselves as not adequately meeting the standard. 99\% of participants indicated that they agree or strongly agree with the importance of reducing electricity usage. However, only $48 \%$ agreed or strongly agreed that they are doing enough to reduce electricity usage, showing that many consumers feel like what they are doing is not enough in terms of saving energy.

Participants identified 'lack of functionality' in terms of controlling electricity usage, 'lack of information' when informing consumers of their usage behavior and 'lack of motivation' as the main reasons for their struggle to reduce electricity usage. They showed unmet needs in the following areas: a function that blocks the power leakage of an appliance when not in use, a function that informs the users on the level of electricity usage and the energyefficiency of each device, a function that notifies users of the cumulative rate ${ }^{2}$ of electricity usage and a motivator that pushes consumers to reduce their electricity usage.

2 The Korean electricity rate is a cumulative rate, which means the rate changes depending on the total amount of electricity used. So if there is an alarm system that helps consumers to know when their rate is about to move on to the next rate pricing category, it will significantly help them to save money. 


\begin{tabular}{|c|c|}
\hline Type & Behaviors \\
\hline Constant Use & $\begin{array}{l}\text { - } \quad \text { Refrigerator, telephone, water purifier, etc } \\
\text { - Switch is on all day } \\
\text { - } \quad \text { Do not unplug } \\
\text { - } \quad \text { Come are built-in devices } \\
\text { determining to buy these appliances }\end{array}$ \\
\hline Frequent use & $\begin{array}{l}\text { - TV, PC, washing machine, rice cooker, light, dish washer, air } \\
\text { conditioner, fan, etc } \\
\text { 1) Cut the power supply } \\
\text { - Pull the plug out(if the appliances consume a lot of energy or } \\
\text { conveniently set up so that it is easy to unplug) } \\
\text { - Turn off the switch on extension cord } \\
\text { - Check the appliances and turn off the power when not in use (e.g. } \\
\text { light) } \\
\text { 2) Reduce the use } \\
\text { - Use an alternative appliance instead of a high energy consuming one } \\
\text { (e.g., use a fan instead of an air conditioner). } \\
\text { 3) Cheduce the use of the appliance or abstain from using it } \\
\text { - Carefully check the bill and learn of one's own electricity use } \\
\text { - Compare the bill with that of neighbors and find any mistakes or } \\
\text { points for improvement }\end{array}$ \\
\hline Occasional Use & $\begin{array}{l}\text { - } \quad \text { Microwave, electric oven, hair dryer, video game console } \\
\text { - } \quad \text { Usually unplugged, for the safety and cleaner look of the house }\end{array}$ \\
\hline
\end{tabular}

Table 1. Consumers' attempted behaviors of saving the use of electricity

\begin{tabular}{|c|c|}
\hline Pain point & Unmet Needs \\
\hline Lack of functions & $\begin{array}{l}\text { - A function that blocks power leakage of unused appliances after } \\
\text { turning of the power } \\
\text { - A function that cuts the power supply of multiple appliances at once } \\
\text { - A timer function to control the use of electricity more conveniently }\end{array}$ \\
\hline $\begin{array}{l}\text { Lack of } \\
\text { Information }\end{array}$ & $\begin{array}{l}\text { - Information on the electricity usage level of each appliance and of } \\
\text { - } \\
\text { - Infotal } \\
\text { - Information on the level of energy-efficiency on each appliance } \\
\text { Information on efficient ways to reduce the use of electricity (e.g., } \\
\text { knowing which appliances can be operated on reduced energy and } \\
\text { which appliances consume electricity even when in not use) }\end{array}$ \\
\hline $\begin{array}{l}\text { Lack of } \\
\text { Motivator }\end{array}$ & $\begin{array}{l}\text { - A system that evaluates whether the efforts made in an attempt to } \\
\text { reduce the use of electricity have been effective and identifies the } \\
\text { effective ones } \\
\text { - A motivator that stimulates consumers to reduce the use of electricity }\end{array}$ \\
\hline
\end{tabular}

Table 2. Unmet needs of saving use of electricity 


\subsection{Attitude towards the safe use of electricity}

Consumers were worried about the potential threats of using electricity and they engaged in activities geared toward the safe use of electricity. For example, some used products advertised as having the power to protect against harmful electromagnetic waves, such as stickers, coals and plants, while others paid closer attention to arranging appliances in a living room or kitchen to prevent electric shocks and some individuals even got into a habit of unplugging appliances in order to avoid short circuits.

\begin{tabular}{|c|c|}
\hline Potential danger & Behaviors \\
\hline $\begin{array}{l}\text { Harmful } \\
\text { electromagnetic } \\
\text { waves }\end{array}$ & $\begin{array}{l}\text { - Attempt to prevent being exposed to harmful electromagnetic waves } \\
\text { - Appliances on watch-out list: devices that is used over a long period } \\
\text { of time like PC and TV or appliances with high emission of } \\
\text { electromagnetic waves like microwave } \\
\text { - Attempted behaviors to thwart the potential danger: 1) use of } \\
\text { mediums advertised to block the harmful waves such as stickers, } \\
\text { coals and plants 2) avoid being too close to appliances emitting } \\
\text { harmful waves }\end{array}$ \\
\hline Electric & $\begin{array}{l}\text { - Careful with uses of appliances placed in a bathroom or a kitchen } \\
\text { where they may get wet (e.g., hair dryer, bidet, etc) } \\
\text { - Attempted behaviors to thwart the potential danger: being careful } \\
\text { not to get appliances wet }\end{array}$ \\
\hline Short-circuit & $\begin{array}{l}\text { - } \quad \text { Fire or spark due to short circuit (caused by dusts on the outlet) } \\
\text { - } \quad \text { Consider the following as a potential danger: too many cords being } \\
\text { plugged in one outlet, twisted and complicated wiring } \\
\text { - Attempted behaviors to thwart the potential danger: unplug cords } \\
\text { from outlet when leaving house }\end{array}$ \\
\hline
\end{tabular}

Table 3. Behaviors concerning the safe use of electricity

With regards to the safe use of electricity, all agreed with its importance but evaluated themselves as not adequately meeting the standard. All of the polled participants indicated that they agree or strongly agree with the importance of the safe use of electricity. However, only $66 \%$ agreed or strongly agreed that they are currently using electricity safely. Although the feeling of inadequacy was not as strong as it was in the energy reduction case (48\%), this data shows that consumers feel like they are not doing enough to prevent themselves from the potential danger of using electricity.

Although consumers constantly made an effort to protect themselves from the potential dangers of using electricity including harmful electromagnetic waves, electric shocks and short circuits, they still displayed a feeling of uneasiness. They pinpointed unmet needs in the following areas: device safeguards against the emission of harmful electromagnetic waves, a guide on how to safely handle appliances in bathrooms and in kitchens, and a device that automatically shuts off the power if there is a hazard.

\section{Directions for the provision of successful home services in a Smart Grid}

Our study shows that, in relation to saving electricity, consumers have unmet needs in the areas of 'functionality' in terms of aids for saving electricity. They also need 'information' that tells consumers about their usage behavior and 'motivation' that pushes consumers to 


\begin{tabular}{|l|l|}
\hline Pain point & \multicolumn{1}{|c|}{ Unmet Needs } \\
\hline $\begin{array}{l}\text { Danger of } \\
\text { electromagnetic } \\
\text { waves }\end{array}$ & $\bullet \begin{array}{l}\text { Needs to measure the level of emission of electromagnetic waves } \\
\text { by each appliance }\end{array}$ \\
\hline $\begin{array}{l}\text { A device safeguards against the emission of harmful } \\
\text { electromagnetic waves } \\
\text { electric shock }\end{array}$ & $\bullet \begin{array}{l}\text { Guide on how to safely handle electronic appliances, particularly } \\
\text { around water (e.g., in a bathroom or in a kitchen) }\end{array}$ \\
\hline $\begin{array}{l}\text { Danger of short } \\
\text { circuit }\end{array}$ & $\begin{array}{l}\text { A function that auto-cleanses outlet (e.g., extension cord with a } \\
\text { function of self-removing dusts) } \\
\text { A device/tool to organize convoluted wirings } \\
\text { A device that automatically shuts off the entire power when } \\
\text { leaving house }\end{array}$ \\
\hline
\end{tabular}

Table 4. Unmet needs in the areas of safe use of electricity

take actions. Also, in regards to the safe use of electricity, they have unmet needs in terms of protecting against electromagnetic waves, electric shocks and short circuits.

However, in order to provide a home service that satisfies the needs mentioned above, smart grid devices like a smart meter or a smart appliance must be connected to a home network system. Connecting a home network onto a smart grid opens the door for many new helpful services for consumers as a result of the positive synergy effect from combining electrical services with IT.

Suppliers simply need to know the total amount of electricity used in order to control their supply level. However, this information is not enough for consumers. In order to be an efficient electricity consumer, they need to know the level of usage for each appliance. Since each appliance consumes a different level of electricity, the total amount of electricity used after a given time can vary depending on the appliance. Many people may be aware of the fact that one air conditioner consumes the same amount of electricity required for operating thirty fans. However, not many may be aware of the fact that making a toast in an electronic oven consumes 33 times as much electricity as is required to make a toast in a toaster. If monitoring the electric usage of individual appliance becomes possible, consumers will save electricity by voluntarily using an appliance with a higher energy-efficiency level.

If a smart grid is connected to a home network system, consumers could reduce their electricity usage because appliances could be turned off automatically during peak electricity usage periods. Both consumers and suppliers could benefit from this, by increasing the generation efficiency. Consumers could use electricity at a lower rate and suppliers could reduce wasteful consumption during critical peak times when the demand is high.

There are various players in the business of providing home energy management systems that helps consumers to become better consumers of electricity. Google and Microsoft, two of the biggest IT corporations, recently launched their home energy management systems, called Google PowerMeter and MS Hohm, respectively. Their home energy management portals show the energy consumption of each home appliance, calculates the average electricity consumption of a consumer in real time, and provides consulting information on how to reduce energy consumption and $\mathrm{CO} 2$ emissions.

Combining a smart grid with a home network should proceed as a team effort including various players such as power suppliers, telecom companies, and electronic manufacturers. 
An open partnership between different players is crucial to foster the invention of converged smart services and to avoid overlapping capital investments.

There are various players in the business of providing home energy management systems that helps consumers to become better consumers of electricity. Google and Microsoft, two of the biggest IT corporations, recently launched their home energy management systems, called Google PowerMeter and MS Hohm, respectively. Their home energy management portals show the energy consumption of each home appliance, calculates the average electricity consumption of a consumer in real time, and provides consulting information on how to reduce energy consumption and $\mathrm{CO} 2$ emissions.

In addition to IT corporations, electrical power companies are also entering this business area. For example, General Electric (GE) has made smart appliances - devices that can take commands from utility companies or consumers to power down and save energy. Also, GE's consumer and industrial division has teamed up with Tendril to develop a system that will essentially allow utility companies employing Tendril's TREE (Tendril Residential Energy Ecosystem) platform to turn GE dryers, refrigerators, washing machines and other energy-gobbling appliances off or on to conserve power consumption.

Aside from their partnership with GE, Tendril also provides their own service as well. Tendril partners with utility companies and supplies smart meters, smart outlets and smart gateways to consumers. Consumers can remotely control each appliance at home using an iPhone or PC when a smart outlet is plugged in between a cord and an outlet. They can also shut down the power of less important appliances automatically during peak times.

Combining a smart grid with a home network should proceed as a team effort including various players such as power suppliers, telecom companies, and electronic manufacturers. An open partnership between different players is crucial to foster the invention of converged smart services and to avoid overlapping capital investments.

\section{Suggestion for a Smart Green Service and the willingness to use it}

\subsection{Service concept}

Based on the result of our earlier study, we developed a service called the 'Smart Green Service' with four distinct functions as shown in Table 5. As illustrated in Figure 5, the Smart Green Service allows an individual to discover their total amount of electric usage for individual appliances by setting up a 'Smart box' and 'Smart Tags' at home.

A Smart Box gathers the data of electrical devices from some Smart Tags inside the house and the household electricity usage from Smart Meters via ZigBee or PLC, and sends the data to the SGC (Smart Green Center). In addition, the Smart Box receives control messages from the center through Ethernet, and transmits them to the Smart Tags, thus acting as a gateway. A Smart Tag monitors and controls the power usage of each electrical device. The measurement is conducted by placing the Smart Tag between an outlet and an electrical device which monitors its electricity usage with a CT (Current Transformer) sensor. The measured data is transmitted to a Smart Box through ZigBee or PLC. In addition, the Smart Tag embedded with an actuator is possible to be powered on or off according to commands from the Smart Box. Figure 6 illustrate Smart Box and Smart Tag.

A Smart Meter measures the household electricity usage and sends the data to the SGC or a Smart Box via any networking module. Generally, it receives control commands from the SGC, and passes them to Tags or Boxes. However, the smart meter in the KT Smart Green Service is not able to receive and to pass on the commands so the command handling function is taken over by the Smart Box. 


\begin{tabular}{|l|l|}
\hline $\begin{array}{l}\text { Energy } \\
\text { Monitoring }\end{array}$ & $\begin{array}{l}\text { This service monitors the total energy usage and the energy usage of each } \\
\text { appliance in real time using a PC, SoIP 3, IPTV and mobile phone. } \\
\text { Consumers can monitor their electricity usage/rate and } \mathrm{CO}_{2} \text { emissions } \\
\text { and get notified before a higher cumulative rate is applied. }\end{array}$ \\
\hline $\begin{array}{l}\text { Standby Power } \\
\text { Cut Service }\end{array}$ & $\begin{array}{l}\text { This service helps consumers to save electricity by automatically cutting } \\
\text { off standby power when a smart tagged appliance is on standby. } \\
\text { Consumers can choose various settings such as 'out' 'bedtime', etc. so } \\
\text { that for example, when a consumer chooses the 'out' setting, the standby } \\
\text { power on appliances can be cut off. }\end{array}$ \\
\hline $\begin{array}{l}\text { Remote Control } \\
\text { Service }\end{array}$ & $\begin{array}{l}\text { This service lets consumers control their smart tagged home appliances } \\
\text { remotely via PC, SoIP, IPTV or mobile phone. }\end{array}$ \\
\hline $\begin{array}{l}\text { Energy } \\
\text { Consulting }\end{array}$ & $\begin{array}{l}\text { This service provides customized consulting services to consumers, } \\
\text { based on a careful analysis of their electricity usage via e-mail, mobile } \\
\text { SMS and an internet portal. }\end{array}$ \\
\hline
\end{tabular}

Table 5. The functions of Smart Green Service

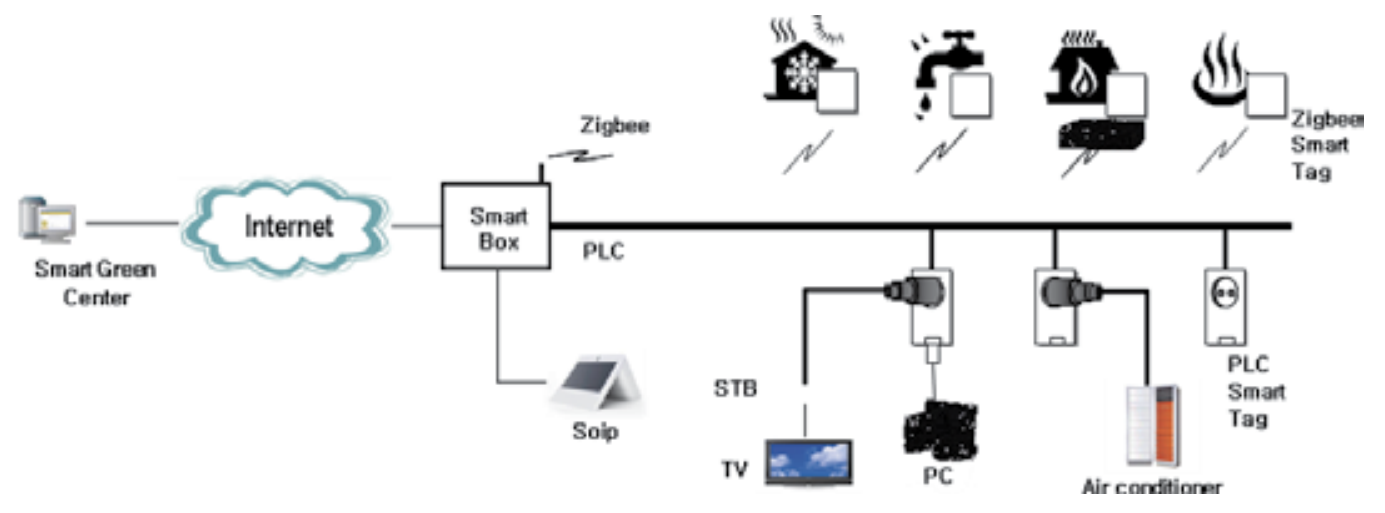

Fig. 5. Components of the Smart Green Service
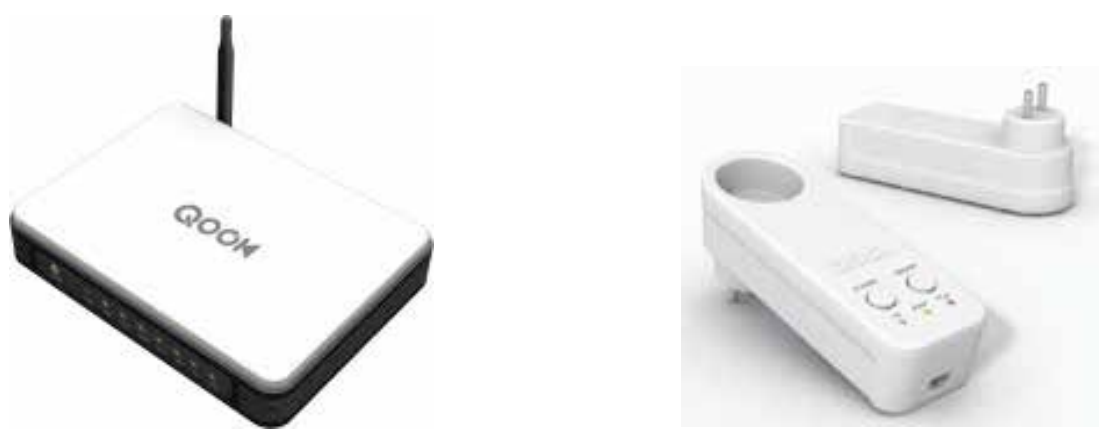

Fig. 6. Smart Box (left) and Smart Tag (right)

\footnotetext{
${ }^{3}$ SoIP (Service over IP) is an advanced VoIP(voice over IP) system that has many functions like call, videophone, SMS, e-banking and searching via IP.
} 
The SGC are mainly divided into the management system monitoring Smart Boxes/Tags, the application server system providing customer services, and data warehouse system maintaining data for managing network elements and providing services. The center handles and stores metered and measured data, analyze electric usage in various time intervals (hourly, daily, monthly or annually) and by categories such as neighborhood, region, and living standard. Not only system operators but also customers can determine statistical results, and set commands to control their electricity usage automatically.

KT (Korea Telecom) already made Smart Box and Smart Tag prototypes and will participate in the Jeju Smart Grid Demonstration Project from 2010 to 2013 in order to test and improve the equipment. The Jeju Smart Grid Demonstration Project is a vast project that tests stateof-the-art smart grid technology in five different fields - smart place, smart transport, smart renewable energy, smart electricity service and smart power grid. Over 150 companies will participate in the project, testing 6000 homes over the next four years.

\subsection{Service evaluation (by qualitative survey)}

Participants evaluated the Energy Monitoring Service positively for the following reasons: By informing them of the exact amount of electricity usage on each device, their curiosity is satisfied, they are more motivated and better strategized. However, some suggested that too detailed information may be a cause of stress. They also made additional suggestions such as an alarming function that rings when a child touches an outlet, a system that notes the amount of electromagnetic waves emitted by each appliances and a service that sends out a text message in case of electric outage.

Participants evaluated Standby Power Cut Service positively for the fact that it removes them of hassles to unplug. However, willingness to pay for the service was much lower because they could not see a distinct benefit of using the service apart from using an extension cord.

Although Remote Control Service received some positive feedback for aiding safe use of electricity by removing threats of potential danger, overall, participants showed very low willingness to pay for the service since they considered the occasions to use such service to be very rare. Additionally, they showed needs for a service that also controls the power of lights, not just the smart tagged appliances, and for a service that notifies when a person uses electricity via mobile service.

Participants showed interested in immediate and professional problem solving Energy Consulting Service can provide, but they considered such service to be going overboard and worried that it may be a cause of stress. However, they showed needs for consulting service via online chatting and online portal where they can share information on using electricity.

\subsection{Willingness to use suggested services (by quantitative survey)}

As shown in Table 6, all four of the Smart Green Services offerings got a high willingness-touse rating of over $60 \%$, showing that consumers are highly interested in home-based electricity services.

Among the four services, the Energy Monitoring and Standby Power Cut Service received the highest willingness-to-use ratings, $78.3 \%$ and $76.3 \%$, respectively. The Remote Control Service and the Energy Consulting Service received a lower, but still high willingness-to-use rating, $64.7 \%$ and $60.3 \%$, respectively. 


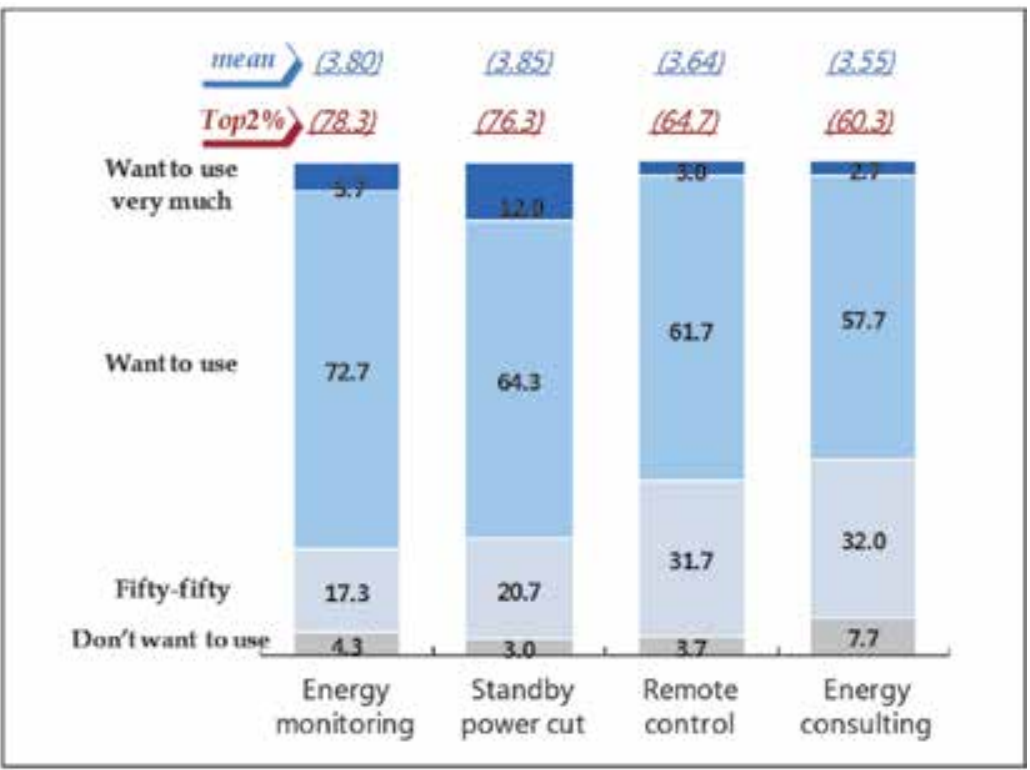

Table 6. Willingness to use

As shown in Table 7, participants who chose no willingness-to-use did not want to use the service because they expected the service to be difficult and complex to use and ineffective for saving energy. They thought the Energy Consulting Service would be particularly difficult and complex to use. They also had doubts about the Remote Control Service; they did not think that the service could actually work smoothly.

\begin{tabular}{|c|c|}
\hline Reasons & Services \\
\hline It seems too difficult to understand and use & 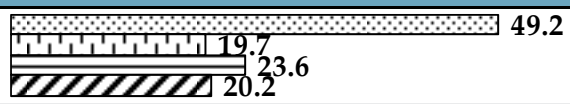 \\
\hline It's not useful for saving energy and money & 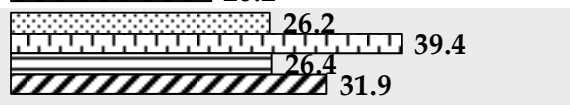 \\
\hline $\begin{array}{l}\text { It's not useful for using electricity safely and } \\
\text { conveniently }\end{array}$ & $\begin{array}{l}16.9 \\
Z Z Z 10.9 \\
17.9\end{array}$ \\
\hline $\begin{array}{r}\text { It's not necessary, I'm already using similar } \\
\text { services }\end{array}$ & 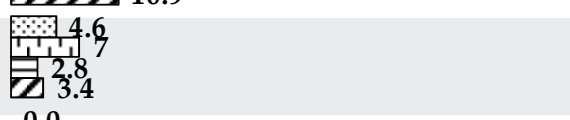 \\
\hline I'm not interested in such services & $\begin{array}{l}0.0 \\
2.8 \\
0.9\end{array}$ \\
\hline I can't trust it work well, safely & 28.3 \\
\hline $\begin{array}{r}\text { It's so annoying and complicated so I'll not } \\
\text { use those services }\end{array}$ & $\begin{array}{l}\frac{0.0}{1,1,1,1,1,1,1,1,1} 21.1 \\
0, Z Z Z Z Z Z Z Z Z Z Z Z 32.8\end{array}$ \\
\hline
\end{tabular}

图 Energy monitoring 回 Standby power cut 目 Remote control $\square$ Energy consulting

Table 7. The reasons for not wanting to use the service 


\section{Conclusion}

In this study, we have explored consumers' perceptions and attitudes toward using electricity by observing their behavior using HV and we studied a cognitive map derived from a FGD model. Based on the result, we have developed smart grid home services and tested consumers' willingness to use our services using a survey.

Consumers perceived electricity as an indispensable necessity that makes the use of home appliances easier and they wanted electricity saving activities to be both easier-tounderstand and stress-free, and also wanted to use electricity safely without being exposed to the potential dangers of using electricity such as harmful electromagnetic waves, short circuits and electric shocks.

Consumers made varied efforts to be aware of their electricity usage in order to reduce their electricity usage; they unplugged appliances that are occasionally used like TVs or PCs, thoroughly studied electricity bills and compared them with that of their neighbors. However, they found that conserving electricity is difficult due to a lack of functionality, information and motivation. They showed unmet needs in the following areas: a function that cuts off standby power, a function that informs the user of the level of electricity usage and the energy efficiency of each device, an alarm that notifies the user of changes in the electricity rate, and a motivation system that pushes consumers to conserve electricity.

Consumers were also taking various steps to protect themselves from the potential threats of using electricity. However they still felt uneasy about safety. In terms of the safe use of electricity, they showed unmet needs in the following areas: the need for a simple device that protects against harmful electromagnetic waves, a guide on how to safely handle electronic appliances in a bathroom and a kitchen and a function that shuts off the entire power supply.

To satisfy the unmet needs in the aforementioned areas, KT has developed Smart Green Services that can monitor the aggregated and individual electricity consumption rate of consumers in real time using a smart box and tag. All four services received a high willingness to use rating of over $60 \%$.

KT will participate in the Jeju Smart Grid Demonstration Project from 2010 to 2013 and test prototype equipment examples - like the smart box and tag - that are enabled with services like Energy Monitoring and Remote Control.

When our Smart Green Service, which was developed based on consumers' opinions and unmet needs, integrates several technical innovations and gets the active participation of consumers, it will come closer to the ultimate goal of a smart grid, allowing the efficient use of electricity.

\section{References}

Lee, H. \& Park, H. (2009), To Estabilish Smart Grid Roadmap, pp. 139-154, Ministry of Knowledge Economy, Seoul, Korea, pp.139-154

Kim, M. (2009), The estabilishment of Korean smart grid, TechnologyManagement, August 2009, pp. 36-41

Moon, S. (unpublished), Green economy and Smart Grid, Seoul National University, Seoul, Korea

Haaser, B. (2008), Connecting Smart Homes and Smart Grids to Save Energy, LonmarkMagazine, Vol. 4, Issue 3, 2008, pp. 20-23 
Leeds, D. (2009), The smart grid in 2010: Market segments, applications and industry players, GTM research

John, J. (2009), Microsoft Hohm: First Seattle City Light, Now Xcel Energy, Greentech Media Kanellos, M. (2009), GE, Tendril Team Up on Smart Home Technology, Greentech Media

John, J. (2009), Top Ten Smart Grid, Greentech Media

Kanellos, M. (2009), Tendril Targets Meter Makers, Greentech Media

http://www.tendrilinc.com/consumers/products/ 


\section{Part 2}

Energy Management 



\title{
Management Crisis in Partial Deregulation of Energy Sector and Modeling the Technical and Economic Results of Organizational Management Structure
}

\author{
Joseph Yakubu Oricha \\ Department of Electrical and Computer Engineering, Ahmadu Bello University, Zaria \\ Nigeria
}

\section{Introduction}

Decentralization through reduction of the state property share aimed at attracting loan and joint stock capital, including foreign capital, in the conditions of continual acute shortage of primary energy sources such as coal, gas and petroleum as well as lack of investment for modernization could be termed as 'partial deregulation' [Nedin \& Oricha 1998]. The transition from vertical integrated utility to unbundle utilities made inevitable a number of basic rearrangements of the power industry management both in developed and developing countries.

The practical implementation of restructuring the branch was reflected in energy market creation and partial denationalization of the power industries of different types [Oricha, 1998\}. Restructuring was aimed at a number of goals which were unattainable in the conditions of the former organizational management structure. The main goals of the restructuring were to create the competitive environment (first of all in the field of energy production transmission and distribution), as well as to attract foreign investing capital (IC), that will be needed for the large-scale modernization of the fixed capital stock (FCS). The development of competition was substantiated by the opportunity to raise the effectiveness of power production, reduce costs of production and improve reliability of power supply. Unfortunately the above mentioned goals are always unattainable in partial deregulation and could even lead to management crisis with some negative consequences. The main risk in deregulation of energy sector is the risk of unfavourable regulatory decisions and cost overruns due to bad project management [Nedin \& Oricha]. It is clear that the best approach toward energy cost reduction is investment and technological modernization. However, introduction of liberalisation in energy markets is removing the regulatory risk shield and thereby exposing investors to various business risks. The level of risk anticipated by an investor in a power plant will be reflected in the level of return expected on that investment [ Daniel S.K \& Garan .S, 2004]. This means the greater the business and financial risk, the higher the return that would be demanded. The management crisis that would eventually occurs and will also affect the volume of investments in liberalized markets is the inherent uncertainty about electricity prices in electricity markets. This chapter discusses this 
management crisis and some of its negative consequences and give suggestions on how to modelling the organizational management structure in power industries.

\section{Management crisis and its consequences in deregulation of energy sector in Ukraine}

During the transition and breakdown of Soviet Union, Ukrainian energy sector was subjected to deep economic crisis that calls for immediate restructuring and re-organization of various forms of ownership. The failure to achieve the main goals of restructuring the energy sector leads to management crisis in power industry with some negative consequences. One of the first steps in restructuring energy sector in Ukraine by then was to amend some of the normative acts inherited from the monopolistic forms of management. However, decentralization of the state property share to attract loans including foreign investments, created a further management crises in the power industry of Ukraine. The development of competition by creating energy market to raise the effectiveness of power production, reduce expenses and prevent increase in the energy products also were not achieved but resulted in further increase in the prices of energy products.

The three major problems identified as the main reason for restructuring are:

- The basic production assets - fixed capital stock (FCS) were won out and the low efficiency of their uses

- Serious acute shortage of primary energy sources;

- Low caloric nature of the imported fuels.

These three problems mentioned above refer in particular to the coal thermal power plants, which constitute the basis of the Ukrainian power industry. Thus, $48 \%$ of the thermal power plants generating units have passed their utilization threshold and at least \$30 million and extensive time period is needed for their modernization that would meet the up-to-date safety, economic and ecological requirements. On the other hand, 200 and $300 \mathrm{MW}$ coal generating units are compelled to be used to cover the alternating part of the load schedule because of insufficient maneuver capability in the Ukrainian energy system. It resulted in increased fuel consumption, 30-45 \% increase in internal energy consumption, decrease in efficiency factor and increase in fuel unit cost, rising wear level and reduced generating equipment reliability [.Nedin , Ryzhov., Oricha \& Chastockolenko, 2001].

The above stated conditions caused constant increase of energy production costs that are not paid off by means of present day rates, which consequently undermines the financial state of the power enterprises. However restructuring held in the conditions of continual acute economic crisis not only failed to eliminate the destabilizing factors, mentioned above, but provoked in addition a number of negative consequences, including:

1. Application of foreign loans;

Investing loans of the international financial companies proved insufficient for visible improvement of the technical and economic indicators of the power industry, and their use proved irrational [ Nedin, Senko \& Shetrenko, 1999]. During the energy crisis in Ukraine, the World Bank provided credits to Ukrainian ministry of energy for thermal power station modernization, which has appeared too minor to improve the economic situation of the power sector in appreciable measure.

Loan repayment and the payment for the expenses on their services according to the lending terms must be made by means of surcharges to the corresponding rates. It overlaps with the general decrease in domestic manufacturing and paying capability of the energy consumers, 
posing the threat of increasing loan debt and further destabilisation of the the financial status of power industry.

2. Privatization through the sales of public utilities assets;

Attracting the investment capital (IC) through sales of the 'public power utilities' share holdings without clear regulated and controlled liabilitites of the new share holders that cannot guarantee the necessary fixed capital stock (FCS) modernization will leads to loss of state control over the most important basic economy branch. The loss of state control over power sector will results to catastophic economic consequences that affects other branch of the economy as a whole. From the experience of restructuring energy sectors in various places such as Chile, Carifonia, Russia and etc. became clear that fairely aged power equipment/infrastructures are not attractive to investors. Thefore, the orientation to sell some of the state share holdings to attract investors always deepen the management and economic crisis of the power industry.

3. Introduction of independent power suppliers (IPS) and Introduction of independent power producers (IPP);

Involving many independent power suppliers (IPS) to discourage monopoly and creating a competitive environment for electricity market failed due to problem of lack of payments. In addition to problem of lack of funds, majority of IPS has no direct relation to power production and IPS according to different estimates $60-80 \%$ control the entire sales volume profits. And this means that the power industry is losing a lot of funds, which could have been partially used for further investments. The involement of many independent power supliers only added to the burden the consumers bear because the cost of power can only be reduced if there are enough power producers who have no mopolistic power over the price but compete on the basis of price bids and offers. In a competetive electricity market where the producers have no control over the price but want to maximize their profits, will be compel to use those generating units where the production costs will be minimized or use more efficient equipment. In partial deregulation, this could not be achieved because majority of the investors will always feel reluctanct to invest in a sector where prices cannot be forecasted as a result of regulatory action that maybe imposed. In practice, deciding to invest in a new generating plant is considerably more complex because of considerable amount of uncertainty. Construction delays and fluctation in the price of fuel can affect the long-run marginal cost. On the other hand, acording to [Fraser \& Huist, 2003], the evolution of wholesale electricity prices over a long period is a nototoriously difficult to forecast because demand might change, competitors might enter the market or new, more efficient generation technologies might be developed. Once the problems mentioned above cannot be predicted and solved, then partial deregulation will always lead to management crisis with catastrphic economics problem of the state.

The idea of allowing IPP to take active part in power generation is to create an environment conducive for electricity markets to exit, with the view that when we have more power producers, there is likely possibilities that there would be surplus in power generation thereby reduces the prices. But unfortunately, competition in partial deregulation is normally limited primarily to the power realization field. This will lead to practically no effective competition that could stimulate tangible decrease in power and heat production costs. This doesn't let stabilize consumers' paying capacities and together with too widely spread and insufficiently grounded use of noncash forms of payment (promissory notes and mutual offset) that cannot create the environment for financial / capital accumulation. 
4. Absense of perfect regulatory and legal framework;

Absense of perfect regulatory and legal framework, which could regulate the interaction of market participants of different forms of ownership in all the power industry fields, is aggravated by no pracctical actions taken to organize mutually beneficial cooperation of the public power sector (PPS) companies with private corporations and enterprises aiming at participating in energy production and investing into it. This essentially restricts the opportunities of private power companies potential use, that could improve the technical and economic indicators of the industry in general. For instance, one of the major problems in deregulation of energy sector in Nigeria is lack of perfect regulatory and legal framework that is discouraging independent power producers (IPP) to participate in the field of electricity generation. It is observed that the largest potential resource for power generation in Nigeria is natural gas, which current estimate of total about 187 trillion cubic feet [The Nigerain Electricity Regulatory Commission, 2008]. Technically speaking, even $10 \%$ of this natural gas reserves can support over 10,000 MW capacity power plants operating at a power factor of $80 \%$ for 22,000 years. However, the total power generation in Nigeria as of 2010 is not up to 4,000 MW, while the minimum total demand was estimated to be 10,000 MW for fairly constant power supply. It was estimated that the total available coal deposit can support $15,700 \mathrm{MW}$ capacity at $80 \%$ capacity utilization for over 50 years but the challenges facing coal power generation in Nigeria include uncertainities in the actual reserves of coal on which long term projects could be based, low productivity of the coal mines, low level of mechanization of production facilities and absence of cost- effective transportation system and lack of investment in coal industry.

The major problems as always explained by Power Holding Company of Nigeria (PHCN) was shortage of gases to power the plants. The reallity of the problem is that over the past 50 years of oil and gas production in Nigeria, the greater part of the gas produced had been flared while investment in the usable gas supply had focused more on exports than on domestic utilization. In this situation, oil and gas in Nigeria still remained vertically intergated while privatization to bring competition was introduced in power sector. But Unfortunately, privatization is not a prequisite for the introduction of competition [Daniel \& Garan, 2004]. The Nigerian Electricity Regulatory Commission introduced various codes that further complicated the right of independent power producers and consumers with additional increase in the cost of power production. The amount of charges that an IPP will have to pay inorder to allowed to be connect to National gride are so much that practically no IPP will have a strong incentive to participate in power generation.

The major negative consequences in restructuring the energy secor in Nigeria is continous high prices of goods and sercices as a result of high cost of electricity genaration.

The experience in South Australia suggests that markets can respond to price signal and meet demand and reliability requirements if government policies are consistent with the development of effcient and sustainable electricity markets, and where they are implemented transparently and consistently [Fraser \& Huist, 2003]. However, in the examples of restructuring of Ukrainian, Nigerian energy sectors as cited above does not have a conssitent and transparent policies that would attract investments for modernization of the basic production assets. These factors mantioned above are the basis for management crisis phenomena in partial deregulation of energy sector.

5. Establishment of power markets;

Power market establishment with non-existent coal and gas market, nonequivalent exchange of coal for power regulated by subjective negotiated prices deepens the coal and gas industry crisis and endangers the next shortage of coal and gas output. 
The above listed factors are particularly dangerous not only for the power industry, but also for related industries, bacause the stable escalation loop of the crisis phenomena (its fragment shown in the figure below) has been formed. In the given loop investment capital (IC) and current capital deficit stimulates further fixed capital stock (FCS) wear escalation and worsening of their technical and economic indicators.

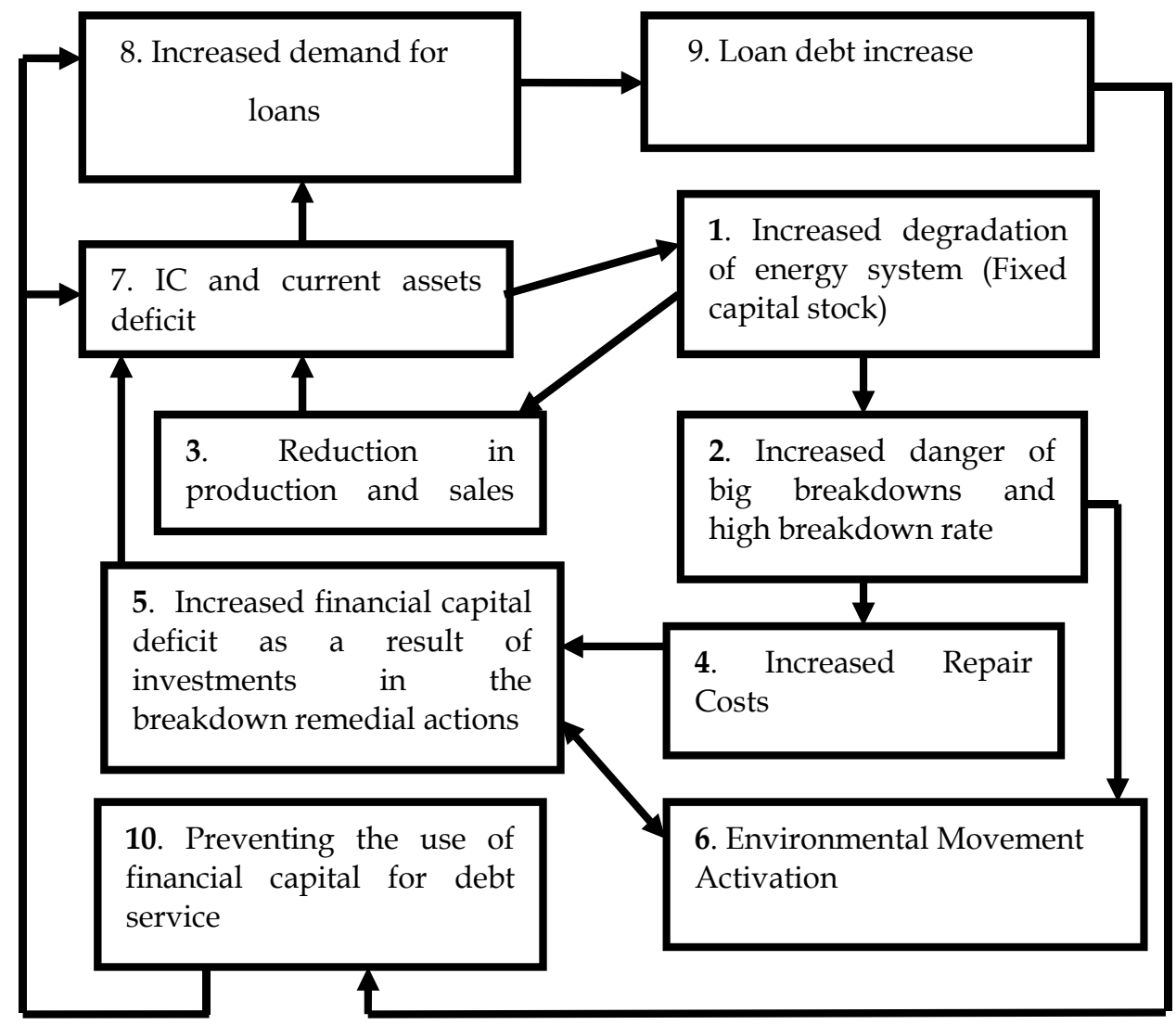

Fig. 1. Degradation factors interplay in the crisis phenomena escalation loop

\section{Escalation loop of the crisis phenomena as a result of partial deregulation of energy sector}

The figure above illustrate the escalation of the crisis phenomena that normally exists as a result of partial deregulation of energy sector. The direction of the arrows indicate how one action or consequence will stimulate the others to happen. In this case there will be continious crisis phenomena in form of loop. Consequently, competitive ability of the power systems in the market economy deteriorates, the danger of big breakdowns and actual breakdown rate increase, which respectively provokes increased spendings on the repair works and, accordingly, prevents the use of resources in other fields of their proper use. In this situation there would be always budget deficit. As a result, the incresed demand for loans and no real prospect to promptly repay them and pay for their servicing, are stimulated. And this means that the further stimulation of such threats to security of energy 
supply (ENS) as "investment stock deficit and its inefficient application", "financial destabilization and non-payment increase", "violation of import equipment, materials and fuel deliveries" etc.

The above noted phenomena in power industry alone due to increased power selliing price and respectively rate increase influence in an extremely negative way the competitive ability of the national economy on the whole, which according to [Nedin., Senko, Shetrenko, 1999] is defined as «the position of the country or the commodity producer at the domestic and foreign markets" (ability to withstand the international competition at these markets). And it is caused by the energy production price rise, what should be considered as reduced competitiveness of energy as a commodity, which seriously complicates realization opportunities of the reproductive processes not only in power industry, but also in other industries. And renewable resources are generally known to be one of the main preconditions to the economic security (ECS) of the state.

The only way to change the established situation is to create preconditions to activate all available investment capital (IC) [6] in order to reach the FCS state when the energy production costs could decrease to the level enough to stabilize consumers' paying ability and power enterprises financial condition. The opportunities of the power companies, belonging to the PPS of Ukraine, are absolutely restricted. That is why the following directions should be considered as priority to meet the present-day management crisis in power industry:

i. The use of more rational (compared to the ones suggested by the World's Bank) schemes to organize investing in power projects, that provide the possibility for prompt loan debt repayment introducing no surcharges to rates and the energy selling price.

ii. Creating the real competitive environment in the power production field, taking into account existing energy market regulations. Hereby the mutually beneficial cooperation of the market participants of different forms of ownership that would enjoy equal rights should be guaranteed, which may further call for specification and improvement of the regulatory and legal framework of their interaction.

iii. Provision of economic and organizational conditions to ensure the high-quality fuel supply to the dust-coal thermal power plants, including the formation of regional multifunctional industrial and financial groups encompassing general purpose thermal power plants, mines, coal cleaning, metallurgical and other power-consuming enterprises.

Concerning the first direction financial leasing should be noted as the most reasonable investment form applicable to the energy projects in Ukraine; it can be combined with the "tax holidays" regime [Nedin \& Oricha, 1998] and applied to the objects, created by means of effective loan irrespective of its receiver's form of ownership. At the same time the "tax holidays" should be considerd not only the form of tax benefits, but first of all the form of establishing the conditions for tax base extended recreation and growth on the basis of production development and efficiency increase beyond the "tax holidays" duration period. The second direction suggests that the PPS cooperate in the power production field with private companies, focused to create their own generating capactites on the basis of the most efficient power production technologies use. JSC "Kontsern Energiya / Concern Energy" [Nedin , Oricha \& Sheverev, 1999] is one of such companies; it developed the project of use of the combined-cycle plant run on the natural gas, having high efficiency coefficient, not less than $52-55 \%$. Under current economic conditions such cooperation will let the PPS to expand its structure and the range of sources of income as well as promote 
competitive ability of the coal thermal power plants. Here are the major advantages of the cooperation worth mentioning [Nedin I.V., Senko I.V., Shetrenko,1999]:

1. Payment for the specialized construction and mounting organizations of the industry for their participation in bulding the private power objects. According to [Oricha., Nedin .1998], approximately $\$ 9,77$ billion is expected to be assigned for investing in the power objects taking into account 15 year period of reinvestment. If at least $20 \%$ of the sum is used to pay for the services of the organizations, then they will be able to annually receive approximately $\$ 130$ million as direct payments as opposed to loans. And the loan liabilities lie with their receiver, not the PPS. At the same time, the sum given substantially exceeds the average annual amount assignable to the industry by international financial organizations.

2. Payment for the traffic through $220-750 \mathrm{~kW}$ electrical networks and for the services of traffic control system, which objects are not subjected to denationalizing. If these services are used by a private power company to transfer $11,4 \mathrm{GW}$ of the rated capacity through the energy system networks with the performance life equal to, for instance, 5000 hours per year, then after all the pre-scheduled capacities have been put into operation about 57 billion $\mathrm{kW} / \mathrm{h}$ could be trafficked annually. If the traffic rate is 0,17 cent per $\mathrm{kW} / \mathrm{h}$ the annual total will reach appr. $\$ 97$ billion. These are also PPS direct payments and not loans. Certainly this sum will differ according to the year of the JSC "Concern Energy" program implementation, but even when $10 \%$ of the capacities have been put into production this payment would be quite appreciable given the presentday financial state of the PPS.

3. Decrease of the production cost of the power released across the energy system on the whole. [9] illustrates that, with the $10 \%$ combined-cycle plant use share in the energy system capacities the decrease of the production cost of the power released could be compensated by the rate increase, that is inevitable compiling with the terms of the World's Bank and the European bank for Reconstruction and Development debt loan repayment [Nedin I.V, Oricha J.Y, Sheverev, 1999]. In this case, notwithstanding the fact that according to the energy market regulations the specific weight of the power produced by the combined-cycle plants of the private energy company will surpass their value in the rated capacity of the energy system and the respective redistribution of the sold energy volume in favor of the private company, the production cost decrease will generally make it possible to avoid ungrounded increase of the corresponding rates. The latter should contribute to the renewal of consumer paying abilities and in the long run stabilize the payment regime.

4. Stabilizing the energy system operating mode. A more stable operating mode will be ensured by the relatively maneuver combined-cycle plants, preplanned capacity of which significantly surpasses that of the existing thermal power plants, as a part of the rated capacity of the energy system. This will allow increasing the consumer power supply reliability, to reduce the costs for the repair of the coal thermal power plant heat-mechanic equipment, as well as to supplant a part of the thermal power plants from alternating component of the energy union load schedule. Owing to this the fuel consumption will be reduced as the result of lower number of starts and stops. At the same time, in spite of the fact that the power delivery to such thermal power plants will fall, their profitability, as it has been illustrated in [Nedin, Ryzhov., Oricha, Chastockolenko, 2001] , can increase rather substantially, being the direct manifestation of their competitive ability. Due to combined-cycle plants more favorable conditions for 
the operation of power generating units equipped by the circulating boiling layer (CBL) boilers will be created, the capabilities of their use in the maneuver regime being still sufficiently studied.

5. Possibility for PPS to take part in the public energy companies property. Losses caused by the suppression of the PPS energy producers from the energy market can be partially compensated by the PPS participation as a shareholder-co-owner of the privately owned energy object, which will allow for certain redistribution of the revenues delivered by combined-cycle plants, that produces cheaper power as compared to the thermal power plants. But this being the case, PPS energy companies must bear a part of the responsibility, i.e. investment loan repayment.

On the whole the result of such cooperation helps play for time and accumulate funds, sufficient for improving coal energy technologies.

The third direction is effective in a way that it reduces the power production cost and the costs of end-produce of the consumer enterprises, belonging to a certain union. In this specific case, according to [Lir ., Nedin Oricha \& Xalyava], energy consumption in the coal output process is considered as the power spent for internal needs, i.e. power is supplied to the coal mining enterprises at its production cost price. As a result the production price of the heat and power end produce should be reduced. At the same time the conditions for guaranteed higher quality fuel, delivered to the thermal power plants, are created. That is because its supplier operating in a united technological complex is interested in the end result - the decreased of the energy production cost, and therefore in the increase of its production efficiency. The task of restricting the number of mediators, whose activities are based on fuel and energy resale, is simultaneously being radically solved. There are other possible significant advantages of introducing a similar organization of multifunctional enterprises interaction - creation of favourable organizational and technological conditions for utilizing the ash-and-slad thermal power plants' waste products and their relocation into the coal mine uses etc.

The above listed ways of improving the management organization have a significant influence on the production and technological sphere of power industry and must be implemented in compliance with economic and technical solutions, aimed at improving energy production efficiency. Complex realization of the listed directions is possible only with the necessary legislative support provided the latter being at the time insufficient to reach the target results. That is why the burning issue remains: to create the regulatory and legal framework, which can ensure mutually beneficial cooperation of market participants of different forms of ownership in energy production, with the obligatory condition to improve the main technical and economical indicators of power indutry (including the production cost of the released energy) and to keep their commercial interests balanced.

\section{Modeling the technical and economic results of organizational management strucure in power industry}

The introduction of market relations in power industry involves the necessary modernization of organizational and functional management structure (OFS) of the energy systems (ES) control. The necessity of such modernization is stipulated by changed terms of financing, decentralization of control functions, diversity of forms of ownership and other factors. The management OFS of the power facilities and their commercial interaction 
influences ES reliability and security, and, consequently, power supply reliability. The structure of interaction of power facilities among themselves, with consumers of energy product, and with the systems ensuring ES operation, is rather complicated. That is why while choosing the OFS version one should not limit himself to intuitive understanding of their possible effectiveness. A meaningful quantitative estimate of the possible consequences of their application is indispensable. Below are some of the principal statements of such estimate.

OFS is considered as a specific case of program-technical complex (PTC), meant to automate ES management, to ensure its reliable operation, etc. That is why to describe the versions of OFS the structural and contingency approach as applied to PTC, examined in [Nedin \& Oricha 1998], is used, according to which technological and commercial interaction of the ES objects is reflected by a corresponding structural model, whose composition and internal structure are chosen as based on the level of territorial and operational hierarchy of the control object. The structural model gives account of material and cash flows with regard to their diversity. Material flows imply exchange of products - fuel, energy and various services, and cash flows imply co-payments, assignments to budgets of different levels, crediting, penalty charges, a.o. Material and cash flows can be of unilateral or two-way direction. The choice of the OFS version should be determined only by assessment of consequences of their possible application.

The evolution of technical and economic indicators which characterize the control object and results of its operation as well as characteristics of external environment, including the characteristics of regulatory and legal framework for the industrial and economic activities, is presented with the help of situational models, as it is shown in [Nedin, Oricha, Sheverev]. It should be taken into account that the composition and the nature of interconnection between the elements in the structural model in general case can change over time and thus is of situational nature.

The Figure shows in less detail one of the possible types of power market participant interaction, which operates in the territory of a large energy union.. It also indicates the major material and cash flows and provides interpretation of the numerical symbols standing for the interacting power objects. The material and cash flows in the Figure designate the following:

- $\quad$ ЭIII-I, ЭIV-I, ЭV-I - power production by PJGC, NPP and APU within the power union network;

- $\quad$ ЭI-II - selling of power by energy union to power-suppliers;

- VI, VII-I, VVI-0, VVI-I, VVI-II, VVI-III,VVI-IV - technical, resource, investment and financial, mediator, a.o. services;

- $\quad$ OЭ0-III, OЭ0-IV, ОЭ0-V, OЭII-0 - payment for power;

- Oy0-III, Oy0-IV, Oy0-VI, OYII-VI, OYIII-VI, OyIV-VI - payment for technical, resource, investment and financial, mediator services.

The first index in the designations of material and cash flows indicated above refers to the object, which markets power, renders services or makes payments, and the second index refers to the object-receiver. Below are the numerical designations of interacting power market participants:

0 control center and its regional departments;

I high voltage 220-750 kW electrical network;

II public joint-stock power supplying companies (PJSC); 
III public joint-stock power generating companies (PJGC), which include thermal power plants and hydropower plants;

IV nuclear power plants;

$\mathrm{V} \quad$ adjacent power unions (APU);

VI repair, transportation, investment and financial, mediator and other organizations and enterprises, which render services to the power industry enterprises.

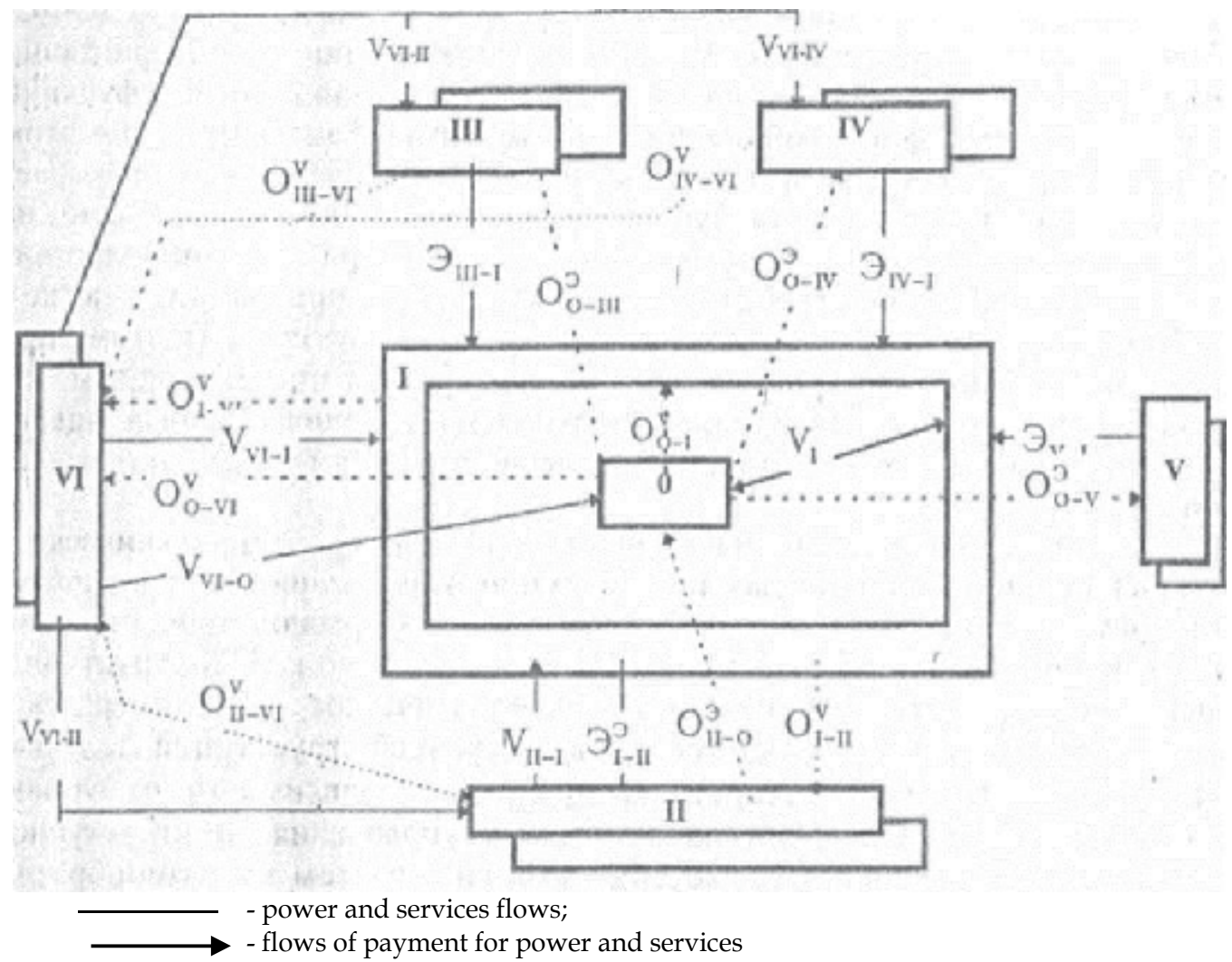

Fig. 2. Organizational and functional structure of commercial interaction between power markets Participants.

Annual profit, calculated according to the formula given below, can be regarded as the measure of effectiveness of OFS commercial interaction with respect to individual power market participants:

$$
\begin{gathered}
\prod_{0}=\sum_{i} O_{I I-0}-\left[O_{0-1}^{y}+\sum_{j} O_{0-I V}^{y}+\sum_{k} O_{0-I I I}^{y}+\sum_{s} O_{0-I V}^{y}+\sum_{t} O_{0-V}^{y}+\ni_{0}\right], \\
\prod_{1}=O_{0-I}^{y}-\left[\sum_{q} O_{I-V I}^{y}+\ni_{I}\right], \\
\prod_{I I I}=O_{0-I I I}^{y}-\left[\sum_{f} O_{I I I-V I}^{y}+\ni_{I I I}\right],
\end{gathered}
$$

where 30, 3I, 3ш stand for total annual expenditure on principal industrial and economic activity; $1, \mathrm{j}, \mathrm{k}, \mathrm{s}, \mathrm{t}, \mathrm{q}, \mathrm{f}$ - indices, which testify multiplicity of possible ties between the subjects of commercial interaction. 
For a higher level of hierarchy, e.g., for a regional level, to assess the effectiveness of a specific OFS version one can use such indicator as the sum of contribution to the budget made by power market participants.

The scheme given in the Figure is used only to illustrate the principle of formalized description of commercial interaction applied to one out of many possible versions. OFS versions in specific calculations can be rather diverse and their variation consists in redistribution of different control functions among them, in changed quantitative characteristics of interaction, etc. The operational reliability of the mentioned objects is in direct relation to the extend to which earnings from payments cover the expenditures on maintenance and repair works (MARW) of the operational equipment, ensure the income accumulation in the amount sufficient for budget contributions, contributions to the centralized funds, whose availability of means can create a possibility to improve the fixed capital stock of the branch, etc. While making an estimate of the considered OFS version effectiveness it is necessary to take into account the conditions, provided in the table below. Factors 1.1-1.9 apply to all the relevant objects. Restrictions 2.1-2.3 apply to PJGC and the energy union integrally, while 2.4 is of a more general character. Restrictions 2.1 and 2.2 determine the lower limit of the PJGC, NPP and high voltage electricity networks operating costs, and restrictions 2.3 and 2.4 - the upper limit of power sales proceeds and earnings from services rendered to customers. Efficiency factors can also be considered selectively depending on what objects the task is related to.

The indicators from (1)-(3), whose interconnection is reflected in the above given structure of interaction, depend on work standards as well as on the numerical level of operating mode indicators of the energy union objects and the consumer power supply reliability. According to the new conditions of economic management, the above mentioned sources and components of payments are practically the only source of funds that ensure industrial and economic activities of the power enterprises.

The process of formation of these means is extended in time. The characteristics of this process depend on a specific content of the OFS modeled, whose structural functional and quantitative parameters are in a general case invariable over time. That is why an indispensible element of the complex of tools used for modeling technical and economic results of OFS implementation must be an appropriate system of situational models, with the help of which it is possible to track and predict the process of returns from the production consumed and services rendered, as well as the accompanying charges in the technical and economic indicators of the control object in a similar way to the one illustrated.. Such tracking makes it possible to get an idea of real possibilities of a specific OFS version within the time interval of a given period of $\mathrm{T} 0$ duration, within the range of which OFS application is planned. To make the situation estimates of the operating mode indicators of energy objects and their reliability, approved standard estimation software tools should be used, and subsequently situational estimate should be generalized for each of the analyzed versions. Meanwhile it must be taken into consideration that the structural model of OFS may be invariable within Tp, but situational nature of results of OFS application is still possible while there might be a change in macroeconomic conditions, technical state of the equipment in use and in other characteristics of the control object, which cause the change of its maintenance costs, of the size of payment components in (1) (3). Generally, it should be noted that the OFS proper, meant to be used during a rather lengthy $\mathrm{Tp}$, is more stable than the indicators estimating it and the organizational economic and technical factors that influence them. 


\begin{tabular}{|c|c|}
\hline $\begin{array}{l}\text { Conditions, } \\
\text { restrictions } \\
\text { and results }\end{array}$ & Contents \\
\hline $\begin{array}{l}\text { 1. Accountable } \\
\text { factors }\end{array}$ & $\begin{array}{l}\text { 1.1. The structure of power objects interaction among themselves and } \\
\text { with the external environment. } \\
\text { 1.2. The forms of ownership of the power complex objects } \\
\text { 1.3. Belonging to administrative and balance units. } \\
\text { 1.4. The level of centralization of control over interrelated power } \\
\text { objects' operating mode. } \\
\text { 1.5. The level of centralization of resources for MARW. } \\
\text { 1.6. The share of administrative expenses in the power object operating } \\
\text { cost. } \\
\text { 1.7. Taxation and other contributions to budgets and centralized funds. } \\
\text { 1.8. Product and service sales proceeds. } \\
\text { 1.9. Production cost value. }\end{array}$ \\
\hline $\begin{array}{l}\text { 2. Accounted } \\
\text { restrictions }\end{array}$ & $\begin{array}{l}\text { 2.1. Fuel cost. } \\
\text { 2.2. The cost of spares, equipment and other services. } \\
\text { 2.3. Power release price. } \\
\text { 2.4. The cost of services rendered by power enterprises (reciprocal } \\
\text { services and services rendered }\end{array}$ \\
\hline $\begin{array}{l}\text { 3. Possible } \\
\text { economic } \\
\text { results }\end{array}$ & $\begin{array}{l}\text { 3.1. Economically sound level of centralization of control over the } \\
\text { operation of power objects. } \\
\text { 3.2. Improvement of power supply reliability. } \\
\text { 3.3. Reduced operating time of power facilities. } \\
\text { 3.4. MARW resource shrinkage if they are ordered and stored centrally. } \\
\text { 3.5. Reduction of general expenses on supply of resources (spares, fuel, } \\
\text { reduced number of personnel of different categories). } \\
\text { 3.6. Increase of power equipment serviceability by means of more } \\
\text { effective use of technical diagnostic systems. } \\
\text { 3.7. Increased efficiency of settling with the consumers by means of } \\
\text { applying respective technical systems calculating energy consumption, } \\
\text { which are centrally controlled. } \\
\text { 3.8. Increased efficiency of power facility control by means of } \\
\text { optimization of MARW Schedule diagram }\end{array}$ \\
\hline
\end{tabular}

Table 1. Conditions of selection and possible results of realization of type of commercial interaction among energy complex.

\section{Conclusion}

In the process of preparing decision on adjusting the normative regulation of industrial and economic activities and managerial decision to the new conditions of energy system operation it is necessary to make a quantitative assessment of the technical and economic results of applying the organizational and functional managerial structures that control 
the power objects and of their commercial interaction. Restructuring of energy sector and creation of competitive environment for smooth operation of the market participant interactions should be done in stages starting with the total deregulation of primary energy sources. The various factors that can affect the efficiency and stability of power supply in developing countries according to [ Oricha, 2009], are government policy, economy factor, society/community factor, natural phenomena factor, efficient technology and skilled personnel. These factors mentioned above should be taken into consideration when preparing for restructuring of energy sector and privatization of public utilities.

\section{References}

John Surrey [1992] Energy policy in the European community The Energy Journal International Association for Energy economics, volume 16 Number 3

David M.N [1995] Power markets and market power, The Energy Journal International Association for Energy economics, volume 16 Number 3

Nedin I. V \& Oricha J.Y (1998), Management Crisis in Power Industry and Priority Ways to Overcome It. "Power Industry and Market" National Technical University of Ukraine Vol.3(4)- 4(8) 1998 - p26-30. ISSN - 40692.

Oricha J.Y. The Influence of Subjective Factors on the Ukrainian Energy Market Efficiency "Herald of the Ukrainian House of Economic and Scientific-Technical Knowledge", 1998. Volume 6 - p104-108.

Nedin I.V, Oricha J.Y, Sheverev V.E. Economic Assessment of Organizational and Functional Structure of Market Participants in Power Industry "Herald of the Ukrainian House of Economic and Scientific-Technical Knowledge-1999 -N5 -p.6471

Oricha.J.Y, Nedin .I.V,. Monitoring Process of Mutual Settlement- Conditions of Effective Commercial Interaction in Energy Production. file://Thesis speech scientific conference" Market and Logic in Management system" Lvov State University" Lvov Polytechnic 1998 p138.

Nedin I.V, Ryzhov V.v., Oricha J.Y, Chastockolenko I.P: Influence of Subjective Factors on Effectiveness of Energy Market Liberalization / Proceeding of the Russian National symposium on Power Engineering (RNSPE) Kazan, Russia, 10-14 Sept.2001, Volume 1l, p.209.

Fraser P,. Huist N. V (2003), Power generation investment in electricity markets. ISSN 75775

Oricha J.Y, Jimoh Boyi, Muhammed B. Mu'azu. Restructuring the Electrical Energy Sector and Analysis of Electricity Market Models in Nigeria. International Conference and Exhibition on Power Systems, University of Lagos July, 2007. ISBN: 978-37052 2-9

Daniel S.K \& Garan .S. (2004), Fundamentals of Power system Economics, ISBN- 0-470-84572-4.

Oricha J.Y, Tolu Akinbulire, Peter Ola, C.O.A. Awosope. Comparative Analysis of Crisis in Electricity Sector in Emerging Economy. Proceedings of The $1^{\text {st }}$ National Engineering Technology Conference (NETeC 2008) p 140- 144

The Nigerian Electricity Regulatory Commission (2008), Guide to the development of independent power plants 
Oricha J.Y. Analysis of Interrelated Factors Affecting Efficiency and Stability of Power Supply in Developing Countries. IEEE Africon conference 2009, Nairobi, Kenya. 


\title{
Methodology Development for a Comprehensive and Cost-Effective Energy Management in Public Administrations
}

\author{
Capobianchi Simona ${ }^{1}$, Andreassi Luca ${ }^{2}$, Introna Vito' ${ }^{2}$, \\ Martini Fabrizio ${ }^{1}$ and Ubertini Stefano ${ }^{3}$ \\ ${ }^{1}$ Green Energy Plus Srl \\ 2University of Rome "Tor Vergata" \\ "University of Naples "Parthenope"
}

Italy

\section{Introduction}

Energy saving represents one of the most relevant research areas because of the several environmental, economical and legislative motivations, especially in the public sector. In fact, the current international legislation aims to incentivize the activity of energy saving and the use of renewable energy sources in this area. The European Union, while recognizing public administration buildings as a large source for potential energy saving, also assigned to them the role of promoters of energy saving.

The SET PLAN constitutes a support to the 20/20/20 objectives and some European Directives clearly assign to the Public Administration (PA) the strategic role to promote energy efficiency in buildings (EU Directive 2006/32/CE) and to underline that the public buildings (occupied by the Public Administration and open to the public) have to be an example and a reference for the citizens in concrete activities in energetic certification and display campaigns (EU Directive 2002/91/CE). In this scenario an effective energy management procedure becomes unavoidable to reach the imposed targets. Energy management, in fact, is a well structured process that is both technical and managerial in nature. In (Kannan \& Boie, 2003) the authors provide a guideline for entrepreneurs in implementing energy management in an industrial field. Using techniques and principles from both fields, energy management monitors, records, investigates, analyzes, changes, and controls energy using systems within the organization. It should guarantee that these systems are supplied with the energy they need as efficiently as possible, at the time and in the form they need and at the lowest possible cost (Petrecca, 1992).

Accordingly, an important figure is the energy manager (a compulsory figure in organization featuring an energy consumption above certain limits, introduced in the Italian legislative system since 1991) (art. 19 law 10/91). Nevertheless, in the public sector he hardly succeeds in reaching important results because of the absence of powerful methodologies and analysis instruments. Energy management procedures in public sector have been illustrated in different work (Na Wei et al., 2009, Feng Yan-Ping et al., 2009, Zia \& Devadas, 2007), focusing on the concept of monitoring and metering consumptions. In the final report 
"Energy Performance Benchmarking of Ontario's Municipal Sector" of the Local Authority Services Ltd. (Association of Municipalities of Ontario) and in the report "Case Studies on Municipal Energy Initiatives" of the Commission of Environmental Cooperation, 2010 various attempts to create energy management system are described. In every case there is the absence of common guidelines and the tendency of proceeding with quick fixes, not integrated operations.

Currently about two-thirds of the global energetic consumptions are attributable to the urban areas, which also result as the major Green House Gas (GHG)-emitters with a critical environmental impact. In fact about the $50 \%$ of the global population lives in urban areas and they are responsible of the $60-80 \%$ of the global GHG-emissions (Dawson, 2007). For these reasons urban areas become important actors on the global decisions about energetic issues (see the C20 and C40 Cities Climate Leadership Group, Clinton initiative, ICLEI, Climate Alliance etc.) (Dawson, 2007).

The concept of Urban Carbon Management originates from these considerations and in many studies it is possible to find interesting energy-efficiency benchmarks developed as valuable tools for governments in managing energy consumption. Olazabal et al., 2008 developed the concept of urban ecosystem with particular attention to energy flows. Bennett \& Newborough, 2001, illustrate a model of energy auditing in urban areas highlighting the role of involved people, areas in which the conurbation is divided and required data. The main employed indicators are the Energy Flow Accounting (EFA), the Life Cycle Assessment (LCA) (Tjahjadi, 1999) and the Energy Footprint (EF) (Plan de uso sostenibile de la energia y prevenciòn del cambio climatico de la ciudad de Madrid, 2008). All these approaches are interesting for their aspects of generality and for their action in large systems but they take into account the whole city and not only the public administration subset.

Attempts to define energy benchmarks for single users, for example schools are carried out, taking into account specific technical and constructive characteristics of the buildings (Hernandez et al., 2008) or comparing different reference specific consumption (Filippìn, 2000). Similar consideration are made for public office buildings, creating a calculated dataset (Nikolaou et al., 2009), using the Energy Use Intensity (EUI) (Chung \& Hui, 2009) or applying the data envelopment analysis (Lee, 2008). These studies allow the definition of indicators of the energy performance of particular types of buildings. Nevertheless the proposed approaches are very specific and absolutely not general.

Other two important examples come from the Energy Star ${ }^{\circledR}$ and the Carbon Trust, two government organizations with the aim of incentivizing studies and methodologies for promoting energy efficiency and energy saving from households appliances to the building sector, through a labeling process (Energy Star $\left.{ }^{\circledR}\right)$ or the definition of benchmark and Good practice (Carbon Trust). In the presented method the indicators for the more detailed level (the efficiency ratios) are revised starting from the ones defined by Energy Star ${ }^{\circledR}$ (in the "ENERGY STAR ${ }^{\circledR}$ Performance Ratings - Technical Methodology", 2007) and Carbon Trust (Good Practice Guide 306, 2001).

In this chapter a comprehensive and innovative methodology for analyzing the energy performance of Public Administrations is illustrated. It takes into account an intermediate field: a local government consisting of different users (buildings and services as public lighting) with different peculiarities. At the same time this field doesn't comprehend all the productive sector of a city (agricultural, industrial, residential and service) as seen in the urban carbon management. The focus is on a specific sector (public administration), a subset of the city as a whole, but extremely heterogeneous. This approach has been developed in a 
general way in order to be applied to every kind of public organization, filling the gap with the industrial applications (Andreassi et al., 2009a, 2009b) and developing a specific methodology for PAs. Besides this method succeeds in obtaining results starting from a condition of a shortage of data. Differently from Chung et al, 2006 or Bohdanowicz \& Martinac, 2007, who define indexes with very detailed information, in this approach we establish our considerations starting from general data commonly available.

To structure this method into a model of analysis, a process consisting in four phases has been developed: the collection of data and information, the benchmark evaluation, the creation of consumption models, the definition of the measures of improvement of the users performance. In particular for realizing the benchmark evaluation phase, a system of composite indicators for mapping the energy performances in different and successive levels of detail is proposed. A case study will demonstrate the methodology reliability.

In conclusion this methodology can be applied to different types of municipalities and allows obtaining immediate and clear results about energy behavior, even more significant results can be when applied to public infrastructures (buildings and services) managed by small-medium municipalities, which usually feature great inefficiencies in the energy management and energy costs forming a consistent part of their budget.

This methodology has been applied and verified in an Italian contest but for its general approach can be adapted to the different European realities; in every case in fact there are approximately the same legislative ties, the same types of users with the same needs and issues. For these reasons the general guidelines of the methodology can be adapted to every specific case.

\section{The public administration}

Public administration can be defined as a group of users which supply services to the citizenry, as the public buildings (schools, offices, sport buildings, health buildings, etc..), the public lighting, the transport system and the industrial service infrastructures (the waste water and garbage treatment plants) regardless of whether they are paid directly by the Public Administration or by other service companies. In Italy the energy cost (VAT exclusive) is about 5\% of a Public Administration's balance. Figure 1 shows the energy costs

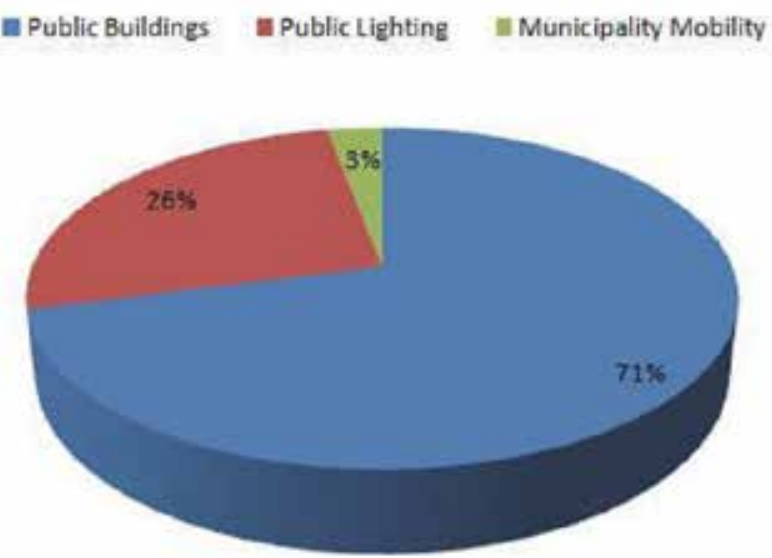

Fig. 1. Allocation of energy costs (example municipality with 200.000 inhabitants) 
distribution of an Italian municipality of about 200000 inhabitants (Picchioluto, 2006): the most relevant cost is attributable to the public buildings, while the public lighting becomes preponderant in smaller towns (constituting about the $60 \%$ of the total energy costs).

In this paper the different users are classified in the following groups (or typologies):

- public lighting;

- schools;

- $\quad$ city hall and other offices;

- $\quad$ sports and recreation buildings (gyms, swimming pools, etc..);

- $\quad$ small health care buildings.

Technical structures as waste water and garbage treatment plants are neglected as this study is addressed to small-medium size administrations in which these plants are not common. All these groups, of course, are extremely heterogeneous and are characterized by different consumption trends. Furthermore, also the correspondent users can present different energy use modalities.

To study a so-complex system, a division of the PA into the following three levels is proposed:

1. the administration on the whole;

2. the administration sectors (formed of the same type users);

3. the single users.

This structure allows rationalizing the study and investing the resources more efficiently and effectively.

There are some aspects that emerged during the realization of this study about the Italian situation of the municipalities which need to be underlined. First of all, the consideration given to the energy cost in general is very limited and it's difficult to find in the organization the responsible figure with the correct knowledge about these themes. This situation is very common in Italian Public Administration, due to a lack of knowledge and skills in the activity of energy management and consequently a gap with the advanced and restrictive European Energy policies is determined.

The installed power could be very large and comparable to the industrial organizations but the control about the invoiced consumptions isn't well developed.

Very often municipalities have to face numerous energetic bills, with different types of contracts and even contractors. The accounting system is often not rationalized (especially with the electrical consumption), with a measurement system which is developed over the years, without a rationalization. Databases of the historical consumptions and the structural changes happened in the different users are rarely available because of the insufficient sensitivity to the energy management.

Considering the energy cost of a municipality we have to underline that the payment falls on the citizenry which faces these operating costs with the local taxation system. A correct management of the energy resources has also positive effects directly on the citizenry which can understand the importance of the savings in a practical way. Last important aspect is the growing interest of the public opinion on the environmental issue that should incentivize the creation of an energy management system. The present methodology can succeed in facing them practically and support responsible in energy matter in these type of organizations.

\section{The methodology}

\subsection{The four phases of the analysis}

First of all, the present approach required the definition of some energy benchmarks to evaluate the energy performance of the various users and to identify the anomalies in the 
way of consuming. Then, this approach allows modeling the PA energy consumption in function of its major affecting factors (i. e. energy drivers), as population, temperature, daylight length etc. The resulting models can be used to resolve the previously identified anomalies and to predict the future trends of the energy usage.

Finally, there is the definition of the energy management activities to improve the users efficiency and to minimize energy consumption. To structure these activities into a model of analysis, a process consisting in four phases has been developed (see Figure 2):

1. data and information collection;

2. benchmark evaluation;

3. consumption models creation;

4. measures of users performance and improvement definition.

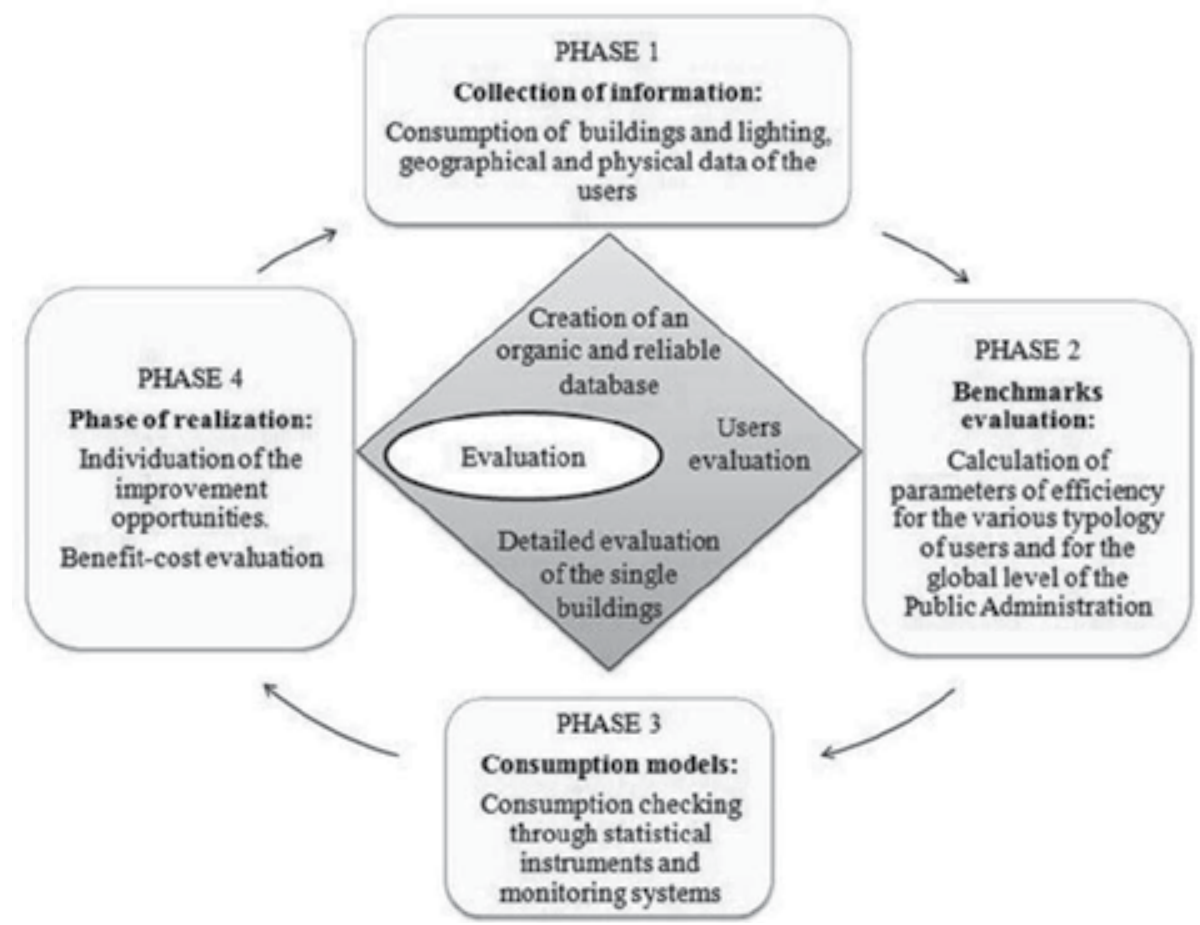

Fig. 2. The four phases of the model

This approach can constitute a guide, a standard procedure, for the energy management activities in a Public Administration supporting who try to rationalize the energy performance of a public organization. One of the most important advantages offered by the presented approach is the capability to obtain results also starting from a condition of poor information. The first step of the procedure is the collection of very general information of the municipality, as those reported in Table 1. A complete list of users information, comprehensive of the total annual energy consumption and the gross heating surface of each user has to be added to these general data.

These data allow assessing the energy performance of the municipality and the various sectors just identified. For a more detailed evaluation, forms to be compiled with all the necessary data for every typology of users have been elaborated, in order to characterize 
them and calculate the efficiency indexes. Table 2 reports only an example of these forms, used to describe the characteristics of the public lighting.

\begin{tabular}{|l|}
\hline General information \\
\hline Surface of the municipality $\left(\mathrm{km}^{2}\right)$ \\
\hline Altitude $(\mathrm{m})$ \\
\hline Road length $(\mathrm{m})$ \\
\hline Number of inhabitants \\
\hline Annual Degree Days \\
\hline Variation of dark hours \\
\hline Number of houses \\
\hline
\end{tabular}

Table 1. Collecting information: the general data

The data collected in the first phase are used for the evaluation of the various performance indicators; in this way a complete screening of the energy performance of a municipality can be obtained: the second phase includes the estimate through convenient spreadsheets of the benchmarks at every level of the organization and this gives the possibility to identify the more critical areas and to focus the attention on them.

\begin{tabular}{|l|}
\hline Public lighting \\
\hline Technical information \\
\hline Number of lamps \\
\hline Number of spot lights \\
\hline Surface of the municipality $\left(\mathrm{km}^{2}\right)$ \\
\hline Annual consumption (MWh) \\
\hline Lamps characteristics: typology, numbers, power (W) \\
\hline Incandescent \\
\hline Mercury-vapor \\
\hline High pressure Sodium-vapor \\
\hline Low pressure Sodium-vapor \\
\hline Fluorescent \\
\hline Led \\
\hline Economical characteristics \\
\hline Economic value of the lamps $(€)$ \\
\hline Investment in public lighting (from municipality's balance sheet) $(€)$ \\
\hline
\end{tabular}

Table 2. Collecting information: Public lighting

The great advantage of this approach is the immediate and easy form in which the evaluation results are obtained: a sort of display for all the municipality areas with the performance evaluation expressed in a symbolic way and a rapid consideration about the room for improvement (Figure 3). For the different levels an efficiency ratio or a user indicator is calculated (and represented with a symbolic color) and a "map" of energy performance is obtained.

The benchmark creation is explained in every methodological aspect in a proper paragraph (3.2): the benchmark is in fact the central tool in the analysis process and in this study innovative energy indicators are developed for this purpose. 
The two following steps of the process can be carried out starting from the more critical areas. In this phase it becomes necessary to design a monitoring system net, to obtain more detailed real time data and to define a flexible and effective control and management system.

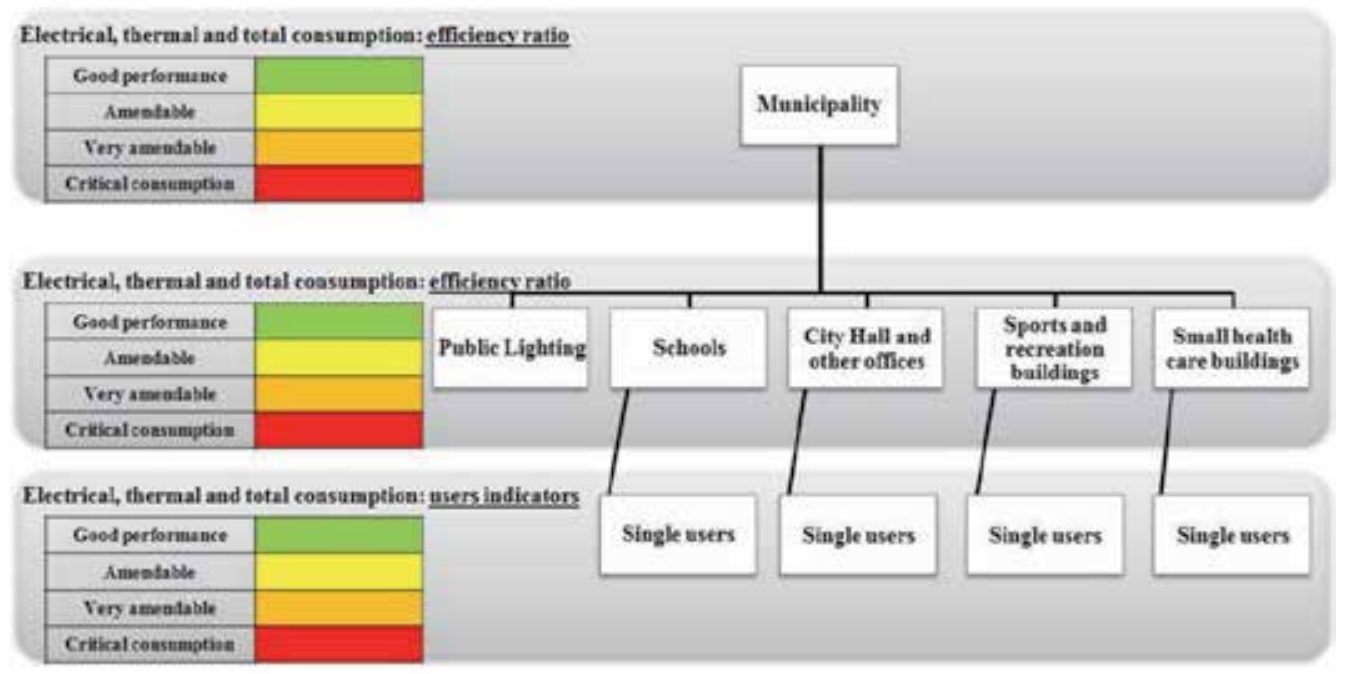

Fig. 3. The energy benchmark system

The availability of more detailed data, not only in an aggregate form as from the energy bills, allows to identify the inefficiencies and to monitor in real time the trends of the consumption. The measurements system integrated with software for the data elaboration creates daily, weekly, monthly and annual consumption profiles; the comparison between the recorded values and the historical profiles allows the individuation of anomalies or changes in the consumption in real time and for all the significant users. The availability of detailed data about the historical consumption also allows an evaluation of the chosen tariff and the choice of a possible alternative (even in this field the room of improvement are next to $10 \%)$.

Moreover, the data obtained by a monitoring system can be processed to model energy consumption in time series. The methodologies investigated in this study are essentially:

- regressions;

- neural networks;

- decisional trees.

Tso \& Yau, 2007 made an interesting comparison between these approaches suggesting that the regression analysis could be considered the most useful for predicting energy consumption. Generally the popularity of the regression models may be attributed to the interpretability of model parameters and easiness of use. A multiple regression analysis, in fact, can be realized with rapid calculation systems and gives an equation as the following one:

$$
Y=a_{0}+a_{1} \cdot x_{1}+a_{2} \cdot x_{2}+\ldots++a_{n} \cdot x_{n}
$$

where $a_{i}$ are the coefficients of the $x_{i}$ (explicatory variables or energy drivers). Such equation gives the possibility to attribute quotes of consumptions to the various variables and in this 
way it is possible to deeply understand the trend of consumption, the affecting variables and their relative importance. The strength of the forecasting models depends on the quality of the used data and on the type of the chosen energy drivers. In public buildings the most useful and suitable drivers are resulted to be the daily Heating and Cooling Degree Days (respectively $\mathrm{DD}_{\mathrm{H}, \mathrm{t}}$ and $\mathrm{DD}_{\mathrm{C}, \mathrm{t}}$ ) calculated as in (2) and (3) (where $\mathrm{T}_{\text {ref_DDc }}$ and $\mathrm{T}_{\text {ref_DDh }}$ are different for each type of user, as reported in Table 3 for the specific Italian case and $T_{\text {mean,t }}$ is the daily mean temperature for the day $t$ ) and dummies variables to represent the day of the week, the month of the year (Pardo et al., 2002, Mirasgedis et al., 2006).

$$
\begin{aligned}
& \mathrm{DD}_{\mathrm{C}, \mathrm{t}}=\max \left(\mathrm{T}_{\text {ref } \mathrm{DD}_{\mathrm{C}}}-\mathrm{T}_{\text {mean }, \mathrm{t}}, 0\right) \\
& \mathrm{DD}_{\mathrm{H}, \mathrm{t}}=\max \left(\mathrm{T}_{\text {mean }, \mathrm{t}}-\mathrm{T}_{\text {ref } \mathrm{DD}_{\mathrm{H}}}, 0\right)
\end{aligned}
$$

\begin{tabular}{|c|c|c|}
\hline Type of building & $\begin{array}{c}\mathbf{T}_{\text {ref }}\left({ }^{\circ} \mathrm{C}\right) \text { - Heating } \\
\text { (legislative reference) }\end{array}$ & $\begin{array}{c}\mathbf{T}_{\text {ref }}\left({ }^{\circ} \mathbf{C}\right) \text { - Cooling } \\
\text { (legislative reference) }\end{array}$ \\
\hline Schools & $\begin{array}{c}20 \pm 2 \text { (Municipal } \\
\text { regulations) }\end{array}$ & 26 (UNI 10339:1995) \\
\hline City hall and other offices & $\begin{array}{c}20 \pm 2 \text { (Municipal } \\
\text { regulations) }\end{array}$ & 26 (UNI 10339:1995) \\
\hline $\begin{array}{c}\text { Sports and recreation buildings } \\
\text { (gyms, swimming pools, etc..) }\end{array}$ & 18 (UNI 10339:1995) & 24 (UNI 10339:1995) \\
\hline Small health care buildings & $\begin{array}{c}20 \pm 2 \text { (Municipal } \\
\text { regulations) }\end{array}$ & 26 (UNI 10339:1995) \\
\hline
\end{tabular}

Table 3. $\mathrm{T}_{\text {ref }}$ and legislative reference for the different type of buildings

The models created and validated by a statistical point of view can be used to put under control the future consumptions, using the CUSUM charts and a system of alerts (Cesarotti et al., 2007).

The final step for the efficiency increase process is the determination of the so called Energy Management Opportunities; the accurate realization of all the previous activities generates the individuation of the anomalies in the way of consumption of all the users, starting from the most critical conditions.

Essentially there are three types of Energy opportunities:

- $\quad$ zero or low cost measures: definition of good practices in using energy;

- investment measures/refurbishment: operations for increasing the efficiency with substantial investment in the energy systems and in the buildings' envelopes;

- non conventional measures: energy certification.

It's clear that this last part of the methodology is the less automatable or standardizable because the choice of energy management or saving opportunities implies an active evaluation by the energy manager and consequently a project analysis which it may be very different depending on the circumstances.

Besides every consideration about the most adapt type of measure has to pair with a complete economic plan, with profit margin, payback and internal rate of return.

\subsection{Benchmark creation}

After collecting all the necessary information, the energy performance of the municipality through a benchmarking tool can be evaluated. 
As previously reminded, for this aim a more complex system of indexes to evaluate an heterogeneous field consisting of very different users is needed. Accordingly, the Energy Star ${ }^{\circledR \prime}$ 's approach has been revised to be adapted to the specific needs and a set of indexes addressed to the entire municipality and every constituting sector has been created.

For establishing the energy performance indexes system, a complete dataset has been firstly defined. Generally datasets may result from measurements or simulations. Nikolaou et al., 2009 developed an energy benchmarking dataset at a national level for the office sector through the modeling process of different sample buildings. Differently, in this study all the necessary information about the consumptions and the users' structural characteristics are extracted from a dataset created by measurements in the study "Audit GIS" realized by the Fondazione Cariplo and available in the web. This project consists of over 650 Energy Audits in municipalities located in the North-West of Italy. These data have been collected during a period of two years (2006-2008). The energy audits realized in these municipalities are available on web through a Web-GIS platform: it is possible to navigate the Web-GIS maps and choose the municipalities to examine. A successive menu shows the existent public structures and the reports with the audit results. In particular there are single report with the consumptions and the carbon emissions data, the structural and usage information and the realized or proposed energy saving measures of the single building and the possibility to consult aggregated data in Excel format. In Table 4 is reported a list example of data reported for each user.

\begin{tabular}{|c|c|}
\hline \multicolumn{2}{|c|}{ AUDIT RESULTS } \\
\hline Audit date & Audit typology (level of detail) \\
\hline \multicolumn{2}{|c|}{ Buildings characteristics } \\
\hline Type of construction & Transparent surface $\left(\mathrm{m}^{2}\right)$ \\
\hline Destination of use & Daily usage (hour) \\
\hline Year of construction & Weekly usage (day) \\
\hline Year of renovation & Yearly usage (hour) \\
\hline Renovation description & Number of occupant \\
\hline Thermal gross area $\left(\mathrm{m}^{2}\right)$ & Climatic Area \\
\hline Thermal gross volume $\left(\mathrm{m}^{3}\right)$ & Energy efficiency class \\
\hline Form factor $(\mathrm{S} / \mathrm{V})$ & \\
\hline & \\
\hline Type of plant & Type of fuel \\
\hline Power (W) & Annual consumption (kWh) \\
\hline Year of installation & Annual $\mathrm{CO}_{2}$ emission $\mathrm{kg}_{\mathrm{CO} 2 \mathrm{eq}}$ \\
\hline \multicolumn{2}{|c|}{ Electrical system } \\
\hline Annual consumption (kWh) & Annual $\mathrm{CO}_{2}$ emission $\mathrm{kg}_{\mathrm{CO} 2 \mathrm{eq}}$ \\
\hline \multicolumn{2}{|c|}{ Energy saving opportunities } \\
\hline Description & Annual saving $\left(\mathrm{CO}_{2}\right.$ emissions $)$ \\
\hline Annual saving $(€)$ & \\
\hline
\end{tabular}

Table 4. Audit result of the project Audit GIS: typical report

In this study, the consumption data and all the other necessary related information are extracted from this dataset even if, for sake of honesty, two weak spots have to be underlined: first of all, these data are elaborated by third and we haven't control about the 
presence of error of statistical unreliability; secondly, these municipalities are all concentrated in a relatively small geographical area and of small-medium size.

To increase the database reliability all the included information have been pre-processed, as in the Energy Star ${ }^{\circledR}$ 's procedure ("ENERGY STAR ${ }^{\circledR}$ Performance Ratings - Technical Methodology", 2007) for eliminating outliers, making more robust the statistical analysis and detrending the available datasets (Pardo et al., 2002). In particular, we locate the outliers of the distribution of consumption data through the elimination of all the values which were smaller or bigger than three times the standard deviation of the distribution. Then we elaborate the data through their natural logarithm, for making more robust the analysis and limiting the effects of heteroscedasticity.

After the pre-processing phase, a linear regression between the consumption data and the more significant energy drivers has been realized.

The first defined indexes are finalized to the energy rating of the entire municipality. The necessary data are the total annual electrical (sum of the electrical consumption of all the municipality's users, except the public lighting) and thermal consumption of the administration (sum of the thermal consumption of all the municipality's users): we calculate the mean value of energy consumptions over a period of three years. As energy drivers the sum of the thermal gross areas of the users (Sur, expressed in $\left.\mathrm{m}^{2}\right)$, the annual Heating Degree Days $(D D)$ and the population (Pop) are used. The obtained relationships are:

$$
\begin{gathered}
\ln (E)=6,72+0,78 \cdot \ln (\text { Sur })-0,52 \cdot \ln (\mathrm{DD})+0,28 \cdot \ln (\text { Pop }) \\
\mathrm{R}^{2}=0,817 \\
\ln (\mathrm{Q})=5,52+0,72 \cdot \ln (\text { Sur })-0,09 \cdot \ln (\mathrm{DD})+0,33 \cdot \ln (\text { Pop }) \\
\mathrm{R}^{2}=0,868
\end{gathered}
$$

where $E$ and $Q$ represent the annual electric and thermal energy consumption, respectively. Clearly there is an anomaly referring to the thermal consumption because the Degree Days coefficient is strangely negative. Furthermore, all the statistical tests ( $p$-value, Test F) confirm the unreliability of this variable. To justify this fact, we can consider that the switching on of the heating systems in the public buildings is automatically settled by law (D.P.R. 26 August 1993 n. 412), depending on the climatic areas. They are geographical areas designed by law on the basis of the annual Heating degree days recorded in a particular year and indicated by letters (A area has the warmest climate, $\mathrm{F}$ the coldest).

Therefore, a more correct procedure could be creating a different consumption model for each climatic area. In the data that we elaborate, all the municipalities belong to the climatic areas $\mathrm{E}$ and $\mathrm{F}$, so we create models only for these types of municipalities. The following equations have been obtained:

$$
\begin{gathered}
\ln (\mathrm{Q})=4,77+0,72 \cdot \ln (\text { Sur })+0,34 \cdot \ln (\text { Pop }) \quad \text { Climatic Area } E \\
\mathrm{R}^{2}=0,868 \\
\ln (\mathrm{Q})=5,27+0,79 \cdot \ln (\text { Sur })+0,2 \cdot \ln (\text { Pop }) \quad \text { Climatic Area } F \\
\mathrm{R}^{2}=0,724
\end{gathered}
$$


While the model created for the climatic area $\mathrm{E}$ is reliable, the one for the climatic area $\mathrm{F}$ needs more accurate information because the predicted consumptions are always lower than the one predicted by the other model and it is unacceptable because the Area F has a colder climate. Fig. 4 reports the predicted consumptions for both the climatic areas in ascending order: it's clear that the class F's consumptions are always lower. This problem is due to the availability of a little number of data belonging to the climatic area F. Our approach is however addressed to the creation of different models for every climatic area, obviously taking into proper account a correct number of data.

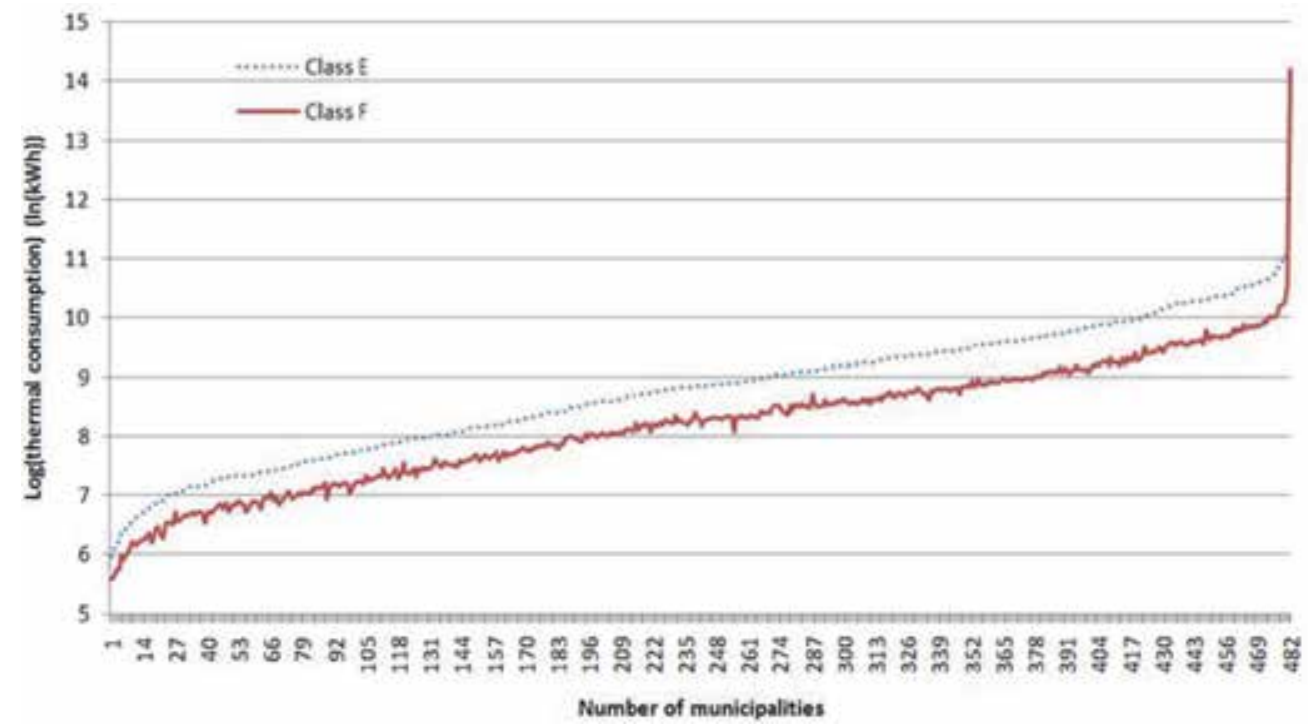

Fig. 4. Comparison between the model consumption of the climatic area $\mathrm{E}$ and $\mathrm{F}$

Then, the ratio between the real consumptions and the predicted ones is calculated. In this way the energy performance of every municipality can be classified through the Efficiency Ratio (ER) defined as follows:

$$
\mathrm{ER}=\frac{\ln \left(\mathrm{E}_{\mathrm{c}, \mathrm{r}}\right)}{\ln \left(\mathrm{E}_{\mathrm{c}, \mathrm{p}}\right)}
$$

where $E_{c, r}$ and $E_{c, p}$ represent the real and the predicted energy consumption, respectively. The greater is ER, the worst is the PA energy management.

The successive step is to fit the results of the efficiency ratio data in a cumulative percentage profile: the result for the total annual electrical consumption is reported in Figure 5. These curves allow determining the performance indexes or attributing the PA to a specific class of consumption. We could decide to assign a score to every municipality corresponding to the complement to 100 of the percentage value of the considered municipality, depending on the ER value. In this study four classes of consumption, identified by efficiency ratio thresholds corresponding to $0.25,0.50$ and 0.75 have been defined. Two positive results can be immediately achieved: on the one hand the attribution to a particular class of efficiency (i.e. labeled by a color) is an immediate result for the municipality and on the other hand this is a powerful approach to compare different municipalities and to assess future targets. 


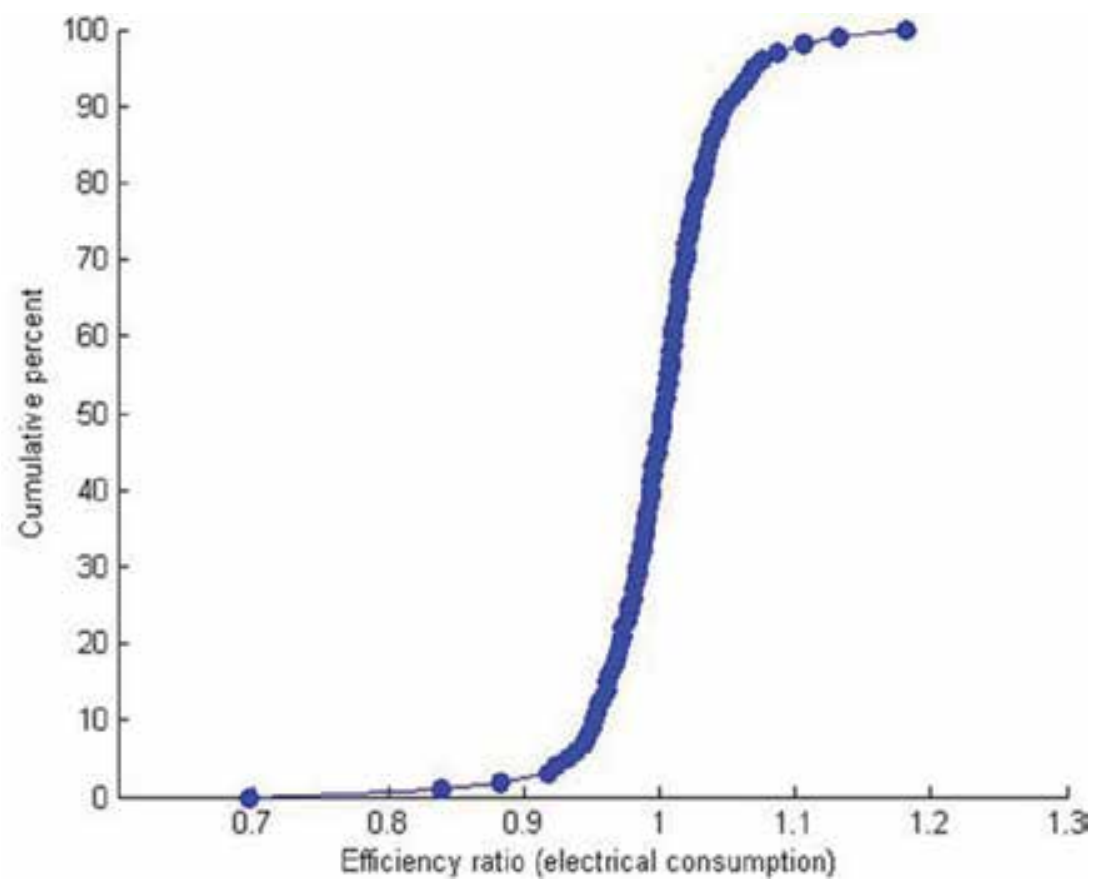

Fig. 5. Cumulative percentage curve for the efficiency ratio of the electrical consumptions

In Table 5 the ER boundary values of the four classes for both electrical and thermal consumption is reported. The final result is a complete and detailed overview about the energy consumption of the administration.

\begin{tabular}{|c|c|c|}
\hline Efficiency ratios-Electrical consumption & Lower limit & Upper limit \\
\hline Good performance & 0.6967 & 0.9778 \\
\hline Amendable & 0.9778 & 1.0023 \\
\hline Very amendable & 1.0023 & 1.0229 \\
\hline Critical consumption & 1.0229 & 1.1811 \\
\hline Efficiency ratios-Thermal consumption & Lower limit & Upper limit \\
\hline Good performance & 0.8445 & 0.9835 \\
\hline Amendable & 0.9835 & 0.9998 \\
\hline Very amendable & 0.9998 & 1.0153 \\
\hline Critical consumption & 1.0153 & 1.1257 \\
\hline
\end{tabular}

Table 5. Class of consumption for the municipality

Considering the other two levels, two different approaches have to be distinguished: a topdown or a bottom-up approach. A top-down approach is necessary when the PA has only aggregate data: it's an easy implementable method but it has a great inertia in modifying the benchmark results as a consequence of changes in the way of consuming. On the contrary a bottom-up approach requires detailed information about the user characteristics, which are often not available. Here the present method has been employed to develop indexes for the top-down approach and different benchmarks available in literature for the bottom-up approach are revised. 


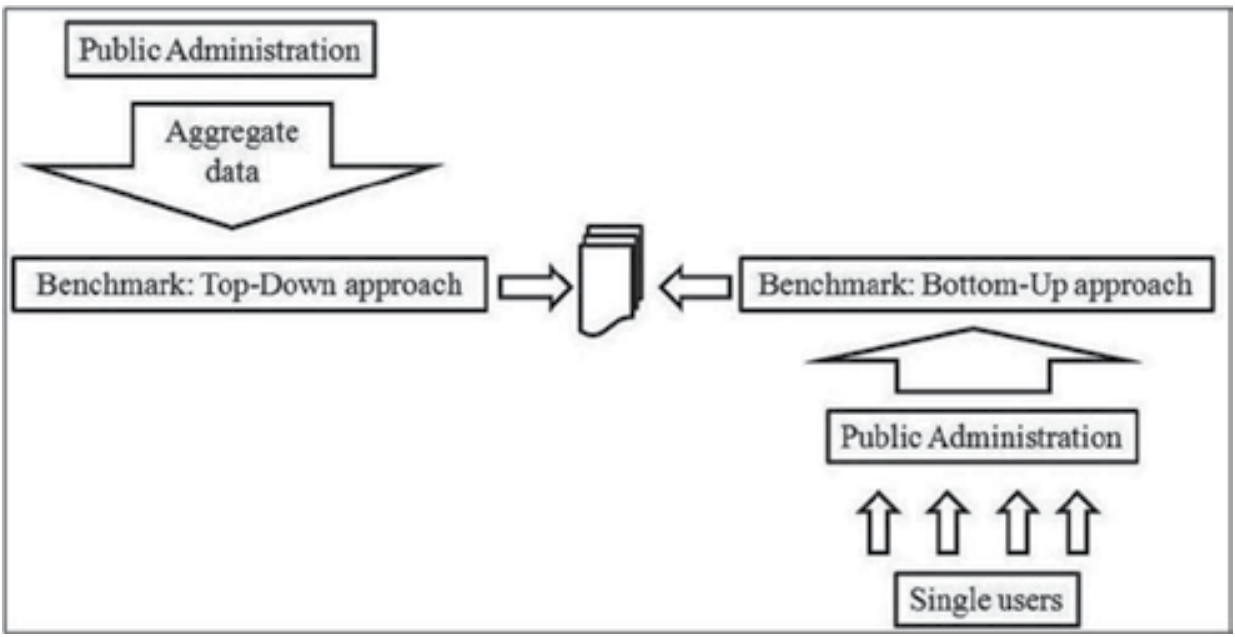

Fig. 6. Comparison between bottom-up and top-down approach

Using the same benchmark procedures for the whole administration, the regression equations for forecasting the electrical and thermal consumptions in each sector of the municipality can be calculated as indicated in paragraph 2 . The data used in these cases are the total annual electrical and thermal consumptions for each sector: the mean consumption value is calculated over three years. The considered energy drivers are the sum of heating gross surfaces of the users and the annual Heating Degree Days (general data which can be applied in every organization).

As in the previous case the regression equations and the four classes of consumption are determined.

Then, to validate the approach, a comparison between the results obtained by the efficiency ratios (which classify the performance of the entire sector) and what emerges from the evaluation of the single user indexes is performed. The result (in terms of class of energy performance of the whole sector) of the efficiency ratios should coincide with the mean result of the users indicators.

As indexes for the so-defined bottom-up approach for the single users we decide to revise and adapt to our specific aim some indicators found in literature.

In general these indexes use detailed information to normalize the energy consumption with respect to the climate conditions, the level and the type of usage, the structural characteristics of the buildings or of the plants.

We report an interesting example of this type of comparison, illustrating the case of "schools" but the same reasoning has been made for the other types of users.

The indexes for the schools, defined by the FIRE (Italian Federation for the Rational use of Energy), are the $\operatorname{IEN}_{\mathrm{E}}$ and the $\mathrm{IEN}_{\mathrm{R}}$ (respectively Energetic Normalized Index for electricity and thermal consumption).

These indexes are calculated for every school formulas as it follows:

$$
\operatorname{IEN}_{R}=\frac{C_{\text {Ther }} \cdot 1000 \cdot F_{h} \cdot F_{e}}{D D \cdot V} \quad \operatorname{IEN}_{E}=\frac{C_{E l} \cdot F_{h}}{S}
$$

where $\mathrm{C}_{\text {Ther }}$ is the annual heating consumption $(\mathrm{kWh}$ therm $), \mathrm{C}_{\mathrm{El}}$ is the annual electrical consumption $\left(\mathrm{kWh}_{\mathrm{el}}\right), \mathrm{F}_{\mathrm{h}}$ is a corrective factor concerning the hours of work, $\mathrm{F}_{\mathrm{e}}$ is a 
corrective factor concerning the characteristics of the building (form factor S/V), DD are the annual Heating Degree days, $V$ the heating gross volume $\left(\mathrm{m}^{3}\right), \mathrm{S}$ the heating gross surface $\left(\mathrm{m}^{2}\right)$. The FIRE provides three classes of consumption regarding these indexes.

For the validation process the IEN $\mathrm{E}_{\mathrm{E}}$ and the $\mathrm{IEN}_{\mathrm{R}}$ for a group of 48 schools homogeneously distributed have been calculated and a class of efficiency for every school has been assigned. This result has to coincide with one from the efficiency ratio (that is an efficiency but averaged on the total of schools). In Table 6 the results are reported.

As it's clear in the Table 6 the values concerning the thermal consumption show a great correspondence between the two different approaches, instead of the electrical indexes which give very different results in term of assessment of the performance. For understanding this problem we observe the distribution of our sample of data according to the IENE and the IEN $N_{R}$ and we note that our sample is concentrated in an inefficient evaluation in term of IEN $\mathrm{E}_{\text {. }}$

\begin{tabular}{|l|l|l|l|}
\hline Indice & & & \\
\hline IEN $_{R}$ & $47.92 \%$ & $29.17 \%$ & $22.92 \%$ \\
\hline $\mathrm{IEN}_{\mathrm{E}}$ & $12.50 \%$ & $22.92 \%$ & $64.58 \%$ \\
\hline
\end{tabular}

Table 6. Distribution in the IEN $\mathrm{E}_{\mathrm{E}}$ and $\mathrm{IEN}_{\mathrm{R}}$ classes

To realize a correct comparison we have to adapt our sample of data and re-define the limit values of the IEN ${ }_{\mathrm{E}}$ 'S classes: the IEN $\mathrm{E}_{\mathrm{E}}$ is in fact the result of a study of simulation of the energy performance of the schools instead our efficiency ratio gives a correct comparison between the performance of a particular set of data. The scaled limit values are obtained centering our dataset on the IEN $\mathrm{E}_{\mathrm{E}}$ values.

Finally the pie graphs in Figure 7 show the final repartition of the consumption (respectively thermal and electrical) of 7 schools existing in an example municipality; each school is represented in the pie graphs with the color correspondent of the efficiency class defined by the user's indicator. It's clear that the class with the major incidence in the total consumption is correspondent of the class defined by the efficiency ratio

The same considerations have been developed on the other users typologies, creating indexes allowing the sector's classification and analyzing the most powerful benchmark in literature for the classification of the single users. The results are reported in Table 7. A different approach has been used only for the public lighting where the distinction between sector and user indexes doesn't make practical sense. In this case the most powerful benchmarks come from an Italian research, making a technical and economic evaluation of the lighting system of the municipality.

\section{The case study}

This method has been applied to the case study of two small towns close to Rome in the region of Lazio, in Italy, called in this paragraph as municipalities A and B. These towns don't present any control in the energy management and for this reason the phase of the real time monitoring net couldn't be insert in this project.

The aim of this project has been the mapping process of the energy efficiency of the different sectors and end-users and the evaluation of the possible energy saving opportunities.

The phases of project, according the procedure previously described, have been:

1. the data collection; 


\section{Thermal Efficiency ratio $\quad \mathbf{1 . 0 1 0 7}$}

$\square$ Elementary school $\square$ Elementary school $\square$ Elementary school $\square$ Elementary school

$\square$ Elementaryschool $\square$ Nurseyschool $\square$ Secondaryschool

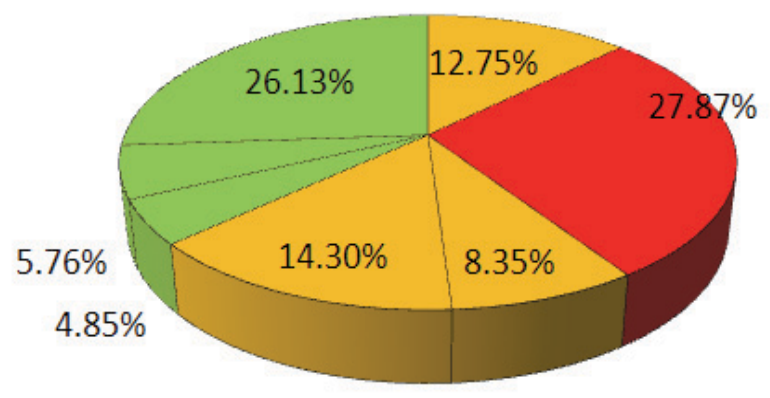

\section{\begin{tabular}{ll|} 
Electrical Efficiency ratio & $\mathbf{1 . 0 2 1 2}$
\end{tabular}}

$\square$ Elementary school $\square$ Elementary school $\square$ Elementary school $\square$ Elementary school

$\square$ Elementaryschool $\square$ Nurseyschool $\square$ Secondaryschool

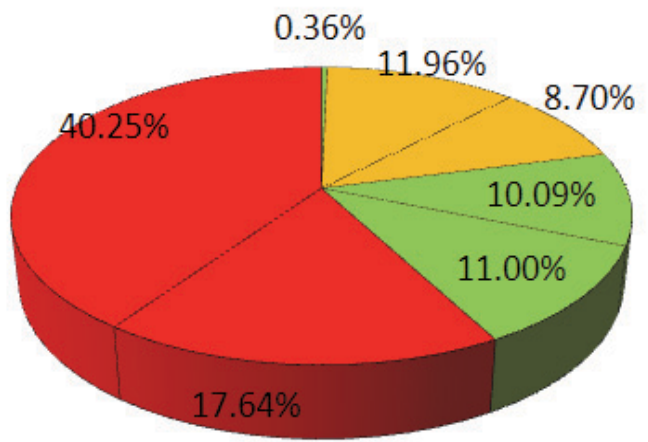

Fig. 7. Comparison between efficiency ratios and users indicators: sector of schools 


\begin{tabular}{|c|c|c|c|}
\hline \multirow{3}{*}{$\begin{array}{l}\text { Typologies of } \\
\text { users }\end{array}$} & \multicolumn{3}{|c|}{ Electrical consumption } \\
\hline & \multirow{2}{*}{ Sector index } & \multicolumn{2}{|c|}{ Single user index } \\
\hline & & Index & Ref. \\
\hline Schools & $\frac{\ln (\mathrm{E})}{11.13+0.98 \cdot \ln (\text { Sur })-1.035 \cdot \ln (\mathrm{DD})}$ & $\begin{array}{c}\mathrm{IEN}_{\mathrm{E}} \\
\left(\mathrm{kWh}_{\mathrm{e}} / \mathrm{m}^{2}\right)\end{array}$ & $(1)$ \\
\hline $\begin{array}{l}\text { City Hall and } \\
\text { offices }\end{array}$ & $\frac{\ln (\mathrm{E})}{14.7+0.94 \cdot \ln (\text { Sur })-1.37 \cdot \ln (\mathrm{DD})}$ & $\begin{array}{l}\text { El. benchmark } \\
\left(\mathrm{kWh} / \mathrm{m}^{2}\right)\end{array}$ & $(2)$ \\
\hline $\begin{array}{l}\text { Sports } \\
\text { buildings }\end{array}$ & $\frac{\ln (\mathrm{E})}{9.13+0.86 \cdot \ln (\text { Sur })-0.62 \cdot \ln (\mathrm{DD})}$ & $\begin{array}{l}\text { El. benchmark } \\
\left(\mathrm{kWh} / \mathrm{m}^{2}\right)\end{array}$ & (3) \\
\hline $\begin{array}{c}\text { Health } \\
\text { buildings }\end{array}$ & $\frac{\mathrm{E}}{426.58+55.10 \cdot \ln (\text { Sur })}$ & $\begin{array}{c}\text { El. benchmark } \\
\left(\mathrm{kWh} / \mathrm{m}^{3}\right)\end{array}$ & (4) \\
\hline Public lighting & $\begin{array}{r}\text { Luminous efficiency (lu } \\
\text { Municipality surface on annual cons } \\
\text { Number of lighting spots on annual } \\
\text { Mean economic value of the li } \\
\text { Investment on installed po }\end{array}$ & $\begin{array}{l}\left.\mathrm{km}^{2} / \mathrm{kWh}\right) \\
\text { on }\left(\mathrm{kWh}^{-1}\right) \\
(€)\end{array}$ & (5) \\
\hline
\end{tabular}

\begin{tabular}{|c|c|c|c|}
\hline \multirow{2}{*}{$\begin{array}{c}\text { Typologies of } \\
\text { users }\end{array}$} & Sector index & \multicolumn{2}{|c|}{ Thermal consumption } \\
\cline { 2 - 4 } & $\frac{\ln (\mathrm{Q})}{5.51+0.95 \cdot \ln (\text { Sur })}$ & \multicolumn{2}{|c|}{ Index } \\
\cline { 2 - 4 } Schools & $\frac{\ln (\mathrm{Q})}{6.5+0.79 \cdot \ln (\text { Sur })}$ & $\begin{array}{c}\mathrm{IEN}_{\mathrm{R}} \\
\left(\mathrm{kWh}_{\mathrm{t}} /\left(\mathrm{m}^{3} \times{ }^{\circ} \mathrm{C}\right)\right)\end{array}$ & $(1)$ \\
\hline $\begin{array}{c}\text { City Hall and } \\
\text { offices }\end{array}$ & $\begin{array}{c}\text { Ther. benchmark } \\
\left(\mathrm{kWh} / \mathrm{m}^{2}\right)\end{array}$ & $(2)$ \\
\hline $\begin{array}{c}\text { Sports } \\
\text { buildings }\end{array}$ & $\frac{\ln (\mathrm{Q})}{5.84+0.91 \cdot \ln (\text { Sur })}$ & $\begin{array}{c}\text { Ther. benchmark } \\
\left(\mathrm{kWh} / \mathrm{m}^{2}\right)\end{array}$ & $(3)$ \\
\hline $\begin{array}{c}\text { Health } \\
\text { buildings }\end{array}$ & $\mathrm{Q}$ & $\begin{array}{c}\text { Ther. benchmark } \\
\left(\mathrm{kWh} / \mathrm{m}^{3}\right)\end{array}$ & $(4)$ \\
\hline
\end{tabular}

(1) Guida per il contenimento della spesa energetica nelle scuole, ENEA; FIRE

(2) Good Practice Guide 286, 2000

(3) Energy Consumption Guide 78, 2001

(4) Murray et al., 2008

(5) Facciamo piena luce. Indagine nazionale sull' efficienza nell'illuminazione pubblica, 2006

Table 7. Sectors and users indicators for the municipalities

2. the benchmark evaluation (for both the sector and single users levels);

3. the individuation of anomalies and inefficiencies;

4. the definition of the measures of improvement of the users performance.

For the data collection the forms of the paragraph 3.1 have been used. The first information collected for the towns have been:

- general geographical and demographic information;

- the annual electrical and thermal consumptions of all the municipal structures (and their sum);

- $\quad$ the heating gross surface of all the municipal structures (and their sum).

Table 8 reports the general information of both municipalities and clearly highlights that they are small towns with a cold climate and a limited number of users. 


\begin{tabular}{|l|l|l|}
\hline General information & Municipality A & Municipality B \\
\hline Surface of the municipality $\left(\mathrm{km}^{2}\right)$ & 86.4 & 61.25 \\
\hline Altitude $(\mathrm{m})$ & 600 & 668 \\
\hline Number of inhabitants & 4787 & 9392 \\
\hline Number of houses & 2872 & 4506 \\
\hline Annual Degree Days & 2063 & 2331 \\
\hline Climatic area & D & E \\
\hline
\end{tabular}

Table 8. General information of the two municipalities

For the municipality A the individuated structures are:

- 2 schools: a nursery-elementary school and a middle school;

- 1 office: the city hall;

- 3 sports buildings: two football pitches and a tennis pitch;

- 3 leisure buildings: a library and two recreational centres.

For the municipality B the individuated structures are:

- 5 schools: a nursery school, a nursery-elementary school, a middle school, an elementary school and an high school;

- 1 office: the city hall;

- 1 health care building: a consulting room,;

- 4 sports buildings: two football pitches, a rugby pith and a tennis pitch;

- 2 leisure buildings: two recreational centres.

Obviously for both the municipalities the public lighting has been analyzed and evaluated.

From this first macroscopic analysis, it can be observed the total absence of renewable energy power plants. Energy is consumed as electrical energy, natural gas and LPG.

Basing the analysis of this initial data, some interesting elaboration can be obtained. The proportion between thermal and electrical consumption is reported in Fig. 8 where a preponderance of the electrical consumption for both the municipalities can be observed.

The comparison is possible using the conversion factors in TEP (Tons Equivalent of Petroleum)

$$
1 \mathrm{TEP}=11628 \mathrm{kWh} \mathrm{thermal}=5347,6 \mathrm{kWh} \text { electrical }
$$
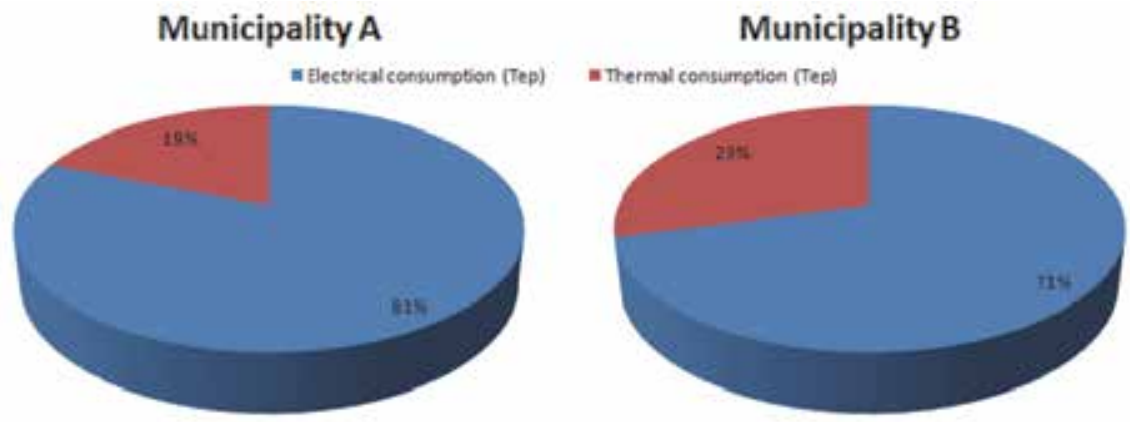

Fig. 8. Consumptions distribution

This is due to the great consumption of the public lighting that, as we previously remembered, usually constitutes a major cost for small municipalities. 
The aggregated data allow the evaluation of the energy benchmark of the whole municipality as reported in the Table 9 .

\begin{tabular}{|c|c|c|}
\cline { 2 - 3 } \multicolumn{1}{c|}{} & Municipality A & Municipality B \\
\hline Electrical ER & 0.9980 & 1.0146 \\
\hline Thermal ER & 0.9146 & 0.9594 \\
\hline Total ER & 0.9443 & 0.9747 \\
\hline
\end{tabular}

Good performance Amendable

Very amendable

Critical consumption

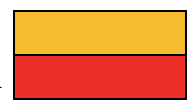

Table 9. Efficiency ratios of the whole municipalities

Considering the entire municipality, the $\mathrm{B}$ town $\left(\mathrm{ER}_{\mathrm{el}}=1.0146\right.$ and $\left.\mathrm{ER}_{\mathrm{th}}=0.9594\right)$ shows a worst performance compared to our sample of data in terms of electric energy and a better performance in terms of thermal energy, while the town $\mathrm{A}\left(\mathrm{ER}_{\mathrm{el}}=0.9980\right.$ and $\left.\mathrm{ER}_{\mathrm{th}}=0.9146\right)$ is more efficient. In fact, the $B$ results in a "very amendable" class and the $A$ in an "amendable" one for the electrical consumption and they are both in the "good performance" class for the thermal energy usage.

Than the consumptions of the single sectors of the municipalities have been examinated. The repartition of energy consumption per sectors for both municipalities has been evaluated, as reported in Figure 9: this analysis confirms the previous consideration. About $50-60 \%$ of the whole energy consumption is used for public lighting.

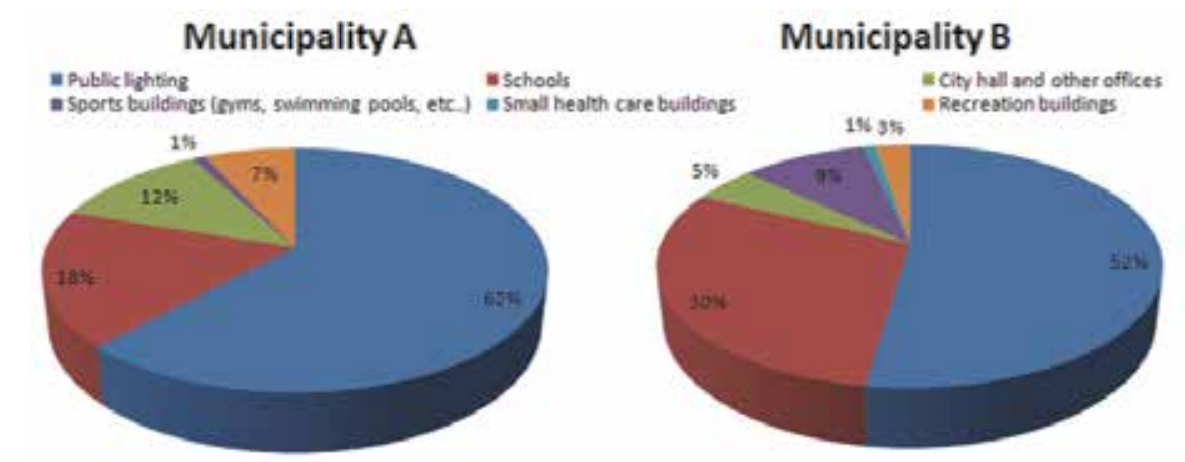

Fig. 9. Repartition of consumption per sector

For each sector the specific consumption (electrical and thermal) have been evaluated and the results are in Figure 10 and Figure 11.

From these graphs interesting considerations may be obtained but not absolute, because an high values not necessary coincide with an anomaly. In particular for the municipality A the most energy intensive sectors are the one of offices and leisure buildings. Differently for the municipality B the most energy intensive sector is constituted by sports buildings. Obviously these are preliminary considerations, for a general overview and characterization of the energy performance of the municipalities.

Successively the thermal and electrical ERs for each sector for both municipalities have been calculated using the general data collected in this phase. The results are reported in Table 10, where different colours have been employed to identify the energy classes. 


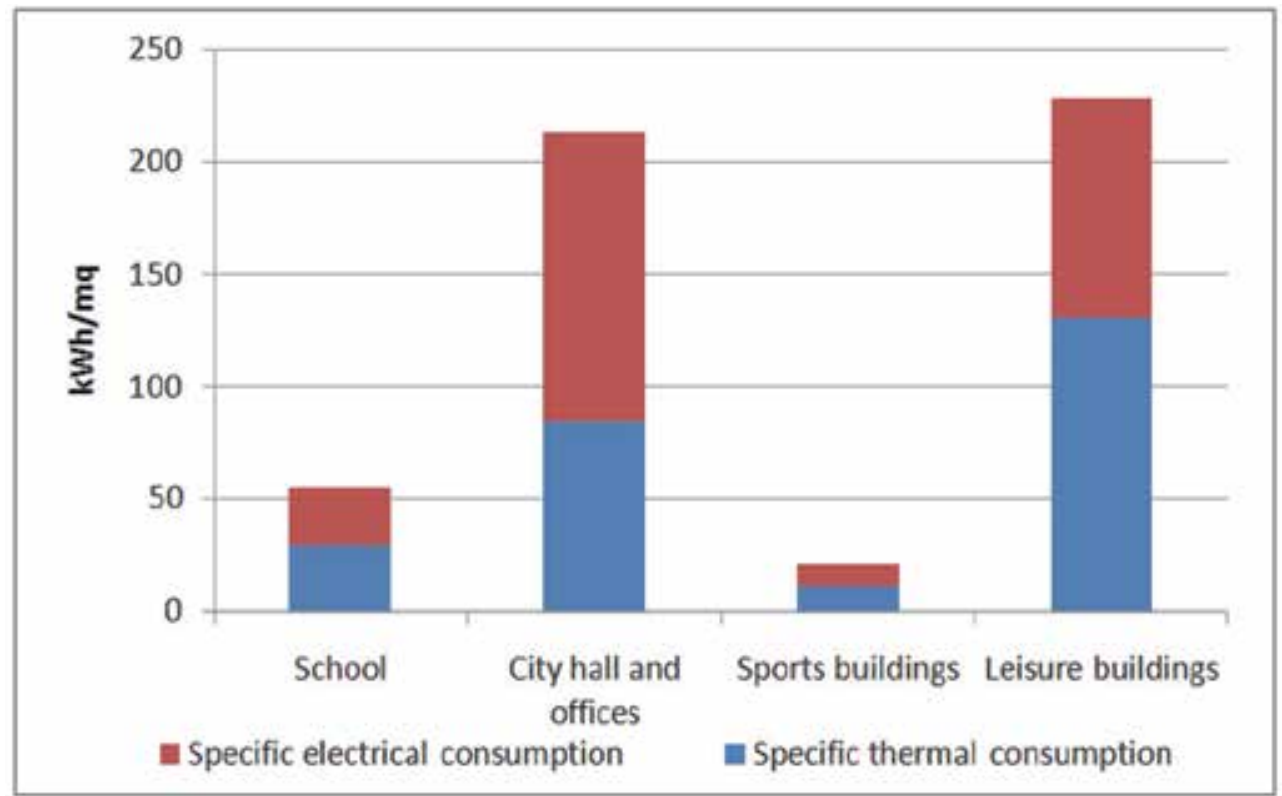

Fig. 10. Repartition specific consumption for the municipality A

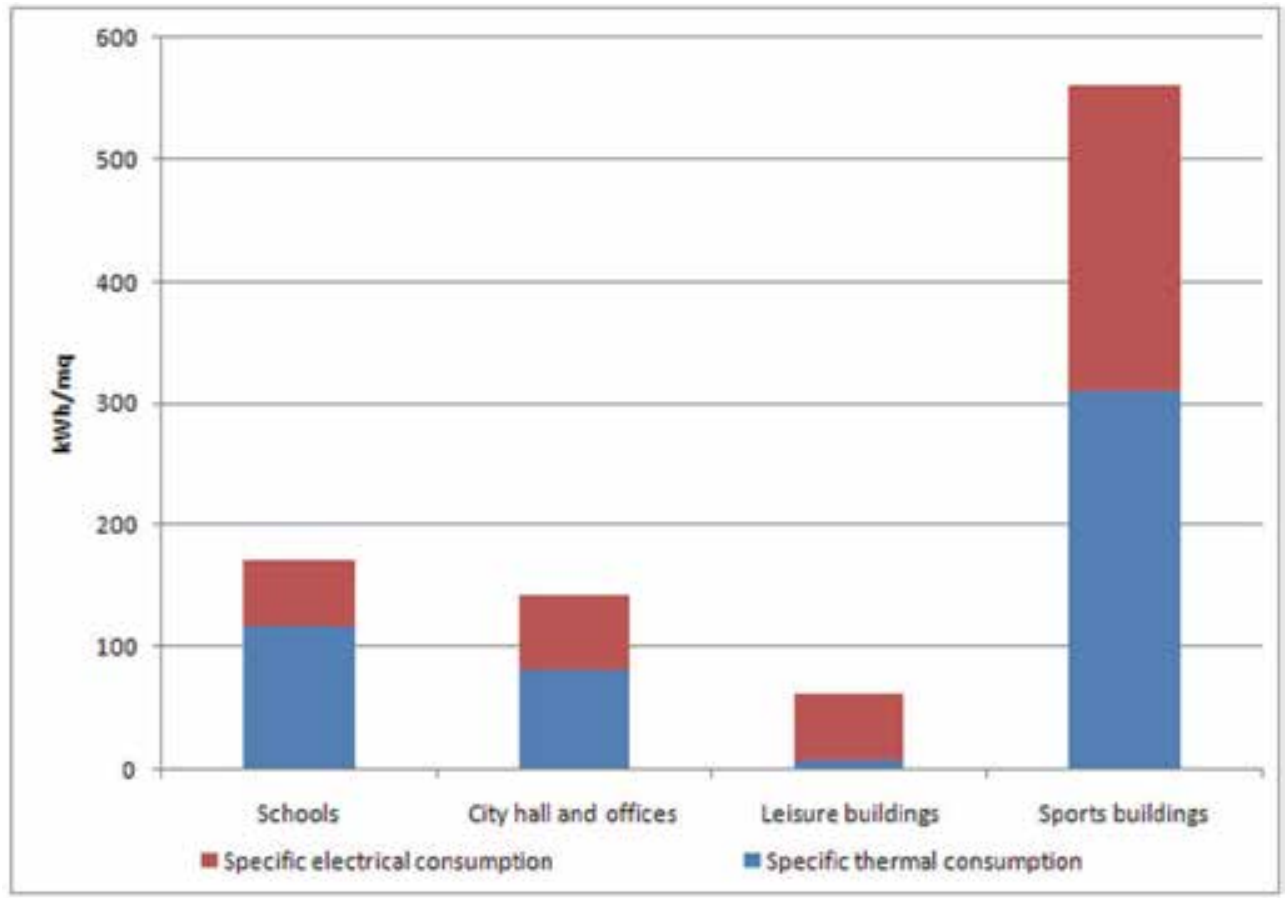

Fig. 11. Repartition specific consumption for the municipality B 
By this way, a map of the municipalities performance can be obtained and the more critical areas individuated: the city hall (electrical consumption) for the municipality A and the sports buildings (both thermal and electrical consumptions) and the schools (electrical consumption) for the municipality $\mathrm{B}$.

\begin{tabular}{|l|l|l|l|l|}
\hline Municipality A & $\begin{array}{l}\text { Electrical } \\
\text { ER }\end{array}$ & $\begin{array}{l}\text { Percentage } \\
\text { repartition of the } \\
\text { electrical } \\
\text { consumption }\end{array}$ & $\begin{array}{l}\text { Thermal } \\
\text { ER }\end{array}$ & $\begin{array}{l}\text { Percentage } \\
\text { repartition of the } \\
\text { thermal } \\
\text { consumption }\end{array}$ \\
\hline Schools & 0.9732 & $44 \%$ & 0.8934 & $51 \%$ \\
\hline City hall and other offices & 1.0218 & $38 \%$ & 0.9475 & $25 \%$ \\
\hline Sports buildings & 0.8516 & $3 \%$ & 0.7855 & $3 \%$ \\
\hline Leisure buildings & 0.9919 & $15 \%$ & 0.9715 & $21 \%$ \\
\hline Total & 0.998 & & 0.9146 & \multicolumn{2}{l}{} \\
\cline { 2 - 3 } & &
\end{tabular}

\begin{tabular}{|l|l|l|l|l|}
\hline Municipality B & $\begin{array}{l}\text { Electrical } \\
\text { ER }\end{array}$ & $\begin{array}{l}\text { Percentage } \\
\text { repartition of the } \\
\text { electrical } \\
\text { consumption }\end{array}$ & $\begin{array}{l}\text { Thermal } \\
\text { ER }\end{array}$ & $\begin{array}{l}\text { Percentage } \\
\text { repartition of the } \\
\text { thermal } \\
\text { consumption }\end{array}$ \\
\hline Schools & 1.0235 & $52 \%$ & 0.977 & $71 \%$ \\
\hline City hall and other offices & 0.9714 & $12 \%$ & 0.945 & $9 \%$ \\
\hline Sports buildings & 1.1404 & $24 \%$ & 1.0391 & $19 \%$ \\
\hline Leisure buildings & 0.9616 & $12 \%$ & 0.7484 & $1 \%$ \\
\hline Total & 1.0146 & & 0.9594 & \multicolumn{2}{l|}{} \\
\cline { 1 - 2 } & &
\end{tabular}

Good performance Amendable

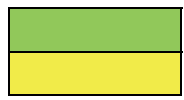

Very amendable

Critical consumption

Table 10. Efficiency ratios of the two municipalities

A similar evaluation has been made for the public lighting and the results are reported in Table 11: the global index, calculated as linear combination of the other indicators reported in the table, gives a good assessment on the municipality A's public lighting, but the second and third sub-indexes show the possibility to improve lighting's performance with a better distribution of lighting spots on the territory or the use of regulation of lighting intensity systems.

A little worst performance is attributed to the municipality B's plant by the global index; in particular in this case an improvement also of the lamps' efficiency is necessary. In general this sector isn't very critical even if we have to remember that it's the major cost for both the municipalities and for this reason a saving in this area will generate a more substantial improvement.

The first result of this analysis is the individuation of the more critical areas in which concentrate the more detailed evaluations; these are:

- City hall and other offices for the municipality A;

- Leisure buildings for the municipality A;

- $\quad$ Sports buildings for the municipality B;

- Schools for the municipality B;

- City hall and other offices for the municipality B. 


\begin{tabular}{|l|l|l|l|l|}
\hline Municipality A & $\begin{array}{l}\text { Calculated } \\
\text { value }\end{array}$ & $\begin{array}{l}\text { Minimal } \\
\text { value }\end{array}$ & $\begin{array}{l}\text { Benchmark } \\
\text { value }\end{array}$ & $\begin{array}{l}\text { Good } \\
\text { Practice }\end{array}$ \\
\hline 1) Luminous efficiency (lumen/W) & 129.91 & 55.3 & 86.065 & 116.83 \\
\hline $\begin{array}{l}\text { 2) Municipality surface on annual } \\
\text { consumption }\left(\mathrm{km}^{2} / \mathrm{kWh}\right)\end{array}$ & 0.00017 & 0.00018 & 0.00595 & 0.01173 \\
\hline $\begin{array}{l}\text { 3) No. of lighting spots on annual } \\
\text { consumption }\left(\mathrm{kWh}^{-1}\right)\end{array}$ & 1.352 & 0.353 & 1.469 & 2.585 \\
\hline Global index & 73.82 & 31.38 & 49.03 & 66.69 \\
\hline
\end{tabular}

\begin{tabular}{|l|l|l|l|l|}
\hline Municipality B & $\begin{array}{l}\text { Calculated } \\
\text { value }\end{array}$ & $\begin{array}{l}\text { Minimal } \\
\text { value }\end{array}$ & $\begin{array}{l}\text { Benchmark } \\
\text { value }\end{array}$ & $\begin{array}{l}\text { Good } \\
\text { Practice }\end{array}$ \\
\hline 1) Luminous efficiency (lumen/watt) & 101.49 & 55.3 & 86.065 & 116.83 \\
\hline $\begin{array}{l}\text { 2) Municipality surface on annual } \\
\text { consumption }\left(\mathrm{km}^{2} / \mathrm{kWh}\right)\end{array}$ & 0.000074 & 0.00018 & 0.00595 & 0.01173 \\
\hline $\begin{array}{l}\text { 3) Number of lighting spots on annual } \\
\text { consumption }\left(\mathrm{kWh}^{-1}\right)\end{array}$ & 1.3869 & 0.353 & 1.469 & 2.585 \\
\hline Global index & 57.75 & 31.38 & 49.03 & 66.69 \\
\hline
\end{tabular}

\section{Good performance Amendable \\ Very amendable \\ Critical consumption}

\section{Table 11. Performance indicators of the public lighting}

Through energy audits realized in the single structures and the use of the appropriate form for the data collections, the benchmarks of each user, in order to identify the possible inefficiencies in more detail have been calculated. Only the sector of leisure buildings for the municipality A has been neglected because of the total absence of detailed information about the structures which constitute it.

The results obtained from these indexes on the one hand confirm the validity of our efficiency ratios and on the other hand allow a precise localization of the problem.

\begin{tabular}{|l|l|l|}
\hline Normalized thermal consumption $\left(\mathrm{kWh}_{\text {term }} / \mathrm{m}^{2}\right)$ & Typical Value & Good Practice \\
\hline 146.54 & 151 & 79 \\
\hline Normalized electrical consumption $\left(\mathrm{kWh} / \mathrm{m} \mathrm{m}^{2}\right)$ & Typical Value & Good Practice \\
\hline 185.51 & 85 & 54 \\
\hline
\end{tabular}

\section{Good performance}

$$
\text { Amendable }
$$

\section{Critical consumption}

Table 12. Energy Indicators for offices in the municipality A

The values of the IEN $\mathrm{E}_{\mathrm{E}}$ and the $\mathrm{IEN}_{\mathrm{R}}$ for each school allow the individuation of the users where energy saving measures must be applied.

The application of the two first phases of this model and the use of the innovative sector energy benchmarks realize a complete mapping of the energy performances and a first assessment of the possible measures. 


\begin{tabular}{|l|l|l|}
\hline Normalized thermal consumption $\left(\mathrm{kWh}_{\mathrm{term}} / \mathrm{m}^{2}\right)$ & Typical Value & Good Practice \\
\hline 309.68 & 237 & 162 \\
\hline Normalized electrical consumption $\left(\mathrm{kWh}_{\mathrm{el}} / \mathrm{m}^{2}\right)$ & Typical Value & Good Practice \\
\hline 115.58 & 56 & 31.7 \\
\hline
\end{tabular}

\begin{tabular}{|c|c|c|}
\hline Good performance & Amendable & Critical consumption \\
\hline
\end{tabular}

Table 13. Energy Indicators for sports buildings in the municipality B

\begin{tabular}{|l|l|l|l|l|}
\cline { 2 - 5 } \multicolumn{1}{c|}{} & IEN & $\begin{array}{l}\text { Percentage repartition of } \\
\text { the electrical consumptions }\end{array}$ & IEN $_{\mathbf{R}}$ & $\begin{array}{l}\text { Percentage } \\
\text { repartition of } \\
\text { the thermal } \\
\text { consumptions }\end{array}$ \\
\hline Nursery school & 115 & $14.53 \%$ & 54.4 & $14.34 \%$ \\
\hline Nursery school-Pantano & 100 & $12.49 \%$ & 67.1 & $17.36 \%$ \\
\hline Primary school & 24.4 & $33.54 \%$ & 8.0 & $24.21 \%$ \\
\hline Primary school -Pantano & 15.9 & $9.05 \%$ & 11.0 & $13.67 \%$ \\
\hline Junior high school & 23.1 & $30.39 \%$ & 11.3 & $30.42 \%$ \\
\hline
\end{tabular}

Good performance

Amendable

Critical consumption

Table 14. Energy Indicators for schools in the municipality B

\begin{tabular}{|l|l|l|}
\hline Normalized thermal consumption $\left(\mathrm{kWh}_{\text {term }} / \mathrm{m}^{2}\right)$ & Typical Value & Good Practice \\
\hline 90.01 & 151 & 79 \\
\hline Normalized electrical consumption $\left(\mathrm{kWh}_{\mathrm{el}} / \mathrm{m}^{2}\right)$ & Typical Value & Good Practice \\
\hline 84.46 & 85 & 54 \\
\hline
\end{tabular}

\begin{tabular}{|l|l|l|l|l|}
\hline Good performance & & Amendable & & Critical consumption \\
\hline
\end{tabular}

Table 15. Energy Indicators for offices in the municipality B

It's clear that the successive actions will plan to create a more capillary system of measurements and the creation of the model of consumption for forecasting the trend and individuate changes.

The case study ended with the definition of the more convenient energy saving opportunities, basing this evaluation on the priority ranking previously obtained. Every proposed activity has also an economic plan which is a fundamental support in the decisional process for the realization of the energy saving opportunities.

For individuating the most adapt activity to reduce energy consumptions, a block diagram approach has been used.

In Figure 12 and Figure 13 the decisional process for the individuation of the energy saving opportunities is highlighted, for the more critical sectors with the highest efficiency ratio values (electrical consumption of offices and sports buildings for municipality A, thermal consumptions of sports buildings for municipality B). 
The variables which take part of the decisional process are both technical and economic; for example for thermal consumption after the evaluation of the correct dimension and of the efficiency of the boilers (technical consideration) the choice between the use of energy saving equipments (e.g. for the showers) or the installation of solar thermal collectors is necessarily based on the bankroll of the organization.

For this reason the municipality A decides for a more substantial investment and on the contrary the municipality B prefers a low impact energy saving opportunity.

From this reasoning approach the results are the following proposed activities:

1. installation of a solar photovoltaic plant on the city hall structure;

2. installation of energy and water saving equipments in the shower of the sports buildings and substitution of the boiler because oversized after the energy saving equipments installation.

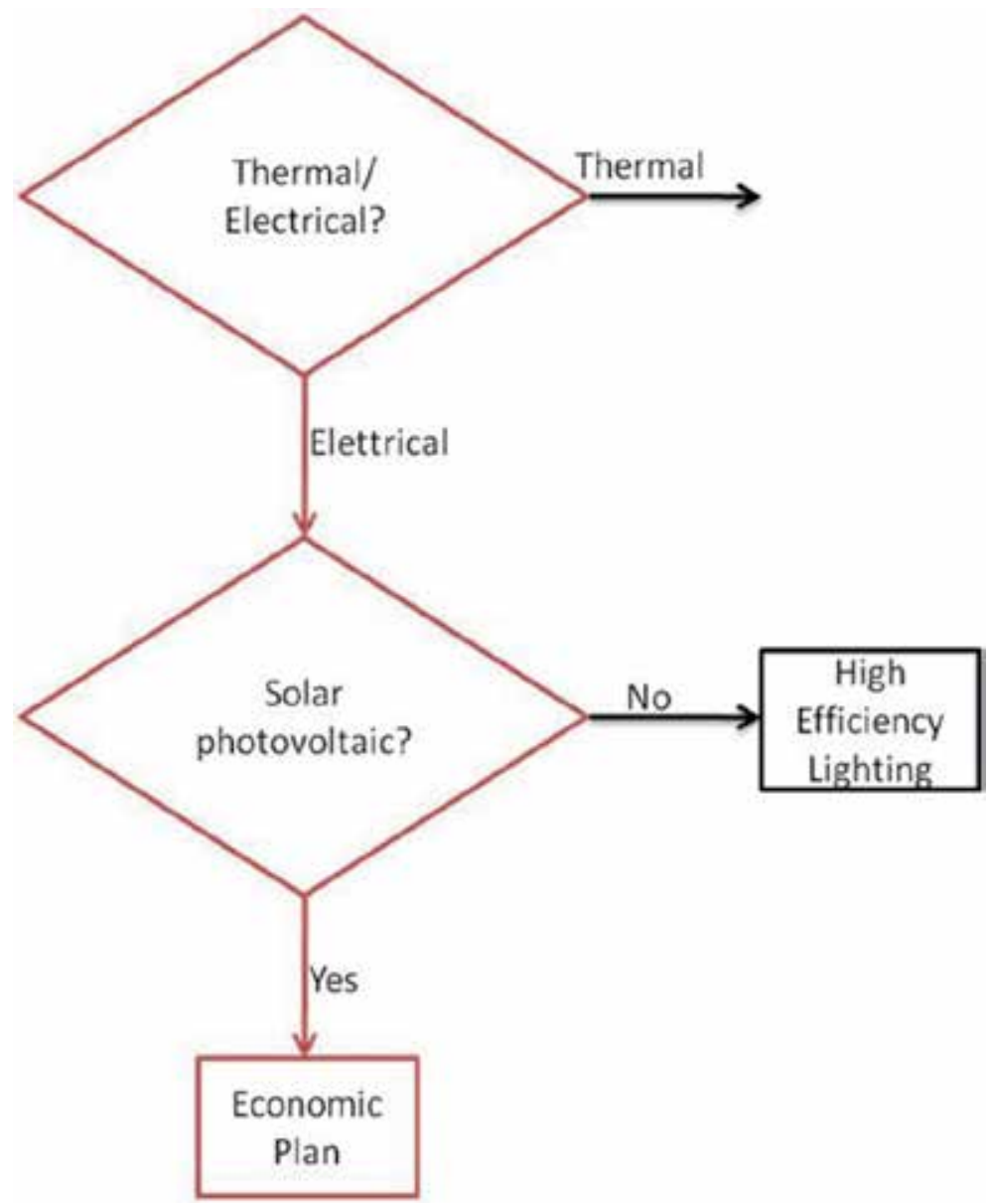

Fig. 12. Block diagram for electrical consumption of offices and sports buildings 


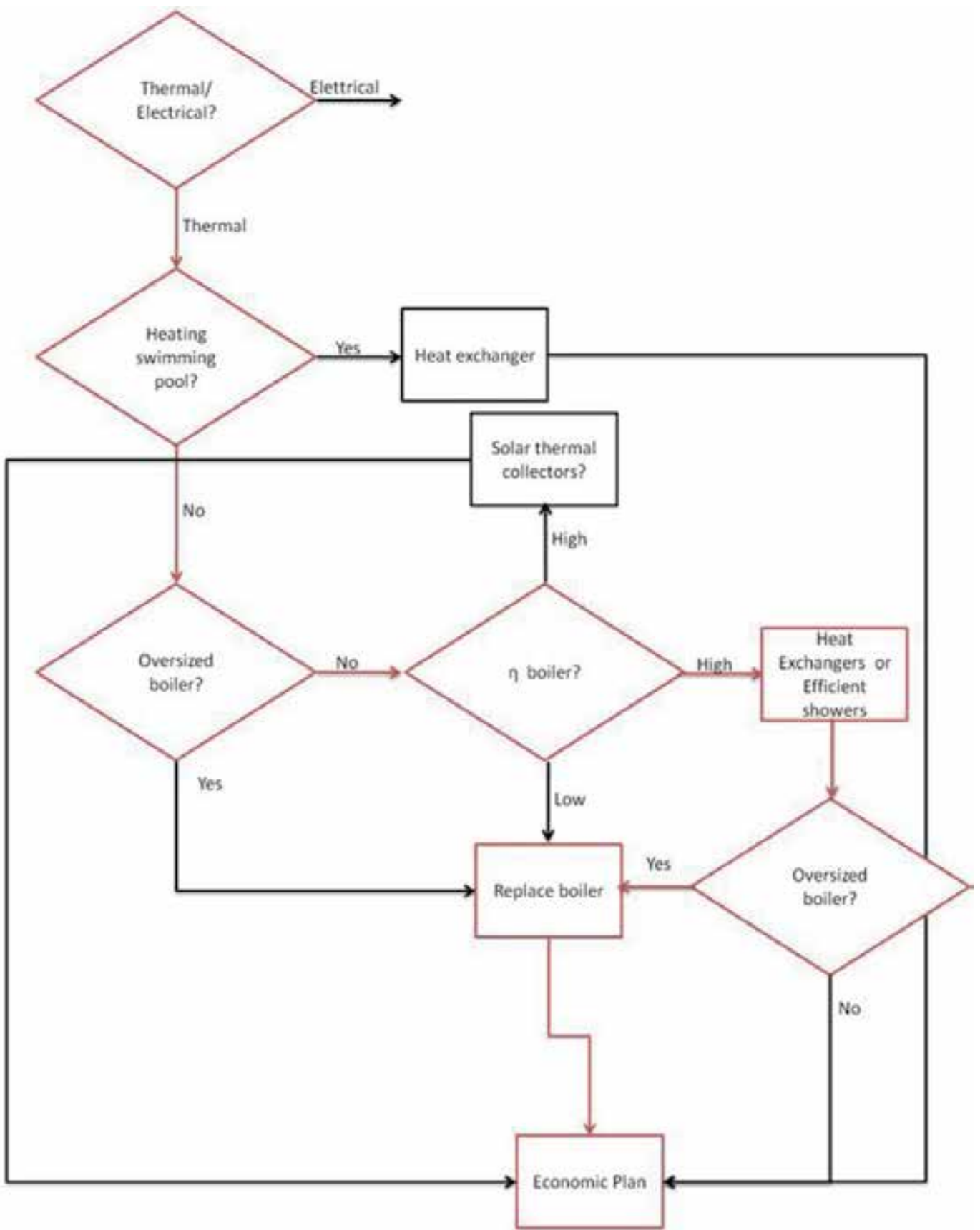

Fig. 13. Block diagram for thermal consumptions of sports buildings

The economic evaluations of these proposed activities are reported in the following figures and tables.

The photovoltaic plant has $5 \mathrm{~kW}$ of installed power and through an appropriate software (PVGIS, Potential Estimation Utility) an estimate of the annual producibility is obtained; 
then, the Pay Back period, the Net Present Value, and the Internal Rate of Return has been calculated. In Table 16 the data used for the calculation are reported

\begin{tabular}{|c|c|}
\hline \multirow{3}{*}{ Module characteristics } & $\begin{array}{c}\text { Conergy Power Plus } 230 \\
\text { Polycrystalline silicon } \\
\text { Efficiency: } 14.3 \%\end{array}$ \\
& Efficiency decrease: $1 \%$ per year \\
\hline Module dimension & Weight: $22 \mathrm{kilos}$ \\
& Surface: $1.63 \mathrm{~m}^{2}$ \\
Thickness: $46 \mathrm{~mm}$
\end{tabular}

Table 16. Data for photovoltaic evaluation

In particular in Figure 14 there is the representation of the PBP, which is of 8.7 years.

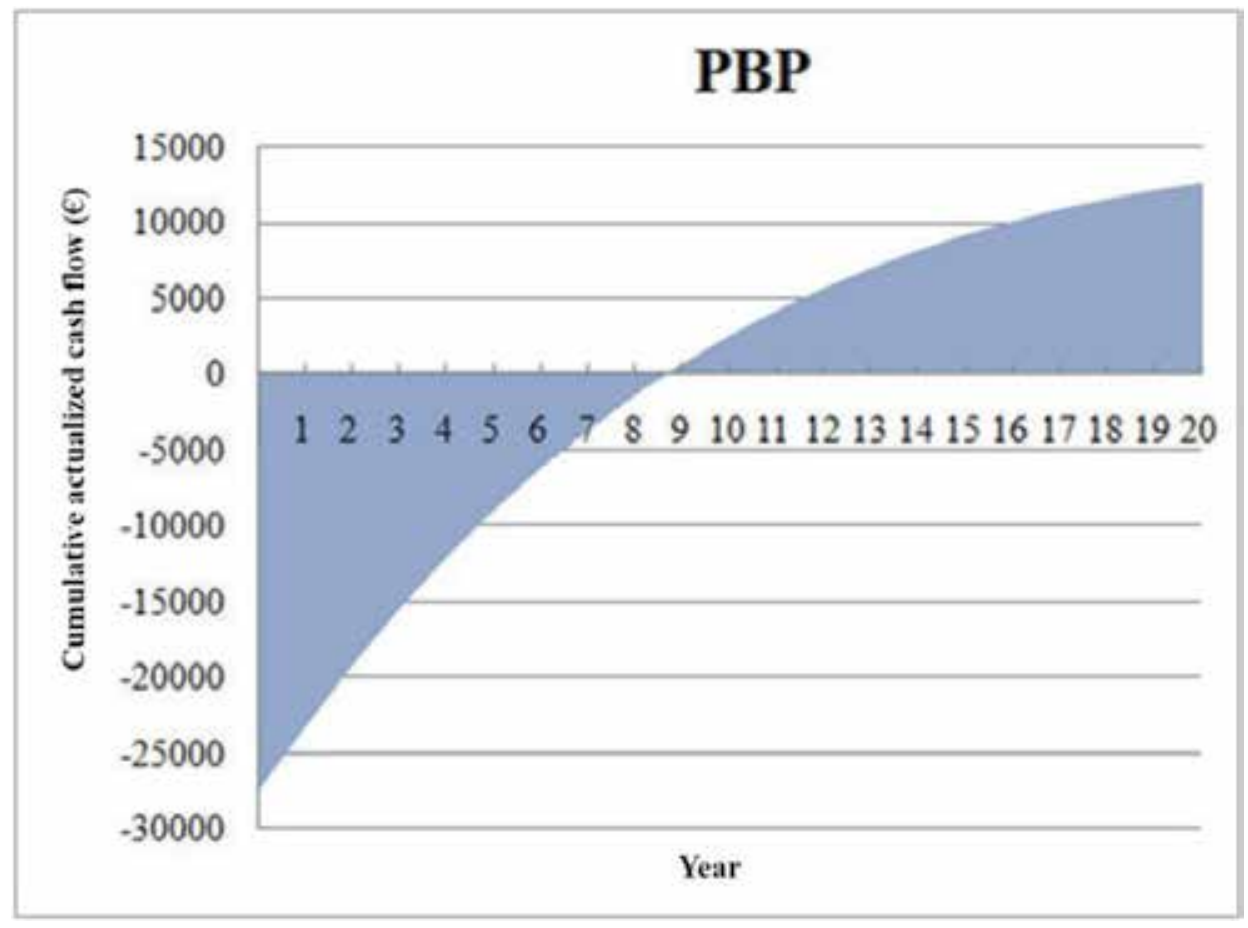

Fig. 14. Pay-Back Period for the photovoltaic plant of the municipality

Instead, for the thermal energy improvements of the sports buildings of the municipality B, the information about the investment are reported in the Table 17. The PBP in this case is lower than 1 year. 


\begin{tabular}{|l|l|}
\hline Cost installation of two condensing boilers - 32 kW( $€)$ & 4760 \\
\hline Fiscal deduction $(\%)$ & 55 \\
\hline Final cost of boilers $(€)$ & 2142 \\
\hline Cost of timer (shower) $(€)$ & 1501.5 \\
\hline Final cost of thermal improvement $(€)$ & 3643.5 \\
\hline Previous annual consumption (m³/ year) & 18674 \\
\hline Actual annual consumption $\left(\mathrm{m}^{3} /\right.$ year) & 8039.2 \\
\hline Annual saving in natural gas consumption $\left(\mathrm{m}^{3} /\right.$ year) & 10634.8 \\
\hline Specific cost of natural gas (€/m³) & 0.442283 \\
\hline Previous annual cost of natural gas (€/year) & 8259 \\
\hline Actual annual cost of natural gas $(€ /$ year) & 3556 \\
\hline Annual saving post improvement $(€ /$ year) & 4703 \\
\hline
\end{tabular}

Table 17. Investments and savings for the municipalities B: sports buildings

\section{Conclusion}

A method for the realization of the energy management activities in the Public Administrations (in particular in the local government organizations) has been developed. All the necessary indicators for mapping the energy performance of this kind of organizations are defined: the system of indicators is hierarchical and it is differentiated on the basis of the various levels of detail.

By this way, an assessment on the efficiency of the energy users can be obtained and all the anomalies can be identified.

These indicators are used in an integrated approach which starts from the data collection and terminates with the identification of the main energy management opportunities.

The strength of the approach is the capability to obtain benchmarking evaluation starting from a total absence of energy management approach, a very common situation in Italian municipalities.

The application of this method to a case study of two small Italian towns shows this potentiality to rapidly understand the energy performance of an administration, even if we are starting from a shortage of data.

Even if the designed approach in the case study isn't realized in each phase, the achieved results permit to delineate a map of energy performance of the municipalities, a benchmark evaluation in terms of efficiency classes and the determination of initial and general energy management opportunities for the more inefficient areas (electrical consumption of city hall, thermal consumptions of sports buildings and public lighting).

The successive actions will be addressed to the acquisition of the historical consumption data, the design of monitoring system nets, the determination of predictive models and the creation of an alarm system which keeps under the consumptions.

In conclusion, we want to underline that a possible improvement of the method could still be possible if a dataset on national scale would be available, in order to create the most reliable energy indicators. 


\section{References}

Kannan, R., Boie, W. (2003). Energy management practices in SME-case study of a bakery in Germany. Energy Conversion and Management, vol.44, pp. 945-959

Petrecca, G. (1992). Industrial Energy Management: principles and Applications, Springer, ISBN 0-7923-9305-8, NY

$\mathrm{Na}$ Wei, Wu Yong, Song Yan, Dong Zhongcheng, (2009). Government management and implementation of national real-time energy monitoring system for China largescale public building. Energy Policy, vol. 37, pp. 2087-2091

Feng Yan-ping, Wu Yong, Liu Chang-bin, (2009). Energy-efficiency supervision systems for energy management in large public buildings: Necessary choice for China. Energy Policy, vol. 37, pp.2060-2065

Zia, H., Devadas, V., (2007). Energy management in Lucknow city. Energy Policy, vol. 35, pp. 4847-4868

LAS (Local Authority Services), Energy Performance Benchmarking of Ontario's Municipal Sector, Available from http://www.amo.on.ca/AM/Template.cfm?Section=Final_ Report\&Template $=/ \mathrm{CM} /$ ContentDisplay.cfm\&ContentID $=157911$

Commission of Environmental Cooperation (2010), Case Studies on Municipal Energy Initiatives, Available from http://www.cec.org/municipalenergy/docs/QA0945Municipal-Case-Studies_en.pdf

Dawson ,R., (2007) Cities and climate change: The Tyndall Centre Cities Program, National Institute for Environmental Studies, Tsukuba, Japan, Available from http://www.gcp-urcm.org/files/A20071102/Dawson-TyndallCities-NIES.pdf

Olazabal, M., Garcia, G., Abajo, B., Alonso, A., Garcia, I, Herranz, K., Feliù, E., Izaola, B., Aspuru, I, Santa Coloma, O., (2008), Urban system metabolism analysis: an approach for the definition of urban strategic actions, ConAccount 2008, Urban metabolism: measuring the ecological city, Available from http://www.conaccount.cuni.cz/storage/1223556930_sb_urbansystem.pdf

Bennett, M., Newborough, M., (2001). Auditing energy use in cities. Energy Policy, vol. 29, pp. 125-134

Tjahjadi, B., Schafer, D., Radermacher, W., Hoh, H., (January 1999). Material and energy flow accounting in Germany-Data base for applying the National accounting matrix including environmental accounts concept. Structural Change and Economic Dynamics, vol. 10, pp. 73-97

Plan de uso sostenibile de la energia y prevenciòn del cambio climàtico de la ciudad de Madrid, (2008), approved by the government of the city of Madrid

Hernandez, P., Burke, K., Lewis, J. O., (2008). Development of energy performance benchmarks and building energy ratings for non-domestic buildings: An example for Irish primary schools. Energy and Buildings, vol. 40, pp. 249-254

Filippìn, C., (2000). Benchmarking the energy efficiency and greenhouse gases emissions of school buildings in central Argentina. Building and Environment, vol.35, pp. 407414

Nikolaou, T., Skias, I., Kolokotsa, D., Stavrakakis, G. (2009). Virtual Building Dataset for energy and indoor thermal comfort benchmarking of office buildings in Greece. Energy and Buildings, vol. 41, pp. 1409-1416 
Chung, W., Hui, Y.V., (2009). A study of energy efficiency of private office buildings in Hong Kong. Energy and Buildings, vol. 41, pp. 696-701

Lee, W. S., (2008). Benchmarking the energy efficiency of government buildings with data envelopment analysis. Energy and Buildings, vol. 40, pp. 891-895

Carbon trust, (2001), Good Practice Guide 306, Energy management priorities: a self assessment tool

Andreassi, L., Ciminelli, M.V., Feola, M. and Ubertini, S.,(2009). Innovative method for energy management: Modeling and optimal operation of energy systems. Energy $\mathcal{E}$ Buildings, vol. 41, Issue 4, pp. 436-444

Andreassi, L., Ciminelli, M.V., Di Silvio, B., Introna, V. and Ubertini, S., (2009), Systematic approach to Energy Saving: an integrated methodology for industrial plant. Proceedings of the Fourth International Exergy, Energy and Environment Symposium, April 19-23, 2009, AUS, Sharjah, UAE

Chung, W., Hui, Y.V., Miu Lam, Y., (2006). Benchmarking the energy efficiency of commercial buildings. Applied Energy, vol. 83 pp. 1-14

Bohdanowicz, P., Martinac, I., (2007). Determinants and benchmarking of resource consumption in hotels Case study of Hilton International and Scandic in Europe. Energy and Buildings, vol. 39, pp. 82-95

Picchioluto, S., (2006), Workshop Il nuovo ruolo del Responsabile dell'Energia secondo le indicazioni del D. Lgs. 192/2005, Il ruolo degli EE.LL. nell'utilizzo delle Energie Rinnovabili: dalla riduzione dei costi alla promozione del mercato, Available from http://www.fire-italia.it/convegni/pon/EM/picchiolutto.pdf

Tso, G. K. F., Yau, K. K. W., (2007). Predicting electricity energy consumption: A comparison of regression analysis, decision tree and neural networks. Energy, vol. 32, pp. 17611768

Pardo, A., Meneu, V., Valor, E., (2002). Temperature and seasonality influences on Spanish electricity load. Energy Economics, vol. 24, pp. 55-70

Mirasgedis, S., Sarafidis, Y., Georgopoulou, E., Lalas, D. P., Moschovits, M., Karagiannis, F., Papakonstantinou, D., (2006). Models for mid-term electricity demand forecasting in corporating weather influences. Energy, vol. 31, pp. 208-227

Cesarotti, V., Ciminelli, M. V., Di Silvio, B., Fedele, T., Introna, V., Energy budgeting and control for industrial plant through consumption analysis and monitoring. 7th IASTED International Conference on Power and Energy Systems (EuroPES 2007) Proceedings, pp. 389-394, Palma de Maiorca, 29 - 31 August 2007

AuditGIS Database, Available from http://www.webgis.fondazionecariplo.it/public/

Legambiente, (2006), Facciamo piena luce. Indagine nazionale sull'efficienza nell'illuminazione pubblica, Padova Fiere Spa, realized in collaboration with the Electric Engineering Department of the University of Padova, Available from http:/ / www.fire-italia.it/eell/illuminazione/facciamoPienaLuce.pdf

Carbon Trust, (2000), Good Practice Guide 286, Energy performance in the government's civil estate

Carbon Trust, (2001), Energy Consumption Guide 78, Energy use in sports and recreation buildings

Murray, J., Pahl, O., Burek, S., (2008). Evaluating the scope for energy-efficiency improvements in the public sector: Benchmarking NHSScotland's smaller health buildings. Energy Policy, vol. 36, pp. 1236-1242 



\section{Edited by Tauseef Aized}

The civilization of present age is predominantly dependent on energy resources and their utilization. Almost every human activity in today's life needs one or other form of energy. As world's energy resources are not unlimited, it is extremely important to use energy efficiently. Both energy related technological issues and policy and planning paradigms are highly needed to effectively exploit and utilize energy resources. This book covers topics, ranging from technology to policy, relevant to efficient energy utilization. Those academic and practitioners who have background knowledge of energy issues can take benefit from this book.

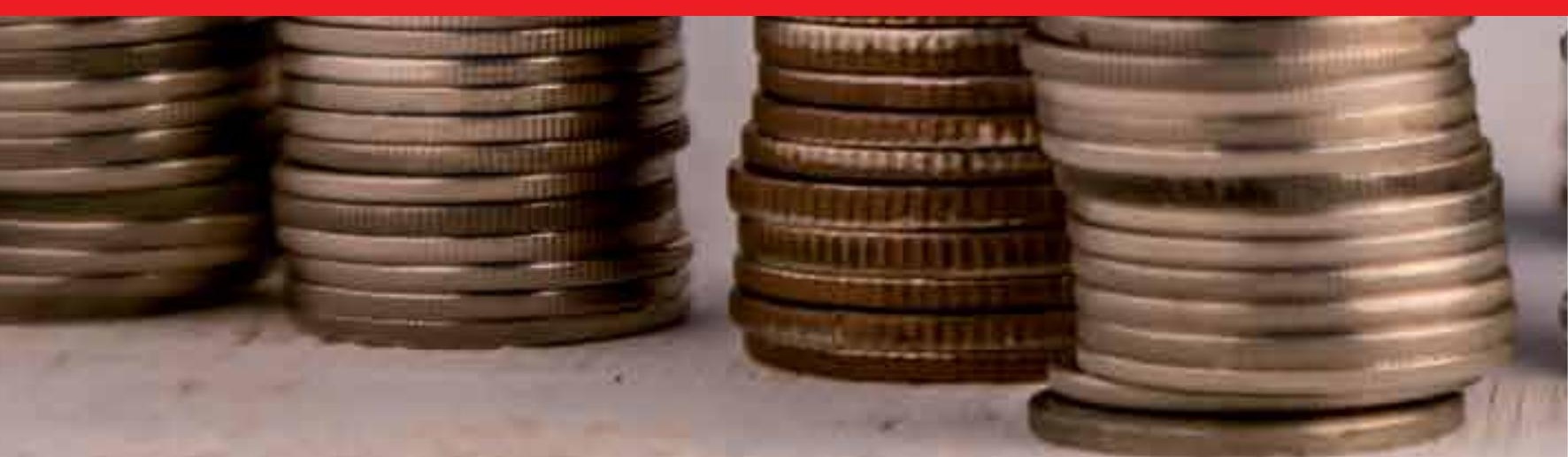

